PNL-7336

UC-903

5C

\title{
Geohydrology of the 218-W-5 Burial Ground, 200-West Area, Hanford Site
}

B. N. Bjornstad

May 1990

Prepared for the U.S. Department of Energy under Contract DE-AC06-76RLO 1830

Pacific Northwest Laboratory Operated for the U.S. Department of Energy by Battelle Memorial Institute 


\title{
DISCLAIMER
}

This report was prepared as an account of work sponsored by an agency of the United States Government. Neither the United States Government nor any agency thereof, nor Battelle Memorial Institute, nor any of their employees, makes any warranty, expressed or implied, or assumes any legal liability or responsibilityfor the accuracy, completeness, or usefulness of any information, apparatus, product, or process disclosed, or represents that its use would not infringe privately owned rights. Reference herein to any specific commercial product, process, or service by trade name, trademark, manufacturer, or otherwise, does not necessarily constitute or imply its endorsement, recommendation, or favoring by the United States Government of any agency thereof, or BattelleMemorial Institute. The views and opinions of authors expressed herein do not necessarily state or reflect those of the United States Government or any agency thereof.

\section{PACIFIC NORTHWEST LABORATORY operated by \\ BATTELLEMEMORIAL INSTITUTE for the \\ UNITED STATES DEPARTMENT OF ENERGY under Contract DE-AC06-76RLO 1830}

\author{
Printed in the United States of America
}

Available to DOE and DOE contractors from the

Office of Scientific and Technical Information, P.O. Box 62, Oak Ridge, TN 37831; prices available from (615) 576-8401. FTS 626-8401.

Available to the public from the National Technical Information Service,

U.S. Department of Commerce, 5285 Port Royal Rd., Springfield, VA 22161.

NTIS Price Codes, Microfiche A01

Printed Copy

\begin{tabular}{lrrrr}
\hline Price Code & Page Range & & Price Code & Page Range \\
\cline { 5 - 5 } A02 & $1-10$ & & A15 & $326-350$ \\
A03 & $11-50$ & A16 & $351-375$ \\
A04 & $\mathbf{5 1 - 7 5}$ & A17 & $376-400$ \\
A05 & $\mathbf{7 6 - 1 0 0}$ & A18 & 401425 \\
A06 & $101-125$ & A19 & $426-450$ \\
A07 & $\mathbf{1 2 6 - 1 5 0}$ & A20 & 451475 \\
A08 & $\mathbf{1 5 1 - 1 7 5}$ & A21 & $\mathbf{4 7 6 - 5 0 0}$ \\
A09 & $\mathbf{1 7 6 - 2 0 0}$ & A22 & $501-525$ \\
A10 & $\mathbf{2 0 1 - 2 2 5}$ & A23 & $526-550$ \\
A11 & $\mathbf{2 2 6 - 2 5 0}$ & A24 & $551-575$ \\
A12 & $\mathbf{2 5 1 - 2 7 5}$ & A25 & $576-600$ \\
A13 & $\mathbf{2 7 6 - 3 0 0}$ & A99 & $601-U p$ \\
A14 & $\mathbf{3 0 1 - 3 2 5}$ & &
\end{tabular}


PNL-7336

UC-903

33679000465775

GEOHYDROLOGY OF THE 218-W-5 BURIAL GROUND, 200-WEST AREA, HANFORD SITE

B. N. Bjornstad

May 1990

Prepared for

the U.S. Department of Energy

under Contract DE-AC06-76RLO 1830

Pacific Northwest Laboratory

Richland, Washington 99352 
to $30 \%$ for the Hanford formation. A lot of data are available for the transmissivity and hydraulic conductivity of the middle Ringold unit, based on aquifer testing. There is very little information, however, on the hydrologic properties of other units, such as the early "Palouse" soil, the Plio-Pleistocene unit, and the upper Ringold unit, which together make up the central third of the stratigraphic sequence present in the unsaturatedzone at W-5. 


\section{CONTENTS}

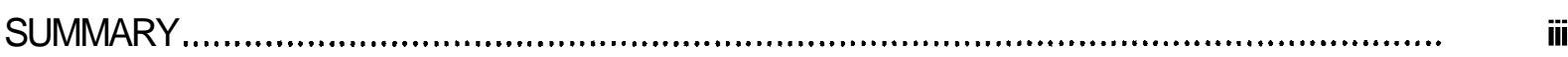

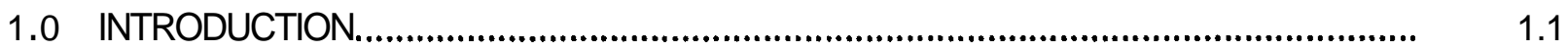

2.0 INVESTIGATIVE METHODS..................................................................................

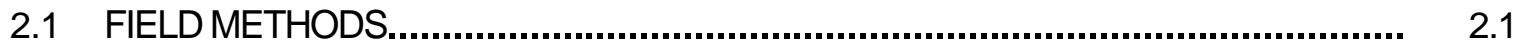

2.1.1 Sample Collection..................................................................... 2.6

2.1.2 Geologic Logging...................................................................... 2.6

2.1.3 Borehole Geophysical Logging .......................................................... 2.7

2.1.4 Aquifer Testing.......................................................................................... 2.7

2.1.5 Water-Level Measurements............................................................. 2.15

2.2 LABORATORY METHODS ...................................................................... 2.15

2.2.1 Particle-Size Distribution .......................................................................... 2.15

2.2.2 Calcium Carbonate Content................................................................... 2.17

2.2.3 Field Moisture Content......................................................................... 2.17

2.2.4 Permeameter ……......................................................................... 2.17

2.2.5 Water Retention ......................................................................... 2.18

2.2.6 Cation Exchange Capacity .................................................................. 2.20

2.2.7 Mineralogical Analysis ........................................................................ 2.20

2.2.8 Bulk Geochemistry....................................................................... 2.21

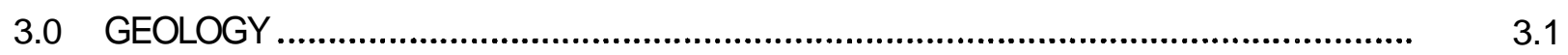

3.1 STRATIGRAPHY AND LITHOLOGY ............................................................

3.1.1 Saddle Mountains Basalt......................................................................

3.1.2 Ringold Formation ................................................................................... 3.2

3.1.3 Plio-Pleistocene Unit ..............................................................................

3.1.4 Early "Palouse" Soil ............................................................................ 3.11

3.1.5 Hanford Formation............................................................................. 3.12

3.2 MINERALOGY AND BULK GEOCHEMISTRY................................................

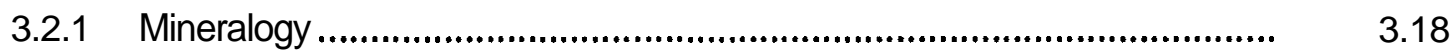


3.2.2 Bulk Geochemistry........................................................... $\quad 3.27$

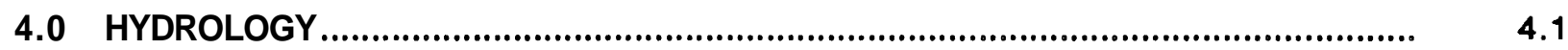

4.1 REGIONAL HYDROLOGY ................................................................. 4.2

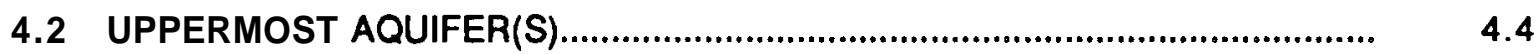

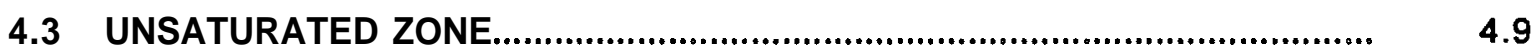

4.4 HYDROLOGIC PROPERTIES.................................................................. 4.10

4.4.1 Hydraulic Conductivity.................................................................. $\quad 4.10$

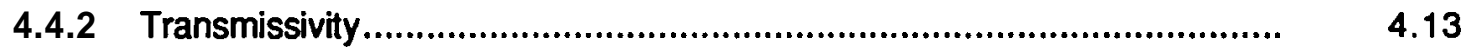

4.4.3 Storativity............................................................................. $\quad 4.13$

4.4.4 Porosity ........................................................................... 4.13

4.4.5 Field Moisture Content................................................................... $\quad 4.14$

4.4.6 Soil Bulk Density.................................................................................. 4.14

4.4.7 Water-Retention Characteristics.................................................... 4.14

4.4.8 Cation Exchange Capacity ...................................................... 4.17

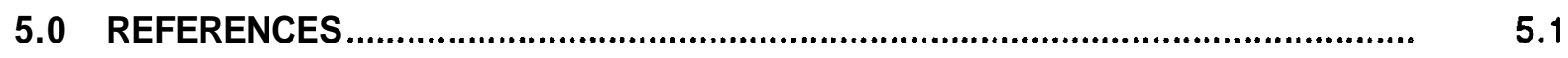

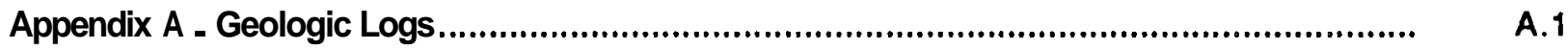

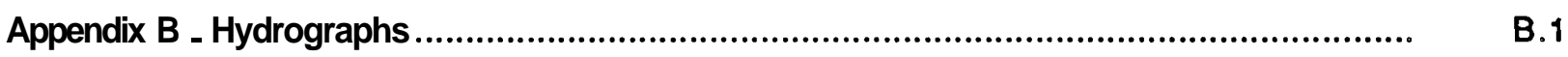

Appendix C . Particle-Size Distribution $/ \mathrm{CaCO}_{3}$ Analyses ........................................ C.1

Appendix D _ Moisture Characteristic Cuwes................................................................ D.1 


\section{FIGURES}

1 Map Showing Location of Hanford Site and the 200 Areas Low-Level Burial Grounds.

2 Locations of Boreholes Within $1000 \mathrm{ft}$ of the W-5 Burial Ground.

3 Stratigraphic Chart for the 200-West Area......

4 Cross Section $\mathbf{A}$ -

5 Cross Section B-

6 Cross Section C-C'

7 Sediment Classification According to Particle-Size Distribution.

8 Hanging Water Column Apparatus Used to Measure Water Retention.

9 Pressure Plate Apparatus Used to Measure Water Retention...................................... $\quad 2.20$

10 Comparison of X-Ray Fluoresence Analyses from WSU and PNL Laboratories.

11 Fence Diagram of the Area near W-5

12 Surface of the Elephant Mountain Member Beneath the 200-West Area....

13 Thickness of the Lower Ringold Unit Beneath the 200-West Area.

14 Top of the Plio-Pleistocene Unit

15 Geomorphic Features in the West-Central Portion of the Hanford Site.

16 Thickness of the Hanford Formation Beneath the 200-West Area

17 Example of Lateral Fades Variations Within the Upper Stratigraphic Section Beneath the W-5 Burial Ground.

18 Clastic Dike.

19 Ternary Diagram Showing the Ratio of Quartz. Feldspar. and Rock Fragments for Various Stratigraphic Units.....

20 Temary Diagram Showing the Ratio of Quartz. Plagioclase. and Potassium Feldspar for Various StratigraphicUnits.

21 Ternary Diagram Showing the Ratio of Three Common Types of Rock Fragments for Various Stratigraphic Units.

22 Predominant Clay Minerals.

23 Average Distribution of $\mathrm{Al}_{2} \mathrm{O}_{3}$ and $\mathrm{SiO}_{2}$ for Stratigraphic Units Analyzed Within the 200 Areas. 
Average Distribution of $\mathrm{K}_{2} \mathrm{O}, \mathrm{CaO}, \mathrm{TiO}_{2}$, and $\mathrm{FeO}$ for Stratigraphic Units Analyzed Within the 200 Areas.

25 Bivariate Plot of $\mathrm{Al}_{2} \mathrm{O}_{3}$ and $\mathrm{SiO}_{2}$ for the Hanford Formation and Middle Ringold Unit......

26 Bivariate Plot of $\mathrm{SiO}_{2}$ Versus $\mathrm{FeO}$.

27 Bivariate Plot of FeO Versus Total Organic Carbon ............................................. 3.32

28 Bivariate Plot of Rubidium Versus Zinc ..........................................................

29 Bivariate Pbt of Zirconium Versus Barium.........................................................

30 Bivariate Plot of Arsenic Versus Rubidium ...................................................... 3.34

31 Water-Table Map for the 200 Areas Measured in December 1987 ............................... 4.3

32 Estimated 1944 Water-Table Map ................................................................... 4.4

33 Depth to Water Table in the Vicinity of the W-5 Burial Ground ....................................

34 Surface of the Water Table in the Vicinity of the W-5 Burial Ground. Measured on February 27. 1988

35 Generalized Hydrogeologic Cross Section Beneath the 200-West Area...................... 4.8

36 Saturated Thickness of the Unconfined Aquifer....................................................

37 Distribution and Range of Hydraulic Conductivities for the Hanford and Ringold Formations.

38 Moisture Characteristic Curves for the Hanford Formation ..................................... 4.17

39 Moisture Characteristic Curves of Four Stratigraphic Intervals from Well 299-W7-2......... $\quad 4.18$ 


\section{TABLES}

1 Special. Non-Routine Sample Inventory from Wells near the W-5 Burial Ground.............

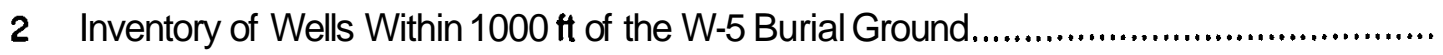

3 Hydrometer Analyses from Wells in the Vicinity of the W-5 Burial Ground

4 Petrographic Data from 200-West Area Wells.

5 Results of Clay Mineralogy Analyses

6 Statistical Parameters for Mapr Elements According to Stratigraphic Unit.

7 Summary of Hydraulic Properties Reported for the Hanford Site

8 Hydraulic Parameters Measured and/or Derived near the W-5 Burial Ground

9 Field Moisture Data Collected from Wells near the W-5 Burial Ground......

10 Water-Retention Data from the 200 Areas.

11 Cation Exchange Capacity of Sediment Samples Collected near the W-5 Burial Ground 


\subsection{INTRODUCTION}

Construction of a disposal facility for solid, mixed low-level radioactive and hazardous wastes in the 218-W-5 Burial Ground (W-5) in the 200-West Area of the U.S. Department of Energy's (DOE's) Hanford Site in southeastern Washington State (Figure 1) is planned. DOE Order $\mathbf{5 8 2 0 . 2 A}$ requires a site-specific performanceassessmentfor each new disposal facility to ensure that wastes will be isolated from the environment. To demonstrate the adequacy of the facility for isolatingthe wastes, computer codes are used to simulate the physical processes that could cause the waste to migrate to underground water supplies or to the land's surface.

The purpose of this report is provide a compilation and interpretation of geologic and hydrologic data available for the area near W- 5 for use in the performance assessment modelling. A variety of data are needed to model flow and transport from a solid-waste burial trench. These data include soil water content, soil moisture potential, saturated and unsaturated hydraulic conductivity, and phase mineralogy of the soils and sediments within the vadose zone. The hydrologic data that are critical for quantifying the water storage and transport properties for unsaturated soils require a characterization of the heterogeneities of various soil layers and the moisture characteristiccurves for these layers. Hydraulicproperties and mineralogic data for the saturated sediments are also important for modelling the flow and transport of wastes in the unconfined aquifer.

This report begins with a discussion of the procedures and methods used to gather data both in the field and in the laboratory. This is followed by a summary of the geology, including the stratigraphic framework, lithofacies, and mineralogic/geochemical characteristics of the suprabasalt sediments. The hydrology of the region and of the W-5 site is discussed next. In this discussion, the characteristics of the uppermost aquifer(s), unsaturated zone, and the various hydrogeologic units are presented. 


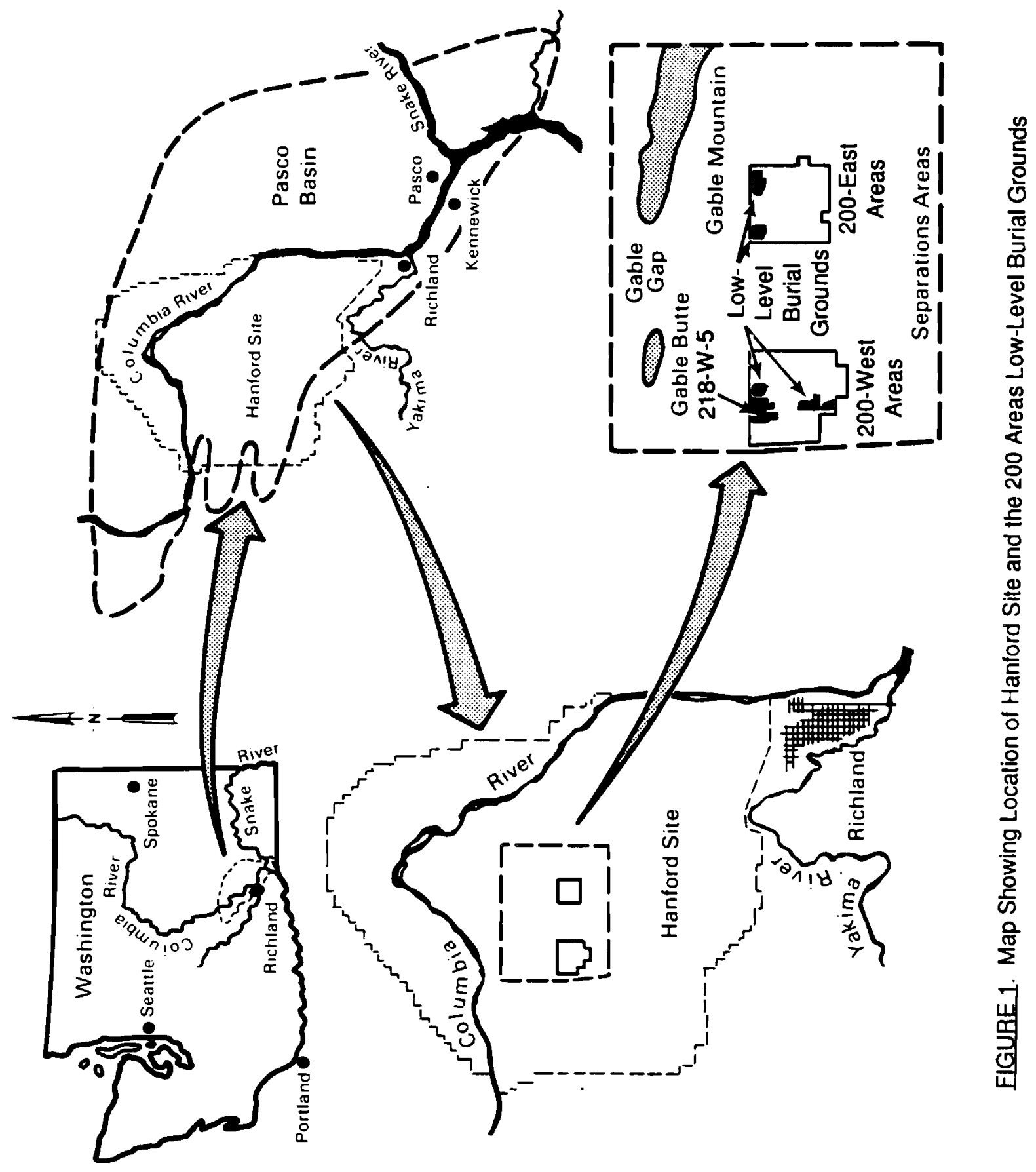


A variety of investigative methods have been used to evaluate the geology and hydrology of W-5. The methods used in the field have included sample collection, geologic logging, geophysical logging, aquifer testing, and water-level measurements. In the laboratory, several physical and chemical analyses of borehole samples were routinely performed; these include analyses of grain-size distribution, field moisture, and calcium carbonate content. Results of other, less routine laboratory analyses available for sediment samples include hydrometer and permeameter tests, as well as determination of cation exchange capacity, mineralogy, and bulk geochemistry. Most of these data were originally documented by Last et al. (1989); however, many of the data collected were not completely analyzed in time for that publication. Those data, listed in Table 1 and unavailable for publication by Last et al. (1989), are presented for the first time in this document.

A total of 11 boreholes, all within $1000 \mathrm{ft}$ of $\mathrm{W}-5$, were selected for use in the evaluation of the geohydrology of the area (Figure 2). Table 2 lists the 11 boreholes used in the present study, with their locations, elevations, total depths, screened intervals, dates of completion, and other data available. Eight of the 11 boreholes were drilled in the late 1980s for ground-water monitoring to comply with the Resource Conservationand Recovery Act (RCRA). The geohydrology and installation of these wells has been documented by Last et al. (1989) and Last and Bjornstad (1989). There are two sets of well clusters (299-W7-2/3 and 299-W10-13/14). Individual wells in these clusters are $<50 \mathrm{ft}$ apart; one well in the cluster is developed at the top of the unconfined aquifer and the other is developed at the base. The remaining boreholes include an older ground-water monitoring well (299-W15-2), and two (699-45-78 and 699-47-808) that were drilled for geologic investigation for DOE's Basalt Waste Isolation Project (BWIP). The only information used from well 699-47-808 is the level of the top of basalt and the gross gamma ray log. Other geologic data from this borehole are suspect and therefore were not used in the interpretation. A stratigraphic chart showing the sedimentary units present in the 200-West Area, which includes W-5, is presented in Figure 3.

\subsection{FIELD METHODS}

A variety of field methods have been used to obtain data from wells drilled in the vicinity of W-5. These include sample collection, geologic logging, borehole geophysical logging, aquifer testing, and water-level measurements. The methods used are described in this section. 


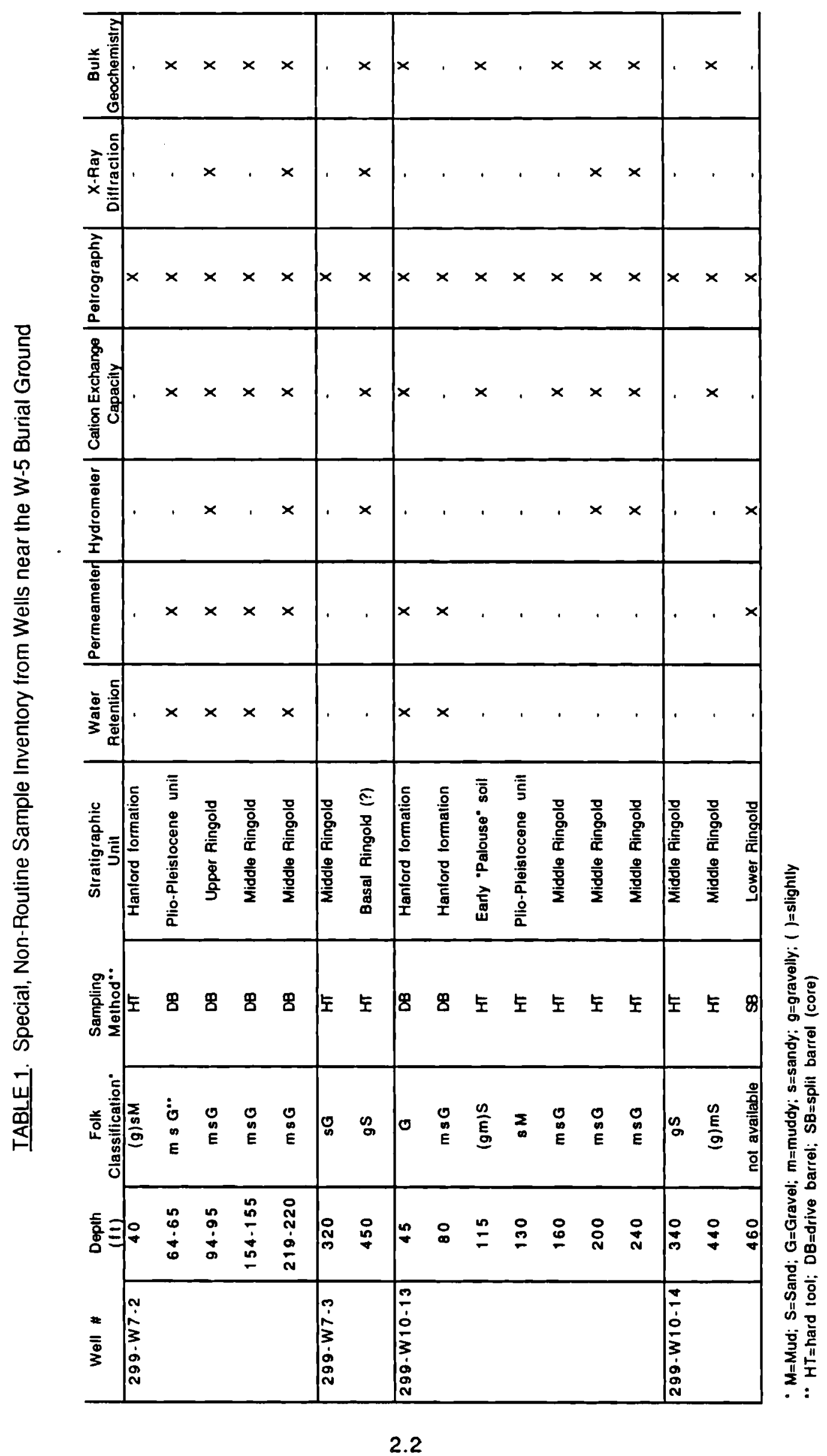




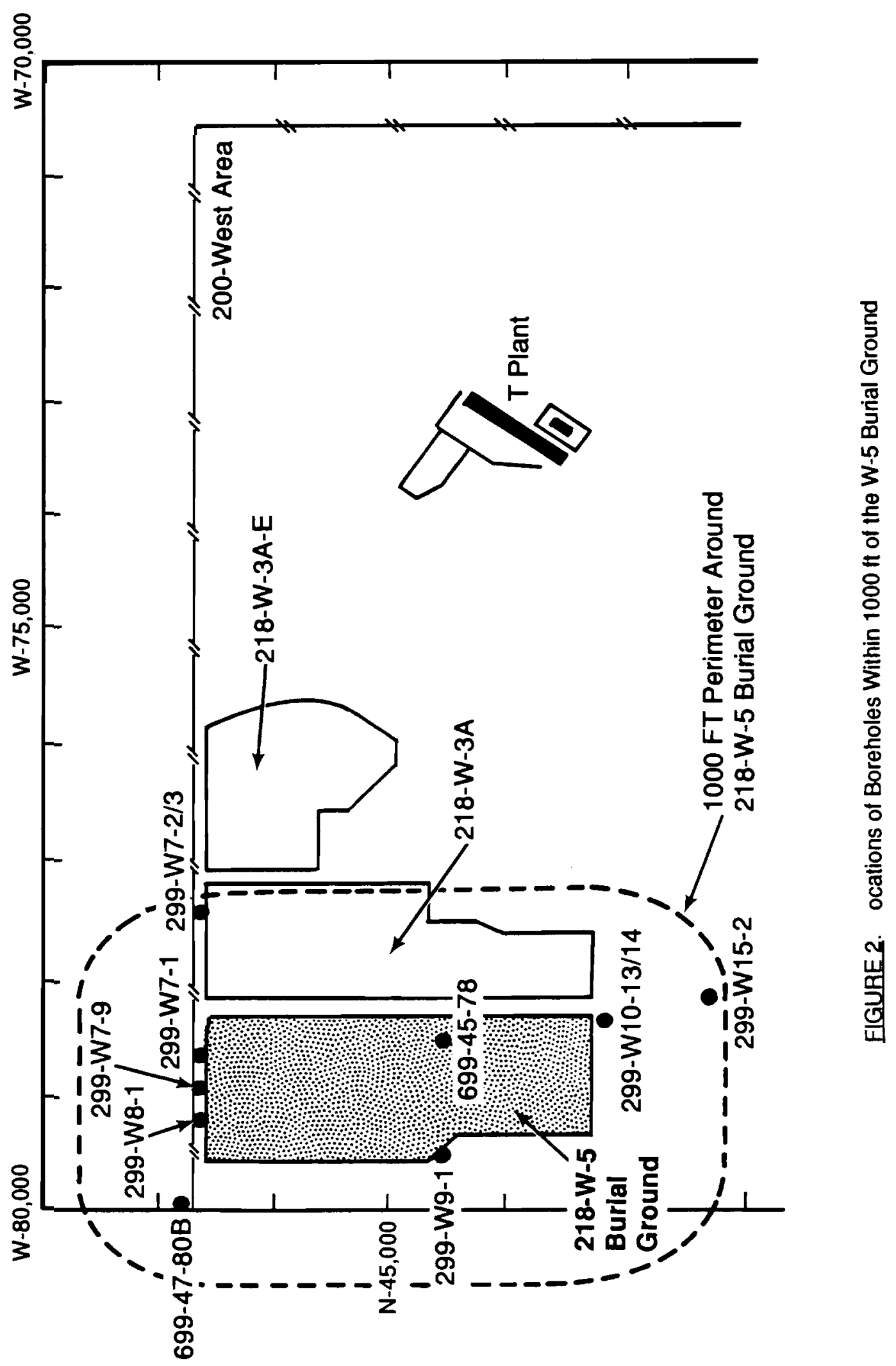


TABLE 2. Inventory of Wells Within $1000 \mathrm{ft}$ of the W-5 Burial Ground

\begin{tabular}{|c|c|c|c|c|c|c|c|c|c|c|}
\hline \multirow{2}{*}{\begin{tabular}{|l} 
BORETOE \\
$200 \cdot W 7 \cdot \mid$
\end{tabular}} & \multicolumn{2}{|c|}{$\begin{array}{c}\text { HANFORD PLANT } \\
\text { DOORONATES }\end{array}$} & \multirow{2}{*}{$\begin{array}{c}\text { DATE } \\
\text { COMPLEIED }\end{array}$} & \multirow{2}{*}{\begin{tabular}{|c|} 
CAsngelev. \\
(11)
\end{tabular}} & \multirow{2}{*}{$\begin{array}{c}\text { OPENINTERNAL } \\
\text { UIU }\end{array}$} & \multirow{2}{*}{$\begin{array}{c}\text { AOUIFERMONITORED } \\
\text { Middle Ringold }\end{array}$} & $\left|\begin{array}{c}\text { TOTAL DEPTH } \\
\text { IIII }\end{array}\right|$ & \multirow{2}{*}{$\begin{array}{c}\text { DEPTH } \\
\text { (1) }\end{array}$} & $\begin{array}{c}\text { WATER DEPTH } \\
\text { (iti) }\end{array}$ & \multirow{2}{*}{\begin{tabular}{|c|} 
PEFEPENCE \\
Last el al. (1989)
\end{tabular}} \\
\hline & N46551 & W78601 & & & & & 245 & & 228.61 & \\
\hline $209 \cdot W 7 \cdot 2$ & $\mathrm{~N} 46519$ & W77385 & $09 / 87$ & 675.59 & $202 \cdot 222$ & Middle Fingold & 236 & NP & 214.48 & Last el al. (1989) \\
\hline 209.67 .9 & N46520 & $W 77420$ & 11187 & 676.14 & $449 \cdot 470$ & Middle/Basal Ringold & 475 & 476 & 216.28 & |Last of al. (1989) \\
\hline 200.67 .0$. & N46551 & W78900 & $12 / 89$ & 690 & $221 \cdot 241$ & Middle Fingold & 248 & NA & 227 & \\
\hline $299-W 8.1$ & N46551 & W79200 & $07 / 87$ & 701.33 & 257.267 & Middle Ringold & 270.5 & NA & 238.82 & Last ot al. (1989) \\
\hline $209 \cdot w \cdot \cdot 1$ & N44508 & W79507 & $10 / 87$ & 737.73 & $266 \cdot 286$ & Middle Ringold & 295 & NA & 272.09 & Last ot al. (1989) \\
\hline 299-W15-2 & N42251 & W78082 & $08 / 54$ & 690.71 & 210.258 & Middle Ringold & 260 & NF & 221.00 & Last el al. (1989) \\
\hline $290 . W 10.13$ & N43137 & W78297 & $09 / 87$ & 699.04 & 227.247 & Middle Ringold & 250 & NP & 232.11 & Last et al. (1989) \\
\hline $299 . W 10.14$ & $N 43143$ & W78330 & $11 / 87$ & 699.43 & $427 \cdot 447$ & Middle Ringold (base) & 462 & NF & 232.90 & Last et al. (1989) \\
\hline 699.45.78 (RRL-3) & N44575 & W78294 & $10 / 81$ & 689.81 & None & & 730 & 508 & uncenain & Bjornstad (1984) \\
\hline 699.47.80B (DC.208) & N46910 & W79835 & $09 / 83$ & 712.10 & None & & 1632 & 531 & uncertain & $\begin{array}{l}\text { Ledgerwood (1986) } \\
\text { McGhan (1989) }\end{array}$ \\
\hline
\end{tabular}




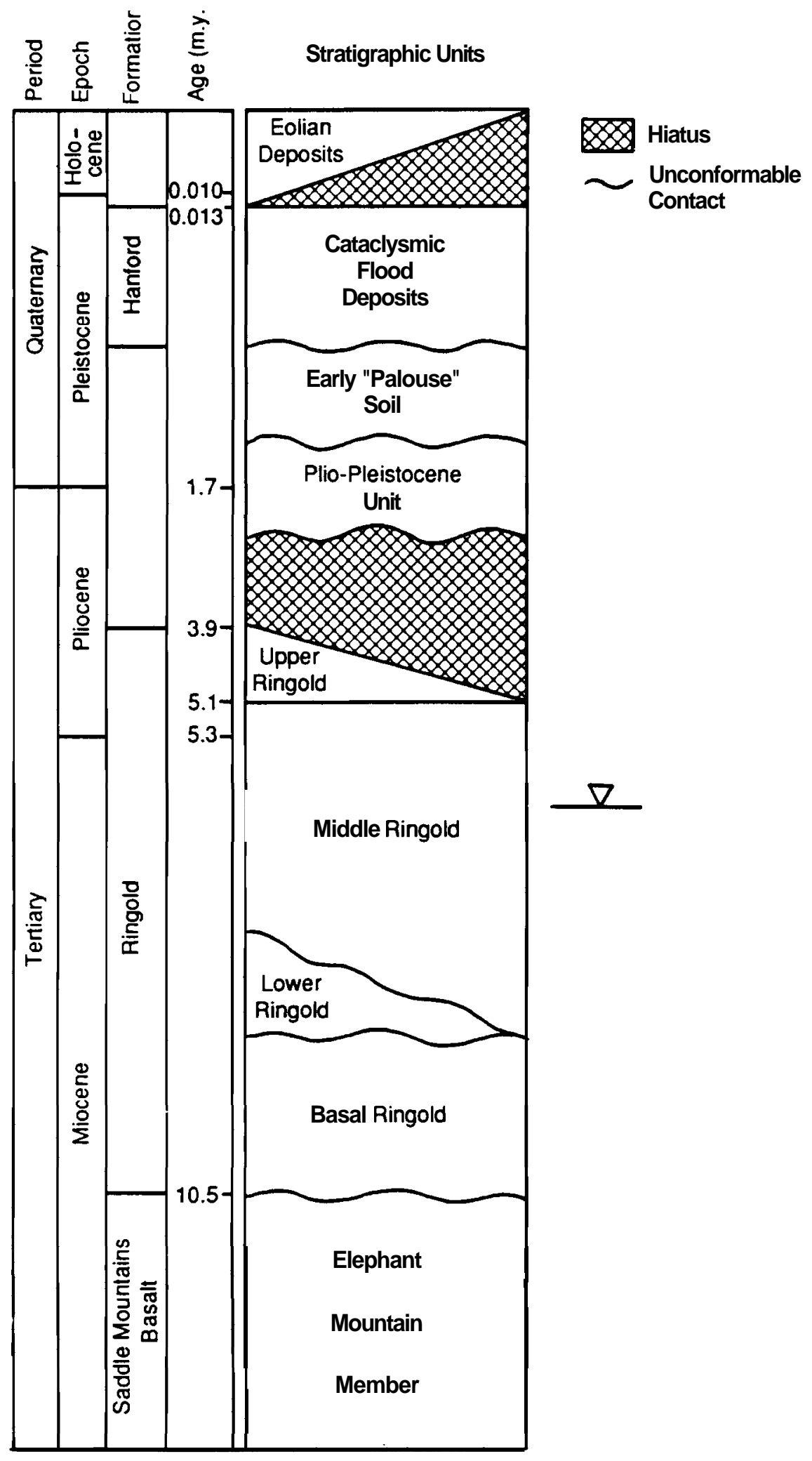

FIGURE 3. Stratigraphic Chart for the 200-West Area 


\subsubsection{Sample Collection}

Geologic samples provide the materials necessary to perform laboratory analysis of the physical and chemical properties of the geohydrologic media. Two pint-jar geologic samples of drill cuttings were collected from RCRA ground-water monitoring wells every $5 \mathrm{ft}$ and/or at changes in lithology (Last et al. 1989). Where possible, the sampled materials were recovered from the borehole using a drive barrel. Another sample for moisture content analysis was collected from each sampled interval above the water table from which samples were retrieved with a drive barrel or split barrel (i.e., core). Where hard-tool drilling was necessary, the sampled materials were recovered with a bailer. Additional samples for other types of analyses (e.g., water retention, hydrometer, permeameter, cation exchange capacity, mineralogy, and bulk geochemistry) were collected at irregular intervals.

The drive-barrel and split-barrel methods, which were the preferred methods of drilling and collecting samples, drive a hollow cylinder into the formation, immediately bringing the sample to the surface. Drive-barreland split-barrel samples are similar except that split-barrel samples are collected intact within a plastic or metal liner, and drive-barrel samples are loose mixtures of the material hammered out of the drive barrel. These methods provide the most representative samples, which, when analyzed for grain size, most accurately represent the true particle-size distribution.

When drilling through flowing sands or consolidated and/or extremely coarse-grained strata, drillers often resort to drilling with the hard-tool method. The hard-tool drilling method uses a heavy metal bit to penetrate the formation while water is added to form a mud slurry, which is removed with a bailer. Therefore, the hard-tool samples produce granulometric results that are skewed toward the finer grained fractions more than results from an undisturbed sample would be. Thus hard-tool samples are representative of neither in situ grain-size distribution nor moisture content.

\subsubsection{Geologic Logaing}

Geologic logs provide a cursory means of visual inspection and description of retrieved samples, as well as a record of drilling activities, unusual occurrences, or problems.

Geologic logs are of two types. The first type is recorded by geologists and based on observation of drill cuttings removed from the hole every $5 \mathrm{ft}$ and at changes in lithology. The other type of geologic log is recorded by the driller and focuses on drilling properties, although it may also provide a general description of geologic materials.

Geologic samples from RCRA monitoring wells were collected and described according to standardized procedures (PNL 1989). Sample descriptions by geologists included a textural classification (after Folk 1974) and estimates of particle-size distribution, sorting, gross mineralogy, roundness, color, 
calcium carbonate content, and relative consolidation. Summary geologic logs for each borehole, other than 699-47-80B, which is suspect, are presented in Appendix A. Data on several of these boreholes were originally presented by Last et al. (1989).

\subsubsection{Borehole Geophysical Logoing}

Borehole geophysical logs (i.e., gross gamma ray logs) provide useful information for interpreting and correlating stratigraphy. Fine-grained sediments normally produce a higher gamma response than coarse-grained sediments. Gross gamma ray logs are available for all 11 boreholes within $1000 \mathrm{ft}$ of W-5. Geophysical logging of the RCRA monitoring wells was conducted with a gross gamma probe, according to procedure GL-7 or GL-7A (PNL 1989), to assist in the identification of fine-grained sedimentary layers.

Gross gamma ray logs for the wells near W-5 are shown both on the cross sections (Figures 4 to 6 ) and on the individual summary logs presentedin Appendix A. These logs are not suitable for quantitative purposes. Instead they are used qualitatively, in combination with geologic logs and granulometric and geochemical data, to aid in the interpretation and correlation of stratigraphicunits.

\subsubsection{Aquifer Testing}

Aquifer tests provide in situ aquifer measurements of transmissivity, from which hydraulic conductivity and storativity can be derived. Several types of aquifer tests have been performed during the installation of RCRA ground-water monitoring wells in the vicinity of W-5 (Last et al. 1989). These tests include 1) constant-discharge withdrawal, 2) recovery, and 3) slug injection/withdrawal tests.

Constantdischargewithdrawaltests at the wells were designedto stress the aquifer as much as possible by pumping at a maximum constant rate. The wells tested were drilled to depth (at least $10 \mathrm{ft}$ below the water table) and cased with temporary, 8-in.-diameter carbon steel casing and screened with a 10-ft section of 8-in. telescoping stainless steel screen. The lower 8 to $9 \mathrm{ft}$ of screen were exposed by pulling back the temporary casing. The wells were generally pumped using a 20-hp submersible pump at a constant rate for up to $8 \mathrm{~h}$. Discharge was measured using in-line flow meters. Totalizers on largediameter flow meters read to the nearest $100 \mathrm{gal}$; the small flow meter reads to the nearest 1 gal. A measured 5-gal bucket and/or 55-gal drum was used to check the performance of the in-line flow meters. Discharge was stabilized at a constant rate with variations of no more than $\pm 10 \%$. Water pumped from wells during aquifer testing was disposed of at least $1000 \mathrm{ft}$ away from the wells and W-5. Constantdischarge tests were performed at the top of the unconfined aquifer at wells 299-W7-1 and 299-W7-2.

Water-level responses were measured during both the drawdown period and the recovery period (after pumping had been terminated). These measurements were made using an electric water-level indicator (E-tape) and/or a pressure transducer. The E-tape measurements may be no more accurate than 
$0.5 \mathrm{ft}$ relative to the top of the well casing, but they are accurate to within at least $0.10 \mathrm{ft}$ between successivemeasurements. The E-tape was placed in a stilling well wherever possible. Like the E-tapes, the pressure transducers were used only for relative measurements. The transducers were capable of measuring pressure ranges of $\mathbf{0}$ to 5,0 to 10 , or $\mathbf{0}$ to $15 \mathrm{psi}$. Steel tapes were used to obtain accurate measurements at the beginning and end of each test. Recovery tests were performed at wells 299-W7-1, 299-W7-2, 299-W8-1, 299-W10-13, and 299-W10-14.

A slug injection/withdrawal test was performed on well 200-W9-1 because the aquifer yield of the tested interval was too low for conducting a constant-discharge test. This well was completed with 4-in. stainless steel casing and screen and was developed only by bailing before the test. The slug consisted of a 10-ft-long, 2.375-in.-diameter pipe filled with sand and sealed at both ends. This pipe displaced $0.31 \mathrm{ft}^{3}$ of water. The slug was lowered instantly into the aquifer until completely submerged. After the water level reached equilibrium, the slug was rapidly removed from the aquifer. The aquifer response was measured using a 0 - to 10-psi pressure transducer and verifiedusing an E-tape. Both the injection and the withdrawal tests were monitored for $180 \mathrm{~min}$.

Two methods were used to analyze the drawdown and recovery data obtained from the constantdischarge and recovery tests:

- Theis method (Theis 1935)

- Cooper-Jacob straight-line method (Cooper and Jacob 1946; Lohman 1972).

The following assumptions underlie the use of these methods:

- The aquifer is of infinite areal extent.

- The aquifer is homogeneous, isotropic, and of uniform thickness within the radius of influence of the aquifer test.

- The tested well penetrates and receives water from the full thickness of the aquifer by horizontal flow.

- The well is pumped at a constant discharge rate.

- The static level of the piezometric surface is horizontal within the radius of influence of the well.

- The well diameter is infinitesimal (i.e., storage in the well is neglected).

- The water removed from storage is discharged instantaneously with decline of head.

Although some of these assumptions were obviously violated (e.g., full penetration, homogeneity), the results obtained using these techniques do allow for a comparison of the hydraulic properties between the Hanford and Ringold Formations, the primary hydrogeologicunits tested. 


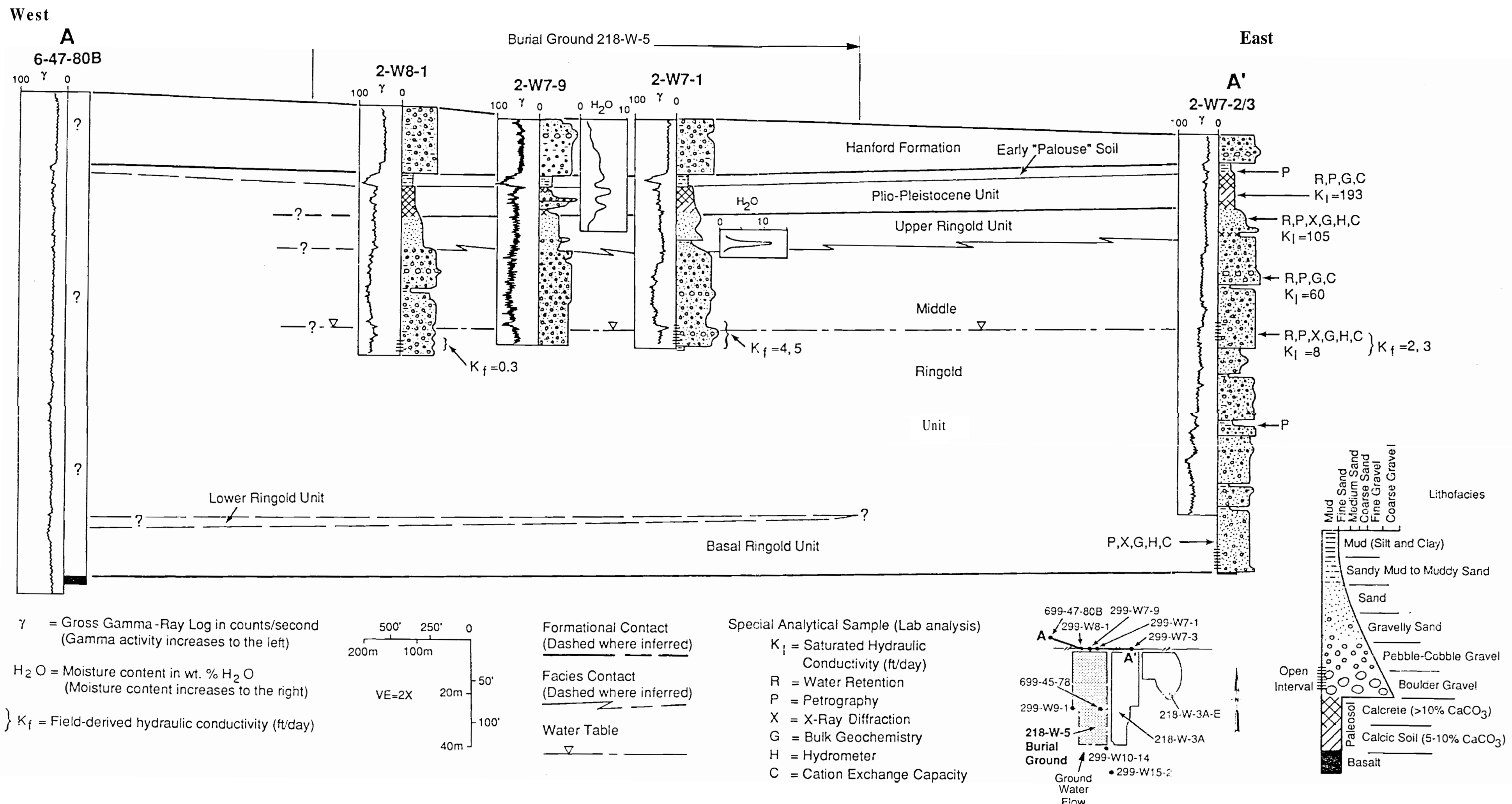




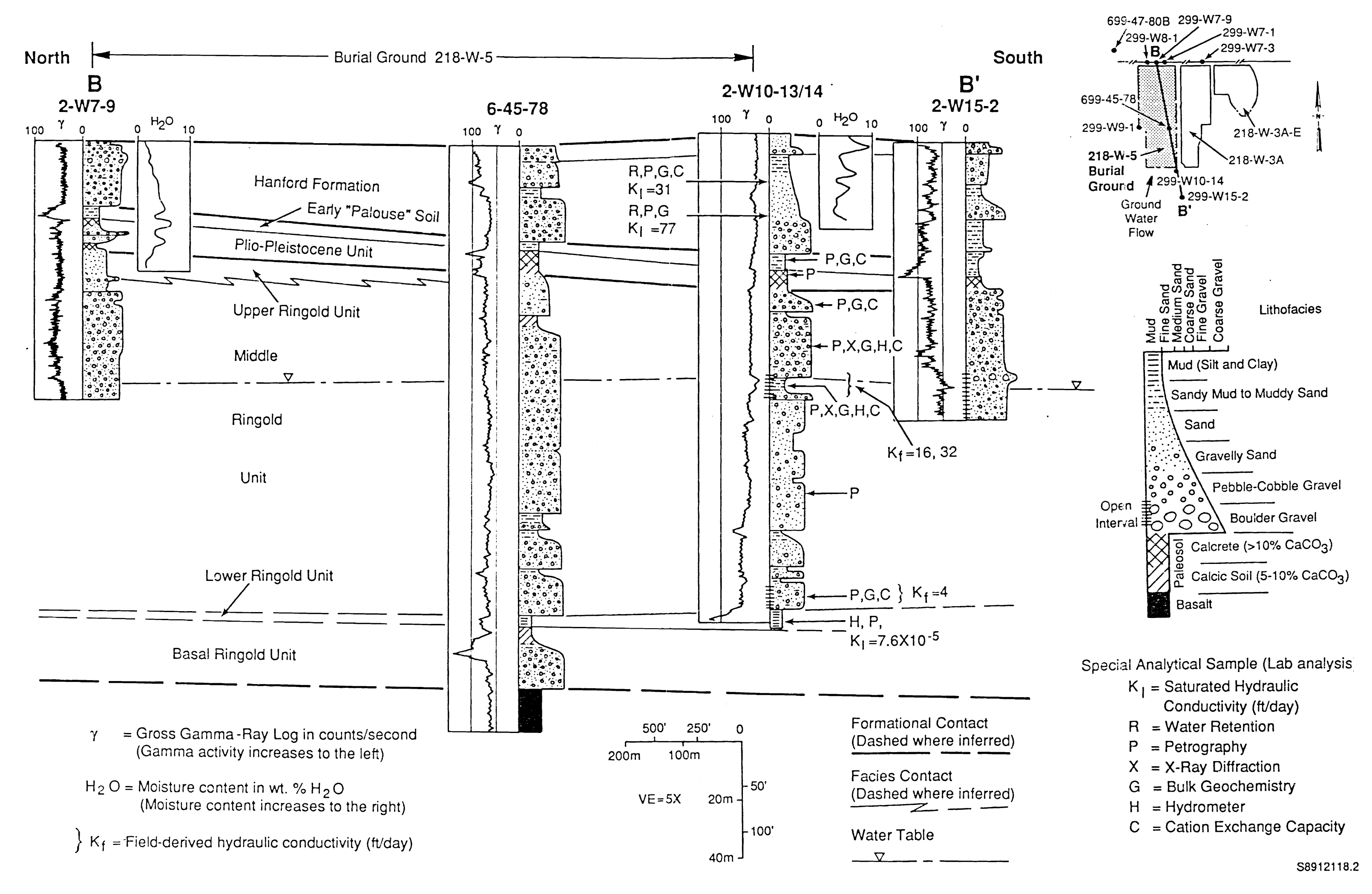

FIGURE 5. Cross Section B-B' 


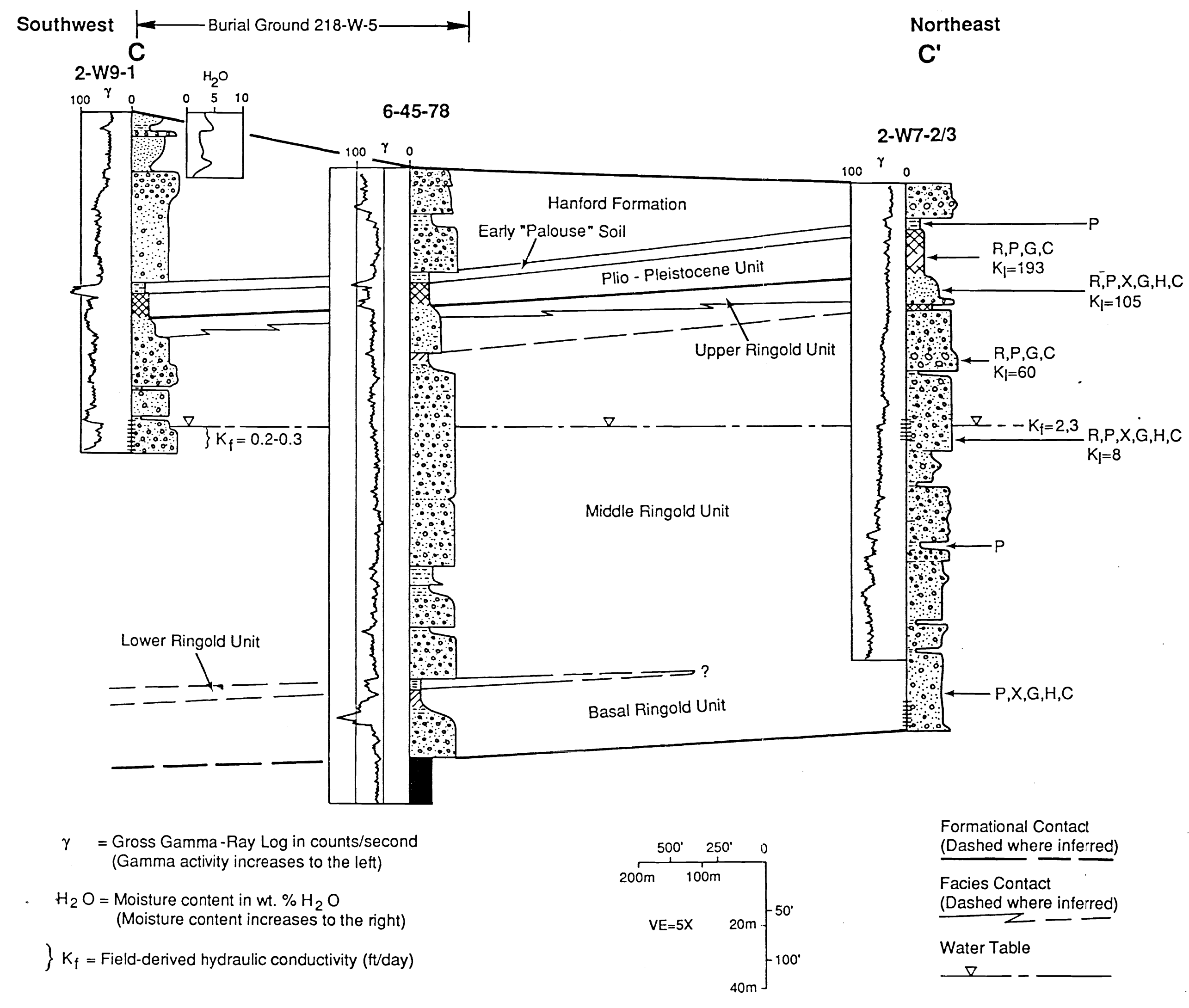

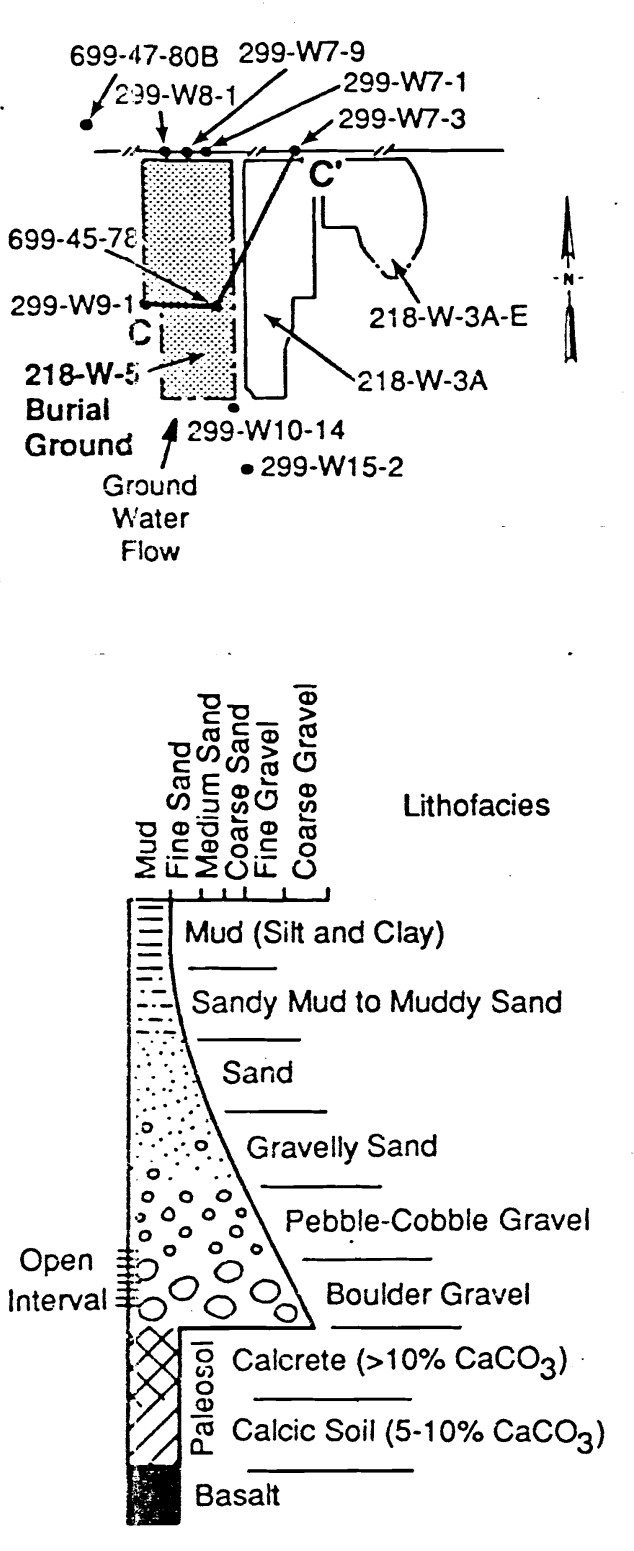

Special Analytical Sample (Lab analysis

$K_{1}=$ Saturated Hydraulic

Conductivity (ft/day)

$R=$ Water Retention

$P=$ Petrography

$X=X-$ Ray $\mid$ Diffraction

$G=$ Bulk Geochemistry .

$\mathrm{H}=$ Hydrometer

$\mathrm{C}=$ Cation Exchange Capacity

S8912118.3 


\subsubsection{Water-Level Measurements}

An interpretation of water-level measurements over time provides valuable information, such as the vertical component of ground-waterflow, aquifer communication, and aquifer behavior in response to artificial recharge. Therefore, monthly water-level measurements are made for most ground-water monitoring wells in the vicinity of W-5. These measurements are made in duplicate using a steel tape in accordance with PNL procedures WL-1 through WL-5 (PNL 1989). Each steel tape is standardized against a steel tape to $\pm 0.10 \mathrm{ft}$. Hydrographs available for ground-water monitoring wells within $1000 \mathrm{ft}$ of the burial ground are presented in Appendix B.

\subsection{LABORATORY METHODS}

A variety of laboratory analyses have been performed on samples from wells in the vicinity of W-5. Dry-sieve analysis and calcium carbonate analysis were conducted on a routine basis for most 5-ft samples from ten of the wells within $1000 \mathrm{ft}$ of W-5 (the exception was well 699-47-808). Additional samples underwent, on a non-routine basis, a variety of other tests, including moisture content, water retention, permeameter, hydrometer analysis, cation exchange capacity, petrography, X-ray diffraction, and bulk geochemistry (Last et al. 1989). Sieve and calcium carbonate data are presented in Appendix C. Moisture content data are presented elsewhere in the text. Locations and types of analyses performed on other non-routine samples are summarizedin Table 1.

\subsubsection{Particle-Size Distribution}

Particle-sizeanalyses provide a quantitative measure of the size, distribution, and sorting relationships among individual particle grains. Particle-size analyses of the sand-to-gravel-sized particles were conducted using the dry-sieve method for 5 -ft intervals of most of the wells near W-5. The dry-sieve procedure is presented in PNL (1989). Dry-sieve analysis is normally performed on one of the two pint-jar samples from all split-barrel, drive-barrel, or hard-tool samples. Six nested sieves had 2.00-, 1.00-, 0.50-, 0.25-, 0.125-, and 0.063-mrn sieve openings. After the weight of material retained on each sieve was determined, the data were entered into the ROCSAN data base described by Tallman et al. (1979, p. 107109). The ROCSAN (originally ROC) program calculates the total weight percent and particle-size distribution of the sample and classifies the sample according to one of the 19 sediment classes shown in Figure 7. Output on particle-size distributions generated by the ROCSAN program as of December 1 , 1989, is presented in Appendix C. 


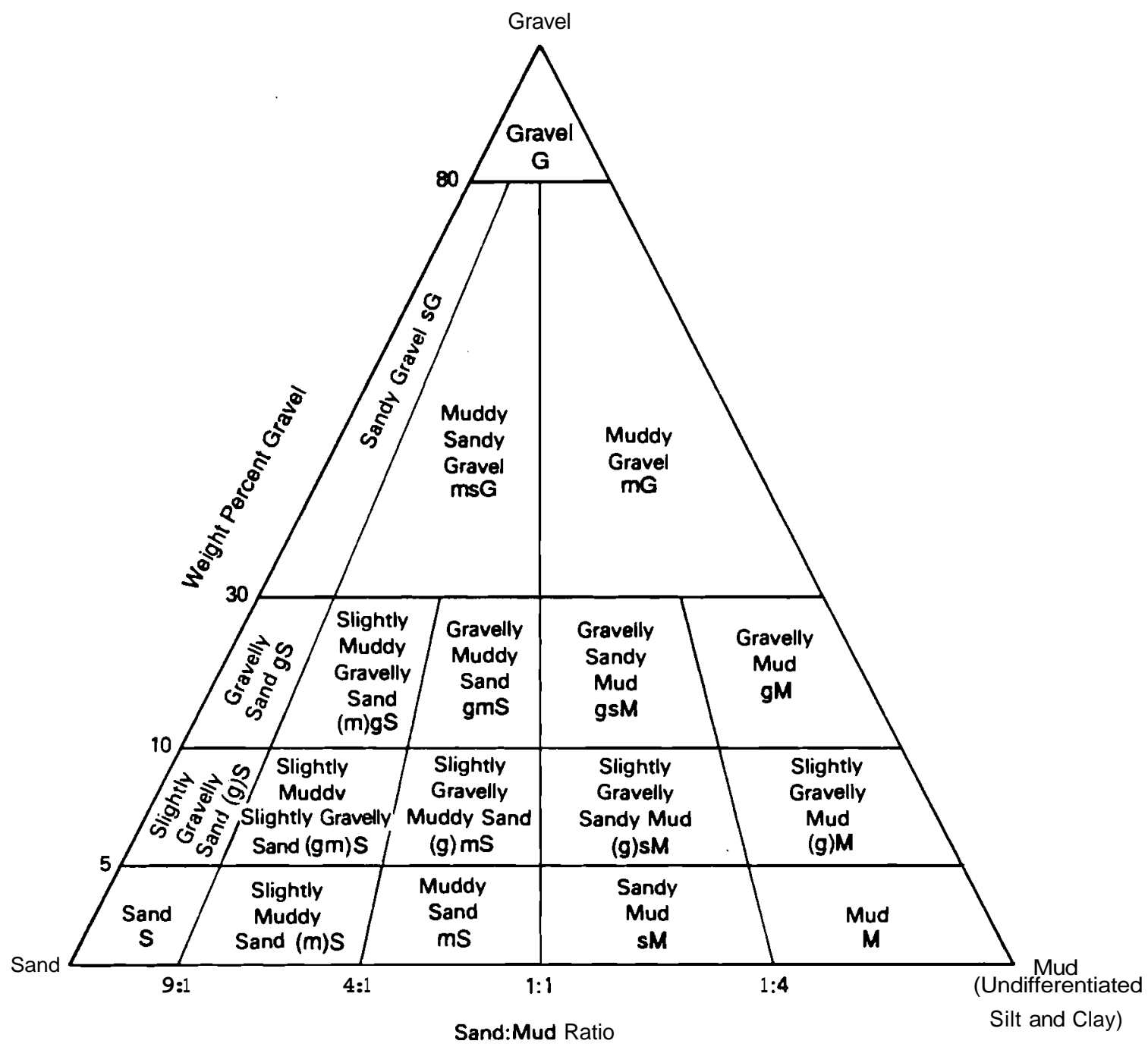

ElGURE7. Sediment Classification According to Particle-SizeDistribution

The fine-grained fraction of a few samples was also analyzed via hydrometer/wet-sieve analysis to determine the distribution of clay-to-silt-sized particles, using the methods described by ASTM (1986a) and Gee and Bauder (1986).

Particle-size distribution data are not absolute measurements and must be used qualitatively in combination with other data. While the results of dry-sieving may be representative for most samples collected with a drive-barrel or a split-banel sampler, the results for samples collected with a bailer after 
hard-tool drilling are skewed toward finer grain sizes because of crushing and pulverizing by the hard-tool bit. Therefore, particle-size distribution data must be examined in combination with geologic logs and the gross gamma ray logs to arrive at an estimate of the in situ particle-sizedistribution.

\subsubsection{Calcium Caibonate Content}

Concentrations of calcium carbonate in sediments are often a function of cementation and soil development, and they are therefore a useful tool for identifying cemented zones and differentiating among stratigraphicunits. Calcium carbonate analyses have been routinely conducted for the same samples as were analyzed for particle-size distribution. The calcium carbonate content is determined from a portion of the fine-grained fraction left over from dry-sieving. The weight percent of calcium carbonate present is analyzed by measuring the amount of carbon dioxide gas generated by the dissolution of calcium carbonate in acid. Procedures for measuring calcium carbonate in soils have been presented by Alison and Moodie (1965) and Nelson (1982). Results of calcium carbonate analysis are also entered into the ROCSAN data base and are included, along with particle-size data, in the ROCSAN output presented in Appendix C.

\subsubsection{Field Moisture Content}

In situ moisture content in the unsaturated zone is an important parameter for modelling unsaturated flow conditions. Samples for determiningin situ water content were collected when drilling with a drive barrel or split barrel in the unsaturated zone. Field moisture samples were collected at 5 -ft intervals along with the samples for particle-size analysis. Hard-tool samples were not analyzed for moisture content because the drilling method adds water to the formation. Because sediment samples are saturated below the water table, moisture samples were not collected below the level of the water table. Moisture content is plotted on the summary geologic logs presented in Appendix $A$.

Samples collected for moisture analyses were placed in airtight containers, sealed with tape, and enclosed in plastic bags to prevent moisture loss. At the end of each day, the samples were placed in a refrigerator, where they remained until the next working day, when analysis was initiated. After the plastic bag was removed, the entire sample was weighed, oven-dried at $105^{\circ} \mathrm{C}$ for $24 \mathrm{~h}$, and reweighedin accordance with ASTM procedures (ASTM 1986b).

\subsubsection{Permeameter}

Permeameter measurements provide data on saturated hydraulic conductivity, an important parameter for modelling water movement through the saturated and unsaturated zones. Perrneameter tests on two types of samples were performed in the laboratory. One type of sample consisted of repacked drive-barrel material; the other consisted of intact split-barrel (core) samples. 


\section{Hydraulic Conductivity of Drive-Barrel Samples}

Saturated hydraulic conductivity was determinedfor six repacked sediment samples collected from the unsaturated zone in the vicinity of W-5. These measurements were made using the constant-head method describedby Klute and Dirksen (1986). During this procedure, the loose sediment was packed into a cell $5.36 \mathrm{~cm}$ in diameter by $3 \mathrm{~cm}$ high, until a bulk density of $1.6 \mathrm{~g} / \mathrm{cm}^{3}$ was attained. The ends of the cell were closed with lids, with an inflow valve at one end and outflow valve at the opposite end. The inflow valve was then connected to the constant-head device, and the outflow valve was connected to a collection vessel. Starting with a saturated sample, an initial time was recorded and the water allowed to flow through the sample for a set amount of time. The amount of water discharged from the sample was then recorded and the saturated hydraulic conductivity calculated.

\section{Hxdraulic Conductivity of Split-Barrel Samples}

Three split-barrel samples from fine-grained Ringold material were analyzed for their vertical hydraulic conductivity using the falling-head method also described by Klute and Dirksen (1986). In this procedure, a 4-in.-long section was cut from the end of each split-barrel core while it was still encased in the sample liner. The sides of the core were then sealed to the liner, which was used as the containing cell. End caps, one with an inflow valve and one with an outflow valve, were placed on opposite ends of the cell. The inflow valve was then connected to a standpipe of known cross-sectional area and height. The samples were saturated before testing by slowly wetting them from the bottom. A solution of $0.01-\mathrm{M}$ calcium chloride was used to ensure no interaction with the fine-grained sediment. Because the flow rates were extremely low, this saturation process took several months. At the start of the test, the hydraulic head in the standpipe was measured. The dilute calcium chloride solution was then allowed to flow from the standpipe through the sample for a set time. An ending head level in the standpipe was then recorded. Test runs were repeated five times and the values averaged to calculate the hydraulic conductivity.

\subsubsection{Water Retention}

The functional relation between soil water content and soil water potential, as displayedin moisture characteristic curves, and hydraulic conductivity are important parameters for ground-water transport models.

A total of six samples collectedfrom wells near W- 5 have been analyzed for water retention characteristics (Table 1). Water retention characteristics were measured at 5-, 10-, 20-, 30-, 40-, 50-, and 75cm head pressure using hanging water columns (Figure 8). At 510-, 1020-, and 3060-cm head pressure, 


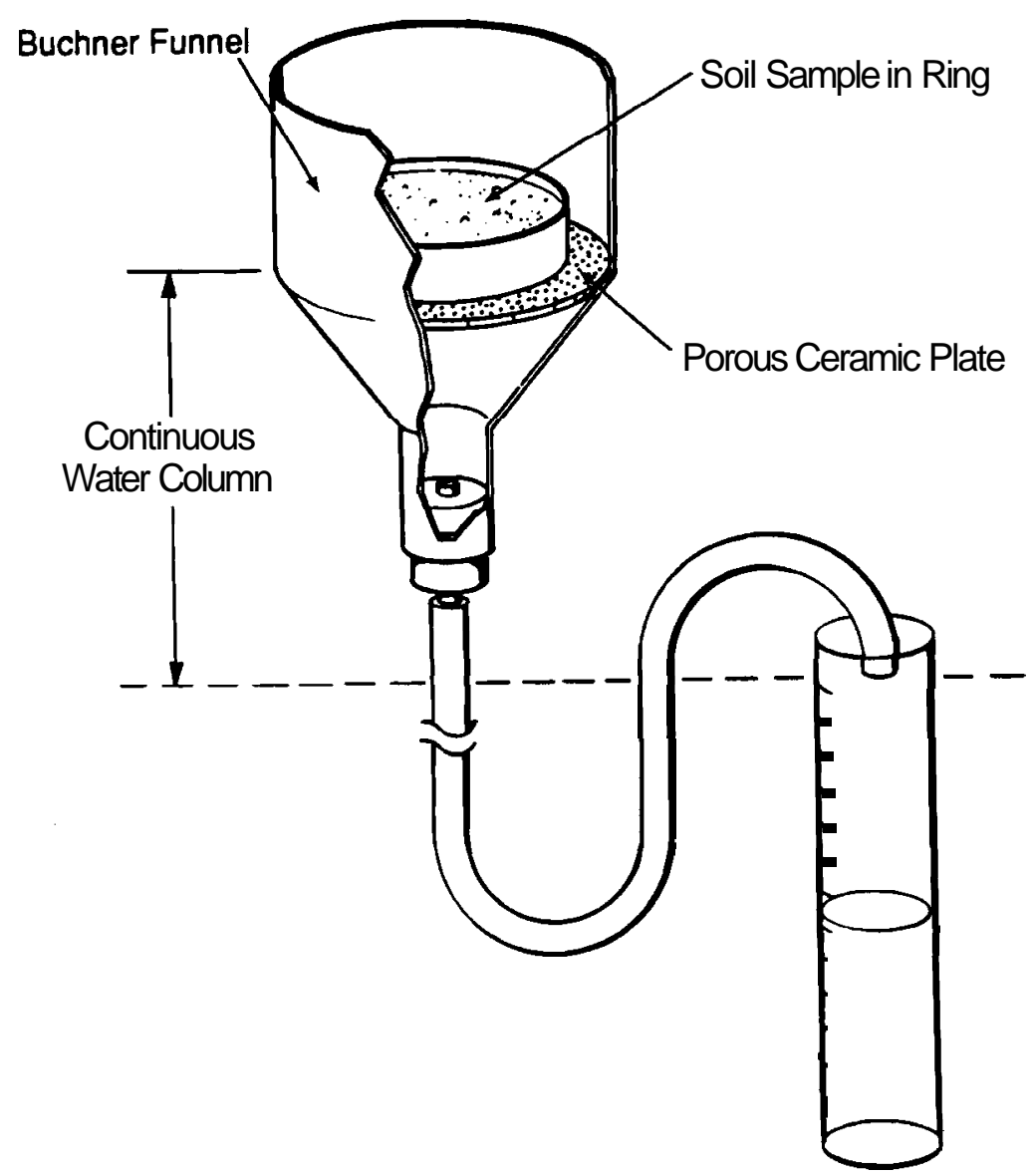

Graduated Cylinder

EIGURE 8. Hanging Water Column Apparatus Used to Measure Water Retention

the characteristics were determined using a pressure plate apparatus (Figure 9) and the method of Rawlins and Campbell (1986) and Klute (1986). Moisture characteristic curves, derived from the results of water-retention analyses, are presented in Appendix D.

Hanging water column analyses (Figure 8) were performed using the same saturated core-barrel samples used in the hydraulic conductivity analyses. These samples were transferred to the porous plates in Buchner funnels while they were still in their containingrings. The hanging water column is made by creating a continuous column of water up from the bottom of the porous plate through a cork in the neck of the funnel and into an narrow open-ended tube that is long enough to allow measurement of the pressure heads desired. The pressure head is measured from the center of the soil cell, which is in continuous contact with the column of water to the open end of the tubing (i.e., the open water surface). 


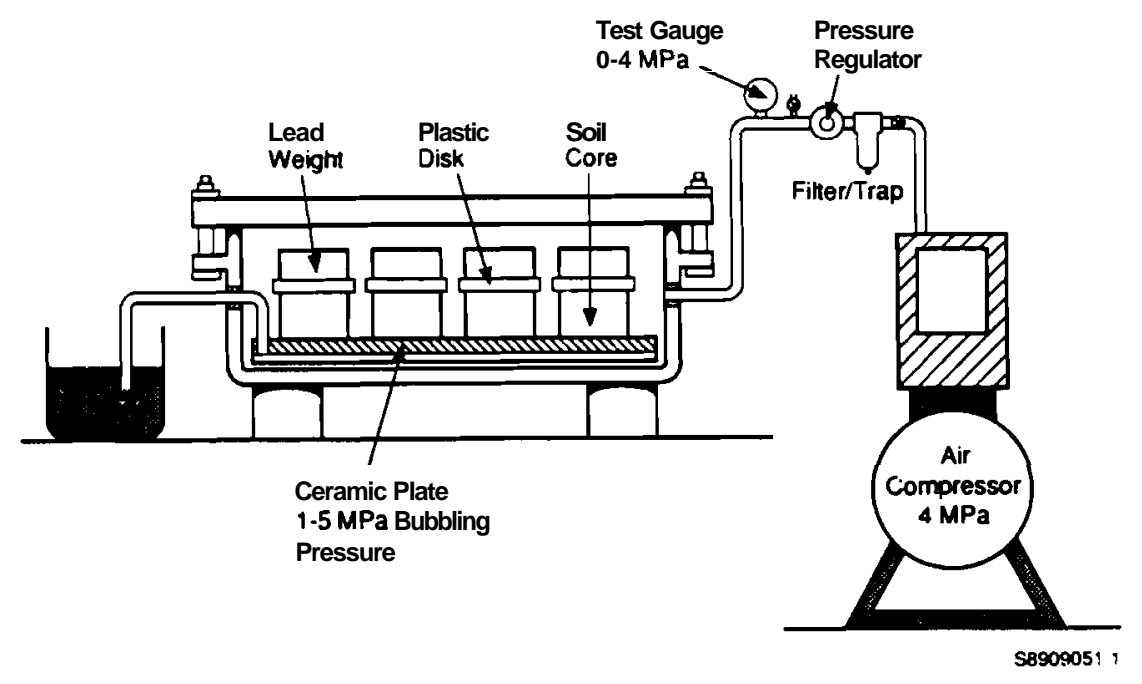

EIGURE9. Pressure Plate Apparatus Used to Measure Water Retention

After equilibrium is reached at each head level, the soil cell is weighed, and the weight is recorded. After final head-value equilibrium has been achieved, the sample is oven-dried, and the water content at each level is calculated.

For pressure plate extractor analyses (Figure 9), samples were packed into containing rings on a porous plate and allowed to stand for $\mathbf{2 4} \mathrm{h}$. Equilibrium water contents were obtained by pressuredraining the samples in the extractor at the desired test level. At the end of each pressure run, the sample was weighed and oven-dried to determine the moisture content at that pressure (Klute 1986).

\subsubsection{Cation Exchanoe Capacity}

Cation exchange capacity (CEC) provides information on the relative ability of sediments to exchange one type of positive ion for others, such as radionuclides.

Cation exchange capacity analyses were performed by the Soil Testing Laboratory at Oregon State University, using the ammonium acetate method modified after Schollenberger and Simon (1945). Aliquots of borehole samples were sieved to recover the $<2-\mathbf{m m}$ size fraction. This fraction was then analyzed for CEC. The results of the CEC analysis are discussed in Section 4.3.8.

\subsubsection{Mineralogical Analysis}

The mineralogy of the sediments through which contaminants flow is important for determining the amount and extent of sediment/waste interaction. Of particular interest is the potential for sediments to adsorb radionuclides or hazardous constituents. 
Sample splits were analyzed mineralogically using X-ray diffraction and petrographic techniques. In preparation for X-ray diffractionanalysis, the sand- and silt-sized fractions were ground and randomly oriented. Twelve samples were run by PNL on an X-ray diffractometer and the mineralogies determined, qualitatively for the sand- to silt-sizedparticles and quantitatively for the clay-sizedfraction.

A total of 33 petrographic analyses of the sand-sized fraction were performed by Washington State University (WSU) on loose, grain-mounted thin sections with a polarizing optical microscope. A total of $\mathbf{3 0 0}$ grains were identified per sample. Samples were stained to help differentiate plagioclase from potassium feldspar.

The results of the mineralogical analyses are discussed in Section 3.2.1.

\subsubsection{Bulk Geochemistrx}

The bulk geochemistry of sediment samples provides useful information on the relative amounts of major and trace elements for the various hydrostratigraphicunits. Bulk geochemical analyses provide 1) background values for the major and trace elements present in the sediments and 2) a potential method to refine stratigraphiccorrelations.

Bulk geochemical analyses of borehole samples have been determined by PNL and WSU using the X-ray fluorescence (XRF) method. Samples were separated by grain size, and powdered specimens of selected grain sizes were analyzed by XRF. A total of 55 samples were analyzed for bulk geochemistry; most were duplicate samples analyzed independently by PNL and WSU laboratories. Sample results from the two laboratories were in general agreement, as demonstratedin Figure 10. Major elements analyzed for included Al, Ca, Fe, K, Mn, Si, and Ti. Trace elements included As, Ba, Br, Ce, Co, Cr, Cu, Ga, I, In, La, Mo, Nb, Ni, Pb, Rb, Rh, Ru, Se, Sr, Sn, U, V, Y, Zn, and Zr. The results of the bulk geochemicalanalyses are discussed in detail in Section 3.2.2. 
Well \#: 299-W7-5

Depth = 10'

Stratigraphic Unit: Hanford Formation

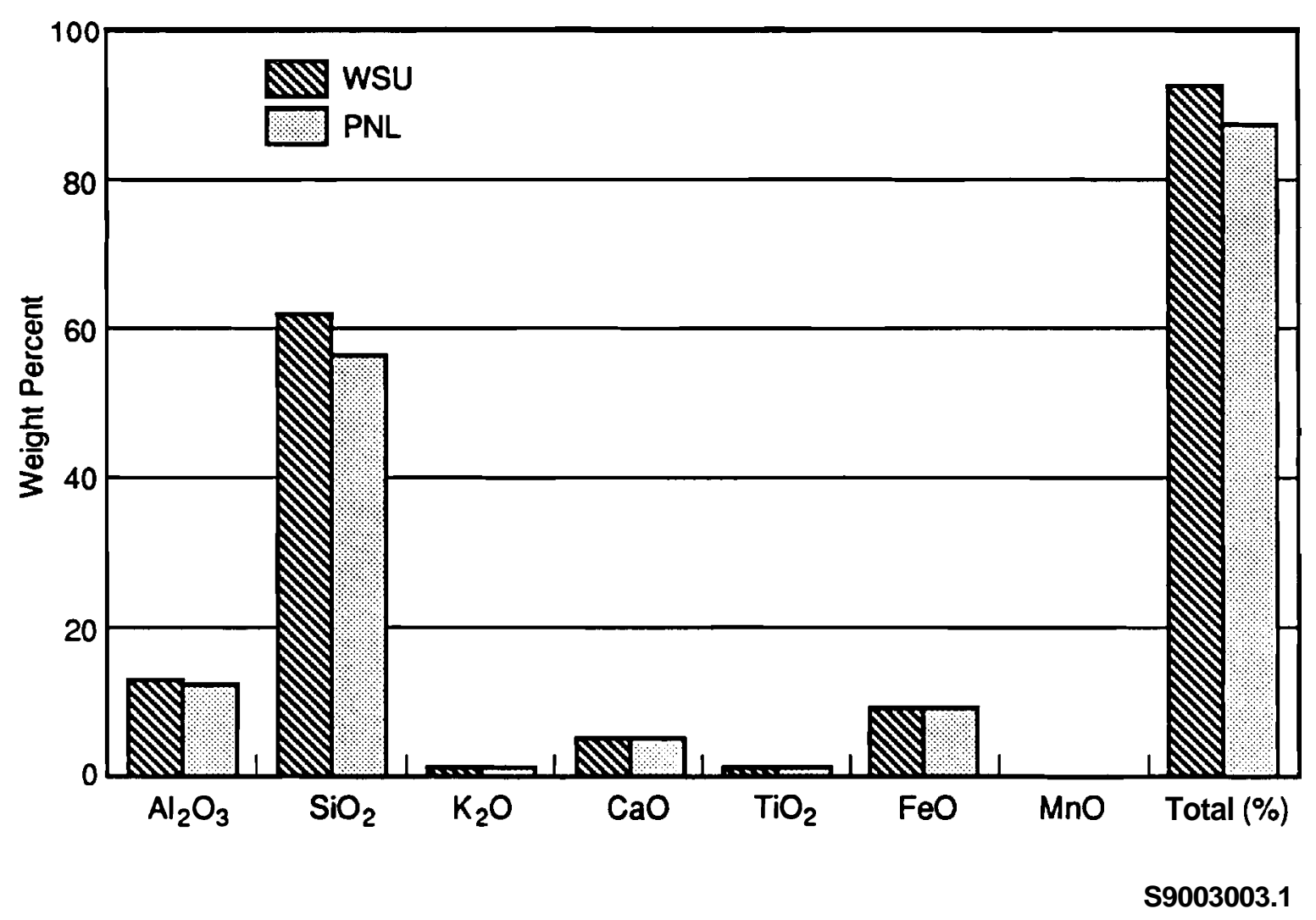

ElGURE 10. Comparison of X-Ray Fluorescence Analyses from WSU and PNL Laboratories 


\subsection{GEOLOGY}

This interpretation of the geology is based on information from 11 wells located within $1000 \mathrm{ft}$ of W-5 (Figure 2). These include seven wells installed as RCRA-compliance, ground-water monitoring wells, as well as three older wells documented by Last et al. (1989) and Last and Bjomstad (1989). The geology of the eleventh well, a recently drilledRCRA well (299-W7-9) that yielded nearly continuous split-barrel core samples to the top of the middle Ringold unit, is included with this report even though the well is incomplete.

Of the 11 wells used for the interpretation of geology and hydrology, seven are shallow wells primarily drilled to intercept the water table (210- to 310-ft depth), one terminates within the confining lower Ringold unit, and three intercept the uppermost basalt flow.

The stratigraphy of the area around W-5 is typical for that of the 200-West Area, as presented in Figure 3. The regional geology is discussed in other reports (Myers/Price et al. 1979; Myers and Price 1981; DOE 1986, 1988). Previous reports on the geology in the vicinity of W-5 include those by Tallman et al. (1979, 1981), Van Luik (1980), DOE (1988), and Last et al. (1989), and Last and Bjornstad (1989).

Surface soils in the vicinity of W-5 are classifiedas Ruport Sand (Hajek 1966) and support a dominantly sagebrush/cheatgrass plant community (Van Luik et al. 1980).

\subsection{STRATIGRAPHYANDUTHOLOGY}

The subsurface geology of the W-5 site has been interpreted from field descriptions and laboratory analyses of borehole cuttings and cores. For each well, these included two or more of the following: 1) geologist's logs, 2) gross gamma logs, 3) driller's logs, 4) granulometric analyses, and 5) calcium carbonate analyses. These data were evaluated in accordance with the characteristics used by Bjomstad (1985) and DOE (1988) to distinguish between the various geologic units.

An analysis of the geology and facies distributionbegan with construction of the three detailed cross sections represented in Figures 4 to 6 . Where data were not available for depths to the lower Ringold unit and the top of basalt, they were determined indirectly from structure contour maps presented by Last et al. (1989) and plotted onto the cross sections. From these cross sections, stratigraphic relationships were evaluated and correlations were made. From these correlations, a fence diagram (Figure 11) was generated. 
The sedimentary units overlying the Elephant Mountain Member of the Saddle Mountains Basalt in this area include the four Ringold lithologic units (basal, lower, middle, and upper), as well as the PlioPleistocene unit (Bjomstad 1984, 1985), the early "Palouse" soil (Brown 1960; Tallman et al. 1979), and the glaaofluvial Hanford formation (Tallman et al. 1979). Locally, a thin veneer of Holocene sand may overlie the Hanford formation. Except for complex facies relationshipstypical of the Hanfordformation, most of these stratigraphic units appear to be continuous beneath W-5. A more detailed discussion of each of these units except the Holocene sands is presented below.

\subsubsection{Saddle Mountains Basalt}

The Saddle Mountains Basalt is the uppermost formation of the Columbia River Basalt Group (Swanson et al. 1979). The uppermost basalt unit in the vicinity of W-5 is the Elephant Mountain Member (Van Luik et al. 1980; Last et al. 1989), which is dated at about 10.5 million years. W-5 lies along the northern flank of the Cold Creek syncline. Because it does, the basalt surface slopes gently to the southwest, ranging in elevation from $-190 \mathrm{ft}$ in the northeast to $-160 \mathrm{ft}$ in the southwest corner of W-5 (Figure 12).

\subsubsection{Binoold Formation}

Overlying the Elephant Mountain Member are fluvial-lacustrine deposits belonging to the Ringold Formation (Tallman et al. 1979, 1981). The Ringold Formation represents deposits from the ancestral Columbia and Snake rivers (Fecht et al. 1985). The Ringold Formation in the vicinity of the 200-West Area appears to be mostly of fluvial origin and contains a wide variety of sediment types (i.e., lithofacies), ranging from coarse sandy gravel (channel faaes) to fine-grainedmud (overbank-lacustrinefades) (Bjornstad 1985). For convenience and for consistency with past terminology, the Ringold Formation is subdivided into the basal, lower, middle, and upper Ringold units (Tallman et al. 1979).

These Ringold units, however, may not be correlative with the similarly defined units everywhere else in the Pasco Basin. For example, it has not been demonstrated, nor is it likely, that what is defined as the lower Ringold in the 200-West Area is correlative with what was originally defined as the lower Ringold unit in the east-central Pasco Basin (see Figure 1) by Newcomb (1958). Fluvial faaes, like those forming the Ringold Formation, may not be expected to correlate over more than a few kilometers (Walker and Cant 1984). For this reason, readers should not assume that the Ringold units referred to in this report are the same as those with the same names in other parts of the basin. Furthermore, considerable variation can occur, both within units of the Ringold Formation and within the Hanford formation. Because lithofacies better represent the lithologic heterogeneity inherent in fluvial depositional environments (Lindsey 


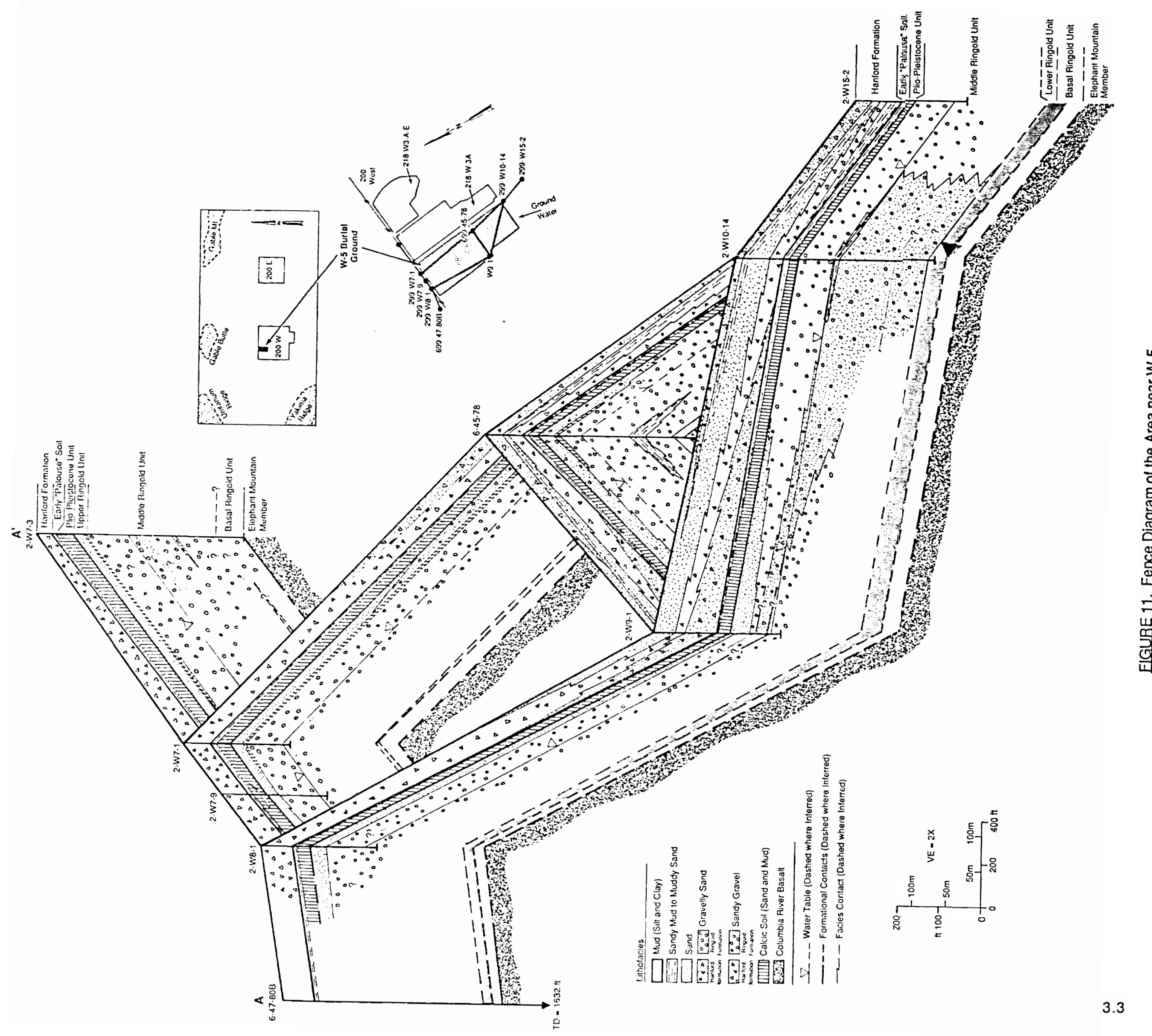

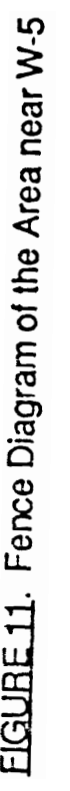




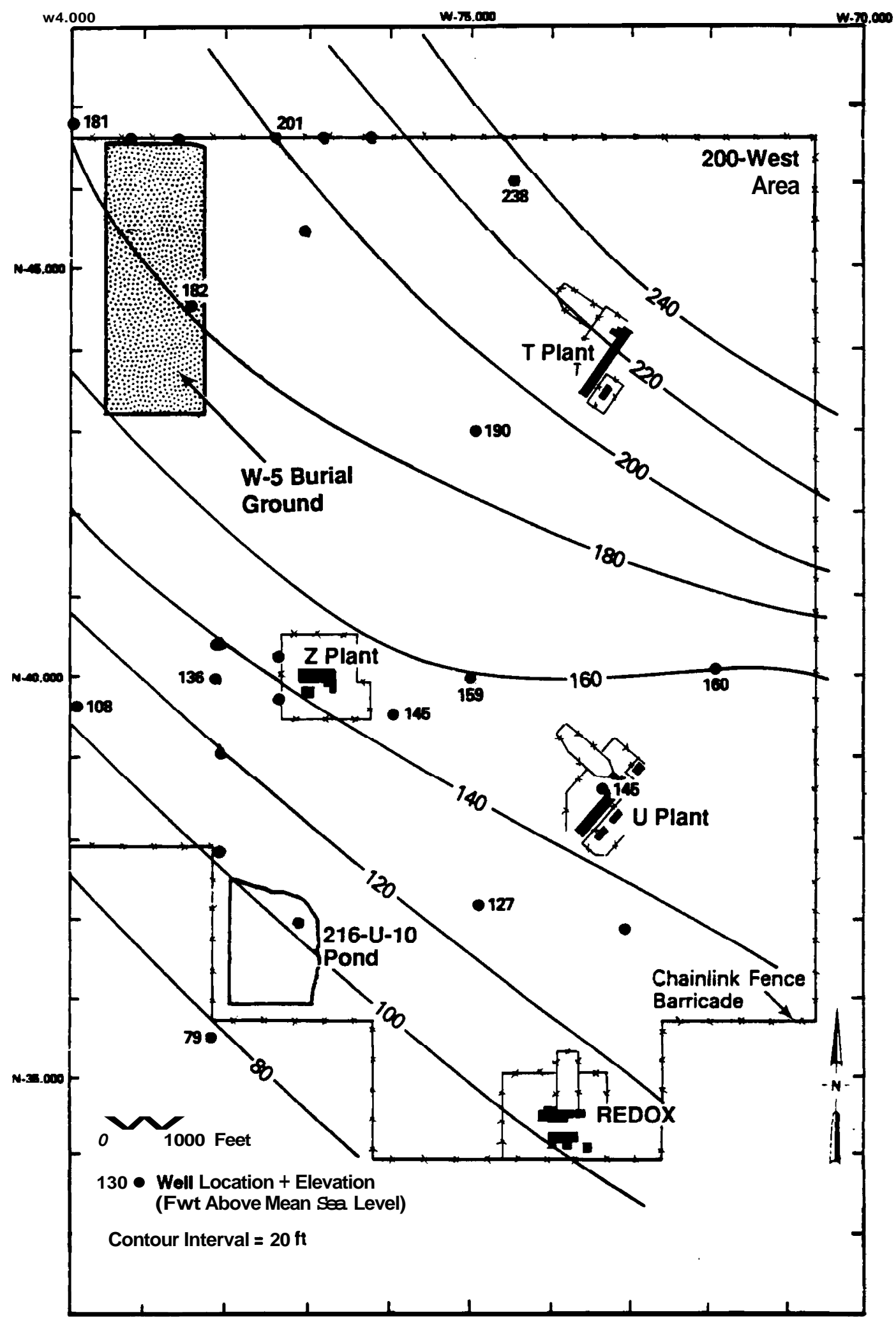

ElGURE 12. Surface of the Elephant Mountain Member Beneath the 200-West Area 
and Gaylord 1989; Lindsey et al. 1989), the use of lithofaaes such as those in the fence diagram (Figure 11) is a more appropriate method for describing and correlating stratigraphic units than is the use of traditional Ringold units (i.e., upper, middle, lower, basal).

\section{Basal Ringold Unit}

The basal Ringold unit is further subdivided into a coarse-grained and a fine-grained subunit (Bjornstad 1985; DOE 1988). The basal Ringold deposits show a moderate to strong consolidation, a result of either compaction or cementation. The coarse-grained subunit is composed of deposits of sandy gravel to gravelly sand. The coarse-grained Ringold sediments, which represent high-energy riverchannel deposits, are usually moderately to well sorted, the composition of gravel clasts is $<50 \%$ basalt, and neither the gravel nor the matrix generally reacts with dilute aad. The coarse-grainedbasal Ringold unit is overlain by increasingly finer sand and mud, which is capped by a well-developed, argillic (i.e., mudrich) paleosol; this sequence is referred to as the finegrained subunit of the basal Ringold unit. The thickness and extent of this unit is inferred from regional isopach and structure contour maps presented by Last et al. (1989) and Last and Bjornstad (1989).

\section{Lower Ringold Unit}

Above the basal Ringold unit is another fine-grained unit -the lower Ringold unit. The results of a hydrometer analysis from the lower Ringold unit corroborate the high mud content; the unit consists predominantly of silt (44\%), with approximately equal amounts of sand (29\%) and clay (27\%) (Table 3). The fine-grained lower Ringold unit is distinguished from the similar-textured basal Ringold paleosol by 1) the presence of welldeveloped laminae, 2) a distinct gray (as opposed to olive) color, and 3) a significantly higher natural-gamma response in geophysical logs (DOE 1988). The lower Ringold unit is generally less than $10 \mathrm{ft}$ thick beneath W-5 and, along with the basal Ringold fine-grained subunit, pinches out near the northeast corner of W-5 (Figure 13). Where these fine-grained strata are missing, it is not possible to differentiate between the middle and coarse-grainedbasal Ringold units, which share a common texture and mineralogy. The lower and fine-grained basal Ringold units form a confining layer, which separates the upper unconfined aquifer from a semiconfined aquifer (i.e., this coarse-grained basal Ringold subunit). This subject is discussed further in Section 4.0.

\section{Middle Ringold Unit}

The middle Ringold unit is about $300 \mathrm{ft}$ thick and is the thickest geologic unit beneath $\mathrm{W}-5$. The middle Ringold unit consists of mostly coarse-grained gravel and sand deposited in a high-energy fluvial 
IABLE 3. Hydrometer Analyses from Wells in the Vicinity of the W-5 Burial Ground

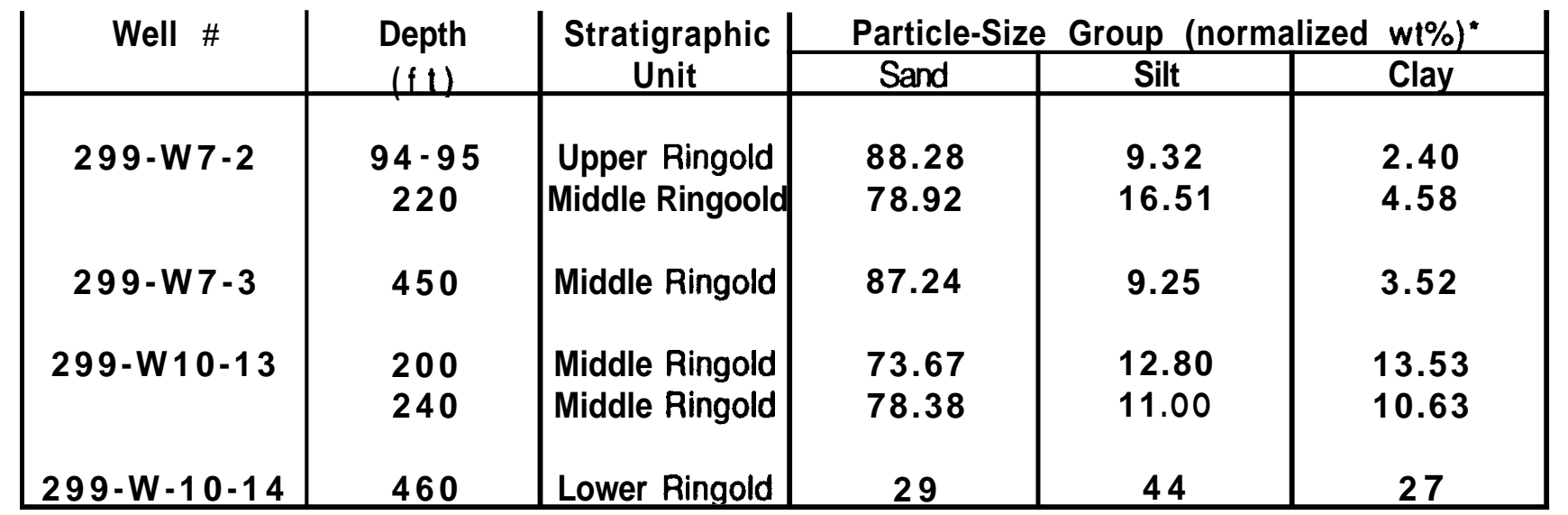

- Gravel-sized particles separated out prior to hydrometer analysis 


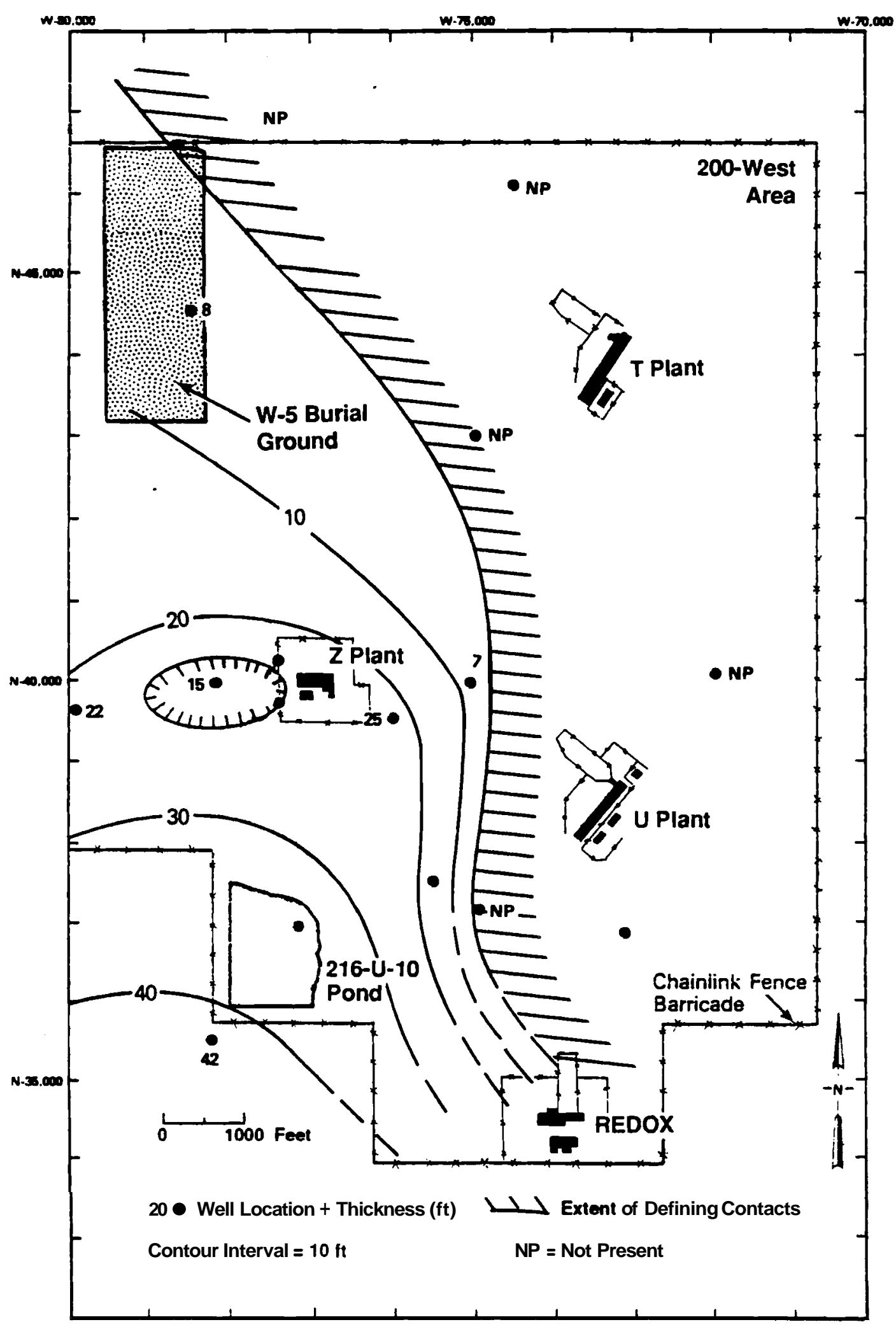

EIGURE 13. Thickness of the Lower Ringold Unit Beneath the 200-West Area 
environment. As in the case of the coarse-grained basal Ringold subunit, the characteristics used to define the middle Ringold are its 1) coarse texture, 2) relatively high proportion of quartzite and granitic clasts, 3) relatively low calcium carbonate content, 4) partial consolidation, and 5) relatively low naturalgamma response. Hydrometer analyses of the sandy matrix from four samples of the middle Ringold unit indicate that they may contain from 9 to $16 \%$ silt and 3 to $13 \%$ clay in the non-gravel fraction (Table 3 ). The uniform clast-supported texture of the middle Ringold unit is sometimes interrupted with what appear to be thin, probably discontinuous layers of mud and/or sand.

\section{Upper Bingold Unit}

The upper Ringold unit, which is $-35 \mathrm{ft}$ thick along the northern margin of $\mathrm{W}-5$, pinches out to the south (Van Luik et al. 1980; Last et al. 1989); this is shown in cross section B-B' (Figure 5). The upper Ringold unit consists of finer-grained deposits, mostly muddy sand to gravelly sand, which represent a transition to a lower-energy fluvial environment than in the middle Ringold. Hydrometer analysis of one <2-mm sample from the upper Ringold unit (Table 3) indicates that it is mostly sand (88.3\%), with only minor amounts of silt (9.3\%) and clay (2.4\%). The contact with the middle Ringold unit appears to be gradational and is generally defined by the interval above the middle Ringold where the amount of lightcolored arkosic sand exceeds the amount of gravel. Characteristics of the upper Ringold unit are its 1) abundance of well-sorted sand, 2) light color, caused primarily by the predominance of quartz and feldsparover basalt; and 3) variable natural-gamma response.

\subsubsection{Plio-Pleistocene Unit}

The Plio-Pleistoceneunit (Bjomstad 1984, 1985) consists of a chemically altered (i.e., calcareous) mixture of sand and mud, which represents a highly weathered paleosurface that developed atop the Ringold Formation (Brown 1959, 1960). While some aggradation of new material may be associated with this unit, much of the material is the result of in situ weathering of the uppermost Ringold Formation (i.e., middle or upper Ringold units). The characteristics of the Plio-Pleistocene unit, which suggest it is pedogenic in nature (Gile et al. 1966), are its almost white color, its high degree of cementation, and the presence of animal burrows and root traces in cores. Natural-gamma activity within the Plio-Pleistocene unit is erratic, high in places and moderate to low elsewhere.

The thickness of the Plio-Pleistoceneunit ranges from $-40 \mathrm{ft}$ along the north edge of $\mathrm{W}-5$ to $-20 \mathrm{ft}$ at the south, and the top of this unit dips approximated $1.5^{\circ}$ to the southwest (Figure 14). This is significant hydrologically, because highly cemented zones within this unit could inhibit the downward percolation of water in the unsaturated zone. If unsaturated flow moved downward along this surface, it would be moving opposite to the regional northeasterly trend of movement of ground water in the saturated zone (see Section 4.2). 


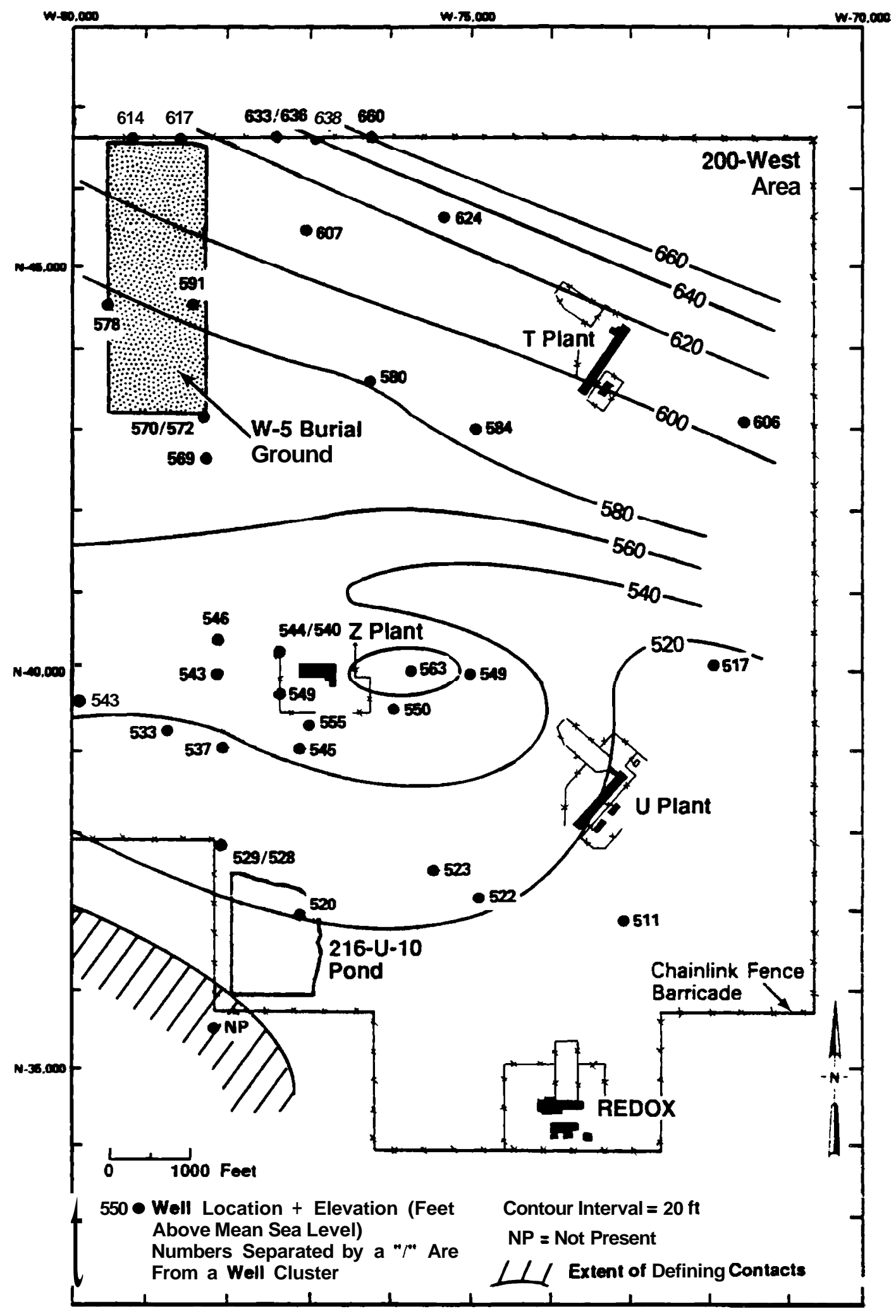

FIGURE 14. Top of the Plio-Pleistocene Unit 
The concentration of secondary calcium carbonate cement, a common soil-development product in arid environments, exceeds $30 \mathrm{wt} \%$ in some samples. In places, this secondary cement may completely fill the voids between sedimentary particles, making it relatively impermeable. The Plio-Pleistocene unit is defined as that interval below the early "Palouse" soil where the concentration of calcium carbonate exceeds $-5 \%$ by weight. Those areas in the Plio-Pleistocene unit that contain between 5 and $10 \%$ calcium carbonate are referred to as calcic soil; zones containing $>10 \%$ calcium carbonate are usually very firmly cemented and are referred to as calcrete (Machette 1985). Whether the Plio-Pleistocene unit in a particular area consists of a calcic soil or calcrete is indicated on the geologic cross sections (Figures 4 to 6). From the cross sections, it is apparent that the thickness and degree of calcic-soil development within the Plio-Pleistocene unit varies considerably from one location to another beneath W-5. This suggests that the impermeable calcrete layers are discontinuous and might cause only local perched-water even if there were enough recharge available for saturated conditions.

Interestingly, the Plio-Pleistocene unit in some places appears to be a single massive unit [e.g., at wells 299-W8-1 and 299-W7-1 (Figure 4)]. However, between these two wells (about a 600-ft distance), the two calcrete layers are separated by $15 \mathrm{ft}$ of non-calcareous, basaltic gravel and sand. This suggests that, locally, channels transporting sidestream alluvium incised into the weathered Ringold paleosurface.

\subsubsection{Earlv "Palouse" Soil}

Overlying the Plio-Pleistocene unit is the early "Palouse" soil, an unconsolidatedmuddy fine sand to fine sandy mud layer believed to be an early Pleistocene loess (windblown silt and sand). This deposit is derived from either reworked Plio-Pleistocene unit or upper Ringold material (Brown 1960). Characteristics of the early "Palouse" soil include 1) uniform fine-grainedtexture, 2) unconsolidated nature, 3) high mica content, and 4) moderate calcium carbonate content (2-5\%). The most distinctivecharacteristic of the early "Palouse" soil is its high natural-gamma log response; this is particularly apparent in cross section A-A' (Figure 4).

Compared to the Plio-Pleistocene unit, the early "Palouse" soil is relatively unconsolidated and less calcareous, and it displays a consistently higher natural-gamma response. Although thin (-10-15ft), the early "Palouse" soil appears to form a continuous, relatively uniform layer beneath W-5. Van Luik et al. (1980) reported a thickness of at least $25 \mathrm{ft}$ for the early "Palouse" soil beneath W-5. However, it is likely that they merged the early "Palouse" soil and Plio-Pleistocene units, because without a careful analysis of calcium carbonate data and gross gamma logs it can be difficult to differentiatethese units. 


\subsubsection{Hanford Formation}

The Hanford formation was deposited by Pleistocene cataclysmic floods (Myers/Price et al. 1979), which first inundated eastern Washington as early as 1 million years ago (Bjornstad and Fecht 1989). These floods were derived from the sudden release of glacial Lake Missoula near the northern Idaho/Montana border and covered eastern Washington perhaps dozens of times between 1 million and 13,000 years ago (Baker 1973; Waitt 1980). In the Pasco Basin, these floods eroded into the Ringold Formation and blanketed the area with mostly coarse-grained, poorly sorted glaaofluvialdeposits that are now referred to, informally, as the Hanford formation.

The Hanford formation is divided into three facies. Coarser sand and gravels were deposited where the current energy of the flooding was greatest; these deposits are referred to as the Pasco Gravels (Myers/Price et al. 1979). In what were slack-water areas during flooding, deposits consist predominantly of sand and silt belonging to the Touchet Beds (Flint 1938; Bjornstad 1980). The third facies, referred to here as the "transitionalfacies," is predominantly plane-laminatedsand (Bjornstad et al. 1987), but it may have characteristics of the Pasco Gravels or the Touchet Beds or both. The "transitional facies" occurs in depositional environments where flood gravels do not occur or where the intensity of the current energy was intermediate between those that deposited the Pasco Gravels and Touchet Beds.

The thicknesses and facies distribution within the Hanford formation are highly variable because of the dynamic nature of the flood currents in the vicinity of W-5. A detailed facies analysis of the Hanford formationis not possible with the present distribution of boreholes. This is a result of the highly variable nature of the Hanford lithofaaes, which can alternate and grade from coarse-grained cobbles and boulders to fine-grained sand and silt within a few hundred feet.

Geomorphically, W-5 lies along the northern edge of a giant flood bar (Cold Creek bar) and near the intersection of two flood channels (Figure 15). A major east-west flood channel lies north of Cold Creek bar, and a secondary north/south-trending channel runs parallel to the eastern boundary of W-5.

During the development of the relatively flat-topped Cold Creek bar, aggradation of flood deposits covered a sloping paleosurface. This resulted in less accumulation $(-50 \mathrm{ft})$ of sediments to the north of W-5 than to the south, where as much as $150 \mathrm{ft}$ of sediments were deposited in the Hanford formation (Figure 16).

Along the northern edge of Cold Creek bar, the Hanford formation appears to consist of a single sequence of coarse-grained bouldery gravel and sand (Pasco Gravels fades). To the south, on the other hand, where flood current energy was less vigorous, these coarse-grained deposits are replaced with "transitional" and/or Touchet Beds facies (Figure17). 


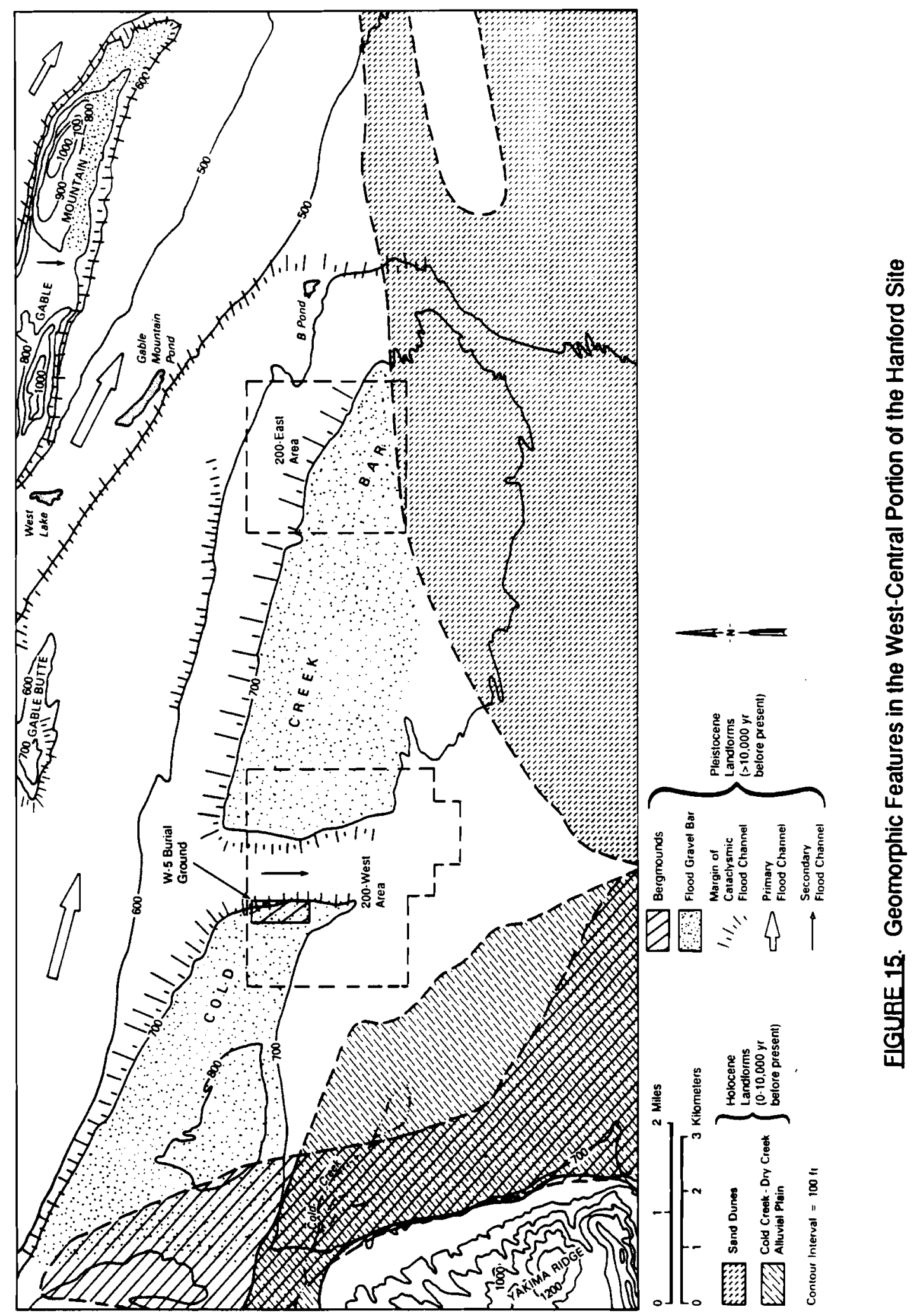




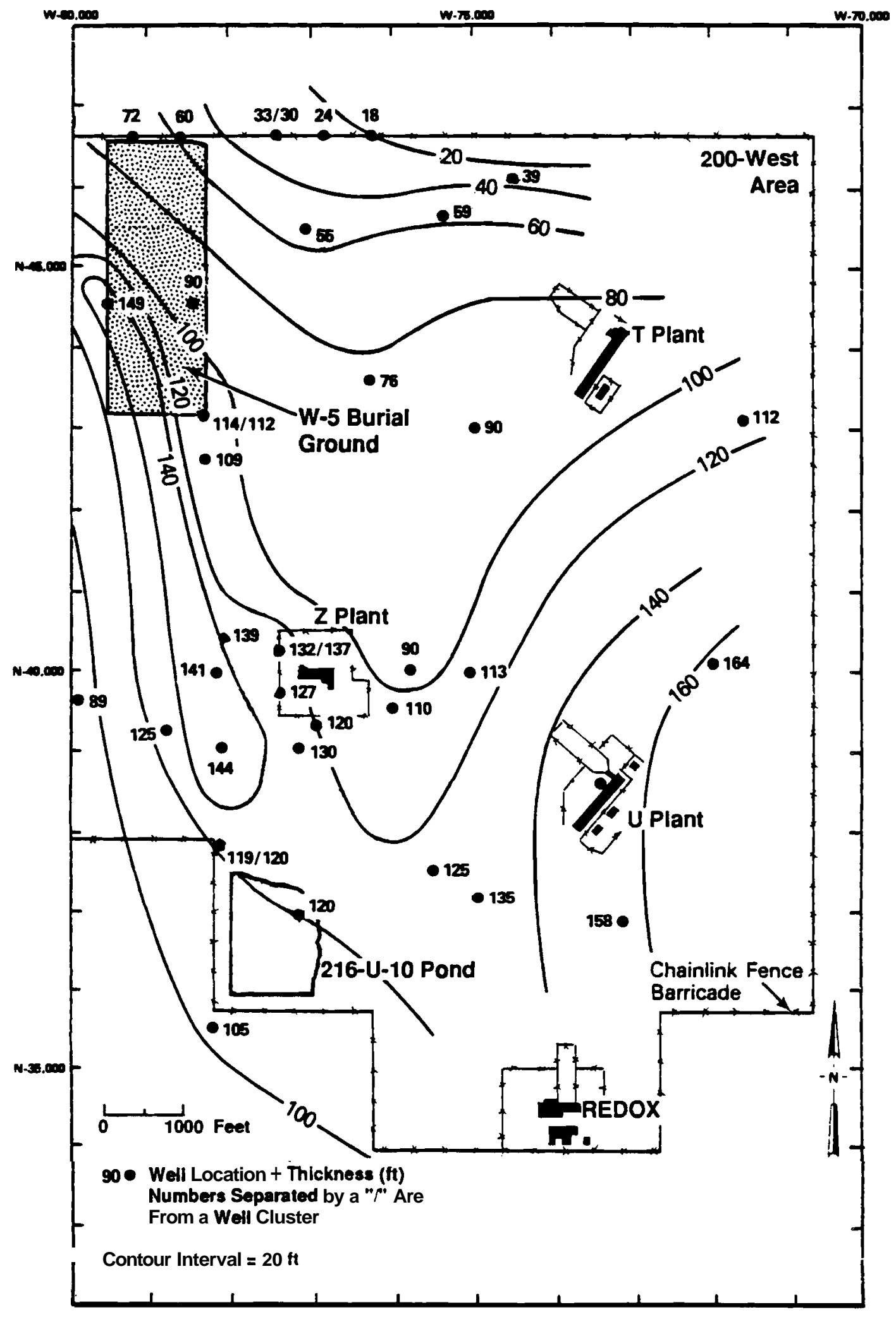

ElGURE 16. Thickness of the Hanford Formation Beneath the 200-West Area 


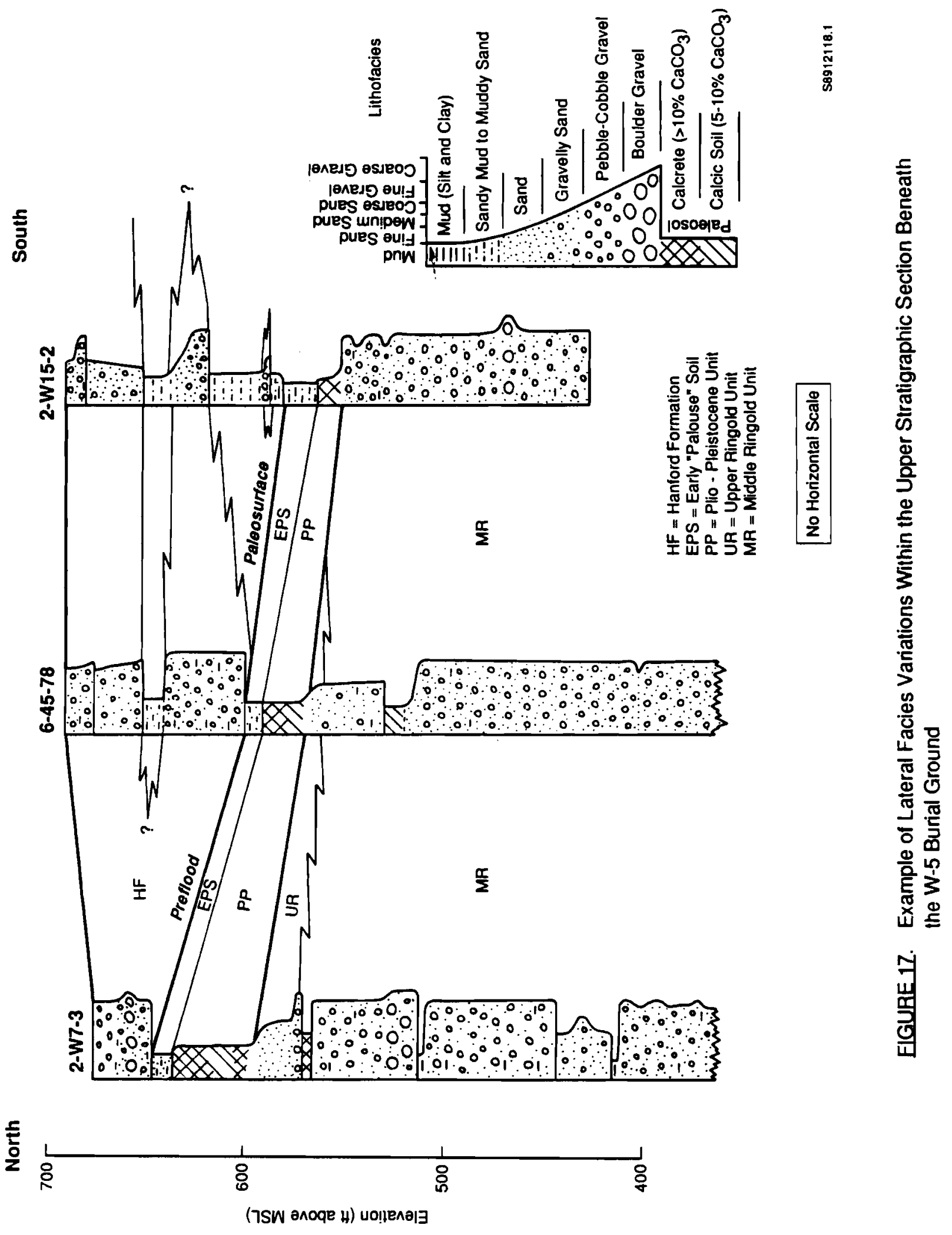


Clastic dikes (Figure 18) are a ubiquitous sedimentary structure observedin, but not restricted to, outcrops and trenches that expose the Hanford formation. These dikes are thought to represent dewatering structures that developedduring compaction and settling of cataclysmic flood deposits during or soon after floodwaters drained from the Pasco Basin (Bergeron et al. 1987).

The true nature and extent of clastic dikes are poorly understood, however, because the dikes are rarely detected or observedin vertically oriented boreholes. This occurs because the clastic dikes are usually oriented vertically, although some may extend horizontally as well. Often they form a polygonal pattern where they intersect the ground surface; patterned ground is reported near the southern boundary of the 200-West Area (Lillie et al. 1978). Clastic dikes are significant hydrologically because they occur as alternating, vertically laminated layers of sand and mud, which may total a meter or more in thickness. They could act as hydrologic barriers to the lateral migration of water, in both the saturated and the unsaturated zones. At the same time, clastic dikes could act as vertical conduits whereby vadose-zone water would preferentially flow downward within the coarser-grained laminae in the dikes, as shown in Figure 18.

Clastic dikes are more common in slack-water flood sequences, although they may be present in the Pasco Gravels faaes as well. No clastic dikes are visible in burial trench \#40 in the 216-W-3A burial ground just east of W-5; the trench exposes coarse-grained Hanford formation gravels. The presence of clastic dikes at $\mathrm{W}-5$ is more likely to the south, where the texture of the Hanford formation is finer grained (Figure 5).

Locally, the Hanford formation may be overlain by a few feet or less of windblown sand to silty sand.

\subsection{MINERALOGY AND BUIK GEOCHEMISTRY}

Quantitative mineralogical and geochemical data for the sediments within the Pasco Basin are limited. Most of the following discussion is based on recent petrographic, X-ray diffraction(XRD) and X-ray fluorescence (XRF) analyses performed at PNL and WSU laboratories. The only previous mineralogical work was that of Tallman et al. (1979), who reported semiquantitative XRD and microprobe analyses on sediment samples from the 200 Areas. The Tallman study revealed 1) no significant differences in the types of minerals detected in the fine-grainedportions of samples (<74 microns) and 2) the presence of quartz, feldspar, mica, srnectite, and chlorite in all stratigraphic units sampled from several different wells. The results of microprobe analyses reported by Tallman et al. (1979) corroboratedthe identification of an 


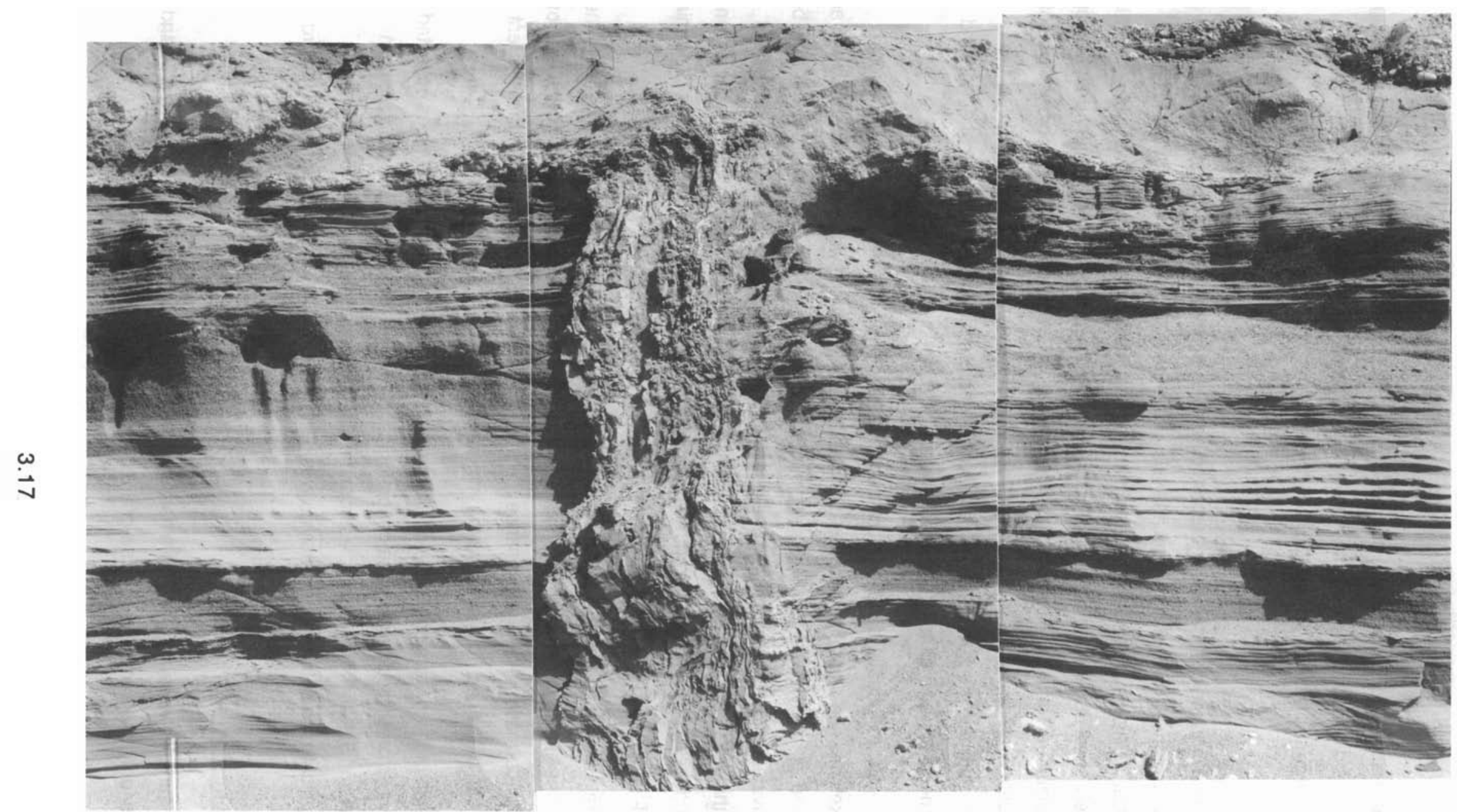

FIGURE 18. Clastic Dike 
arkosic (i.e., predominantly quartz + feldspar) composition for the suprabasalt sediments. The following subsections present and discuss the results of more comprehensive mineralogical and geochemical analyses recently performed by PNL and WSU.

\subsubsection{Mineralogy}

Two types of mineralogical analysis are reported here: 1) petrographic analysis of sand- to gravelsized particles (>62 microns) and 2) clay mineral analysis, using the XRD method, of particles less than 4 microns in diameter. Results of petrographic analysis are presented in Table 4. Overall, clay minerals represent a relatively small percentage of the total volume for most suprabasalt sedimentary units, except the lower Ringold unit (see Table 3). Because of their small size, silt particles (3.9-62.5 microns) are not usually analyzed petrographically. Based on the petrography of the sand and coarser particles, the suprabasalt sediments within the 200 -West Area are considered predominantly mixtures of quartz and feldspar; rock fragments are also a major constituent (Figure 19).

Rock fragments are usually identified from the gravel-size portion of the sample. Once rock fragments are broken down to sand-size particles, they usually appear as individual mineral grains and are no longer recognized as rock fragments. For example, during hard-tool drilling, a granite boulder may be reduced to the individual minerals (monocrystalline quartz, potassium feldspar, mica, hornblende, and opaque minerals) that together constitute a granitic rock. Hard-tool drilling through a basalt boulder, on the other hand, produces abundant basalt fragments, in addition to individual grains of mafic rock-forming minerals, such as plagioclase feldspar and pyroxene.

Clay minerals are also present. Although they are a relatively minor constituent compared to the total volume, the types of clay minerals present serve as important indicators of the geochemical environment. Thus, much information on the provenance (i.e., source) and diagenetic history of the different stratigraphic units can be gained from studying the mineralogy.

A discussion of the mineralogy of the different stratigraphic units, based on samples collected from 200-West Area, is presented below. The mineralogical data used for this interpretation are summarized in Tables 4 and 5. All the XRD samples from the Ringold units listed in Table 5 were also analyzed petrographically; no petrographic analysis was performed for XRD samples from the Hanford formation.

\section{Bingold Formation}

A total of 17 samples were analyzed from hard-tool drill cuttings of the coarse-grainedmiddle and basal Ringold units; only one sample of the fine-grained lower Ringold unit was analyzed. 
TABLE 4. Petrographic Data from 200-West Area Wells

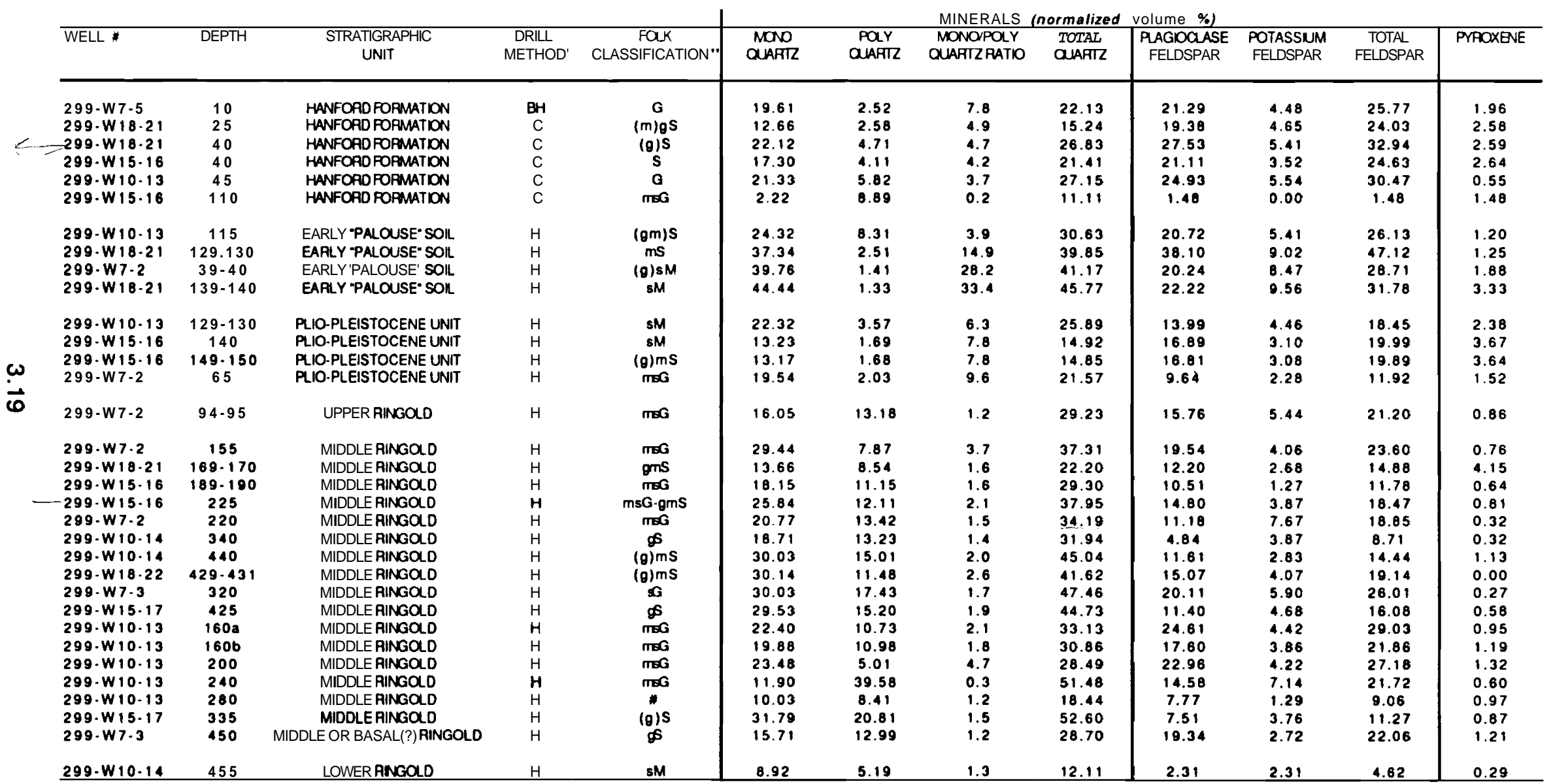

BH-backhoe, C=drive barrel. H-hard tool

-M=mud. Susand, G-gravel, m= muddy, 8- sandy. g-gravelly, ( )=slighlly

- No grain-size data 
TABLE 4. (contd)

\begin{tabular}{|c|c|c|c|c|c|c|c|c|c|c|c|c|c|}
\hline & & & INERAL & & & & & & ROCK FRAG & ENTS (normal & volume $\%$ & & \\
\hline $\begin{array}{l}\text { HORN- } \\
\text { BLENDE }\end{array}$ & BIOTITE & MUSCONITE & $\begin{array}{l}\text { TOTAL } \\
\text { MICA }\end{array}$ & OPAOUES & GARNET & EPDOTE & $\begin{array}{l}\text { Malic } \\
\text { clasts }\end{array}$ & $\begin{array}{c}\text { Granitic } \\
\text { clasts }\end{array}$ & $\begin{array}{l}\text { Metamorphic } \\
\text { clasts (exclud. } \\
\text { ino quartzit } \theta \text { ) }\end{array}$ & $\begin{array}{l}\text { Sedimentary } \\
\text { aggregates }\end{array}$ & $\begin{array}{c}\text { Carbonate } \\
\text { clasis }\end{array}$ & $\begin{array}{l}\text { Other } \\
\text { clasts }\end{array}$ & Total \\
\hline 1.12 & 3.08 & 0.00 & 3.08 & 1.96 & 0.00 & 0.00 & 29.41 & 2.80 & 0.56 & 1.40 & 1.12 & 8.68 & 43.97 \\
\hline 1.55 & 4.85 & 0.78 & 5.43 & 1.55 & 0.00 & 0.00 & 33.85 & 5.43 & 0.52 & 2.33 & 1.55 & 5.94 & 49.82 \\
\hline 1.18 & 2.35 & 0.24 & 2.59 & 2.59 & 0.94 & 0.24 & 11.29 & 9.65 & 1.42 & 1.41 & 0.24 & 6.35 & 30.38 \\
\hline 1.17 & 1.47 & 1.17 & 2.64 & 2.35 & 0.29 & 0.00 & 32.55 & 3.52 & 1.17 & 1.47 & 0.29 & 5.88 & 44.86 \\
\hline 1.68 & 6.09 & 0.28 & 6.37 & 1.11 & 0.00 & 0.00 & 8.92 & 11.91 & 1.39 & 1.66 & 2.22 & 8.59 & 32.69 \\
\hline 0.00 & 0.74 & 0.00 & 0.74 & 0.00 & 0.00 & 0.00 & 84.44 & 7.41 & 0.00 & 3.33 & 0.00 & 8.89 & 84.07 \\
\hline 0.90 & 5.41 & 0.90 & 6.31 & 0.90 & 0.30 & 0.00 & 11.71 & 11.71 & 0.90 & 2.70 & 1.20 & 5.40 & 33.62 \\
\hline 1.25 & 1.25 & 0.25 & 1.50 & 0.00 & 0.00 & 0.50 & 0.50 & 0.75 & 0.25 & 4.51 & 0.75 & 1.75 & 8.51 \\
\hline 0.71 & 8.12 & 2.35 & 8.47 & 1.18 & 0.24 & 0.00 & 4.24 & 1.85 & 0.24 & 8.94 & 1.85 & 0.94 & 17.66 \\
\hline 2.44 & 4.22 & 0.44 & 4.66 & 1.33 & 0.67 & 0.00 & 1.33 & 1.33 & 0.22 & 0.22 & 1.78 & 2.00 & 6.88 \\
\hline 1.79 & 2.38 & 1.19 & 3.57 & 1.19 & 0.30 & 0.00 & 3.27 & 3.27 & 0.30 & 25.30 & 11.61 & 2.39 & 46.14 \\
\hline 0.28 & 0.57 & 0.28 & 0.85 & 1.13 & 0.28 & 0.00 & 10.42 & 2.53 & 0.00 & 0.28 & 44.76 & 0.85 & 58.84 \\
\hline $\begin{array}{l}0.28 \\
0.28\end{array}$ & 0.58 & 0.28 & $\begin{array}{l}.85 \\
0.84\end{array}$ & 1.12 & 0.28 & 0.00 & 10.36 & 2.52 & 0.00 & 0.28 & 44.54 & 0.84 & 58.54 \\
\hline 0.78 & 1.52 & 0.25 & 1.77 & 1.78 & 0.25 & 0.00 & 17.01 & 5.84 & 0.00 & 31.98 & 2.03 & 3.55 & 60.41 \\
\hline 0.29 & 2.29 & 0.00 & 2.29 & 0.29 & 0.00 & 0.29 & 20.34 & 16.05 & 1.15 & 1.15 & 0.00 & 6.87 & 45.58 \\
\hline 0.25 & 1.27 & 0.76 & 2.03 & 0.76 & 0.25 & 0.25 & 9.65 & 16.24 & 0.25 & 1.02 & 0.00 & 7.61 & 34.77 \\
\hline 0.24 & 0.73 & 0.00 & 0.73 & 1.95 & 0.00 & 0.49 & 38.05 & 5.85 & 0.00 & 1.46 & 0.00 & 7.08 & 52.44 \\
\hline 0.32 & 0.84 & 0.32 & 0.96 & 0.84 & 0.00 & 0.00 & 18.80 & 11.15 & 2.55 & 8.60 & 0.00 & 15.29 & 58.39 \\
\hline 4.81 & 0.54 & 0.27 & 0.81 & 1.07 & 0.27 & 0.00 & 12.92 & 13.73 & 5.37 & 2.96 & 0.00 & 5.65 & 40.63 \\
\hline 0.32 & 0.84 & 0.00 & 0.64 & 0.00 & 0.00 & 0.00 & 13.42 & 18.21 & 4.15 & 3.83 & 0.00 & 4.79 & 44.40 \\
\hline 0.00 & 0.32 & 0.65 & 0.97 & 0.00 & 0.00 & 0.00 & 23.22 & 9.68 & 3.22 & 16.45 & 0.00 & 5.48 & 58.05 \\
\hline 0.85 & 1.13 & 0.85 & 1.98 & 0.85 & 0.85 & 0.00 & 10.49 & 5.95 & 2.55 & 10.20 & 0.28 & 4.82 & 34.29 \\
\hline 0.98 & 1.44 & 0.00 & 1.44 & 0.72 & 0.72 & 0.00 & 7.42 & 10.77 & 1.87 & 11.24 & 0.00 & 2.63 & 33.73 \\
\hline 0.80 & 2.14 & 0.27 & 2.41 & 0.80 & 0.27 & 0.00 & 8.04 & 6.97 & 1.34 & 1.81 & 0.27 & 3.76 & 21.99 \\
\hline 0.58 & 1.75 & 0.00 & 1.75 & 0.58 & 0.29 & 0.29 & 8.14 & 9.08 & 2.83 & 11.11 & 0.29 & 4.37 & 33.60 \\
\hline 0.63 & 2.21 & 0.32 & 2.53 & 2.21 & 0.00 & 0.32 & 10.72 & 12.82 & 2.52 & 0.63 & 0.32 & 4.31 & 31.12 \\
\hline 0.59 & 0.59 & 0.30 & 0.89 & 0.89 & 0.00 & 0.00 & 18.99 & 11.87 & 1.18 & 2.97 & 1.19 & 7.72 & 43.92 \\
\hline 1.06 & 1.85 & 0.00 & 1.85 & 0.53 & 0.00 & 0.26 & 8.44 & 20.32 & 1.06 & 4.75 & 0.00 & 4.75 & 39.32 \\
\hline 0.30 & 2.88 & 0.30 & 2.98 & 0.00 & 0.60 & 0.00 & 3.87 & 10.71 & 0.30 & 3.57 & 0.00 & 3.87 & 22.32 \\
\hline 0.00 & 0.97 & 0.32 & 1.29 & 0.97 & 0.32 & 0.00 & 26.88 & 9.39 & 1.94 & 22.98 & 1.94 & 5.50 & 88.81 \\
\hline 0.29 & 0.58 & 0.00 & 0.58 & 0.29 & 0.00 & 0.29 & 8.38 & 8.87 & 1.18 & 11.85 & 0.29 & 3.47 & 33.82 \\
\hline 0.60 & 0.30 & 0.00 & 0.30 & 0.00 & 0.80 & 0.60 & 28.09 & 6.95 & 1.81 & 0.91 & 0.00 & 7.88 & 45.62 \\
\hline 0.00 & 0.00 & 0.29 & 0.29 & 0.00 & 0.00 & 0.00 & 2.01 & 4.61 & 0.29 & 75.22 & 0.00 & 0.58 & 82.71 \\
\hline
\end{tabular}




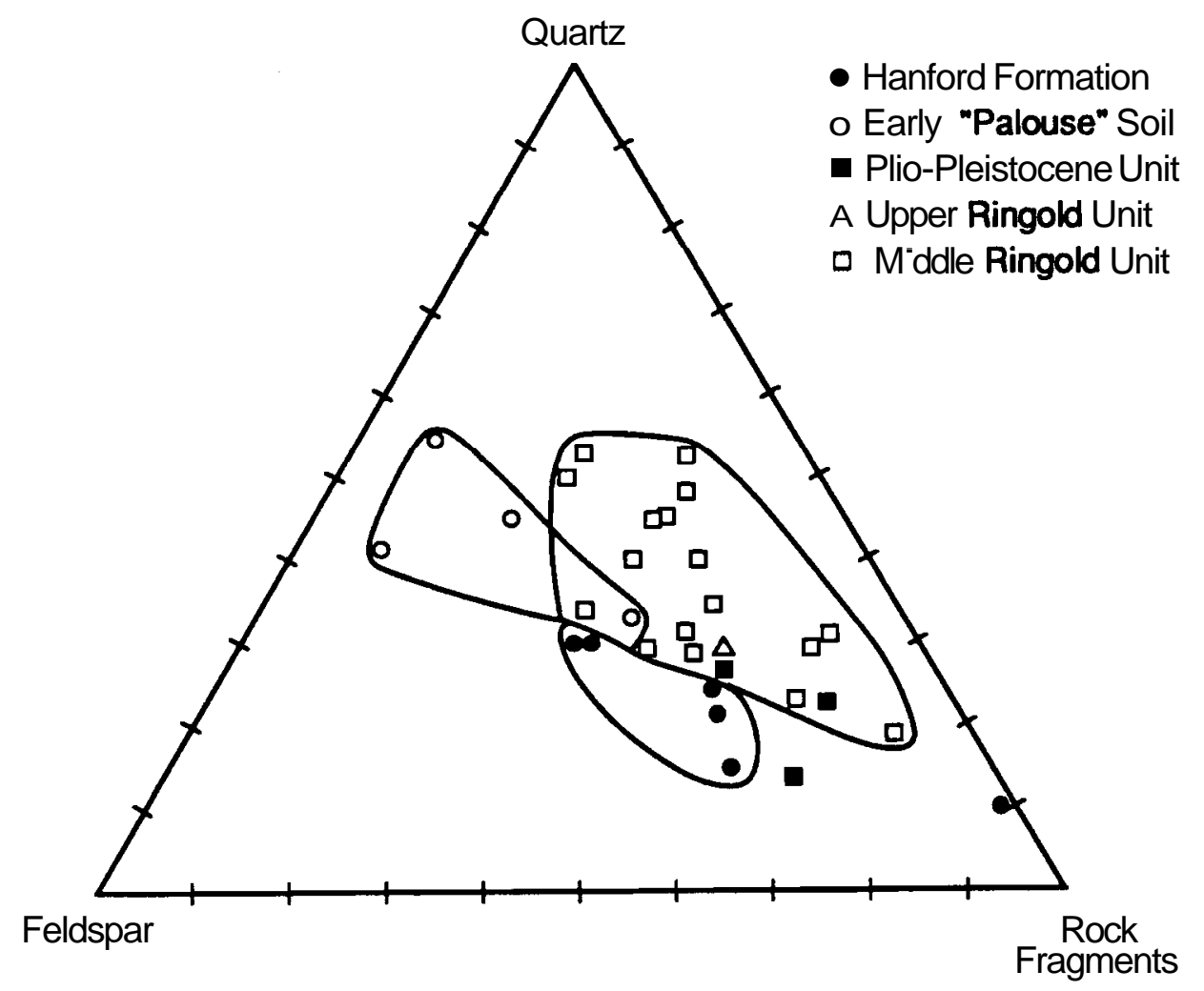

S9002061.4

EIGURE 19. Ternary Diagram Showing the Ratio of Quartz, Feldspar, and Rock Fragments for Various Stratigraphic Units

Petrographically, the lower Ringold sample consists predominantly of sedimentary aggregates (73\% by volume), followed by quartz (12\%), and feldspar (5\%). The high proportion of sedimentary aggregates actually represents mud clumps, which result from hard-tool drilling through the soft, cohesive muds of the lower Ringold unit.

Within the coarse-grained Ringold deposits, quartz is the predominant mineral (up to 53\%). The higher quartz content relative to other stratigraphic units (Figures 19 and 20) is probably a result of the higher proportion of arkosic sand and quartzite gravel clasts, which were transportedinto the Pasco Basin from the margins of the Columbia Plateau by the ancestral Columbia and Snake rivers (Fecht et al. 1985). Quartzite is a metamorphosed rock consisting of tightly bonded grains of quartz; as a result, it is very resistant to weathering and abrasion. During hard-tool drilling, quartzite pebbles and cobbles are pulverized by the hard-tool bit and reduced to sand grains, which, when observed under a petrographic 
IABLE 5. Results of Clay Mineralogy Analyses

\begin{tabular}{|c|c|c|c|c|c|c|c|c|}
\hline Woll * & \multirow{2}{*}{$\begin{array}{l}\text { Depth } \\
(\mathrm{ft})\end{array}$} & \multirow{2}{*}{$\begin{array}{c}\text { Folk } \\
\text { Classiflcation *t }\end{array}$} & \multirow{2}{*}{$\underset{\text { Unit }}{\text { Stratigraphic }}$} & \multicolumn{5}{|c|}{ CLAY MINERALS (normalized weight \%) } \\
\hline & & & & Illite & $\begin{array}{c}\text { Kaolinite } \\
\text { Group }\end{array}$ & $\begin{array}{c}\text { Chlorite } \\
\text { Group }\end{array}$ & $\begin{array}{c}\text { Vermiculite } \\
\text { Group }\end{array}$ & $\begin{array}{c}\text { Smectite } \\
\text { Group }\end{array}$ \\
\hline $\begin{array}{l}299-E 28-26 \\
299-E 34-3 \\
299-W 15-16 \\
299-E 28-26\end{array}$ & $\begin{array}{c}294-295 \\
49-50^{\prime} \\
110^{\prime} \\
319-320\end{array}$ & $\begin{array}{l}m \otimes G \\
m \otimes G \\
m \otimes G \\
\mathbf{s} G\end{array}$ & $\begin{array}{l}\text { Hanford Formation } \\
\text { Hanlord Formation } \\
\text { Hanford Formation } \\
\text { Hanford Formation? }\end{array}$ & $\begin{array}{l}11 \\
11 \\
13 \\
16\end{array}$ & $\begin{array}{l}22 \\
13 \\
15 \\
27\end{array}$ & $\begin{array}{l}26 \\
11 \\
15 \\
27\end{array}$ & $\begin{array}{l}19 \\
10 \\
10 \\
9\end{array}$ & $\begin{array}{l}23 \\
55 \\
47 \\
21\end{array}$ \\
\hline & & & Average & 13 & 19 & 20 & 12 & 37 \\
\hline 299-W7-2 & $94-95$ & $m \in G$ & Upper Rinqold Unit & 17 & 21 & 27 & 5 & 30 \\
\hline $\begin{array}{l}299-W 7-2 \\
299 \cdot W 10 \cdot 13 \\
299-W 10-13 \\
299-W 18-22\end{array}$ & $\begin{array}{c}220 \\
200^{\prime} \\
240 \\
\mathbf{4 2 9 \cdot 4 3 0}\end{array}$ & $\begin{array}{c}m E G \\
m \in G \\
m \& G \\
(g) m S\end{array}$ & $\begin{array}{l}\text { Middle Ringold Unit } \\
\text { Middle Ringold Unit } \\
\text { Middle Ringold Unit } \\
\text { Middle Ringold Unit }\end{array}$ & $\begin{array}{l}2 \\
4 \\
1 \\
2\end{array}$ & $\begin{array}{l}5 \\
8 \\
2 \\
3\end{array}$ & $\begin{array}{l}6 \\
2 \\
0 \\
3\end{array}$ & $\begin{array}{l}1 \\
7 \\
1 \\
0\end{array}$ & $\begin{array}{l}86 \\
79 \\
96 \\
95\end{array}$ \\
\hline & & & Average & 2 & 5 & 3 & 2 & 89 \\
\hline $299-W 7-3$ & 450 & gs & Basal Ringold Unit & 1 & 5 & 4 & 5 & 85 \\
\hline
\end{tabular}

'Sample is from unsaturated zone

•"M=mud, $\mathbf{S = s a n d , ~} \mathbf{G = g r a v e l ,} \mathbf{m =}$ muddy, $\mathbf{s =}$ sandy, $\mathbf{g}=$ gravelly, ( )=slightly 


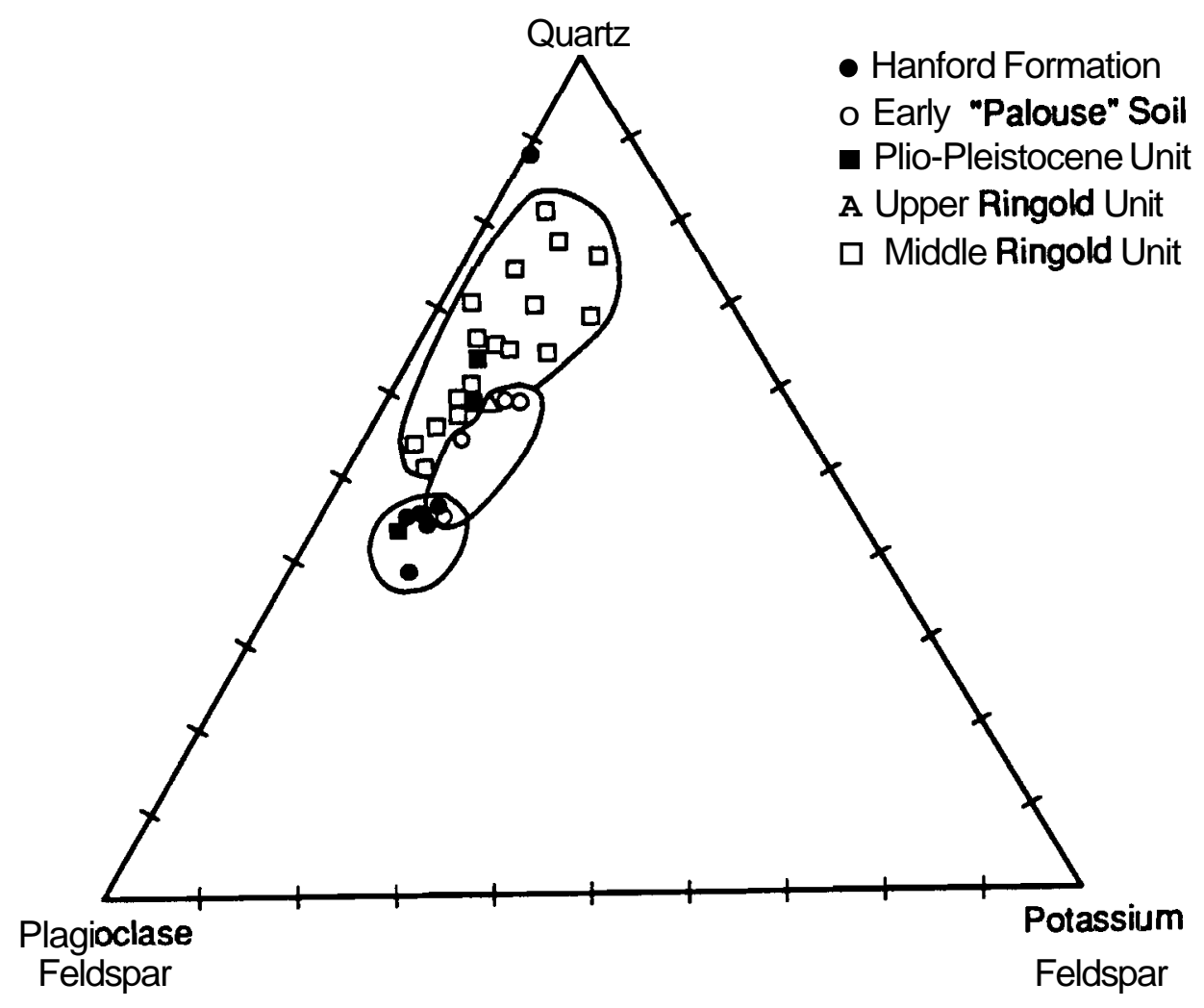

S9002061.3

EIGURE 20. Temary Diagram Showing the Ratio of Quartz, Plagioclase, and Potassium Feldspar for Various Stratigraphic Units

microscope may appear as polycrystalline quartz. The results of this pulverization are reflectedin the ratio of polycrystallineto monocrystalline quartz (Table 4), which is considerably higher for the middle Ringold unit than for younger stratigraphic units.

The second most predominant mineral is feldspar, which accounts for up to $29 \%$ of the volume of Ringold sediments; plagioclase feldspar is significantly more abundant than potassium feldspar (Figure 20). Coarse-grainedRingold deposits have less mica (biotite and muscovite) than the younger stratigraphic units. The proportions and types of rock fragments vary considerably within the coarsegrained Ringold units, which range from 21 to $69 \%$ of the total volume. In general, mafic rock fragments (principallybasalt) are the most abundant type of rock fragment; quartzite clasts might be as abundant but drilling probably reduced most quartzite clasts to smaller grains of polycrystallinequartz. Other lithic constituents include granitic clasts (Figure 21), which are followed in abundance by sedimentary aggregates. 


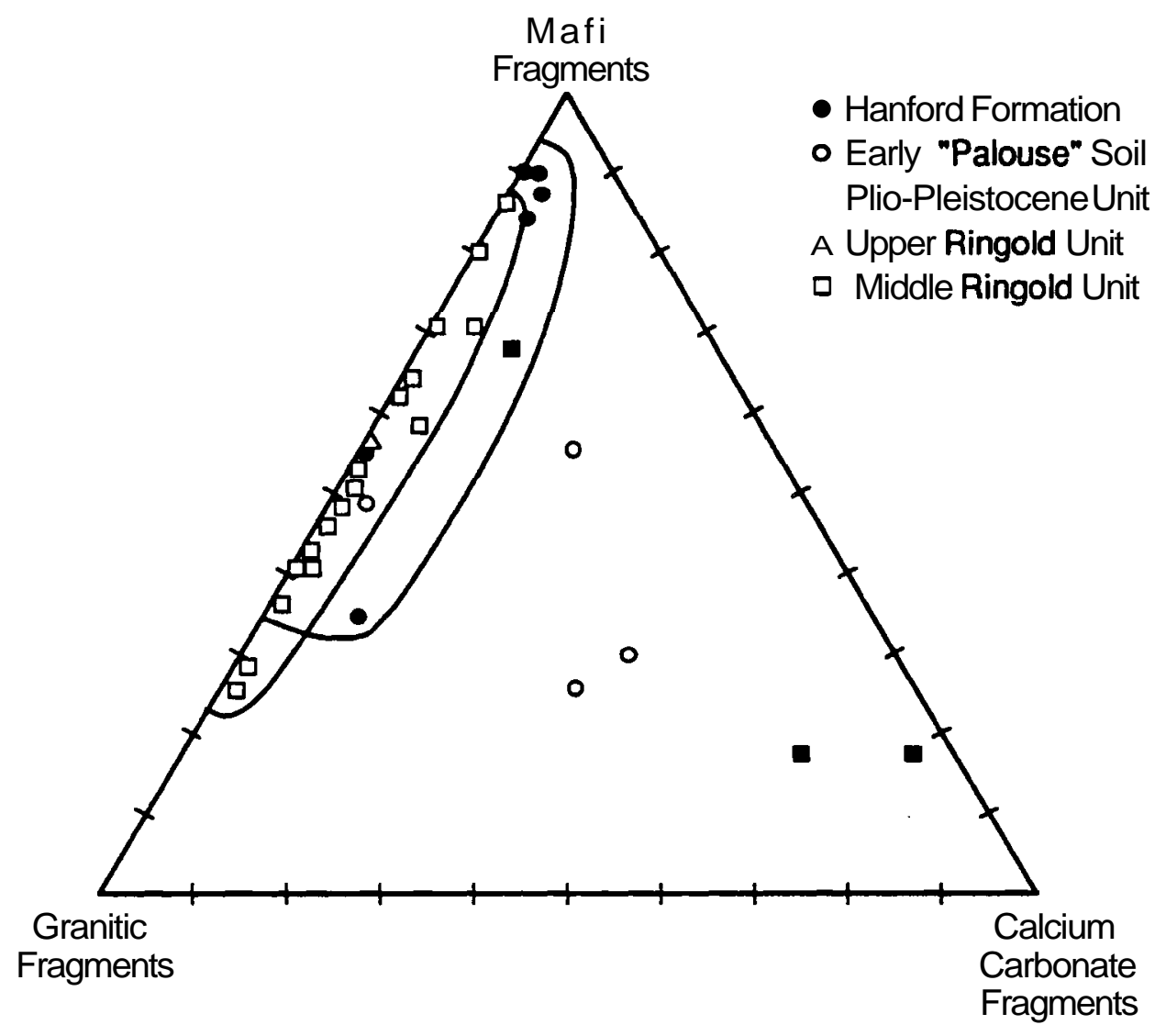

59002061.2

EIGUIRE 21. Temary Diagram Showing the Ratio of Three Common Types of Rock Fragments for Various Stratigraphic Units

Clay mineralogicalanalysis of four samples from the middle Ringold unit and one from the basal Ringold unit shows smectite to be the dominant clay mineralin these units of the Ringold Formation (Table 5, Figure 22). The very high amounts present (79-96 wt?! of the clay fraction) suggest that smectite is authigenic and that geochemical conditions favor it as the thermodynamically stable clay mineral in the Ringold Formation. Typically, smectites form in neutral- to high-pH environments that are rich in soluble $\mathrm{Si}$ and $\mathrm{Mg}$ and that are not extensively leached. The presence of authigenic srnectite therefore suggests a stable weathering regime involving contact with a nearly stagnant water reservoir

In contrast, the upper Ringold unit contains a diverse assemblage of clay minerals. X-ray diffraction analysis of a single sample shows that smectite, chlorite, and kaolinite each make up about 20$30 w t \%$ of the total, with lesser amounts of illite (17 wt\%) and vermiculite (5wt\%) being present. This diversity suggests that perhaps several sources contributed to the primary mineralogy, and that the sediments are in a relatively early stage of weathering compared to the older Ringold units. Vermiculite is a 


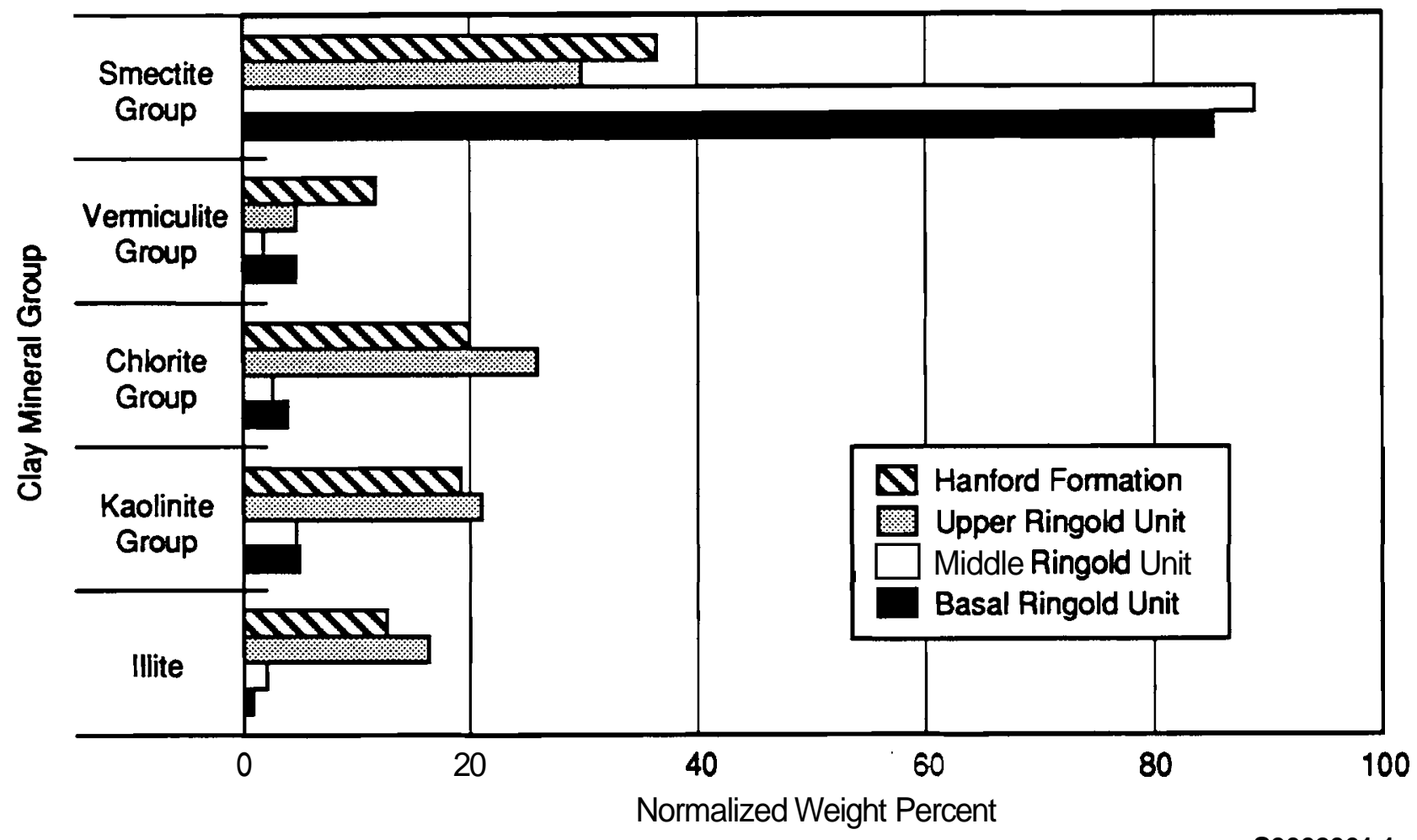

S9002061.1

EIGURE 22. Predominant Clay Minerals

metastable weathering product of illite and will eventually weather to form smectite or kaolinite. The chlorite present is a "swelling"chlorite (meaning that only part of the hydroxyl interlayer is present, enough to prevent collapse to a 1.0-nm spacing as vermiculites and smectites, yet not enough to prevent swelling like a srnectite). It is probable that this swelling chlorite is a weathering product of ferruginous illite or vermiculite and that it will eventually transform to smectite with continued leaching of $\mathbf{A l}$ and Fe from the unit. The substantial presence of the illite and its metastable weathering products (vermiculite and swelling chlorite) is good evidence for a slow weathering regime in a well-drained position and is typical of arid climates with xeric moisture regimes.

Kaolinite is normally formed under neutral to slightly aadic weathering conditions where moderate leaching is prevalent. Because the upper Ringold sample appears to be well-drained, the kaolinite present may be authigenic, although a detrital origin is also possible. Weathering of ferrous-iron-containing minerals (e.g., pyroxenes and biotites) is an aad-producing reaction and may provide sufficient localized acidity to allow kaolinite to form. This possibility is supported by the presence of biotite in the sand fraction 
(2.3 vol\%) and the absence of muscovite. It is probable, therefore, that the kaolinite is a direct weathering product of a biotitic illite, which is abundant in this sample. However, kaolinite is also a direct weathering product of potassium feldspar, which is relatively abundant in the sand fraction as well. No evidence for carbonates was found in the coarser fractions of this sample, helping to confirm that the geochemical environment in the region from which this sample was taken is neutral or slightly acidic and that it is subject to more leaching than the older units in the Ringold formation. The coexistence of smectite and kaolinite suggests that the weathering solution composition lies along the intersection of their thermodynamic stability fields (i.e., lower concentrations of $\mathrm{OH}, \mathrm{Si}$, and $\mathrm{Mg}$ than in the older Ringold units).

\section{Plio-Pleistocene Unit}

The Plio-Pleistocene unit in the 200-West Area represents a weathering surface developed on top of the Ringold Formation, characterizedby mostly fine-grained sand and silt cemented together with a calcium-carbonate precipitate. Mineralogical analysis of four samples from the Plio-Pleistocene unit (Table 4) corroborates that this unit consists predominantly of calcium carbonate and/or sedimentary rock fragments, with lesser amounts of quartz (monocrystalline) and feldspar (plagioclase) in roughly equal proportions (Figure 19). The relatively high proportion of plagioclase feldspar and pyroxene compared to other stratigraphic units indicates that the Plio-Pleistocene unit may be largely derived from either reworked and weathered Ringold deposits containing a relatively high percentage of basalt or eolian (wind-transported) material derived from the basaltic highlands surrounding the Pasco Basin. No clay mineralogy analyses are available for the Plio-Pleistocene unit.

\section{Earlv "Palouse" Soil}

The early "Palouse" soil contains higher concentrations of quartz (up to $46 \%$ ), feldspar (up to $47 \%$ ), and mica (up to $8.5 \%$ ) than the other stratigraphic units present in the 200 -West Area. The quartz is almost entirely the monocrystalline variety, and plagioclase is the predominant feldspar, although there are higher concentrations of potassium feldspar in the early "Palouse" soil than in the other units. The early "Palouse" soil represents a buried loess (windblown silt and sand) deposit. Apparently aggradation of this unit occurred relatively quickly; otherwise, carbonate-cemented horizons, similar to those in the Plio-Pleistocene unit, would have formed. The mineral assemblage indicates that, in contrast to the PlioPleistocene unit, the early "Palouse" soil is derived primarily from the weathering and reworking of granitic material by eolian transport.

A moderate amount of carbonate rock fragments is present, which may account for the field tests often indicating that hand samples are calcareous. Other types of rock fragments are generally sparse, which is consistent with the typically fine grain sizes associated with this unit. As in the case of the PlioPleistocene unit, no clay mineralogy analyses are available for the early "Palouse" soil. 


\section{Hanford Formation}

Deposits of the Hanford formation were laid down instantly, relative to geologic time, during giant cataclysmic floods originating along the northem margin of the Columbia Plateau. Unlike the other stratigraphic units, the Hanford formation was derived from a wide variety of sources, including massive reworking and transport of Ringold Formation and other unconsolidated suprabasalt sediments, scouring of Columbia River Basalt, as well as erosion of a wide variety of other rock types surroundingthe Columbia Plateau. As a result, the Hanford formation contains a more diverse mineral and rock-fragment assemblage, any one of which is present in moderate amounts compared to other units. An exception is indigenous mafic (i.e., basalt) clasts, which usually occur in greater amounts in the Hanford formation (up to $64 \%$ ) (Figures 19 and 21). Weathering profiles are not well developed in the Hanford formation beneath the 200 Areas, and therefore significant amounts of calcium carbonate fragments or sedimentary aggregates are also poorly developed.

The clay mineralogy of the Hanford formation is similar to that of the upper Ringold unit, except that vermiculite is more prevalent in the Hanford formation, at the expense of illite and swelling chlorite (Table5, Figure 22). Whether these differences are significant cannot be judged on the basis just of four Hanford samples and one upper Ringold sample. As in the case of the upper Ringold unit, the diverse mineralogy of the Hanford formation is indicative of limited weathering in a relatively welldrained xeric environment.

\subsubsection{Bulk Geochemistry}

More than 60 bulk sediment samples were analyzed using XRF for major and trace elements. The major-element geochemistry is discussed first, followed by the trace-element geochemistry.

\section{Maior Elements}

Some statistical parameters for the major elements by stratigraphicunit are presented in Table 6; these data are presented graphically in Figures 23 and 24. For consistency, the concentration of the major elements has been converted to weight percent of oxide compound. Silicon is the most abundant element, followed by aluminum (Figure 23), iron, calcium, potassium, and titanium (Figure 24).

The total amount of siliconis greatest in the Ringold Formation, averaging 75-80\% (Table 6), which is consistent with the formation's quartz-rich mineralogy. The amount of silicon is least (-65\%) in the Plio-Pleistocene unit, apparently because of enrichment with calcium carbonate, which partially to completely filled the particle matrices during soil formation. This enrichment with calcium carbonate is indicated in Figure 24. 
IABLE 6. Statistical Parameters for Major Elements According to Stratigraphic Unit

\begin{tabular}{|c|c|c|c|c|c|c|c|c|}
\hline & & \multicolumn{3}{|c|}{ MAJOR } & \multicolumn{4}{|c|}{ ELEMENT (normalized weight \%) } \\
\hline & & $A 1203$ & $\mathrm{SiO2}$ & $K 2 O$ & $\mathrm{COO}$ & Ti02 & $F O O$ & MnO \\
\hline HANFORD FORMATION & $\begin{array}{r}\text { Samples } \\
\text { Average } \\
\text { Standard Deviation } \\
\text { Minimum } \\
\text { Maximum } \\
\end{array}$ & $\begin{array}{c}14 \\
\mathbf{1 4 . 5 0} \\
0.46 \\
13.63 \\
15.17 \\
\end{array}$ & $\begin{array}{c}14 \\
69.47 \\
4.25 \\
64.19 \\
76.77 \\
\end{array}$ & $\begin{array}{l}14 \\
2.10 \\
0.37 \\
1.54 \\
2.79 \\
\end{array}$ & $\begin{array}{c}14 \\
5.42 \\
1.49 \\
3.26 \\
7.63 \\
\end{array}$ & $\begin{array}{c}14 \\
\mathbf{1 . 2 6} \\
0.50 \\
0.52 \\
1.85 \\
\end{array}$ & $\begin{array}{l}14 \\
7.12 \\
2.37 \\
3.15 \\
9.73 \\
\end{array}$ & $\begin{array}{c}14 \\
0.11 \\
0.04 \\
0.06 \\
0.16 \\
\end{array}$ \\
\hline $\begin{array}{c}\text { EARLY } \\
\text { "PALOUSE" } \\
\text { SOIL }\end{array}$ & $\begin{array}{r}\text { Samples } \\
\text { Average } \\
\text { Standard Deviation } \\
\text { Minimum } \\
\text { Maximum } \\
\end{array}$ & $\begin{array}{c}6 \\
14.00 \\
0.71 \\
13.07 \\
15.00 \\
\end{array}$ & $\begin{array}{c}6 \\
73.25 \\
2.23 \\
70.26 \\
76.54 \\
\end{array}$ & $\begin{array}{c}6 \\
2.37 \\
0.18 \\
2.15 \\
2.63 \\
\end{array}$ & $\begin{array}{c}6 \\
4.69 \\
0.72 \\
3.51 \\
5.64 \\
\end{array}$ & $\begin{array}{c}6 \\
0.86 \\
0.19 \\
0.53 \\
1.06 \\
\end{array}$ & $\begin{array}{l}6 \\
4.74 \\
0.99 \\
3.19 \\
5.96 \\
\end{array}$ & $\begin{array}{c}6 \\
0.08 \\
0.01 \\
0.06 \\
0.10 \\
\end{array}$ \\
\hline $\begin{array}{c}\text { PLIO-PLEISTOCENE } \\
\text { UNIT }\end{array}$ & $\begin{array}{r}\text { Samples } \\
\text { Average } \\
\text { Standard Deviation } \\
\text { Minimum } \\
\text { Maximum } \\
\end{array}$ & $\begin{array}{c}6 \\
\mathbf{1 2 . 8 7} \\
1.84 \\
9.56 \\
14.34 \\
\end{array}$ & $\begin{array}{c}6 \\
64.45 \\
6.64 \\
53.41 \\
70.77 \\
\end{array}$ & $\begin{array}{l}6 \\
\mathbf{1 . 7 5} \\
0.26 \\
1.33 \\
2.11 \\
\end{array}$ & $\begin{array}{c}6 \\
\mathbf{1 2 . 4 8} \\
8.24 \\
6.43 \\
27.07 \\
\end{array}$ & $\begin{array}{c}6 \\
\mathbf{1 . 4 0} \\
0.27 \\
0.93 \\
1.66 \\
\end{array}$ & $\begin{array}{c}6 \\
6.93 \\
1.54 \\
4.45 \\
9.22 \\
\end{array}$ & $\begin{array}{c}6 \\
0.11 \\
0.02 \\
0.08 \\
0.13 \\
\end{array}$ \\
\hline $\begin{array}{c}\text { UPPER } \\
\text { RINGOLD } \\
\text { UNIT }\end{array}$ & $\begin{array}{r}\text { Samples } \\
\text { Average } \\
\text { Standard Deviation } \\
\text { Minimum } \\
\text { Maximum } \\
\end{array}$ & $\begin{array}{c}2 \\
13.25 \\
0.47 \\
12.92 \\
13.58 \\
\end{array}$ & $\begin{array}{c}2 \\
75.52 \\
0.75 \\
74.99 \\
76.05 \\
\end{array}$ & $\begin{array}{c}2 \\
2.06 \\
0.07 \\
2.01 \\
2.11 \\
\end{array}$ & $\begin{array}{l}2 \\
3.76 \\
0.19 \\
3.63 \\
3.90 \\
\end{array}$ & $\begin{array}{c}2 \\
0.72 \\
0.00 \\
0.72 \\
0.73 \\
\end{array}$ & $\begin{array}{c}2 \\
4.61 \\
0.04 \\
4.58 \\
4.64 \\
\end{array}$ & $\begin{array}{c}2 \\
\mathbf{0 . 0 7} \\
0.01 \\
0.06 \\
0.08 \\
\end{array}$ \\
\hline $\begin{array}{l}\text { MIDDLE } \\
\text { RINGOLD } \\
\text { UNIT }\end{array}$ & $\begin{array}{r}\text { \# Samples } \\
\text { Average } \\
\text { Standard Deviation } \\
\text { Minimum } \\
\text { Maximum } \\
\end{array}$ & $\begin{array}{c}29 \\
11.71 \\
1.32 \\
9.92 \\
14.03 \\
\end{array}$ & $\begin{array}{c}29 \\
78.35 \\
3.22 \\
69.13 \\
82.65 \\
\end{array}$ & $\begin{array}{c}29 \\
\mathbf{1 . 8 2} \\
0.17 \\
1.55 \\
2.27 \\
\end{array}$ & $\begin{array}{l}29 \\
3.20 \\
0.83 \\
2.05 \\
5.75 \\
\end{array}$ & $\begin{array}{l}29 \\
0.66 \\
0.16 \\
0.48 \\
1.31 \\
\end{array}$ & $\begin{array}{l}29 \\
\mathbf{4 . 1 9} \\
1.06 \\
2.72 \\
8.01 \\
\end{array}$ & $\begin{array}{l}29 \\
0.07 \\
0.02 \\
0.05 \\
0.13 \\
\end{array}$ \\
\hline $\begin{array}{l}\text { LOWER } \\
\text { RINGOLD } \\
\text { UNIT }\end{array}$ & $\begin{array}{r}\text { Samples } \\
\text { Average } \\
\text { Standard Deviation } \\
\text { Minimum } \\
\text { Maximum } \\
\end{array}$ & $\begin{array}{c}4 \\
\mathbf{1 1 . 0 2} \\
0.33 \\
10.67 \\
11.45 \\
\end{array}$ & $\begin{array}{c}4 \\
80.80 \\
0.45 \\
80.18 \\
81.14 \\
\end{array}$ & $\begin{array}{c}4 \\
\mathbf{1 . 7 5} \\
0.11 \\
1.66 \\
1.89 \\
\end{array}$ & $\begin{array}{l}4 \\
2.50 \\
0.20 \\
2.24 \\
2.68 \\
\end{array}$ & $\begin{array}{l}4 \\
0.55 \\
0.03 \\
0.53 \\
0.58 \\
\end{array}$ & $\begin{array}{l}4 \\
3.31 \\
0.26 \\
3.06 \\
3.59 \\
\end{array}$ & $\begin{array}{c}4 \\
0.07 \\
0.02 \\
0.06 \\
0.09 \\
\end{array}$ \\
\hline $\begin{array}{l}\text { BASAL } \\
\text { RINGOLD } \\
\text { UNIT }\end{array}$ & $\begin{array}{r}\text { \# Samples } \\
\text { Average } \\
\text { Standard Deviation } \\
\text { Minimum } \\
\text { Maximum }\end{array}$ & $\begin{array}{c}2 \\
12.17 \\
0.13 \\
12.08 \\
12.26\end{array}$ & $\begin{array}{c}2 \\
75.32 \\
0.03 \\
75.30 \\
75.34\end{array}$ & $\begin{array}{l}2 \\
\mathbf{1 . 5 8} \\
0.07 \\
1.53 \\
1.63\end{array}$ & $\begin{array}{l}2 \\
4.25 \\
0.08 \\
4.19 \\
4.30\end{array}$ & $\begin{array}{l}2 \\
0.91 \\
0.03 \\
0.89 \\
0.93\end{array}$ & $\begin{array}{l}2 \\
5.68 \\
0.06 \\
5.63 \\
5.72\end{array}$ & $\begin{array}{l}2 \\
0.09 \\
0.01 \\
0.09 \\
0.10\end{array}$ \\
\hline
\end{tabular}




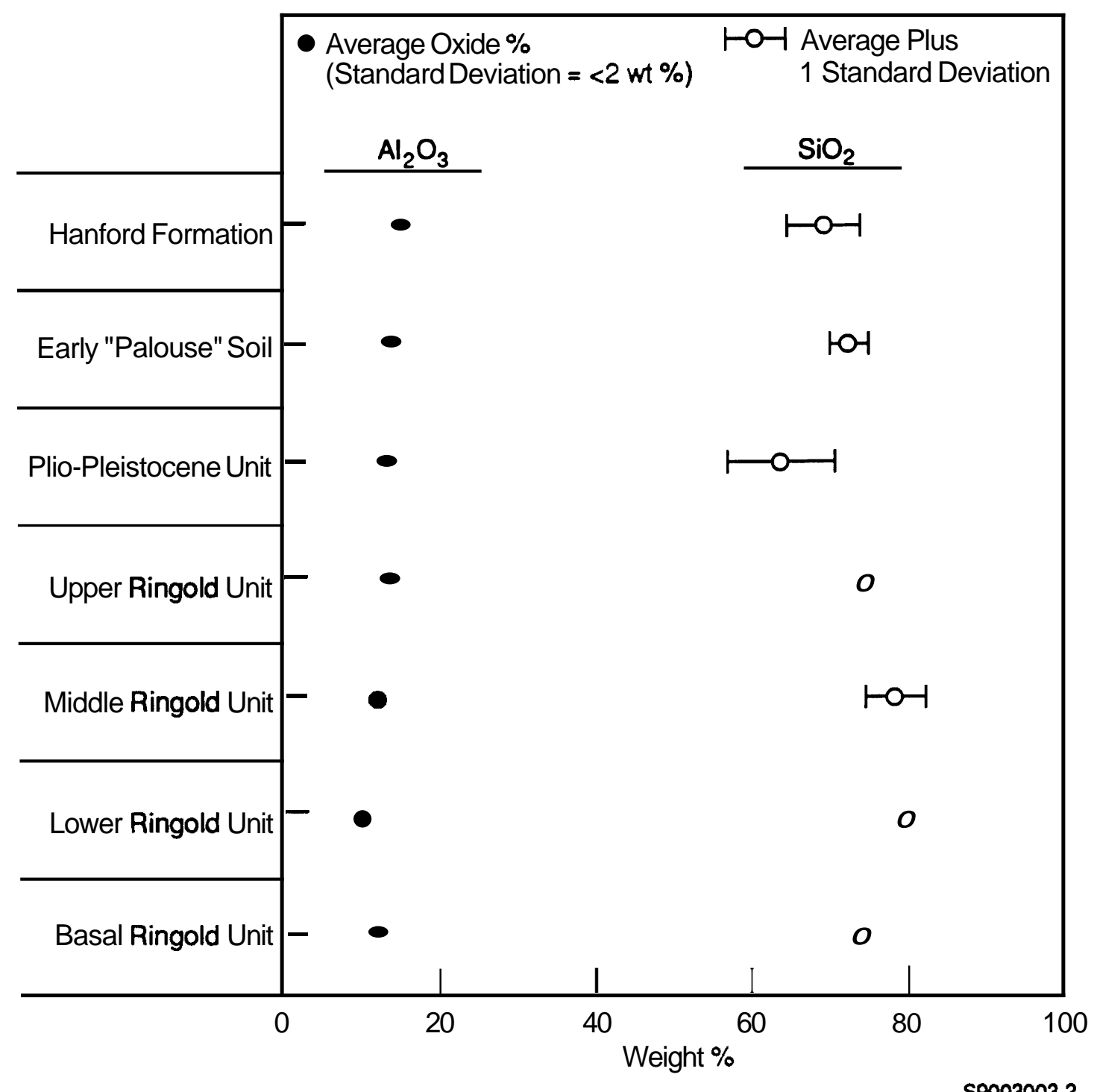

ElGURE 23. Average Distribution of $\mathrm{Al}_{2} \mathrm{O}_{3}$ and $\mathrm{SiO}_{2}$ for Stratigraphic Units Analyzed Within the 200 Areas

Among the other major elements, aluminum ranges between 10 and 15\%; averages are slightly higher within the Hanford formation and early "Palouse" soil than in the underlying stratigraphicunits (Figure 23). The total amount of iron is greatest ( $7 \%$ ) in the Hanfordformation as well, although there is a wide range in the distribution of iron values. On the average, slightly less iron is present in the early "Palouse" soil and Plio-Pleistoceneunit; the least amount of iron (3.3 to 4.6\%) is present in the Ringold Formation, although the basal Ringold unit has an average about $5.7 \%$ iron oxide (Figure 24). Amounts of titanium are greatest (1.3-1.4\%) within the Plio-Pleistocene unit and Hanford formation. 


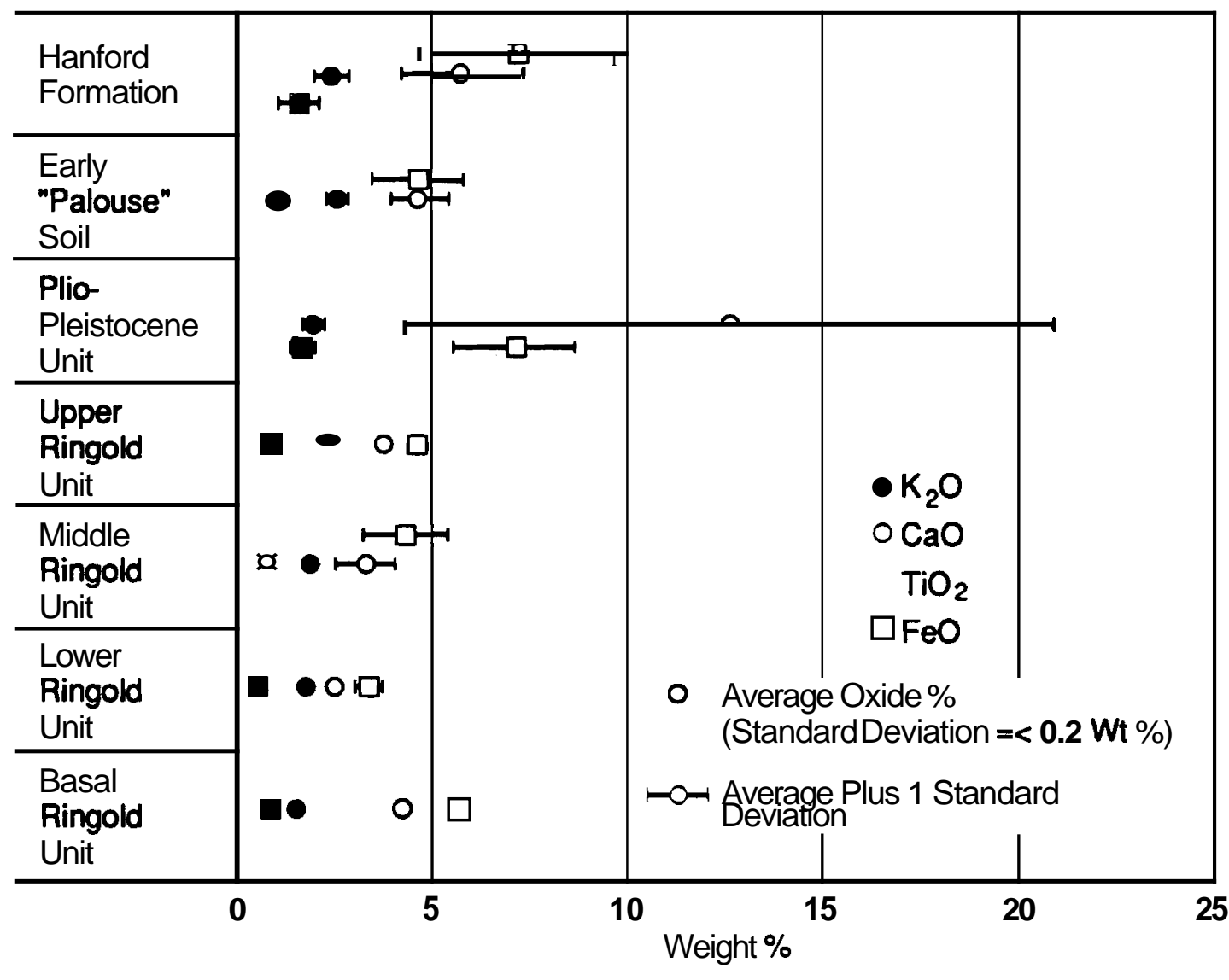

59003003.3

ElGURE 24. Average Distribution of $\mathrm{K}_{2} \mathrm{O}, \mathrm{CaO}, \mathrm{TiO}_{2}$, and $\mathrm{FeO}$ for Stratigraphic Units Analyzed Within the 200 Areas

Some geochemical characteristics of the different stratigraphic units can be identified through bivariate plots. For example, by plotting the relative amounts of $\mathrm{Al}_{2} \mathrm{O}_{3}$ versus $\mathrm{SiO}_{2}$, it becomes apparent that the Hanford formation and middle Ringold unit occur in two semidistinct fields (Figure 25). In the middle Ringold unit, silicon is distributed over a narrower range than that for aluminum, whereas the opposite occurs in for the Hanford formation. Furthermore, there appears to be a linear relationship between the amounts of silicon and aluminum for the middle Ringold unit. However, no such function is apparent for the Hanford formation. The Hanford formation does not lie in a totally separate, welldefined field possibly because the formation consists of a mixture of reworked Ringold and other sedimentary deposits.

A linear relationship between the distributions of silicon and iron is apparent for both the middle Ringold unit and the Hanford formation (Figure 26). The amount of iron is generally greater and occurs over a wider range in the Hanford formation, however. 


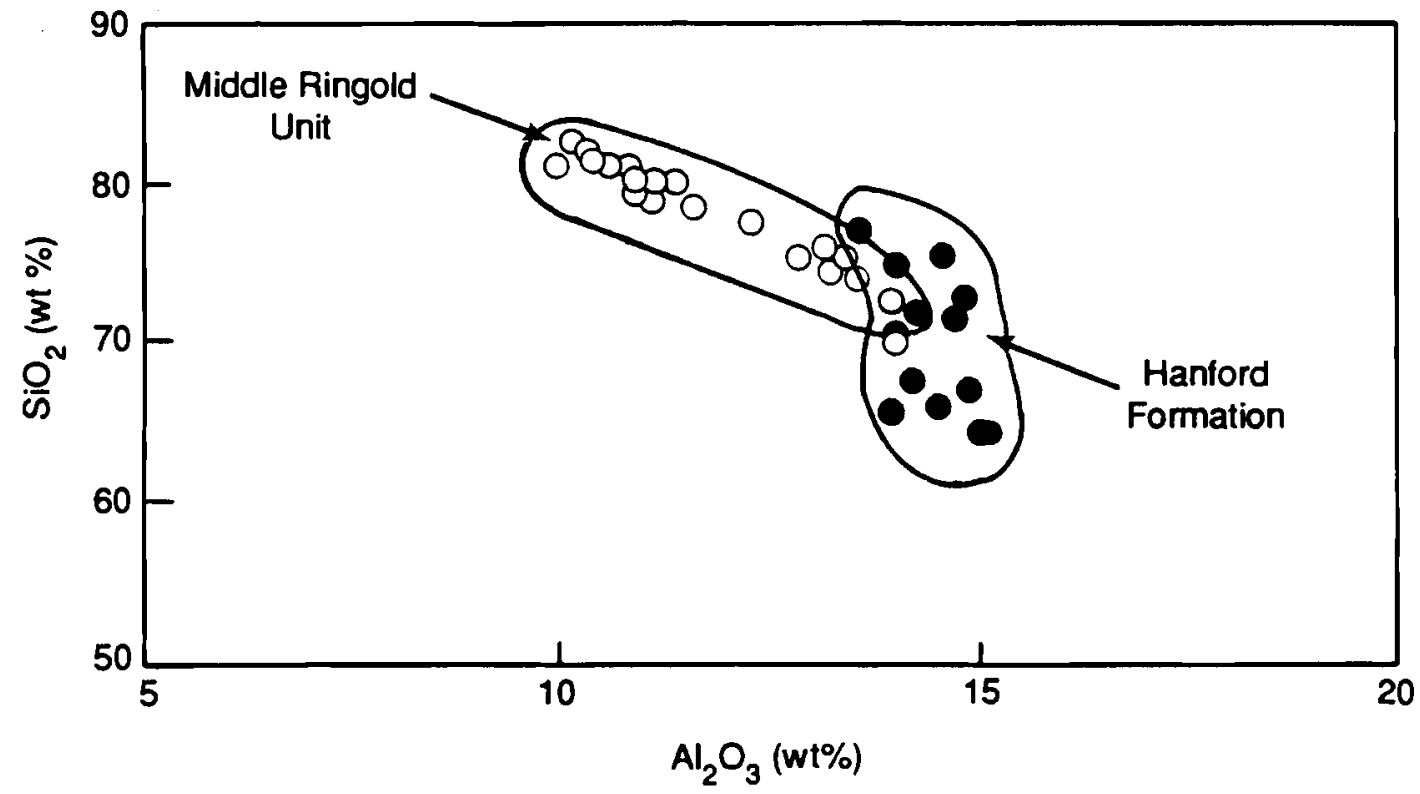

ElGURE 25. Bivariate Plot of $\mathrm{Al}_{2} \mathrm{O}_{3}$ and $\mathrm{SiO}_{2}$ for the Hanford Formation and Middle Ringold Unit

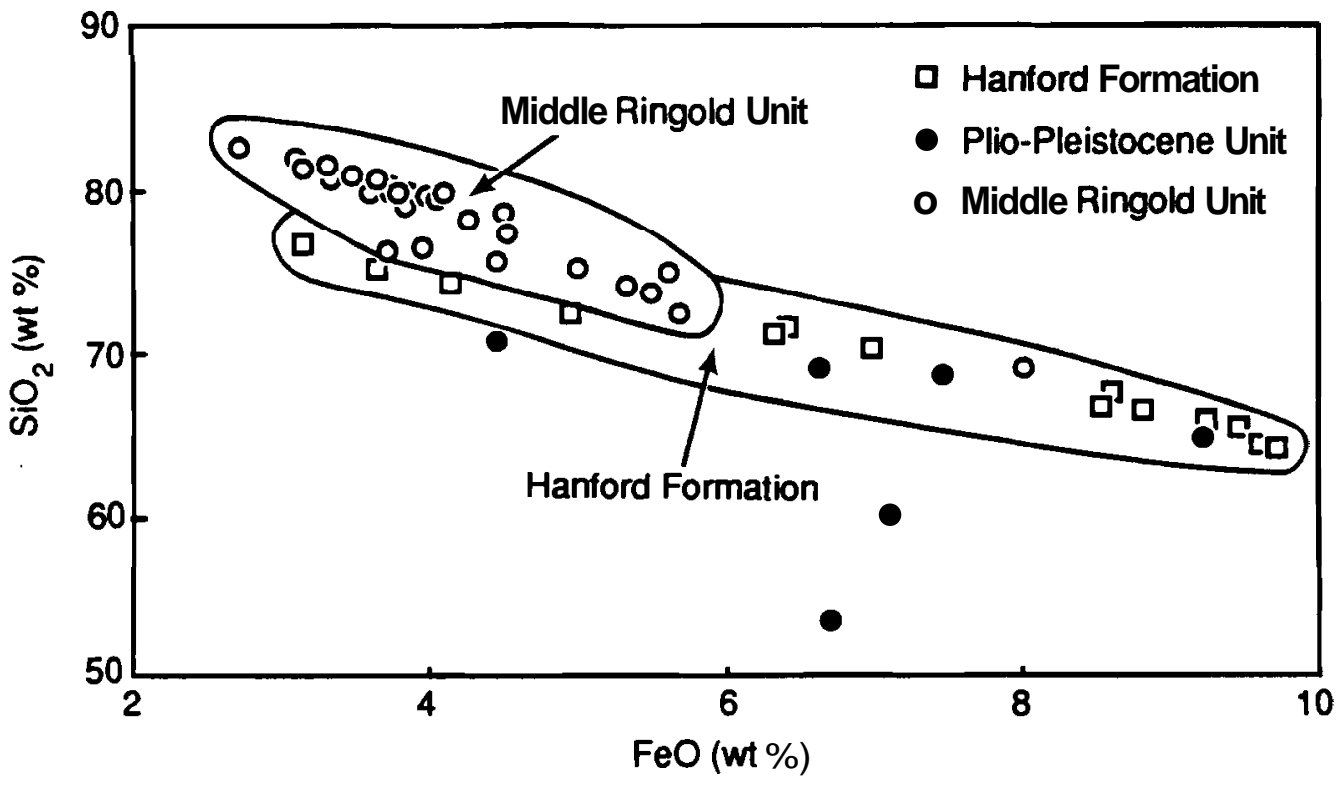

ElGURE26. Bivariate Plot of $\mathrm{SiO}_{2}$ Versus $\mathrm{FeO}$ 
The total organic carbon present in the suprabasalt sediments is low (Figure 27). The organic carbon content for the Plio-Pleistocene unit and Hanford formation is highly variable; some samples contain up to $\mathbf{0 . 2} \mathbf{w} \%$ organic carbon, while other samples do not contain any detectable organic carbon. The middle Ringold unit and early "Palouse" soil, however, are consistently very low ( 0.05\%i) organic carbon.

\section{Irace Elements}

Trace elements, while perhaps not important from a standpoint of geochemicalstability, appear to be a useful tool for identifying and distinguishing among stratigraphic units. In general, the post-Ringold units (Plio-Pleistocene unit, early "Palouse" soil, and the Hanford formation) contain higher concentrations of trace elements than the Ringold units do. Three bivariate plots are presented as examples; these are rubidium vs. zinc (Figure 28), barium vs. zirconium (Figure 29 ), and arsenic vs. rubidium (Figure 30). In Figures 28 and 29, the fields for the Hanford and Ringold Formations are almost mutually exclusive. The multiple sources from which the Hanford formation derives are indicated in these figures by larger fields, in contrast to the Ringold Formation, which is derived from a more restricted source area and whose fields cluster into a tighter group in these plots.

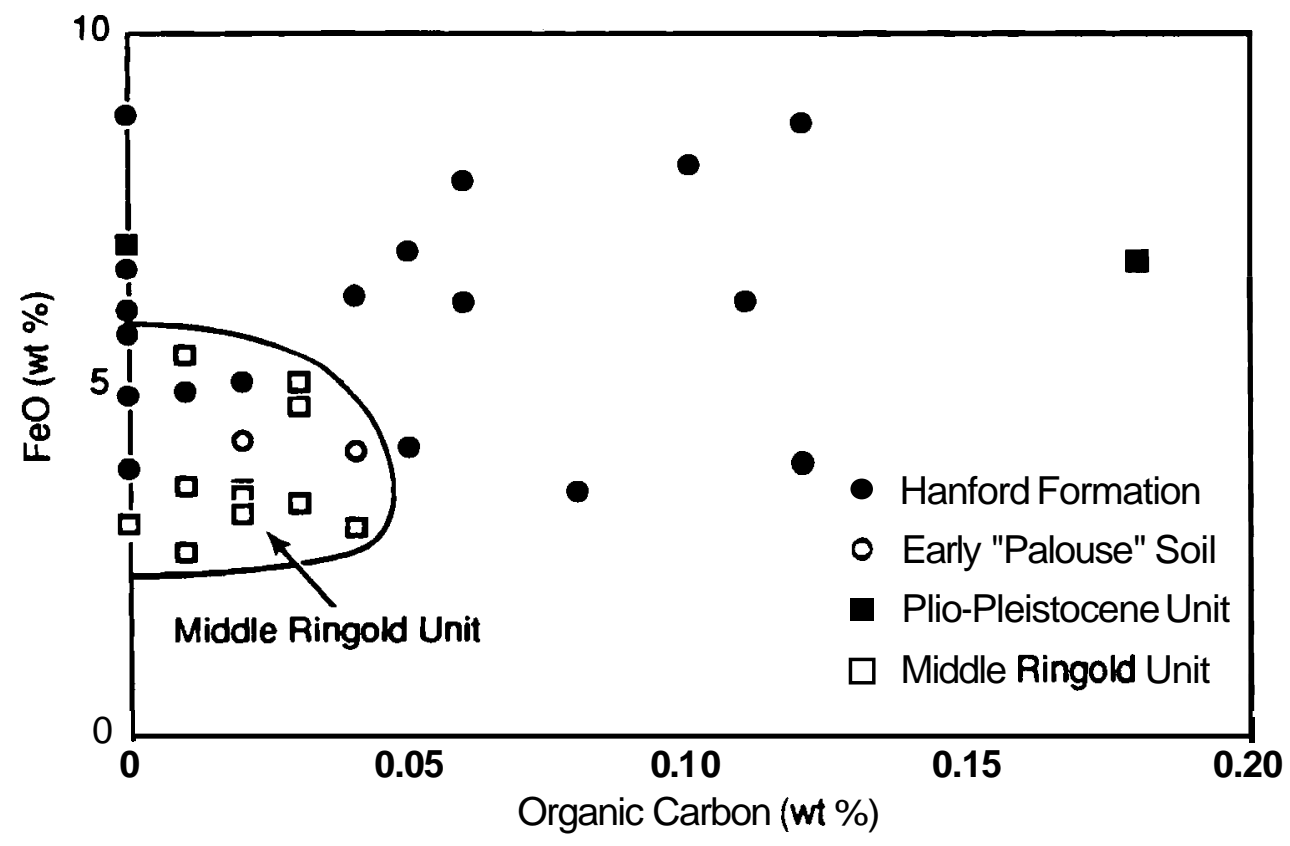

FIGURE27. Bivariate Plot of FeO Versus Total Organic Carbon 


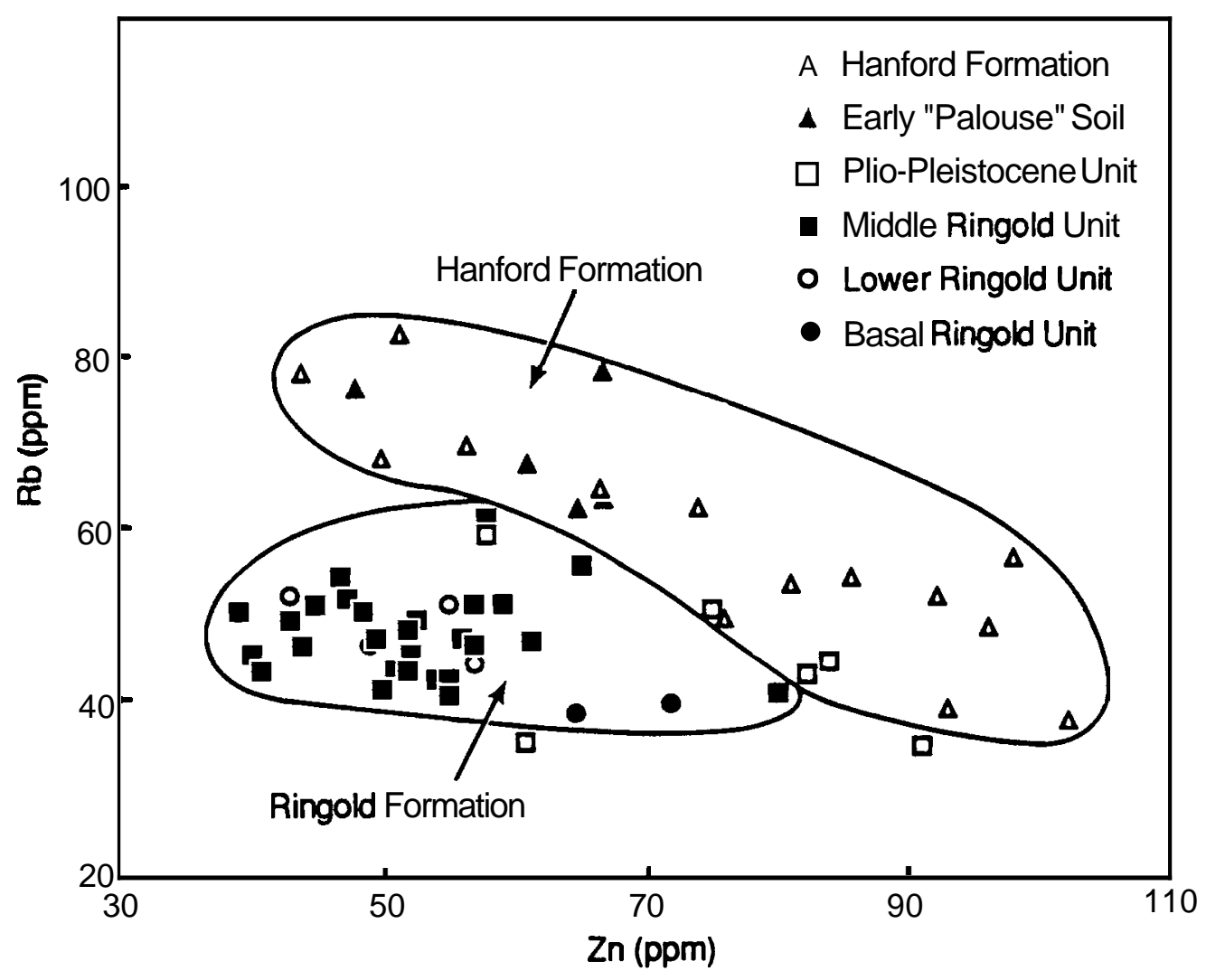

EIGURE 28. Bivariate Plot of Rubidium Versus Zinc

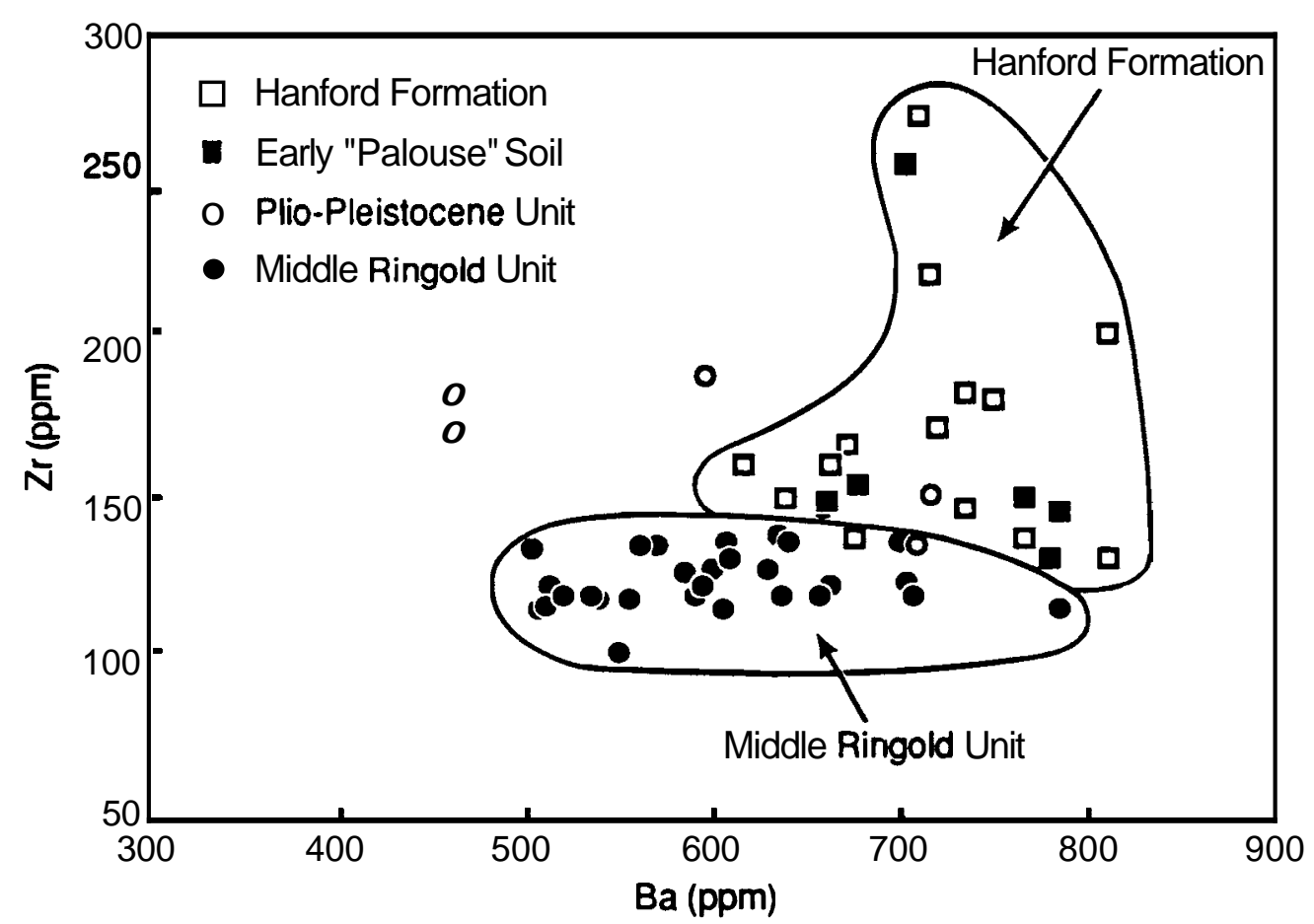

EIGURE 29. Bivariate Plot of Zirconium Versus Barium 


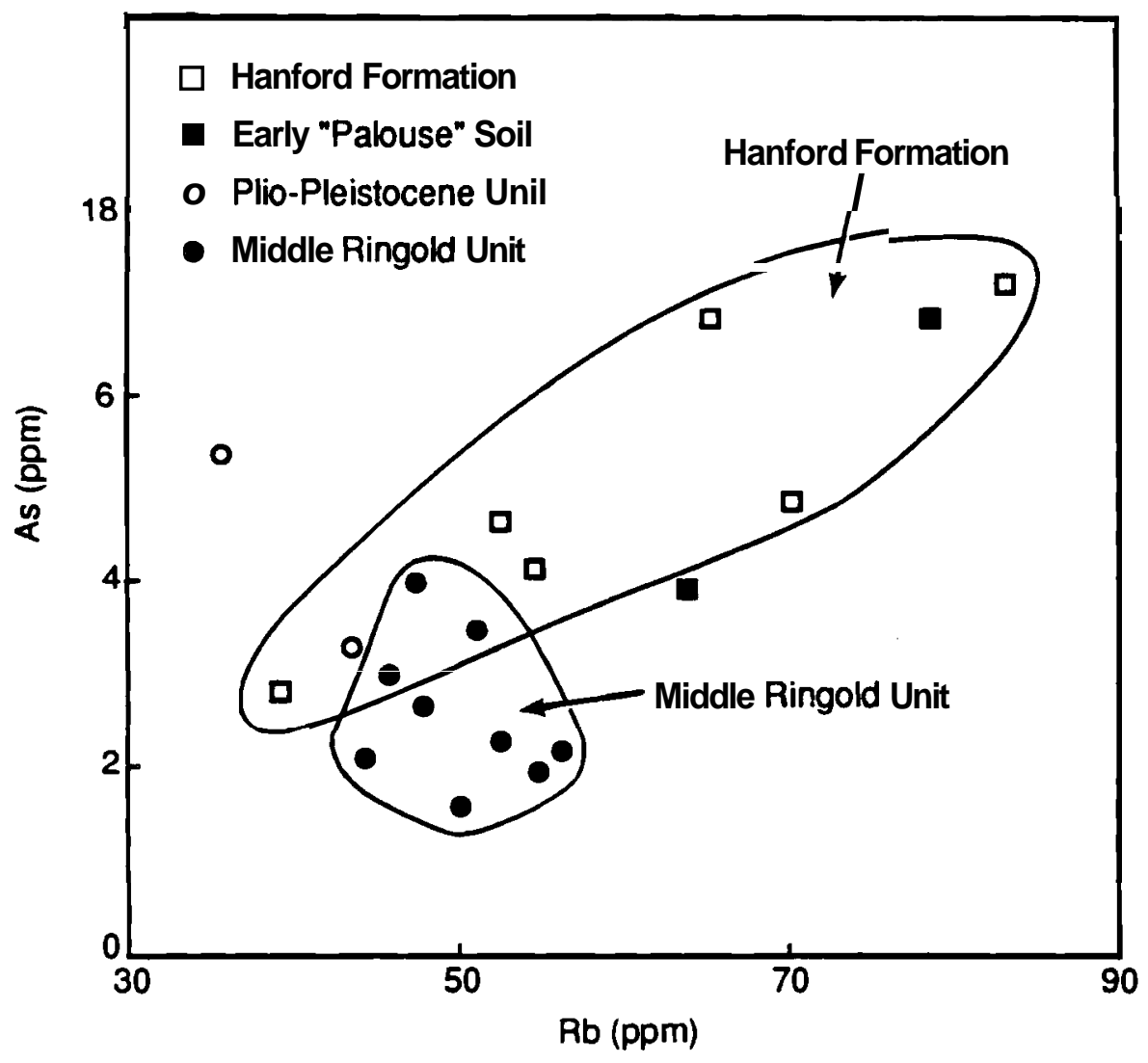

FIGURE 30. Bivariate Pbt of Arsenic Versus Rubidium 


\subsection{HYDROLOGY}

The following discussion of hydrology is based on a multitude of previous geohydrologic studies performedin the Pasco Basin and 200 Areas (Newcomb et al. 1972; Gephart et al. 1979; Graham et al. 1981, 1984; Strait and Mercer 1987; DOE 1986, 1988). Hydrologic data for the area in the immediate vicinity of W-5 have been presentedby Last et al. (1989).

As discussed in Section 3.0, several diverse hydrogeologic units are present, including several Ringold units (basal, lower, middle, upper Ringold), the Plio-Pleistocene unit, the early "Palouse" soil, and several facies of the Hanford formation. As a result, a wide variety of hydrogeologicunits of variable thicknesses are present beneath W-5. In general, most units are relatively homogeneous, except for the Hanford formation.

Units in the saturated zone include the coarse-grained facies and the fine-grainedfacies of the basal Ringold unit, the fine-grainedlower Ringold unit, and the relatively thick coarse-grainedmiddle Ringold unit, which extends 50 to $60 \mathrm{ft}$ or more above the water table. The basal, lower, and middle Ringold units appear to be continuous across W-5; however, the fine-grainedbasal and lower Ringold units pinch out near the northeastern boundary.

Overlying the middle Ringold unit in the unsaturated zone are the upper Ringold unit, the PlioPleistocene unit, the early "Palouse" soil, and the Hanford formation. The upper Ringold is a unit of mostly sand to gravelly sand that pinches out in the northern portion of W-5. The Plio-Pleistocene unit consists of a fine-grained, calcareous, weakly to strongly cemented mixture of mud and sand; locally, however, it may contain lenses of basaltic gravel and sand. Above this is a uniform layer (10 to $15 \mathrm{ft}$ ) of loose, sandy mud (early "Palouse" soil). The Hanford formation ranges in thickness from 50 to $150 \mathrm{ft}$ across W-5, thickening to the south. Internally, the Hanford formation is extremely heterogeneous; it consists of bouldery gravel along the northern boundary, and grades southward into finer-grained gravels interbedded with sand and mud.

The following discussion of hydrology will cover 1) regional hydrology, 2) the characteristics of the aquifer(s) and confining beds in the suprabasalt sediments, 3) the unsaturatedzone, and 4) hydrologic properties of the various local hydrogeologic units. 


\subsection{BEGIONAI_HYDROIOGY}

Principal sources of natural recharge to the unconfined aquifer, where precipitation and surface runoff infiltrate to the water table, occur west of the 200-West Area. Several small streams, such as Cold Creek and Dry Creek that are located between the Rattlesnake Hills and Umtanum Ridge, drain the western slopes of the Pasco Basin, losing water to the subsurface as they spread across the lower valley plains. From here, ground water moves through the sediments generally from west to east (DOE 1988). Studies performed on the Hanford Site indicate that some recharge to the water table (0-4 in./year) may occur locally depending on surface conditions, especially during the winter months (Gee 1987); most precipitation, however, is returned to the atmosphere through evapotranspiration.

Artificial or synthetic recharge to the unconfined aquifer from liquid-waste disposal operations occurs at the Hanford Site, mainly in the 200 Areas. Recharge from the 200 Areas wastewater-disposal facilities is estimated to be approximately 10 times the natural recharge at the Hanford Site (Graham et al. 1981).

The present direction of ground-water flow in the vicinity of the 200-West Area is highly influenced by a ground-water mound associated with past artificial recharge at 216-U-10 Pond (U Pond) and with current recharge to the $216-U-14$ ditch, which lies just east of $U$ Pond. The influence of the $U$ Pond mound on ground-water flow beneath $\mathrm{W}-5$ is demonstratedon a regional water table map that also indicates generalized ground-water flow directions (Figure 31). Artificial recharge at $U$ Pond raised the level of the unconfined aquifer $55 \mathrm{ft}$ between 1944, which was prior to the construction of U Pond, and 1987. Even though $U$ Pond has been decommissioned since 1984, the ground-water mound is expected to persist for a number of years. As the ground-water mound is allowed to dissipate, the direction of ground-water flow beneath W-5 will likely swing to the east, perhaps returning to the due-east regional ground-water flow direction recorded in 1944 (Figure 32).

Based on water-level measurements collected at well 299-W15-2 (Appendix B), it appears that the water table has fluctuated as much as $15 \mathrm{ft}$ over the last 35 years. The fluctuationsare probably the result of varied volumes of process water being delivered to $U$ Pond. Since about the time $U$ Pond was decommissioned, water levels have been in general decline, having fallen a total of $-7 \mathrm{tt}$ in the last 6 years at 299-W15-2. Hydrographs from the other wells located within $1000 \mathrm{ft}$ of W-5 corroborate a general drop in water levels over the last two years relative to those measured ever since the water levels have been measured at the site (Appendix B).

The relatively young, uncompacted and unconsolidated sands and gravels belonging to the Hanford formation are the most conductive unit, transporting water at rates ranging from 500 to $90,000 \mathrm{ft} /$ day across the Pasco Basin (Table 7). Within the 200-East Area, located 5 to 6 miles east of W-5, Last et al. 


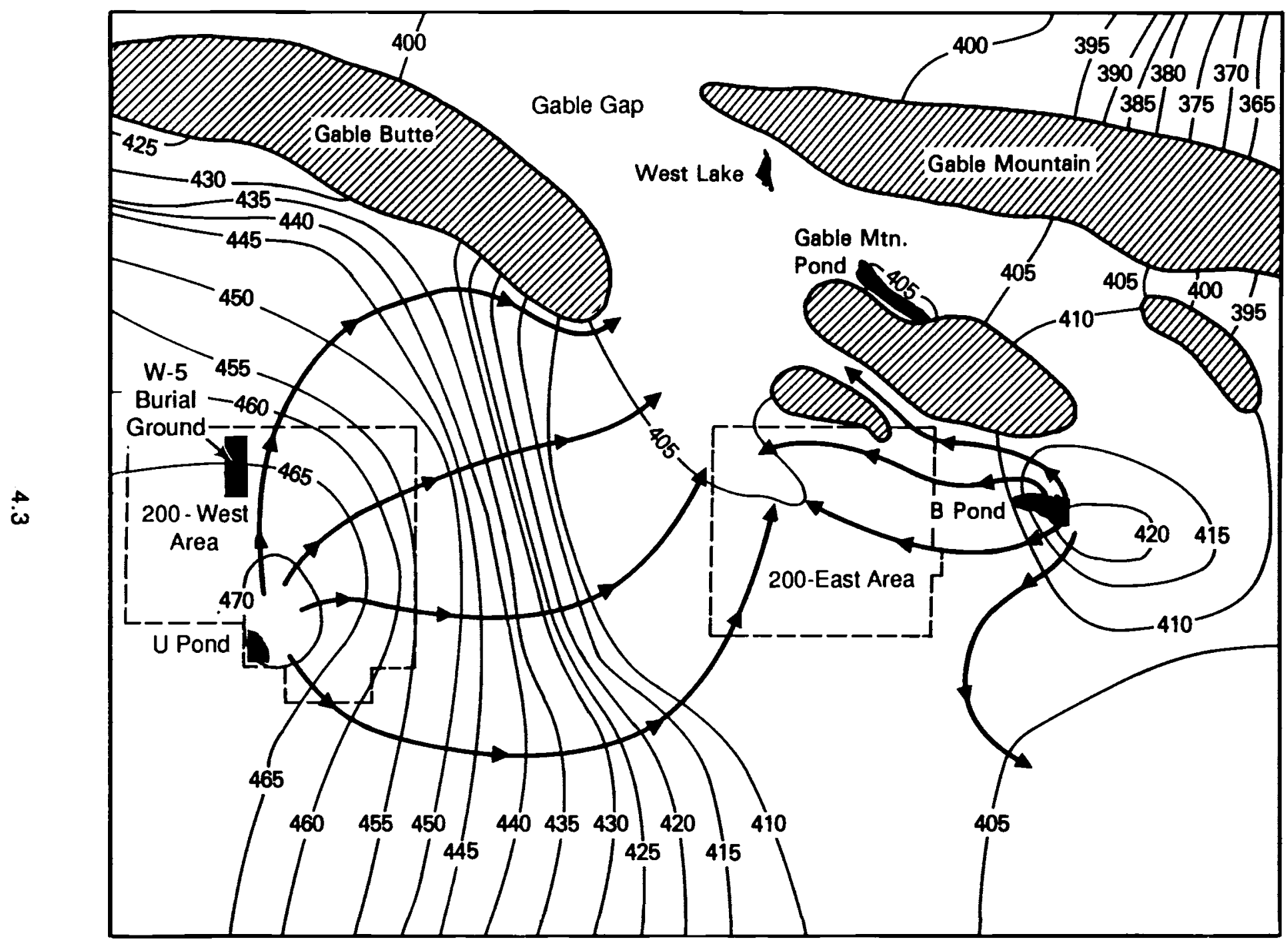

December 1987

Water-Table Contours

in Feet Above Mean

Sea Level

- 5-ft Contour

$\rightarrow$ Flow Path

$\underbrace{0}_{1}{ }_{1}^{1}$ Kilometer

Estimated Basalt

Above Water

Table

$\left.\right|_{-N} ^{-1}$

FIGURE 31. Water-Table Map for the 200 Areas Measured in December 1987 


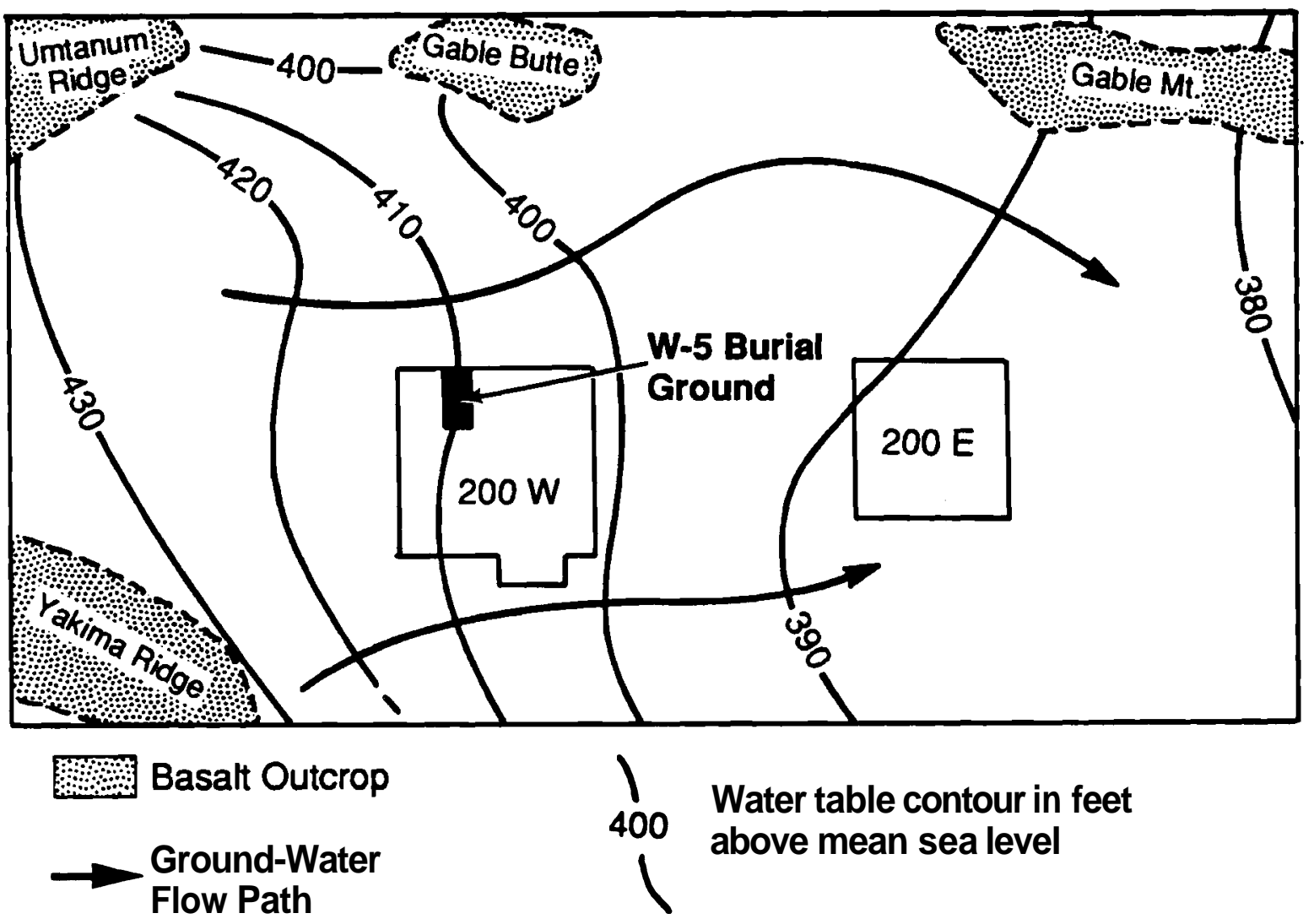

ElGURE 32. Estimated 1944 Water-Table Map

(1989) used aquifer testing to measure hydraulic conductivities for the Hanford formation ranging from 1500 to 90,000 ft/day and hydraulic conductivities for the upper part of the Ringold Formation ranging from 0.1 to $200 \mathrm{ft}$ day. Elsewhere, Bergeron et al. (1987, p. 41) have reported saturated hydraulic conductivities between 1.5 and $125 \mathrm{ft}$ day for relatively fine-grained sands and muds of the Hanford formation present at the U.S. Ecology Site. The compact silts and clays of the lower Ringold unit transmit water at rates of only 0.1 to $12 \mathrm{ft}$ day. The hydraulic conductivity of the middle Ringold gravels, the most voluminous and extensive hydrogeologic unit, range from 9 to $600 \mathrm{ft} /$ day, whereas the hydraulic conductivity of the basal Ringold ranges from 0.004 to $20 \mathrm{tt}$ day (Table 7).

\subsection{UPPERMOST AQUIFER(S)}

The top of the unconfined aquifer lies between 225 and $270 \mathrm{ft}$ below ground surface (Figure 33). The depths to water are greatest to the west of W- 5 because of the higher topography in this area. The 


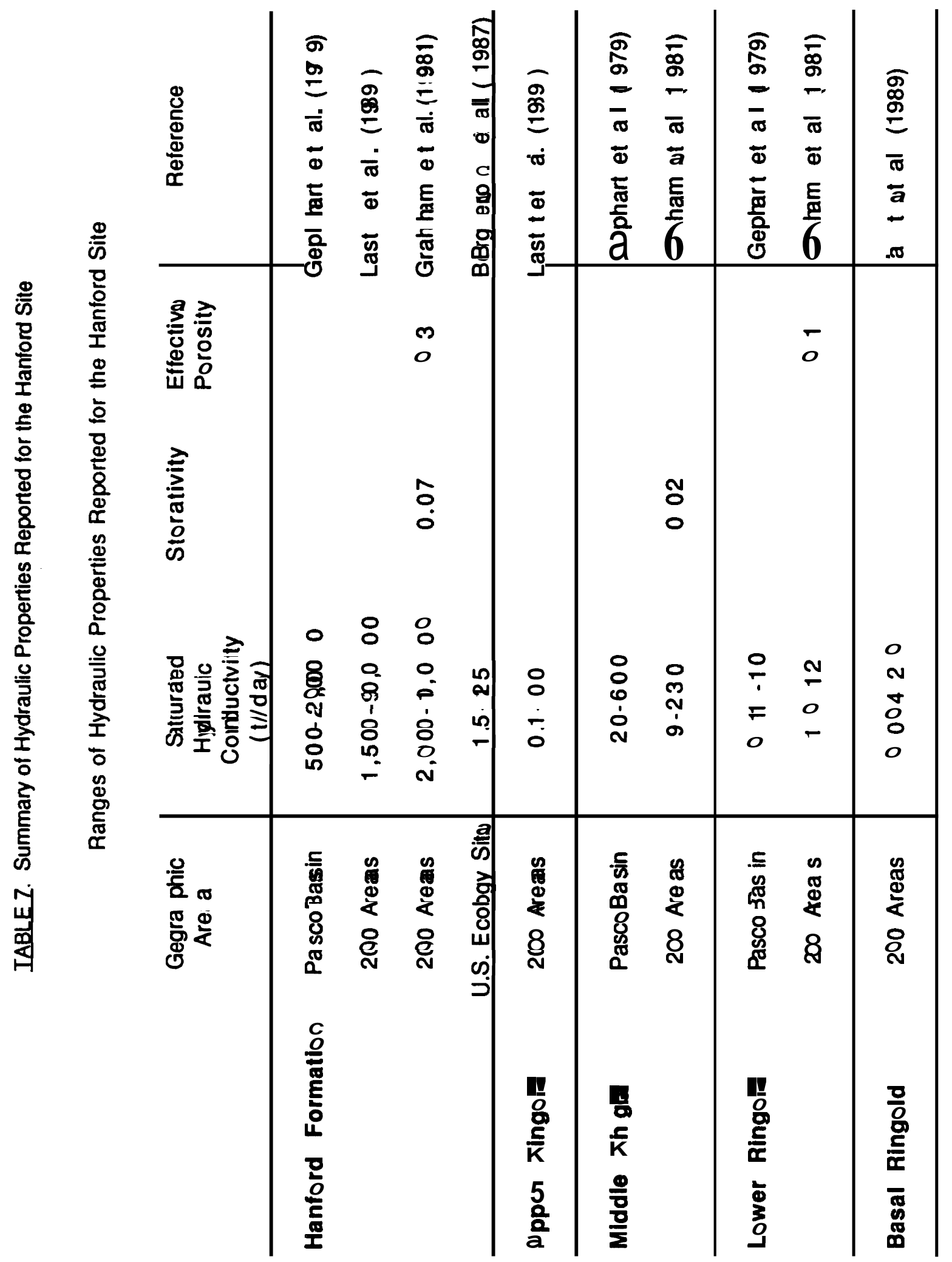




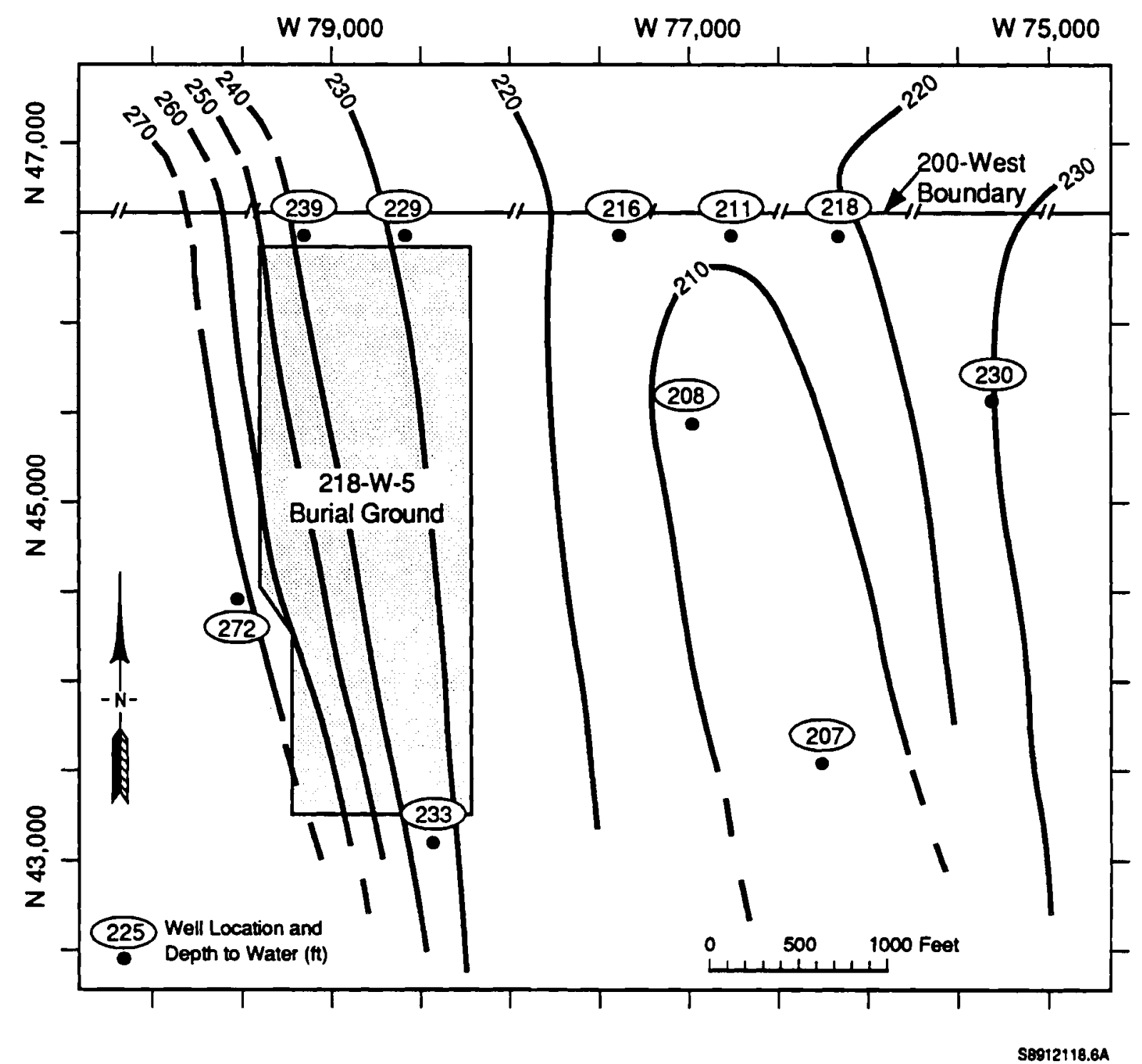

FIGURE 33. Depth to Water Table in the Vicinity of the W-5 Burial Ground

water table lies within the upper portion of the coarse-grainedmiddle Ringold unit everywhere in the vicinity of W-5 (Figures 4 to 6). Changes in elevation of the water table are shown in hydrographs presented in Appendix B. Water-table maps indicate that the present direction of ground-water flow is to the north-northeast (Figure 34).

The Elephant Mountain Member, which is continuous across the central Pasco Basin, acts as a confining unit that separates the unconfined aquifer system from the lower confined aquifers found within the Columbia River basalts. The base of the unconfined aquifer lies atop the lower Ringold unit, except to the north and east where the lower and the fine-grained basal Ringold units appear to pinch out (Figure 35). 


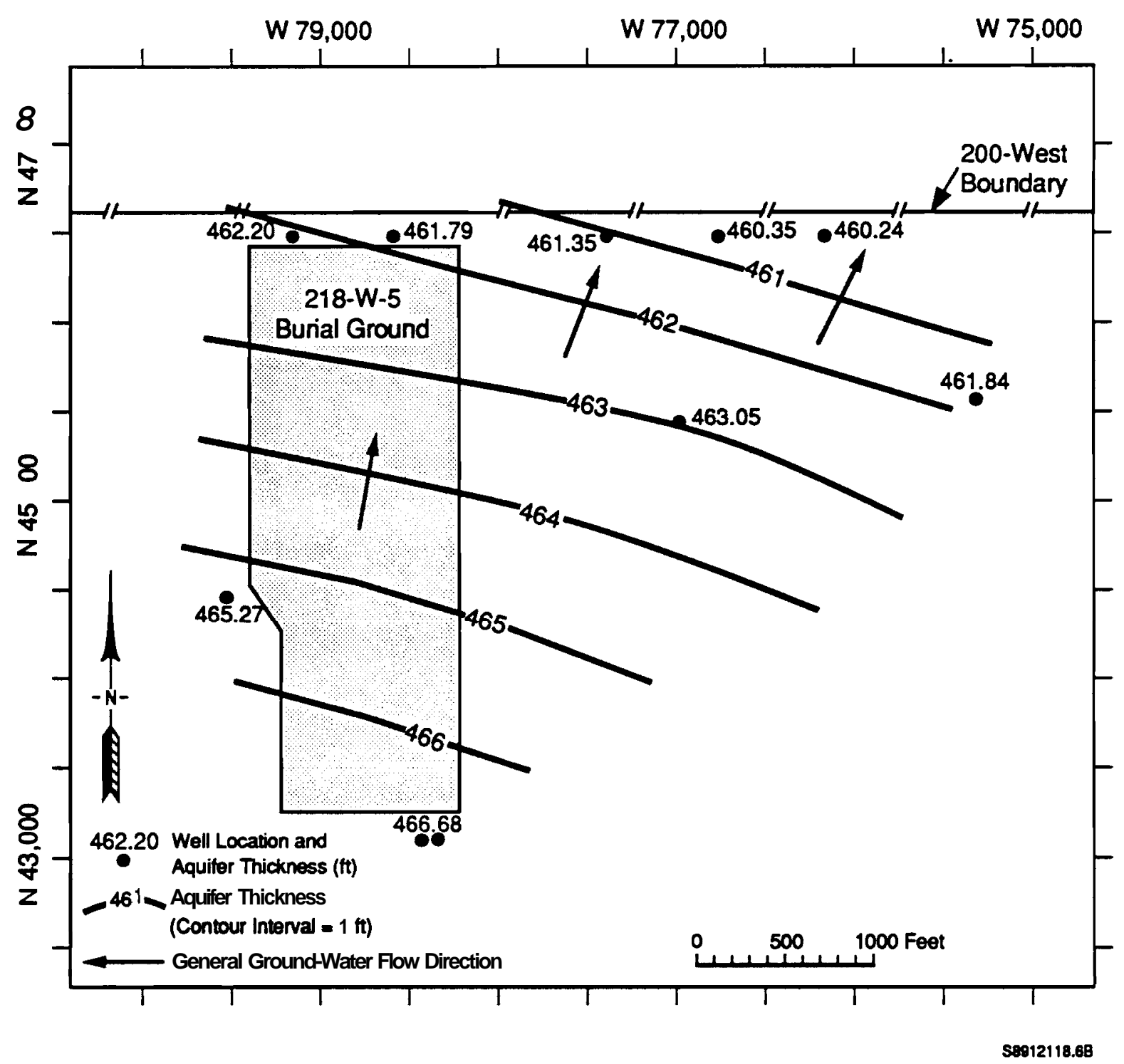

FIGURE 34. Surface of the Water Table in the Vicinity of the W-5 Burial Ground, Measured on February 27,1988

The lower Ringold unit and the fine-grained facies of the basal Ringold unit combined consist of up to $70 \mathrm{ft}$ of what is predominantly mud (i.e., silt and clay). These muds a d as a single confining unit separating the middle unit of the Ringold Formation from the coarse-grained faaes of the basal Ringold unit. A confined aquifer exists locally within the coarse-grained faaes of the basal Ringold unit. Where the confining Ringold layers are present beneath W-5, the saturated thickness of the unconfined aquifer ranges from about $215 \mathrm{ft}$ along the eastern boundary to about $230 \mathrm{ft}$ along the western boundary of W-5 (Figure 36).

Where lower and fine-grained basal Ringold faaes are missing, the undifferentiatedsandy gravel sequence of the basal and middle Ringold units extends downward to the top of the of the Elephant 


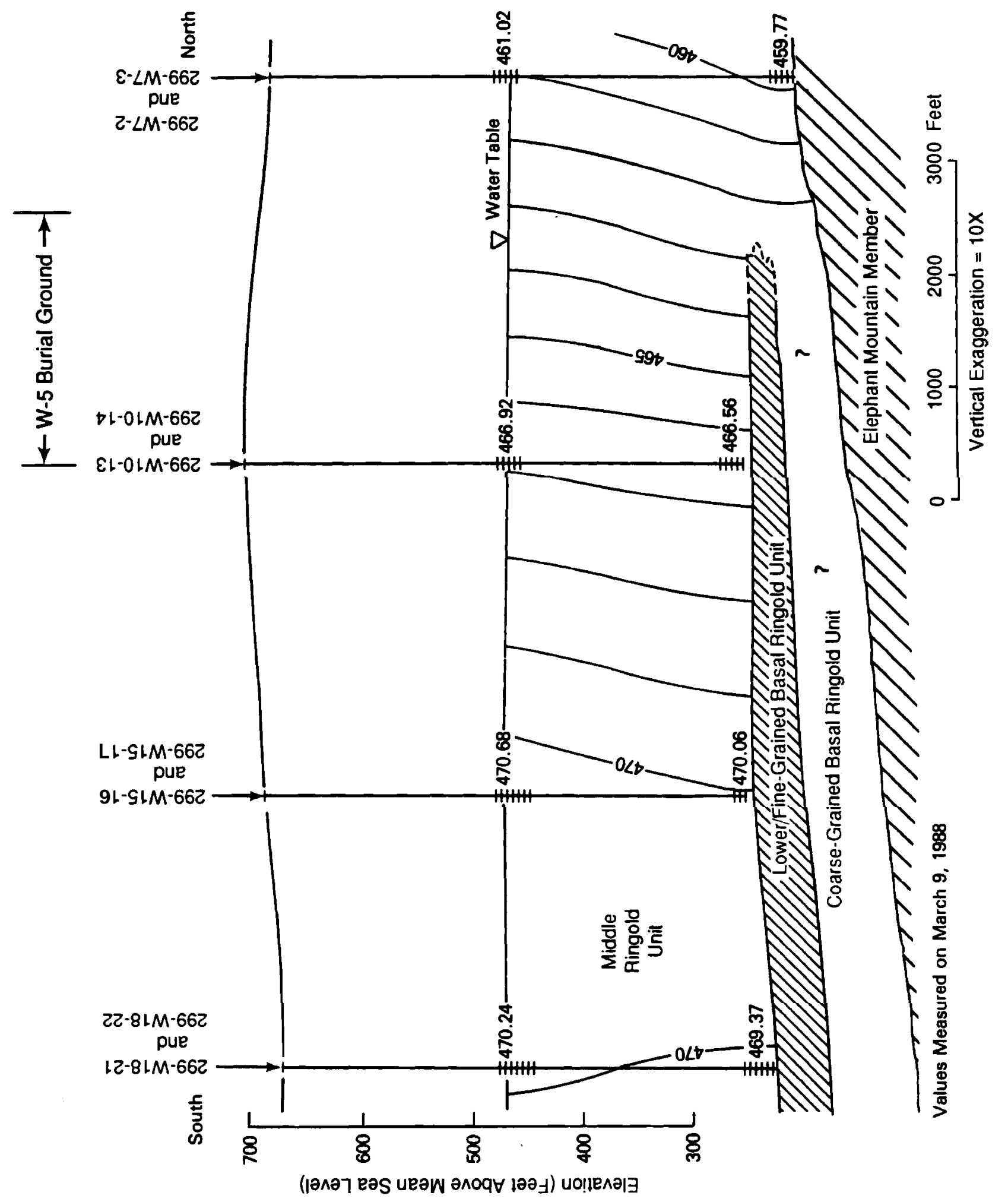

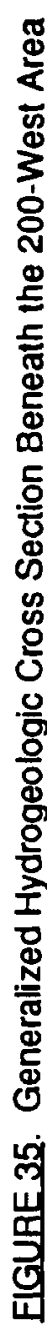




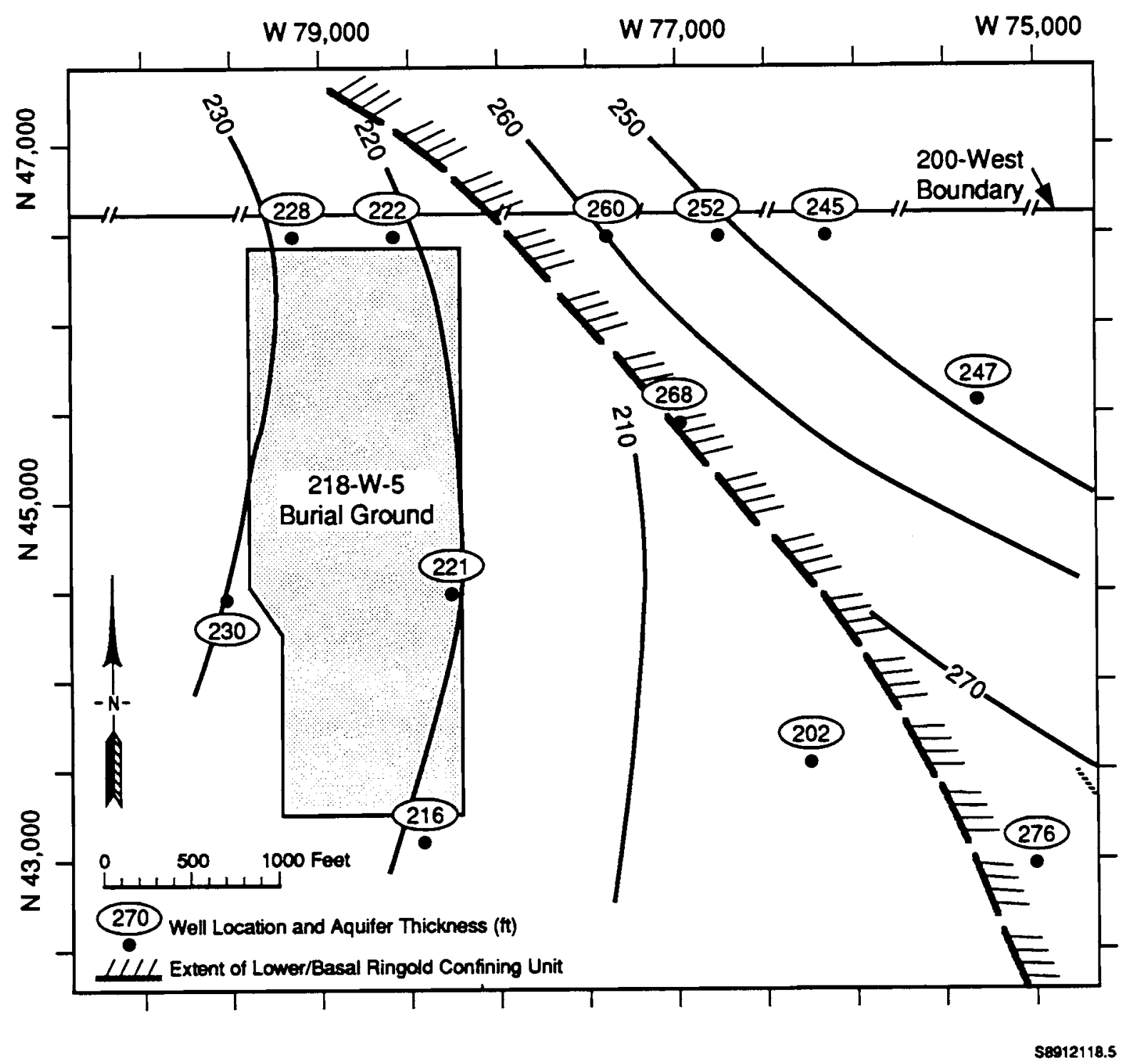

ElGURE 36. Saturated Thickness of the Unconfined Aquifer

Mountain Member. Without the confining layers, it is difficult to distinguish the middle Ringold unit from the coarse-grained basal Ringold unit. Here the saturated thickness of the aquifer is $\mathbf{- 2 6 0} \mathrm{ft}$ (Figure 36) .

\subsection{UNSATURATEDZONE}

The movement of water through the unsaturated zone to the underlying aquifer is controlled by many factors, including the thickness of the unsaturated zone (Figure 33), the hydraulic properties of sediments in the unsaturated zone, and the moisture content of these sediments (Last et al. 1989). Any excess recharge water not removed by evapotranspiration could move downward through the 
unsaturated and relatively permeable, coarse-grained Hanford formation deposits. Upon reaching the relatively fine-grained early "Palouse" soil and the carbonate-rich Plio-Pleistocene unit, water may move within these units and/or collect on them and move laterally. Water in the unsaturatedzone may also collect atop or within fine-grained lenses within the Hanford and Ringold Formations.

Because grain size generally increases northward across the Hanford formation, it is expected that water might move downward more rapidly in the northern portion of W-5. To the south, there are more fine-grained layers of sand and mud within the Hanford formation. These fine-grained layers have lower hydraulic conductivities and greater water retention capacities and so would retard water movement.

An estimate of the length of time it would take for water from the bottom of the U.S. Ecology waste facility to reach the water table is 1060 to 1400 years, based on a model presented by Bergeron et al. (1987). This depth to the water table in the area for which the estimate was made is $2300 \mathrm{ft}$. Furthermore, the geohydrology in that area is considerably different from that at W-5, so these travel times are not necessarily applicable to W-5.

\subsection{HYDROLOGIC PROPERTIES}

Most of the information on hydrologic properties is from the unsaturated Hanford formation (Routson and Fecht 1979; Bergeron et al. 1987). Facies of the Hanford formation vary considerably from one part of the Hanford Site to another. Therefore, data from one site may not be representative of the Hanford formation at other sites. A lot of information for the transmissivity and hydraulic conductivity of the middle Ringold unit is based on aquifer tests (Gephart et al. 1979; Graham et al. 1981; Last et al. 1989). There is very little information, however, on the hydrologic properties of other units, such as the early "Palouse" soil, the Plio-Pleistocene unit, and the upper Ringold unit, which make up the central third of the stratigraphic sequence within the unsaturated zone at W-5 (Figure 3).

The following discussion presents the information available on physical and chemical properties of the sediments represented at W-5. Included are data on hydraulic conductivity, transmissivity, storativity, effective porosity, field moisture content, soil bulk density, water-retentioncharacteristics, and cation exchange capacity.

\subsubsection{Hydraulic Conductivity}

A large amount of hydraulic conductivity data is available for sediments in the Pasco Basin. In general, a one- to three-order-of-magnitude difference exists in hydraulic conductivity between the Hanford and Ringold Formations (DOE 1988, p. 3.9-88), yet large differences in the range of conductivities also exist within units (Figure 37). 


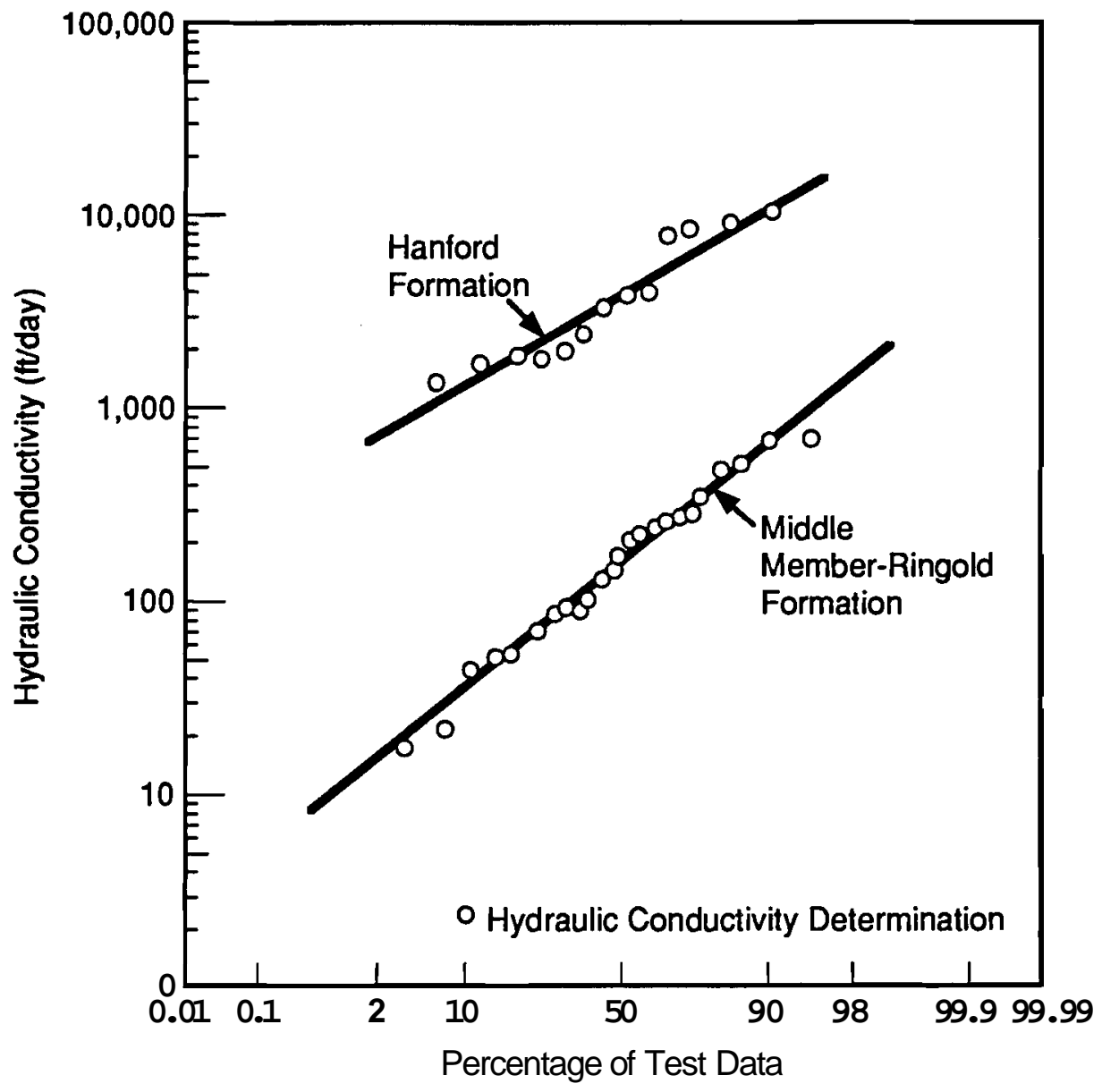

$\mathbf{S 8 9 1 2 1 1 8 . 7}$

EIGURE 37. Distribution and Range of Hydraulic Conductivities for the Hanford and Ringold Formations

Results of aquifer tests performedin wells open to the Ringold Formation near W- 5 are summarized in Table 8. Transrnissivity and hydraulic conductivity apparently decrease with depth within the basal and middle Ringold units. This is corroborated by slower drill rates, which suggest that the Ringold Formation becomes increasingly more compacted and indurated with depth.

Hydraulic conductivity values for the middle Ringold unit, calculated from transmissivities measured during aquifer testing in the vicinity of $\mathrm{W}-5$, range from 0.2 to $32 \mathrm{ft} /$ day (Table 8). These values are at the low end of the range given for hydraulic conductivity elsewhere on the Hanford Site (see Table 7). 
TABLE 8. Hydraulic Parameters Measured and/or Derived near the W-5 Burial Ground

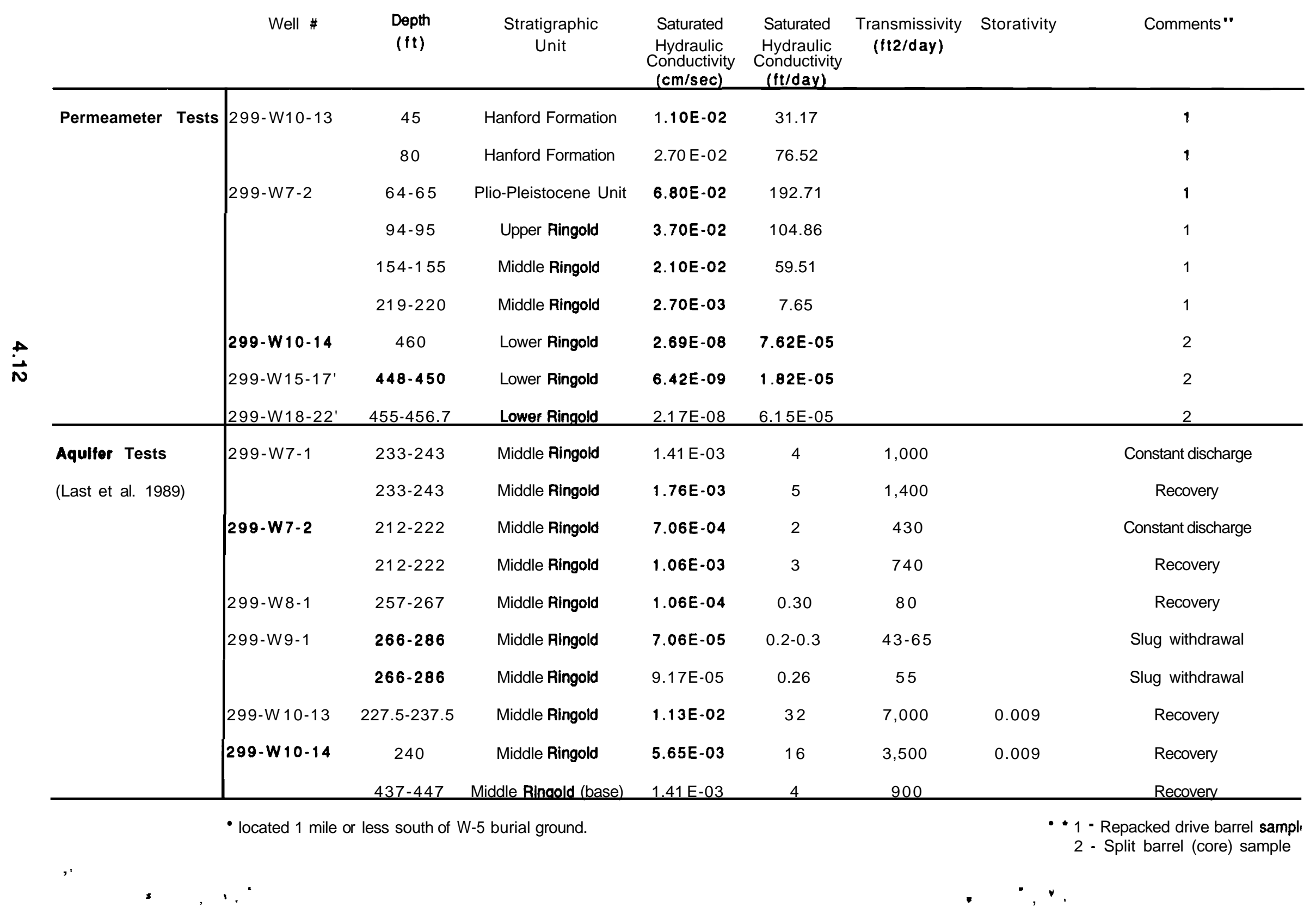


Hydraulic conductivity values calculated from permeameter tests performed in the laboratory range from about 30 to $200 \mathrm{ftlday}$ for the Hanford formation, $104 \mathrm{ft} /$ day for the upper Ringold unit, and 7 to 60 ftlday for the middle Ringold unit.

The vertical hydraulic conductivities determined from split-barrel (core) samples of the lower Ringold unit in the 200-West Area are four to seven orders of magnitude less than values reported for the lower Ringold elsewhere in the Pasco Basin (see Table 7). These values are based on three permeameter measurements, all of which are on the order of $10^{-5} \mathrm{ftlday}$ (Table 8). One of the lower Ringold samples was collected immediately south of W-5 (299-W10-14; 460-ft depth); the other two lower Ringold samples are from wells located within a mile south of W-5.

A permeameter measurement performed on the Plio-Pleistocene unit (64-ft depth, well 299-W7-2) yielded a value of $192 \mathrm{ftlday}$ (Table 8), which seems anomalously high considering the degree of cementation and normally fine-grainedtexture of this unit. This anomaly might result from the fact that the repacked drive-barrel material that was placed in the permeameter consisted of mostly broken up graveland sand-sized aggregates of finer-grained calcrete.

\subsubsection{Iransmissivity}

Transmissivity values in the vicinity of W-5 obtained from nine aquifer tests performed within the upper portion of the middle Ringold unit (Table 8) range from a low of $50 \mathrm{ft}^{2} /$ day to a maximum of $7000 \mathrm{ft}^{2} /$ day (Last et al. 1989). A transmissivity value of $900 \mathrm{ft}^{2} /$ day was obtained from the base of the middle Ringold unit (well 299-W10-14; 437- to 447-ft depth). Transmissivities measured from the middle Ringold unit a few thousand feet south of W-5 were as high as $51,000 \mathrm{ft}^{2} /$ day (Last et al. 1989).

\subsubsection{Storativity}

Only a few storativity values are available for wells near W-5; these values are based on aquifer tests of the middle Ringold unit. A storativity of 0.009 was obtained from the 299-W10-13/14 well cluster (Table 8). Storativity values of 0.001 and 0.038 were obtained from other well clusters located a few thousand feet south of W-5 (Last et al. 1989). Elsewhere on the Hanford Site, storage coefficients range from 0.02 to 0.07 ; the lower values are associated with the Ringold Formation and the higher values with the Hanfordformation (Graham et al. 1981).

\subsubsection{Porosity}

Very few data on porosity are available for sediments within the Pasco Basin. The effective porosity (i.e., interconnected pore space) of the unconfined aquifer within the 200 Areas ranges between 10 and $30 \%$. The lower value is correlated with the lower Ringold unit, and the upper value approaches the total porosity of the Hanford formation(Graham et al. 1981). 
The total porosity calculated from mean particle and bulk densities ranges from 0.256 to $0.477 \mathrm{~cm}^{3} / \mathrm{cm}^{3}$, measured from predominantly sandy facies of the Hanford formation at the Grout Treatment Facility.

\subsubsection{Field Moisture Content}

Moisture contents vary depending on sediment grain-size distribution and stratigraphic relationships to adjacent units. In general, finer-grainedsediments, particularly those with significant amounts of silt and/or clay, contain the most moisture. This is shown in Table 9 , in which most samples with $>5 \%$ water are associated with mud. Moisture contents in the unsaturated zone may range from as little as $1-2 \%$ to as much as $18 \%$ water (Last et al. 1989). Most measured water contents are within the range of 2 to $6 \%$ water. Zones with the highest moisture content are usually concentrated along significant textural boundaries where large contrasts in average grain size exist. For example, in well 299-W7-1, a moisture content of $12 \%$ water occurs at the $\sim 140$-tt depth within a poorly sorted, calcareous sandy mud layer where it is overlain by a clean sand, within the unsaturated upper Ringold unit (see Figure 4).

\subsubsection{Soil Bulk Density}

Soil bulk density and particle density are important parameters for determining porosity of the sediments. Accurate measurement of bulk density requires intact, undisturbed sediment samples. A procedure to determine soil bulk density has been given by Blake and Hartage (1986). No soil bulk density measurements have been performed on samples near W- 5 to date. Bulk density has been measured in sediment cores collected from the Grout Treatment Faality located -7 miles east of W- 5 . In that area, the mean bulk density ranged from 1.27 to $1.97 \mathrm{~g} / \mathrm{cm}^{3}$ for the predominantly sandy facies of the Hanford formation.

\subsubsection{Water-Retention Characteristics}

A total of six water-retention analyses are available for wells in the immediate vicinity of W-5. Waterretention data recently obtained from wells in the 200 Areas are presented in Table 10. Plots of each of the characteristiccurves for moisture are presented in Appendix D. Figure 38 shows the contrast in characteristic curves for four different-textured samples of the Hanford formation collected from the 200-West Area. Saturated, these samples contained up to $40 \%$ water.

Moisture characteristic curves from other hydrogeologic units sampled from well 299-W7-2 are shown in Figure 39. The sample from the Plio-Pleistocene unit contains the highest saturated water volume (almost 50\%). One sample from the middle Ringold unit (at a depth of $219 \mathrm{ft}$ ) is probably significantly more compacted or cemented, judging by its relatively low saturated volumetric water content. 
IABLE9. Field Moisture Data Collected from Wells near the W-5 Burial Ground

Moisture Data from Wells Within $1000 \mathrm{ft}$ of $\mathrm{W}-5$

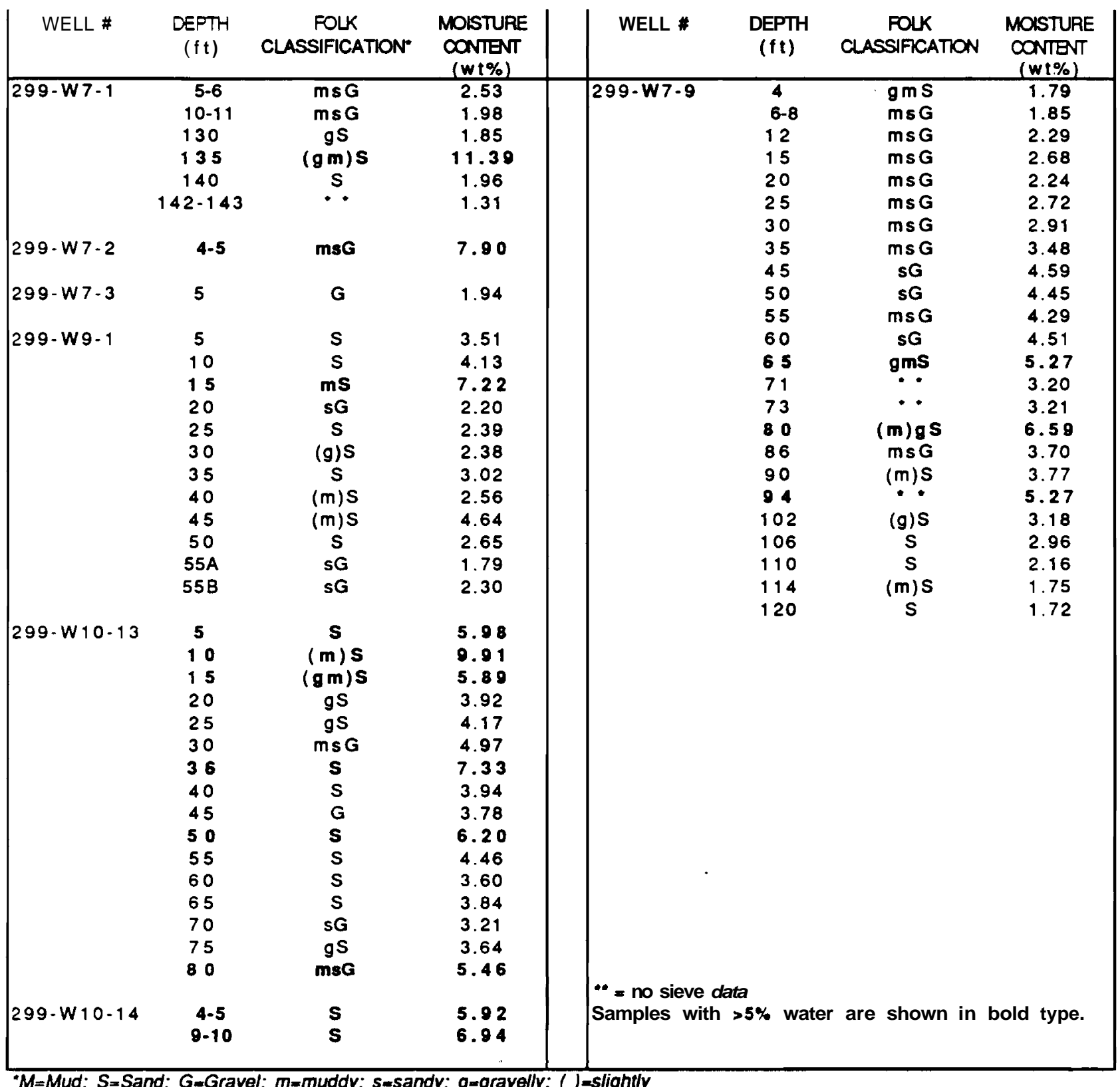




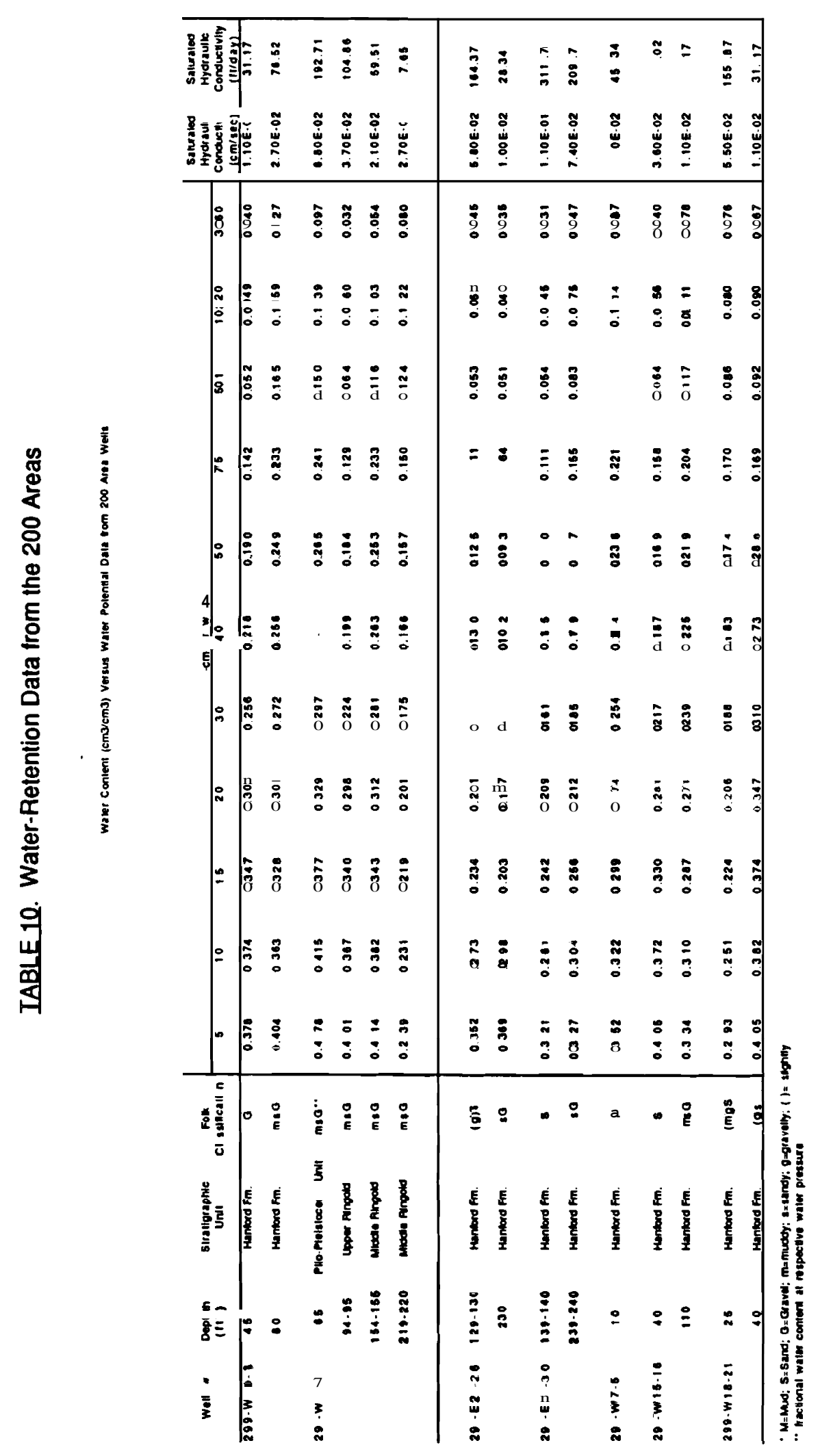

4.16 


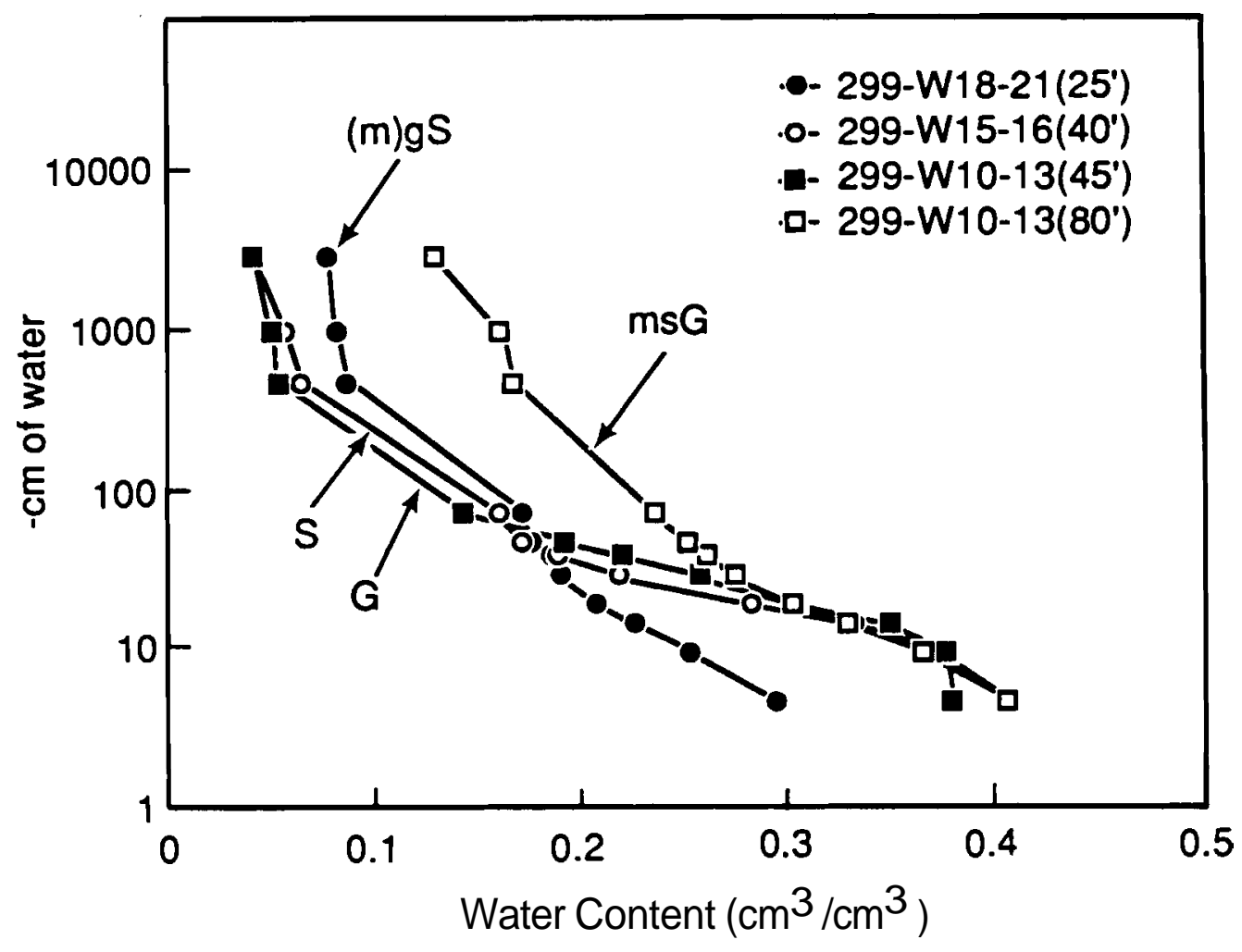

EIGURE 38. Moisture Characteristic Curves for the Hanford Formation

Like those shown for the Hanford formation (Figure 38), the samples representedin Figure 39 lose the most water between 10 and $100 \mathrm{~cm}$ of head pressure.

Water-retention characteristics were measured on 24 samples from sands and muds (transitional facies) of the Hanfordformation just south of the 200-East Area (Bergeronet al. 1987, p. 39). Some of these data may be representative of the finer-grainedportions of the Hanford formation in the southern portion of W-5.

\subsubsection{Cation Exchange Capacity}

A total of 11 samples from wells near W-5 have been analyzed for cation exchange capacity (CEC; Table 11). The stratigraphic locations that these samples came from are shown in the cross sections (Figures 4 to 6). 


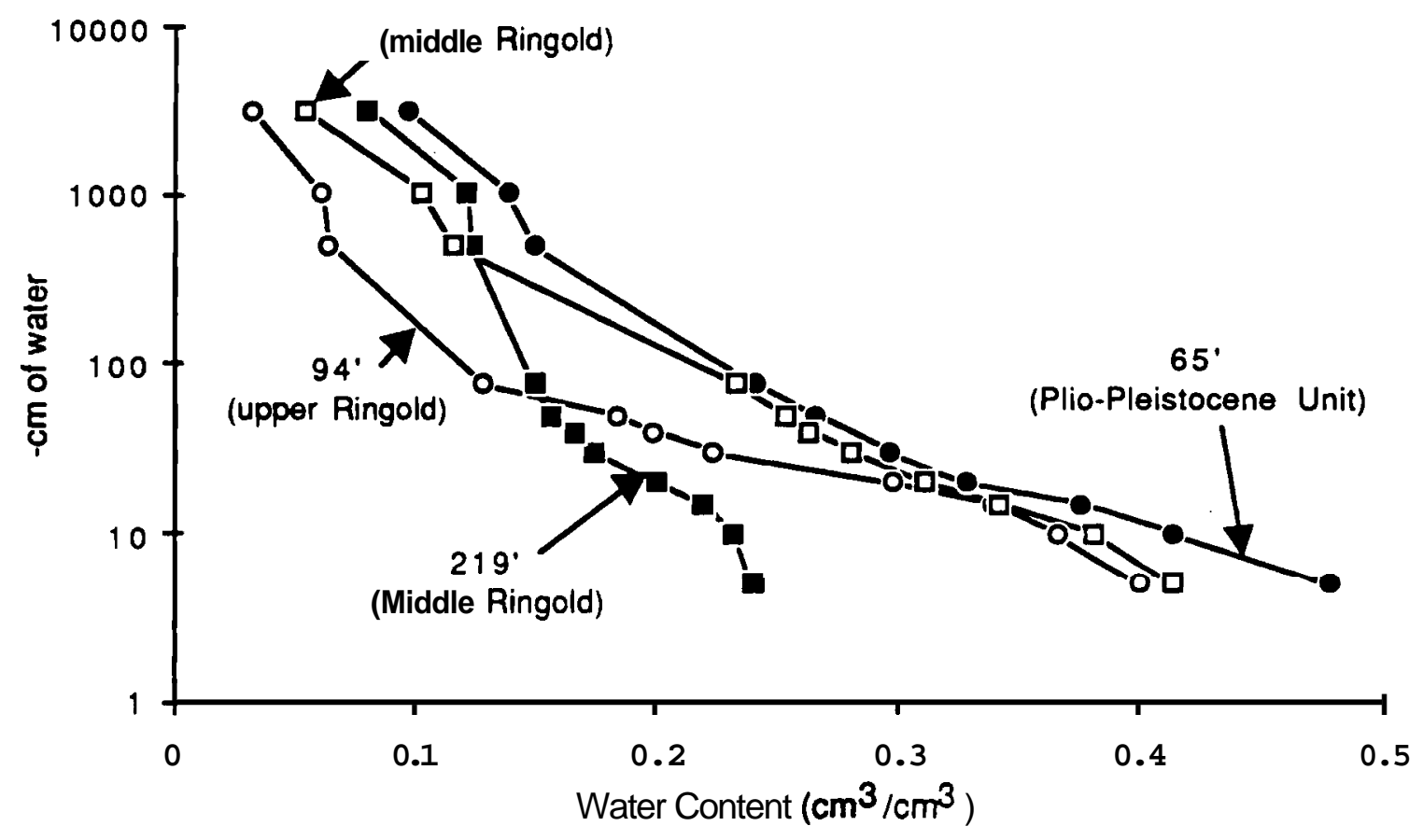

EIGURE 39. Moisture Characteristic Curves of Four Stratigraphic Intervals from Well 299-W7-2

$\underline{T A B I}$ E 11. Cation Exchange Capacity of Sediment Samples Collected near the W-5 Burial Ground

\begin{tabular}{|c|c|c|c|}
\hline Well \# & $\begin{array}{l}\text { Depth } \\
(\mathrm{ft})\end{array}$ & $\begin{array}{c}\text { Stratigraphic } \\
\text { Unit }\end{array}$ & $\begin{array}{l}\text { Cation Exchange } \\
\text { Capacity } \\
\text { (meq/100 q soil) }\end{array}$ \\
\hline 299-W7-2 & $\begin{array}{c}64-65 \\
94-95 \\
155 \\
220\end{array}$ & \begin{tabular}{|} 
Plio-Pleistocene Unit \\
Upper Ringold \\
Middle Ringold \\
Middle Ringold
\end{tabular} & $\begin{array}{l}6.0 \\
2.3 \\
2.2 \\
3.6\end{array}$ \\
\hline 299-W7-3 & 450 & Basal Ringold (?) & 3.2 \\
\hline $299 \cdot W 10 \cdot 13$ & $\begin{array}{c}45 \\
115 \\
160 \\
200 \\
240\end{array}$ & $\begin{array}{l}\text { Hanford Formation } \\
\text { Early "Palouse" Soil } \\
\text { Middle Ringold } \\
\text { Middle Ringold } \\
\text { Middle Ringold }\end{array}$ & $\begin{array}{l}2.9 \\
4.1 \\
1.8 \\
3.2 \\
6.2\end{array}$ \\
\hline 299-W 10-14 & 440 & Middle Rinaold & 6.2 \\
\hline
\end{tabular}


With the limited data available, it is difficult to identify any trends relating CEC and hydrogeologic unit. The highest CEC values came from the Plio-Pleistocene unit $(6.0 \mathrm{meq} / 100 \mathrm{~g})$ and the middle Ringold unit (6.2 meq/100 g), yet the middle Ringold unit also yielded some of the lowest CEC values. Only one sample each was analyzed from the Hanford formation, early "Palouse" soil, and basal Ringold units, and all yielded intermediate CEC values (2.9 to $4.1 \mathrm{meq} / 100 \mathrm{~g})$. 


\subsection{REFERENCES}

Alison, L. E., and C. D. Moodie. 1965. "Carbonate." In Methods of Soil Analysis, Part 2, ed. C. A. Black, pp. 1379-1396. American Society of Agronomy, Madison, Wisconsin.

ASTM. 1986a. "Specific Gravity of Soils." Annual Book of ASTM Standards, Vol. 4.08, ASTM D854. American Society for Testing and Materials, Philadelphia, Pennsylvania.

ASTM. 1986b. "Laboratory Determination of Water (Moisture) Content of Soil, Rock, and Soil-Aggregate Mixtures." Annual Book of ASTM Standards, Vol. 4.08, ASTM D2216. American Society for Testing and Materials, Philadelphia, Pennsylvania.

Baker, V. R. 1973. "Paleohydrology and Sedimentology of Lake Missoula Flooding in Eastern Washington." Special Paper \# 144, Geological Society of America, Boulder, Colorado.

Bergeron, M. P., G. V. Last, and A. E Reisenauer. 1987. Geohydrology of a Commercial $\perp$ ow-Level Radioactive Waste Disoosal Facility near Richland. Washinoton. Report prepared for U.S. Ecology, Inc., Richland, Washington.

Bjornstad, B. N. 1980. Sedimentology and Depositional Environment of the Touchet Beds. Walla Walla Biver Basin. Washington. RHO-BWI-SA-44, Rockwell Hanford Operations, Richland, Washington.

Bjornstad, B. N. 1984. Suprabasalt Stratioraphy Within and Adjacent to the Beference Repository Location. SD-BWI-DP-039, Rockwell Hanford Operations, Richland, Washington.

Bjornstad, B. N. 1985. "Late-Cenozoic Stratigraphy and Tectonic Evolution Within a Subsiding Basin, South-Central Washington." Geological Society of America, Abstracts with Programs, Vol. 17, p. 524.

Bjornstad, B. N., and K. R. Fecht. 1989. "Pre-Wisconsin Glacial-Outburst Floods: Pedogenic and Paleomagnetic Evidence from the Pasco Basin and Adjacent Channeled Scabland." Geological Society of America, Abstracts with Programs, Vol. 21, No. 5, p. 58.

Bjornstad, B. N., K. R. Fecht, and A. M. Tallman. 1987. Quaternary Stratioraphy of the Pasco Basin. South-Central Washinoton. RHO-BW-563A, Rockwell Hanford Operations, Richland, Washington

Blake, G. R., and K. H. Hartage. 1986. "Bulk Density." In Methods of Soil Analysis, Part 1, ed. A. Klute, pp. 383-409. American Society of Agronomy, Madison, Wisconsin.

Brown, D. J. 1959. Subsurface Geoloav of the Hanford Separation Areas. HW-61780, General Electric Company, Richland, Washington.

Brown, D. J. 1960. An Eolian Deposit Beneath the 200-West Area. HW-67549, General Electric Company, Richland, Washington.

Cooper, H. H., and C. E. Jacob. 1946. "A Generalized Graphical Method for Evaluating Formation Constants and Summarizing Well-Field History." In Iransactions of the American Geophysical Union 27:526-534.

DOE. 1986. Environmental Assessment. Beference Bepository location Hanford Site Washington. DOE/RW-0070, U.S. Department of Energy, Washington D.C. 
DOE. 1988. Consultation Draft. Site Characterization Plan. Reference Repository Location. Hanford Site. Washington. DOWRW-0164, Vol. 1, U.S. Department of Energy, Washington, D.C.

Fecht, K. R, S. P. Reidel, and A. M. Tallman. 1985. Paleodrainage of the Columbia Biver System on the Columbia Plateau of Washinoton State: A Summary. RHO-BW-SA-318P, Rockwell Hanford Operations, Richland, Washington.

Flint, R. F. 1938. "Origin of the Cheney-Palouse Scabland Tract." Geological Society of America Bulletin 49:461-524.

Folk, R. L. 1974. Petrologr of Sedimentary Rocks. Hemphill's, Austin, Texas.

Gee, G. W. 1987. Recharge at the Hanford Site: Status Repert. PNL-6403, Pacific Northwest Laboratory, Richland, Washington.

Gee, G. W., and J. W. Bauder. 1986. "Particle-Size Analysis." In Methods of Soil Analysis, Part 1, ed. A. Klute, pp. 383-409. American Society of Agronomy, Madison, Wisconsin.

Gephart, R. E, R. C. Arnett, R. G. Baca, L. S. Leonhart, and F. A Spane, Jr. 1979. .Hydrologic Studies within the Columbia Plateau. Washinoton: An Intearation of Current Knowledge. RHO-BWI-ST-5, Rockwell Hanford Operations, Richland, Washington.

Gile, L. H., F. F. Peterson, and R. B. Grossman. 1966. "Morphologicaland Genetic Sequences of Carbonate Accumulation in Desert Soils." Soil Science 101:347-360.

Graham, M. J., M. D. Hall, S. R. Strait, and W. R. Brown. 1981. Hydrology of the Separations Area. RHO-ST-42, Rockwell Hanford Operations, Richland, Washington.

Graham, M. J., G. V. Last, and K. R. Fecht. 1984. An Assessment of Aquifer Intercommunication in the B Pond-Gable Mountain Pond Area of the Hanford Site. RHO-RE-ST-12P, Rockwell Hanford Operations, Richland, Washington.

Hajek, B. F. 1966. Soil Survey. Hanford Project in Benton County. Washingten. BNWL-243, Pacific Northwest Laboratory, Richland, Washington.

Klute, A. 1986. "Water Retention: Laboratory Methods." In Methods of Soil Analysis, Part 1, ed. A. Klute, pp. 635-660. American Society of Agronomy, Madison, Wisconsin.

Klute, A, and C. Dirksen. 1986. "Hydraulic Conductivity and Diffusivity: Laboratory Methods." In Methods of Soil Analysis, Part 1, ed. A Klute, pp. 687-732. American Society of Agronomy, Madison, Wisconsin.

Last, G. V, and B. N. Bjomstad. 1989. Bevised Ground-Water Monitoning Plan for the 200 Areas I owLevel Burial Grounds. WHC-SD-EN-AP-015, Rev. 0, Westinghouse Hanford Company, Richland, Washington.

Last, G. V., B. N. Bprnstad, M. P. Bergeron, D. W. Wallace, D. R. Newcomer, J. A. Schramke, M. A Chamness, C. S. Cline, S. P. Airhart, and J. S. Wilbur. 1989. Hydrogeology of the 200 Areas Low-Level Burial Grounds - An Interim Repert. PNL-6820, 2 volumes, Pacific Northwest Laboratory, Richland, Washington. 
Ledgerwood, R. K. 1986. Borehole Depths and Thicknesses for Saddle Mountains and Wanapum Basalts. SD-BWI-DP-068, Rockwell Hanford Operations, Richland, Washington.

Lillie, J. T., A. M. Tallman, and J. A. Caggiano. 1978. Preliminary Geologic Map of the Late Cenozoic Sediments of the Western Half of the Pasco Basin. RHO-BWI-LD-8, Rockwell Hanford Operations, Richland, Washington.

Lindsey, K. A, and D. R. Gaylord. 1989. Sedimentoleay and Stratioraphy of the Miocene-Pliocene Bingold Formation. Hanford Site, South-Central Washington. WHCWA-0740-FP, Westinghouse Hanford Company, Richland, Washington.

Lindsey, K. A., Gaylord, D. R., and Poeter, E. P. 1989. "Sedimentary and Stratigraphic Examination of the Ringold Formation, Hanford Nuclear Reservation, Washington: Applied Lithofacies Analysis." Geelegical Society of America. Abstracts with Programs, Vol. 21, No. 5, p. 107.

Lohman, S. W. 1972. Ground-WaterHydraulics. Professional Paper 708, U.S Geological Survey, Washington, D.C.

Machette, M. N. 1985. "Calcic Soils of the Southwestem United States." In Soils and Quatemary Geology of the Southwestern United States, ed. D. L. Weide, pp. 1-21. Special Paper 203, Geological Society of America, Boulder, Colorado.

McGhan, V. L. 1989. Hanford Wells. PNL-6907, Pacific Northwest Laboratory, Richland, Washington.

Myers, C.W., and S.M. Price, eds. 1979. Geologic Studies of the Columbia Plateau: A Status Repert. RHO-BWI-ST-4, Rockwell Hanford Operations, Richland, Washington.

Nelson, R. E. 1982. "Carbonate and Gypsum." In Methods of Soil Analysis, Part 2, eds. A G. Page, R. H. Miller, and D. R. Keeney, pp. 181-197. American Society of Agronomy, Madison, Wisconsin.

Newcomb, R. C. 1958. "Ringold Formation of Pleistocene Age in Type Locality, the White Bluffs, Washington." American Journal of Science, 256:328-340.

Newcomb, R. C., J. P. Strand, and F. J. Frank. 1972. Geoboy and Ground-Water Characteristics of the Hanford Beservation of the U.S.Atomic Eneroy Commission. Washinoton. Professional Paper 717, U.S. Geological Survey, Washington, D.C.

PNL. 1989. Procedures for Ground-Water Investigations. PNL-6894, Pacific Northwest Laboratory, Richland, Washington.

Rawlins, S. L., and G. S. Campbell. 1986. "Water Potential: Thermocouple Psychrometry." In Methods gf SoilAnalysis, Part 1, ed. A. Klute, pp. 597-618. American Society of Agronomy, Madison, Wisconsin.

Routson, R. C., and K. R. Fecht. 1979. Soil(Sediment) Properties of Twelve Hantord Wells, with Geologic Interpretation. RHO-LD-82, Rockwell Hanford Operations, Richland, Washington.

Schatz, A L, and J. J. Ammerman. 1988. Ground-Water Maps of the Hanford Site Separations Area. December 1987. WHC-EP-0142, Westinghouse Hanford Company, Richland, Washington.

Schollenberger, C. A., and R. H. Simon. 1945. "Determination of Exchange Capacity and Exchangeable Bases in Soil--Ammonium Acetate Method." Soil Scjence 59:13-25. 
Strait, S. R, and R. B. Mercer. 1987. Hydraulic Property Data from Selected Test Zenes on the Hanford Site. SD-BWI-DP-051, Rev. 2, Rockwell Hanford Operations, Richland, Washington.

Swanson, D. A, T. L. Wright, P. R. Hooper, and R. D. Bentley. 1979. Bevisions in Stratiaraphic Nomenclature of the Columbia River Basalt Group. USGS Bulletin 1457-G, U.S. Geological Survey, Washington, D.C.

Tallman, A. M., J. T. Lillie, and K. R. Fecht. 1981. "Suprabasalt Stratigraphy of the Cold Creek Syncline Area." In Subsurface of the Cold Creek Syncline, eds. C. W. Myers and S. M. Price, pp. 2-1 to 2-27, RHO-BWI-ST-14, Rockwell Hanford Operations, Richland, Washington.

Tallrnan, A M., K. R. Fecht, M. C. Marratt, and G. V. Last. 1979. Geoloov of the Separations Area. Hanford Site South-Central Washington. RHO-ST-23, Rockwell Hanford Operations, Richland, Washington.

Theis, C. V. 1935. "The Relation Between the Lowering of the Piezometric Surface and the Rate and Duration of Discharge at a Well Using Ground-Water Storage." Iransactions of the American Geophysical Union 16:519-524.

Van Luik, A E, R. M. Mitchell, G. V. Last, R. R. Routson, D. S. Landeen, and K. R. Fecht. 1980. Characterization of the W-5 Burial Ground Expansion Site. RHO-CD-941, Rockwell Hanford Operations, Richland, Washington.

Waitt, R. B., Jr. 1980. "About Forty Last Glacial Lake Missoula Jokulhlaups through Southern Washington." Journal of Geology 88:653-679.

Walker, R. G., and D. J. Cant. 1984. "Sandy Fuvial Systems." In Facies Models, 2nd ed., ed. R. G. Walker, pp. 71-89. Geological Association of Canada, Toronto, Ontario. 
APPENDIX A

GEOLOGIC LOGS 


\section{APPENDIX A}

\section{GEOLOGIC LOGS}

This appendix provides a summary of geologic and geophysical data available for existing boreholes within $1000 \mathrm{ft}$ of $\mathrm{W}-5$. These logs were used to construct cross sections (Figures 4 through 6 ) and the fence diagram (Figure 11) presented in the text. The locations of these boreholes are shown in Figure 2. Specifically, these logs include plots of percent gravel, percent sand, percent mud, dominant sand size, sand/silt ratio, percent basalt, weight percent calcium carbonate, moisture content, and/or gross gammaray log. The shaded or blackened areas appearing in these plots are arbitrary and intended to visually enhance those areas for which a given parameter is higher. The symbols used on the geologic logs are defined below.

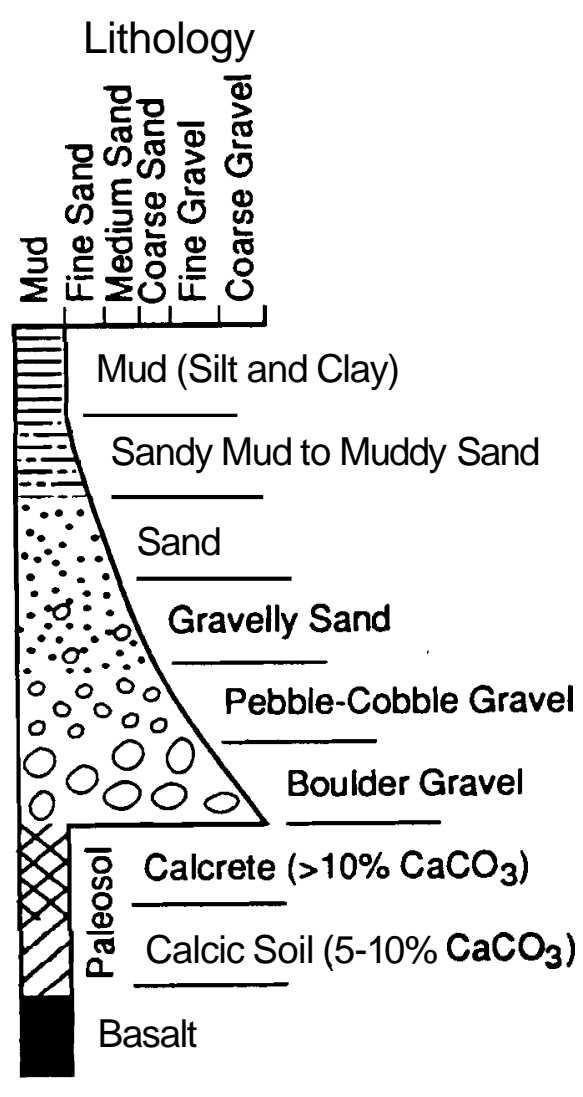

Drill Method

$\mathrm{BH}=$ Backhoe

$\mathrm{HT}=$ Hard Tool

$\mathrm{DB}=$ Drive Barrel

$\mathrm{SB}=$ Split Barrel

VIIn $=$ Mud Rotary

Water Table 


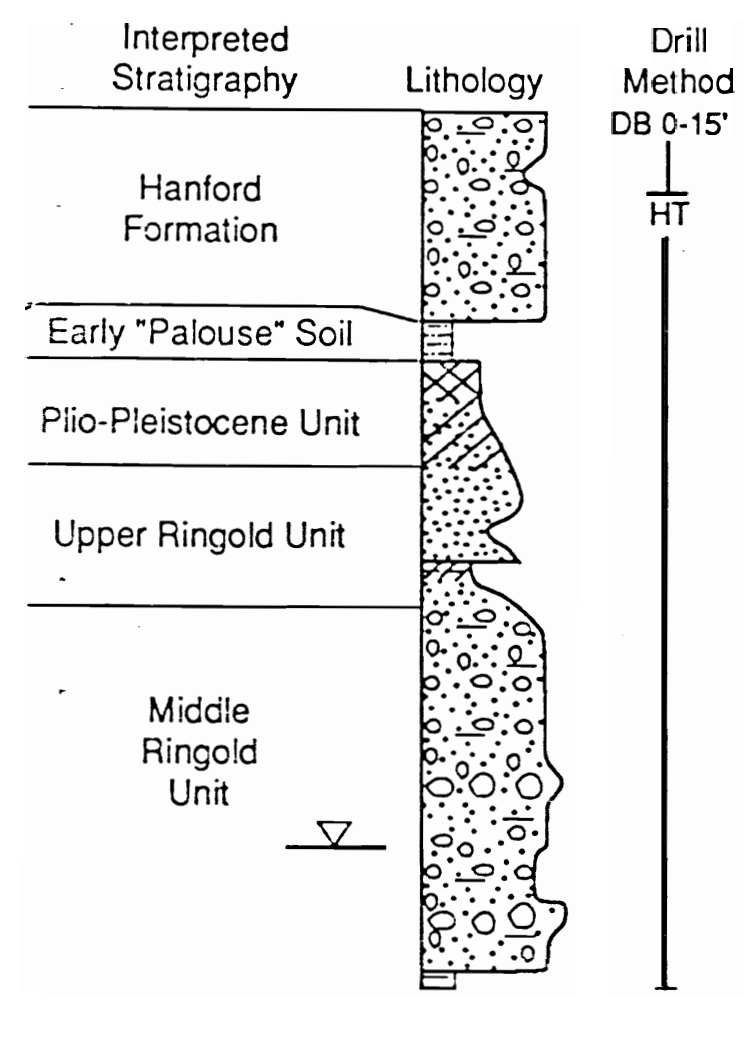
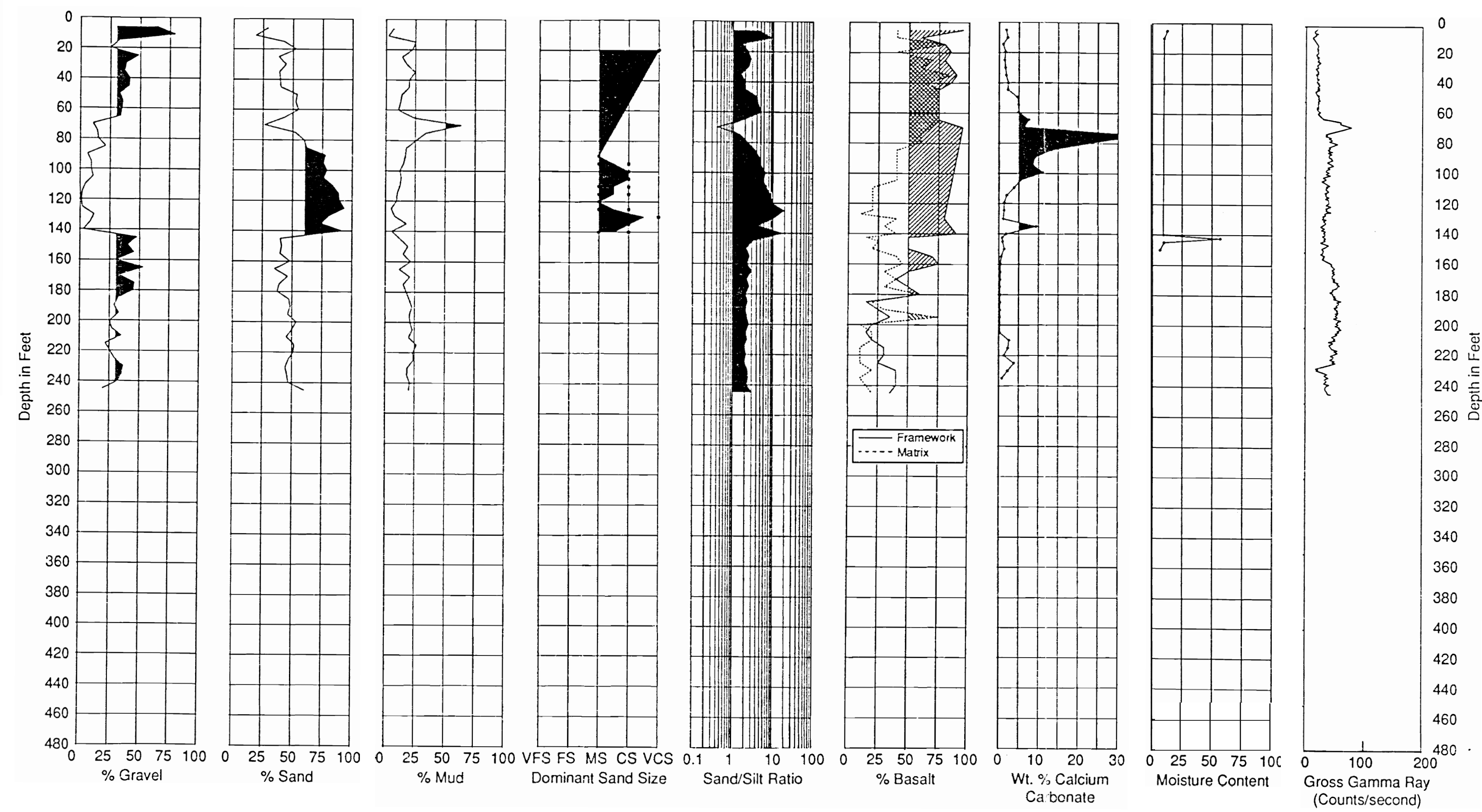
299-W7-2
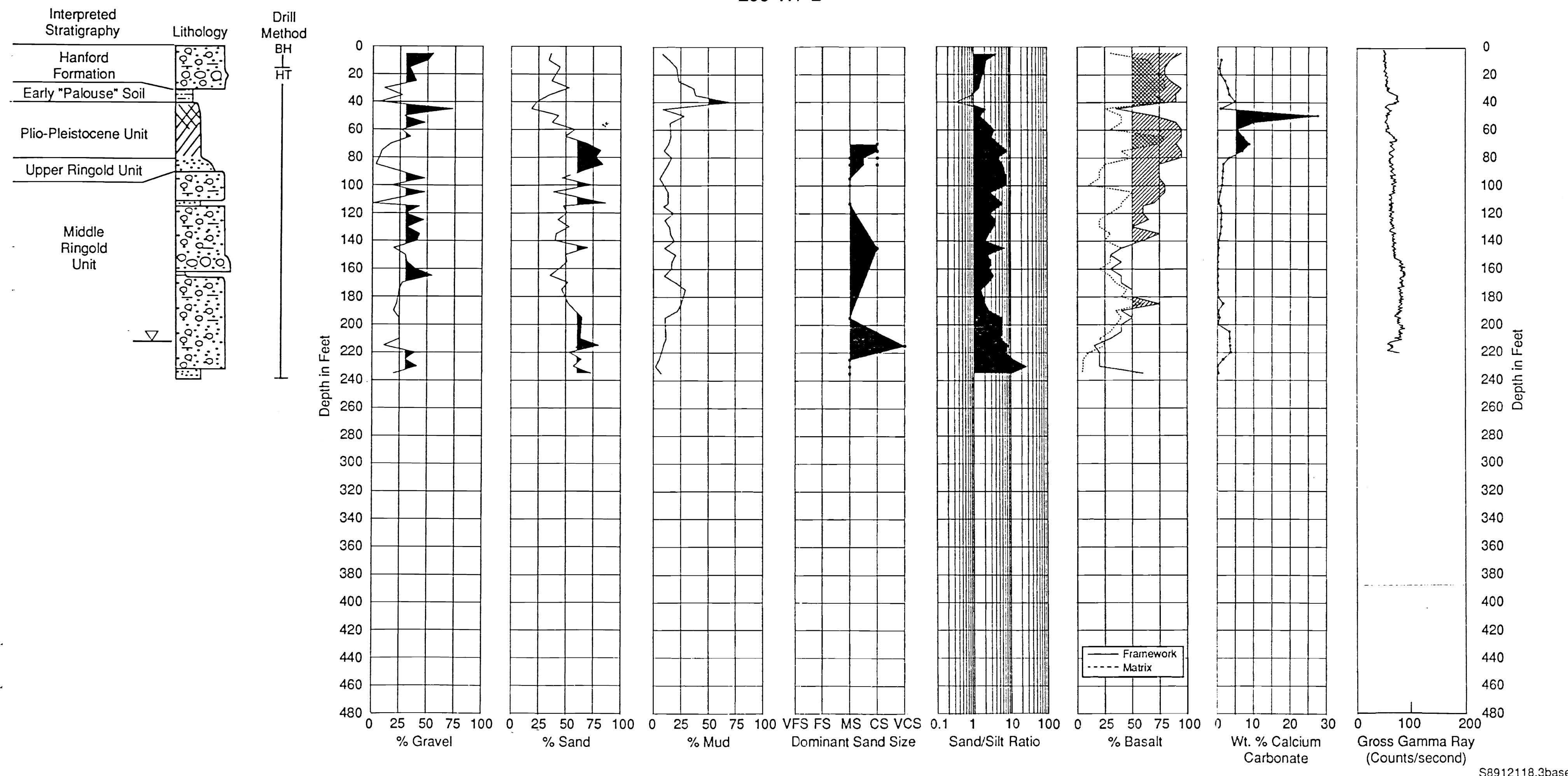
299-W7-3

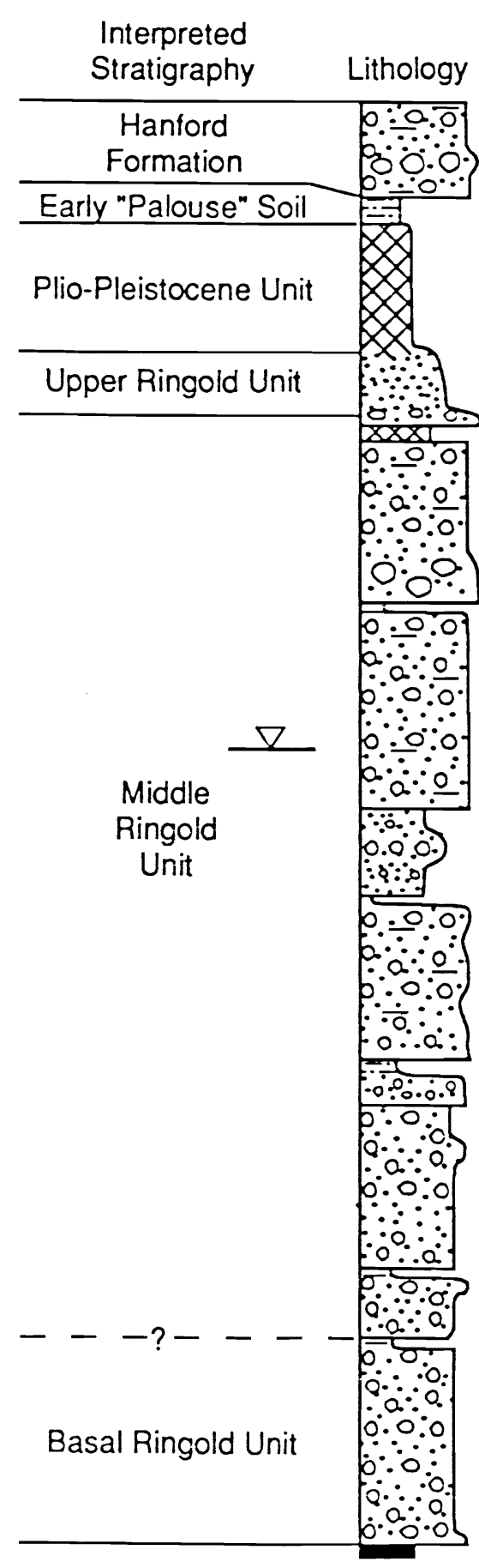

Drill
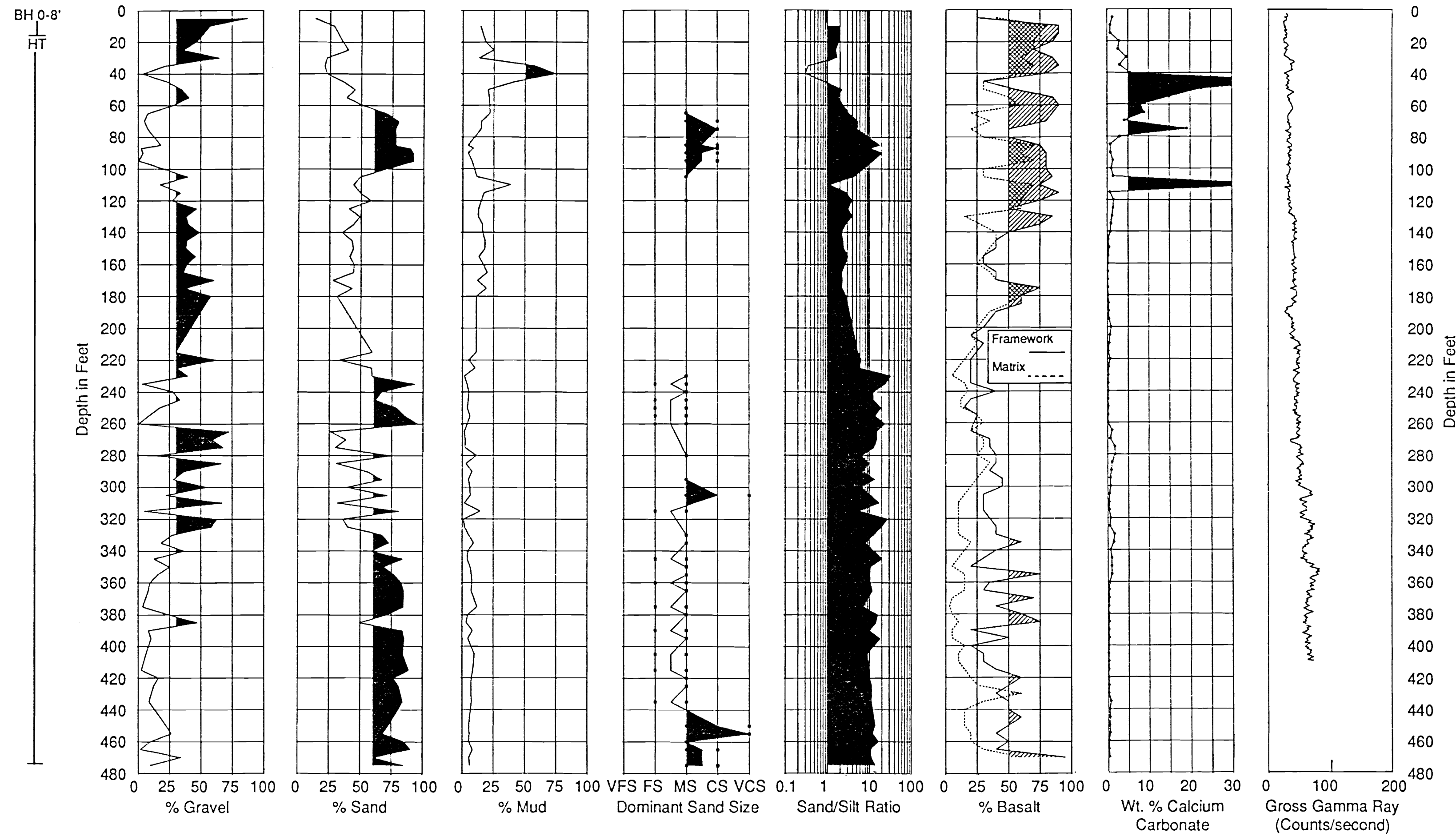
299-W7-9

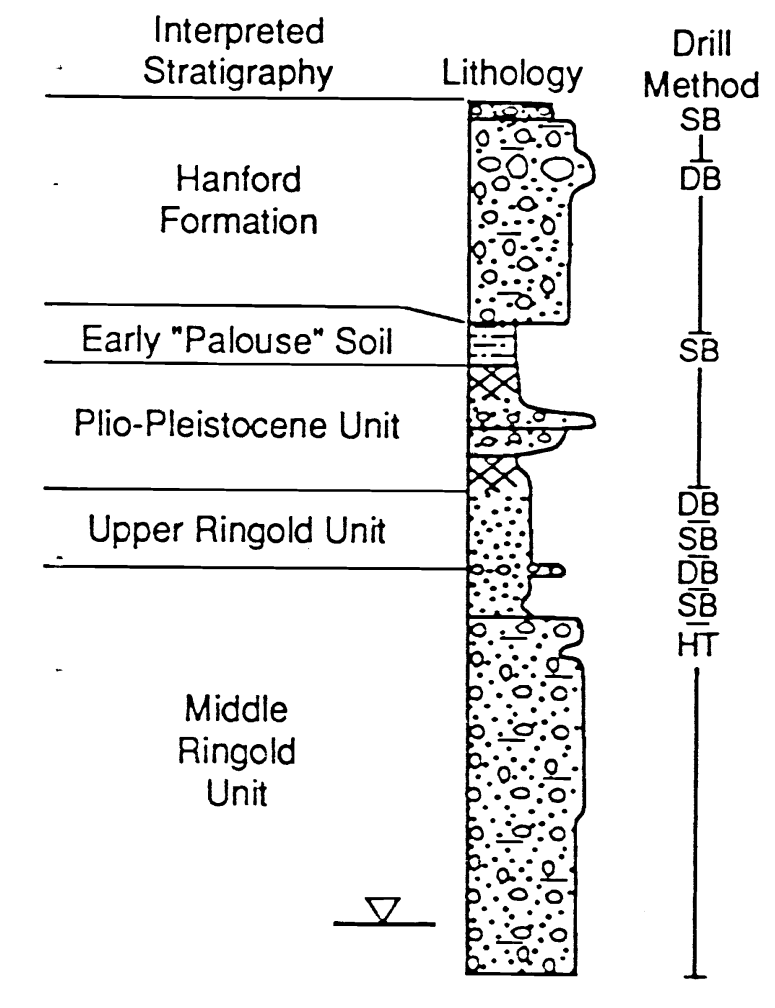
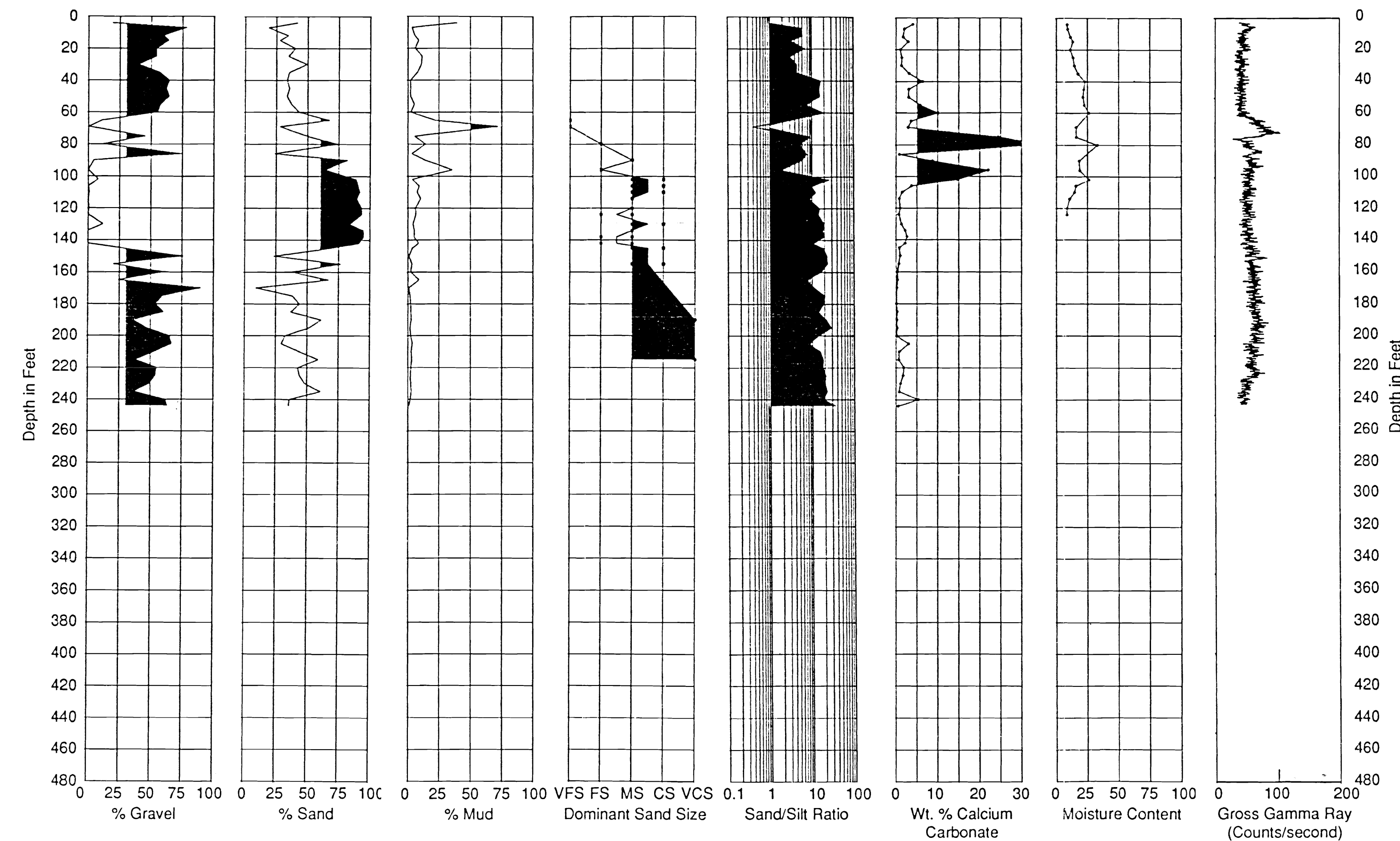
299-W8-1

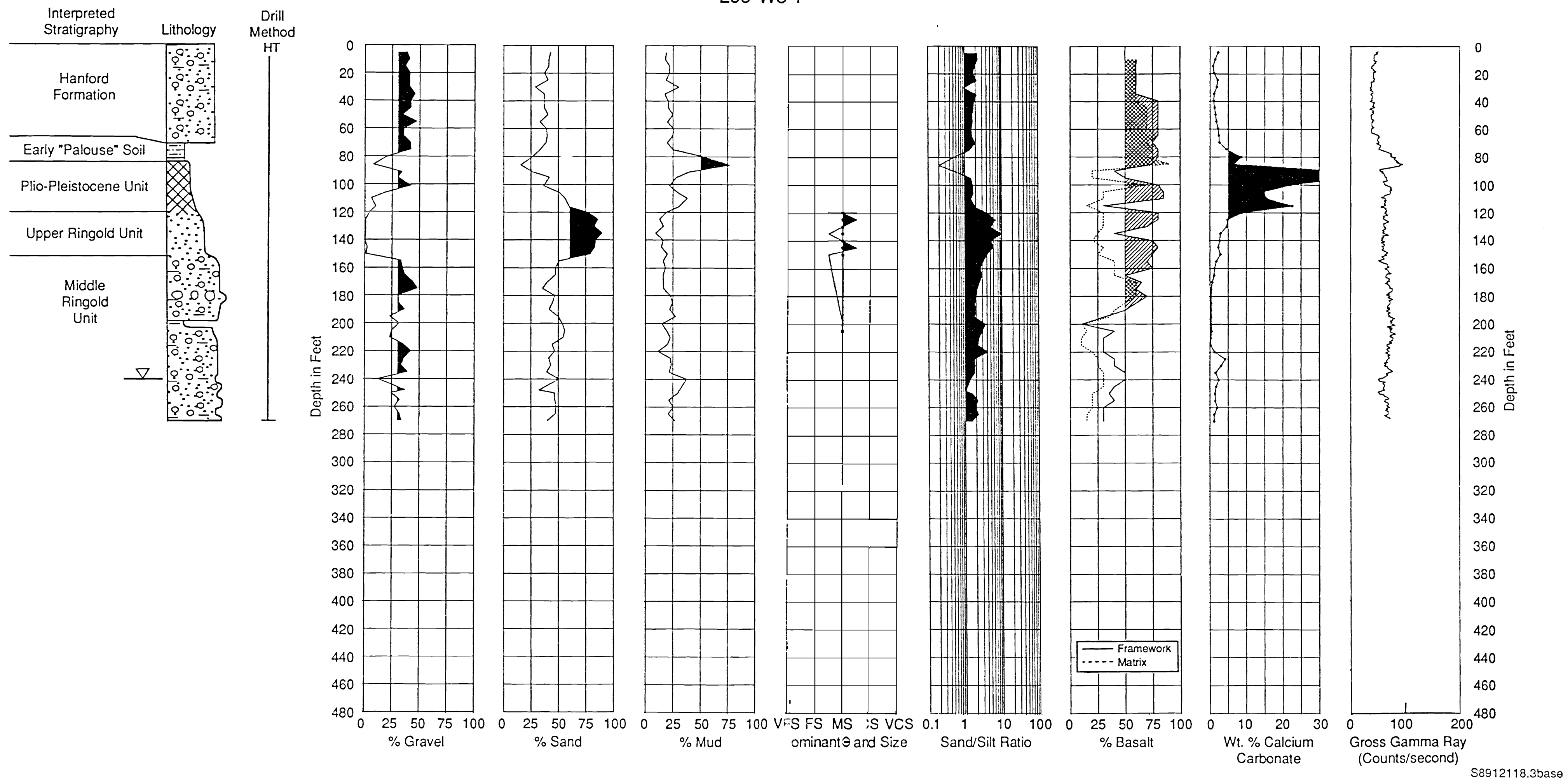




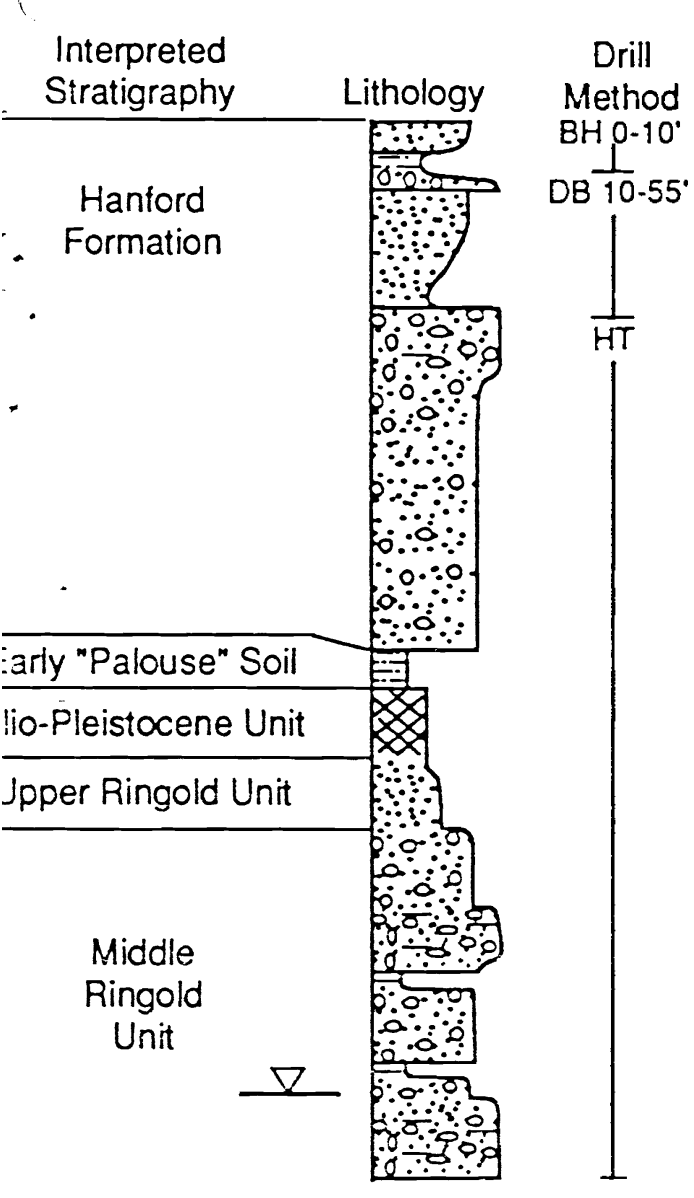

S8912118.4base

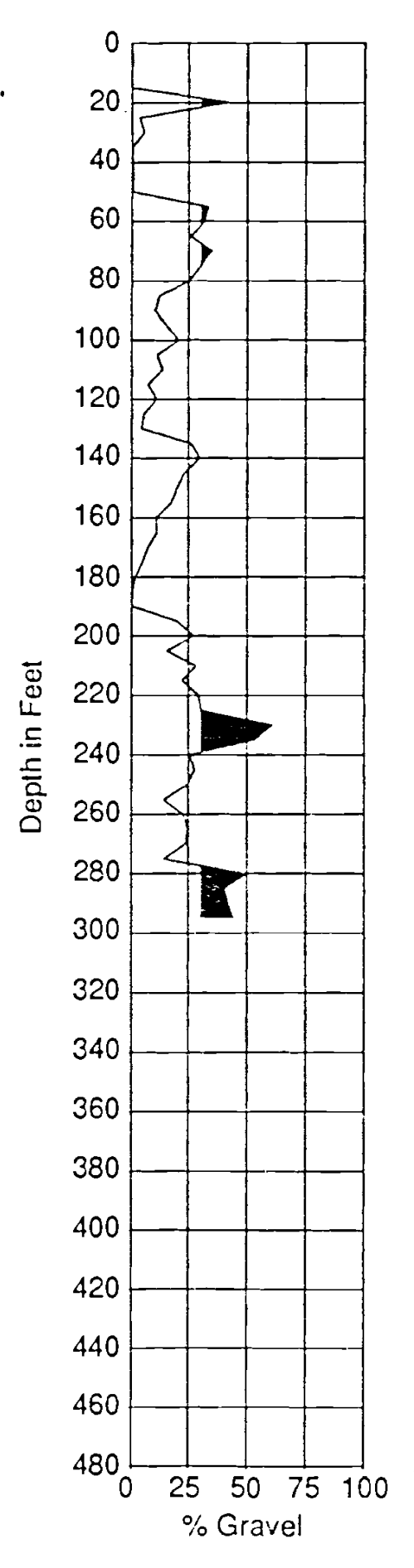

299-WV9-1

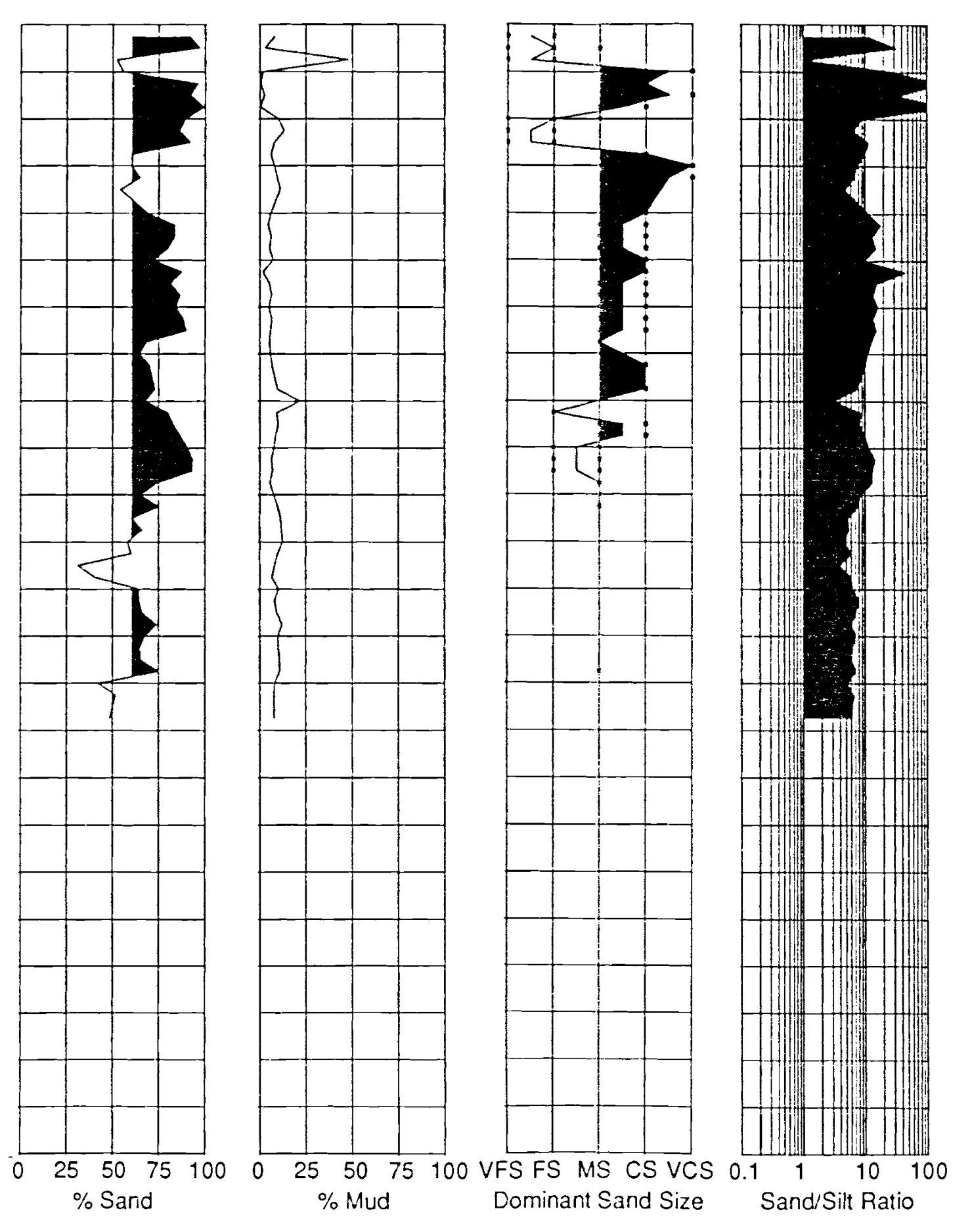

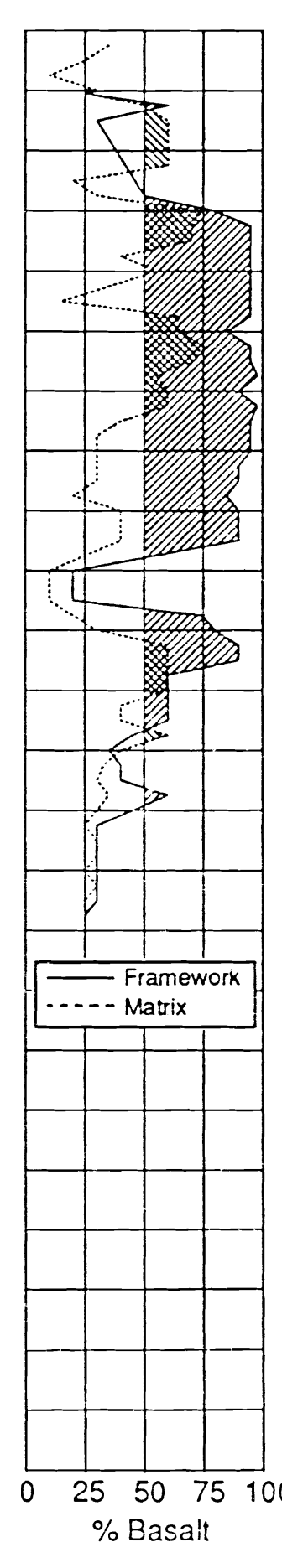
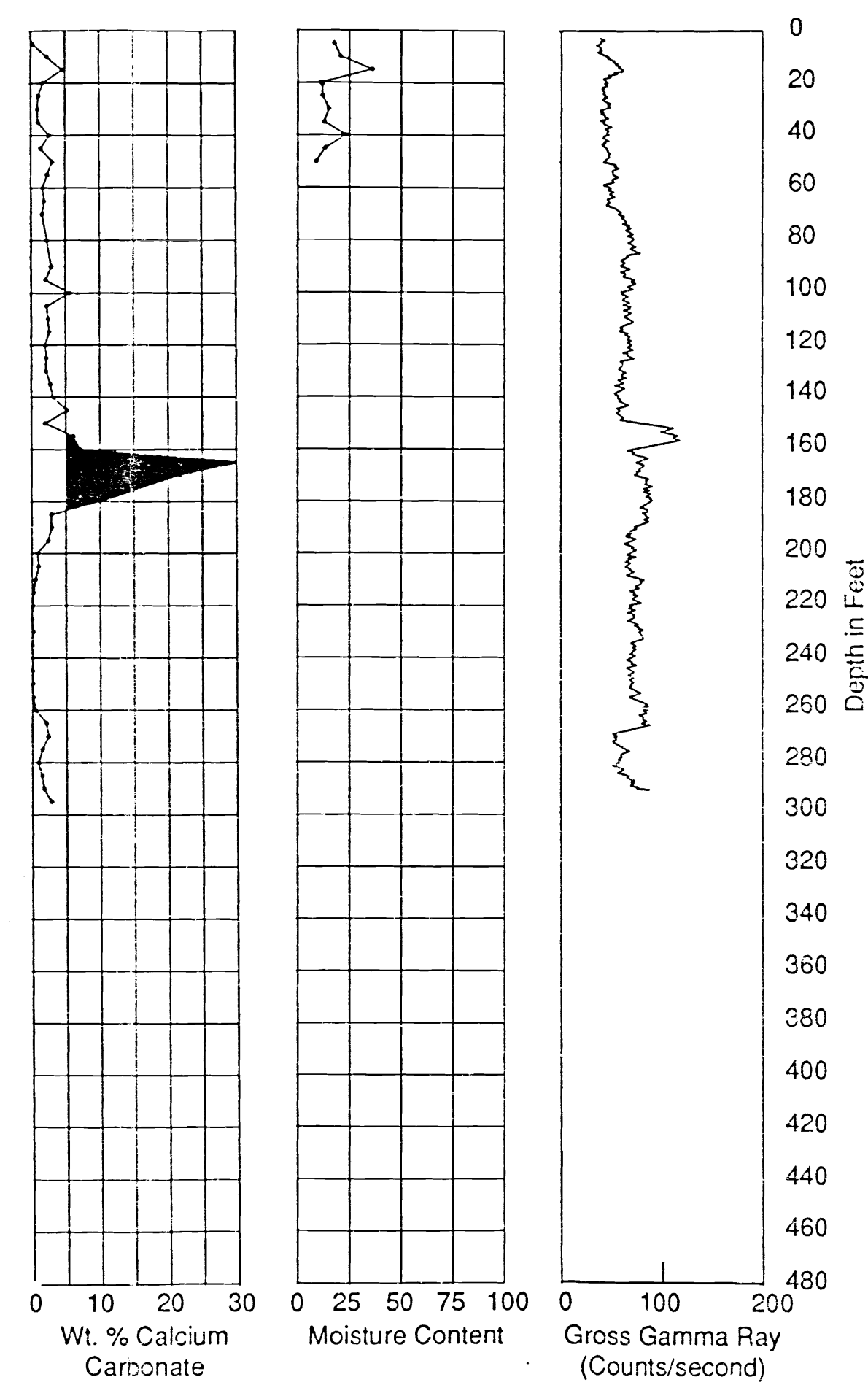
299-W/10-13

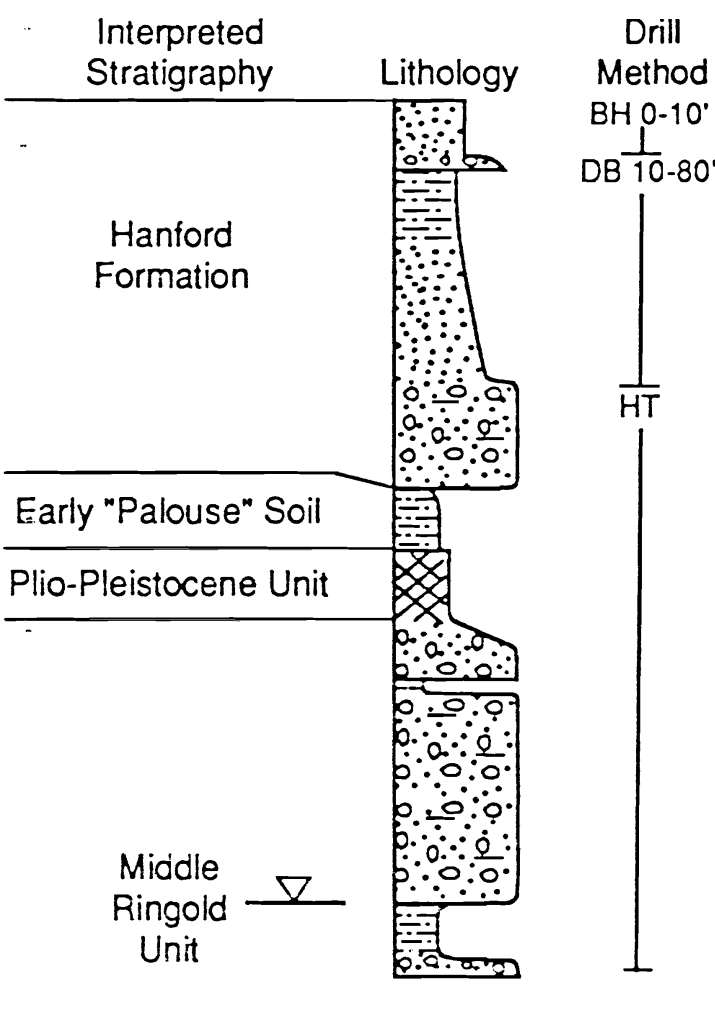
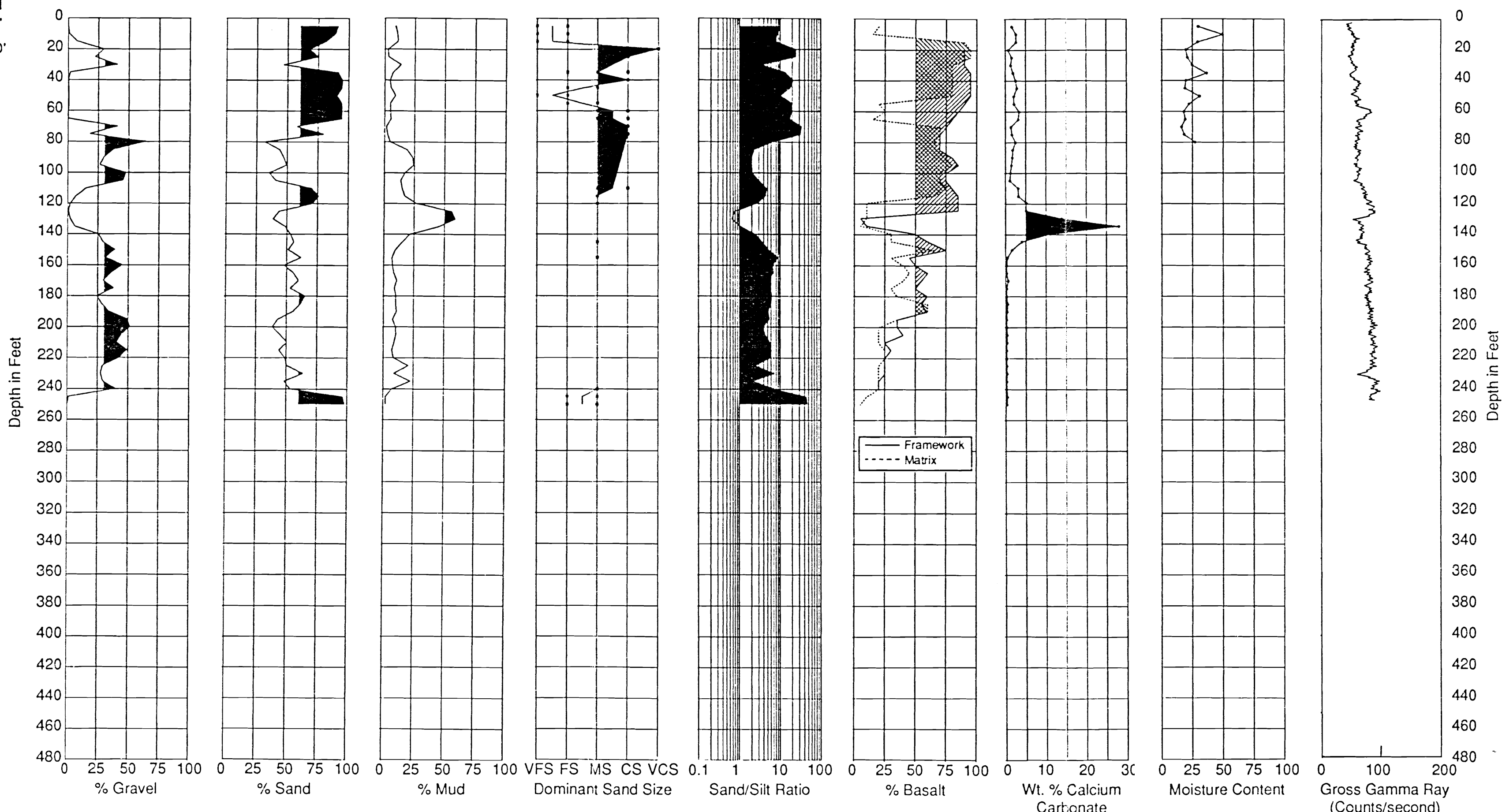
299-W10-14

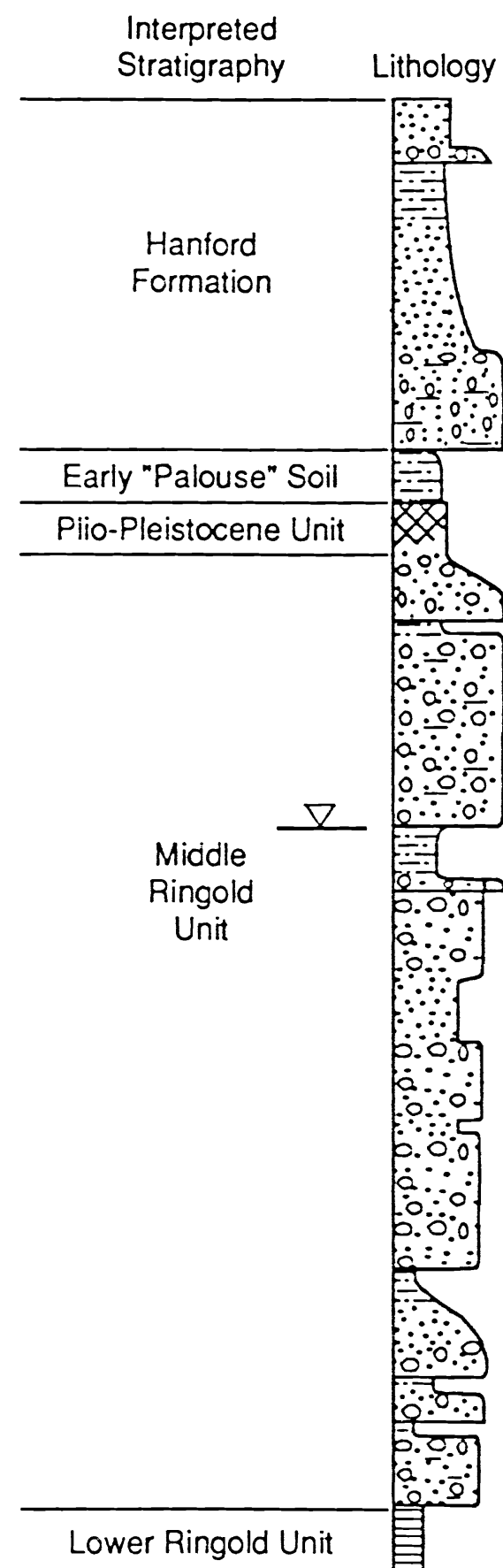

Drill

Method
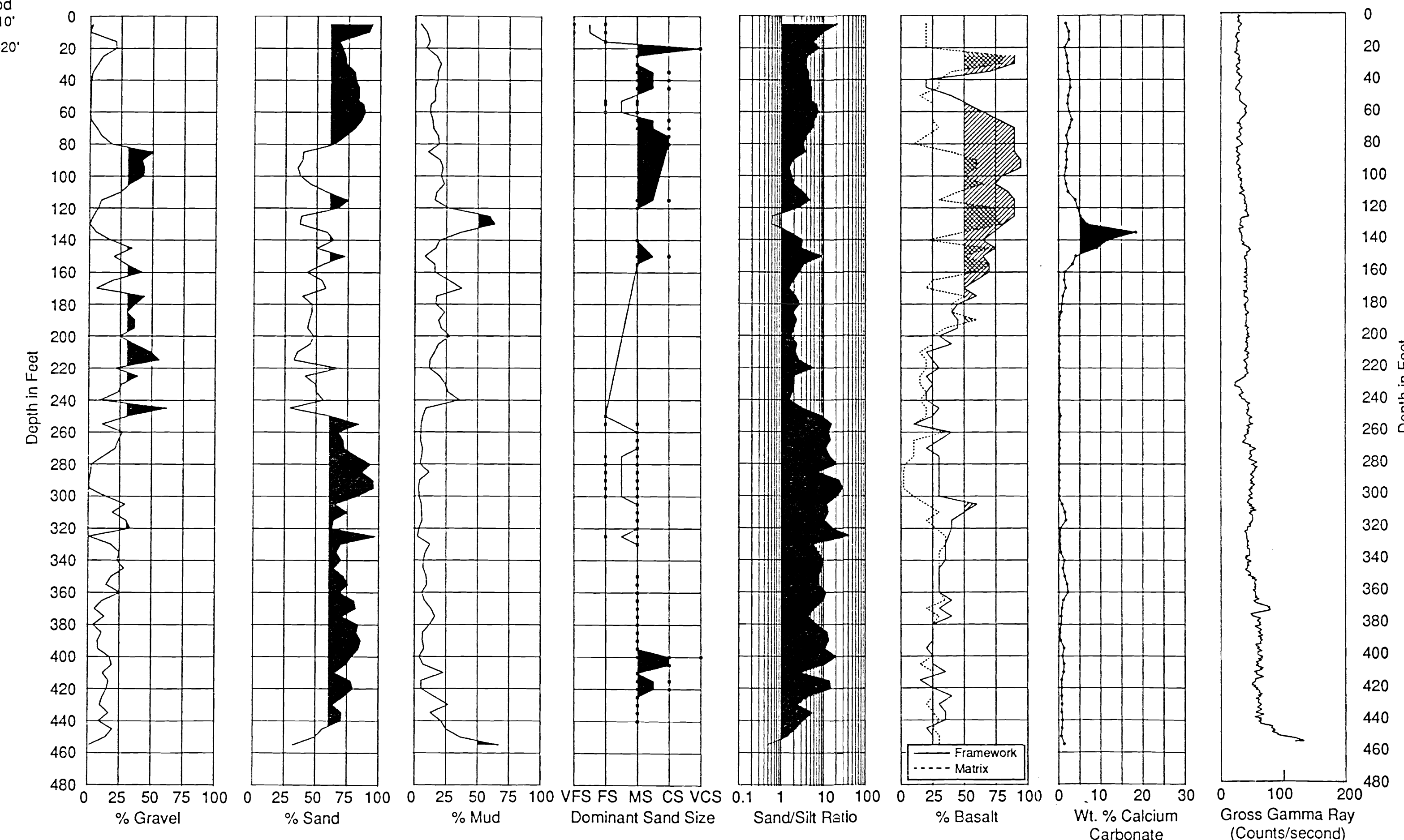

Dominant Sand Size

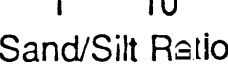

$\%$ Basalt Carbonate

Gross Gamma Ray 


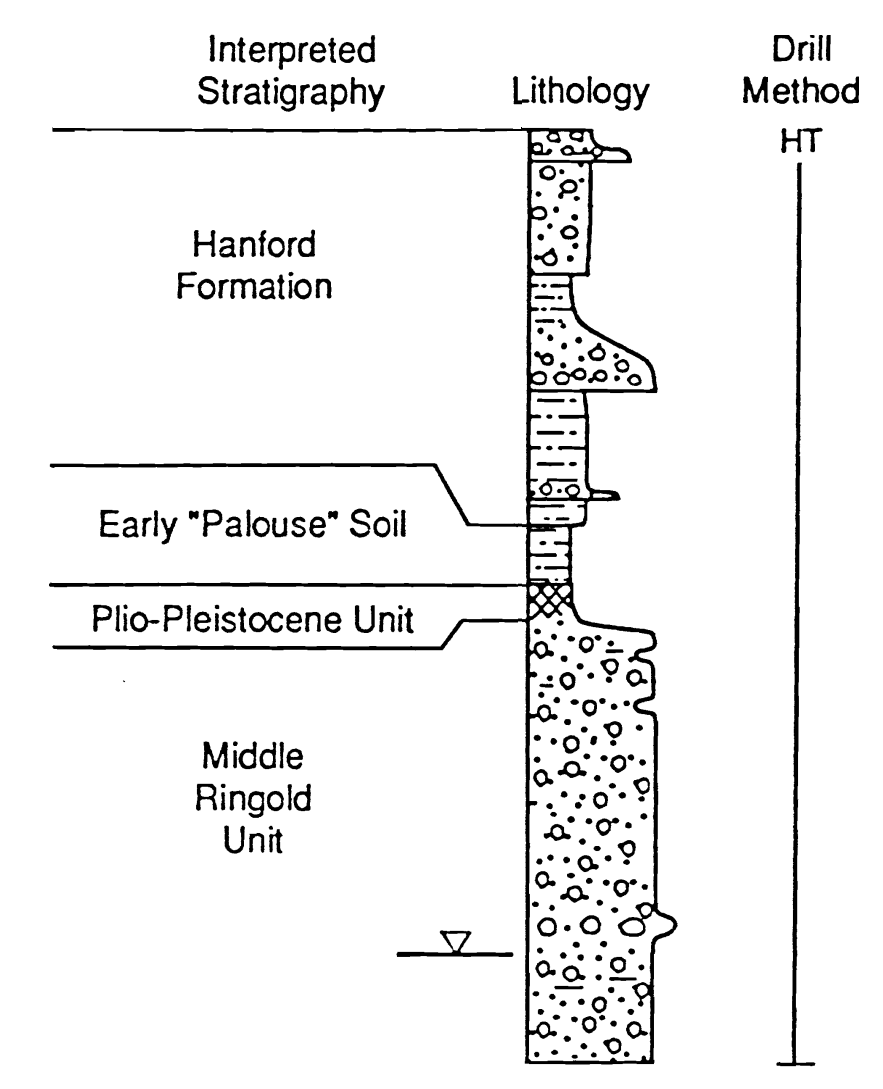
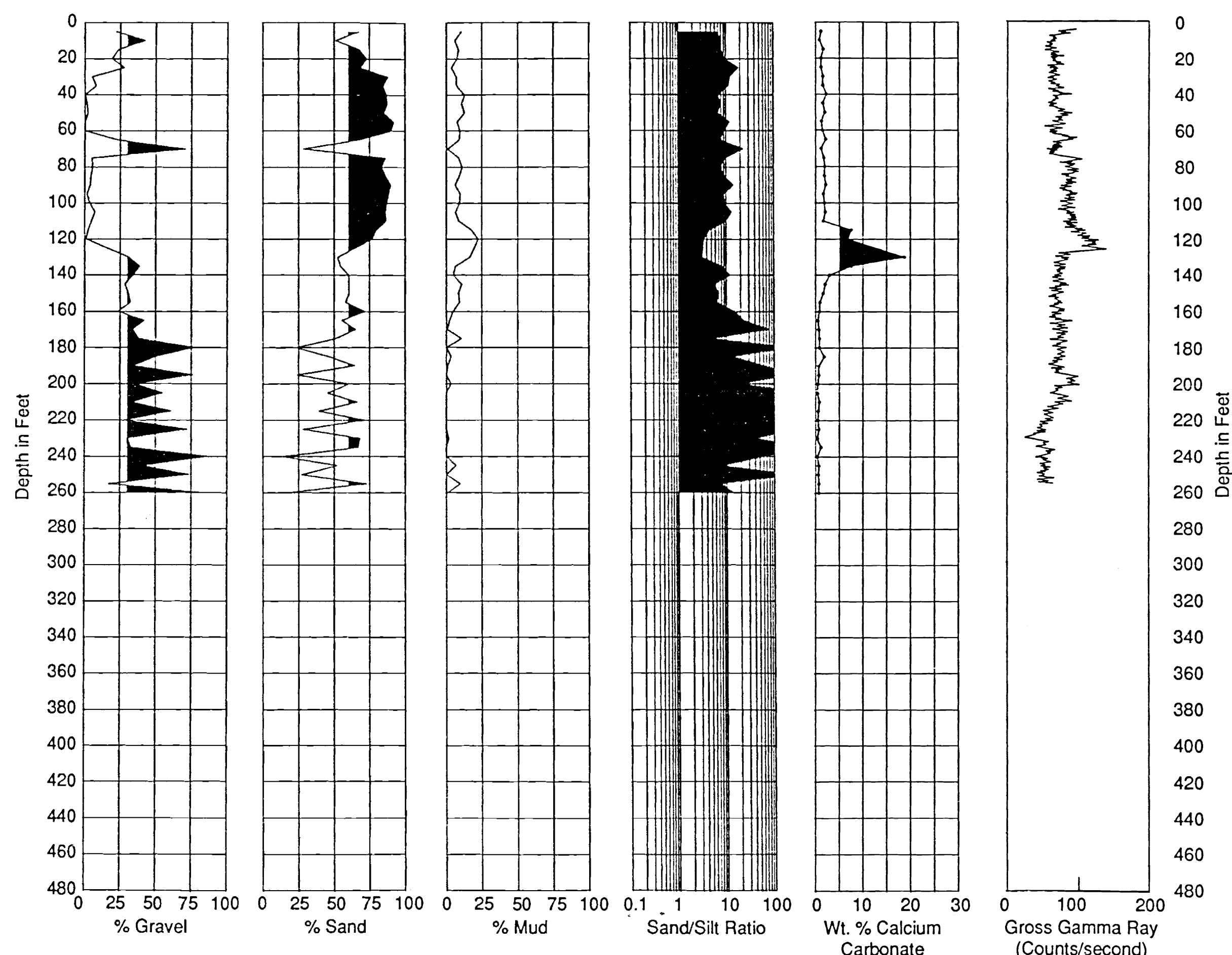

S8912118.2base 


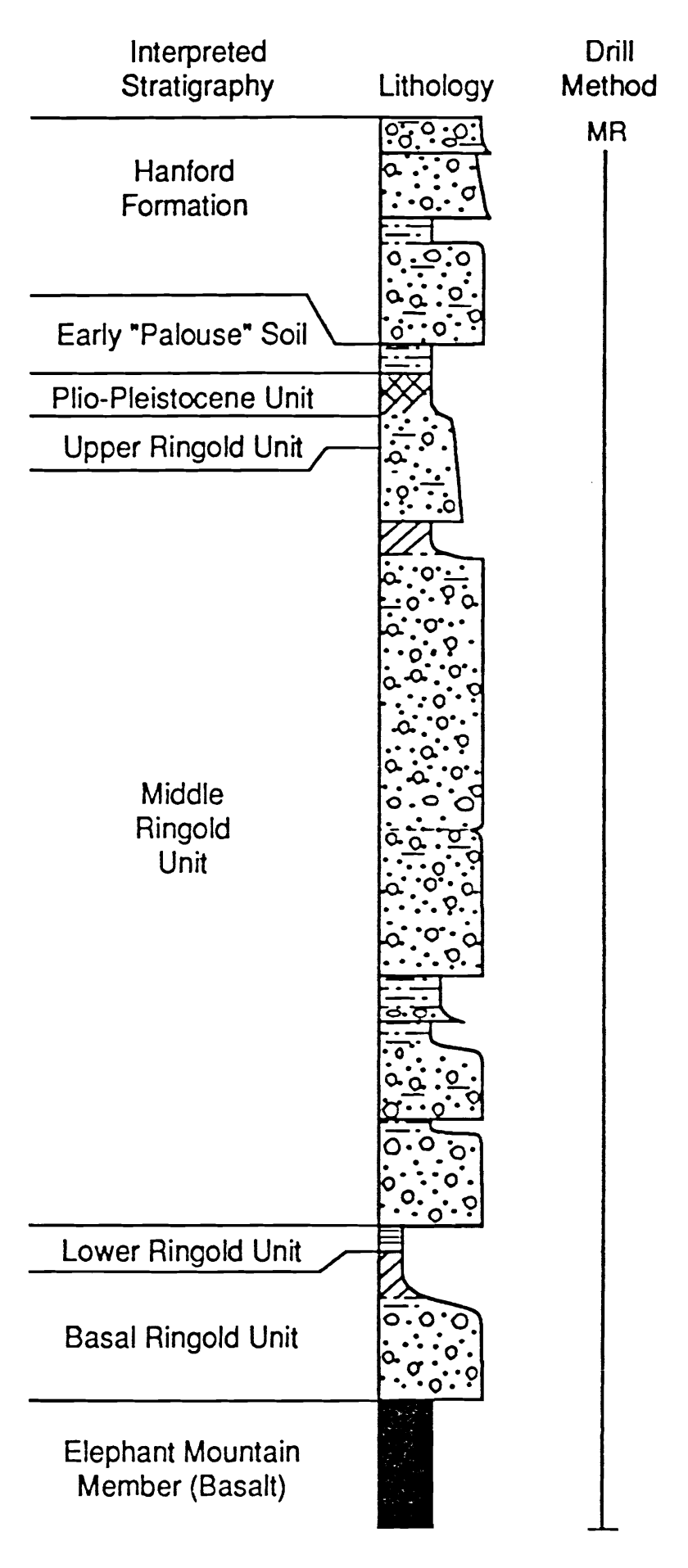
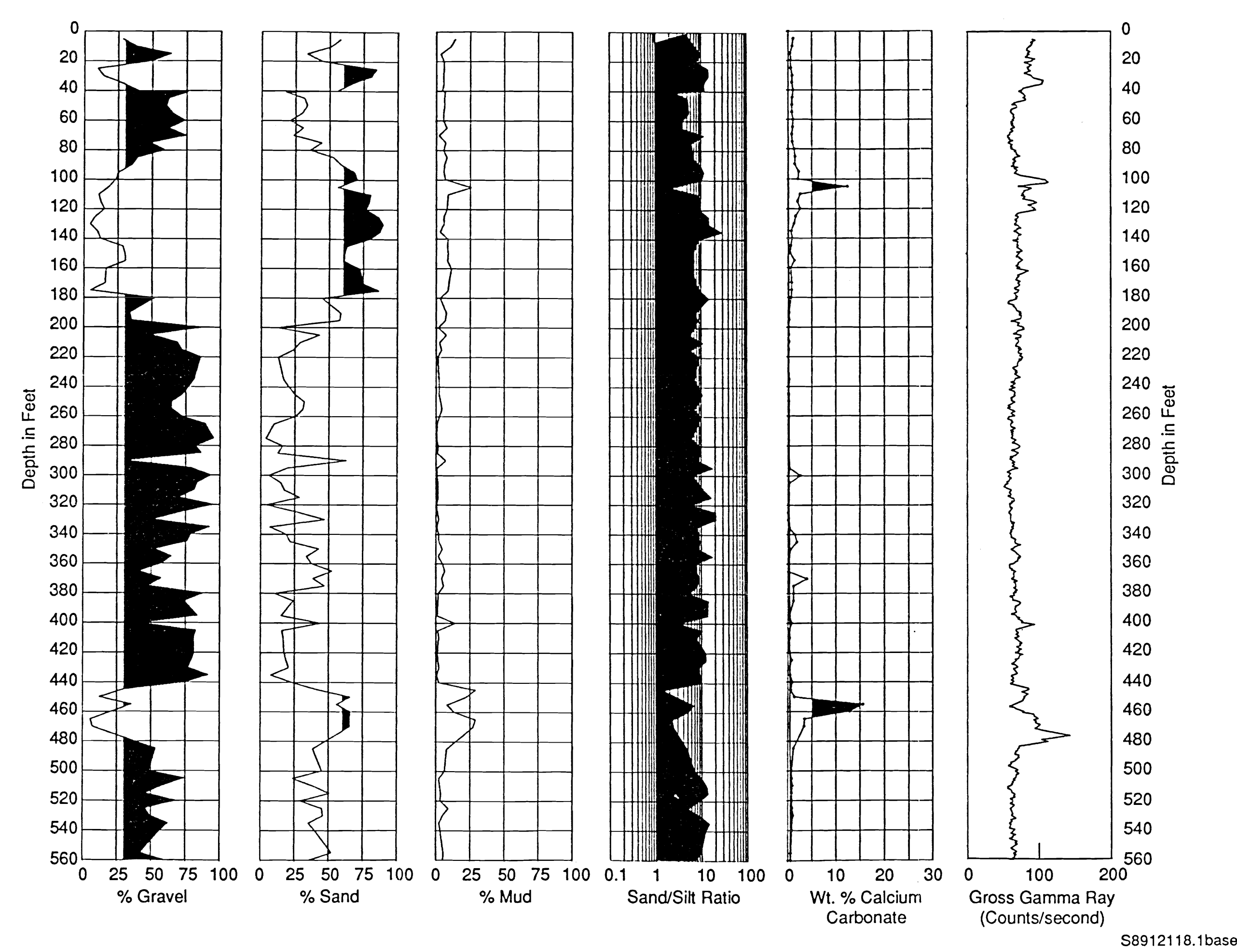
APPENDIX B

HYDROGRAPHS 


\section{APPENDIX B}

\section{HYDROGRAPHS}

This appendix presents hydrographs from eight wells located within $1000 \mathrm{ft}$ of W5 where water-level measurements have been collected. The first two hydrograph plots are from RCRA wells that penetrate the top (page B.2) and bottom (page B.3) of the unconfined aquifer, respectively. Because RCRA wells are relatively new, there are only about two years of water-leveldata available.

The last figure (page B.4), on the other hand, is a hydrograph plot from well 299-W15-2, where water levels have been measured over the last 35 years (1954 to present). 


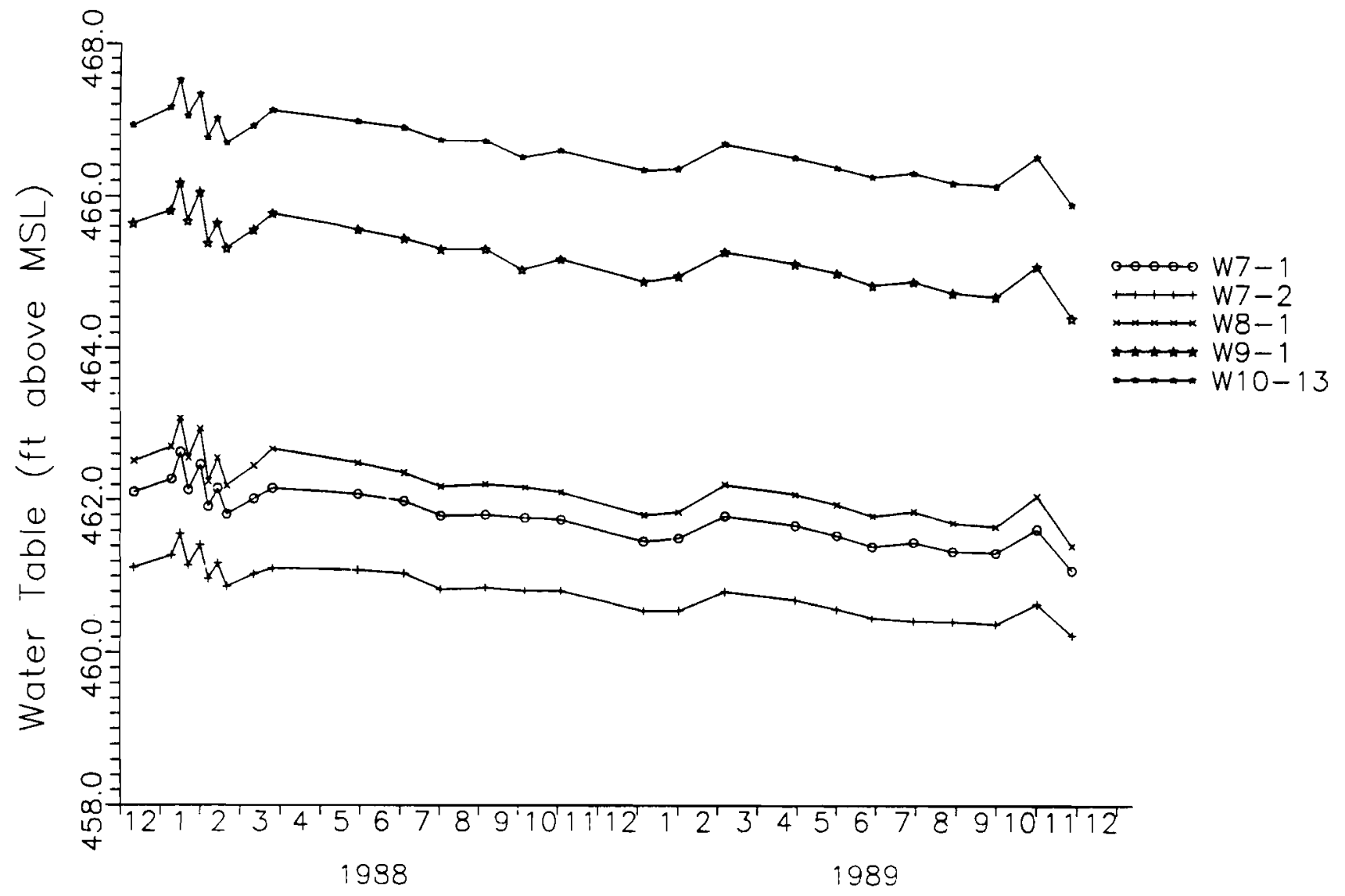




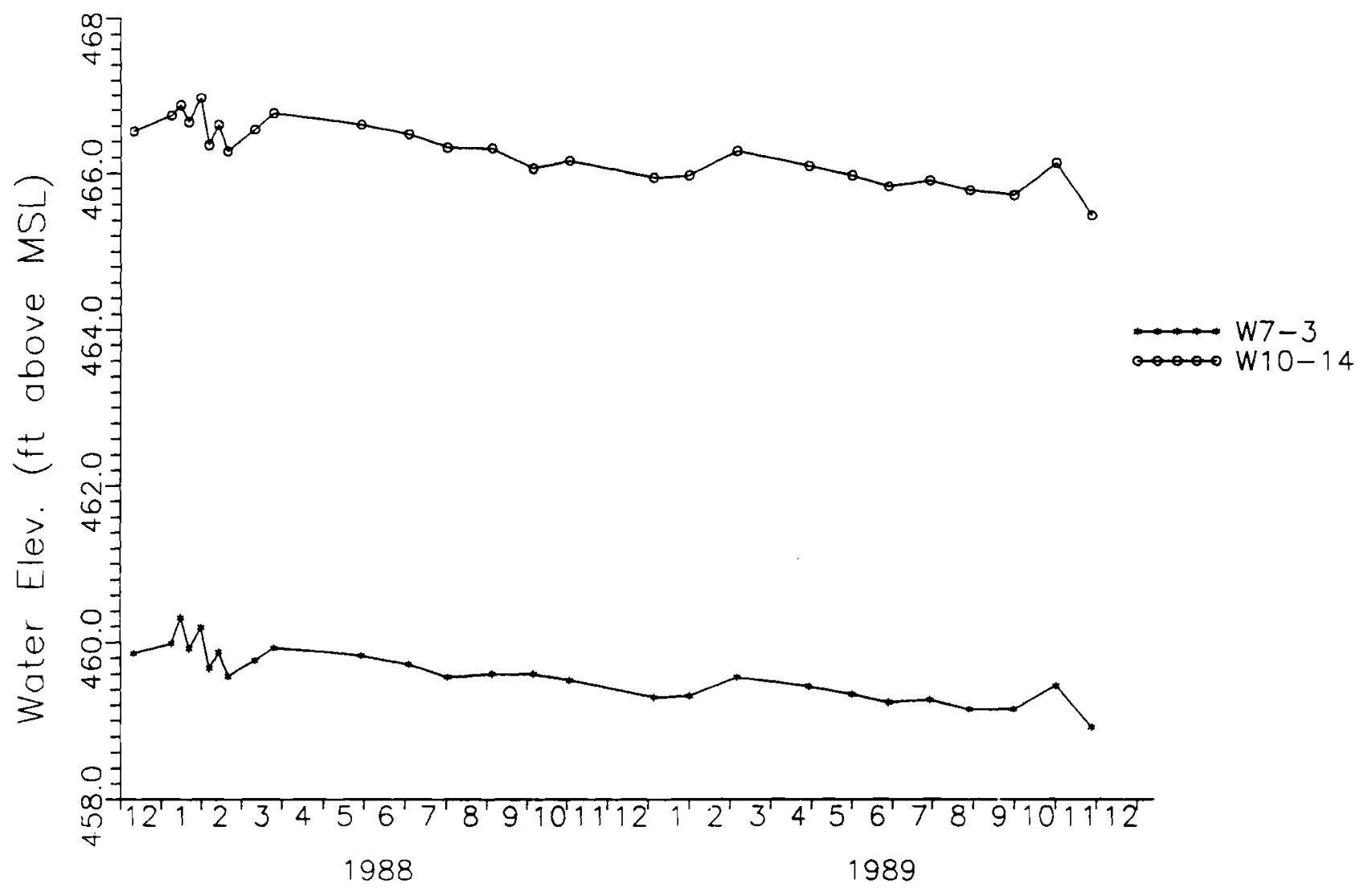




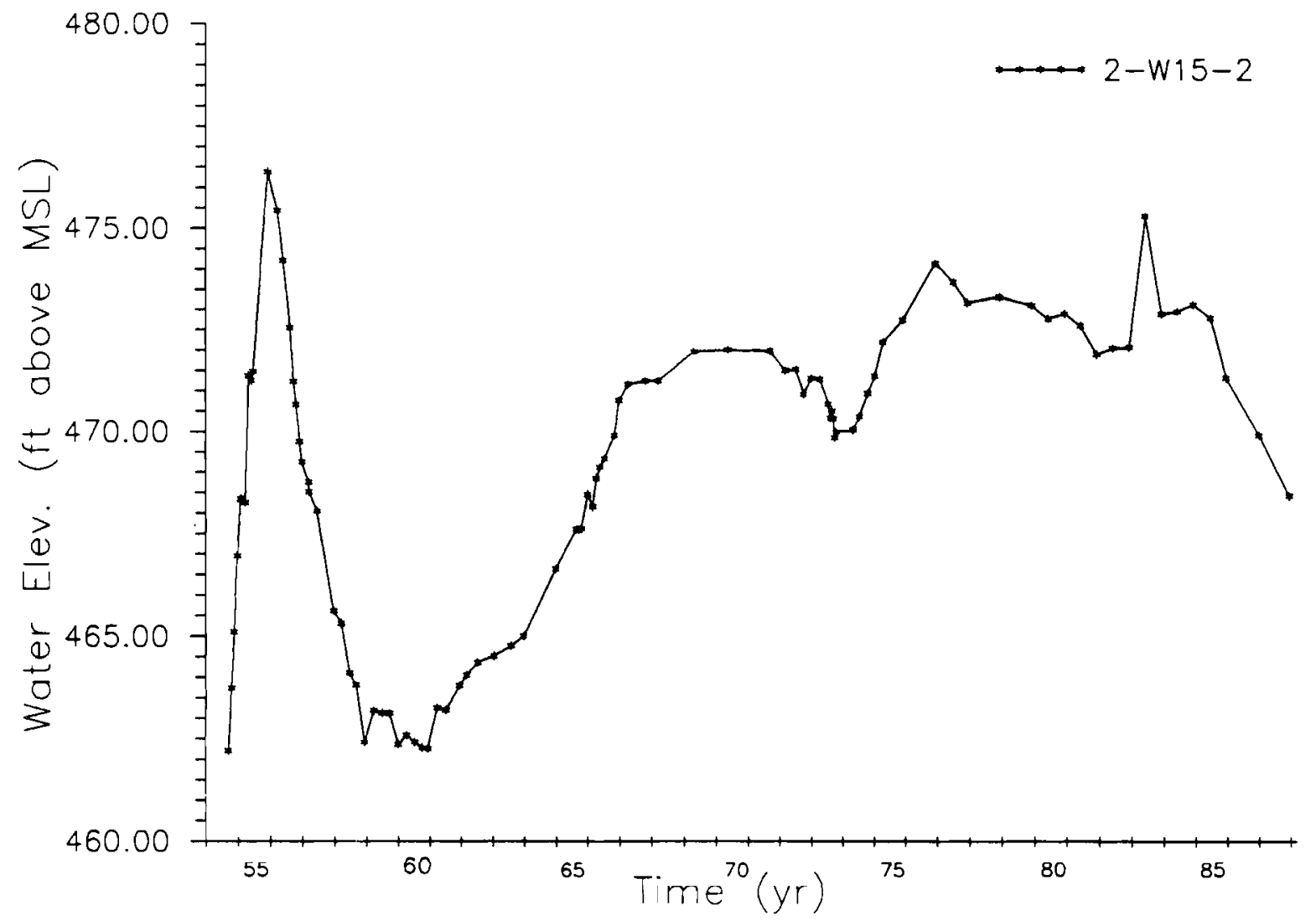


APPENDIX C

PARTICLE-SIZE DISTRIBUTION/CACO 3 ANALYSES 


\section{APPENDIX C}

\section{PARTICLE-SIZE DISTRIBUTION/CaCO3 ANALYSES}

This appendix presents particle-sizeand calcium carbonate data available for samples collected from boreholes within $1000 \mathrm{ft}$ of $\mathrm{W}-5$. These data were output from the ROCSAN computer program.

The headings on the output have the following meanings:

$\% \mathrm{CACO}_{3}$ indicates that calcium carbonate is expressedin weight percent.

DM stands for drill method $(\mathrm{H}=$ hard tool; $\mathrm{C}=$ drive barrel; $\mathrm{S}=$ split barrel).

CLASS indicates the Folk classification symbol, which is an abbreviated form of the grain-size distribution. According to the Fold classification system, $M=$ mud; $S=$ sand; $G=$ gravel; $m=$ muddy; $\mathbf{s}=$ sandy; $\mathrm{g}$ = gravelly; ( ) = slightly. Using this system, all sediment samples can be described as one of the 19 Folk classification types shown in Figure 7.

The nine grain-size classes (fine peb, vfine peb, very coars, coars, med, fine, very fine, silt, and pan) are expressed in phi units (in parentheses), which correspond respectively to 4.0-, 2.0-, 1.0-, 0.5-, 0.25-, 0.125-, 0.063-, 0.037-, and <0.037-mm sieve openings.

In the table below the headings, the phrases SORT (for sorting), MEDIAN, MODE, and MEAN refer to calculated statistical parameters. 


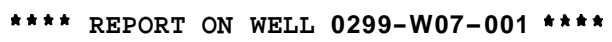

$12 / 11 / 89$

\begin{tabular}{|c|c|c|c|c|c|c|c|c|c|c|c|c|c|c|c|c|c|c|}
\hline EPTH & $8 \mathrm{CACO3}$ & & IMUD & ISAND & :GRAVEL & CLAS & & & & $\begin{array}{l}\text { FINE } \\
\text { PEB } \\
(<--2) \\
-----\end{array}$ & $\begin{array}{l}\text { VFINE } \\
\text { PEB } \\
(-1)\end{array}$ & $\begin{array}{l}\text { VERY } \\
\text { COARS } \\
(0)\end{array}$ & $\begin{array}{c}\text { COARS } \\
(1)\end{array}$ & $\begin{array}{l}\text { MED } \\
(2)\end{array}$ & $\begin{array}{l}\text { FINE } \\
\text { (3) }\end{array}$ & $\begin{array}{l}\text { VERY } \\
\text { FINE } \\
\text { (4) }\end{array}$ & $\begin{array}{c}\text { SILT } \\
(4.75)\end{array}$ & $\begin{array}{c}\text { PAN } \\
(>4.75)\end{array}$ \\
\hline $\begin{array}{r}5 \\
\text { SORT- } \\
\text { SPLIT }\end{array}$ & $\begin{array}{c}1.1 \\
\text { N/A MED } \\
\text { WT= } 807.7\end{array}$ & $\underset{7}{C}$ & $\begin{array}{c}6.7 \\
-\quad-1.00\end{array}$ & $\begin{array}{r}29.0 \\
\text { MODE- }\end{array}$ & $\begin{array}{l}64.3 \\
-1.00 \mathrm{MEAN}\end{array}$ & msG & N/A & CUM & $\begin{array}{l}\text { WT } \\
\text { WT } \\
\text { WT }\end{array}$ & $\begin{array}{l}\text { N/A } \\
\text { N/A } \\
\text { N/A }\end{array}$ & $\begin{array}{r}519.3 \\
64.3 \\
64.3\end{array}$ & $\begin{array}{r}68.5 \\
8.5 \\
72.8\end{array}$ & $\begin{array}{r}40.5 \\
5.0 \\
77.8\end{array}$ & $\begin{array}{r}24.3 \\
3.0 \\
80.8\end{array}$ & $\begin{array}{r}41.9 \\
5.2 \\
86.0\end{array}$ & $\begin{array}{r}59.3 \\
7.3 \\
93.3\end{array}$ & $\begin{array}{l}\text { N/A } \\
\text { N/A } \\
\text { N/A }\end{array}$ & $\begin{array}{r}53.8 \\
6.7 \\
100.0\end{array}$ \\
\hline $\begin{array}{r}10 \\
\text { SORT- } \\
\text { SPLIT }\end{array}$ & $\begin{array}{c}1.3 \\
\text { N/A MED } \\
\text { WT- } 870.2\end{array}$ & ${ }_{2}^{\mathrm{C}}$ & $\begin{array}{c}2.1 \\
-1.00\end{array}$ & $\begin{array}{r}18.7 \\
\text { MODE- }\end{array}$ & -1.00 MEAN & $\begin{array}{l}\mathbf{m s G} \\
\mathrm{N}-\end{array}$ & N/A & CUM & $\begin{array}{l}\text { WT } \\
\text { WT } \\
\text { WT }\end{array}$ & $\begin{array}{l}\text { N/A } \\
\text { N/A } \\
\text { N/A }\end{array}$ & $\begin{array}{r}689.7 \\
79.3 \\
79.3\end{array}$ & $\begin{array}{r}68.9 \\
7.9 \\
87.2\end{array}$ & $\begin{array}{r}52.0 \\
6.0 \\
93.1\end{array}$ & $\begin{array}{r}16.0 \\
1.8 \\
95.0\end{array}$ & $\begin{array}{r}10.7 \\
1.2 \\
96.2\end{array}$ & $\begin{array}{r}14.9 \\
1.7 \\
97.9\end{array}$ & $\begin{array}{l}\text { N/A } \\
\text { N/A } \\
\text { N/A }\end{array}$ & $\begin{array}{r}18.1 \\
2.1 \\
100.0\end{array}$ \\
\hline $\begin{array}{r}15 \\
\text { SORT- } \\
\text { SPLIT }\end{array}$ & $\begin{array}{c}0.6 \\
\text { N/A MED } \\
\text { WT= } 456.2\end{array}$ & $\underbrace{\mathrm{H}}_{2}$ & $\begin{array}{c}25.2 \\
-\quad 1.00\end{array}$ & $\begin{array}{r}42.4 \\
\text { MODE- }\end{array}$ & $\begin{array}{c}32.4 \\
-1.00 \mathrm{MEAN}\end{array}$ & $\begin{array}{l}\mathbf{m s G} \\
\mathrm{v}-\end{array}$ & N/A & CUM & $\begin{array}{l}\text { WT } \\
\text { WT } \\
\text { WT } \\
\text { I }\end{array}$ & $\begin{array}{l}N / A \\
N / A \\
N / A\end{array}$ & $\begin{array}{r}148.0 \\
32.4 \\
32.4\end{array}$ & $\begin{array}{l}53.8 \\
11.0 \\
44.2\end{array}$ & $\begin{array}{l}47.3 \\
10.4 \\
54.6\end{array}$ & $\begin{array}{r}35.8 \\
7.9 \\
62.5\end{array}$ & $\begin{array}{r}32.9 \\
7.2 \\
69.7\end{array}$ & $\begin{array}{r}23.5 \\
5.2 \\
74.8\end{array}$ & $\begin{array}{l}\text { N/A } \\
\text { N/A } \\
\text { N/A }\end{array}$ & $\begin{array}{r}114.9 \\
25.2 \\
100.0\end{array}$ \\
\hline $\begin{array}{r}20 \\
\text { SORT- } \\
\text { SPIIT }\end{array}$ & $\begin{array}{c}0.9 \\
\text { N/A MED } \\
\text { WT }-436.1\end{array}$ & $\underset{1}{\stackrel{\mathrm{H}}{\text { DIAN- }}}$ & $\begin{array}{c}22.3 \\
-\quad 1.00\end{array}$ & $\begin{array}{r}52.6 \\
\text { MODE- }\end{array}$ & -1.00 MEAN & gms & N/A & CUM & $\begin{array}{l}\text { WTT } \\
\text { WT } \\
\text { I }\end{array}$ & $\begin{array}{l}N / A \\
N / A \\
N / A\end{array}$ & $\begin{array}{r}109.4 \\
25.1 \\
25.1\end{array}$ & $\begin{array}{l}92.8 \\
21.3 \\
46.4\end{array}$ & $\begin{array}{l}60.4 \\
13.9 \\
60.2\end{array}$ & $\begin{array}{r}34.0 \\
7.0 \\
68.0\end{array}$ & $\begin{array}{r}25.9 \\
5.9 \\
74.0\end{array}$ & $\begin{array}{r}16.1 \\
3.7 \\
77.7\end{array}$ & $\begin{array}{l}\text { N/A } \\
\text { N/A } \\
\text { N/A }\end{array}$ & $\begin{array}{r}97.4 \\
22.3 \\
100.0\end{array}$ \\
\hline $\begin{array}{r}25 \\
\text { SORT- } \\
\text { SPIIT }\end{array}$ & $\begin{array}{c}0.8 \\
\mathrm{~N} / \mathrm{A} \text { MED } \\
\mathrm{WT}=302.6\end{array}$ & $\begin{array}{l}\mathrm{H} \\
6 \\
\text { DIAN- }\end{array}$ & $\begin{array}{c}13.6 \\
-\quad 0.00\end{array}$ & $\begin{array}{r}38.0 \\
\text { MODE- }\end{array}$ & $\begin{array}{c}48.4 \\
-1.00 \text { MEAN }\end{array}$ & msG & N/A & CUM & $\begin{array}{ll} & \text { WT } \\
\text { WT } & \text { I } \\
\text { WT } & I\end{array}$ & $\begin{array}{l}N / A \\
N / A \\
N / A\end{array}$ & $\begin{array}{r}146.5 \\
48.4 \\
48.4\end{array}$ & $\begin{array}{l}36.9 \\
12.2 \\
60.6\end{array}$ & $\begin{array}{r}27.2 \\
9.0 \\
69.6\end{array}$ & $\begin{array}{r}21.5 \\
7.1 \\
76.7\end{array}$ & $\begin{array}{r}18.2 \\
6.0 \\
82.7\end{array}$ & $\begin{array}{r}11.1 \\
3.7 \\
86.4\end{array}$ & $\begin{array}{l}\text { N/A } \\
\text { N/A } \\
\text { N/A }\end{array}$ & $\begin{array}{r}41.2 \\
13.6 \\
100.0\end{array}$ \\
\hline $\begin{array}{r}30 \\
\text { SORT- } \\
\text { SPIIT }\end{array}$ & $\begin{array}{c}0.9 \\
\text { N/A MED } \\
\text { WT }=388.5\end{array}$ & $\begin{array}{l}\text { DIAN- } \\
5\end{array}$ & $\begin{array}{c}17.7 \\
-\quad 0.00\end{array}$ & $\begin{array}{r}44.0 \\
\text { MODE- }\end{array}$ & -1.00 MEAN & $\begin{array}{l}\text { msG } \\
\mathrm{N}-\end{array}$ & $\mathbf{N} / \mathbf{A}$ & CUM & $\begin{array}{l}\text { WT } \\
\text { WT } \\
\text { WT } \\
\text { W }\end{array}$ & $\begin{array}{l}N / A \\
N / A \\
N / A\end{array}$ & $\begin{array}{r}148.7 \\
38.3 \\
38.3\end{array}$ & $\begin{array}{l}57.4 \\
14.0 \\
53.0\end{array}$ & $\begin{array}{l}10.9 \\
10.5 \\
63.6\end{array}$ & $\begin{array}{r}28.0 \\
7.2 \\
70.8\end{array}$ & $\begin{array}{r}25.8 \\
6.6 \\
77.4\end{array}$ & $\begin{array}{r}18.9 \\
1.9 \\
82.3\end{array}$ & $\begin{array}{l}\text { N/A } \\
\text { N/A } \\
\text { N/A }\end{array}$ & $\begin{array}{r}68.9 \\
17.7 \\
100.0\end{array}$ \\
\hline $\begin{array}{r}35 \\
\text { SORT- } \\
\text { SPLIT }\end{array}$ & $\begin{array}{c}1.1 \\
\text { N/A MED } \\
W T-406.8\end{array}$ & ${ }_{B}^{\stackrel{H}{\text { DIAN- }}}$ & $\begin{array}{c}24.6 \\
-\quad 1.00\end{array}$ & $\begin{array}{r}38.6 \\
\text { MODE- }\end{array}$ & $\begin{array}{c}36.8 \\
-1.00 \text { MEAN }\end{array}$ & $\begin{array}{l}\text { msG } \\
N-\end{array}$ & N/A & CUM & $\begin{array}{l}\text { WT } \\
\text { WT }\end{array}$ & $\begin{array}{l}N / A \\
N / A \\
N / A\end{array}$ & $\begin{array}{r}149.8 \\
36.8 \\
36.8\end{array}$ & $\begin{array}{l}50.5 \\
12.4 \\
49.2\end{array}$ & $\begin{array}{r}36.3 \\
8.9 \\
58.2\end{array}$ & $\begin{array}{r}27.2 \\
6.7 \\
64.8\end{array}$ & $\begin{array}{r}25.3 \\
6.2 \\
71.1\end{array}$ & $\begin{array}{r}17.8 \\
1.4 \\
75.4\end{array}$ & $\begin{array}{l}\text { N/A } \\
\text { N/A } \\
\text { N/A }\end{array}$ & $\begin{array}{r}99.9 \\
24.6 \\
100.0\end{array}$ \\
\hline $\begin{array}{r}40 \\
\text { SORT- } \\
\text { SPIIT }\end{array}$ & $\begin{array}{c}1.3 \\
N / A \text { MED } \\
W T=366.0\end{array}$ & $\begin{array}{l}\stackrel{\text { HAN- }}{\mathrm{DIAN}-} \\
0\end{array}$ & $\begin{array}{c}19.6 \\
-\quad 0.00\end{array}$ & $\begin{array}{r}39.2 \\
\text { MODE- }\end{array}$ & $-1.00 \mathrm{MEAN}$ & ${ }_{N-}^{m s G}$ & $N / A$ & CUM & $\begin{array}{ll}\text { WT } \\
\text { WT } \\
\text { WT }\end{array}$ & $\begin{array}{l}N / A \\
N / A \\
N / A\end{array}$ & $\begin{array}{r}150.5 \\
41.1 \\
11.1\end{array}$ & $\begin{array}{l}48.7 \\
13.3 \\
54.4\end{array}$ & $\begin{array}{r}33.7 \\
9.2 \\
63.6\end{array}$ & $\begin{array}{r}25.6 \\
7.0 \\
70.6\end{array}$ & $\begin{array}{r}21.3 \\
5.8 \\
76.5\end{array}$ & $\begin{array}{r}14.3 \\
3.9 \\
80.4\end{array}$ & $\begin{array}{l}\text { N/A } \\
\text { N/A } \\
\text { N/A }\end{array}$ & $\begin{array}{r}71.9 \\
19.6 \\
100.0\end{array}$ \\
\hline $\begin{array}{r}45 \\
\text { SORT- } \\
\text { SPIIT }\end{array}$ & $\begin{array}{c}1.3 \\
\text { N/A MED } \\
\text { WT }-369.7\end{array}$ & $\underset{7}{\mathrm{DIAN}-}$ & $\begin{array}{c}19.5 \\
-\quad 0.00\end{array}$ & $\begin{array}{r}39.7 \\
\text { MODE- }\end{array}$ & $\begin{array}{c}40.8 \\
-1.00 \text { MEAN }\end{array}$ & $\stackrel{m g G}{\mathrm{~m}}$ & N/A & CUM & $\begin{array}{l}\text { WT } \\
\text { WT }\end{array}$ & $\begin{array}{l}\text { N/A } \\
\text { N/A } \\
\text { N/A }\end{array}$ & $\begin{array}{c}150.9 \\
40.8 \\
40.8\end{array}$ & $\begin{array}{l}47.5 \\
12.9 \\
53.7\end{array}$ & $\begin{array}{r}32.0 \\
8.7 \\
62.3\end{array}$ & $\begin{array}{r}26.9 \\
7.3 \\
69.6\end{array}$ & $\begin{array}{r}24.4 \\
6.6 \\
76.2\end{array}$ & $\begin{array}{r}16.0 \\
4.3 \\
80.6\end{array}$ & $\begin{array}{l}\text { N/A } \\
\text { N/A } \\
\text { N/A }\end{array}$ & $\begin{array}{r}71.9 \\
19.5 \\
100.0\end{array}$ \\
\hline $\begin{array}{r}50 \\
\text { SORT- } \\
\text { SPIIT }\end{array}$ & $\begin{array}{c}2.7 \\
\text { N/A MED } \\
\text { พT= } 321.7\end{array}$ & $\begin{array}{l}\text { H } \\
7\end{array}$ & $\begin{array}{c}13.4 \\
-\quad 1.00\end{array}$ & $\begin{array}{r}53.5 \\
\text { MODE- }\end{array}$ & -1.00 MEAN & $\stackrel{\text { mSG }}{\mathrm{N}-}$ & N/A & CUM & $\begin{array}{l}\text { WT } \\
\text { WT } \\
\text { WT }\end{array}$ & $\begin{array}{l}\text { N/A } \\
\text { N/A } \\
\text { N/A }\end{array}$ & $\begin{array}{r}106.4 \\
33.1 \\
33.1\end{array}$ & $\begin{array}{l}50.8 \\
15.8 \\
48.9\end{array}$ & $\begin{array}{l}49.4 \\
15.4 \\
64.2\end{array}$ & $\begin{array}{l}32.8 \\
10.2 \\
74.4\end{array}$ & $\begin{array}{r}24.2 \\
7.5 \\
82.0\end{array}$ & $\begin{array}{r}14.9 \\
4.6 \\
86.6\end{array}$ & $\begin{array}{l}\text { N/A } \\
\text { N/A } \\
\text { N/A }\end{array}$ & $\begin{array}{r}43.1 \\
13.4 \\
100.0\end{array}$ \\
\hline $\begin{array}{r}55 \\
\text { SORT- } \\
\text { SPLIT }\end{array}$ & $\begin{array}{c}2.9 \\
\text { N/A MED } \\
\text { WT- } 399.6\end{array}$ & $\underbrace{\text { H }}_{\text {DIAN- }}$ & $\begin{array}{c}12.2 \\
-\quad 0.00\end{array}$ & $\begin{array}{r}52.3 \\
\text { MODE- }\end{array}$ & $\begin{array}{c}35.5 \\
-1.00 \text { MEAN }\end{array}$ & $\underset{N-}{\mathbf{m s G}}$ & N/A & 1 & $\begin{array}{l}\text { WT } \\
\text { WT } \\
\text { WT }\end{array}$ & $\begin{array}{l}\text { N/A } \\
\text { N/A } \\
\text { N/A }\end{array}$ & $\begin{array}{r}141.8 \\
35.5 \\
35.5\end{array}$ & $\begin{array}{l}69.3 \\
17.3 \\
52.8\end{array}$ & $\begin{array}{l}56.5 \\
14.1 \\
67.0\end{array}$ & $\begin{array}{r}39.2 \\
9.8 \\
76.8\end{array}$ & $\begin{array}{r}27.8 \\
7.0 \\
83.7\end{array}$ & $\begin{array}{r}16.1 \\
4.0 \\
87.8\end{array}$ & $\begin{array}{l}\text { N/A } \\
\text { N/A } \\
\text { N/A }\end{array}$ & $\begin{array}{r}48.9 \\
12.2 \\
100.0\end{array}$ \\
\hline
\end{tabular}


\#, REPORT ON WHL 0299-พ07-001 „\#*

$12 / 11 / 89$

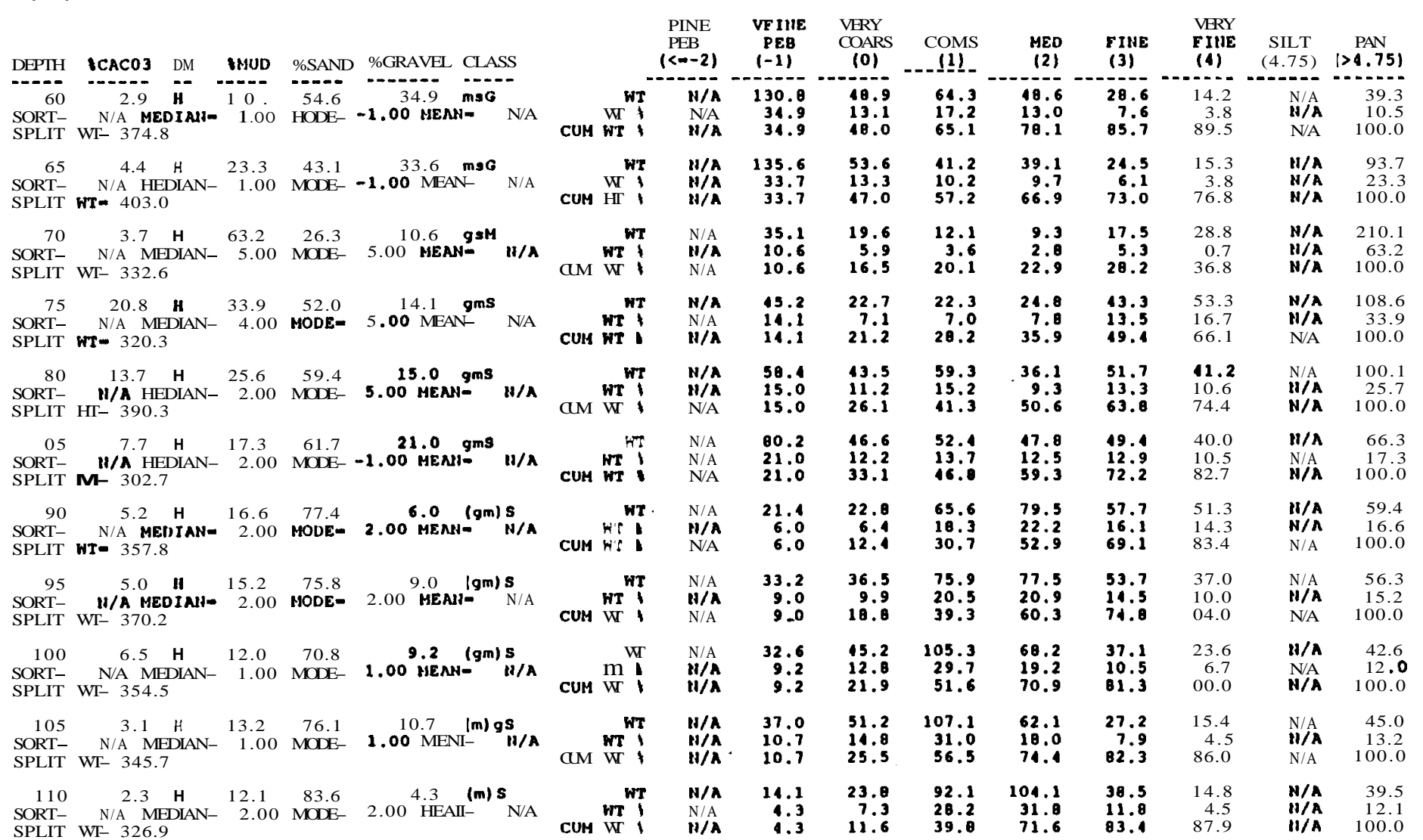


WESTINGHOUSE HANFORD OPERATIONS SIEVE ANALYSIS ROCSAN REPORT

$* * *$ REPORT ON WELL 0299-w07-001 $\star * \star \star$

$12 / 11 / 89$

\begin{tabular}{ccccccc} 
DEPTH & ICACO3 & DM & IMUD & ISAND & IGRAVEL & CLASS \\
\hdashline 115 & 1.2 & H & 9.9 & 88.5 & 1.6 & (m) $\mathrm{s}$
\end{tabular}

SORT- N/A MEDIAN- $\mathbf{2 . 0 0}$.MODE- 2.00 MEAN- N/A

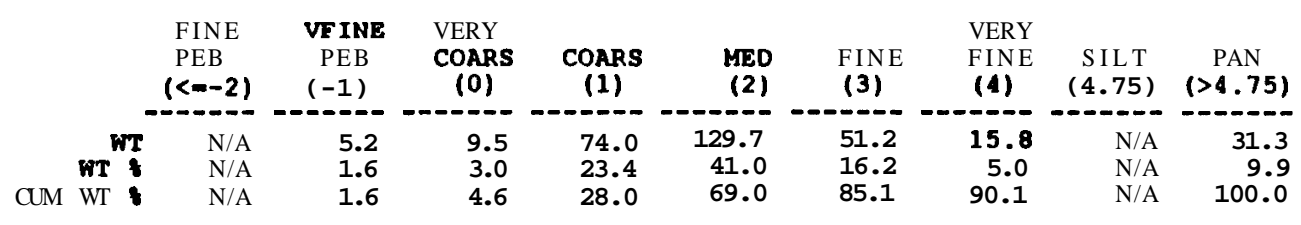

$\begin{array}{ccccccc}120 & \mathbf{0 . 8} & \mathrm{H} & \mathbf{9 . 4} & \mathbf{8 9 . 4} & \mathbf{1 . 1} \mathbf{s} \\ \text { SORT- } & \text { N/A MEDIAN- } & \mathbf{2 . 0 0} & \text { MODE- } & \mathbf{2 . 0 0} & \text { MEAN- } & \text { N/A }\end{array}$

$\begin{array}{lll}\text { WT } & \text { N/A } & 3.8 \\ \text { WT } & \text { N/A } & 1.2\end{array}$

$\begin{array}{rrrrrrr}3.0 & 56.5 & 172.6 & 45.1 & 19.0 & \text { N/A } & 31.2 \\ 0.9 & 17.1 & 52.1 & 13.6 & 5.7 & \text { N/A } & 9.4\end{array}$

SPLIT WT- 331.

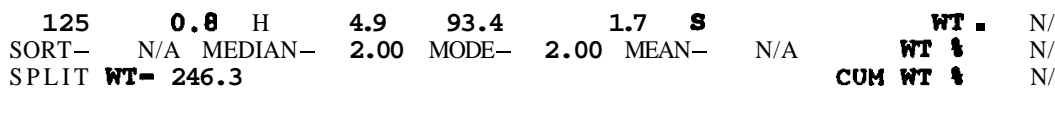

$\begin{array}{lllllll}130 & 0.6 & \mathrm{C} & 7.7 & 80.8 & 11.5 & \mathrm{gs}\end{array}$

SORT - N/A M

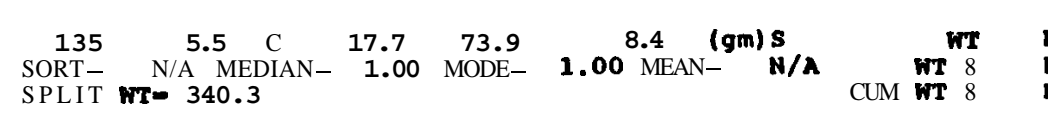

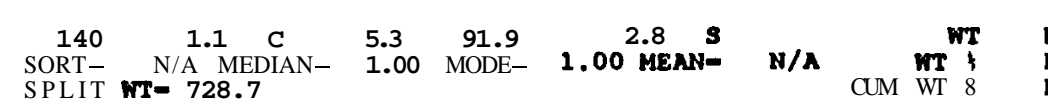

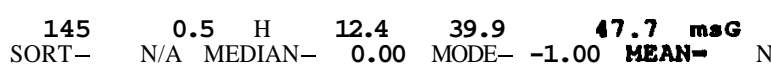
SPLIT WT- 463.8

พr: ${ }^{\text {WT }}$

$\begin{array}{cccccc}150 & 0.8 \mathrm{H} & 19.0 & 41.0 & 40.0 & \text { mac }\end{array}$

SORT- N/A MEDIAN- 1.00 MODE- $\mathbf{- 1 . 0 0}$ MEAN- N/A
SPLIT MT- 464.6

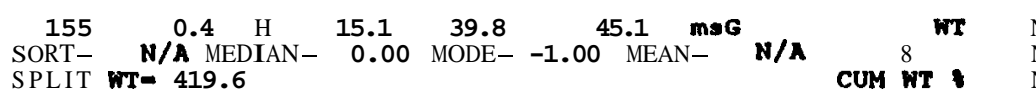

$$
\begin{array}{lll}
\mathbf{W T} & \text { N/A } & \text { N/A } \\
\text { CUM } & \text { NT } & \text { N/A }
\end{array}
$$

$\begin{array}{lll}1.2 & 2.1 & 17.1 \\ & & 19.1\end{array}$

$\begin{array}{rrrrr}52.1 & 13.6 & 5.7 & \text { N/A } & 9.4\end{array}$

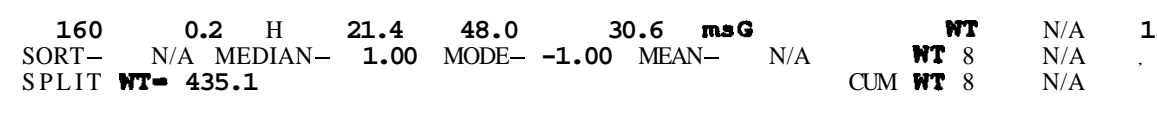

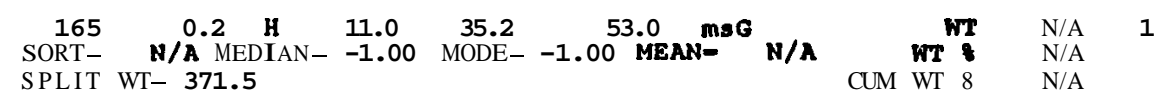

$\begin{array}{rrrrrrrr}4.2 & 13.0 & 70.0 & 111.1 & 28.9 & 7.3 & \text { N/A } & 12.0 \\ 1.7 & 5.3 & 28.4 & 45.1 & 11.7 & 3.0 & \text { N/A } & 4.9\end{array}$

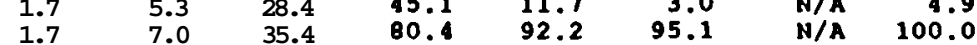

$\begin{array}{llllllll}92.9 & 177.9 & 243.9 & 121.8 & 71.1 & 36.7 & \mathrm{~N} / \mathrm{A} & 62.1\end{array}$

$\begin{array}{rrrrrrrr}92.9 & 177.9 & 243.9 & 121.8 & 71.1 & 36.7 & \text { N/A } & 62.1 \\ 11.5 & 22.1 & 30.3 & 15.1 & 8.8 & 1.6 & \text { N/A } & 7.7 \\ 11.5 & 33.6 & 63.8 & 78.9 & 87.8 & 92.3 & \text { N/A } & 100.0\end{array}$

$\begin{array}{lllllllll}A & 28.7 & 31.4 & 128.6 & 49.1 & 28.6 & 13.8 & N / A & 60.1\end{array}$

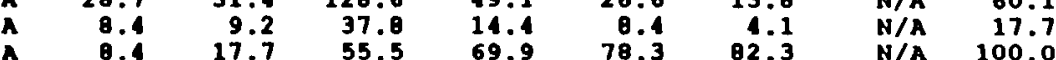

$\begin{array}{lll}68.3 & 02.3\end{array}$

A 100.0

$\begin{array}{rrrr}0.1 & 72.3 & 329.1 & 187 \\ 2.8 & 9.9 & 15.2 & 25\end{array}$

$\begin{array}{lllll}.6 & 56.9 & 23.8 & \text { N/A } & 30.0\end{array}$

$\begin{array}{rrrrrrrr}.0 & 12.7 & 57.9 & 03.6 & 91.4 & 94.7 & \text { N/A } & 100.0\end{array}$ 
WESTINGHOUSE HANEORD OPERATIONS SIEVE ANALYSIS

*** REPORT ON WELL 0299-W07-001 ***

$12 / 11 / 89$

\begin{tabular}{|c|c|c|c|c|c|c|c|c|c|c|c|c|c|c|c|c|c|c|}
\hline DEPTH & $8 \mathrm{CACO} 3$ & DM & IMUD & ISAND & IGRAVEL & CLAS & & & & $\begin{array}{l}\text { FINE } \\
\text { PEB } \\
(<--2)\end{array}$ & $\begin{array}{c}\text { VFINE } \\
\text { PEB } \\
(-1)\end{array}$ & $\begin{array}{l}\text { VERY } \\
\text { COARS } \\
\text { (0) }\end{array}$ & $\begin{array}{c}\text { COARS } \\
\text { (1) }\end{array}$ & $\begin{array}{l}\text { MED } \\
(2)\end{array}$ & $\begin{array}{l}\text { FINE } \\
\text { (3) }\end{array}$ & $\begin{array}{l}\text { VERY } \\
\text { FINE } \\
\text { (4) }\end{array}$ & $\begin{array}{l}\text { SILT } \\
(4.75)\end{array}$ & $\begin{array}{c}\text { PAN } \\
(>4.75)\end{array}$ \\
\hline $\begin{array}{l}170 \\
\text { SORT- } \\
\text { SPLIT }\end{array}$ & $\begin{array}{c}0.2 \\
\text { N/A MED } \\
\text { WT= } 373.2\end{array}$ & ${ }^{\mathrm{H}}$ & $\begin{array}{c}20.7 \\
1.00\end{array}$ & $\begin{array}{r}46.1 \\
\text { MODE- }\end{array}$ & $\begin{array}{c}33.2 \\
-1.00 \mathrm{MEAN}\end{array}$ & msG & N/A & CUM & WT & $\begin{array}{l}\text { N/A } \\
\text { N/A } \\
\text { N/A }\end{array}$ & $\begin{array}{r}123.8 \\
33.2 \\
33.2\end{array}$ & $\begin{array}{l}40.9 \\
11.0 \\
44.1\end{array}$ & $\begin{array}{l}44.0 \\
11.8 \\
55.9\end{array}$ & $\begin{array}{r}35.6 \\
9.5 \\
65.5\end{array}$ & $\begin{array}{r}31.4 \\
0.4 \\
73.9\end{array}$ & $\begin{array}{c}20.3 \\
5.4 \\
79.3\end{array}$ & $\begin{array}{l}\text { N/A } \\
\text { N/A } \\
\text { N/A }\end{array}$ & $\begin{array}{r}77.2 \\
20.7 \\
100.0\end{array}$ \\
\hline $\begin{array}{r}175 \\
\text { SORT- } \\
\text { SPLIT }\end{array}$ & $\begin{array}{c}0.2 \\
N / A \text { MED } \\
\text { WT }=396.9\end{array}$ & ${ }_{9}^{\stackrel{H}{\text { DIAN- }}}$ & $\begin{array}{c}15.2 \\
0.00\end{array}$ & $\begin{array}{r}39.2 \\
\text { MODE- }\end{array}$ & -1.00 MEAN & msG & N/A & CUM & $\begin{array}{l}\text { WT } \\
\text { WT } \\
\text { WT }\end{array}$ & $\begin{array}{l}\text { N/A } \\
\text { N/A } \\
\text { N/A }\end{array}$ & $\begin{array}{r}181.1 \\
45.6 \\
45.6\end{array}$ & $\begin{array}{l}46.8 \\
11.8 \\
57.4\end{array}$ & $\begin{array}{r}34.3 \\
8.6 \\
66.1\end{array}$ & $\begin{array}{r}31.0 \\
7.8 \\
73.9\end{array}$ & $\begin{array}{r}26.3 \\
6.6 \\
80.5\end{array}$ & $\begin{array}{r}17.0 \\
8.3 \\
04.8\end{array}$ & $\begin{array}{l}N / A \\
N / A \\
N / A\end{array}$ & $\begin{array}{r}60.4 \\
15.2 \\
100.0\end{array}$ \\
\hline $\begin{array}{r}180 \\
\text { SORT- } \\
\text { SPLIT }\end{array}$ & $\begin{array}{c}0.4 \\
\text { N/A MED } \\
\text { WT- } 503.2\end{array}$ & $\begin{array}{l}\underset{2}{\mathbf{H}} \\
\text { DIAN- }\end{array}$ & $\begin{array}{c}17.8 \\
0.00\end{array}$ & $\begin{array}{r}37.5 \\
\text { MODE- }\end{array}$ & $-1.00^{44.7}$ & ${ }_{N-}^{m s G}$ & N/A & CUM & WT & $\begin{array}{l}\text { N/A } \\
\text { N/A } \\
\text { N/A }\end{array}$ & $\begin{array}{r}224.7 \\
44.7 \\
14.7\end{array}$ & $\begin{array}{r}45.6 \\
9.1 \\
53.7\end{array}$ & $\begin{array}{r}36.0 \\
7.2 \\
60.9\end{array}$ & $\begin{array}{r}39.3 \\
7.8 \\
68.7\end{array}$ & $\begin{array}{r}43.4 \\
8.6 \\
77.3\end{array}$ & $\begin{array}{r}24.3 \\
4.8 \\
62.2\end{array}$ & $\begin{array}{l}N / A \\
N / A \\
N / A\end{array}$ & $\begin{array}{r}89.8 \\
17.9 \\
100.0\end{array}$ \\
\hline $\begin{array}{r}185 \\
\text { SORT- } \\
\text { SPLIT }\end{array}$ & $\begin{array}{c}0.3 \\
\text { N/A MED } \\
\text { WT- } 401.0\end{array}$ & ${ }_{\text {DIAN- }}^{\mathrm{H}}$ & $\begin{array}{c}20.3 \\
-\quad 1.00\end{array}$ & $\begin{array}{r}47.4 \\
\text { MODE- }\end{array}$ & $-1.00^{32.3}$ MEAN & ms G & N/A & CUM & $\begin{array}{l}\text { WT } \\
\text { WT } \\
\text { WT }\end{array}$ & $\begin{array}{l}\text { N/A } \\
\text { N/A } \\
\text { N/A }\end{array}$ & $\begin{array}{r}129.5 \\
32.3 \\
32.3\end{array}$ & $\begin{array}{l}11.9 \\
10.5 \\
42.7\end{array}$ & $\begin{array}{r}33.4 \\
8.3 \\
51.1\end{array}$ & $\begin{array}{l}12.7 \\
10.7 \\
61.7\end{array}$ & $\begin{array}{l}48.3 \\
12.0 \\
73.0\end{array}$ & $\begin{array}{r}23.7 \\
5.9 \\
79.7\end{array}$ & $\begin{array}{l}N / A \\
N / A \\
N / A\end{array}$ & $\begin{array}{r}81.5 \\
20.3 \\
100.0\end{array}$ \\
\hline $\begin{array}{r}190 \\
\text { SORT- } \\
\text { SPLIT }\end{array}$ & $\begin{array}{c}0.3 \\
\text { N/A MED } \\
\mathrm{HT}=439.1\end{array}$ & ${ }_{1}^{\stackrel{H}{\text { DIAN- }}}$ & $\begin{array}{c}22.7 \\
-\quad 2.00\end{array}$ & $\begin{array}{c}48.4 \\
\text { MODE- }\end{array}$ & $-1.00^{28.9}$ WAN & gms & N/A & CUM & $\underset{W T}{W T}$ & $\begin{array}{l}\text { N/A } \\
\text { N/A } \\
\text { N/A }\end{array}$ & $\begin{array}{r}126.9 \\
28.9 \\
28.9\end{array}$ & $\begin{array}{l}14.7 \\
10.2 \\
39.1\end{array}$ & $\begin{array}{r}34.6 \\
7.9 \\
17.0\end{array}$ & $\begin{array}{l}16.0 \\
10.5 \\
57.4\end{array}$ & $\begin{array}{l}52.3 \\
11.9 \\
69.1\end{array}$ & $\begin{array}{r}34.9 \\
0.0 \\
77.3\end{array}$ & $\begin{array}{l}N / A \\
N / A \\
N / A\end{array}$ & $\begin{array}{r}99.7 \\
22.7 \\
100.0\end{array}$ \\
\hline $\begin{array}{r}195 \\
\text { SORT- } \\
\text { SPLIT }\end{array}$ & $\begin{array}{c}0.2 \\
\text { N/A MED } \\
\text { WT }=398.7\end{array}$ & $\begin{array}{l}\mathrm{H} \\
\text { DIAN- }\end{array}$ & $\begin{array}{c}20.6 \\
-\quad 1.00\end{array}$ & $\begin{array}{r}46.5 \\
\text { MODE- }\end{array}$ & $-1.00^{32.9}$ & $\operatorname{mag}_{\mathrm{N}=}$ & N/A & CUM & WT & $\begin{array}{l}\text { N/A } \\
\text { N/A } \\
\text { N/A }\end{array}$ & $\begin{array}{r}131.2 \\
32.9 \\
32.9\end{array}$ & $\begin{array}{l}14.3 \\
11.1 \\
14.0\end{array}$ & $\begin{array}{r}29.7 \\
7.5 \\
51.5\end{array}$ & $\begin{array}{l}15.2 \\
11.3 \\
62.8\end{array}$ & $\begin{array}{l}13.3 \\
10.9 \\
73.7\end{array}$ & $\begin{array}{r}22.8 \\
5.7 \\
79.4\end{array}$ & $\begin{array}{l}N / A \\
N / A \\
N / A\end{array}$ & $\begin{array}{r}82.1 \\
20.6 \\
100.0\end{array}$ \\
\hline $\begin{array}{r}200 \\
\text { SORT- } \\
\text { SPLIT }\end{array}$ & $\begin{array}{c}0.3 \\
N / A \text { MED } \\
\text { WT- } 349.7\end{array}$ & $\begin{array}{l}\text { H } \\
\text { DIAN- }\end{array}$ & $\begin{array}{c}21.0 \\
-\quad 2.00\end{array}$ & $\begin{array}{r}53.5 \\
\text { MODE- }\end{array}$ & $-1.00 \mathrm{MEAN}$ & $N^{-}$ & $\mathrm{N} / \mathrm{A}$ & CUM & $\begin{array}{l}W T \\
W T\end{array}$ & $\begin{array}{l}\text { N/A } \\
\text { N/A } \\
\text { N/A }\end{array}$ & $\begin{array}{l}89.2 \\
25.5 \\
25.5\end{array}$ & $\begin{array}{l}16.1 \\
13.2 \\
38.7\end{array}$ & $\begin{array}{r}31.4 \\
9.0 \\
47.7\end{array}$ & $\begin{array}{l}14.4 \\
12.7 \\
60.4\end{array}$ & $\begin{array}{l}44.3 \\
12.7 \\
73.0\end{array}$ & $\begin{array}{r}21.1 \\
6.0 \\
79.0\end{array}$ & $\begin{array}{l}N / A \\
N / A \\
N / A\end{array}$ & $\begin{array}{r}73.3 \\
21.0 \\
100.0\end{array}$ \\
\hline $\begin{array}{r}205 \\
\text { SORT- } \\
\text { SPLIT }\end{array}$ & $\begin{array}{c}0.3 \\
\text { N/A MED } \\
\mathrm{WT}-342.4\end{array}$ & $\underset{4}{\stackrel{\mathbf{H}}{\text { DIAN- }}}$ & $\begin{array}{r}23.1 \\
-\quad 2.00\end{array}$ & $\begin{array}{r}50.0 \\
\text { MODE- }\end{array}$ & -1.00 MEAN & $\mathrm{gms}$ & $\mathrm{N} / \mathrm{A}$ & CUM & WT: & $\begin{array}{l}N / \boldsymbol{A} \\
N / \boldsymbol{A} \\
\mathbf{N} / \mathbf{A}\end{array}$ & $\begin{array}{l}92.0 \\
26.9 \\
26.9\end{array}$ & $\begin{array}{l}11.5 \\
12.1 \\
39.0\end{array}$ & $\begin{array}{r}28.9 \\
0.4 \\
47.4\end{array}$ & $\begin{array}{l}12.9 \\
12.5 \\
60.0\end{array}$ & $\begin{array}{l}39.3 \\
11.5 \\
71.4\end{array}$ & $\begin{array}{r}18.7 \\
5.5 \\
76.9\end{array}$ & $\begin{array}{l}\text { N/A } \\
\text { N/A } \\
\text { N/A }\end{array}$ & $\begin{array}{r}79.2 \\
23.1 \\
100.0\end{array}$ \\
\hline $\begin{array}{r}210 \\
\text { SORT- } \\
\text { SPLIT }\end{array}$ & $\begin{array}{c}2.6 \\
\text { N/A MED } \\
\text { WT- } 378.7\end{array}$ & $\underset{7}{\mathrm{H}}$ & $\begin{array}{c}19.9 \\
-\quad 1.00\end{array}$ & $\begin{array}{r}45.3 \\
\text { MODE- }\end{array}$ & $-1.00^{34.8}$ & mo 0 & $\mathrm{~N} / \mathrm{A}$ & CUM & $\begin{array}{l}\text { WT } \\
\text { WT }\end{array}$ & $\begin{array}{l}\text { N/A } \\
\text { N/A } \\
\text { N/A }\end{array}$ & $\begin{array}{r}131.9 \\
34.8 \\
34.8\end{array}$ & $\begin{array}{l}15.1 \\
11.9 \\
16.7\end{array}$ & $\begin{array}{r}29.7 \\
7.8 \\
54.6\end{array}$ & $\begin{array}{l}39.8 \\
10.5 \\
65.1\end{array}$ & $\begin{array}{l}38.0 \\
10.0 \\
75.1\end{array}$ & $\begin{array}{r}18.9 \\
5.0 \\
80.1\end{array}$ & $\begin{array}{l}N / A \\
N / A \\
N / A\end{array}$ & $\begin{array}{r}75.3 \\
19.9 \\
100.0\end{array}$ \\
\hline $\begin{array}{r}215 \\
\text { SORT- } \\
\text { SPIIT }\end{array}$ & $\begin{array}{c}2.4 \\
\mathrm{~N} / \mathrm{A} \text { MED } \\
\mathrm{wT}=416.5\end{array}$ & $\begin{array}{l}\mathrm{H} \\
\text { DIAN- }\end{array}$ & $\begin{array}{c}26.6 \\
2.00\end{array}$ & $\begin{array}{r}51.9 \\
\text { MODE- }\end{array}$ & $5.00^{21.5}$ MEAN & gms & N/A & CUM & $\begin{array}{l}\mathrm{WT} \\
\mathrm{WT} \\
\mathrm{WT}\end{array}$ & $\begin{array}{l}\text { N/A } \\
\text { N/A } \\
\text { N/A }\end{array}$ & $\begin{array}{l}89.3 \\
21.5 \\
21.5\end{array}$ & $\begin{array}{l}50.8 \\
12.2 \\
33.7\end{array}$ & $\begin{array}{r}37.6 \\
9.0 \\
42.7\end{array}$ & $\begin{array}{l}17.6 \\
11.6 \\
54.1\end{array}$ & $\begin{array}{l}52.7 \\
12.7 \\
66.8\end{array}$ & $\begin{array}{r}27.4 \\
6.6 \\
73.4\end{array}$ & $\begin{array}{l}N / \mathbf{A} \\
N / \mathbf{A} \\
\mathbf{N} / \mathbf{A}\end{array}$ & $\begin{array}{r}110.9 \\
26.6 \\
100.0\end{array}$ \\
\hline $\begin{array}{r}220 \\
\text { SORT- } \\
\text { SPLIT }\end{array}$ & $\begin{array}{c}1.3 \\
\text { N/A MED } \\
\text { WT- } 356.3\end{array}$ & $\underbrace{\mathrm{H}}$ & $\begin{array}{c}23.6 \\
-\quad 2.00\end{array}$ & $\begin{array}{r}51.1 \\
\text { MODE- }\end{array}$ & $\begin{array}{c}25.4 \\
-1.00 \quad \text { MEAN }\end{array}$ & $\mathrm{gms}$ & $\mathbf{N} / \mathbf{A}$ & CUM & $\begin{array}{l}\text { WT } \\
\text { WT } \\
\text { WT }\end{array}$ & $\begin{array}{l}\text { N/A } \\
\text { N/A } \\
\text { N/A }\end{array}$ & $\begin{array}{l}90.4 \\
25.4 \\
25.4\end{array}$ & $\begin{array}{l}19.6 \\
13.9 \\
39.3\end{array}$ & $\begin{array}{r}33.1 \\
9.3 \\
48.6\end{array}$ & $\begin{array}{l}12.3 \\
11.9 \\
60.5\end{array}$ & $\begin{array}{l}36.0 \\
10.1 \\
70.6\end{array}$ & $\begin{array}{r}20.9 \\
5.9 \\
76.4\end{array}$ & $\begin{array}{l}N / A \\
N / A \\
N / A\end{array}$ & $\begin{array}{r}84.0 \\
23.6 \\
100.0\end{array}$ \\
\hline
\end{tabular}


WESTINGHOUSE HANFORD OPERATIONS SIEVE ANALYSIS

$$
\text { ROCSAN REPORT }
$$

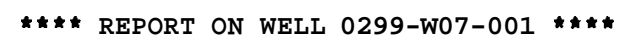

$12 / 11 / 89$

\begin{tabular}{|c|c|c|c|c|c|c|c|c|c|c|c|c|c|c|c|c|c|c|}
\hline DEPTH & ICACO3 & DM & IMUD & \SAND & TGRAVEL & CLAS & & & & $\begin{array}{l}\text { FINE } \\
\text { PEB } \\
(<--2)\end{array}$ & $\begin{array}{l}\text { W I NE } \\
\text { PEB } \\
(-1)\end{array}$ & $\begin{array}{l}\text { VERY } \\
\text { COARS } \\
(0)\end{array}$ & $\begin{array}{l}\text { COARS } \\
\text { (1) }\end{array}$ & $\begin{array}{l}\text { MED } \\
(2)\end{array}$ & $\begin{array}{l}\text { FINE } \\
(3)\end{array}$ & $\begin{array}{l}\text { VERY } \\
\text { FINE } \\
\text { (d) }\end{array}$ & $\begin{array}{c}\text { SILT } \\
(4.75)\end{array}$ & $\begin{array}{c}\text { PAN } \\
(>4.75)\end{array}$ \\
\hline $\begin{array}{r}225 \\
\text { SORT- } \\
\text { SPIIT }\end{array}$ & $\begin{array}{c}3.9 \\
\text { N/A MED } \\
\text { WT- } 349.4\end{array}$ & $\begin{array}{l}\stackrel{\mathrm{H}}{\mathrm{DIAN}-} \\
4\end{array}$ & $\begin{array}{r}24.0 \\
2.00\end{array}$ & $\begin{array}{r}46.9 \\
\text { MODE- }\end{array}$ & $-1.00^{29.1}$ & gms & N/A & CuM & $\begin{array}{l}\text { WT } \\
\text { WT } \\
\text { WT }\end{array}$ & $\begin{array}{l}\text { N/A } \\
\text { N/A } \\
\text { N/A }\end{array}$ & $\begin{array}{r}101.5 \\
29.1 \\
29.1\end{array}$ & $\begin{array}{l}41.4 \\
11.9 \\
40.9\end{array}$ & $\begin{array}{r}29.5 \\
0.5 \\
49.4\end{array}$ & $\begin{array}{l}40.3 \\
11.5 \\
60.9\end{array}$ & $\begin{array}{l}35.3 \\
10.1 \\
71.0\end{array}$ & $\begin{array}{r}17.3 \\
5.0 \\
76.0\end{array}$ & $\begin{array}{l}\text { N/A } \\
\text { N/A } \\
\text { N/A }\end{array}$ & $\begin{array}{r}04.0 \\
24.1 \\
100.0\end{array}$ \\
\hline $\begin{array}{r}230 \\
\text { SORT- } \\
\text { SPIIT }\end{array}$ & $\begin{array}{c}2.3 \\
\text { N/A MED } \\
\text { WT }=353.6\end{array}$ & $\begin{array}{l}\text { DIAN- } \\
5\end{array}$ & $\begin{array}{c}19.0 \\
1.00\end{array}$ & $\begin{array}{r}44.4 \\
\text { MODE- }\end{array}$ & $-1.00^{36.6}$ MEAN & $\mathbf{m s} \mathbf{G}$ & N/A & CUM & $\begin{array}{l}\text { KT } \\
\text { KT } \\
\text { WT Z }\end{array}$ & $\begin{array}{l}\text { N/A } \\
\text { N/A } \\
\text { N/A }\end{array}$ & $\begin{array}{r}129.6 \\
36.6 \\
36.6\end{array}$ & $\begin{array}{l}42.9 \\
12.1 \\
40.0\end{array}$ & $\begin{array}{c}29.4 \\
8.3 \\
57.1\end{array}$ & $\begin{array}{l}39.2 \\
11.1 \\
60.2\end{array}$ & $\begin{array}{r}30.8 \\
0.7 \\
76.9\end{array}$ & $\begin{array}{r}14.7 \\
4.2 \\
81.0\end{array}$ & $\begin{array}{l}\text { N/A } \\
\text { N/A } \\
\text { N/A }\end{array}$ & $\begin{array}{r}67.1 \\
19.0 \\
100.0\end{array}$ \\
\hline $\begin{array}{r}235 \\
\text { SORT- } \\
\text { SPLIT }\end{array}$ & $\begin{array}{c}0.8 \\
\text { N/A MED } \\
\mathrm{WT}=326.6\end{array}$ & $\begin{array}{l}\text { H } \\
\text { DIAN- }\end{array}$ & $\begin{array}{c}10.6 \\
1.00\end{array}$ & $\begin{array}{r}45.0 \\
\text { MODE= }\end{array}$ & $-1.00^{35.6}$ MEAN & $\mathbf{m a g}$ & N/A & CUM & $\begin{array}{l}\text { WT } \\
\text { WT } \\
\text { WT }\end{array}$ & $\begin{array}{l}N / \lambda \\
N / A \\
N / A\end{array}$ & $\begin{array}{r}116.2 \\
35.6 \\
35.6\end{array}$ & $\begin{array}{l}43.2 \\
13.2 \\
48.8\end{array}$ & $\begin{array}{r}25.6 \\
7.8 \\
56.7\end{array}$ & $\begin{array}{r}30.9 \\
9.5 \\
66.1\end{array}$ & & $\begin{array}{r}17.2 \\
5.3 \\
81.4\end{array}$ & $\begin{array}{l}\text { N/A } \\
N / A \\
N / A\end{array}$ & $\begin{array}{r}60.9 \\
18.7 \\
100.0\end{array}$ \\
\hline $\begin{array}{r}240 \\
\text { SORT- } \\
\text { SPLIT }\end{array}$ & $\begin{array}{c}\text { N/A } \\
\text { N/A MED } \\
\text { WT }=375.6\end{array}$ & $\begin{array}{l}\text { H } \\
\text { DIAN- } \\
6\end{array}$ & $\begin{array}{c}21.0 \\
1.00\end{array}$ & $\begin{array}{r}46.0 \\
\text { MODE- }\end{array}$ & $-1.00^{32.2}$ MEAN & $m=G$ & N/A & cum & $\begin{array}{l}\text { WT } \\
\text { WT } \\
\text { WT }\end{array}$ & $\begin{array}{l}N / \lambda \\
N / A \\
N / \lambda\end{array}$ & $\begin{array}{r}120.9 \\
32.2 \\
32.2\end{array}$ & $\begin{array}{r}36.6 \\
9.8 \\
42.0\end{array}$ & $\begin{array}{r}31.1 \\
8.3 \\
50.2\end{array}$ & $\begin{array}{l}45.9 \\
12.2 \\
62.5\end{array}$ & $\begin{array}{l}38.9 \\
10.4 \\
72.8\end{array}$ & $\begin{array}{r}23.3 \\
6.2 \\
79.0\end{array}$ & $\begin{array}{l}\text { N/A } \\
\text { N/A } \\
\text { N/A }\end{array}$ & $\begin{array}{r}78.7 \\
21.0 \\
100.0\end{array}$ \\
\hline $\begin{array}{r}245 \\
\text { SORT- } \\
\text { SPLIT }\end{array}$ & $\begin{array}{c}\text { N/A } \\
\text { N / A MED } \\
\text { WT- } 354.6\end{array}$ & $\begin{array}{l}\mathbf{n} \\
\text { DIAN- }\end{array}$ & $\begin{array}{r}20.2 \\
2.00\end{array}$ & $\begin{array}{r}60.3 \\
\text { MODE- }\end{array}$ & $\begin{array}{r}19.4 \\
5.00 \quad \mathrm{ME}\end{array}$ & gms & N/A & CUM & WT & $\begin{array}{l}N / \lambda \\
N / \lambda \\
N / \lambda\end{array}$ & $\begin{array}{l}68.0 \\
19.4 \\
19.4\end{array}$ & $\begin{array}{l}38.1 \\
10.8 \\
30.2\end{array}$ & $\begin{array}{r}29.2 \\
8.2 \\
38.5\end{array}$ & $\begin{array}{l}68.4 \\
19.3 \\
57.8\end{array}$ & $\begin{array}{l}52.5 \\
14.8 \\
72.6\end{array}$ & $\begin{array}{r}25.5 \\
7.2 \\
79.8\end{array}$ & $\begin{array}{l}N / A \\
N / A \\
N / A\end{array}$ & $\begin{array}{r}71.0 \\
20.3 \\
100.0\end{array}$ \\
\hline
\end{tabular}


$07 / 19 / 80$

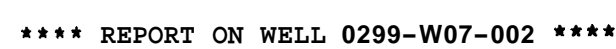

\begin{tabular}{|c|c|c|c|c|c|c|c|c|c|c|c|c|c|c|c|c|c|c|}
\hline $\mathrm{PTH}$ & $8 \mathrm{CACO} 3$ & DM & IMUD & CSAND & GRAVEL & CLAS: & & & & $\begin{array}{l}\text { FINE } \\
\text { PEB } \\
(<--2)\end{array}$ & $\begin{array}{c}\text { VFINE } \\
\text { PEB } \\
(-1)\end{array}$ & $\begin{array}{l}\text { VERY } \\
\text { COARS } \\
(0)\end{array}$ & $\begin{array}{l}\text { COARS } \\
\text { (1) }\end{array}$ & $\begin{array}{l}\text { MED } \\
\text { (2) }\end{array}$ & $\begin{array}{l}\text { FINE } \\
\text { (3) }\end{array}$ & $\begin{array}{l}\text { VERY } \\
\text { FINE } \\
\text { (4) }\end{array}$ & $\begin{array}{c}\text { SILT } \\
(4.75)\end{array}$ & $\begin{array}{c}\text { PAN } \\
(>4.75)\end{array}$ \\
\hline $\begin{array}{r}\text { SORT- } \\
\text { SPLIT }\end{array}$ & $\begin{array}{c}\mathrm{N} / \mathrm{A} \\
\mathrm{N} / \mathrm{A} \text { MED } \\
\mathrm{WT}=629.0\end{array}$ & DIAN- & $\begin{array}{c}8.4 \\
-1.00\end{array}$ & $\begin{array}{r}36.5 \\
\text { MODE- }\end{array}$ & $\begin{array}{l}55.1 \\
00 \text { MEAN- }\end{array}$ & ms G & N/A & CUM & $\begin{array}{l}\text { WT } \\
\text { WT }\end{array}$ & $\begin{array}{l}\text { N/A } \\
\text { N/A } \\
\text { N/A }\end{array}$ & $\begin{array}{r}346.5 \\
55.1 \\
55.1\end{array}$ & $\begin{array}{r}59.2 \\
9.4 \\
64.5\end{array}$ & $\begin{array}{r}51.9 \\
8.3 \\
72.8\end{array}$ & $\begin{array}{r}34.9 \\
5.6 \\
78.3\end{array}$ & $\begin{array}{c}44.4 \\
7.1 \\
85.4\end{array}$ & $\begin{array}{r}39.1 \\
6.2 \\
91.6\end{array}$ & $\begin{array}{l}\text { N/A } \\
\text { N/A } \\
\text { N/A }\end{array}$ & $\begin{array}{r}53.0 \\
8.4 \\
100.0\end{array}$ \\
\hline $\begin{array}{r}10 \\
\text { SORT- } \\
\text { SPLIT }\end{array}$ & $\begin{array}{c}1.0 \\
N / A \text { MED } \\
\text { WT= } 470.4\end{array}$ & $\begin{array}{l}\text { N/ } \\
\text { DIAN- } \\
4\end{array}$ & $\begin{array}{l}15.3 \\
-1.00\end{array}$ & $\begin{array}{r}34.2 \\
\text { MODE- }\end{array}$ & $\begin{array}{c}50.5 \\
.00 \mathrm{MEAN}-\end{array}$ & $\mathbf{m g G}$ & N/A & CUMI & 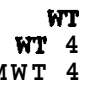 & $\begin{array}{l}\text { N/A } \\
\text { N/A } \\
\text { N/A }\end{array}$ & $\begin{array}{r}237.3 \\
50.5 \\
50.5\end{array}$ & $\begin{array}{r}40.7 \\
8.7 \\
59.1\end{array}$ & $\begin{array}{r}45.5 \\
9.7 \\
68.8\end{array}$ & $\begin{array}{r}34.0 \\
7.2 \\
76.0\end{array}$ & $\begin{array}{r}23.4 \\
5.0 \\
81.0\end{array}$ & $\begin{array}{r}17.3 \\
3.7 \\
04.7\end{array}$ & $\begin{array}{l}N / A \\
N / A \\
N / A\end{array}$ & $\begin{array}{r}72.1 \\
15.3 \\
100.0\end{array}$ \\
\hline $\begin{array}{r}15 \\
\text { SORT- } \\
\text { SPLIT }\end{array}$ & $\begin{array}{c}0.5 \\
\text { N/A MED } \\
\text { WT- } 435.2\end{array}$ & $\begin{array}{l}\underbrace{H}_{2} \\
{ }^{\text {IAN- }-}\end{array}$ & $\begin{array}{l}21.3 \\
1.00\end{array}$ & $\begin{array}{r}44.6 \\
\text { MODE- }\end{array}$ & $\begin{array}{c}34.0 \\
.00 \mathrm{MEAN}-\end{array}$ & $\mathbf{m s}_{-}^{\mathbf{m s}}$ & $N / A$ & CUM" & WT & $\begin{array}{l}\text { N/A } \\
\text { N/A } \\
\text { N/A }\end{array}$ & $\begin{array}{r}148.1 \\
34.0 \\
34.0\end{array}$ & $\begin{array}{l}57.4 \\
13.2 \\
47.2\end{array}$ & $\begin{array}{l}45.4 \\
10.4 \\
57.7\end{array}$ & $\begin{array}{r}35.1 \\
8.1 \\
65.7\end{array}$ & $\begin{array}{r}30.2 \\
6.9 \\
72.7\end{array}$ & $\begin{array}{r}26.2 \\
6.0 \\
78.7\end{array}$ & $\begin{array}{l}\text { N/A } \\
\text { N/A } \\
\text { N/A }\end{array}$ & $\begin{array}{r}92.8 \\
21.3 \\
100.0\end{array}$ \\
\hline $\begin{array}{r}25 \\
\text { SORT- } \\
\text { SPLIT }\end{array}$ & $\begin{array}{c}2.0 \\
\text { N/A MED } \\
\text { WT }=471.2\end{array}$ & $\begin{array}{l}\text { H } \\
2\end{array}$ & $\begin{array}{c}23.3 \\
0.00\end{array}$ & $\begin{array}{r}36.9 \\
\text { MODE- }\end{array}$ & $\begin{array}{l}39.8 \\
.00 \mathrm{MEAN}-\end{array}$ & $\mathbf{m s G}$ & N/A & CUM & $\begin{aligned} & \text { WT } \\
& \text { WT } 4 \\
& \text { WT }\end{aligned}$ & $\begin{array}{l}\text { N/A } \\
\text { N/A } \\
\text { N/A }\end{array}$ & $\begin{array}{r}187.6 \\
39.8 \\
39.8\end{array}$ & $\begin{array}{l}52.4 \\
11.1 \\
50.9\end{array}$ & $\begin{array}{r}39.4 \\
0.4 \\
59.3\end{array}$ & $\begin{array}{r}31.5 \\
6.7 \\
66.0\end{array}$ & $\begin{array}{r}27.0 \\
5.7 \\
71.7\end{array}$ & $\begin{array}{r}23.7 \\
5.0 \\
76.7\end{array}$ & $\begin{array}{l}N / A \\
N / A \\
N / A\end{array}$ & $\begin{array}{r}109.8 \\
23.3 \\
100.0\end{array}$ \\
\hline $\begin{array}{r}30 \\
\text { SORT- } \\
\text { SPLIT }\end{array}$ & $\begin{array}{c}2.8 \\
\text { N/A MED } \\
\text { WT- } 340.6\end{array}$ & $\begin{array}{l}\text { H } \\
6\end{array}$ & $\begin{array}{r}36.7 \\
3.00\end{array}$ & $\begin{array}{r}53.0 \\
\text { MODE- }\end{array}$ & | 10.3 & $g m s$ & $\mathbf{N} / \mathbf{A}$ & CUM & ${ }_{W T}^{W T}$ & $\begin{array}{l}N / A \\
N / A \\
N / A\end{array}$ & $\begin{array}{l}35.2 \\
10.3 \\
10.3\end{array}$ & $\begin{array}{l}50.8 \\
14.9 \\
25.2\end{array}$ & $\begin{array}{l}15.2 \\
13.3 \\
38.5\end{array}$ & $\begin{array}{l}36.0 \\
10.6 \\
49.1\end{array}$ & $\begin{array}{r}27.5 \\
8.1 \\
57.2\end{array}$ & $\begin{array}{r}21.0 \\
6.2 \\
63.3\end{array}$ & $\begin{array}{l}N / A \\
N / A \\
N / A\end{array}$ & $\begin{array}{r}124.9 \\
36.7 \\
100.0\end{array}$ \\
\hline $\begin{array}{r}35 \\
\text { SORT- } \\
\text { SPIIT }\end{array}$ & $\begin{array}{c}3.2 \\
\text { N/A MED } \\
\text { WT- } 381.4\end{array}$ & $\begin{array}{l}\text { H } \\
4\end{array}$ & $\begin{array}{c}38.4 \\
\quad 2.00\end{array}$ & $\begin{array}{r}34.7 \\
\text { MODE- }\end{array}$ & $\begin{array}{c}26.9 \\
5.00 \mathrm{MEAN}\end{array}$ & gsM & N/A & CUM & $\begin{array}{l}\text { WT } \\
\text { WT } \\
\text { WT }\end{array}$ & $\begin{array}{l}N / A \\
N / A \\
\text { N/A }\end{array}$ & $\begin{array}{r}102.6 \\
26.9 \\
26.9\end{array}$ & $\begin{array}{l}42.4 \\
11.1 \\
38.0\end{array}$ & $\begin{array}{r}30.4 \\
8.0 \\
16.0\end{array}$ & $\begin{array}{r}25.1 \\
6.6 \\
52.6\end{array}$ & $\begin{array}{r}18.4 \\
4.8 \\
57.4\end{array}$ & $\begin{array}{r}15.9 \\
1.2 \\
61.6\end{array}$ & $\begin{array}{l}N / A \\
N / A \\
N / A\end{array}$ & $\begin{array}{r}146.6 \\
38.4 \\
100.0\end{array}$ \\
\hline $\begin{array}{r}40 \\
\text { SORT- } \\
\text { SPLIT }\end{array}$ & $\begin{array}{c}4.0 \\
\text { N/A MED } \\
\text { WT- } 267.7\end{array}$ & $\underset{7}{\mathrm{H}}$ & $\begin{array}{c}70.7 \\
5.00\end{array}$ & $\begin{array}{r}23.3 \\
\text { MODE- }\end{array}$ & $5.00^{6.0} \mathrm{MEAN}$ & $(g)=$ & $\stackrel{S M}{N / A}$ & CUM & $\begin{aligned} & \text { WT } \\
\text { WT } & 4 \\
\text { WT } & \text { t }\end{aligned}$ & $\begin{array}{l}\mathrm{N} / \mathrm{A} \\
\mathrm{N} / \mathrm{A} \\
\mathrm{N} / \mathrm{A}\end{array}$ & $\begin{array}{r}16.1 \\
6.0 \\
6.0\end{array}$ & $\begin{array}{l}9.0 \\
3.0 \\
9.0\end{array}$ & $\begin{array}{r}6.5 \\
2.4 \\
11.4\end{array}$ & $\begin{array}{r}5.1 \\
1.9 \\
13.3\end{array}$ & $\begin{array}{r}5.1 \\
1.9 \\
15.2\end{array}$ & $\begin{array}{l}37.8 \\
14.1 \\
29.3\end{array}$ & $\begin{array}{l}N / A \\
N / A \\
N / A\end{array}$ & $\begin{array}{r}189.4 \\
70.7 \\
100.0\end{array}$ \\
\hline $\begin{array}{r}45 \\
\text { SORT- } \\
\text { SPLIT }\end{array}$ & $\begin{array}{c}0.9 \\
\text { N/A MED } \\
\text { WT- } 564.3\end{array}$ & ${ }_{3}^{\mathrm{H}}$ & $\begin{array}{c}0.4 \\
-1.00\end{array}$ & $\begin{array}{c}18.4 \\
\text { MODE- }\end{array}$ & .00 MEAN & $\begin{array}{l}\mathrm{mso} \\
\mathrm{N}=\end{array}$ & $N / A$ & CUM & $\begin{array}{l}\text { WT } \\
\text { WT } \\
\text { WT }\end{array}$ & $\begin{array}{l}\text { N/A } \\
\text { N/A } \\
\text { N/A }\end{array}$ & $\begin{array}{r}113.3 \\
73.2 \\
73.2\end{array}$ & $\begin{array}{r}29.7 \\
5.3 \\
78.5\end{array}$ & $\begin{array}{r}22.2 \\
3.9 \\
82.4\end{array}$ & $\begin{array}{r}20.6 \\
3.7 \\
86.1\end{array}$ & $\begin{array}{r}17.5 \\
3.1 \\
89.2\end{array}$ & $\begin{array}{r}13.6 \\
2.4 \\
91.6\end{array}$ & $\begin{array}{l}\text { N/A } \\
\text { N/A } \\
\text { N/A }\end{array}$ & $\begin{array}{r}47.6 \\
8.4 \\
100.0\end{array}$ \\
\hline $\begin{array}{r}50 \\
\text { SORT- } \\
\text { SPLIT }\end{array}$ & $\begin{array}{c}27.5 \\
\text { N/A MED } \\
\text { WT- } 350.2\end{array}$ & $\begin{array}{l}\text { DIAN- } \\
2\end{array}$ & $\begin{array}{c}28.1 \\
1.00\end{array}$ & $\begin{array}{r}43.2 \\
\text { MODE- }\end{array}$ & $\begin{array}{c}28.7 \\
.00 \mathrm{MEAN}\end{array}$ & gms & N/A & CUM & $\begin{array}{l}\mathrm{WT} \\
\mathrm{WT} \\
\mathrm{WT}\end{array}$ & $\begin{array}{l}\text { N/A } \\
\text { N/A } \\
\text { N/A }\end{array}$ & $\begin{array}{r}100.1 \\
28.7 \\
28.7\end{array}$ & $\begin{array}{l}38.6 \\
11.0 \\
39.7\end{array}$ & $\begin{array}{l}30.4 \\
11.0 \\
50.7\end{array}$ & $\begin{array}{r}24.8 \\
7.1 \\
57.7\end{array}$ & $\begin{array}{r}23.4 \\
6.7 \\
64.4\end{array}$ & $\begin{array}{r}26.1 \\
7.5 \\
71.9\end{array}$ & $\begin{array}{l}N / A \\
N / A \\
N / A\end{array}$ & $\begin{array}{r}98.5 \\
28.1 \\
100.0\end{array}$ \\
\hline $\begin{array}{r}55 \\
\text { SORT- } \\
\text { SPIIT }\end{array}$ & $\begin{array}{c}9.9 \\
\text { N } / A \text { MED } \\
\mathrm{MT}=400.3\end{array}$ & $\begin{array}{l}\text { H } \\
3\end{array}$ & $\begin{array}{l}15.3 \\
0.00\end{array}$ & $\begin{array}{r}37.1 \\
\text { MODE- }\end{array}$ & $.00_{\text {MEAN- }}^{17.7}$ & ms & $N / A$ & CUM & $\begin{aligned} \text { WT } & \text { HT } \\
\text { WT } & 4\end{aligned}$ & $\begin{array}{l}\text { N/A } \\
\text { N/A } \\
\text { N/A }\end{array}$ & $\begin{array}{r}194.6 \\
47.7 \\
17.7\end{array}$ & $\begin{array}{l}51.2 \\
12.5 \\
60.2\end{array}$ & $\begin{array}{r}36.2 \\
8.9 \\
69.1\end{array}$ & $\begin{array}{r}28.8 \\
7.1 \\
76.1\end{array}$ & $\begin{array}{r}19.0 \\
4.7 \\
80.8\end{array}$ & $\begin{array}{r}16.1 \\
3.9 \\
84.7\end{array}$ & $\begin{array}{l}N / A \\
N / A \\
N / A\end{array}$ & $\begin{array}{r}62.4 \\
15.3 \\
100.0\end{array}$ \\
\hline $\begin{array}{r}60 \\
\text { SORT- } \\
\text { SPIIT }\end{array}$ & $\begin{array}{c}5.3 \\
\text { N/A MED } \\
\text { WT- } 415.9\end{array}$ & $\begin{array}{l}\text { H } \\
\text { DIAN- }\end{array}$ & $\begin{array}{l}15.5 \\
1.00\end{array}$ & $\begin{array}{r}58.3 \\
\text { MODE- }\end{array}$ & $1.00 \mathrm{MEAN}$ & gms & $N / A$ & CUM & $\begin{array}{rr} & \text { WT } \\
\text { HT } & 4 \\
\text { WT } & 4\end{array}$ & $\begin{array}{l}\text { N/A } \\
\text { N/A } \\
\text { N/A }\end{array}$ & $\begin{array}{r}109.7 \\
26.2 \\
26.2\end{array}$ & $\begin{array}{l}53.6 \\
12.8 \\
39.0\end{array}$ & $\begin{array}{l}78.7 \\
18.8 \\
57.8\end{array}$ & $\begin{array}{l}63.5 \\
15.2 \\
72.9\end{array}$ & $\begin{array}{r}30.0 \\
7.2 \\
80.1\end{array}$ & $\begin{array}{r}18.4 \\
4.4 \\
84.5\end{array}$ & $\begin{array}{l}\text { N/A } \\
\text { N/A } \\
\text { N/A }\end{array}$ & $\begin{array}{r}65.1 \\
15.5 \\
100.0\end{array}$ \\
\hline
\end{tabular}




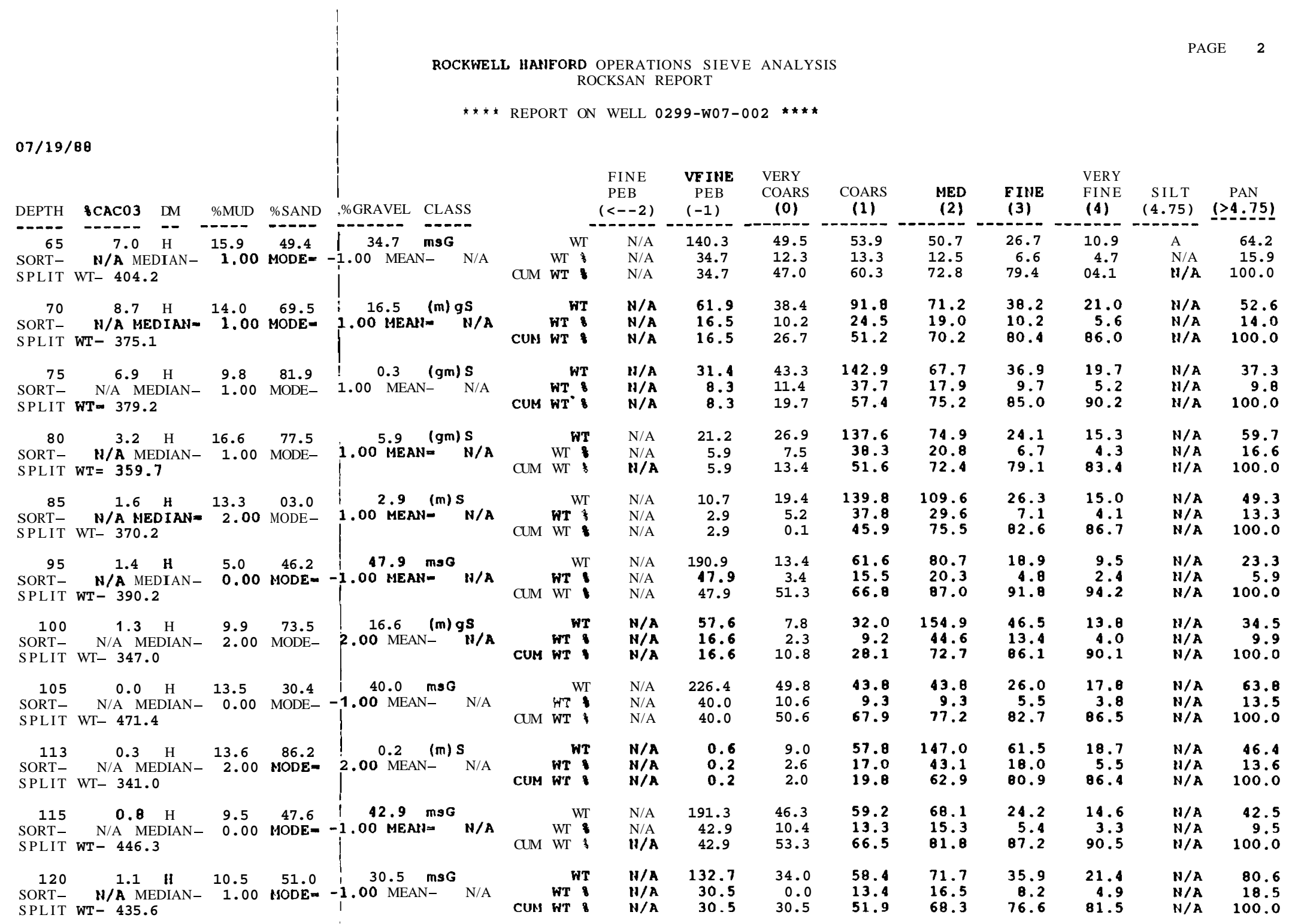




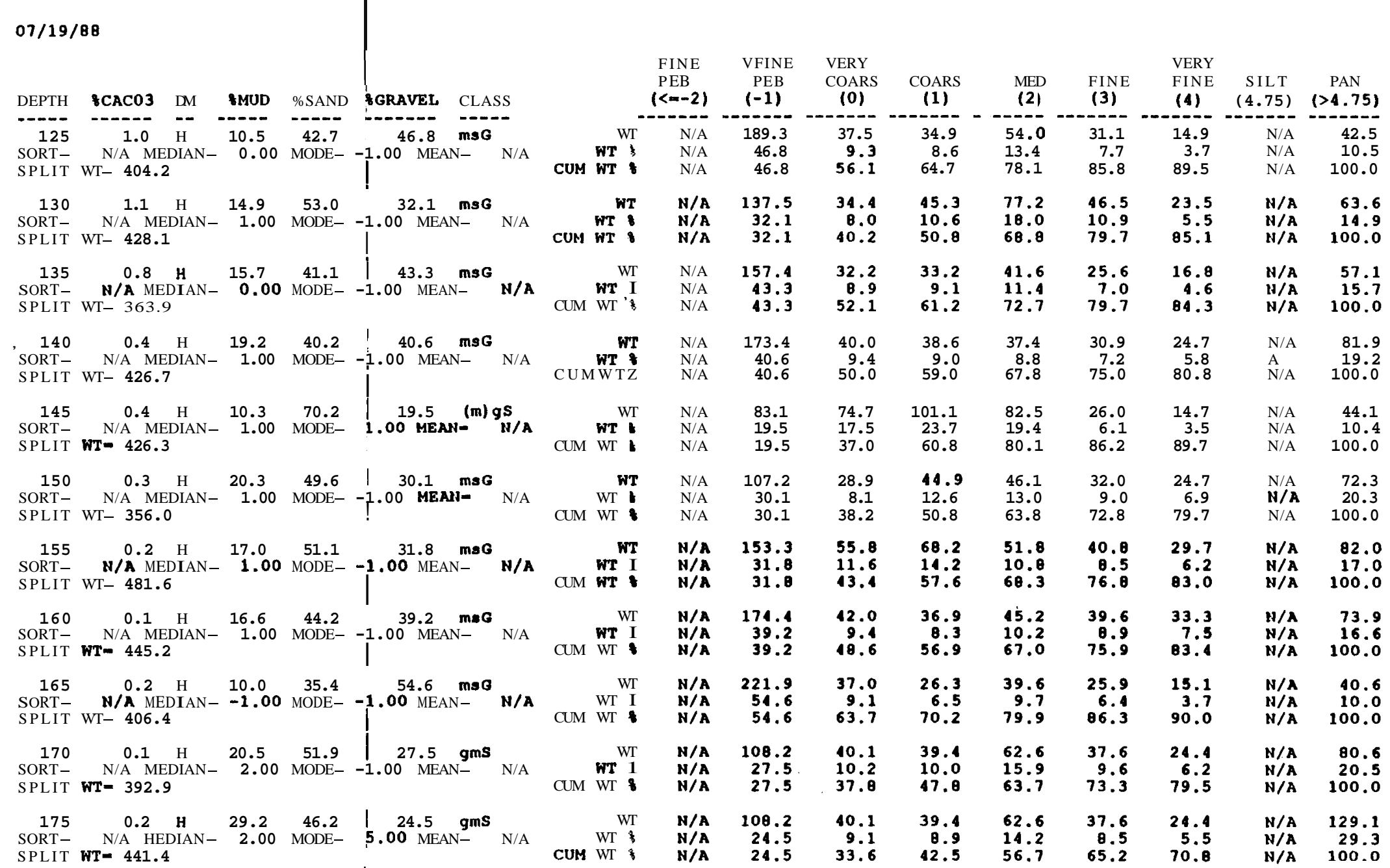


ROCKWELL HANFORD OPERATIONS SIEVE ANALYSIS ROCKSAN REPORT

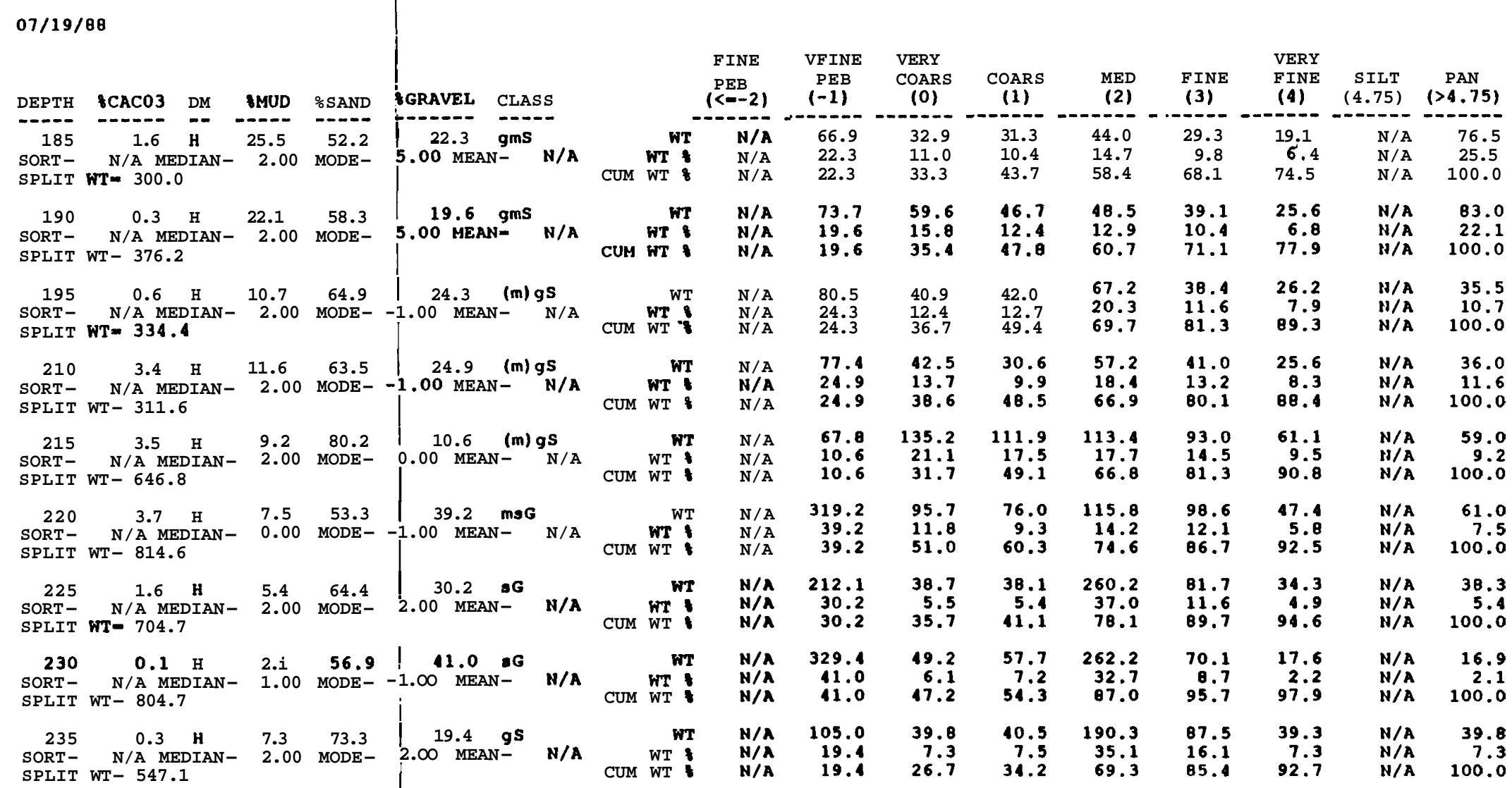




\begin{tabular}{|c|c|c|c|c|c|c|c|c|c|c|c|c|c|c|c|c|c|c|}
\hline DEPTH & BCACO3 & DM & IMUD & $\because$ SAND & \%GRAVEL C & CLAS & & & & $\begin{array}{l}\text { INE } \\
E B \\
E--2)\end{array}$ & $\begin{array}{l}\text { VF INE } \\
\text { PEB } \\
(-1)\end{array}$ & $\begin{array}{l}\text { VERY } \\
\text { COARS } \\
(0)\end{array}$ & $\begin{array}{l}\text { COARS } \\
(1)\end{array}$ & $\begin{array}{l}\text { MED } \\
\text { (2) }\end{array}$ & $\begin{array}{l}\text { FINE } \\
\text { (3) }\end{array}$ & $\begin{array}{l}\text { VERY } \\
\text { FINE } \\
\text { (4) }\end{array}$ & $\begin{array}{l}\text { SILT } \\
(4.75)\end{array}$ & $\begin{array}{c}\text { PAN } \\
(>4.75)\end{array}$ \\
\hline $\begin{array}{r}5 \\
\text { SORT } \\
\text { SPIIT }\end{array}$ & $\begin{array}{c}0.8 \\
\text { N/A MED } \\
\text { WT }=832.3\end{array}$ & $\begin{array}{l}\mathbf{N} / \\
\text { DIAN- } \\
3\end{array}$ & $\begin{array}{c}\mathbf{N} / \mathbf{A} \\
-1.00\end{array}$ & $\begin{array}{r}12.9 \\
\text { MODE- }\end{array}$ & $\begin{array}{l}87.1 \\
\text { N/A MEAN- }\end{array}$ & N/A & N/A & CUM & $\begin{array}{l}\text { WT } \\
\text { WT } \\
\text { WT }\end{array}$ & $\begin{array}{l}\text { N/A } \\
\text { N/A } \\
\text { N/A }\end{array}$ & $\begin{array}{r}719.9 \\
87.1 \\
87.1\end{array}$ & $\begin{array}{c}51.0 \\
6.2 \\
93.3\end{array}$ & $\begin{array}{r}39.1 \\
4.7 \\
98.0\end{array}$ & $\begin{array}{r}10.3 \\
1.3 \\
99.3\end{array}$ & $\begin{array}{r}3.9 \\
0.5 \\
99.7\end{array}$ & $\begin{array}{r}2.1 \\
0.3 \\
100.0\end{array}$ & $\begin{array}{l}\mathrm{H} / \mathrm{A} \\
\mathrm{N} / \mathrm{A} \\
\mathrm{N} / \mathrm{A}\end{array}$ & $\begin{array}{l}N / A \\
N / A \\
N / A\end{array}$ \\
\hline $\begin{array}{r}10 \\
\text { SORT- } \\
\text { SPIIT }\end{array}$ & $\begin{array}{c}0.4 \\
\text { N/A MED } \\
\text { WT- } 495.8\end{array}$ & $\begin{array}{l}\text { H } \\
\text { DIAN- } \\
8\end{array}$ & $\begin{array}{l}14.4 \\
-1.00\end{array}$ & $\begin{array}{l}28.1 \\
\text { MODE- }\end{array}$ & $-1.00^{57.5}$ MEAN- & $\mathbf{m s G}$ & N/A & CUM & $\begin{array}{l}\text { WT } \\
\text { WT }\end{array}$ & $\begin{array}{l}N / A \\
N / A \\
N / A\end{array}$ & $\begin{array}{r}285.2 \\
57.5 \\
57.5\end{array}$ & $\begin{array}{r}42.8 \\
8.6 \\
66.1\end{array}$ & $\begin{array}{r}30.0 \\
6.1 \\
72.2\end{array}$ & $\begin{array}{r}24.9 \\
5.0 \\
77.2\end{array}$ & $\begin{array}{r}22.0 \\
4.4 \\
81.7\end{array}$ & $\begin{array}{c}19.7 \\
4.0 \\
85.6\end{array}$ & $\begin{array}{l}N / A \\
N / A \\
N / A\end{array}$ & $\begin{array}{r}71.3 \\
14.4 \\
100.0\end{array}$ \\
\hline $\begin{array}{r}15 \\
\text { SORT- } \\
\text { SPLIT }\end{array}$ & $\begin{array}{c}0.5 \\
\text { N } / \mathrm{A} \text { MED } \\
\mathrm{WT}=502.8\end{array}$ & $\begin{array}{l}\text { H } \\
\text { DIAN- } \\
3\end{array}$ & $\begin{array}{l}16.2 \\
-1.00\end{array}$ & $\begin{array}{l}31.5 \\
\text { MODE- }\end{array}$ & $-1.00^{52.3}$ MEAN- & ms G & N/A & CUM & $\begin{array}{l}\text { WT. } \\
\text { WT } \\
\text { WT }\end{array}$ & $\begin{array}{l}\text { N/A } \\
\text { N/A } \\
\text { N/A }\end{array}$ & $\begin{array}{r}263.2 \\
52.3 \\
52.3\end{array}$ & $\begin{array}{r}41.1 \\
8.2 \\
60.5\end{array}$ & $\begin{array}{r}37.6 \\
7.5 \\
68.0\end{array}$ & $\begin{array}{r}30.2 \\
6.0 \\
74.0\end{array}$ & $\begin{array}{r}26.8 \\
5.3 \\
79.3\end{array}$ & $\begin{array}{r}22.6 \\
4.5 \\
83.8\end{array}$ & $\begin{array}{l}N / A \\
N / A \\
N / A\end{array}$ & $\begin{array}{r}81.4 \\
16.2 \\
100.0\end{array}$ \\
\hline $\begin{array}{r}20 \\
\text { SORT- } \\
\text { SPLIT }\end{array}$ & $\begin{array}{c}1.7 \\
N / A M E D \\
\text { WT }=421.4\end{array}$ & $\begin{array}{l}\text { H } \\
\text { DIAN- } \\
4\end{array}$ & $\begin{array}{c}18.4 \\
0.00\end{array}$ & $\begin{array}{c}35.0 \\
\text { MODE- }\end{array}$ & $-1.00^{46.6}$ MEAN & msG & N/A & CUM & $\begin{array}{l}\text { WT } \\
\text { WT } \\
\text { WT }\end{array}$ & $\begin{array}{l}\text { N/A } \\
\text { N/A } \\
\text { N/A }\end{array}$ & $\begin{array}{r}196.3 \\
46.6 \\
46.6\end{array}$ & $\begin{array}{r}40.6 \\
9.6 \\
56.2\end{array}$ & $\begin{array}{r}36.0 \\
8.5 \\
64.8\end{array}$ & $\begin{array}{r}28.8 \\
6.8 \\
71.6\end{array}$ & $\begin{array}{r}23.0 \\
5.5 \\
77.0\end{array}$ & $\begin{array}{r}19.0 \\
4.5 \\
81.6\end{array}$ & $\begin{array}{l}N / A \\
N / A \\
N / A\end{array}$ & $\begin{array}{r}77.7 \\
18.4 \\
100.0\end{array}$ \\
\hline $\begin{array}{r}25 \\
\text { SORT- } \\
\text { SPIIT }\end{array}$ & $\begin{array}{c}1.5 \\
N / A \text { MED } \\
W T=402.5\end{array}$ & $\begin{array}{l}\text { H } \\
5 \text { IAN- } \\
5\end{array}$ & $\begin{array}{r}24.7 \\
-\quad 1.00\end{array}$ & $\begin{array}{r}39.2 \\
\text { MODE- }\end{array}$ & $-1.00^{36.1}$ MEAN & msG & $\mathrm{N} / \mathrm{A}$ & CUM & $\begin{array}{l}\text { WT } \\
\text { WT } \\
\text { WT }\end{array}$ & $\begin{array}{l}\text { N/A } \\
\text { N/A } \\
\text { N/A }\end{array}$ & $\begin{array}{r}145.5 \\
36.2 \\
36.2\end{array}$ & $\begin{array}{l}44.7 \\
11.1 \\
47.3\end{array}$ & $\begin{array}{r}37.3 \\
9.3 \\
56.5\end{array}$ & $\begin{array}{r}30.2 \\
7.5 \\
64.0\end{array}$ & $\begin{array}{r}23.9 \\
5.9 \\
70.0\end{array}$ & $\begin{array}{r}21.6 \\
5.4 \\
75.3\end{array}$ & $\begin{array}{l}N / A \\
N / A \\
N / A\end{array}$ & $\begin{array}{r}99.3 \\
24.7 \\
100.0\end{array}$ \\
\hline $\begin{array}{r}30 \\
\text { SORT- } \\
\text { SPIIT }\end{array}$ & $\begin{array}{c}2.7 \\
\text { N/A MED } \\
\text { WT- } 450.2\end{array}$ & $\begin{array}{l}\stackrel{H}{D^{2}} \\
2^{\text {IAN- }}\end{array}$ & $\begin{array}{l}13.0 \\
-1.00\end{array}$ & $\begin{array}{r}22.3 \\
\text { MODE- }\end{array}$ & -1.00 MEAN- & $\mathbf{m s} \mathbf{G}$ & $\mathrm{N} / \mathrm{A}$ & CUM & $\begin{array}{l}\text { WT } \\
\text { HT } \\
\text { WT }\end{array}$ & $\begin{array}{l}\text { N/A } \\
\text { N/A } \\
\text { N/A }\end{array}$ & $\begin{array}{r}291.4 \\
64.7 \\
64.7\end{array}$ & $\begin{array}{r}34.0 \\
7.6 \\
72.3\end{array}$ & $\begin{array}{c}21.8 \\
4.8 \\
77.1\end{array}$ & $\begin{array}{r}18.1 \\
4.0 \\
81.2\end{array}$ & $\begin{array}{r}14.4 \\
3.2 \\
04.4\end{array}$ & $\begin{array}{c}12.0 \\
2.7 \\
87.0\end{array}$ & $\begin{array}{l}N / A \\
N / A \\
N / A\end{array}$ & $\begin{array}{r}58.4 \\
13.0 \\
100.0\end{array}$ \\
\hline $\begin{array}{r}35 \\
\text { SORT- } \\
\text { SPIIT }\end{array}$ & $\begin{array}{c}1.8 \\
N / A \\
W T=382.3\end{array}$ & $\begin{array}{l}\text { H } \\
\text { DIAN- } \\
3\end{array}$ & $\begin{array}{c}58.0 \\
5.00\end{array}$ & $\begin{array}{l}20.4 \\
\text { MODE- }\end{array}$ & $5.00^{21.6}$ & gaH & $\mathbf{N} / \mathbf{A}$ & CUM & $\begin{array}{l}\text { WT } \\
\text { WT }\end{array}$ & $\begin{array}{l}\text { N/A } \\
N / A \\
\text { H/A }\end{array}$ & $\begin{array}{l}82.5 \\
21.6 \\
21.6\end{array}$ & $\begin{array}{c}18.0 \\
4.7 \\
26.3\end{array}$ & $\begin{array}{r}17.2 \\
4.5 \\
30.8\end{array}$ & $\begin{array}{r}13.9 \\
3.6 \\
34.4\end{array}$ & $\begin{array}{r}11.6 \\
3.0 \\
37.5\end{array}$ & $\begin{array}{r}17.3 \\
4.5 \\
42.0\end{array}$ & $\begin{array}{l}\text { N/A } \\
\text { N/A } \\
\text { N/A }\end{array}$ & $\begin{array}{r}221.0 \\
58.0 \\
100.0\end{array}$ \\
\hline $\begin{array}{r}40 \\
\text { SORT- } \\
\text { SPLIT }\end{array}$ & $\begin{array}{c}3.2 \\
\text { N/A MED } \\
\text { WT- } 309.2\end{array}$ & $\begin{array}{l}\stackrel{\text { H }}{\text { DIAN- }} \\
2\end{array}$ & $\begin{array}{l}74.3 \\
5.00\end{array}$ & $\begin{array}{r}22.3 \\
\text { MODE- }\end{array}$ & $5.00^{3.4}=$ & $\operatorname{sM}$ & N/A & CUM & $\begin{array}{l}\text { WT } \\
\text { WT }\end{array}$ & $\begin{array}{l}\text { N/A } \\
\text { N/A } \\
\text { N/A }\end{array}$ & $\begin{array}{r}10.6 \\
3.4 \\
3.4\end{array}$ & $\begin{array}{l}3.9 \\
1.3 \\
4.7\end{array}$ & $\begin{array}{l}4.0 \\
1.3 \\
6.0\end{array}$ & $\begin{array}{l}3.2 \\
1.0 \\
7.0\end{array}$ & $\begin{array}{l}5.5 \\
1.8 \\
8.8\end{array}$ & $\begin{array}{l}52.4 \\
17.0 \\
25.7\end{array}$ & $\begin{array}{l}N / A \\
N / A \\
N / A\end{array}$ & $\begin{array}{r}229.6 \\
74.3 \\
100.0\end{array}$ \\
\hline $\begin{array}{r}45 \\
\text { SORT- } \\
\text { SPLIT }\end{array}$ & $\begin{array}{c}26.1 \\
\text { N/A MED } \\
\text { WT- } 267.0\end{array}$ & $\begin{array}{l}\mathrm{H} \\
\text { DIAN- } \\
0\end{array}$ & $\begin{array}{r}41.8 \\
-\quad 4.00\end{array}$ & $\begin{array}{c}35.3 \\
\text { MODE- }\end{array}$ & $5.00^{23.0}$ MEAN & gst & N/A & CUM & $\begin{array}{l}\text { WT } \\
\text { WT }\end{array}$ & $\begin{array}{l}N / A \\
N / A \\
N / A\end{array}$ & $\begin{array}{l}61.3 \\
23.0 \\
23.0\end{array}$ & $\begin{array}{r}18.7 \\
7.0 \\
30.0\end{array}$ & $\begin{array}{r}13.6 \\
5.1 \\
35.1\end{array}$ & $\begin{array}{r}12.9 \\
4.8 \\
39.9\end{array}$ & $\begin{array}{r}17.0 \\
6.4 \\
46.3\end{array}$ & $\begin{array}{l}31.9 \\
12.0 \\
58.3\end{array}$ & $\begin{array}{l}N / A \\
N / A \\
N / A\end{array}$ & $\begin{array}{r}111.4 \\
41.8 \\
100.0\end{array}$ \\
\hline $\begin{array}{r}50 \\
\text { SORT- } \\
\text { SPLIT }\end{array}$ & $\begin{array}{c}13.1 \\
\text { N/A MED } \\
\text { WT- } 363.8\end{array}$ & $\begin{array}{l}\mathrm{H} \\
\text { DIAN- } \\
8\end{array}$ & $\begin{array}{c}20.3 \\
1.00\end{array}$ & $\begin{array}{r}44.7 \\
\text { MODE- }\end{array}$ & $-1.00^{35.0}$ MEAN- & $\mathbf{m s G}$ & H/A & CUM & $\begin{array}{l}\text { WT } \\
\text { WT }\end{array}$ & $\begin{array}{l}N / A \\
N / A \\
N / A\end{array}$ & $\begin{array}{r}127.5 \\
35.0 \\
35.0\end{array}$ & $\begin{array}{l}51.8 \\
14.2 \\
49.3\end{array}$ & $\begin{array}{l}44.2 \\
12.2 \\
61.4\end{array}$ & $\begin{array}{r}23.4 \\
6.4 \\
67.9\end{array}$ & $\begin{array}{r}21.3 \\
5.9 \\
73.7\end{array}$ & $\begin{array}{r}21.8 \\
6.0 \\
79.7\end{array}$ & $\begin{array}{l}N / A \\
N / A \\
N / A\end{array}$ & $\begin{array}{r}73.9 \\
20.3 \\
100.0\end{array}$ \\
\hline $\begin{array}{l}55 \\
\text { SORT- } \\
\text { SPLIT }\end{array}$ & $\begin{array}{c}8.7 \\
\text { N/A MED } \\
\text { WT- } 394.1\end{array}$ & $\underset{\text { DIAN- }}{\mathbf{H}}$ & $\begin{array}{c}21.2 \\
0.00\end{array}$ & $\begin{array}{r}38.1 \\
\text { MODE- }\end{array}$ & $\begin{array}{c}40.7 \\
-1.00^{\text {MEA }}\end{array}$ & $\mathbf{m s G}$ & N/A & CUM & $\begin{aligned} & \text { WT } \\
\text { WT } & \text { \& } \\
\text { WT } & \%\end{aligned}$ & $\begin{array}{l}\text { N/A } \\
\text { N/A } \\
\text { N/A }\end{array}$ & $\begin{array}{r}160.3 \\
40.7 \\
40.7\end{array}$ & $\begin{array}{l}56.0 \\
14.2 \\
54.9\end{array}$ & $\begin{array}{l}42.4 \\
10.8 \\
65.6\end{array}$ & $\begin{array}{r}20.8 \\
5.3 \\
70.9\end{array}$ & $\begin{array}{r}16.3 \\
4.1 \\
75.0\end{array}$ & $\begin{array}{r}14.8 \\
3.8 \\
78.8\end{array}$ & $\begin{array}{l}N / A \\
N / A \\
N / A\end{array}$ & $\begin{array}{r}83.6 \\
21.2 \\
100.0\end{array}$ \\
\hline
\end{tabular}


WESTINGHOUSE HANFORD OPERATIONS SIEVE ANALYSIS ROCSAN REPORT

$\star \star \star *$ REPORT ON WELL 0299-W07-003 $\star * * *$

$12 / 11 / 89$

\begin{tabular}{|c|c|c|c|c|c|c|c|c|c|c|c|c|c|c|c|c|c|}
\hline DEPTH & $8 \mathrm{CAC03} \mathrm{DM}$ & YMUD & ISAND & VRAVEL & CLASS & & & & $\begin{array}{l}\text { FINE } \\
\text { PEB } \\
(<=-2)\end{array}$ & $\begin{array}{l}\text { VFINE } \\
\text { PEB } \\
(-1)\end{array}$ & $\begin{array}{l}\text { VERY } \\
\text { COARS } \\
(0)\end{array}$ & $\begin{array}{l}\text { COARS } \\
\text { (1) }\end{array}$ & $\begin{array}{l}\text { MED } \\
\text { (2) }\end{array}$ & $\begin{array}{l}\text { FINE } \\
\text { (3) }\end{array}$ & $\begin{array}{l}\text { VERY } \\
\text { FINE } \\
\text { (4) }\end{array}$ & $\begin{array}{c}\text { SILT } \\
(4.75)\end{array}$ & $\begin{array}{c}\text { PAN } \\
(>4.75)\end{array}$ \\
\hline $\begin{array}{r}60 \\
\text { SORT- } \\
\text { SPLIT }\end{array}$ & $\begin{array}{c}\mathbf{4 . 7} \mathbf{H} \\
\text { N/A MEDIAN- } \\
\text { WT- } \mathbf{4 4 1 . 2}\end{array}$ & $\begin{array}{r}21.5 \\
-\quad 1.00\end{array}$ & $\begin{array}{r}51.0 \\
\text { MODE- }\end{array}$ & $-1.00^{27.5}$ MEAN & gms & N/A & CUH & $\begin{array}{l}\text { WT } \\
\text { WT } \\
\text { WT }\end{array}$ & $\begin{array}{l}\text { N/A } \\
\text { N/A } \\
\text { N/A }\end{array}$ & $\begin{array}{r}121.4 \\
27.5 \\
27.5\end{array}$ & $\begin{array}{l}61.3 \\
13.9 \\
41.4\end{array}$ & $\begin{array}{l}57.7 \\
13.1 \\
54.5\end{array}$ & $\begin{array}{l}45.2 \\
10.2 \\
64.7\end{array}$ & $\begin{array}{r}28.1 \\
6.4 \\
71.1\end{array}$ & $\begin{array}{r}32.5 \\
7.4 \\
78.5\end{array}$ & $\begin{array}{l}\text { W/A } \\
\text { N/A } \\
\text { N/A }\end{array}$ & $\begin{array}{r}95.0 \\
21.5 \\
100.0\end{array}$ \\
\hline $\begin{array}{l}\quad 65 \\
\text { SORT- } \\
\text { SPLIT }\end{array}$ & $\begin{array}{c}5.2 \underset{\mathbf{n}}{\mathbf{n}} \\
\text { N/A MEDI AN- } \\
\text { WT- } 113.4\end{array}$ & $\begin{array}{r}21.9 \\
-\quad 2.00\end{array}$ & $\begin{array}{c}70.4 \\
\text { MODE- }\end{array}$ & 2.00 ${ }^{7.7}$ MEAN & $(g) \mathrm{ms}$ & $\stackrel{n S}{N / A}$ & CUM & $\begin{array}{l}\text { WT } \\
\text { WT } \\
\text { WT }\end{array}$ & $\begin{array}{l}\text { N/A } \\
\mathrm{A} \\
\mathrm{N} / \mathrm{A}\end{array}$ & $\begin{array}{l}8.7 \\
7.7 \\
7.7\end{array}$ & $\begin{array}{r}10.7 \\
9.5 \\
17.1\end{array}$ & $\begin{array}{l}21.8 \\
19.3 \\
36.4\end{array}$ & $\begin{array}{l}25.5 \\
22.5 \\
58.9\end{array}$ & $\begin{array}{r}10.0 \\
8.8 \\
67.8\end{array}$ & $\begin{array}{l}11.7 \\
10.3 \\
78.1\end{array}$ & $\begin{array}{l}\text { N/A } \\
\text { N/A } \\
N / A\end{array}$ & $\begin{array}{c}24.8 \\
21.9 \\
100.0\end{array}$ \\
\hline $\begin{array}{r}70 \\
\text { SORT- } \\
\text { SPLIT }\end{array}$ & $\begin{array}{c}2.4 \mathrm{H} \\
\text { N/A MEDIAN- } \\
\text { WT }=368.0\end{array}$ & $\begin{array}{r}15.1 \\
-\quad 2.00\end{array}$ & $\begin{array}{r}80.0 \\
\text { MODE- }\end{array}$ & $\begin{array}{c}4.8 \\
1.00 \text { MEAN }\end{array}$ & $(m) s$ & N/A & CUM & $\begin{array}{l}\text { WT } \\
\text { WT } \\
\text { WT }\end{array}$ & $\begin{array}{l}\text { N/A } \\
\text { N/A } \\
\text { N/A }\end{array}$ & $\begin{array}{r}17.8 \\
4.8 \\
4.8\end{array}$ & $\begin{array}{l}39.7 \\
10.8 \\
15.6\end{array}$ & $\begin{array}{r}117.4 \\
31.9 \\
47.5\end{array}$ & $\begin{array}{l}81.6 \\
22.2 \\
69.7\end{array}$ & $\begin{array}{r}32.4 \\
8.8 \\
78.5\end{array}$ & $\begin{array}{r}23.5 \\
6.4 \\
84.9\end{array}$ & $\begin{array}{l}\text { N/A } \\
\text { N/A } \\
\text { N/A }\end{array}$ & $\begin{array}{r}55.7 \\
15.1 \\
100.0\end{array}$ \\
\hline $\begin{array}{r}75 \\
\text { SORT- } \\
\text { SPLIT }\end{array}$ & $\begin{array}{l}11.1 \mathrm{H} \\
\text { N/A MED IAN- } \\
\text { WT- } 350.6\end{array}$ & $\begin{array}{c}14.8 \\
-\quad 1.00\end{array}$ & $\begin{array}{r}77.5 \\
\text { MODE- }\end{array}$ & $1.00 \stackrel{7.7}{\text { MEAN }}$ & $\mathrm{N}-{ }^{(g m)}$ & $\begin{array}{l}S \\
11 / A\end{array}$ & CUM & $\begin{array}{l}\text { WT } \\
\text { WT } \\
\text { WT }\end{array}$ & $\begin{array}{l}\text { N/A } \\
\text { N/A } \\
\text { N/A }\end{array}$ & $\begin{array}{r}27.1 \\
7.7 \\
7.7\end{array}$ & $\begin{array}{l}38.1 \\
10.9 \\
18.6\end{array}$ & $\begin{array}{r}115.2 \\
32.9 \\
51.5\end{array}$ & $\begin{array}{l}60.4 \\
17.2 \\
68.7\end{array}$ & $\begin{array}{r}31.6 \\
9.0 \\
77.7\end{array}$ & $\begin{array}{r}26.3 \\
7.5 \\
85.2\end{array}$ & $\begin{array}{l}\text { N/A } \\
\text { N/A } \\
\text { N/A }\end{array}$ & $\begin{array}{r}51.9 \\
114.8 \\
100.0\end{array}$ \\
\hline $\begin{array}{r}85 \\
\text { SORT- } \\
\text { SPLIT }\end{array}$ & $\begin{array}{l}0.5 \mathrm{H} \\
\text { N/A MEDIAN- } \\
\text { WT- } 381.1\end{array}$ & $\begin{array}{ll}4.4 \\
-\quad 1.00\end{array}$ & $\begin{array}{r}77.7 \\
\text { MODE- }\end{array}$ & $1.00^{17.9}$ MEAN & $\mathbf{g}-{ }_{-}$ & N/A & CUM & $\begin{array}{l}\text { WT } \\
\text { WT }\end{array}$ & $\begin{array}{l}\text { N/A } \\
\text { N/A } \\
\text { N/A }\end{array}$ & $\begin{array}{l}68.4 \\
17.9 \\
17.9\end{array}$ & $\begin{array}{r}33.5 \\
0.8 \\
26.7\end{array}$ & $\begin{array}{r}140.6 \\
36.9 \\
63.6\end{array}$ & $\begin{array}{l}93.7 \\
24.6 \\
88.2\end{array}$ & $\begin{array}{r}18.7 \\
4.9 \\
93.1\end{array}$ & $\begin{array}{r}9.8 \\
2.6 \\
95.6\end{array}$ & $\begin{array}{l}\text { N/A } \\
\text { N/A } \\
\text { N/A }\end{array}$ & $\begin{array}{r}16.6 \\
4.4 \\
100.0\end{array}$ \\
\hline $\begin{array}{r}87 \\
\text { SORT- } \\
\text { SPLIT }\end{array}$ & $\begin{array}{c}\text { N/A H } \\
\text { N/A MEDIAN- } \\
\text { WT- } \mathbf{3 3 4 . 1}\end{array}$ & $\begin{array}{l}0.5 \\
1.00\end{array}$ & $\begin{array}{r}89.3 \\
\text { MODE- }\end{array}$ & $1.00^{2.2}$ MEAN & s- & N/A & CUM & $\begin{array}{l}\text { WT } \\
\text { WT } \\
\text { WT }\end{array}$ & $\begin{array}{l}\text { N/A } \\
\text { N/A } \\
\text { N/A }\end{array}$ & $\begin{array}{l}7.3 \\
2.2 \\
2.2\end{array}$ & $\begin{array}{r}20.9 \\
8.7 \\
10.8\end{array}$ & $\begin{array}{r}172.4 \\
51.6 \\
62.4\end{array}$ & $\begin{array}{l}66.6 \\
19.9 \\
82.4\end{array}$ & $\begin{array}{r}10.9 \\
5.7 \\
88.0\end{array}$ & $\begin{array}{r}11.8 \\
3.5 \\
91.5\end{array}$ & $\begin{array}{l}\text { N/A } \\
N / A \\
N / A\end{array}$ & $\begin{array}{r}28.3 \\
8.5 \\
100.0\end{array}$ \\
\hline $\begin{array}{r}90 \\
\text { SORT- } \\
\text { SPLIT }\end{array}$ & $\begin{array}{l}0.5 \mathrm{H} \\
\text { N/A MEDIAN- } \\
\text { WT- } 321.9\end{array}$ & $\begin{array}{l}4.5 \\
1.00\end{array}$ & $\begin{array}{r}91.7 \\
\text { MODE- }\end{array}$ & $1.00^{3.8} \mathrm{MEAN}$ & & N/A & CUM & $\underset{\text { WT }}{\text { WT }}$ & $\begin{array}{l}\text { N/A } \\
\text { N/A } \\
\boldsymbol{H} / \mathbf{A}\end{array}$ & $\begin{array}{c}12.3 \\
3.8 \\
3.8\end{array}$ & $\begin{array}{r}22.1 \\
6.9 \\
10.7\end{array}$ & $\begin{array}{r}129.7 \\
40.3 \\
51.0\end{array}$ & $\begin{array}{r}106.2 \\
33.0 \\
84.0\end{array}$ & $\begin{array}{r}23.5 \\
7.3 \\
91.3\end{array}$ & $\begin{array}{r}13.6 \\
4.2 \\
95.5\end{array}$ & $\begin{array}{l}\text { N/A } \\
\text { A } \\
\text { N/A }\end{array}$ & $\begin{array}{r}14.5 \\
4.5 \\
100.0\end{array}$ \\
\hline $\begin{array}{r}95 \\
\text { SORT- } \\
\text { SPLIT }\end{array}$ & $\begin{array}{c}0.8 \text { H } \\
\text { N/A MEDIAN- } \\
\mathbf{W T}=350.3\end{array}$ & $\begin{array}{l}7.4 \\
2.00\end{array}$ & $\begin{array}{l}92.0 \\
\text { MODE- }\end{array}$ & $2.00^{0.6}$ MEAN & $\mathrm{S}$ & N/A & CUM & $\begin{array}{l}\text { WT } \\
\text { WT } \\
\text { WT }\end{array}$ & $\begin{array}{l}\text { N/A } \\
\text { N/A } \\
\text { N/A }\end{array}$ & $\begin{array}{l}2.1 \\
0.6 \\
0.6\end{array}$ & $\begin{array}{l}4.3 \\
1.2 \\
1.8\end{array}$ & $\begin{array}{r}128.3 \\
36.6 \\
38.5\end{array}$ & $\begin{array}{r}150.7 \\
43.0 \\
81.5\end{array}$ & $\begin{array}{r}24.0 \\
6.9 \\
88.3\end{array}$ & $\begin{array}{r}15.0 \\
4.3 \\
92.6\end{array}$ & $\begin{array}{l}\text { N/A } \\
\text { N/A } \\
\text { N/A }\end{array}$ & $\begin{array}{r}25.9 \\
7.4 \\
100.0\end{array}$ \\
\hline $\begin{array}{r}105 \\
\text { SORT } \\
\text { SPLIT }\end{array}$ & $\begin{array}{c}\mathbf{0 . 8} \mathrm{H} \\
\text { N/A MEDIAN- } \\
\text { WT- } \mathbf{3 7 1 . 8}\end{array}$ & $\begin{array}{l}11.4 \\
1.00\end{array}$ & $\begin{array}{r}49.0 \\
\text { MODE- }\end{array}$ & $-1.00^{39.6}$. NEAN & $\mathrm{N}={ }_{\mathrm{I}}^{\mathbf{m g G}}$ & N/A & CUM & $\begin{array}{l}\text { WT } \\
\text { WT } \\
\text { WT }\end{array}$ & $\begin{array}{l}\text { N/A } \\
\text { N/A } \\
\text { N/A }\end{array}$ & $\begin{array}{r}146.6 \\
39.6 \\
39.6\end{array}$ & $\begin{array}{r}20.6 \\
5.6 \\
45.2\end{array}$ & $\begin{array}{r}28.2 \\
7.6 \\
52.8\end{array}$ & $\begin{array}{l}85.1 \\
23.0 \\
75.8\end{array}$ & $\begin{array}{r}32.3 \\
8.7 \\
84.6\end{array}$ & $\begin{array}{r}15.1 \\
4.1 \\
88.6\end{array}$ & $\begin{array}{l}\text { N/A } \\
\text { N/A } \\
\text { N/A }\end{array}$ & $\begin{array}{r}42.0 \\
11.4 \\
100.0\end{array}$ \\
\hline $\begin{array}{l}110 \\
\text { SORT- } \\
\text { SPLIT }\end{array}$ & $\begin{array}{c}24.1 \mathrm{H} \\
\text { N/A MEDIAN- } \\
\text { พT- } 349.3\end{array}$ & $\begin{array}{c}38.4 \\
-\quad 4.00\end{array}$ & $\begin{array}{r}43.9 \\
\text { MODE- }\end{array}$ & $5.00^{17.6}$ MEAN & gms & N/A & CUM & $\begin{array}{l}\text { WT } \\
\text { WT } \\
\text { WT }\end{array}$ & $\begin{array}{l}\text { N/A } \\
\text { W/A } \\
\text { R/A }\end{array}$ & $\begin{array}{l}61.6 \\
17.6 \\
17.6\end{array}$ & $\begin{array}{r}26.6 \\
7.6 \\
25.2\end{array}$ & $\begin{array}{r}24.0 \\
6.9 \\
32.1\end{array}$ & $\begin{array}{r}23.3 \\
6.7 \\
38.0\end{array}$ & $\begin{array}{r}31.3 \\
9.0 \\
47.7\end{array}$ & $\begin{array}{l}48.4 \\
13.9 \\
61.6\end{array}$ & $\begin{array}{l}\text { N/A } \\
\text { N/A } \\
\text { N/A }\end{array}$ & $\begin{array}{r}134.3 \\
38.4 \\
100.0\end{array}$ \\
\hline $\begin{array}{l}115 \\
\text { SORT- } \\
\text { SPLIT }\end{array}$ & $\begin{array}{c}0.4 \mathrm{H} \\
\text { N/A MEDIAN- } \\
\text { WT- } 469.1\end{array}$ & $\begin{array}{c}17.2 \\
-\quad 0.00\end{array}$ & $\begin{array}{r}49.2 \\
\text { MODE- }\end{array}$ & $-1.00 \quad 33.6$ & $\begin{array}{l}\mathbf{m s G} \\
\mathrm{N}-\end{array}$ & N/A & & $\begin{array}{l}\text { WT } \\
\text { WT } \\
\text { WT }\end{array}$ & $\begin{array}{l}\boldsymbol{H} / \mathbf{A} \\
\text { N/A } \\
\text { N/A }\end{array}$ & $\begin{array}{r}157.6 \\
33.6 \\
33.6\end{array}$ & $\begin{array}{l}78.7 \\
16.8 \\
50.4\end{array}$ & $\begin{array}{l}81.7 \\
17.4 \\
67.8\end{array}$ & $\begin{array}{r}33.0 \\
7.0 \\
74.8\end{array}$ & $\begin{array}{r}21.4 \\
4.6 \\
79.4\end{array}$ & $\begin{array}{c}16.1 \\
3.4 \\
82.8\end{array}$ & $\begin{array}{l}\text { N/A } \\
\text { N/A } \\
\text { N/A }\end{array}$ & $\begin{array}{r}80.6 \\
17.2 \\
100.0\end{array}$ \\
\hline
\end{tabular}




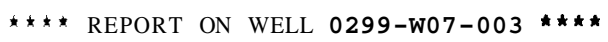

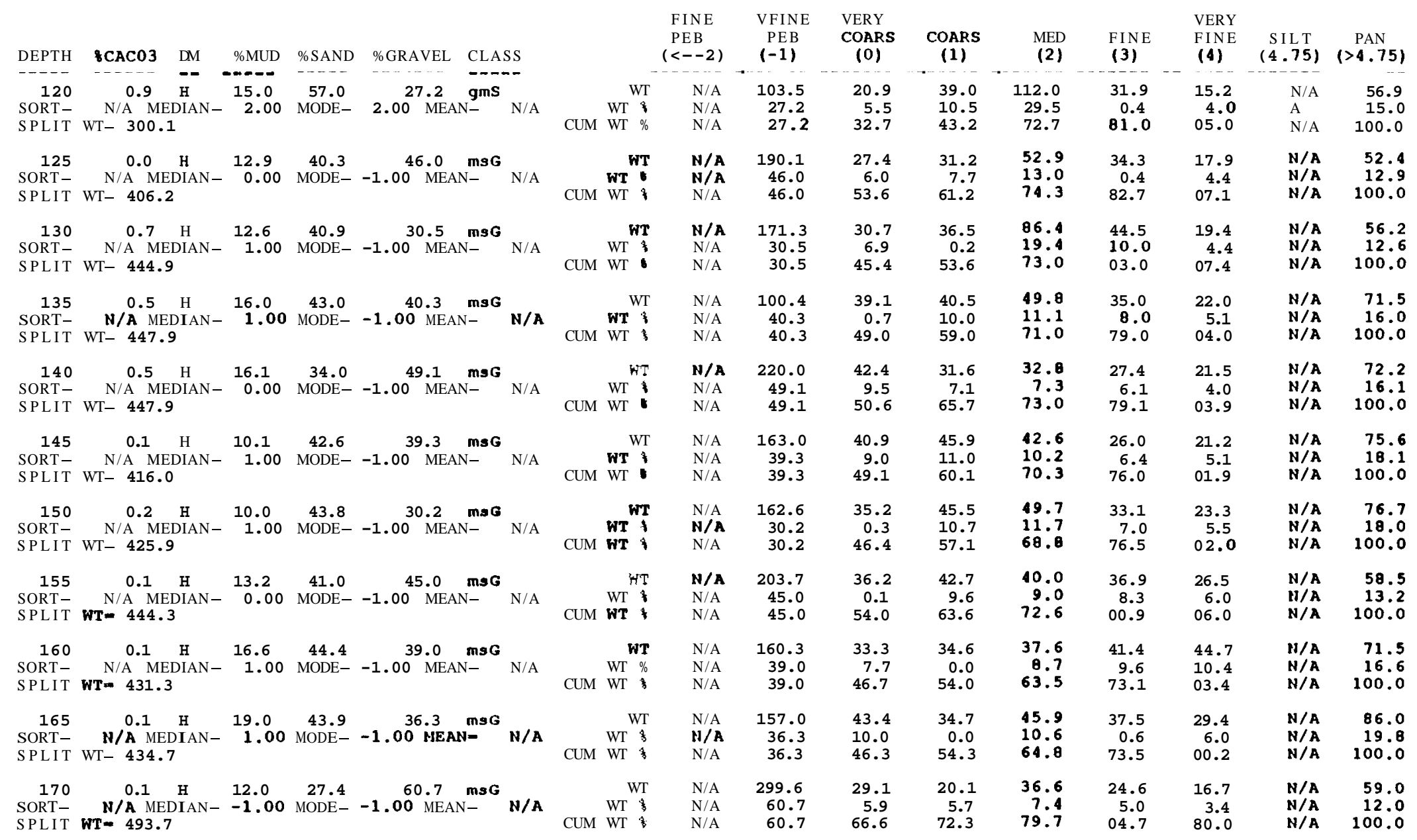




\begin{tabular}{|c|c|c|c|c|c|c|c|c|c|c|c|c|c|c|c|c|c|c|}
\hline EPTH & $8 \mathrm{CACO} 3$ & DM & 8MUD & \&SAND & GRAVEL & CLASS & & & & $\begin{array}{l}\text { FINE } \\
\text { PEB } \\
(<=-2)\end{array}$ & $\begin{array}{l}\text { VFINE } \\
\text { PEB } \\
(-1)\end{array}$ & $\begin{array}{l}\text { VERY } \\
\text { COARS } \\
(0)\end{array}$ & COARS & $\begin{array}{c}\text { MED } \\
(2)\end{array}$ & FINE & $\begin{array}{l}\text { VERY } \\
\text { FINE } \\
\text { (4) }\end{array}$ & $\begin{array}{c}\text { SILT } \\
(4.75)\end{array}$ & $\begin{array}{c}\text { PAN } \\
(>4.75)\end{array}$ \\
\hline $\begin{array}{r}175 \\
\text { SORT- } \\
\text { SPLIT }\end{array}$ & $\begin{array}{c}0.1 \\
\text { N/A MED } \\
\text { WT- } 438.4\end{array}$ & H & $\begin{array}{c}19.2 \\
1.00\end{array}$ & $\begin{array}{r}43.0 \\
\text { MODE- }\end{array}$ & $\begin{array}{c}37.8 \mathrm{~m} \\
-1.00 \text { MEAN- }\end{array}$ & ms G & N/A & CUM & $\begin{array}{l}\text { WT } \\
\text { WT } \\
\text { WT }\end{array}$ & $\begin{array}{l}\text { N/A } \\
\text { N/A } \\
\text { N/A }\end{array}$ & $\begin{array}{r}165.9 \\
37.8 \\
37.8\end{array}$ & $\begin{array}{r}38.6 \\
8.8 \\
46.6\end{array}$ & $\begin{array}{r}38.5 \\
8.8 \\
55.4\end{array}$ & $\begin{array}{l}55.2 \\
12.6 \\
68.0\end{array}$ & $\begin{array}{r}33.3 \\
7.6 \\
75.6\end{array}$ & $\begin{array}{r}22.9 \\
5.2 \\
80.8\end{array}$ & $\begin{array}{l}\text { N/A } \\
\text { N/A } \\
\text { N/A }\end{array}$ & $\begin{array}{r}84.0 \\
19.2 \\
100.0\end{array}$ \\
\hline $\begin{array}{r}180 \\
\text { SORT- } \\
\text { SPLIT }\end{array}$ & $\begin{array}{c}0.1 \\
N / A \text { MED } \\
W T=440.0\end{array}$ & $\begin{array}{l}\text { H } \\
\text { IAN- }\end{array}$ & $\begin{array}{l}11.0 \\
-1.00\end{array}$ & $\begin{array}{l}30.8 \\
\text { MODE- }\end{array}$ & $-1.00^{58.1}{ }^{\prime \prime}$ HEAN" & $\operatorname{mog}^{\mathrm{mg} G}$ & N/A & CUM & $\begin{array}{l}\text { WT } \\
\text { WT } \\
\text { WT }\end{array}$ & $\begin{array}{l}N / A \\
N / A \\
N / A\end{array}$ & $\begin{array}{r}255.8 \\
58.1 \\
58.1\end{array}$ & $\begin{array}{r}24.6 \\
5.6 \\
63.7\end{array}$ & $\begin{array}{r}23.8 \\
5.4 \\
69.1\end{array}$ & $\begin{array}{l}48.4 \\
11.0 \\
80.1\end{array}$ & $\begin{array}{r}23.1 \\
5.3 \\
85.4\end{array}$ & $\begin{array}{r}15.7 \\
3.6 \\
89.0\end{array}$ & $\begin{array}{l}M / A \\
N / A \\
N / A\end{array}$ & $\begin{array}{r}48.6 \\
11.1 \\
100.0\end{array}$ \\
\hline $\begin{array}{r}215 \\
\text { SORT- } \\
\text { SPIIT }\end{array}$ & $\begin{array}{c}0.1 \\
\text { N/A MED } \\
\text { WT- } 673.2\end{array}$ & PIAN- & $\begin{array}{r}11.1 \\
1.00\end{array}$ & $\begin{array}{r}59.0 \\
\text { NODE- }\end{array}$ & -1.00 MEAN & $(m) g s$ & N/A & CUM & $\begin{array}{l}\text { WT } \\
\text { WT } \\
\text { WT }\end{array}$ & $\begin{array}{l}N / A \\
N / A \\
N / A\end{array}$ & $\begin{array}{r}200.8 \\
29.9 \\
29.9\end{array}$ & $\begin{array}{l}91.5 \\
13.6 \\
43.6\end{array}$ & $\begin{array}{r}61.6 \\
9.2 \\
52.8\end{array}$ & $\begin{array}{l}09.5 \\
13.3 \\
66.1\end{array}$ & $\begin{array}{l}97.5 \\
14.5 \\
80.6\end{array}$ & $\begin{array}{r}55.5 \\
8.3 \\
88.9\end{array}$ & $\begin{array}{l}N / A \\
N / A \\
N / A\end{array}$ & $\begin{array}{r}74.4 \\
11.1 \\
100.0\end{array}$ \\
\hline $\begin{array}{r}220 \\
\text { SORT- } \\
\text { SPIIT }\end{array}$ & $\begin{array}{c}0.4 \\
\text { N/A MED } \\
\text { WT- } 793.2\end{array}$ & $\begin{array}{l}\text { H } \\
\text { PIAN- }\end{array}$ & $\begin{array}{c}5.1 \\
-1.00\end{array}$ & $\begin{array}{r}32.8 \\
\text { MODE- }\end{array}$ & $-1.00^{62.1}{ }^{n}{ }^{n}-$ & $\mathbf{m s G}_{-}$ & N/A & CUM & $\begin{array}{l}\text { WT } \\
\text { WT } \\
\text { WT }\end{array}$ & $\begin{array}{l}N / A \\
N / A \\
N / A\end{array}$ & $\begin{array}{r}489.6 \\
62.1 \\
62.1\end{array}$ & $\begin{array}{r}42.9 \\
5.4 \\
67.5\end{array}$ & $\begin{array}{r}33.1 \\
4.2 \\
71.7\end{array}$ & $\begin{array}{r}65.6 \\
0.3 \\
80.1\end{array}$ & $\begin{array}{l}92.8 \\
10.5 \\
90.6\end{array}$ & $\begin{array}{r}34.6 \\
4.4 \\
94.9\end{array}$ & $\begin{array}{l}N / A \\
N / A \\
N / A\end{array}$ & $\begin{array}{r}39.9 \\
5.1 \\
100.0\end{array}$ \\
\hline $\begin{array}{r}225 \\
\text { SORT- } \\
\text { SPIIT }\end{array}$ & $\begin{array}{c}0.2 \\
\text { N/A MED } \\
\text { WT- } 706.3\end{array}$ & $\begin{array}{l}\text { H } \\
\text { PAN- }\end{array}$ & $\begin{array}{c}10.3 \\
2.00\end{array}$ & $\begin{array}{r}58.7 \\
\text { MODE- }\end{array}$ & $-1.00^{31.0}$ MEAN & ms G $_{-}$ & N/A & CUM & $\begin{array}{l}\text { WT } \\
\text { WT } \\
\text { WT }\end{array}$ & $\begin{array}{l}\text { N/A } \\
\text { N/A } \\
\text { N/A }\end{array}$ & $\begin{array}{r}217.6 \\
31.0 \\
31.0\end{array}$ & $\begin{array}{r}68.9 \\
9.8 \\
40.8\end{array}$ & $\begin{array}{r}50.2 \\
7.2 \\
48.0\end{array}$ & $\begin{array}{l}99.6 \\
14.2 \\
62.1\end{array}$ & $\begin{array}{r}131.6 \\
18.7 \\
80.9\end{array}$ & $\begin{array}{r}62.2 \\
8.9 \\
89.7\end{array}$ & $\begin{array}{l}\text { N/A } \\
\text { N/A } \\
\text { N/A }\end{array}$ & $\begin{array}{r}72.1 \\
10.3 \\
100.0\end{array}$ \\
\hline $\begin{array}{r}230 \\
\text { SORT- } \\
\text { SPLIT }\end{array}$ & $\begin{array}{c}0.1 \\
\text { N/A MED } \\
\text { WT- } 802.9\end{array}$ & $\underset{\text { IIAN- }}{\mathrm{H}}$ & $\begin{array}{l}1.8 \\
0.00\end{array}$ & $\begin{array}{r}58.8 \\
\text { MODE- }\end{array}$ & $-1.00^{39.4}$ MEAN & sG & N/A & CUM & $\begin{array}{l}\text { WT } \\
\text { WT } \\
\text { WT }\end{array}$ & $\begin{array}{l}\text { N/A } \\
\text { N/A } \\
\text { N/A }\end{array}$ & $\begin{array}{c}315.9 \\
39.5 \\
39.5\end{array}$ & $\begin{array}{l}96.3 \\
12.0 \\
51.5\end{array}$ & $\begin{array}{r}63.6 \\
7.9 \\
59.4\end{array}$ & $\begin{array}{r}214.1 \\
26.7 \\
86.2\end{array}$ & $\begin{array}{l}79.7 \\
10.0 \\
96.1\end{array}$ & $\begin{array}{r}17.0 \\
2.1 \\
98.2\end{array}$ & $\begin{array}{l}\text { N/A } \\
\text { N/A } \\
\text { N/A }\end{array}$ & $\begin{array}{c}14.2 \\
1.8 \\
100.0\end{array}$ \\
\hline $\begin{array}{r}235 \\
\text { SORT- } \\
\text { SPLIT }\end{array}$ & $\begin{array}{c}0.1 \\
\text { N/A MED } \\
\text { WT- } 661.4\end{array}$ & PIAN- & $\begin{array}{l}3.8 \\
2.00\end{array}$ & $\begin{array}{r}92.9 \\
\text { MODE- }\end{array}$ & $2.00_{\text {MEAN- }}^{3.2}$ & $s$ & N/A & CUM & $\begin{array}{l}\mathrm{WT} \\
\mathrm{WT}\end{array}$ & $\begin{array}{l}\text { N/A } \\
\text { N/A } \\
\text { N/A }\end{array}$ & $\begin{array}{r}21.3 \\
3.2 \\
3.2\end{array}$ & $\begin{array}{r}34.1 \\
5.2 \\
8.4\end{array}$ & $\begin{array}{l}95.8 \\
13.0 \\
21.4\end{array}$ & $\begin{array}{r}228.2 \\
34.5 \\
55.9\end{array}$ & $\begin{array}{r}208.6 \\
31.6 \\
87.5\end{array}$ & $\begin{array}{r}57.3 \\
8.7 \\
96.2\end{array}$ & $\begin{array}{l}\text { N/A } \\
\text { N/A } \\
\text { N/A }\end{array}$ & $\begin{array}{r}25.4 \\
3.8 \\
100.0\end{array}$ \\
\hline $\begin{array}{r}240 \\
\text { SORT- } \\
\text { SPLIT }\end{array}$ & $\begin{array}{c}0.1 \\
\text { N/A MED } \\
\text { WT- } 560.7\end{array}$ & $\underset{\text { DIAN- }}{\text { H }}$ & $\begin{array}{l}5.3 \\
1.00\end{array}$ & $\begin{array}{r}66.7 \\
\text { MODE- }\end{array}$ & $-1.00^{28.0}$ MEAN & $g s$ & N/A & CUM & $\begin{array}{l}\text { WT } \\
\text { WT }\end{array}$ & $\begin{array}{l}\text { N/A } \\
\text { N/A } \\
\text { N/A }\end{array}$ & $\begin{array}{r}155.9 \\
28.0 \\
28.0\end{array}$ & $\begin{array}{l}75.9 \\
13.6 \\
41.6\end{array}$ & $\begin{array}{r}50.5 \\
9.1 \\
50.7\end{array}$ & $\begin{array}{r}142.0 \\
25.5 \\
76.1\end{array}$ & $\begin{array}{l}73.5 \\
13.2 \\
89.3\end{array}$ & $\begin{array}{r}29.8 \\
5.4 \\
94.7\end{array}$ & $\begin{array}{l}N / A \\
N / A \\
N / A\end{array}$ & $\begin{array}{r}29.7 \\
5.3 \\
100.0\end{array}$ \\
\hline $\begin{array}{r}245 \\
\text { SORT- } \\
\text { SPLIT }\end{array}$ & $\begin{array}{c}0.1 \\
\text { N/A MED } \\
\text { WT- } 533.3\end{array}$ & DIAN- & $\begin{array}{l}4.9 \\
2.00\end{array}$ & $\begin{array}{r}61.9 \\
\text { MODE- }\end{array}$ & $-1.00^{33.1}$ MEAN & SG & N/A & CUM & $\begin{array}{l}\text { WT } \\
\text { WT } \\
\text { WT }\end{array}$ & $\begin{array}{l}\text { N/A } \\
\text { N/A } \\
\text { N/A }\end{array}$ & $\begin{array}{r}174.3 \\
33.1 \\
33.1\end{array}$ & $\begin{array}{r}31.4 \\
6.0 \\
39.1\end{array}$ & $\begin{array}{r}24.7 \\
4.7 \\
43.8\end{array}$ & $\begin{array}{r}134.7 \\
25.6 \\
69.4\end{array}$ & $\begin{array}{r}106.4 \\
20.2 \\
89.6\end{array}$ & $\begin{array}{r}28.7 \\
5.5 \\
95.1\end{array}$ & $\begin{array}{l}N / A \\
N / A \\
N / A\end{array}$ & $\begin{array}{r}26.0 \\
4.9 \\
100.0\end{array}$ \\
\hline $\begin{array}{r}250 \\
\text { SORT- } \\
\text { SPLIT }\end{array}$ & $\begin{array}{c}0.1 \\
N / A \text { MED } \\
\text { WT- } 600.5\end{array}$ & PIAN- & $\begin{array}{l}4.1 \\
2.00\end{array}$ & $\begin{array}{r}79.0 \\
\text { MODE- }\end{array}$ & $2.00^{16.9}$ MEAN & gs & N/A & CUM & $\begin{array}{l}\text { WT } \\
\text { WT } \\
\text { WT }\end{array}$ & $\begin{array}{l}\text { N/A } \\
\text { N/A } \\
\text { N/A }\end{array}$ & $\begin{array}{r}101.0 \\
16.9 \\
16.9\end{array}$ & $\begin{array}{r}44.3 \\
.7 .4 \\
24.3\end{array}$ & $\begin{array}{r}31.9 \\
5.3 \\
29.7\end{array}$ & $\begin{array}{r}239.1 \\
40.0 \\
69.7\end{array}$ & $\begin{array}{c}126.3 \\
21.1 \\
90.8\end{array}$ & $\begin{array}{r}30.6 \\
5.1 \\
95.9\end{array}$ & $\begin{array}{l}\text { N/A } \\
\text { N/A } \\
\text { N/A }\end{array}$ & $\begin{array}{r}24.4 \\
4.1 \\
100.0\end{array}$ \\
\hline $\begin{array}{r}255 \\
\text { SORT- } \\
\text { SPLIT }\end{array}$ & $\begin{array}{c}0.1 \\
\text { N/A MED } \\
\text { WT }-443.3\end{array}$ & $\begin{array}{l}\mathrm{H} \\
\text { IAN- }\end{array}$ & $\begin{array}{l}6.2 \\
2.00\end{array}$ & $\begin{array}{r}85.3 \\
\text { MODE- }\end{array}$ & $2.00^{8.5}$ MEAN- & (g) $s$ & $N / A$ & CUM & $\begin{array}{l}\text { WT } \\
\text { WT } \\
\text { WT }\end{array}$ & $\begin{array}{l}\text { N/A } \\
\text { N/A } \\
\text { N/A }\end{array}$ & $\begin{array}{c}37.4 \\
8.5 \\
8.5\end{array}$ & $\begin{array}{r}33.9 \\
7.7 \\
16.3\end{array}$ & $\begin{array}{r}32.7 \\
7.5 \\
23.7\end{array}$ & $\begin{array}{r}164.2 \\
37.5 \\
61.2\end{array}$ & $\begin{array}{r}104.2 \\
23.8 \\
84.9\end{array}$ & $\begin{array}{r}38.8 \\
6.9 \\
93.8\end{array}$ & $\begin{array}{l}N / A \\
N / A \\
N / A\end{array}$ & $\begin{array}{r}27.2 \\
6.2 \\
100.0\end{array}$ \\
\hline
\end{tabular}



ROCSAN REPORT

$12 / 11 / 89$

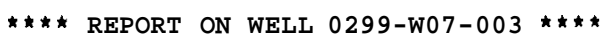

\begin{tabular}{|c|c|c|c|c|c|c|c|c|c|c|c|c|c|c|c|c|c|c|}
\hline DEPTH & $8 \mathrm{CACO} 3$ & DM & ZMUD & $\because$ SAND & ○GRAVEL & CLAS & & & & $\begin{array}{l}\text { FINE } \\
\text { PEB } \\
(<=-2)\end{array}$ & $\begin{array}{l}\text { W I N E } \\
\text { PEB } \\
(-1)\end{array}$ & $\begin{array}{l}\text { VERY } \\
\text { COARS } \\
(0)\end{array}$ & $\begin{array}{l}\text { COARS } \\
\text { (1) }\end{array}$ & $\begin{array}{l}\text { MED } \\
(2)\end{array}$ & $\begin{array}{l}\text { FINE } \\
\text { (3) }\end{array}$ & $\begin{array}{l}\text { VERY } \\
\text { FINE } \\
\text { (4) }\end{array}$ & $\begin{array}{r}\text { SILT } \\
(4.75)\end{array}$ & $\begin{array}{c}\text { PAN } \\
(>4.75)\end{array}$ \\
\hline $\begin{array}{r}260 \\
\text { SORT- } \\
\text { SPLIT }\end{array}$ & $\begin{array}{c}0.1 \\
\text { N/A MED } \\
\text { WT- } 623.1\end{array}$ & $\begin{array}{l}\mathrm{H} \\
1 \\
1\end{array}$ & $\begin{aligned} & 4.1 \\
- & 2.00\end{aligned}$ & $\begin{array}{r}95.7 \\
\text { MODE- }\end{array}$ & $2.00 \quad$ MEAN & d & $\mathbf{N} / \mathbf{A}$ & CUM & $\begin{array}{l}\text { WT } \\
\text { WT } \\
\text { WT }\end{array}$ & $\begin{array}{l}\text { N/A } \\
N / A \\
\text { N/A }\end{array}$ & $\begin{array}{l}1.1 \\
0.2 \\
0.2\end{array}$ & $\begin{array}{l}4.4 \\
0.7 \\
0.9\end{array}$ & $\begin{array}{r}26.8 \\
4.3 \\
5.2\end{array}$ & $\begin{array}{r}334.6 \\
53.8 \\
59.0\end{array}$ & $\begin{array}{r}189.8 \\
30.5 \\
89.6\end{array}$ & $\begin{array}{r}39.4 \\
6.3 \\
95.9\end{array}$ & $\begin{array}{l}\mathrm{H} / \mathrm{A} \\
\mathrm{N} / \mathrm{A} \\
\mathrm{N} / \mathrm{A}\end{array}$ & $\begin{array}{r}25.6 \\
4.1 \\
100.0\end{array}$ \\
\hline $\begin{array}{r}265 \\
\text { SORT- } \\
\text { SPLIT }\end{array}$ & $\begin{array}{c}0.6 \\
\text { N/A MED } \\
\text { WT- } 770.1\end{array}$ & $\begin{array}{l}\stackrel{H}{\mathrm{H}} \\
1\end{array}$ & $\begin{array}{c}1.7 \\
-1.00\end{array}$ & $\begin{array}{r}25.8 \\
\text { MODE- }\end{array}$ & $\begin{array}{c}72.5 \\
-1.00 \text { MEAN }\end{array}$ & s- & $\mathrm{N} / \mathrm{A}$ & CUM & $\begin{array}{l}\text { WT } \\
\text { WT } \\
\text { WT }\end{array}$ & $\begin{array}{l}\text { N/A } \\
\text { N/A } \\
\text { N/A }\end{array}$ & $\begin{array}{r}556.8 \\
72.5 \\
72.5\end{array}$ & $\begin{array}{r}64.2 \\
8.4 \\
80.8\end{array}$ & $\begin{array}{r}30.7 \\
4.0 \\
84.8\end{array}$ & $\begin{array}{r}55.7 \\
7.3 \\
92.1\end{array}$ & $\begin{array}{r}33.6 \\
4.4 \\
96.4\end{array}$ & $\begin{array}{r}14.0 \\
1.8 \\
98.3\end{array}$ & $\begin{array}{l}\text { N/A } \\
\text { N/A } \\
\text { N/A }\end{array}$ & $\begin{array}{r}13.4 \\
1.7 \\
100.0\end{array}$ \\
\hline $\begin{array}{r}270 \\
\text { SORT- } \\
\text { SPLIT }\end{array}$ & $\begin{array}{c}0.4 \\
\text { N/A MED } \\
\text { WT- } 840.2\end{array}$ & $2^{\mathrm{H}}$ & $\begin{array}{c}2.5 \\
-1.00\end{array}$ & $\begin{array}{l}38.2 \\
\text { MODE- }\end{array}$ & $-1.00^{59.3}$ MEAN & $\mathbf{s G}$ & N/A & CUM & $\begin{array}{r}\text { WT } \\
\text { W T } \\
\text { WT }\end{array}$ & $\begin{array}{l}\text { N/A } \\
\text { N/A } \\
\text { N/A }\end{array}$ & $\begin{array}{r}496.5 \\
59.3 \\
59.3\end{array}$ & $\begin{array}{r}119.8 \\
14.3 \\
73.6\end{array}$ & $\begin{array}{r}59.2 \\
7.1 \\
80.6\end{array}$ & $\begin{array}{r}78.0 \\
9.3 \\
89.9\end{array}$ & $\begin{array}{r}43.8 \\
5.2 \\
95.2\end{array}$ & $\begin{array}{r}19.6 \\
2.3 \\
97.5\end{array}$ & $\begin{array}{l}N / A \\
H / A \\
N / A\end{array}$ & $\begin{array}{r}21.0 \\
2.5 \\
100.0\end{array}$ \\
\hline $\begin{array}{r}275 \\
\text { SORT- } \\
\text { SPLIT }\end{array}$ & $\begin{array}{c}1.0 \\
\text { N/A MED } \\
\text { WT- } 774.2\end{array}$ & $2^{\stackrel{H}{\mathrm{IAN}}-}$ & $\begin{array}{c}2.3 \\
-1.00\end{array}$ & $\begin{array}{l}29.8 \\
\text { MODE- }\end{array}$ & -1.00 MEAN & $s G$ & $\mathrm{~N} / \mathrm{A}$ & CUM & $\begin{array}{l}\text { WT } \\
\text { WT } \\
\text { WT }\end{array}$ & $\begin{array}{l}\text { N/A } \\
\text { N/A } \\
\text { N/A }\end{array}$ & $\begin{array}{r}524.9 \\
67.9 \\
67.9\end{array}$ & $\begin{array}{r}75.6 \\
9.0 \\
77.7\end{array}$ & $\begin{array}{r}34.3 \\
4.4 \\
82.1\end{array}$ & $\begin{array}{r}67.9 \\
8.8 \\
90.9\end{array}$ & $\begin{array}{r}36.7 \\
4.8 \\
95.7\end{array}$ & $\begin{array}{r}15.9 \\
2.1 \\
97.7\end{array}$ & $\begin{array}{l}\text { N/A } \\
\text { N/A } \\
\text { N/A }\end{array}$ & $\begin{array}{r}17.5 \\
2.3 \\
100.0\end{array}$ \\
\hline $\begin{array}{r}280 \\
\text { SORT- } \\
\text { SPLIT }\end{array}$ & $\begin{array}{c}1.1 \\
\text { N/A MED } \\
\text { WT }=578.6\end{array}$ & ${ }^{\text {DIAN- }}$ & $\begin{array}{c}11.5 \\
-\quad 2.00\end{array}$ & $\begin{array}{r}74.2 \\
\text { MODE }\end{array}$ & $2.00^{14.3} \mathrm{MEAN}$ & $g^{(m) g}$ & $\underset{N}{g S} / \mathbf{A}$ & CUM & $\begin{array}{l}\text { WT WT } \\
\text { WT }\end{array}$ & $\begin{array}{l}\text { N/A } \\
\text { N/A } \\
\text { N/A }\end{array}$ & $\begin{array}{l}82.2 \\
14.3 \\
14.3\end{array}$ & $\begin{array}{r}31.4 \\
5.5 \\
19.8\end{array}$ & $\begin{array}{r}49.2 \\
8.6 \\
28.4\end{array}$ & $\begin{array}{r}158.4 \\
27.6 \\
56.0\end{array}$ & $\begin{array}{r}114.6 \\
20.0 \\
76.0\end{array}$ & $\begin{array}{l}71.9 \\
12.5 \\
88.5\end{array}$ & $\begin{array}{l}\text { N/A } \\
\text { N/A } \\
\text { N/A }\end{array}$ & $\begin{array}{r}66.0 \\
11.5 \\
100.0\end{array}$ \\
\hline $\begin{array}{r}285 \\
\text { SORT- } \\
\text { SPIIT }\end{array}$ & $\begin{array}{c}0.6 \\
\text { N/A MED } \\
\text { WT- } 837.9\end{array}$ & $\begin{array}{l}\stackrel{H}{\mathrm{H}} \\
9\end{array}$ & $\begin{array}{c}3.1 \\
-1.00\end{array}$ & $\begin{array}{r}30.5 \\
\text { MODE- }\end{array}$ & -1.00 MEAN & $s G$ & N/A & CUM & $\begin{array}{l}\text { WT } \\
\text { WT } \\
\text { WT }\end{array}$ & $\begin{array}{l}\text { N/A } \\
\text { N/A } \\
\text { N/A }\end{array}$ & $\begin{array}{r}553.3 \\
66.4 \\
66.4\end{array}$ & $\begin{array}{l}92.0 \\
11.0 \\
77.4\end{array}$ & $\begin{array}{r}41.9 \\
5.0 \\
82.4\end{array}$ & $\begin{array}{r}59.9 \\
7.2 \\
89.6\end{array}$ & $\begin{array}{r}38.6 \\
4.6 \\
94.2\end{array}$ & $\begin{array}{r}22.0 \\
2.6 \\
96.9\end{array}$ & $\begin{array}{l}\mathrm{N} / \mathrm{A} \\
\mathrm{M} / \mathrm{A} \\
\mathrm{N} / \mathrm{A}\end{array}$ & $\begin{array}{r}26.1 \\
3.1 \\
100.0\end{array}$ \\
\hline $\begin{array}{r}290 \\
\text { SORT- } \\
\text { SPLIT }\end{array}$ & $\begin{array}{c}0.5 \\
\text { N/A MED } \\
\text { WT- } 508.0\end{array}$ & ${ }_{0}^{\mathrm{H}}$ & $\begin{aligned} & 7.8 \\
-\quad & 1.00\end{aligned}$ & $\begin{array}{r}55.3 \\
\text { MODE- }\end{array}$ & $\begin{array}{c}36.9 \\
-1.00 \mathrm{MEAN}\end{array}$ & $\operatorname{mgG}$ & N/A & CUM & WT & $\begin{array}{l}\text { N/A } \\
\text { N/A } \\
\text { N/A }\end{array}$ & $\begin{array}{r}186.3 \\
36.9 \\
36.9\end{array}$ & $\begin{array}{r}45.2 \\
9.0 \\
45.9\end{array}$ & $\begin{array}{r}35.2 \\
7.0 \\
52.9\end{array}$ & $\begin{array}{l}94.8 \\
18.8 \\
71.7\end{array}$ & $\begin{array}{l}66.0 \\
13.1 \\
84.7\end{array}$ & $\begin{array}{r}37.8 \\
7.5 \\
92.2\end{array}$ & $\begin{array}{l}N / A \\
N / A \\
N / A\end{array}$ & $\begin{array}{r}39.2 \\
7.8 \\
100.0\end{array}$ \\
\hline $\begin{array}{r}295 \\
\text { SORT- } \\
\text { SPIIT }\end{array}$ & $\begin{array}{c}0.4 \\
N / A \text { MED } \\
\text { WT- } 808.2\end{array}$ & $2^{\text {HIAN- }}$ & $\begin{array}{ll} & 5.0 \\
- & 1.00\end{array}$ & $\begin{array}{l}67.1 \\
\text { MODE- }\end{array}$ & -1.00 MEAN & $9 s$ & N/A & CUM & $\begin{array}{l}\text { WT } \\
\text { WT } \\
\text { WT }\end{array}$ & $\begin{array}{l}\text { N/A } \\
\text { N/A } \\
\text { N/A }\end{array}$ & $\begin{array}{r}223.8 \\
27.9 \\
27.9\end{array}$ & $\begin{array}{r}118.3 \\
14.8 \\
12.7\end{array}$ & $\begin{array}{l}99.2 \\
11.1 \\
53.0\end{array}$ & $\begin{array}{r}201.8 \\
25.2 \\
79.0\end{array}$ & $\begin{array}{l}85.8 \\
10.7 \\
89.7\end{array}$ & $\begin{array}{r}42.4 \\
5.3 \\
95.0\end{array}$ & $\begin{array}{l}N / A \\
N / A \\
N / A\end{array}$ & $\begin{array}{r}40.1 \\
5.0 \\
100.0\end{array}$ \\
\hline $\begin{array}{r}300 \\
\text { SORT- } \\
\text { SPIIT }\end{array}$ & $\begin{array}{c}0.4 \\
\mathrm{~N} / \mathrm{A} \text { MED } \\
\text { WT- } 634.4\end{array}$ & ${ }_{4}^{\stackrel{H}{\text { DIAN- }}}$ & $\begin{array}{c}6.2 \\
-1.00\end{array}$ & $\begin{array}{l}38.8 \\
\text { MODE- }\end{array}$ & $\begin{array}{l}55.0 \\
-1.00 \mathrm{MEAN}\end{array}$ & $\mathbf{m s G}_{\mathrm{J}}^{\mathbf{m s G}}$ & $\mathrm{N} / \mathrm{A}$ & CUM & $\begin{array}{l}\text { WT } \\
\text { WT } \\
\text { WT }\end{array}$ & $\begin{array}{l}\text { N/A } \\
\text { N/A } \\
\text { N/A }\end{array}$ & $\begin{array}{r}345.8 \\
55.0 \\
55.0\end{array}$ & $\begin{array}{r}59.3 \\
9.4 \\
64.5\end{array}$ & $\begin{array}{r}10.1 \\
6.1 \\
70.9\end{array}$ & $\begin{array}{l}70.0 \\
11.1 \\
82.0\end{array}$ & $\begin{array}{r}11.8 \\
7.1 \\
09.1\end{array}$ & $\begin{array}{r}29.7 \\
4.7 \\
93.9\end{array}$ & $\begin{array}{l}\text { N/A } \\
\text { N/A } \\
\text { N/A }\end{array}$ & $\begin{array}{r}30.7 \\
6.2 \\
100.0\end{array}$ \\
\hline $\begin{array}{r}305 \\
\text { SORT- } \\
\text { SPIIT }\end{array}$ & $\begin{array}{c}0.3 \\
N / A \text { MED } \\
\text { WT= } 550.4\end{array}$ & ${ }_{4}^{\mathrm{H}}$ & $\begin{array}{ll} & 6.6 \\
- & 1.00\end{array}$ & $\begin{array}{c}71.4 \\
M O D E=\end{array}$ & $0.00^{22.0}$ MEAN & $g s$ & N/A & CUM & $\begin{array}{l}\text { WT } \\
\text { WT } \\
\text { WT }\end{array}$ & $\begin{array}{l}\text { N/A } \\
\text { N/A } \\
\text { N/A }\end{array}$ & $\begin{array}{r}120.8 \\
22.0 \\
22.0\end{array}$ & $\begin{array}{r}121.7 \\
22.1 \\
11.1\end{array}$ & $\begin{array}{l}69.2 \\
12.6 \\
56.7\end{array}$ & $\begin{array}{r}117.1 \\
21.3 \\
78.0\end{array}$ & $\begin{array}{l}56.7 \\
10.3 \\
88.3\end{array}$ & $\begin{array}{r}28.0 \\
5.1 \\
93.4\end{array}$ & $\begin{array}{l}\text { N/A } \\
\text { N/A } \\
\text { N/A }\end{array}$ & $\begin{array}{r}36.4 \\
6.6 \\
100.0\end{array}$ \\
\hline $\begin{array}{r}310 \\
\text { SORT- } \\
\text { SPIIT }\end{array}$ & $\begin{array}{c}0.2 \\
\text { N/A MED } \\
\text { WT }=750.2\end{array}$ & ${ }_{2}^{\text {DIAN- }}$ & $\begin{array}{r}1.8 \\
-1.00\end{array}$ & $\begin{array}{r}31.2 \\
\text { MODE- }\end{array}$ & $\begin{array}{c}66.9 \\
-1.00 \mathrm{MEAN}-\end{array}$ & sG & N/A & CUM & $\begin{array}{ll}\text { WT } \\
\text { WT } \\
\text { WT }\end{array}$ & $\begin{array}{l}\text { N/A } \\
\text { N/A } \\
\text { N/A }\end{array}$ & $\begin{array}{r}497.1 \\
66.9 \\
66.9\end{array}$ & $\begin{array}{l}74.1 \\
10.0 \\
76.9\end{array}$ & $\begin{array}{r}41.1 \\
5.5 \\
82.5\end{array}$ & $\begin{array}{l}77.0 \\
10.4 \\
92.8\end{array}$ & $\begin{array}{r}26.7 \\
3.6 \\
96.4\end{array}$ & $\begin{array}{r}12.9 \\
1.7 \\
98.2\end{array}$ & $\begin{array}{l}\text { N/A } \\
\text { N/A } \\
\text { N/A }\end{array}$ & $\begin{array}{r}13.7 \\
1.0 \\
100.0\end{array}$ \\
\hline
\end{tabular}


WESTINGHOUSE HANFORD OPERATIONS SIEVE ANALYSIS

ROCSAN REPORT

$12 / 11 / 89$

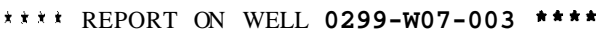

\begin{tabular}{lcccccc} 
DEPTH & ICACO3 & DM & \%MUD & \$SAND & \$GRAVEL & CLASS \\
\hdashline 315 & 0.2 & H & -14.5 & 80.7 & 4.8 & $(\mathrm{~m}) \mathrm{s}$ \\
SORT- & N/A MEDIAN- & 3.00 & MODE- & 3.00 & MEAN - & N/A \\
SPLIT & WT $=365.9$ & & &
\end{tabular}

$\begin{array}{lll}\text { FINE } & \text { VFINE } & \text { VERY } \\ \text { PEE } & \text { PEB } & \text { COARS }\end{array}$

COARS MED FINE FINE

FINE SILT PAN

$\begin{array}{rrrrrrrrrr}\text { WT } & \text { N/A } & 17.3 & 8.2 & 7.4 & 108.0 & 112.8 & 55.2 & \text { A } & 52.4 \\ \text { WT } & \text { N/A } & 4.8 & 2.3 & 2.1 & 29.9 & 31.2 & 15.3 & \text { N/A } & 14.5 \\ \text { CUH WT } & \text { N/A } & 4.8 & 7.1 & 9.1 & 39.0 & 70.2 & 85.5 & \text { N/A } & 100.0\end{array}$

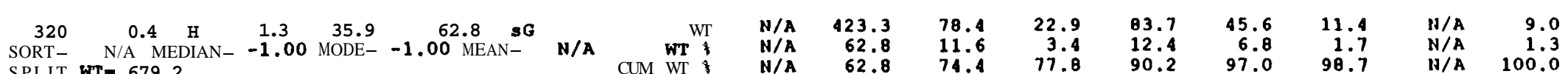

SPLIT WT- 679.2 CUM WT

N/A $\quad 62.8 \quad 74.4$

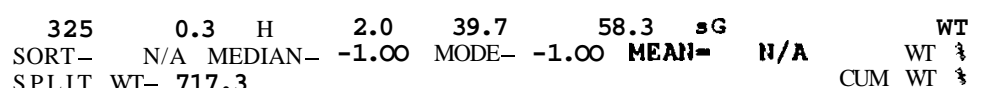

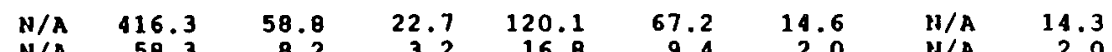

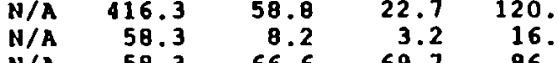

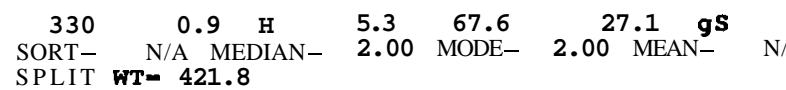

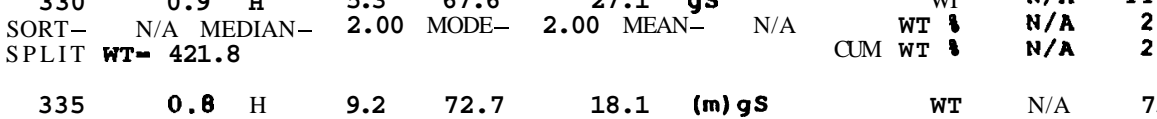

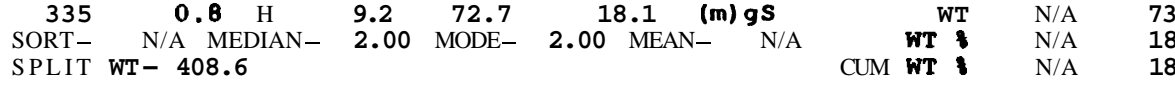

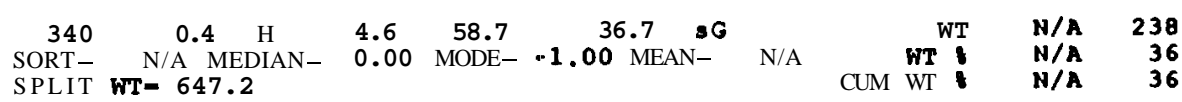

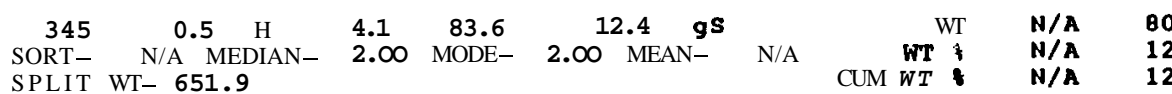

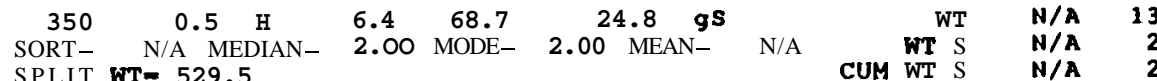

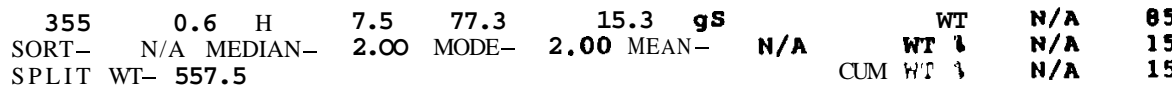

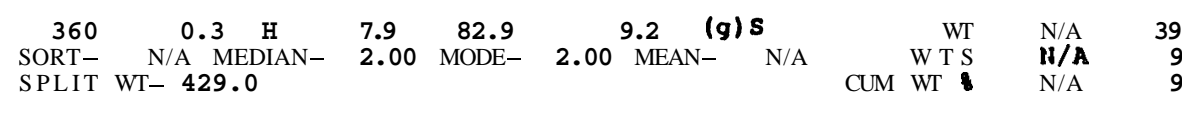

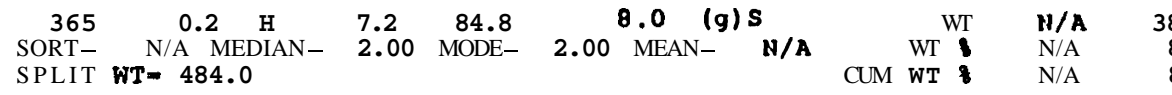
$\begin{array}{lllllll}49.2 & 32.0 & 119.2 & 62.8 & 21.5 & \mathrm{~N} / \mathrm{A} & 22.4\end{array}$ $\begin{array}{rrrrrrr}11.7 & 7.6 & 28.3 & 14.9 & 5.1 & \text { N/A } & 5.3 \\ 3 B .8 & 46.4 & 74.7 & 89.6 & 94.7 & \text { N/A } & 100.0\end{array}$

$\begin{array}{lllllll}9.4 & 65.6 & 97.3 & 60.2 & 32.3 & \mathrm{~N} / \mathrm{A} & 37.4\end{array}$

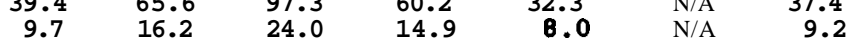
$27.8 \quad 44.0 \quad 68.0$

$94.960 .4 \quad 126.1$

82.8 90.8

N/A $\quad 100.0$

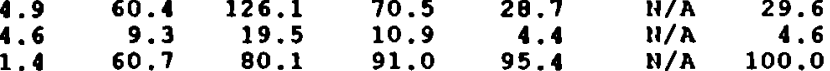

$\begin{array}{lllllll}1.7 & 62.5 & 248.0 & 144.1 & 35.2 & \text { II/A } & 26.3\end{array}$

$\begin{array}{rrrrrrr}51.7 & 62.5 & 248.0 & 144.1 & 35.2 & \text { I/A } & 26.3 \\ 8.0 & 9.6 & 38.3 & 22.2 & 5.4 & \text { N/A } & 4.1\end{array}$

$85.6 \quad 34.7 \quad$ N/A 34.3

$\begin{array}{rrrr}6.1 & 6.5 & \text { N/A } & 6.5 \\ 7.0 & 93.6 & \text { N/A } & 100.0\end{array}$

$\begin{array}{lll}12.3 & 70.9\end{array}$

$\begin{array}{rrr}74.7 & 52.1 & 163 \\ 13.4 & 9.3 & 29.2\end{array}$

\section{$41.2 \quad \mathrm{~N} / \mathrm{A} \quad 41.8$}

$\begin{array}{rrrr}8.0 & 7.4 & \text { N/A } & 7.5 \\ 5.2 & 92.5 & \text { N/A } & 100.0\end{array}$

$\begin{array}{lllllll}32.5 & 42.5 & 152.6 & 92.0 & 35.7 & \text { N/A } & 34.0\end{array}$

$\begin{array}{lllllll}32.5 & 42.5 & 152.6 & 92.0 & 35.7 & \text { N/A } & 34.0\end{array}$ $\begin{array}{lllllll}16.8 & 26.7 & 62.3 & 83.7 & 92.1 & \text { N/A } & 100.0\end{array}$

$\begin{array}{rrrrrrr}34.0 & 52.5 & 205.2 & 80.4 & 35.7 & \text { N/A } & 34.6 \\ 7.1 & 10.9 & 42.7 & 16.7 & 7.4 & \text { N/A } & 7.2\end{array}$




\begin{tabular}{|c|c|c|c|c|c|c|c|c|c|c|c|c|c|c|}
\hline $\begin{array}{l}0^{\circ} \text {.00t } \\
E^{\prime} S \\
0^{\circ} \text { SE }\end{array}$ & $\begin{array}{l}\mathrm{Q} / \mathrm{M} \\
\forall / \mathbb{H} \\
\mathrm{Q} / \mathrm{N}\end{array}$ & $\begin{array}{l}L \cdot b 6 \\
E \cdot S \\
\tau \cdot g E\end{array}$ & $\begin{array}{l}5.68 \\
: .01 \\
L .89\end{array}$ & $\begin{array}{l}0^{\prime} 6 L \\
E^{\prime} O Z \\
0^{\circ} \cup \in \mathbf{I}\end{array}$ & $\begin{array}{l}\qquad \cdot 89 \\
9 \cdot 91 \\
0.601\end{array}$ & $\begin{array}{l}t \cdot z b \\
t \cdot 0 z \\
s \cdot z \varepsilon t\end{array}$ & $\begin{array}{l}O^{\prime} Z Z \\
O^{\prime} Z Z \\
\mathbf{t} \cdot \mathbf{s} \mathbf{I}\end{array}$ & $\begin{array}{l}\mathrm{Q} / \mathrm{N} \\
\mathrm{Q} / \mathrm{N} \\
\mathbf{V} / \mathrm{M}\end{array}$ & $\begin{array}{l}\text { I.M } 1.1113 \\
\text { I.M }\end{array}$ & $Q / N \quad \mathbf{s \sigma}^{\text {ENYGW }} 0^{\prime} Z Z^{\circ} \tau-$ & $\begin{array}{l}=\text { ?aOW } \\
L^{\prime} Z L\end{array}$ & $\begin{array}{r}00^{\circ} \\
\mathbf{E}^{\prime} \cdot \mathbf{g}\end{array}$ & 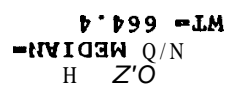 & $\begin{array}{l}\text { LITdS } \\
\text { Lyos } \\
040\end{array}$ \\
\hline $\begin{array}{l}0 \cdot 00 \mathrm{~T} \\
S^{\prime} L \\
9^{\prime} \cdot \mathrm{BE}\end{array}$ & $\begin{array}{l}Q / N \\
\forall / N \\
Q / N\end{array}$ & $\begin{array}{l}9: 26 \\
9 \cdot 8 \\
5: 60\end{array}$ & $\begin{array}{l}6^{\circ} \cdot \varepsilon \boldsymbol{\varepsilon} \\
\mathcal{E} \cdot \tau z \\
0^{\prime} 601\end{array}$ & $\begin{array}{l}L \cdot Z g \\
g \cdot E E \\
E \cdot E L I\end{array}$ & $\begin{array}{l}\tau \cdot 62 \\
2 \cdot 0 \\
2 \cdot 09\end{array}$ & $\begin{array}{l}\cdot: 1 \mathrm{I} \\
0.6 \\
0 \cdot 96\end{array}$ & $\begin{array}{l}S^{\circ} \cdot 8 \\
5^{\circ} \cdot 8 \\
L \cdot E\end{array}$ & $\begin{array}{l}\mathrm{Q} / \mathrm{N} \\
\mathrm{Q} / \mathrm{N} \\
\forall / \mathrm{H}\end{array}$ & $\begin{array}{l}t \text { LM wกว } \\
\text { LMM }\end{array}$ & 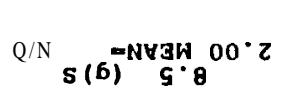 & $\begin{array}{l}-300 \mathrm{~W} \\
\mathrm{I} \cdot 6 \mathrm{~B}\end{array}$ & $\begin{aligned} 00^{\circ} 2 \\
\mathrm{~s} \cdot \mathrm{L}\end{aligned}$ & 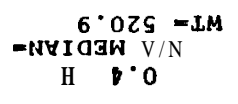 & $\begin{array}{l}\text { LI'IdS } \\
\text {-JuOS } \\
\text { SE P }\end{array}$ \\
\hline $\begin{array}{l}0^{\prime} 001 \\
T^{\prime} L \\
\boldsymbol{\theta}^{\prime} \cdot \mathbf{E E}\end{array}$ & $\begin{array}{l}\mathrm{Q} / \mathrm{N} \\
\mathrm{Q} / \mathrm{N} \\
\mathrm{Q} / \mathrm{N}\end{array}$ & $\begin{array}{l}6: 26 \\
z: 8 \\
0 \cdot 6 \varepsilon\end{array}$ & $\begin{array}{l}L \cdot 68 \\
6 \cdot 9 t \\
L ' S L\end{array}$ & $\begin{array}{l}6: 89 \\
8 \cdot 82 \\
E \cdot L E I\end{array}$ & $\begin{array}{l}\tau \cdot O B \\
6 \cdot 91 \\
9 \cdot O B\end{array}$ & $\begin{array}{l}z \cdot \varepsilon z \\
0 \cdot 1 \mathrm{I} \\
\mathrm{s} \cdot z \boldsymbol{g}\end{array}$ & $\begin{array}{l}2 \cdot 21 \\
2 \cdot 2 I \\
0 \cdot \theta 5\end{array}$ & $\begin{array}{l}\mathbf{Y} / \mathbf{N} \\
\mathbf{W} / \mathbf{W} \\
\mathbf{V} / \mathbf{N}\end{array}$ & $\begin{array}{l}\text { LM NOS } \\
\text { IM IM } \\
\text { LM }\end{array}$ & 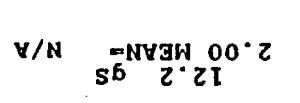 & $=-\mathrm{gaOH}$ & ${ }_{\tau}^{00}: Z$ & 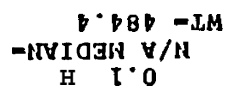 & $\begin{array}{l}\text { LIIdS } \\
\text { = Lyos } \\
\text { S2b }\end{array}$ \\
\hline $\begin{array}{l}0.001 \\
\text { S.L } \\
B^{\circ} \cdot 62\end{array}$ & $\begin{array}{l}\mathrm{V} / \mathrm{N} \\
\mathrm{V} / \mathrm{N} \\
\mathrm{Q} / \mathrm{N}\end{array}$ & $\begin{array}{l}5 \cdot 26 \\
\tau: 6 \\
\tau \cdot 96\end{array}$ & $\begin{array}{l}: \varepsilon 8 \\
0: \angle t \\
L: L 9\end{array}$ & $\begin{array}{l}b \cdot 99 \\
O \cdot L Z \\
\varepsilon \cdot L O \tau\end{array}$ & $\begin{array}{l}\cdot 6 \varepsilon \\
\tau \cdot \varepsilon I \\
9 \cdot \tau S\end{array}$ & $\begin{array}{l}\tau \cdot 9 Z \\
\tau \cdot 0 \tau \\
\varepsilon \cdot 0 \downarrow\end{array}$ & $\begin{array}{l}0.9 \text { I } \\
0.9 I \\
9 \cdot 69\end{array}$ & $\begin{array}{l}v / n \\
v / N \\
v / N\end{array}$ & $\begin{array}{l}\text { IM HIOS } \\
\text { IM } \\
\text { IM }\end{array}$ & $Y / N \quad 5^{-N Y 3 W} 0^{\circ} 0^{\circ} \tau$ & $\begin{array}{ll}-300 \mathrm{H} \\
\mathrm{g} \cdot 9 L\end{array}$ & 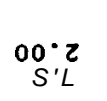 & 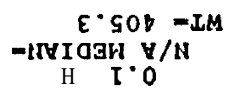 & $\begin{array}{l}\text { Litds } \\
\text { oudos } \\
\text { ozb }\end{array}$ \\
\hline $\begin{array}{l}0.001 \\
8 \cdot 8 \\
6 \cdot 96\end{array}$ & $\begin{array}{l}Q / N \\
\forall / N \\
V / N\end{array}$ & $\begin{array}{l}\varepsilon \cdot 16 \\
L \cdot 1 t \\
6 \cdot 29\end{array}$ & $\begin{array}{l}S^{\cdot} \cdot 6 L \\
6 \cdot z 2 \\
L \cdot 2 z I\end{array}$ & 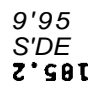 & $\begin{array}{l}T^{\prime} Z Z \\
\theta^{\circ} \cdot 0 \tau \\
8 \cdot L S\end{array}$ & $\begin{array}{l}E \cdot 1 t \\
6 \cdot 8 \\
6 \cdot L b\end{array}$ & $\begin{array}{l}: 2 \\
: 2 \\
0 \cdot 21\end{array}$ & $\begin{array}{l}Y / N \\
Y / N \\
Y / N\end{array}$ & $\begin{array}{l}\text { LM WNO } \\
\text { IM IM } \\
\text { IM }\end{array}$ & 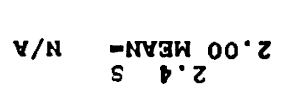 & $\begin{array}{l}-900 \mathrm{~W} \\
6.88\end{array}$ & $00 \cdot 2$ & $\begin{array}{l}\text { b ZBS }-I M \\
\text {-NYIOJW } \forall / N \\
H \quad \tau \cdot O\end{array}$ & $\begin{array}{l}\text { IrTdS } \\
\text {-Iyos } \\
\text { STb }\end{array}$ \\
\hline $\begin{array}{l}0.001 \\
\theta \cdot 6 \\
L \cdot 6 E\end{array}$ & $\begin{array}{l}\mathrm{Q} / \mathrm{N} \\
\mathrm{Q} / \mathrm{N} \\
\mathrm{Q} / \mathrm{N}\end{array}$ & $\begin{array}{l}2 \cdot 06 \\
6 \cdot E I \\
0 \cdot 99\end{array}$ & $\begin{array}{l}E: 9 L \\
E \cdot D 2 \\
E \cdot L 6\end{array}$ & $\begin{array}{l}\varepsilon^{\prime} 25 \\
6^{\prime} 6 Z \\
L^{\circ} 02 \mathrm{r}\end{array}$ & $\begin{array}{l}\cdot 22 \\
0 \cdot 6 \\
2 \cdot 9 \varepsilon\end{array}$ & $\begin{array}{l}S \cdot \varepsilon T \\
i \cdot L \\
T \cdot O E\end{array}$ & $\begin{array}{l}0^{\prime} 9 \\
0,9 \\
\mathbf{C}^{\prime} \cdot \mathbf{Z}\end{array}$ & $\begin{array}{l}\mathrm{Q} / \mathrm{N} \\
\mathrm{Q} / \mathrm{N} \\
\mathrm{Q} / \mathrm{N}\end{array}$ & $\begin{array}{l}\text { IM Kกว } \\
\text { IMM }\end{array}$ & 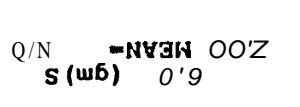 & $z \cdot 68$ & $\begin{aligned} 00: 2 \\
0: 6\end{aligned}$ & 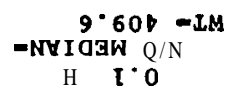 & $\begin{array}{l}\text { LITdS } \\
\text {-Lovs } \\
\text { SOV }\end{array}$ \\
\hline $\begin{array}{l}0 \cdot 00 T \\
L \cdot 0 \\
q \cdot \varepsilon z\end{array}$ & $\begin{array}{l}\forall / n \\
Q / N \\
\forall / w\end{array}$ & $\begin{array}{l}\mathbf{E} \cdot \mathbf{s} \mathbf{6} \\
\mathbf{b} \cdot \mathbf{s} \\
E^{\prime} L Z\end{array}$ & $\begin{array}{l}6.68 \\
0.6 \mathrm{I} \\
8.56\end{array}$ & $\begin{array}{l}6.0 L \\
0^{\circ} 96 \\
9 \cdot 1 E Z\end{array}$ & $\begin{array}{l}8 \cdot b 2 \\
L \cdot L \\
0 \cdot 8 \varepsilon\end{array}$ & $\begin{array}{l}\tau: L \tau \\
0 \cdot L \\
0 \cdot \text { sE }\end{array}$ & $\begin{array}{l}Z \cdot O T \\
z \cdot 0 \tau \\
z \cdot \text { Is }\end{array}$ & $\begin{array}{l}\mathrm{Q} / \mathrm{N} \\
\mathrm{Q} / \mathrm{M} \\
\mathrm{Q} / \mathrm{N}\end{array}$ & $\begin{array}{l}\text { LM Wกว } \\
\text { I.M }\end{array}$ & 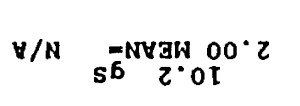 & $\begin{array}{l}-\mathrm{gaOW} \\
\mathrm{i} \cdot \mathrm{SB}\end{array}$ & ${ }_{L}^{00} \cdot ?$ & $\begin{array}{l}S^{\prime} L O S=I M \\
-N \forall I O J W \forall / N \\
\text { H } Z \cdot 0\end{array}$ & $\begin{array}{l}\text { IrTdS } \\
\text {-IUOS } \\
56 E\end{array}$ \\
\hline $\begin{array}{l}0 \cdot 00 \mathrm{r} \\
\varepsilon \cdot 8 \\
L \cdot 26\end{array}$ & $\begin{array}{l}Q / N \\
Y / H \\
Q / N\end{array}$ & $\begin{array}{l}L: 16 \\
\varepsilon \cdot 6 \\
B \cdot L b\end{array}$ & $\begin{array}{l}8: 28 \\
8 \cdot 12 \\
6 \cdot t 1 t\end{array}$ & $\begin{array}{l}9 \cdot 09 \\
9 \cdot 9 E \\
6 \cdot 28 \mathrm{IT}\end{array}$ & $\begin{array}{l}O ' S Z \\
I: 6 \\
\mathrm{~s}: 96\end{array}$ & $\begin{array}{l}0.91 \\
0.98 \\
0.16\end{array}$ & $\begin{array}{l}0.8 \\
0.8 \\
0.16\end{array}$ & $\begin{array}{l}\mathrm{Q} / \mathrm{N} \\
\forall / \mathrm{H} \\
\mathrm{Q} / \mathrm{N}\end{array}$ & $\begin{array}{l}\text { IM WND } \\
\text { IM IM }\end{array}$ & 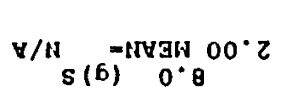 & $\begin{array}{l}=-3 a O W \\
L \cdot E 8\end{array}$ & $\begin{array}{r}00^{\circ}: 2 \\
E^{\circ}: 8\end{array}$ & $\begin{array}{c}z \cdot 0 Z s-L M \\
\text {-IVI IJW } \forall / N \\
H \quad \tau \cdot 0\end{array}$ & $\begin{array}{l}\text { LITdS } \\
\text {-LUOS } \\
\text { O6E }\end{array}$ \\
\hline $\begin{array}{l}0.001 \\
b \cdot \varepsilon \\
6 \cdot 22\end{array}$ & $\begin{array}{l}Q / N \\
\forall / u \\
\forall / t\end{array}$ & $\begin{array}{l}9^{\prime} 96 \\
O^{\prime} E \\
Z^{\prime} 02\end{array}$ & $\begin{array}{l}9 \cdot E 6 \\
0 \cdot 01 \\
L 99\end{array}$ & $\begin{array}{l}9 \cdot E B \\
6: C \mathrm{I} \\
6 \cdot E E I\end{array}$ & $\begin{array}{l}L: E g \\
\varepsilon: 9 \\
Z \cdot V\end{array}$ & $\begin{array}{l}b \cdot 19 \\
z \cdot 0 \mathbf{1} \\
0.89\end{array}$ & $\begin{array}{l}z: \angle b \\
z: \angle b \\
\tau \cdot 9 l \varepsilon\end{array}$ & $\begin{array}{l}Y / \mathbf{H} \\
\mathrm{Q} / \mathrm{N} \\
\mathrm{Q} / \mathrm{N}\end{array}$ & $\begin{array}{l}\text { \& LM NกJ } \\
P L M \\
L_{M}\end{array}$ & 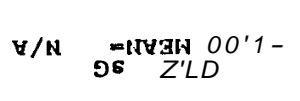 & $\begin{array}{l}=-900 \mathrm{~W} \\
6 \cdot 60\end{array}$ & $00^{\circ} \cdot \mathrm{E}$ & 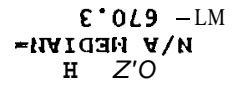 & $\begin{array}{l}\text { LITdS } \\
=\text { IUOS } \\
\text { S日E }\end{array}$ \\
\hline $\begin{array}{l}0.001 \\
c^{\circ} \cdot 8 \\
I \cdot 62\end{array}$ & $\begin{array}{l}Q / N \\
V / N \\
\forall / H\end{array}$ & $\begin{array}{l}c^{\cdot} \cdot 96 \\
\varepsilon \cdot{ }^{6} \\
\tau \cdot 82\end{array}$ & $\begin{array}{l}z \cdot 16 \\
b: 91 \\
i \cdot 901\end{array}$ & $\begin{array}{l}8 \cdot B L \\
B \cdot E E \\
Z \cdot L I Z\end{array}$ & $\begin{array}{l}b \cdot \mathbf{b} \\
O^{\prime} L \\
Z^{\prime} \cdot \mathbf{q b}\end{array}$ & $\begin{array}{l}\text { V. VE } \\
\text { I: OI } \\
9.59\end{array}$ & 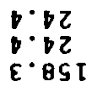 & $\begin{array}{l}Q / N \\
\forall / N \\
Q / N\end{array}$ & $\begin{array}{l}\text { LM ผnว } \\
\text { LMM }\end{array}$ & $\mathrm{se}^{-\mathrm{NYgh}} \mathrm{E}^{\prime} D Z$ & $\begin{array}{l}=-300 \mathrm{~W} \\
\mathrm{z} \cdot \tau l\end{array}$ & $\begin{array}{r}00: 2 \\
\mathrm{~s} \cdot ?\end{array}$ & 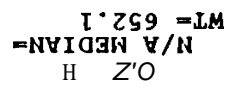 & $\begin{array}{l}\text { LITdS } \\
\text { =LUOS } \\
\text { OBE }\end{array}$ \\
\hline $\begin{array}{l}0.001 \\
6 \cdot 1 \mathrm{rt} \\
0.95 \\
\end{array}$ & $\begin{array}{l}\mathrm{Q} / \mathrm{N} \\
\mathrm{Q} / \mathrm{N} \\
\mathrm{Q} / \mathrm{N}\end{array}$ & 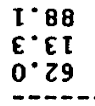 & $\begin{array}{l}8 \cdot V L \\
0.92 \\
Z^{\prime} E Z T \\
-2 .\end{array}$ & $\begin{array}{l}: 86 \\
:-2 E \\
2 \cdot I S I\end{array}$ & $\begin{array}{l}0 \cdot 9 \tau \\
1 \cdot 8 \\
6 . L E\end{array}$ & \begin{tabular}{l}
$\theta^{\circ} L$ \\
$\varepsilon \cdot L$ \\
$0 \cdot 0 z$ \\
\hdashline-0.0
\end{tabular} & 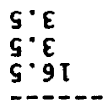 & $\begin{array}{l}\forall / h \\
\forall / h \\
Q / N \\
-\ldots--\end{array}$ & $\begin{array}{l}\text { LM Wnว } \\
\text { LM }\end{array}$ & $\begin{array}{c}\forall / N \quad=N G 3 W 0 \\
S(w)\end{array}$ & $\begin{array}{l}\text {-gaOW } \\
9 \cdot 68 \\
-\cdots-2\end{array}$ & ${ }_{6}^{00} \cdot \frac{E}{11}$ & 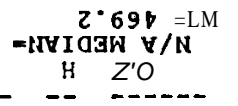 & $\begin{array}{l}\text { LITdS } \\
\text {-LUOS } \\
\text { SLE }\end{array}$ \\
\hline $\begin{array}{c}(2 L \cdot b<) \\
\operatorname{lng} d\end{array}$ & $\begin{array}{l}(g L: b) \\
\text { LIIS }\end{array}$ & $\begin{array}{l}(P I \\
\text { gNI } \\
\text { XYG }\end{array}$ & $\begin{array}{r}(\varepsilon) \\
\operatorname{anI}]\end{array}$ & asw & $\begin{array}{l}\text { (I) } \\
\text { sषYOJ }\end{array}$ & $\begin{array}{l}(0) \\
\text { syvo.3 } \\
\text { xyaA }\end{array}$ & $\begin{array}{l}(\mathrm{I}-) \\
83 \mathrm{~d} \\
\text { INIAS }\end{array}$ & $\begin{array}{c}(2-m) \\
83 \mathrm{~d} \\
\text { anId }\end{array}$ & & 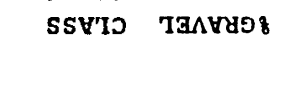 & $\mathrm{a} \mathrm{m} \sim$ & $\$ \mathrm{a} n \sim$ & $\%$ ผ & H山dتa \\
\hline
\end{tabular}

**** EOO-LOM-66ZO TT3M NO L4Od34 **** 
WESTINGHOUSE HANFORD OPERATIONS SIEVE ANALYSIS ROCSAN REPORT

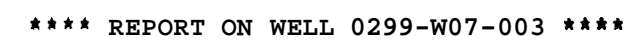

$12 / 11 / 89$

\begin{tabular}{|c|c|c|c|c|c|c|c|c|c|c|c|c|c|c|c|c|c|c|}
\hline PTH & $8 \mathrm{CAC} 03$ & DM & IIFUD & ISAND & ZGRAVEL & CLAS & & & & $\begin{array}{l}\text { FINE } \\
\text { PEB } \\
(<-=-2)\end{array}$ & $\begin{array}{c}\text { VFINE } \\
\text { PEB } \\
(-1)\end{array}$ & $\begin{array}{l}\text { VERY } \\
\text { COARS } \\
(0)\end{array}$ & $\begin{array}{l}\text { COARS } \\
\text { (1) }\end{array}$ & $\begin{array}{l}\text { MED } \\
(2)\end{array}$ & $\begin{array}{l}\text { FINE } \\
\text { (3) }\end{array}$ & $\begin{array}{l}\text { VERY } \\
\text { FINE } \\
\text { (4) }\end{array}$ & $\begin{array}{l}\text { SILT } \\
(4.75)\end{array}$ & $\begin{array}{c}\text { PAN } \\
(>4.75)\end{array}$ \\
\hline $\begin{array}{r}455 \\
\text { SORT- } \\
\text { SPLIT }\end{array}$ & $\begin{array}{c}0.3 \\
N / A \\
W T=649.0\end{array}$ & $\underset{\text { DIAN- }}{\mathrm{H}}$ & $\begin{array}{l}5.7 \\
0.00\end{array}$ & $\begin{array}{r}68.1 \\
\text { MODE- }\end{array}$ & -1.00 MEAN & $g s$ & N/A & CUM & $\begin{array}{l}\text { WT } \\
\text { WT } \\
\text { WT }\end{array}$ & $\begin{array}{l}\text { N/A } \\
\text { N/A } \\
\text { N/A }\end{array}$ & $\begin{array}{r}168.6 \\
26.2 \\
26.2\end{array}$ & $\begin{array}{r}162.2 \\
25.3 \\
51.5\end{array}$ & $\begin{array}{l}85.0 \\
13.2 \\
64.7\end{array}$ & $\begin{array}{l}95.8 \\
14.9 \\
79.6\end{array}$ & $\begin{array}{r}63.6 \\
9.9 \\
89.5\end{array}$ & $\begin{array}{r}30.9 \\
4.8 \\
94.3\end{array}$ & $\begin{array}{l}\text { N/A } \\
\text { N/A } \\
\text { N/A }\end{array}$ & $\begin{array}{r}36.4 \\
5.7 \\
100.0\end{array}$ \\
\hline $\begin{array}{r}460 \\
\text { SORT- } \\
\text { SPIIT }\end{array}$ & $\begin{array}{c}0.1 \\
\text { N/A MEI } \\
\text { WT- } 604 .\end{array}$ & $\begin{array}{l}\mathrm{H} \\
\text { DIAN- }\end{array}$ & $\begin{array}{l}5.2 \\
2.00\end{array}$ & $\begin{array}{r}05.3 \\
\text { MODE- }\end{array}$ & $2.00^{9.6} \mathrm{MEAN}$ & (g) $s$ & $S_{N / A}$ & $\mathrm{CUM}$ & $\begin{aligned} \text { WT } \\
\text { WT } \\
\text { W T } 0\end{aligned}$ & $\begin{array}{l}\text { N/A } \\
\text { N/A } \\
\text { N/A }\end{array}$ & $\begin{array}{r}65.0 \\
9.6 \\
9.6\end{array}$ & $\begin{array}{l}97.2 \\
14.3 \\
23.8\end{array}$ & $\begin{array}{r}124.7 \\
18.3 \\
42.2\end{array}$ & $\begin{array}{r}197.9 \\
29.1 \\
71.3\end{array}$ & $\begin{array}{r}118.9 \\
17.5 \\
88.7\end{array}$ & $\begin{array}{r}41.5 \\
6.1 \\
94.8\end{array}$ & $\begin{array}{l}\text { N/A } \\
\text { N/A }\end{array}$ & $\begin{array}{r}35.1 \\
5.2 \\
100.0\end{array}$ \\
\hline $\begin{array}{r}465 \\
\text { SORT- } \\
\text { SPLIT }\end{array}$ & $\begin{array}{c}0.1 \\
\text { N/A ME } \\
\text { WT- } 732.8\end{array}$ & $\begin{array}{l}\text { H } \\
\text { DIAN- }\end{array}$ & $\begin{array}{l}8.3 \\
2.00\end{array}$ & $\begin{array}{r}90.2 \\
\text { MODE- }\end{array}$ & $2.00 \stackrel{1.5}{\text { MEAN- }}$ & & N/A & CUM & $\begin{array}{l}\text { WT } \\
\text { WT } \\
\text { WT }\end{array}$ & $\begin{array}{l}\text { N/A } \\
N / A \\
N / A\end{array}$ & $\begin{array}{r}10.6 \\
1.5 \\
1.5\end{array}$ & $\begin{array}{r}57.0 \\
7.8 \\
9.3\end{array}$ & $\begin{array}{r}162.5 \\
22.3 \\
31.6\end{array}$ & $\begin{array}{r}241.2 \\
33.1 \\
64.6\end{array}$ & $\begin{array}{r}136.1 \\
18.7 \\
03.3\end{array}$ & $\begin{array}{r}61.1 \\
8.4 \\
91.7\end{array}$ & $\begin{array}{l}\text { N/A } \\
\text { N/A } \\
\text { W/A }\end{array}$ & $\begin{array}{r}60.6 \\
8.3 \\
100.0\end{array}$ \\
\hline $\begin{array}{r}470 \\
\text { SORT- } \\
\text { SPIIT }\end{array}$ & $\begin{array}{r}0.2 \\
\text { N/A ME } \\
\text { WT- } 587 .\end{array}$ & $\begin{array}{l}\text { D } \\
\text { DIAN- }\end{array}$ & $\begin{array}{l}5.5 \\
0.00\end{array}$ & $\begin{array}{l}60.8 \\
\text { MODE- }\end{array}$ & $-1.00^{33.7}$ MEAN & SG & N/A & CUM & $\begin{array}{l}\text { WT } \\
\text { WT } \\
\text { WT }\end{array}$ & $\begin{array}{l}N / A \\
N / A \\
N / A\end{array}$ & $\begin{array}{r}195.2 \\
33.7 \\
33.7\end{array}$ & $\begin{array}{r}104.2 \\
18.0 \\
51.6\end{array}$ & $\begin{array}{l}82.4 \\
14.2 \\
65.9\end{array}$ & $\begin{array}{l}91.2 \\
15.7 \\
01.6\end{array}$ & $\begin{array}{r}46.7 \\
8.1 \\
89.6\end{array}$ & $\begin{array}{r}28.3 \\
4.9 \\
94.5\end{array}$ & $\begin{array}{l}\text { N/A } \\
\text { N/A } \\
\text { A }\end{array}$ & $\begin{array}{r}31.8 \\
5.5 \\
100.0\end{array}$ \\
\hline $\begin{array}{r}475 \\
\text { SORT- } \\
\text { SPLIT }\end{array}$ & $\begin{array}{c}0.2 \\
\text { N/A ME } \\
\text { WT }=497 .\end{array}$ & $\begin{array}{l}\text { H } \\
\text { DIAN- } \\
7\end{array}$ & $\begin{array}{l}6.1 \\
1.00\end{array}$ & $\begin{array}{r}84.3 \\
\text { MODE- }\end{array}$ & $\begin{array}{c}9.6 \\
1.00 \mathrm{MEAN}-\end{array}$ & $(g) s$ & $\mathbf{S}_{\mathrm{N} / \mathrm{A}}$ & M & $\begin{array}{l}\text { WT } \\
\text { WT } \\
\text { WT }\end{array}$ & $\begin{array}{l}\text { N/A } \\
\text { N/A } \\
\text { N/A }\end{array}$ & $\begin{array}{r}46.0 \\
9.6 \\
9.6\end{array}$ & $\begin{array}{l}80.7 \\
16.5 \\
26.0\end{array}$ & $\begin{array}{l}136.9 \\
27.9 \\
54.0\end{array}$ & $\begin{array}{l}120.4 \\
24.6 \\
78.5\end{array}$ & $\begin{array}{l}51.6 \\
10.5 \\
89.1\end{array}$ & $\begin{array}{r}23.6 \\
4.8 \\
93.9\end{array}$ & $\begin{array}{l}\text { N/A } \\
\text { N/A } \\
\text { N/A }\end{array}$ & $\begin{array}{r}30.0 \\
6.1 \\
100.0\end{array}$ \\
\hline
\end{tabular}



ROCSAN REPORT

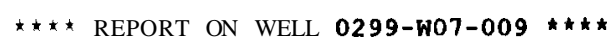

$12 / 05 / 89$

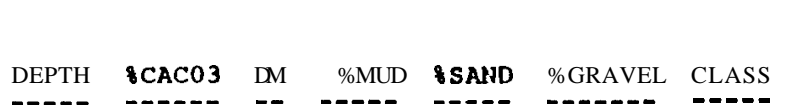

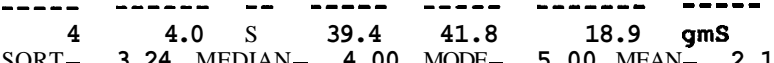

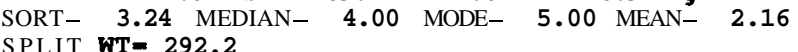

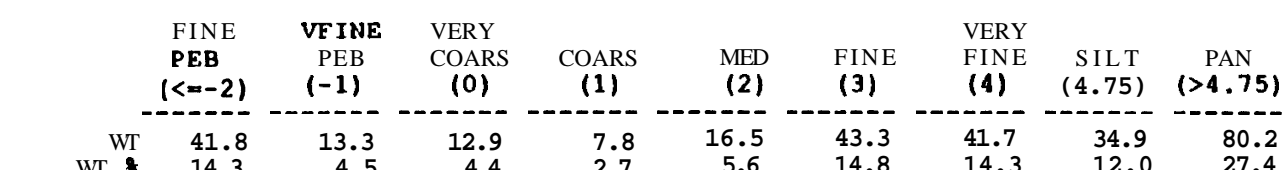

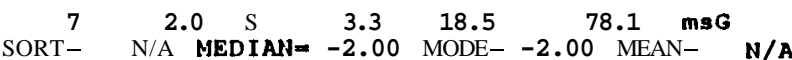

SORT - N/A MED
SPLIT

WT 551.3

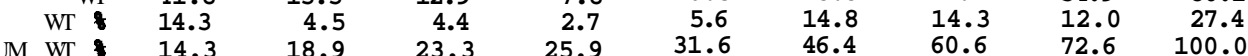

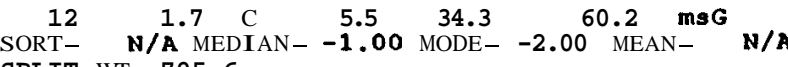

CUM WT 68.7

$75.4 \quad 55.5 \quad 51.3$

$31.6 \quad 46.4$

SPLIT WT- 795.6

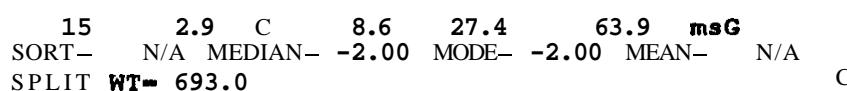

51.3
6.4
91.4

16.913 .7

$11.5 \quad 4.9 \quad 21.8$

$\begin{array}{rrrr}47.2 & 13.0 & 13.2 & 86.4\end{array}$

86.4
10.9
84.3

36.724 .5

$\begin{array}{rrr}1.4 & 0.6 & 2.7\end{array}$

$\begin{array}{lllllll}20 & 1.0 & \mathrm{C} & 5.7 & 40.1 & 54.1 & \mathbf{m s G}\end{array}$

$\begin{array}{lll}47.2 & 60.2 & 73.4\end{array}$

$\begin{array}{rr}4.6 & 3.1 \\ 88.9 & 92.0\end{array}$

$20.1 \quad 8.8 \quad 34.8$

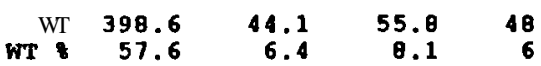

48.0
78.9

$31.7 \quad 26$

2.5
94.5

$\begin{array}{rr}8.8 & 34.8 \\ 1.1 & 4.4\end{array}$

SORT - N/A MEDIAN- -1.00 MODE- -2.00 MEAN-

SPLIT WTP- 794.0

WT 322.1

$107.7 \quad 125.1$

87.2

83.5

87.3

$28.0 \quad 12.4 \quad 47.5$

11.9

s.:.

34.

$91.3 \quad 93.1 \quad 100.0$

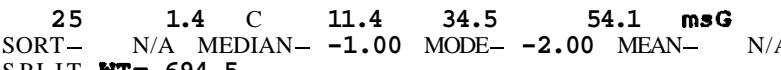

WT 323.4

$52.7 \quad 62.1 \quad 55.3$

86.891 .1

$\begin{array}{rrr}24.9 & 9.3 & 36.2 \\ 3.1 & 1.2 & 4.6\end{array}$

$\begin{array}{rrr}3.1 & 1.2 & 4.6 \\ 4.3 & 95.5 & 100.0\end{array}$

WT- 694.5

CUM WT: 46.5

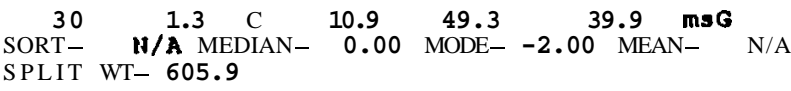

WT 177.7

54.

$\begin{array}{rr}0.9 \\ 63.0 & 8.0\end{array}$

$\begin{array}{lllll}41.9 & 41.2 & 39.8 & 16.0 & 63.1\end{array}$

$\begin{array}{llllll}\text { WT } & 29.4 & 10.5 & 14.8 & 85 \\ & \text { CUM WT } & 29.4 & 39.9 & 54.7 & 68.7\end{array}$

$\begin{array}{lrrrrrrrrr}35 & 3.1 \text { C } & 7.8 & 35.4 & 56.8 \text { msG } & & \text { WT } & 265.0 & 51.9 \\ \text { SORT- } & \text { N/A MEDIAN- } & -1.00 & \text { MODE- } & -2.00 & \text { MEAN- } & \text { N/A } & \text { WT } & 47.5 & 9.3 \\ \text { SPLIT } & \text { WT- } 557.0 & & & & & \text { CUM WT } & 47.5 & 56.8\end{array}$

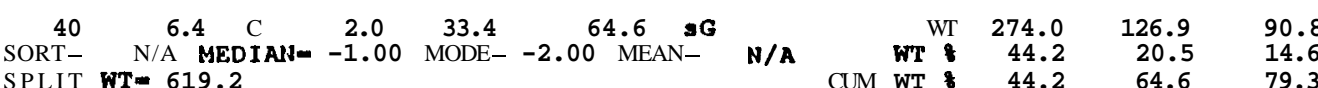

14.0

68.7

77.0

\begin{tabular}{rrrr}
5.9 & 39.8 & 16.0 & 63.1 \\
\hline 5 & 5.7 & 2.3 & 9.1
\end{tabular}

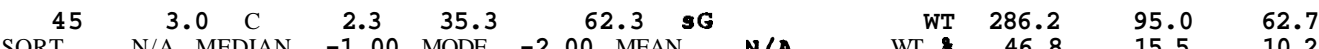

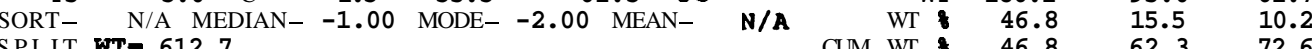

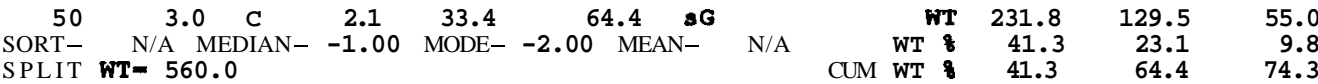

52.0
9.3
75.8

$\begin{array}{lllll}49.1 & 42.0 & 32.5 & 13.1 & 52.6\end{array}$

$\begin{array}{rrrrr}8.1 & 6.9 & 5.4 & 2.2 & 8.7 \\ 6.8 & 83.8 & 89.1 & 91.3 & 100.0\end{array}$

10.2

10.2
89.4

$\begin{array}{rrrrr}36.3 & 30.4 & 24.6 & 10.7 & 32.7 \\ 6.5 & 5.5 & 4.4 & 1.9 & 5.9 \\ 82.3 & 87.8 & 92.2 & 94.1 & 100.0\end{array}$

30 .

$24.6 \quad 10.7 \quad 32.7$

$\begin{array}{rrrrr}9.6 & 14.8 & 9.8 & 3.1 & 9.3 \\ 4.6 & 2.4 & 1.6 & 0.5 & 1.5\end{array}$

$\begin{array}{llllll}62.1 & 56.6 & 22.9 & 11.9 & 3.6 & 10.7\end{array}$

$\begin{array}{rrrrrr}10.2 & 9.3 & 3.7 & 11.9 & 3.6 & 10.7\end{array}$

$3 \quad 100.0$

7.6
81.5

$\begin{array}{rr}54.6 & 26.1 \\ 9.7 & 4.7\end{array}$

$\begin{array}{rrr}11.0 & 3.4 & 8.5 \\ 2.0 & 0.6 & 1.5 \\ 97.9 & 98.5 & \end{array}$ 


\section{$12 / 05 / 89$}

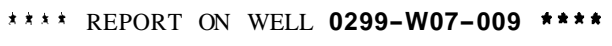

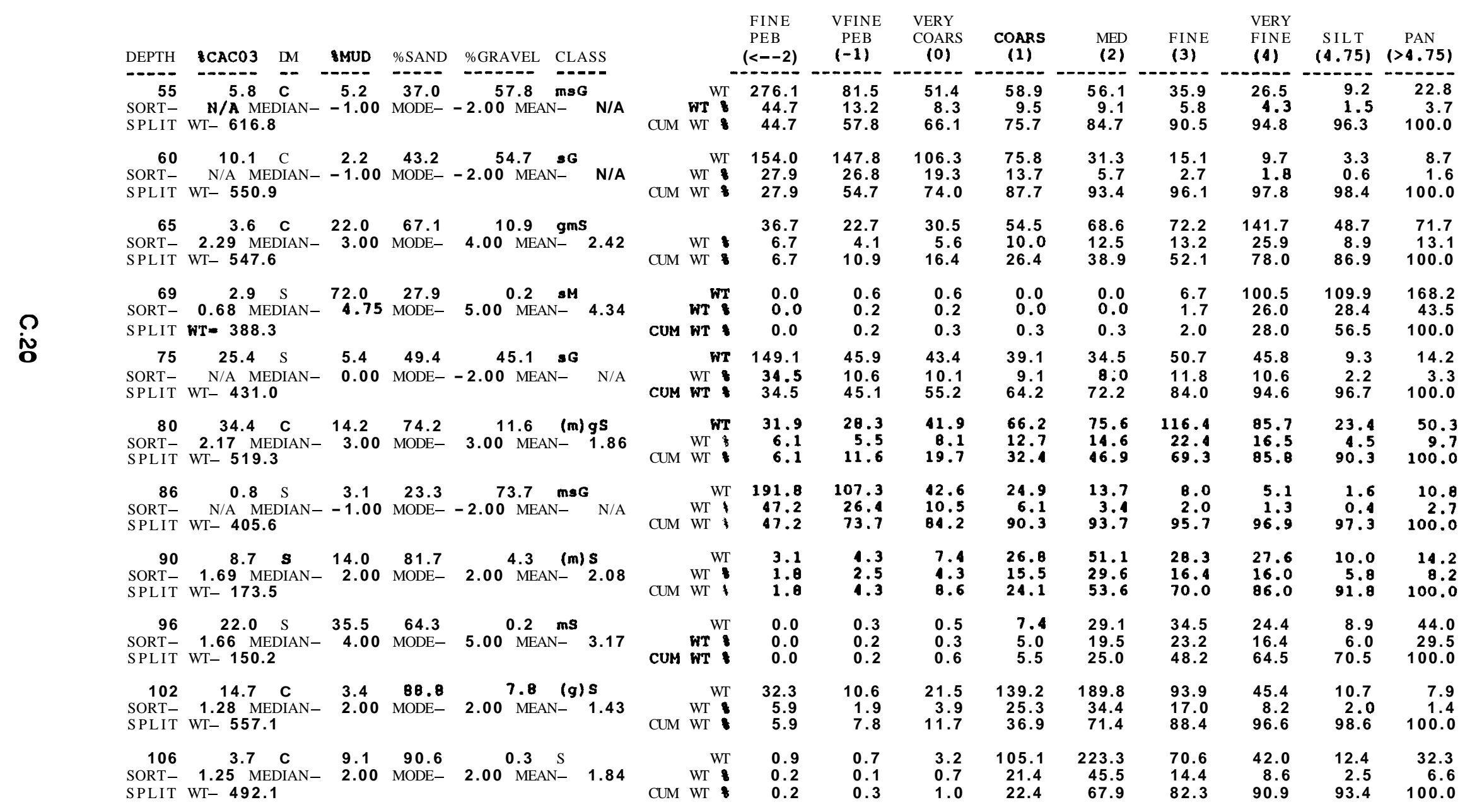


WESTINGHOUSE HAHFORD OPERATIOHS SIEVE ANALYSIS

ROCSAN REPORT

$12 / 05 / 89$

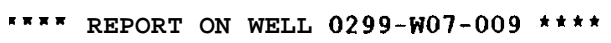

\begin{tabular}{|c|c|c|c|c|c|c|c|c|c|c|c|c|c|c|c|c|c|c|}
\hline DEPTH & \& $\mathrm{CAC} 03$ & DM & $\because \mathrm{MUD}$ & $\frac{\circ}{\partial}$ SAND & $\because$ GRAVEL & CLAS & ss & & & $\begin{array}{l}\text { FINE } \\
\text { PEB } \\
(<=-2)\end{array}$ & $\begin{array}{l}\text { VF INE } \\
\text { PEB } \\
(-1)\end{array}$ & $\begin{array}{l}\text { VERY } \\
\text { COARS } \\
\text { (0) }\end{array}$ & $\begin{array}{l}\text { COARS } \\
(1)\end{array}$ & $\begin{array}{l}\text { MED } \\
\text { (2) }\end{array}$ & $\begin{array}{l}\text { FINE } \\
\text { (3) }\end{array}$ & $\begin{array}{l}\text { VERY } \\
\text { FINE } \\
\text { (4) }\end{array}$ & $\begin{array}{c}\text { SILT } \\
(4.75)\end{array}$ & $\begin{array}{c}\text { PAN } \\
(>4.75)\end{array}$ \\
\hline $\begin{array}{r}110 \\
\text { SORT- } \\
\text { SPIIT }\end{array}$ & $\begin{array}{c}1.5 \\
1.14 \text { MED } \\
\text { WT- } 511.7\end{array}$ & $\begin{array}{l}\text { C } \\
\text { DIAN- }\end{array}$ & $\begin{array}{l}7.4 \\
-\quad 2.00\end{array}$ & $\begin{array}{r}91.9 \\
\text { MODE- }\end{array}$ & $2.00^{0.0} \mathrm{MEAN}-$ & & 1.60 & CUM & $\begin{array}{l}\text { WT } \\
\text { WT } \\
\text { WT }\end{array}$ & $\begin{array}{l}1.2 \\
0.2 \\
0.2\end{array}$ & $\begin{array}{l}2.7 \\
0.5 \\
0.8\end{array}$ & $\begin{array}{l}6.4 \\
1.3 \\
2.0\end{array}$ & $\begin{array}{r}136.1 \\
26.7 \\
28.7\end{array}$ & $\begin{array}{r}234.0 \\
46.0 \\
74.7\end{array}$ & $\begin{array}{l}59.2 \\
11.6 \\
86.3\end{array}$ & $\begin{array}{r}32.6 \\
6.4 \\
92.6\end{array}$ & $\begin{array}{r}10.4 \\
2.0 \\
94.7\end{array}$ & $\begin{array}{r}27.2 \\
5.3 \\
100.0\end{array}$ \\
\hline $\begin{array}{r}114 \\
\text { SORT- } \\
\text { SPLIT }\end{array}$ & $\begin{array}{c}0.8 \\
1.19 \text { MED } \\
\text { WT- } 519.9\end{array}$ & DIAN- & $\begin{array}{c}10.3 \\
-\quad 2.00\end{array}$ & $\begin{array}{r}89.6 \\
\text { MODE- }\end{array}$ & $2.00^{0.1}$ & $(m)$ & $\begin{array}{l}\mathbf{s} \\
1.93\end{array}$ & $\mathrm{CUM}$ & $\begin{array}{r}\text { WT } \\
\text { WT } \\
\text { M T T }\end{array}$ & $\begin{array}{l}0.0 \\
0.0 \\
0.0\end{array}$ & $\begin{array}{l}0.6 \\
0.1 \\
0.1\end{array}$ & $\begin{array}{l}1.3 \\
0.3 \\
0.4\end{array}$ & $\begin{array}{l}92.3 \\
17.8 \\
18.1\end{array}$ & $\begin{array}{r}256.9 \\
49.5 \\
67.6\end{array}$ & $\begin{array}{l}74.2 \\
14.3 \\
81.9\end{array}$ & $\begin{array}{r}40.9 \\
7.9 \\
89.7\end{array}$ & $\begin{array}{r}13.0 \\
2.5 \\
92.2\end{array}$ & $\begin{array}{r}40.4 \\
7.8 \\
100.0\end{array}$ \\
\hline $\begin{array}{r}120 \\
\text { SORT- } \\
\text { SPLIT }\end{array}$ & $\begin{array}{c}1.0 \\
0.95 \text { MED } \\
\text { WT- } 540.0\end{array}$ & $\begin{array}{l}\text { C } \\
\text { DIAN- }\end{array}$ & $\begin{array}{l}6.1 \\
2.00\end{array}$ & $\begin{array}{c}93.3 \\
\text { MODE- }\end{array}$ & 2.00 MEAN- & & 1.68 & CUM & $\begin{array}{l}\text { WT } \\
\text { WT } \% \\
\text { WT }\end{array}$ & $\begin{array}{l}0.9 \\
0.2 \\
0.2\end{array}$ & $\begin{array}{l}2.5 \\
0.5 \\
0.6\end{array}$ & $\begin{array}{l}7.4 \\
1.4 \\
2.0\end{array}$ & $\begin{array}{l}96.5 \\
17.9 \\
19.9\end{array}$ & $\begin{array}{r}288.1 \\
53.3 \\
73.2\end{array}$ & $\begin{array}{l}84.4 \\
15.6 \\
88.8\end{array}$ & $\begin{array}{r}27.4 \\
5.1 \\
93.9\end{array}$ & $\begin{array}{r}8.7 \\
1.6 \\
95.5\end{array}$ & $\begin{array}{r}24.4 \\
4.5 \\
100.0\end{array}$ \\
\hline $\begin{array}{r}124 \\
\text { SORT- } \\
\text { SPLIT }\end{array}$ & $\begin{array}{c}0.7 \\
0.89 \text { MED } \\
\text { WT }=521.7\end{array}$ & $\begin{array}{l}\text { C } \\
\text { DIAN- }\end{array}$ & $\begin{array}{l}6.5 \\
-\quad 2.00\end{array}$ & $\begin{array}{c}93.4 \\
\text { MODE- }\end{array}$ & $2.00^{0.1} \mathrm{MEAN}-$ & s- & 2.04 & CUM & $\begin{array}{l}\text { WT } \\
\text { WT } \\
\text { W'T }\end{array}$ & $\begin{array}{l}0.2 \\
0.0 \\
0.0\end{array}$ & $\begin{array}{l}0.2 \\
0.0 \\
0.1\end{array}$ & $\begin{array}{l}0.7 \\
0.1 \\
0.2\end{array}$ & $\begin{array}{r}44.4 \\
8.5 \\
0.7\end{array}$ & $\begin{array}{r}220.6 \\
42.3 \\
51.0\end{array}$ & $\begin{array}{r}179.7 \\
34.5 \\
85.5\end{array}$ & $\begin{array}{r}41.9 \\
8.0 \\
93.5\end{array}$ & $\begin{array}{r}10.5 \\
2.0 \\
95.5\end{array}$ & $\begin{array}{r}23.4 \\
4.5 \\
100.0\end{array}$ \\
\hline $\begin{array}{r}130 \\
\text { SORT- } \\
\text { SPLIT }\end{array}$ & $\begin{array}{c}1.3 \\
1.38 \text { MED } \\
\text { WT- } 601.6\end{array}$ & $\begin{array}{l}\text { C } \\
\text { DIAN- }\end{array}$ & $\begin{array}{l}4.2 \\
-\quad 1.00\end{array}$ & $\begin{array}{r}03.9 \\
\text { MODE- }\end{array}$ & $1.00^{11.8} \mathrm{MEAN}$ & gs & 0.68 & CUM & $\begin{array}{l}\text { WT } \\
\text { WT } \\
\text { WT }\end{array}$ & $\begin{array}{r}36.2 \\
5.3 \\
5.3\end{array}$ & $\begin{array}{r}44.4 \\
6.5 \\
11.9\end{array}$ & $\begin{array}{r}100.3 \\
14.8 \\
26.6\end{array}$ & $\begin{array}{r}221.8 \\
32.6 \\
59.2\end{array}$ & $\begin{array}{r}167.1 \\
24.6 \\
83.8\end{array}$ & $\begin{array}{r}56.3 \\
8.3 \\
92.0\end{array}$ & $\begin{array}{r}25.5 \\
3.8 \\
95.8\end{array}$ & $\begin{array}{r}7.0 \\
1.0 \\
96.8\end{array}$ & $\begin{array}{r}21.7 \\
3.2 \\
100.0\end{array}$ \\
\hline $\begin{array}{r}134 \\
\text { SORT- } \\
\text { SPLIT }\end{array}$ & $\begin{array}{c}2.2 \\
0.69 \\
\text { WT- } 421.2\end{array}$ & $\begin{array}{l}\text { C } \\
\text { DIAN- }\end{array}$ & $\begin{array}{l}5.2 \\
2.00\end{array}$ & $\begin{array}{r}94.8 \\
\text { MODE- }\end{array}$ & 2.00 HEAN & $s$ & 1.75 & CUM & $\begin{array}{l}\text { WT } \\
\text { WT } \\
\text { WT }\end{array}$ & $\begin{array}{l}0.0 \\
0.0 \\
0.0\end{array}$ & $\begin{array}{l}0.1 \\
0.0 \\
0.0\end{array}$ & $\begin{array}{l}0.7 \\
0.2 \\
0.2\end{array}$ & $\begin{array}{r}29.2 \\
6.9 \\
7.1\end{array}$ & $\begin{array}{r}295.6 \\
70.0 \\
77.1\end{array}$ & $\begin{array}{l}56.1 \\
13.3 \\
90.4\end{array}$ & $\begin{array}{r}18.6 \\
4.4 \\
94.8\end{array}$ & $\begin{array}{r}5.7 \\
1.4 \\
96.2\end{array}$ & $\begin{array}{r}16.2 \\
3.8 \\
100.0\end{array}$ \\
\hline $\begin{array}{r}138 \\
\text { SORT- } \\
\text { SPLIT }\end{array}$ & $\begin{array}{c}2.6 \\
0.77 \text { MED } \\
\text { WT- } 410.2\end{array}$ & $\begin{array}{l}\text { C } \\
\text { DIAN- }\end{array}$ & $\begin{array}{l}5.0 \\
2.00\end{array}$ & $\begin{array}{r}94.9 \\
\text { MODE- }\end{array}$ & $2.00^{0.1} \mathrm{MEAN}-$ & $s-$ & 1.85 & CUM & $\begin{array}{l}\text { WT } \\
\text { WT } \\
\text { WT }\end{array}$ & $\begin{array}{l}0.0 \\
0.0 \\
0.0\end{array}$ & $\begin{array}{l}0.3 \\
0.1 \\
0.1\end{array}$ & $\begin{array}{l}0.0 \\
0.2 \\
0.3\end{array}$ & $\begin{array}{r}28.3 \\
6.9 \\
7.2\end{array}$ & $\begin{array}{r}247.8 \\
60.5 \\
67.7\end{array}$ & $\begin{array}{l}94.8 \\
23.2 \\
90.8\end{array}$ & $\begin{array}{r}17.0 \\
4.2 \\
95.0\end{array}$ & $\begin{array}{r}5.2 \\
1.3 \\
96.3\end{array}$ & $\begin{array}{r}15.3 \\
3.7 \\
100.0\end{array}$ \\
\hline $\begin{array}{r}142 \\
\text { SORT- } \\
\text { SPLIT }\end{array}$ & $\begin{array}{c}2.2 \\
0.88 \text { MED } \\
\text { WT- } 418.2\end{array}$ & DIAN- & $\begin{array}{l}0.6 \\
2.00\end{array}$ & $\begin{array}{r}91.3 \\
\text { MODE- }\end{array}$ & $2.00 \quad 0.0 \mathrm{MEAN}-$ & $s$ & 2.06 & CUM & $\begin{array}{l}\text { WT } \\
\text { WT } \\
\text { WT }\end{array}$ & $\begin{array}{l}0.0 \\
0.0 \\
0.0\end{array}$ & $\begin{array}{l}0.1 \\
0.0 \\
0.0\end{array}$ & $\begin{array}{l}0.4 \\
0.1 \\
0.1\end{array}$ & $\begin{array}{l}0.5 \\
2.0 \\
2.1\end{array}$ & $\begin{array}{r}222.3 \\
53.2 \\
55.4\end{array}$ & $\begin{array}{r}119.1 \\
28.5 \\
83.9\end{array}$ & $\begin{array}{r}31.2 \\
7.5 \\
91.4\end{array}$ & $\begin{array}{r}9.2 \\
2.2 \\
93.6\end{array}$ & $\begin{array}{r}26.9 \\
6.4 \\
100.0\end{array}$ \\
\hline $\begin{array}{r}145 \\
\text { SORT- } \\
\text { SPLIT }\end{array}$ & $\begin{array}{c}0.8 \\
1.92 \mathrm{MED} \\
\mathrm{WT}-593.4\end{array}$ & $\underset{4}{\text { DIAN- }}$ & $\begin{array}{l}3.7 \\
1.00\end{array}$ & $\begin{array}{r}69.5 \\
\text { MODE- }\end{array}$ & $1.00^{26.7}$ MEAN & gs & 0.17 & CUM & $\begin{array}{l}\text { WT } \\
\text { WT } \\
\text { WT }\end{array}$ & $\begin{array}{l}88.6 \\
14.9 \\
14.9\end{array}$ & $\begin{array}{l}70.1 \\
11.8 \\
26.7\end{array}$ & $\begin{array}{l}70.9 \\
11.9 \\
38.7\end{array}$ & $\begin{array}{r}139.3 \\
23.5 \\
62.1\end{array}$ & $\begin{array}{r}137.5 \\
23.2 \\
85.3\end{array}$ & $\begin{array}{r}46.7 \\
7.9 \\
93.2\end{array}$ & $\begin{array}{r}18.5 \\
3.1 \\
96.3\end{array}$ & $\begin{array}{r}6.2 \\
1.1 \\
97.3\end{array}$ & $\begin{array}{r}16.0 \\
2.7 \\
100.0\end{array}$ \\
\hline $\begin{array}{r}150 \\
\text { SORT- } \\
\text { SPLIT }\end{array}$ & $\begin{array}{c}1.0 \\
\text { N/A MED } \\
\text { WT- } 724.4\end{array}$ & $\underset{\text { DIAN- }}{\text { H }}$ & $\begin{array}{l}1.0 \\
-2.00\end{array}$ & $\begin{array}{r}23.0 \\
\text { MODE- }\end{array}$ & $\begin{array}{c}76.0 \\
-2.00 \text { MEAN- }\end{array}$ & 3G & N/A & CUM & $\begin{array}{l}\text { WT } \\
\text { WT } \\
\text { WT }\end{array}$ & $\begin{array}{r}433.3 \\
59.9 \\
59.9\end{array}$ & $\begin{array}{r}116.3 \\
16.1 \\
76.0\end{array}$ & $\begin{array}{r}44.0 \\
6.1 \\
82.1\end{array}$ & $\begin{array}{r}56.2 \\
7.8 \\
89.9\end{array}$ & $\begin{array}{r}45.8 \\
6.3 \\
96.2\end{array}$ & $\begin{array}{r}14.3 \\
2.0 \\
98.2\end{array}$ & $\begin{array}{r}6.0 \\
0.8 \\
99.0\end{array}$ & $\begin{array}{r}1.8 \\
0.2 \\
99.3\end{array}$ & $\begin{array}{r}5.6 \\
0.8 \\
100.0\end{array}$ \\
\hline $\begin{array}{r}155 \\
\text { SORT- } \\
\text { SPLIT }\end{array}$ & $\begin{array}{c}0.7 \\
1.94 \quad \text { MED } \\
\text { WT- } 591.1\end{array}$ & $\begin{array}{l}\text { H } \\
\text { DIAN- }\end{array}$ & $\begin{array}{l}3.3 \\
2.00\end{array}$ & $\begin{array}{c}76.6 \\
\text { MODE- }\end{array}$ & $2.00 \quad 20.1$ & gs & 0.56 & CUM & $\begin{array}{l}\text { WT } \\
\text { WT } \\
\text { WT }\end{array}$ & $\begin{array}{l}79.1 \\
13.4 \\
13.4\end{array}$ & $\begin{array}{c}39.3 \\
6.7 \\
20.1\end{array}$ & $\begin{array}{r}47.5 \\
8.1 \\
20.2\end{array}$ & $\begin{array}{r}126.7 \\
21.5 \\
49.7\end{array}$ & $\begin{array}{r}183.5 \\
31.2 \\
80.8\end{array}$ & $\begin{array}{l}68.2 \\
11.6 \\
92.4\end{array}$ & $\begin{array}{r}25.2 \\
4.3 \\
96.7\end{array}$ & $\begin{array}{r}6.6 \\
1.1 \\
97.8\end{array}$ & $\begin{array}{r}12.9 \\
2.2 \\
100.0\end{array}$ \\
\hline
\end{tabular}




$$
\text { ROCSAN REPORT }
$$

$12 / 05 / 89$

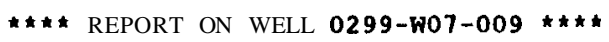

\begin{tabular}{ccccccl} 
& & & & & & \\
DEPTH & \&CAC03 & DM & $\% M U D$ & $\% S A N D$ & $\% G R A V E L$ & CLASS \\
\hdashline 160 & 0.4 & H & -2.3 & 37.3 & 60.4 & sG
\end{tabular} $\begin{array}{ccccccc}160 & 0.4 & \text { H } & 2.3 & 37.3 & 60.4 \text { sG } & \\ \text { SORT }- & \text { N/A MEDIAN- } & \mathbf{- 1 . 0 0} & \text { MODE } & \mathbf{- 2 . 0 0} \text { MEAN- } & \text { N/A }\end{array}$ $\begin{array}{ccccccc}165 & 0.4 & \text { H } & 9.0 & 66.9 & 24.1 & \text { (m) gs } \\ \text { SORT- } & 2.33 & \text { MEDIAN- } & 1.00 & \text { MODE- } & 1.00 & \text { MEAN- }\end{array}$ SPLIT WT- 810.1

$\begin{array}{lllllll}170 & 0.2 & \mathrm{H} & 0.8 & 9.2 & 89.9 & \mathrm{G}\end{array}$ $\begin{array}{lllll}\text { SORT- N/A MEDIAN- } & -2.00 & \text { MODE- } & -2.00 & \text { MEAN- } \\ \text { SPLIT } & \text { WT }=685.7 & \end{array}$

$175-0.0$

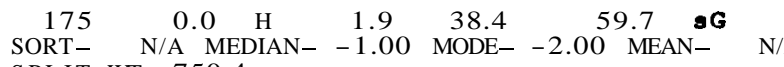

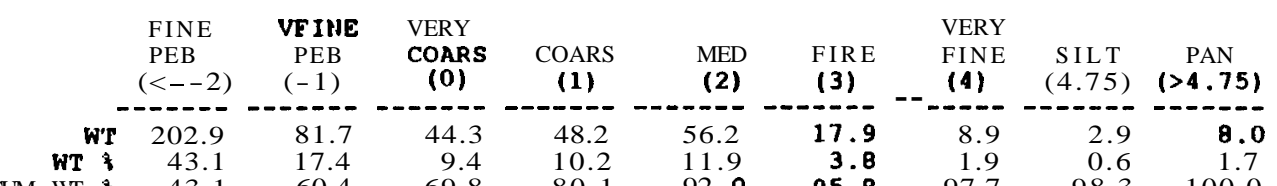

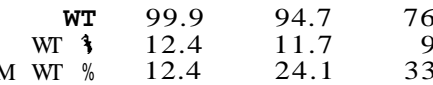

\section{WT $493.9 \quad 121$.} CUL HT

172.2

WT 270

$\begin{array}{lllllll}180 & 0.1 & \mathrm{H} & 2.3 & 43.8 & 53.8\end{array}$ SORT - . N/A MEDIAN- -1.00 MODE- -2.00 MEAN- N/A

$\begin{array}{lrrrrrr}185 & 0.3 & \mathrm{H} & 2.6 & 37.1 & 60.3 & \mathbf{S G}\end{array}$ SPLIT WT- 706.2

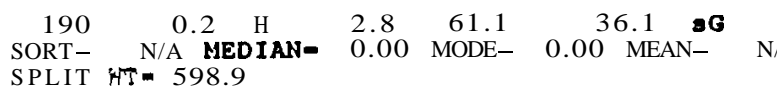

$\begin{array}{ccccccc}195 & 0.3 & \text { H } & \mathbf{1 . 8} & 51.4 & 46.7 & \text { aG } \\ \text { SORT- } & \text { N/A MEDIAN- } & 0.00 & \text { MODE- } & -2.00 & \text { MEAN- } & \text { N/A }\end{array}$

SPLIT WT= 722.1

$\begin{array}{cccccc}200 & 0.2 & \mathrm{H} & 2.3 & 32.5 & 65.2 \text { SG }\end{array}$

SORT - N/A MEDIAI
SPLIT

$\begin{array}{ccccccc}205 & 3.0 & \mathrm{H} & 3.6 & 29.5 & 66.9 & \mathbf{m s G} \\ \text { SORT- } & \text { N/A } & \text { MEDIAN- } & -1.00 & \text { MODE- } & -2.00 & \text { MEAN- }\end{array}$ SPLIT WT- 779.7

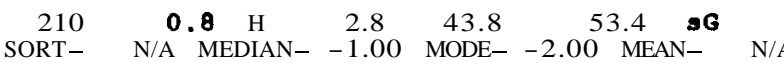
SPLIT WT- 728.3 $\begin{array}{rrr}\text { WT } & 36.1 \\ \text { CUM WT } & 36.1\end{array}$ $\begin{array}{rr}\text { WT } & 286.3 \\ \text { WT } & 35.8 \\ \text { CUM WT } & 35.8\end{array}$

\section{WT $301.8 \quad 123.7$} CUN WT: $\begin{array}{rr}301.8 & 123.7 \\ 42.8 & 17.5 \\ 12.8 & 60.3\end{array}$

\section{พT $104.2 \quad 111.7 \quad 143$.} CUM พт $\begin{array}{rrr}17.4 & 18.7 & 24.0 \\ 17.4 & 36.1 & 60.1\end{array}$

WT 273.0 $\begin{array}{rr}\text { WT } & 38.0 \\ \text { CUM WT } & 38.0\end{array}$

62.7
8.7 46.8

$$
\begin{array}{r}
69.7 \\
9.7 \\
56.4
\end{array}
$$

WT $368.5 \quad 202.9 \quad 104.4$ CUM WT $\quad 42.0 \quad 65.2 \quad 11.9$

$$
\begin{array}{lrrr}
\text { WT } & 265.0 & 255.6 & 99.7
\end{array}
$$
$\begin{array}{rrrr}\text { WT } & 34.1 & 32.9 & 12.8 \\ \text { CUM WT } & 34.1 & 66.9 & 79.7\end{array}$ $\begin{array}{rrrr}\text { WT } & 236.7 & 151.3 & 89.8 \\ \text { WT \% } & 32.6 & 20.8 & 12.4 \\ \text { CUM WT } & 32.6 & 53.4 & 65.8\end{array}$

$\begin{array}{rrrrrr}158.8 & 153.7 & 97.8 & 54.4 & 31.7 & 41.3\end{array}$
$\begin{array}{rrrrrr}19.7 & 19.0 & 12.1 & 6.7 & 3.9 & 5.1 \\ 53.1 & 72.2 & 84.3 & 91.0 & 94.9 & 100.0\end{array}$

$\begin{array}{llllll}13.1 & 10.2 & 5.2 & 3.8 & 1.0 & 4.7\end{array}$

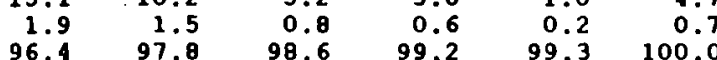
$\begin{array}{llllll}96.4 & 97.8 & 98.6 & 99.2 & 99.3 & 100.0\end{array}$ $\begin{array}{llllll}90.3 & 51.9 & 15.3 & 8.9 & 3.4 & 10.7\end{array}$ $\begin{array}{rrrrrr}12.1 & 6.9 & 2.0 & 1.2 & 0.5 & 1.4 \\ 88.0 & 94.9 & 96.9 & 98.1 & 98.6 & 100.0\end{array}$ $\begin{array}{llllll}57.6 & 99.2 & 79.5 & 26.7 & 6.0 & 12.8\end{array}$ $\begin{array}{rrrrrr}57.6 & 99.2 & 79.5 & 26.7 & 6.0 & 12.8 \\ 7.2 & 12.4 & 9.9 & 3.3 & 0.8 & 1.6\end{array}$ 96.751 .250 .351 .0 $\begin{array}{rrrrrr}6.7 & 51.2 & 58.3 & 31.8 & 7.2 & 11.2 \\ 6.6 & 7.3 & 8.3 & 4.5 & 1.0 & 1.6\end{array}$

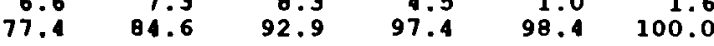
$\begin{array}{llllll}91.2 & 64.4 & 48.2 & 18.2 & 4.6 & 12.1\end{array}$ $\begin{array}{rrrrrr}15.3 & 10.8 & 9.1 & 3.1 & 0.8 & 2.0 \\ 75.3 & 86.1 & 94.2 & 97.2 & 98.0 & 100.0\end{array}$ $\begin{array}{llllll}73.0 & 106.2 & 100.2 & 21.0 & 3.9 & 9.3\end{array}$ $\begin{array}{rrrrrr}10.1 & 14.8 & 13.9 & 2.9 & 0.5 & 1.3 \\ 66.6 & 81.3 & 95.3 & 98.2 & 98.7 & 100.0\end{array}$ 53.8 $\begin{array}{rrrrrr}53.8 & 64.1 & 45.2 & 17.3 & 5.4 & 15.2 \\ 6.1 & 7.3 & 5.2 & 2.0 & 0.6 & 1.7\end{array}$ $\begin{array}{rrrrrr}83.2 & 90.5 & 95.7 & 97.7 & 98.3 & 100.0\end{array}$ $\begin{array}{llllll}44.2 & 38.9 & 28.1 & 18.4 & 7.3 & 20.9\end{array}$ $\begin{array}{rrrrrr}5.7 & 5.0 & 3.6 & 2.4 & 0.9 & 2.7 \\ 05.4 & 90.4 & 94.0 & 96.4 & 97.3 & 100.0\end{array}$ $\begin{array}{llllll}77.2 & 94.6 & 41.1 & 15.5 & 4.5 & 15.7\end{array}$

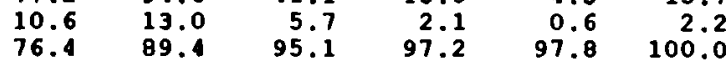


WESTINGIIOUSE HANFORD OPERATIONS SIEVE ANALYSIS

$$
\text { ROCSAN REPORT }
$$

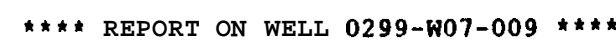

$12 / 05 / 89$

DEPTH \&CACO3 DM IMUD ISAND OGRAVEL CLASS

$\begin{array}{rrrrrrr}215 & 0.7 & \text { H } & -3.2 & -18.8 & -38.1 & \mathbf{s G}\end{array}$

SORT- 1.68 MEDIAN- 0.00 MODE- -1.00 MEAN- -0.21

\begin{tabular}{|c|c|c|c|c|c|c|c|c|}
\hline $\begin{array}{l}\text { FINE } \\
\text { PEB } \\
(<=-2)\end{array}$ & $\begin{array}{c}\text { VFINE } \\
\text { PEB } \\
(-1)\end{array}$ & $\begin{array}{l}\text { VERY } \\
\text { COARS } \\
\text { (0) }\end{array}$ & $\begin{array}{c}\text { COARS } \\
\text { (1) }\end{array}$ & $\begin{array}{l}\text { MED } \\
\text { (2) }\end{array}$ & $\begin{array}{l}\text { FINE } \\
\text { (3) }\end{array}$ & $\begin{array}{l}\text { VERY } \\
\text { FINE } \\
\text { (4) }\end{array}$ & $\begin{array}{c}\text { SILT } \\
(4.75)\end{array}$ & $\begin{array}{c}\text { PAN } \\
(>4.75)\end{array}$ \\
\hline $\begin{array}{r}61.4 \\
8.8\end{array}$ & $\begin{array}{r}204.8 \\
29.3\end{array}$ & $\begin{array}{r}162.3 \\
23.2\end{array}$ & $\begin{array}{r}111.1 \\
15.9\end{array}$ & $\begin{array}{l}77.0 \\
11.0\end{array}$ & $\begin{array}{r}41.2 \\
5.9\end{array}$ & $\begin{array}{r}19.3 \\
2.8\end{array}$ & $\begin{array}{r}6.3 \\
0.9\end{array}$ & $\begin{array}{l}5.9 \\
2.3\end{array}$ \\
\hline
\end{tabular}

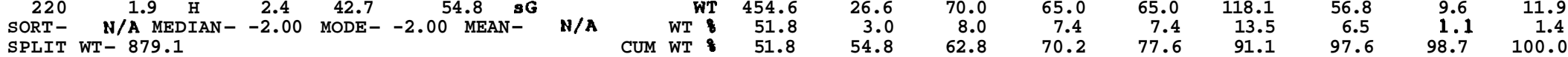

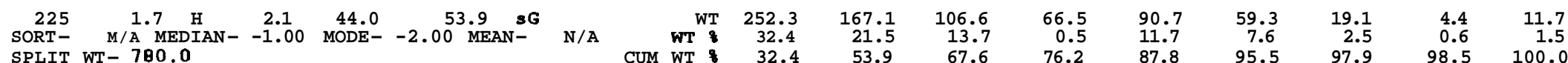

\section{$\begin{array}{ccccccc}230 & 1.1 & H & 2.4 & 48.3 & 49.3 \quad \mathbf{~ s G} & \\ \text { SORT- } & \text { N/A }\end{array}$}

WT 223.3181

$\begin{array}{lllll}\text { SORT- N/A MEDIAN= } & 0.00 \text { MODE- }-2.00 \text { MEAN- N/A CUM WT } 27.2 \\ \text { SPLIT WT- } 821.8 & & & \text { WU }\end{array}$

$\begin{array}{lllllllll}235 & 0.8 \mathrm{H} & 2.7 & 60.5 & 36.8 \text { AG } & \text { WT } & 215.1 & 87.3\end{array}$

$\begin{array}{rrr}11.3 & 114.6 & 93 \\ 22.1 & 14.0\end{array}$

$93.1 \quad 117$

17.253 .3

$6.8 \quad 17$
$\begin{array}{llll}\text { SORT- N/A HEDIAN- } & 1.00 \text { MODE- }-2.00 \text { MEAN- N/A } \\ \text { SPLIT WT- } 819.9 & & & \text { WUM WT: }\end{array}$

$97.2 \quad 101.1 \quad 135.0 \quad 117.4 \quad 45.6 \quad 11.1010 .9$

WT 436.9

36.8

11.

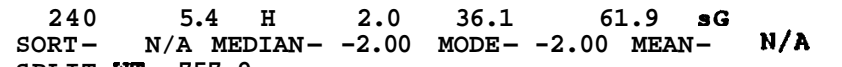

$\begin{array}{rr}436.9 \\ \text { WT } & 56.6\end{array}$

41.4

78.

IT WT- 757.9

WT 451.4 B1.0

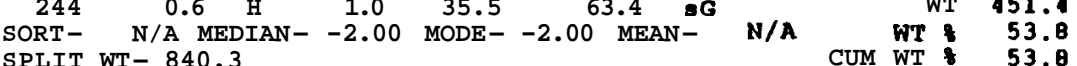

81.0
9.7
63.4

69.0

$\begin{array}{rrrrrr}12.3 & 16.5 & 14.3 & 5.6 & 11.1 & 10.9 \\ 61.0 & 77.5 & 91.8 & 97.4 & 1.4 & 1.3\end{array}$

SPLIT WT- 840.3 


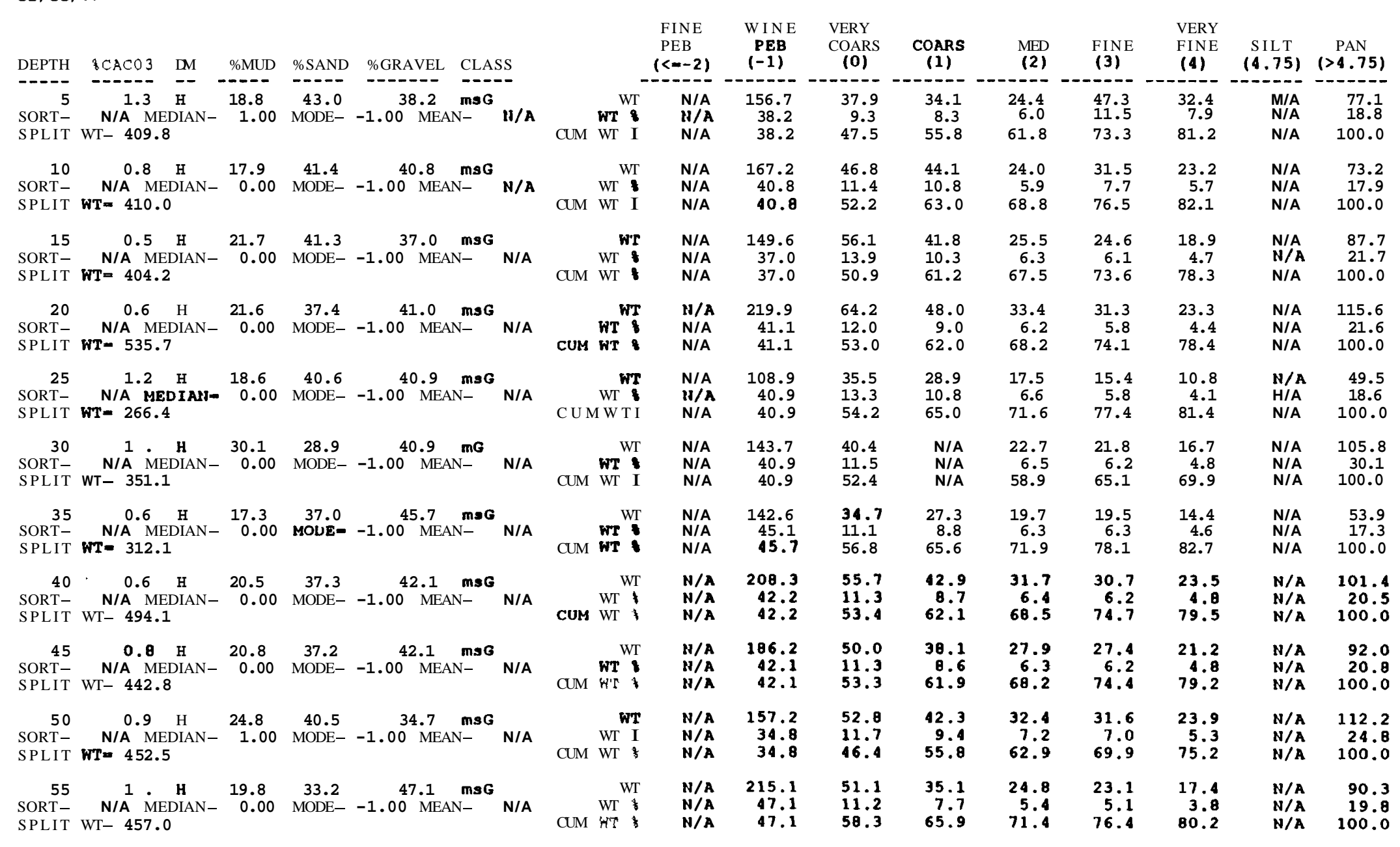




$$
\text { ROCSAN REPORT }
$$

$12 / 11 / 89$

$$
\star \star \star \star \text { REPORT ON WELL 0299-WOB-001 } \star \star \star \star ~
$$

\begin{tabular}{|c|c|c|c|c|c|c|c|c|c|c|c|c|c|c|c|c|c|c|}
\hline DEPTH & $8 \mathrm{CACO} 3$ & DM & ZMUD & \&SAND & $\because$ GRAVEL & CLAS & & & & $\begin{array}{l}\text { FINE } \\
\text { PEB } \\
(<--2)\end{array}$ & $\begin{array}{l}\text { WINE } \\
\text { PEB } \\
(-1)\end{array}$ & $\begin{array}{l}\text { VERY } \\
\text { COARS } \\
(0)\end{array}$ & $\begin{array}{l}\text { COARS } \\
\text { (1) }\end{array}$ & $\begin{array}{l}\text { MED } \\
\text { (2) }\end{array}$ & $\begin{array}{l}\text { FINE } \\
\text { (3) }\end{array}$ & $\begin{array}{l}\text { VERY } \\
\text { FINE } \\
\text { (4) }\end{array}$ & $\begin{array}{l}\text { SILT } \\
(4.75)\end{array}$ & $\begin{array}{c}\text { PAN } \\
(>4.75)\end{array}$ \\
\hline $\begin{array}{r}60 \\
\text { SORT- } \\
\text { SPLIT }\end{array}$ & $\begin{array}{c}1.3 \\
\text { N/A MED } \\
\text { WT }=442.8\end{array}$ & $\begin{array}{l}\mathrm{H} \\
8\end{array}$ & $\begin{array}{r}25.3 \\
1.00\end{array}$ & $\begin{array}{r}39.0 \\
\text { MODE- }\end{array}$ & $\begin{array}{c}35.7 \\
-1.00 \mathrm{MEAN}\end{array}$ & ms G & N/A & CUM & $\begin{array}{l}\text { WT } \\
\text { WT } \\
\text { WT }\end{array}$ & $\begin{array}{l}\text { N/A } \\
N / A \\
\text { N/A }\end{array}$ & $\begin{array}{r}158.1 \\
35.7 \\
35.7\end{array}$ & $\begin{array}{l}59.3 \\
13.4 \\
49.1\end{array}$ & $\begin{array}{r}40.6 \\
9.2 \\
58.3\end{array}$ & $\begin{array}{r}28.5 \\
6.4 \\
64.7\end{array}$ & $\begin{array}{r}25.2 \\
5.7 \\
70.4\end{array}$ & $\begin{array}{r}19.2 \\
4.3 \\
74.7\end{array}$ & $\begin{array}{l}\text { N/A } \\
\text { N/A } \\
\text { N/A }\end{array}$ & $\begin{array}{r}111.9 \\
25.3 \\
100.0\end{array}$ \\
\hline $\begin{array}{r}65 \\
\text { SORT- } \\
\text { SPLIT }\end{array}$ & $\begin{array}{c}1.4 \\
\text { N/A MED } \\
\text { WT- } 444.5\end{array}$ & $\begin{array}{l}\text { H } \\
\text { DIAN- } \\
5\end{array}$ & $\begin{array}{c}24.8 \\
1.00\end{array}$ & $\begin{array}{r}39.8 \\
\text { MODE }=\end{array}$ & $\begin{array}{r}35.4 \\
-1.00 \mathrm{MEAN}\end{array}$ & $\mathbf{m s}^{\mathbf{m g} G}$ & $\mathrm{~N} / \mathrm{A}$ & CUM & $\begin{array}{l}\text { WT } \\
\text { WT \% } \\
\text { WT }\end{array}$ & $\begin{array}{l}\text { N/A } \\
\text { N/A } \\
\text { N/A }\end{array}$ & $\begin{array}{r}157.6 \\
35.5 \\
35.5\end{array}$ & $\begin{array}{l}55.8 \\
12.6 \\
48.0\end{array}$ & $\begin{array}{r}41.2 \\
9.3 \\
57.3\end{array}$ & $\begin{array}{r}30.9 \\
7.0 \\
64.2\end{array}$ & $\begin{array}{r}28.0 \\
6.3 \\
70.5\end{array}$ & $\begin{array}{r}21.0 \\
4.7 \\
75.2\end{array}$ & $\begin{array}{l}\text { N/A } \\
\text { H/A } \\
\text { N/A }\end{array}$ & $\begin{array}{r}110.1 \\
24.8 \\
100.0\end{array}$ \\
\hline $\begin{array}{r}70 \\
\text { SORT- } \\
\text { SPLIT }\end{array}$ & $\begin{array}{c}1.5 \\
\text { N/A MED } \\
\text { WT- } 449.7\end{array}$ & ${ }_{7}^{\mathrm{H}}$ & $\begin{array}{c}19.7 \\
0.00\end{array}$ & $\begin{array}{r}38.7 \\
\text { MODE }\end{array}$ & $\begin{array}{r}41.6 \\
-1.00 \text { MEAN }\end{array}$ & maG & N/A & CUM & $\begin{array}{l}\text { WT } \\
\text { WT } \\
\text { WT }\end{array}$ & $\begin{array}{l}\text { N/A } \\
\text { N/A } \\
\text { H/A }\end{array}$ & $\begin{array}{r}187.0 \\
41.6 \\
41.6\end{array}$ & $\begin{array}{l}60.6 \\
13.5 \\
55.1\end{array}$ & $\begin{array}{r}40.4 \\
9.0 \\
64.1\end{array}$ & $\begin{array}{r}29.7 \\
6.6 \\
70.7\end{array}$ & $\begin{array}{r}25.4 \\
5.7 \\
76.3\end{array}$ & $\begin{array}{r}18.1 \\
4.0 \\
80.4\end{array}$ & $\begin{array}{l}\mathrm{N} / \mathrm{A} \\
\mathrm{A} \\
\mathrm{H} / \mathrm{A}\end{array}$ & $\begin{array}{r}88.4 \\
19.7 \\
100.0\end{array}$ \\
\hline $\begin{array}{r}75 \\
\text { SORT- } \\
\text { SPLIT }\end{array}$ & $\begin{array}{c}2.6 \\
\text { N/A MED } \\
\text { WT- } 442.9\end{array}$ & $\begin{array}{l}\text { H } \\
\text { DIAN- } \\
9\end{array}$ & $\begin{array}{c}25.0 \\
0.00\end{array}$ & $\begin{array}{r}33.2 \\
\text { MODE }\end{array}$ & $\begin{array}{c}41.9 \\
-1.00 \text { MEAN }\end{array}$ & $\underset{d=}{\mathbf{m g G}}$ & N/A & CUM & $\begin{array}{l}\text { WT } \\
\text { WT } \\
\text { WT }\end{array}$ & $\begin{array}{l}\text { N/A } \\
\text { N/A } \\
\text { N/A }\end{array}$ & $\begin{array}{r}185.5 \\
41.9 \\
41.9\end{array}$ & $\begin{array}{l}58.2 \\
13.1 \\
55.0\end{array}$ & $\begin{array}{r}16.2 \\
3.7 \\
58.7\end{array}$ & $\begin{array}{r}31.6 \\
7.1 \\
65.8\end{array}$ & $\begin{array}{r}24.9 \\
5.6 \\
71.5\end{array}$ & $\begin{array}{r}15.9 \\
3.6 \\
75.0\end{array}$ & $\begin{array}{l}\text { N/A } \\
\text { H/A } \\
\text { N/A }\end{array}$ & $\begin{array}{r}110.5 \\
25.0 \\
100.0\end{array}$ \\
\hline $\begin{array}{r}\mathbf{8 0} \\
\text { SORT- } \\
\text { SPLIT }\end{array}$ & $\begin{array}{c}5.0 \\
\text { N/A MED } \\
\text { WT }=361 .\end{array}$ & $\begin{array}{l}\text { H } \\
4 \\
4 \text { IAN- }\end{array}$ & $\begin{array}{c}52.8 \\
5.00\end{array}$ & $\begin{array}{r}26.4 \\
\text { MODE }\end{array}$ & $\begin{array}{c}20.8 \\
5.00 \text { MEAN }\end{array}$ & gsm & $\mathrm{N} / \mathrm{A}$ & CUM & $\begin{array}{l}\text { WT } \\
\text { WT } \\
\text { WT }\end{array}$ & $\begin{array}{l}\text { N/A } \\
\text { N/A } \\
\text { N/A }\end{array}$ & $\begin{array}{l}75.0 \\
20.8 \\
20.8\end{array}$ & $\begin{array}{r}28.7 \\
7.9 \\
28.7\end{array}$ & $\begin{array}{r}16.8 \\
4.7 \\
33.4\end{array}$ & $\begin{array}{r}12.5 \\
3.5 \\
36.8\end{array}$ & $\begin{array}{r}17.5 \\
4.8 \\
41.7\end{array}$ & $\begin{array}{r}20.0 \\
5.5 \\
47.2\end{array}$ & $\begin{array}{l}\text { N/A } \\
\text { H/A } \\
\text { N/A }\end{array}$ & $\begin{array}{r}190.8 \\
52.8 \\
100.0\end{array}$ \\
\hline $\begin{array}{r}86 \\
\text { SORT- } \\
\text { SPLIT }\end{array}$ & $\begin{array}{c}3.8 \\
\text { N/A MEL } \\
\text { WT- } 313 .\end{array}$ & $\begin{array}{l}\underset{D}{D I A N-} \\
5\end{array}$ & $\begin{array}{c}76.9 \\
5.00\end{array}$ & $\begin{array}{r}15.5 \\
\text { MODE }\end{array}$ & $5.00^{7.6}$ & $(g) M$ & ${ }_{N}^{M} / \mathbf{A}$ & CUM & $\begin{array}{l}\text { WT } \\
\text { WT } \\
\text { WT }\end{array}$ & $\begin{array}{l}\text { N/A } \\
\text { N/A } \\
\text { N/A }\end{array}$ & $\begin{array}{r}23.8 \\
7.6 \\
7.6\end{array}$ & $\begin{array}{r}8.8 \\
2.8 \\
10.4\end{array}$ & $\begin{array}{r}5.1 \\
1.6 \\
12.0\end{array}$ & $\begin{array}{r}4.6 \\
1.5 \\
13.5\end{array}$ & $\begin{array}{r}11.4 \\
3.6 \\
17.1\end{array}$ & $\begin{array}{r}18.8 \\
6.0 \\
23.1\end{array}$ & $\begin{array}{l}\text { N/A } \\
H / A \\
\text { N/A }\end{array}$ & $\begin{array}{r}241.1 \\
76.9 \\
100.0\end{array}$ \\
\hline $\begin{array}{r}91 \\
\text { SORT- } \\
\text { SPLIT }\end{array}$ & $\begin{array}{c}20.8 \\
\text { N/A MED } \\
\text { WT- } 364.7\end{array}$ & $\begin{array}{l}\mathrm{H} \\
7\end{array}$ & $\begin{array}{c}39.6 \\
3.00\end{array}$ & $\begin{array}{r}26.4 \\
\text { MODE }\end{array}$ & $5.00^{34.1}$ & $m_{-}$ & $\mathbf{N} / \mathbf{A}$ & CUM & $\begin{array}{l}\text { WT } \\
\text { WT } \\
\text { WT }\end{array}$ & $\begin{array}{l}N / A \\
N / A \\
N / A\end{array}$ & $\begin{array}{r}124.2 \\
34.1 \\
34.1\end{array}$ & $\begin{array}{r}15.8 \\
4.3 \\
38.4\end{array}$ & $\begin{array}{r}10.6 \\
2.9 \\
41.3\end{array}$ & $\begin{array}{r}10.9 \\
3.0 \\
14.3\end{array}$ & $\begin{array}{r}23.4 \\
6.4 \\
50.7\end{array}$ & $\begin{array}{r}35.4 \\
9.7 \\
60.4\end{array}$ & $\begin{array}{l}\text { N/A } \\
\text { N/A } \\
\text { N/A }\end{array}$ & $\begin{array}{r}144.4 \\
39.6 \\
100.0\end{array}$ \\
\hline $\begin{array}{r}95 \\
\text { SORT- } \\
\text { SPLIT }\end{array}$ & $\begin{array}{c}21.1 \\
\text { N/A MED } \\
\text { WT }=420 .\end{array}$ & $\begin{array}{l}\mathrm{H} \\
\mathbf{3}\end{array}$ & $\begin{array}{c}26.0 \\
2.00\end{array}$ & $\begin{array}{r}41.9 \\
\text { MODE }\end{array}$ & $\begin{array}{c}30.2 \\
-1.00 \text { MEAN }\end{array}$ & ms G & N/A & CUM & $\begin{array}{l}\text { WT } \\
\text { WT } \\
\text { WT }\end{array}$ & $\begin{array}{l}\text { N/A } \\
\text { N/A } \\
\text { N/A }\end{array}$ & $\begin{array}{r}126.7 \\
30.2 \\
30.2\end{array}$ & $\begin{array}{l}41.0 \\
10.0 \\
40.1\end{array}$ & $\begin{array}{r}29.6 \\
7.0 \\
47.1\end{array}$ & $\begin{array}{r}32.7 \\
7.8 \\
54.9\end{array}$ & $\begin{array}{r}40.0 \\
9.5 \\
64.4\end{array}$ & $\begin{array}{r}31.8 \\
7.6 \\
72.0\end{array}$ & $\begin{array}{l}N / A \\
N / A \\
N / A\end{array}$ & $\begin{array}{r}117.6 \\
28.0 \\
100.0\end{array}$ \\
\hline $\begin{array}{r}100 \\
\text { SORT- } \\
\text { SPLIT }\end{array}$ & $\begin{array}{c}12.9 \\
\text { N/A MEI } \\
\text { WT }=426 .\end{array}$ & $\begin{array}{l}\text { H } \\
2 \\
2 \text { IAN- }\end{array}$ & $\begin{array}{l}21.6 \\
0.00\end{array}$ & $\begin{array}{r}36.4 \\
\text { MODE- }\end{array}$ & $\begin{array}{c}42.0 \\
-1.00 \mathrm{MEAN}\end{array}$ & $\begin{array}{l}\mathrm{msG} \\
\mathrm{j}-\end{array}$ & $\mathrm{N} / \mathrm{A}$ & CUM & $\begin{array}{l}\text { WT } \\
\text { WT } \\
\text { WT }\end{array}$ & $\begin{array}{l}\text { N/A } \\
\text { N/A } \\
\text { N/A }\end{array}$ & $\begin{array}{r}179.1 \\
42.0 \\
42.0\end{array}$ & $\begin{array}{l}57.5 \\
13.5 \\
55.5\end{array}$ & $\begin{array}{r}32.8 \\
7.7 \\
63.2\end{array}$ & $\begin{array}{r}20.4 \\
4.8 \\
68.0\end{array}$ & $\begin{array}{r}26.1 \\
6.1 \\
74.1\end{array}$ & $\begin{array}{r}18.4 \\
4.3 \\
78.4\end{array}$ & $\begin{array}{l}N / A \\
N / A \\
N / A\end{array}$ & $\begin{array}{r}92.0 \\
21.6 \\
100.0\end{array}$ \\
\hline $\begin{array}{r}105 \\
\text { SORT- } \\
\text { SPLIT }\end{array}$ & $\begin{array}{c}8.4 \\
\text { N/A MEI } \\
\text { WT- } 412.8\end{array}$ & $\begin{array}{l}\text { H } \\
8\end{array}$ & $\begin{array}{r}29.5 \\
2.00\end{array}$ & $\begin{array}{r}51.2 \\
\text { MODE- }\end{array}$ & $5.00^{19.4} \mathrm{MEAN}$ & $g m s$ & $\mathrm{~N} / \mathrm{A}$ & CUM & $\begin{array}{l}\text { WT } \\
\text { WT } \\
\text { WT }\end{array}$ & $\begin{array}{l}\text { N/A } \\
\text { N/A } \\
\text { N/A }\end{array}$ & $\begin{array}{l}79.9 \\
19.4 \\
19.4\end{array}$ & $\begin{array}{r}37.8 \\
9.2 \\
28.5\end{array}$ & $\begin{array}{l}46.0 \\
11.2 \\
39.7\end{array}$ & $\begin{array}{l}49.5 \\
12.0 \\
51.7\end{array}$ & $\begin{array}{r}38.8 \\
9.4 \\
61.1\end{array}$ & $\begin{array}{r}39.1 \\
9.5 \\
70.5\end{array}$ & $\begin{array}{l}\text { N/A } \\
\text { N/A } \\
\text { N/A }\end{array}$ & $\begin{array}{r}121.6 \\
29.5 \\
100.0\end{array}$ \\
\hline $\begin{array}{r}110 \\
\text { SORT- } \\
\text { SPLIT }\end{array}$ & $\begin{array}{c}9.1 \\
\text { N/A MED } \\
\text { WT- } 370.2\end{array}$ & $\begin{array}{l}\text { H } \\
\text { DIAN- } \\
2\end{array}$ & $\begin{array}{l}\mathbf{3 8 . 0} \\
\mathbf{3 . 0 0}\end{array}$ & $\begin{array}{r}56.1 \\
\text { MODE- }\end{array}$ & $5.00^{5.9}$ & $(g) \mathrm{m}$ & $\begin{array}{l}\mathrm{mS} \\
\mathrm{N} / \mathrm{A}\end{array}$ & CUM & $\begin{array}{l}\text { WT } \\
\text { WT } \\
\text { WT }\end{array}$ & $\begin{array}{l}\text { N/A } \\
\text { N/A } \\
\text { N/A }\end{array}$ & $\begin{array}{r}21.9 \\
5.9 \\
5.9\end{array}$ & $\begin{array}{r}21.8 \\
5.9 \\
11.8\end{array}$ & $\begin{array}{l}42.3 \\
11.4 \\
23.2\end{array}$ & $\begin{array}{l}51.3 \\
13.9 \\
37.1\end{array}$ & $\begin{array}{l}48.1 \\
13.0 \\
50.1\end{array}$ & $\begin{array}{l}44.1 \\
11.9 \\
62.0\end{array}$ & $\begin{array}{l}\text { N/A } \\
\text { N/A } \\
\text { N/A }\end{array}$ & $\begin{array}{r}140.8 \\
38.0 \\
100.0\end{array}$ \\
\hline
\end{tabular}


WESTINGHOUSE HANFORD OPERATIONS SIEVE ANALYSIS ROCSAN REPORT

$12 / 11 / 89$

\begin{tabular}{|c|c|c|c|c|c|c|c|c|c|c|c|c|c|c|c|c|c|c|}
\hline DEPTH & $8 \mathrm{CACO} 3$ & DM & 8MUD & OSAND & \%GRAVEL & CLASS & & & & $\begin{array}{l}\text { FINE } \\
\text { PEB } \\
(<=-2)\end{array}$ & $\begin{array}{c}\text { VEINE } \\
\text { PEB } \\
(-1)\end{array}$ & $\begin{array}{c}\text { VERY } \\
\text { COARS } \\
(0)\end{array}$ & $\begin{array}{c}\text { COARS } \\
(1)\end{array}$ & $\begin{array}{l}\text { MED } \\
(2)\end{array}$ & $\begin{array}{l}\text { FINE } \\
\text { (3) }\end{array}$ & $\begin{array}{l}\text { VERY } \\
\text { FINE } \\
\text { (4) }\end{array}$ & $\begin{array}{l}\text { SILT } \\
(4.75)\end{array}$ & $\begin{array}{c}\text { PAN } \\
(>4.75)\end{array}$ \\
\hline $\begin{array}{r}115 \\
\text { SORT- } \\
\text { SPLIT }\end{array}$ & $\begin{array}{c}13.2 \\
\text { N/A MED } \\
\text { WT- } 339.6\end{array}$ & $\begin{array}{l}\text { H } \\
\text { DIAN- }\end{array}$ & $\begin{array}{l}30.0 \\
3.00\end{array}$ & $\begin{array}{l}60.0 \\
\text { MODE- }\end{array}$ & $5.00^{10.0} \mathrm{MEAN}$ & gms & N/A & CUM & $\begin{array}{l}\text { WT } \\
\text { WT } \\
\text { WT }\end{array}$ & $\begin{array}{l}\text { N/A } \\
\text { N/A } \\
\text { N/A }\end{array}$ & $\begin{array}{l}33.9 \\
10.0 \\
10.0\end{array}$ & $\begin{array}{r}22.6 \\
6.7 \\
16.6\end{array}$ & $\begin{array}{l}41.7 \\
12.3 \\
28.9\end{array}$ & $\begin{array}{l}55.3 \\
16.3 \\
45.2\end{array}$ & $\begin{array}{l}44.1 \\
13.0 \\
58.2\end{array}$ & $\begin{array}{l}40.0 \\
11.8 \\
70.0\end{array}$ & $\begin{array}{l}1 / A \\
N / A \\
N / A\end{array}$ & $\begin{array}{r}102.0 \\
30.0 \\
100.0\end{array}$ \\
\hline $\begin{array}{r}120 \\
\text { SORT- } \\
\text { SPLIT }\end{array}$ & $\begin{array}{c}5.1 \\
\text { N/A MED } \\
W T=340.8\end{array}$ & $\begin{array}{l}\mathrm{H} \\
\text { DIAN- } \\
3\end{array}$ & $\begin{array}{c}18.8 \\
2.00\end{array}$ & $\begin{array}{r}76.8 \\
\text { MODE- }\end{array}$ & $2.00 \stackrel{4.4}{\text { MEAN }}$ & $(\mathrm{m}) \mathrm{s}$ & $S_{N / A}$ & $\mathrm{CUM}$ & $\begin{aligned} & \text { WT } \\
& \text { WT } \\
& \text { W T O }\end{aligned}$ & $\begin{array}{l}\text { N/A } \\
\text { N/A } \\
\text { N/A }\end{array}$ & $\begin{array}{c}15.0 \\
4.4 \\
4.4\end{array}$ & $\begin{array}{r}13.4 \\
3.9 \\
8.3\end{array}$ & $\begin{array}{l}58.7 \\
17.2 \\
25.6\end{array}$ & $\begin{array}{r}115.2 \\
33.8 \\
59.4\end{array}$ & $\begin{array}{l}48.4 \\
14.2 \\
73.6\end{array}$ & $\begin{array}{r}25.9 \\
7.6 \\
81.2\end{array}$ & $\begin{array}{l}\text { N/A } \\
\text { N/A } \\
\text { N/A }\end{array}$ & $\begin{array}{r}64.1 \\
18.8 \\
100.0\end{array}$ \\
\hline $\begin{array}{r}125 \\
\text { SORT- } \\
\text { SPLIT }\end{array}$ & $\begin{array}{c}2.9 \\
\text { N/A MED } \\
\text { WT }=373.1\end{array}$ & $\begin{array}{l}\mathrm{H} \\
\text { DIAN- } \\
1\end{array}$ & $\begin{array}{c}12.8 \\
2.00\end{array}$ & $\begin{array}{r}86.2 \\
\text { MODE- }\end{array}$ & $2.00 \stackrel{1.0}{\text { MEAN }-}$ & $(m) S$ & $s_{\mathrm{N} / \mathrm{A}}$ & CUM & $\begin{array}{l}\text { WT } \\
\text { WT } \\
\text { WT }\end{array}$ & $\begin{array}{l}N / A \\
N / A \\
N / A\end{array}$ & $\begin{array}{l}3.7 \\
1.0 \\
1.0\end{array}$ & $\begin{array}{r}14.5 \\
3.9 \\
4.9\end{array}$ & $\begin{array}{r}112.0 \\
30.0 \\
34.9\end{array}$ & $\begin{array}{r}130.4 \\
35.0 \\
69.9\end{array}$ & $\begin{array}{l}45.0 \\
12.1 \\
81.9\end{array}$ & $\begin{array}{r}19.7 \\
5.3 \\
87.2\end{array}$ & $\begin{array}{l}N / A \\
N / A \\
N / A\end{array}$ & $\begin{array}{r}47.7 \\
12.8 \\
100.0\end{array}$ \\
\hline $\begin{array}{r}130 \\
\text { SORT- } \\
\text { SPLIT }\end{array}$ & $\begin{array}{c}2.7 \\
\text { N/A MED } \\
\text { WT- } 369.0\end{array}$ & $\begin{array}{l}\mathrm{H} \\
\text { DIAN- } \\
0\end{array}$ & $\begin{array}{c}16.2 \\
2.00\end{array}$ & $\begin{array}{r}82.9 \\
\text { MODE- }\end{array}$ & $2.00^{0.9}$ MEAN & (m) $s$ & $S_{N / A}$ & CUM & $\begin{array}{l}\text { WT } \\
\text { WT } \\
\text { WT }\end{array}$ & $\begin{array}{l}\text { N/A } \\
\text { II/A } \\
\text { N/A }\end{array}$ & $\begin{array}{l}3.2 \\
0.9 \\
0.9\end{array}$ & $\begin{array}{l}8.1 \\
2.2 \\
3.1\end{array}$ & $\begin{array}{l}67.5 \\
18.3 \\
21.4\end{array}$ & $\begin{array}{r}160.3 \\
43.5 \\
64.8\end{array}$ & $\begin{array}{l}49.2 \\
13.3 \\
78.2\end{array}$ & $\begin{array}{r}20.7 \\
5.6 \\
83.8\end{array}$ & $\begin{array}{l}N / A \\
N / A \\
N / A\end{array}$ & $\begin{array}{r}59.8 \\
16.2 \\
100.0\end{array}$ \\
\hline $\begin{array}{r}135 \\
\text { SORT- } \\
\text { SPLIT }\end{array}$ & $\begin{array}{c}1.7 \\
\text { N/A MED } \\
\text { WT- } 401.9\end{array}$ & $\begin{array}{l}\text { D } \\
\text { DIAN- } \\
9\end{array}$ & $\begin{array}{l}9.3 \\
\quad 2.00\end{array}$ & $\begin{array}{r}89.8 \\
\text { MODE- }\end{array}$ & $2.00^{0.8} \mathrm{MEAN}$ & & N/A & CUM & ${ }_{\mathrm{WT}}^{\mathrm{WT}}$ & $\begin{array}{l}\text { N/A } \\
\text { N/A } \\
\text { N/A }\end{array}$ & $\begin{array}{l}3.4 \\
0.9 \\
0.9\end{array}$ & $\begin{array}{l}1.8 \\
0.5 \\
1.3\end{array}$ & $\begin{array}{r}36.5 \\
9.1 \\
10.4\end{array}$ & $\begin{array}{r}208.6 \\
51.9 \\
62.3\end{array}$ & $\begin{array}{l}95.7 \\
23.8 \\
86.1\end{array}$ & $\begin{array}{r}18.5 \\
4.6 \\
90.7\end{array}$ & $\begin{array}{l}N / A \\
N / A \\
N / A\end{array}$ & $\begin{array}{r}37.5 \\
9.3 \\
100.0\end{array}$ \\
\hline $\begin{array}{r}140 \\
\text { SORT- } \\
\text { SPIIT }\end{array}$ & $\begin{array}{c}1.6 \\
\text { N/A MED } \\
\text { WT }=375.7\end{array}$ & $\begin{array}{l}\text { H } \\
\text { DIAN- } \\
7\end{array}$ & $\begin{array}{c}15.9 \\
2.00\end{array}$ & $\begin{array}{r}84.1 \\
\text { MODE- }\end{array}$ & $2.00^{0.0}$ MEAN & $(m) s$ & $\mathbf{s}_{\mathrm{N} / \mathrm{A}}$ & CUM & $\begin{array}{l}\text { WT } \\
\text { WT }\end{array}$ & $\begin{array}{l}\text { N/A } \\
\text { N/A } \\
\text { N/A }\end{array}$ & $\begin{array}{l}0.0 \\
0.0 \\
0.0\end{array}$ & $\begin{array}{l}2.0 \\
0.5 \\
0.5\end{array}$ & $\begin{array}{l}54.2 \\
14.4 \\
15.0\end{array}$ & $\begin{array}{r}179.6 \\
47.8 \\
62.7\end{array}$ & $\begin{array}{l}63.2 \\
16.8 \\
79.6\end{array}$ & $\begin{array}{r}17.2 \\
4.6 \\
84.1\end{array}$ & $\begin{array}{l}N / A \\
N / A \\
N / A\end{array}$ & $\begin{array}{r}59.6 \\
15.9 \\
100.0\end{array}$ \\
\hline $\begin{array}{r}145 \\
\text { SORT- } \\
\text { SPLIT }\end{array}$ & $\begin{array}{c}1.3 \\
\text { N/A MED } \\
\text { WT- } 423.2\end{array}$ & $\begin{array}{l}\text { H } \\
2\end{array}$ & $\begin{array}{c}14.4 \\
2.00\end{array}$ & $\begin{array}{c}03.4 \\
\text { MODE- }\end{array}$ & $2.00^{2.2}$ MEAN & (m) $s$ & $\mathbf{s}_{\mathrm{N} / \mathrm{A}}$ & CUM & WT & $\begin{array}{l}\text { N/A } \\
\text { N/A } \\
\text { N/A }\end{array}$ & $\begin{array}{l}9.5 \\
2.2 \\
2.2\end{array}$ & $\begin{array}{r}17.2 \\
4.1 \\
6.3\end{array}$ & $\begin{array}{l}85.4 \\
20.2 \\
26.5\end{array}$ & $\begin{array}{r}172.4 \\
40.7 \\
67.2\end{array}$ & $\begin{array}{l}59.4 \\
14.0 \\
81.3\end{array}$ & $\begin{array}{r}18.5 \\
4.4 \\
85.6\end{array}$ & $\begin{array}{l}\text { N/A } \\
\text { N/A } \\
\text { N/A }\end{array}$ & $\begin{array}{r}60.0 \\
14.4 \\
100.0\end{array}$ \\
\hline $\begin{array}{r}150 \\
\text { SORT- } \\
\text { SPLIT }\end{array}$ & $\begin{array}{c}1.7 \\
N / A \text { MED } \\
\text { WT } 377.3\end{array}$ & $\begin{array}{l}\mathrm{H} \\
\text { DIAN- } \\
3\end{array}$ & $\begin{array}{r}19.7 \\
-\quad 2.00\end{array}$ & $\begin{array}{r}79.1 \\
\text { MODE- }\end{array}$ & $2.00 \quad 1.2$ & (m) $s$ & $s_{N / A}$ & CUM & $\begin{array}{l}\text { WT } \\
\text { WT } \\
\text { WT }\end{array}$ & $\begin{array}{l}\text { N/A } \\
\text { N/A } \\
\text { N/A }\end{array}$ & $\begin{array}{l}4.7 \\
1.3 \\
1.3\end{array}$ & $\begin{array}{l}6.4 \\
1.7 \\
3.0\end{array}$ & $\begin{array}{r}37.3 \\
9.9 \\
12.8\end{array}$ & $\begin{array}{r}155.5 \\
41.2 \\
54.1\end{array}$ & $\begin{array}{l}79.3 \\
21.0 \\
75.1\end{array}$ & $\begin{array}{r}19.8 \\
5.3 \\
80.3\end{array}$ & $\begin{array}{l}\text { N/A } \\
\text { N/A } \\
\text { N/A }\end{array}$ & $\begin{array}{r}74.2 \\
19.7 \\
100.0\end{array}$ \\
\hline $\begin{array}{r}155 \\
\text { SORT- } \\
\text { SPLIT }\end{array}$ & $\begin{array}{c}1.1 \\
\text { N/A MED } \\
\text { WT- } 473.4\end{array}$ & $\begin{array}{l}\text { H } \\
\text { DIAN- } \\
4\end{array}$ & $\begin{array}{c}16.1 \\
1.00\end{array}$ & $\begin{array}{r}50.7 \\
\text { MODE- }\end{array}$ & $-1.00 \mathrm{MEAN}-$ & mag & $\mathrm{N} / \mathrm{A}$ & CUM & $\begin{array}{l}\text { WT } \\
\text { WT }\end{array}$ & $\begin{array}{l}\text { N/A } \\
\text { N/A } \\
\text { N/A }\end{array}$ & $\begin{array}{r}157.0 \\
33.2 \\
33.2\end{array}$ & $\begin{array}{r}37.3 \\
7.9 \\
41.1\end{array}$ & $\begin{array}{l}68.1 \\
14.4 \\
55.4\end{array}$ & $\begin{array}{l}74.5 \\
15.7 \\
71.2\end{array}$ & $\begin{array}{r}41.0 \\
8.7 \\
79.8\end{array}$ & $\begin{array}{r}19.0 \\
4.0 \\
83.9\end{array}$ & $\begin{array}{l}\text { N/A } \\
\text { N/A } \\
\text { N/A }\end{array}$ & $\begin{array}{r}76.4 \\
16.1 \\
100.0\end{array}$ \\
\hline $\begin{array}{r}160 \\
\text { SORT- } \\
\text { SPLIT }\end{array}$ & $\begin{array}{r}0.8 \\
\text { N/A HE } \\
\text { WT- } 464 .\end{array}$ & $\begin{array}{l}\text { H } \\
\text { DIANT- }\end{array}$ & $\begin{array}{c}18.2 \\
1.00\end{array}$ & $\begin{array}{r}47.2 \\
\text { MODE- }\end{array}$ & $-1.00^{34.6}$ MEAN" & msG & N/A & CUM & $\begin{array}{l}\text { WT } \\
\text { WT } \\
\text { WT }\end{array}$ & $\begin{array}{l}\text { N/A } \\
\text { II/A } \\
\text { W/A }\end{array}$ & $\begin{array}{r}160.8 \\
34.6 \\
34.6\end{array}$ & $\begin{array}{l}47.7 \\
10.3 \\
44.9\end{array}$ & $\begin{array}{l}61.7 \\
13.3 \\
58.2\end{array}$ & $\begin{array}{l}52.2 \\
11.2 \\
69.4\end{array}$ & $\begin{array}{r}36.6 \\
7.9 \\
77.3\end{array}$ & $\begin{array}{r}21.0 \\
4.5 \\
01.0\end{array}$ & $\begin{array}{l}\text { N/A } \\
\text { N/A } \\
\text { N/A }\end{array}$ & $\begin{array}{r}84.3 \\
18.2 \\
100.0\end{array}$ \\
\hline $\begin{array}{r}165 \\
\text { SORT- } \\
\text { SPLIT }\end{array}$ & $\begin{array}{c}0.7 \\
N / A \text { MED } \\
W T=485.8\end{array}$ & $\begin{array}{l}\text { H } \\
\text { DIAN- } \\
3\end{array}$ & $\begin{array}{l}16.0 \\
1.00\end{array}$ & $\begin{array}{r}47.5 \\
\text { MODE- }\end{array}$ & $\begin{array}{c}36.5 \\
-1.00 \text { MEAN- }\end{array}$ & msG & $\mathrm{N} / \mathrm{A}$ & UM & $\begin{array}{r}\text { WT } \\
\text { W T } 0 \\
\text { WT }\end{array}$ & $\begin{array}{l}\text { N/A } \\
\text { N/A } \\
\text { N/A }\end{array}$ & $\begin{array}{r}177.3 \\
36.5 \\
36.5\end{array}$ & $\begin{array}{r}44.3 \\
9.1 \\
45.6\end{array}$ & $\begin{array}{l}66.6 \\
13.7 \\
59.3\end{array}$ & $\begin{array}{l}65.2 \\
13.4 \\
72.0\end{array}$ & $\begin{array}{r}35.1 \\
7.2 \\
80.0\end{array}$ & $\begin{array}{r}19.7 \\
4.1 \\
84.0\end{array}$ & $\begin{array}{l}N / A \\
A \\
N / A\end{array}$ & $\begin{array}{r}77.6 \\
16.0 \\
100.0\end{array}$ \\
\hline
\end{tabular}




$$
\text { ROCSAN REPORT }
$$

$12 / 11 / 89$

\begin{tabular}{ccccccc} 
DEPTH & CACO3 & DM & \&MD & $\%$ SAND & $\%$ GRAVEL & CLASS \\
\hdashline 170 & 0.4 & H & -16.1 & 40.2 & 43.7 & msG
\end{tabular}

SORT- N/A MEDIAN- 0.00 MODE $=-1.00$ MEAN- $\mathrm{m} / \mathrm{A}$
SPLIT WT- 454.0

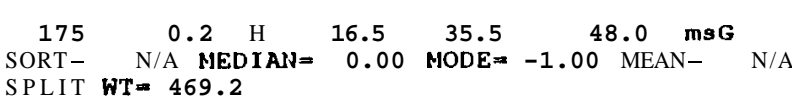

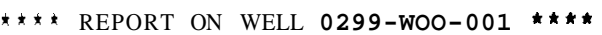

$\begin{array}{rrrrr}180 & 0.2 \mathrm{H} & 22.6 & 46.6 & 30.9 \mathrm{msG}\end{array}$

SPLIT WT- 499.0 N/A WT

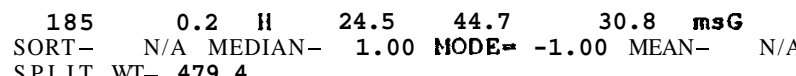

$\begin{array}{llllllll}190 & 0.2 & \mathrm{H} & 22.1 & 41.5 & 36.5 & \mathrm{mgG} & \text { WT }\end{array}$

$\begin{aligned} & 190 \\ & \text { SORT- N/A HEDIAN- }\end{aligned}$ 1.00
SPLIT WT- 441.4

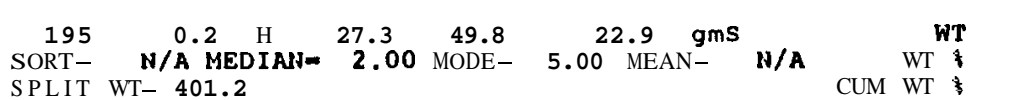

WT

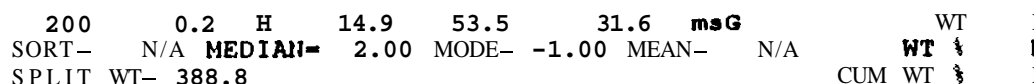

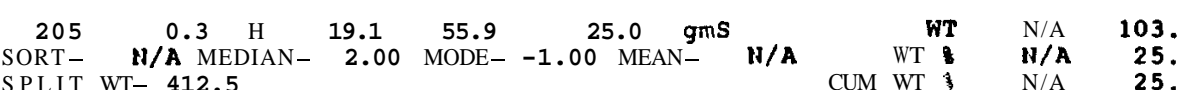

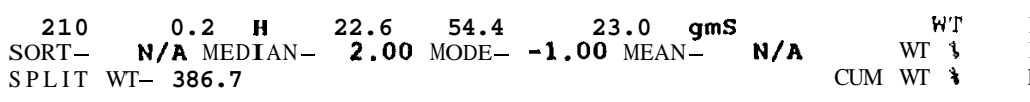

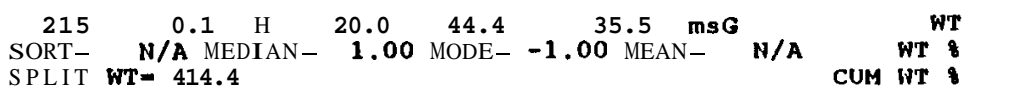

N/A $\quad$ is $\quad 23$.

N/A $\quad \begin{array}{ll}23.0 \\ \text { H/A }\end{array} \quad \mathbf{2 3 . 0}$

$\begin{array}{lr}\text { N/A } & 147.3 \\ \text { N/A } & 35.6\end{array}$

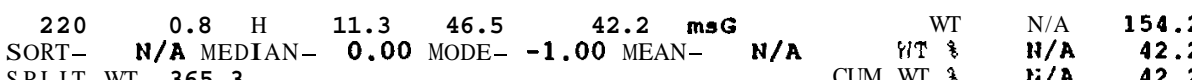

3
.6

SPLIT WT- 365.3

CUM WT 8


WESTINGHOUSE HANFORD OPERATIONS SIEVE ANALYSIS ROCSAN REPORT

$\star \star \star \star$ REPORT ON WELI 0299-W08-001 ****

$12 / 11 / 89$

\begin{tabular}{|c|c|c|c|c|c|c|c|c|c|c|c|c|c|c|c|c|c|c|}
\hline EPTH & $8 \mathrm{CACO} 3$ & DM & \%MUD & $\frac{\circ}{\circ}$ SAND & IGRAVEL & CLAS & & & & $\begin{array}{l}\text { FINE } \\
\text { PEB } \\
(<--2)\end{array}$ & $\begin{array}{l}\text { VF INE } \\
\text { PEB } \\
(-1)\end{array}$ & $\begin{array}{l}\text { VERY } \\
\text { COARS } \\
\text { (0) }\end{array}$ & $\begin{array}{l}\text { COARS } \\
(1)\end{array}$ & $\begin{array}{l}\text { MED } \\
\text { (2) }\end{array}$ & $\begin{array}{l}\text { FINE } \\
\text { (3) }\end{array}$ & $\begin{array}{l}\text { VERY } \\
\text { FINE } \\
\text { (4) }\end{array}$ & $\begin{array}{l}\text { SILT } \\
(4.75)\end{array}$ & $\begin{array}{c}\text { PAN } \\
(>4.75)\end{array}$ \\
\hline $\begin{array}{l}225 \\
\text { SORT- } \\
\text { SPLIT }\end{array}$ & $\begin{array}{c}2.4 \\
N / A \text { MED } \\
W T=374.6\end{array}$ & $\begin{array}{l}\text { DIAN- } \\
6\end{array}$ & $\begin{array}{r}22.7 \\
-\quad 1.00\end{array}$ & $\begin{array}{l}41.2 \\
\text { MODE- }\end{array}$ & -1.00 MEAN & msG & N/A & CUM & $\begin{array}{l}\text { WT } \\
\text { WT } \\
\text { WT }\end{array}$ & $\begin{array}{l}\text { N/A } \\
\text { N/A } \\
\text { N/A }\end{array}$ & $\begin{array}{r}135.3 \\
36.1 \\
36.1\end{array}$ & $\begin{array}{l}37.3 \\
10.0 \\
46.1\end{array}$ & $\begin{array}{r}27.8 \\
7.4 \\
53.5\end{array}$ & $\begin{array}{r}36.9 \\
9.9 \\
63.4\end{array}$ & $\begin{array}{r}33.5 \\
9.0 \\
72.3\end{array}$ & $\begin{array}{r}18.7 \\
5.0 \\
77.3\end{array}$ & $\begin{array}{l}\text { N/A } \\
\text { N/A } \\
\text { N/A }\end{array}$ & $\begin{array}{r}85.0 \\
22.7 \\
100.0\end{array}$ \\
\hline $\begin{array}{r}230 \\
\text { SORT- } \\
\text { SPLIT }\end{array}$ & $\begin{array}{c}1.8 \\
N / A \mathrm{MED} \\
\mathrm{WT}=388.2\end{array}$ & $\underbrace{\stackrel{H}{D I A N}-}_{2}$ & $\begin{array}{c}23.4 \\
-\quad 1.00\end{array}$ & $\begin{array}{r}43.1 \\
\text { MODE- }\end{array}$ & $\begin{array}{c}33.5 \\
-1.00 \mathrm{MEAN}\end{array}$ & ms & N/A & CUM & $\begin{array}{l}\text { WI } \\
\text { WT } \\
\text { WT }\end{array}$ & $\begin{array}{l}\text { N/A } \\
\text { N/A } \\
\text { N/A }\end{array}$ & $\begin{array}{r}130.1 \\
33.5 \\
33.5\end{array}$ & $\begin{array}{l}41.4 \\
10.7 \\
44.2\end{array}$ & $\begin{array}{r}30.4 \\
7.8 \\
52.0\end{array}$ & $\begin{array}{l}38.8 \\
10.0 \\
62.0\end{array}$ & $\begin{array}{r}37.4 \\
9.6 \\
71.6\end{array}$ & $\begin{array}{r}19.3 \\
5.0 \\
76.6\end{array}$ & $\begin{array}{l}\text { N/A } \\
\text { N/A } \\
\text { N/A }\end{array}$ & $\begin{array}{r}90.9 \\
23.4 \\
100.0\end{array}$ \\
\hline $\begin{array}{r}235 \\
\text { SORT- } \\
\text { SPLIT }\end{array}$ & $\begin{array}{c}0.9 \\
N / A \text { MED } \\
\text { WT- } 414.3\end{array}$ & $\begin{array}{l}\text { H } \\
\text { DIAN- } \\
3\end{array}$ & $\begin{array}{r}21.6 \\
-\quad 1.00\end{array}$ & $\begin{array}{r}39.3 \\
\text { MODE- }\end{array}$ & -1.00 MEAN & $\begin{array}{l}m g G \\
N=\end{array}$ & $N / A$ & CUM & $\begin{array}{l}\text { WT } \\
\text { W T B } \\
\text { WT }\end{array}$ & $\begin{array}{l}\text { N/A } \\
\text { N/A } \\
\text { N/A }\end{array}$ & $\begin{array}{r}162.3 \\
39.2 \\
39.2\end{array}$ & $\begin{array}{l}42.6 \\
10.3 \\
49.5\end{array}$ & $\begin{array}{r}30.9 \\
7.5 \\
56.9\end{array}$ & $\begin{array}{r}36.7 \\
8.9 \\
65.8\end{array}$ & $\begin{array}{r}32.7 \\
7.9 \\
73.7\end{array}$ & $\begin{array}{r}19.8 \\
4.8 \\
78.4\end{array}$ & $\begin{array}{l}\text { N/A } \\
\text { N/A } \\
\text { N/A }\end{array}$ & $\begin{array}{r}89.3 \\
21.6 \\
100.0\end{array}$ \\
\hline $\begin{array}{r}240 \\
\text { SORT- } \\
\text { SPLIT }\end{array}$ & $\begin{array}{c}1.4 \\
\text { N/A MED } \\
\text { WT }=360.8\end{array}$ & $\begin{array}{l}\text { H } \\
8 \text { IAN- }\end{array}$ & $\begin{array}{c}37.4 \\
-\quad 3.00\end{array}$ & $\begin{array}{r}50.6 \\
\text { MODE- }\end{array}$ & $5.00^{12.0}$ MEAN & gms & N/A & CUM & $\begin{array}{l}\text { WT } \\
\text { WT } \\
\text { WT }\end{array}$ & $\begin{array}{l}\text { N/A } \\
N / A \\
N / A\end{array}$ & $\begin{array}{l}43.3 \\
12.0 \\
12.0\end{array}$ & $\begin{array}{l}45.4 \\
12.6 \\
24.6\end{array}$ & $\begin{array}{r}35.2 \\
9.8 \\
34.3\end{array}$ & $\begin{array}{l}37.8 \\
10.5 \\
44.8\end{array}$ & $\begin{array}{l}38.0 \\
10.5 \\
55.4\end{array}$ & $\begin{array}{r}26.2 \\
7.3 \\
62.6\end{array}$ & $\begin{array}{l}\text { N/A } \\
\text { N/A } \\
\text { N/A }\end{array}$ & $\begin{array}{r}134.9 \\
37.4 \\
100.0\end{array}$ \\
\hline $\begin{array}{r}248 \\
\text { SORT- } \\
\text { SPIIT }\end{array}$ & $\begin{array}{c}\mathrm{1} \cdot{ }^{\mathrm{M}} \\
\mathrm{NTD} \\
\mathrm{WT}=375.0\end{array}$ & $\begin{array}{l}\mathrm{D}_{0}^{\mathrm{H}}{ }_{0}^{\mathrm{IAN}-} \\
\end{array}$ & $\begin{array}{l}30.8 \\
-\quad 1.00\end{array}$ & MODE & $-1.00^{37} \mathrm{MEAN}^{2}$ & mag & N/A & CUM & $\begin{array}{l}\text { WT } \\
\text { WT }\end{array}$ & $\begin{array}{l}\text { N/A } \\
\text { N/A } \\
\text { N/A }\end{array}$ & $\begin{array}{r}139.5 \\
37.2 \\
37.2\end{array}$ & $\begin{array}{l}37.8 \\
10.1 \\
47.3\end{array}$ & $\begin{array}{r}22.9 \\
6.1 \\
53.4\end{array}$ & $\begin{array}{r}20.8 \\
5.6 \\
58.9\end{array}$ & $\begin{array}{r}24.0 \\
6.4 \\
65.3\end{array}$ & $\begin{array}{l}14.5 \\
3.9 \\
69.2\end{array}$ & $\begin{array}{l}\text { N/A } \\
\text { N/A } \\
\text { N/A }\end{array}$ & $\begin{array}{r}115.6 \\
30.8 \\
100.0\end{array}$ \\
\hline $\begin{array}{r}250 \\
\text { SORT- } \\
\text { SPLIT }\end{array}$ & $\begin{array}{c}0.8 \\
\text { N/A MED } \\
\text { WT- } 307.9\end{array}$ & $\begin{array}{l}\text { H } \\
9\end{array}$ & $\begin{array}{r}29.6 \\
-\quad 2.00\end{array}$ & $\begin{array}{r}46.3 \\
\text { MODE- }\end{array}$ & $5.00^{24.1}$ & gms & N/A & CUM & $\begin{array}{l}\text { WT } \\
\text { WT }\end{array}$ & $\begin{array}{l}\text { N/A } \\
N / A \\
\text { N/A }\end{array}$ & $\begin{array}{l}74.2 \\
24.1 \\
24.1\end{array}$ & $\begin{array}{l}37.6 \\
12.2 \\
36.3\end{array}$ & $\begin{array}{r}26.8 \\
8.7 \\
45.0\end{array}$ & $\begin{array}{r}28.5 \\
9.3 \\
54.3\end{array}$ & $\begin{array}{l}30.9 \\
10.0 \\
64.3\end{array}$ & $\begin{array}{r}18.7 \\
6.1 \\
70.4\end{array}$ & $\begin{array}{l}\text { N/A } \\
\text { N/A } \\
\text { N/A }\end{array}$ & $\begin{array}{r}91.2 \\
29.6 \\
100.0\end{array}$ \\
\hline $\begin{array}{r}255 \\
\text { SORT- } \\
\text { SPIIT }\end{array}$ & $\begin{array}{c}0.9 \\
\text { N/A MED } \\
\text { WT- } 400.1\end{array}$ & $\begin{array}{l}\stackrel{\mathrm{H}}{\mathrm{DIAN}-} \\
1\end{array}$ & $\begin{array}{r}21.2 \\
-\quad 2.00\end{array}$ & $\begin{array}{r}46.8 \\
\text { MODE- }\end{array}$ & $-1.00^{32.1}$ MEAN & ${ }_{\mathrm{N}}^{\mathbf{m s G}}$ & N/A & CUM & $\begin{array}{l}\text { WT } \\
\text { WT }\end{array}$ & $\begin{array}{l}N / A \\
N / A \\
N / A\end{array}$ & $\begin{array}{c}128.3 \\
32.1 \\
32.1\end{array}$ & $\begin{array}{r}30.0 \\
7.5 \\
39.5\end{array}$ & $\begin{array}{r}26.4 \\
6.6 \\
46.1\end{array}$ & $\begin{array}{l}63.0 \\
15.7 \\
61.9\end{array}$ & $\begin{array}{l}46.5 \\
11.6 \\
73.5\end{array}$ & $\begin{array}{r}21.4 \\
5.4 \\
78.9\end{array}$ & $\begin{array}{l}\text { N/A } \\
\text { N/A } \\
\text { N/A }\end{array}$ & $\begin{array}{r}84.7 \\
21.2 \\
100.0\end{array}$ \\
\hline $\begin{array}{r}260 \\
\text { SORT- } \\
\text { SPLIT }\end{array}$ & $\begin{array}{c}1.2 \\
\text { N } / \mathrm{A} \text { MED } \\
\mathrm{WT}-371.5\end{array}$ & $\begin{array}{l}\mathrm{H} \\
5 \mathrm{IAN}-\end{array}$ & $\begin{array}{r}25.1 \\
-\quad 2.00\end{array}$ & $\begin{array}{r}47.8 \\
\text { MODE- }\end{array}$ & $-1.00^{27.0}$ MEAN & gms & N/A & CUM & $\operatorname{wr}_{\mathrm{WT}}^{\mathrm{WT}}$ & $\begin{array}{l}\text { N/A } \\
\text { N/A } \\
\text { N/A }\end{array}$ & $\begin{array}{r}100.4 \\
27.0 \\
27.0\end{array}$ & $\begin{array}{l}39.7 \\
10.7 \\
37.7\end{array}$ & $\begin{array}{r}31.6 \\
8.5 \\
46.2\end{array}$ & $\begin{array}{l}45.9 \\
12.4 \\
58.6\end{array}$ & $\begin{array}{l}38.0 \\
10.2 \\
68.8\end{array}$ & $\begin{array}{r}22.5 \\
6.1 \\
74.9\end{array}$ & $\begin{array}{l}\text { N/A } \\
\text { N/A } \\
\text { N/A }\end{array}$ & $\begin{array}{r}93.4 \\
25.1 \\
100.0\end{array}$ \\
\hline $\begin{array}{r}265 \\
\text { SORT- } \\
\text { SPIIT }\end{array}$ & $\begin{array}{c}0.7 \\
\text { N/A MED } \\
\text { WT- } 379.9\end{array}$ & $\underbrace{\stackrel{\mathrm{H}}{\mathrm{DIAN}-}}_{9}$ & $\begin{array}{c}20.7 \\
-\quad 1.00\end{array}$ & $\begin{array}{r}47.1 \\
\text { MODE- }\end{array}$ & $-1.00^{32.1}$ & msG & $\mathrm{H} / \mathrm{A}$ & CUM & $\begin{array}{l}\text { WT' } \\
\text { WT }\end{array}$ & $\begin{array}{l}\text { N/A } \\
\text { N/A } \\
\text { N/A }\end{array}$ & $\begin{array}{r}122.0 \\
32.2 \\
32.2\end{array}$ & $\begin{array}{l}41.0 \\
10.8 \\
43.0\end{array}$ & $\begin{array}{r}30.5 \\
8.0 \\
51.0\end{array}$ & $\begin{array}{l}49.1 \\
12.9 \\
63.9\end{array}$ & $\begin{array}{l}38.9 \\
10.3 \\
74.2\end{array}$ & $\begin{array}{l}19.4 \\
5.1 \\
79.3\end{array}$ & $\begin{array}{l}\text { N/A } \\
\text { N/A } \\
\text { N/A }\end{array}$ & $\begin{array}{r}78.6 \\
20.7 \\
100.0\end{array}$ \\
\hline $\begin{array}{r}270 \\
\text { SORT- } \\
\text { SPLIT }\end{array}$ & $\begin{array}{c}0.7 \\
\text { N/A MED } \\
\text { WT }=362.7\end{array}$ & $\begin{array}{l}\text { H } \\
\text { DIAN- } \\
7\end{array}$ & $\begin{array}{r}26.7 \\
-\quad 2.00\end{array}$ & $\begin{array}{r}39.8 \\
\text { MODE- }\end{array}$ & $-1.00^{33.6} \mathrm{MEA}$ & msG & $\mathbf{N} / \mathbf{A}$ & CUM & $\begin{array}{l}\text { WT' } \\
\text { WT }\end{array}$ & $\begin{array}{l}\text { N/A } \\
\text { N/A } \\
\text { N/A }\end{array}$ & $\begin{array}{r}121.8 \\
33.6 \\
33.6\end{array}$ & $\begin{array}{r}2.3 \\
0.6 \\
34.2\end{array}$ & $\begin{array}{r}27.4 \\
7.6 \\
41.8\end{array}$ & $\begin{array}{l}57.1 \\
15.7 \\
57.5\end{array}$ & $\begin{array}{l}39.2 \\
10.8 \\
68.3\end{array}$ & $\begin{array}{r}18.2 \\
5.0 \\
73.3\end{array}$ & $\begin{array}{l}\text { N/A } \\
\text { N/A } \\
\text { N/A }\end{array}$ & $\begin{array}{r}96.7 \\
26.7 \\
100.0\end{array}$ \\
\hline
\end{tabular}



ROCSAN REPORT

$12 / 11 / 89$

4444 REPORT ON WELL 0299-W09-001 *k*

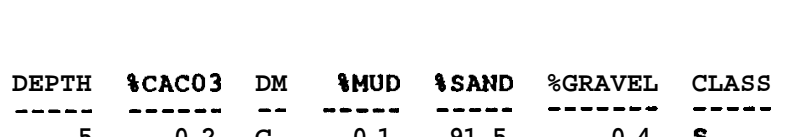

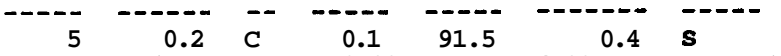
SORT- N/A MEDIAN- 3.00 MODE- 4.00 MEAN- N/A
SPLIT WT- 346.5 $\begin{array}{cccccccc}10 & 1.4 & \mathrm{C} & 3.0 & 97.0 & 0.0 \quad \mathrm{~s} & & \text { WT } \\ \text { SORT- } & \text { N/A MEDIAN- } & 3.00 & \text { MODE- } & 3.00 & \text { MEAN- } & \text { N/A } & \text { WUT } \\ \text { SPIIT } & \text { WT- } 294.4 & & & & \end{array}$

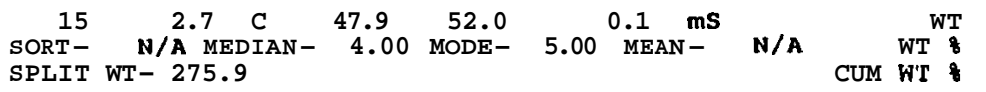

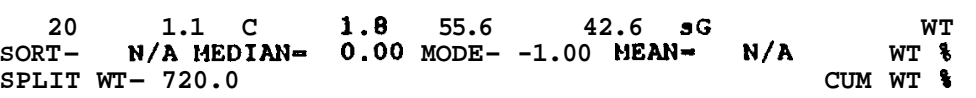

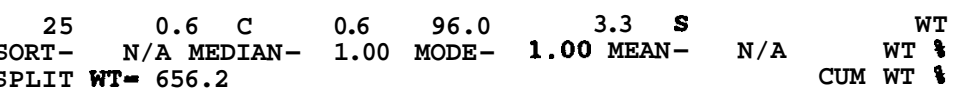
$\begin{array}{rlllllll}30 & 0.6 & \mathrm{C} & 2.6 & 92.1 & 5.2 & (\mathrm{~g}) \mathrm{s} & \\ \text { SORT- } & \text { N/A MEDIAN- } & 1.00 & \text { MODE- } & 1.00 & \text { MEAN } & \text { N/A } & \text { CUM WT }\end{array}$ $\begin{array}{llllllll}35 & 0.6 & \mathrm{C} & 0.5 & 99.3 & 0.2 \mathrm{~s} & & \\ \text { SORT- } & \text { N/A MEDIAN- } & 2.00 & \text { MODE- } & 2.00 & \text { MEAN- } & \text { N/A } & \text { WT } \\ \text { SPLIT WT- } 641.6 & & & & & \text { CUM WT I }\end{array}$ $\begin{array}{rccccccc}40 & 1.6 & \mathrm{C} & 10.1 & 09.4 & 0.4 & \text { (m) s } & \text { WT } \\ \text { SORT- } & \text { N/A MEDIAN- } & 3.00 & \text { MODE- } & 4.00 \mathrm{MEAN}-\mathrm{N}_{\text {N }} & \text { WT I }\end{array}$ SPLIT WT- 550.9 $\begin{array}{lcccccc}45 & 0.9 & \mathrm{C} & 13.2 & 06.3 & 0.5 & (\mathrm{~m}) \mathrm{s} \\ \text { SORT- } & \text { N/A MEDIAN- } & 3.00 & \text { MODE- } & 4.00 & \text { MEAN- } & \text { N/A } \\ \text { SPLIT } & \text { WT- } 312.7\end{array}$

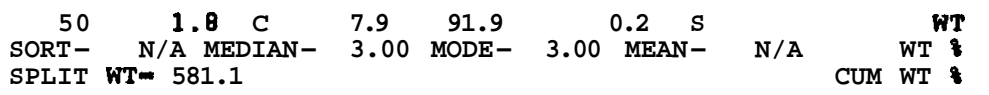

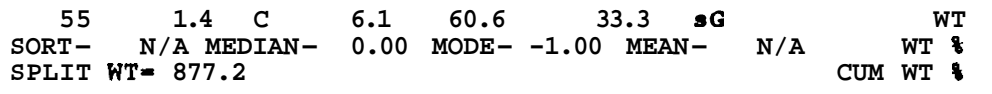

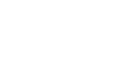

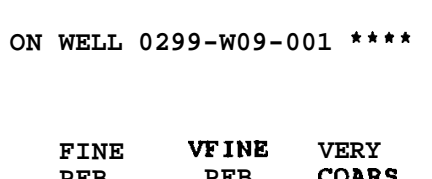


WESTINGHOUSE HANFORD OPERATIONS SIEVE ANALYSIS ROCSAN REPORT

**** REPORT ON WELL 0299-W09-001 ****

$12 / 11 / 89$

\begin{tabular}{ccccccc} 
& & & & & & \\
DEPTH & ICACO3 & DM & IMUD & ISAND & \%GRAVEL & CLASS \\
\hdashline 60 & 1.0 & H & 8.0 & 60.3 & 31.7 & msG
\end{tabular}

SORT- N/A MEDIAN= 0.00 MODE- $-1.00 \mathrm{MEAN}^{\mathrm{msG}} \mathrm{N} / \mathrm{A}$ SPLIT WT- 757.2

$\begin{array}{llllllll}65 & 1 & \text { H } & 9.5 & 64.7 & 25.8 & \text { (m) gS }\end{array}$

FINE VFINE VERY

COARS MED FINE FINE SILT PAN

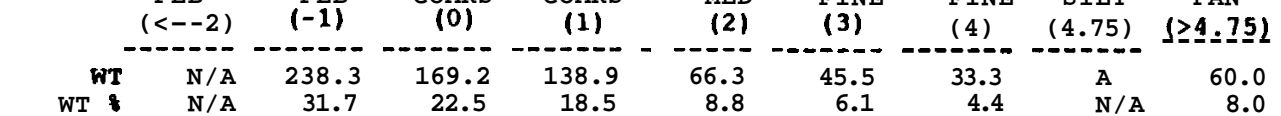

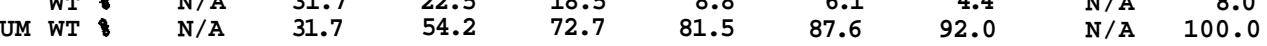
SORT- N/A MEDIAN- 1.00 MODE- -1.00 MEAN- N/A CUM WT
SPLIT WT- 708.9

$\begin{array}{lrrrr}\text { N/A } & 182.6 & 158.1 & 144.2 & 72.3\end{array}$

72.3
10.2

51.

51.0
7.2
85.9

$32.8 \quad$ N/A 67.4 $\begin{array}{ccccccc}70 & 1.0 & \text { H } & 11.2 & 54.0 & 34.8 & \mathrm{mgG} \\ \text { SORT- } & \text { N/A MEDIAN- } & 0.00 & \text { MODE- } & -1.00 & \text { MEAN- } & \text { N/A }\end{array}$ SORT- N/A MEDIAN
SPLIT WT $=810.4$

WT N/A 25.8

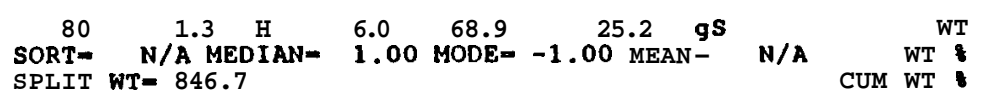
WT $\begin{array}{llllll}\text { SORT- N/A MEDIAN- } & 1.00 & \text { MODE- } & 1.00 \mathrm{MEAN}- & \text { N/A } & \text { WT } \\ \text { CUM WT }\end{array}$

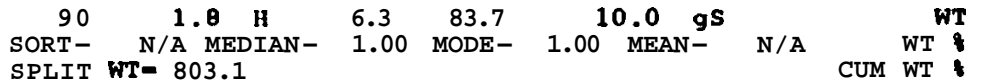

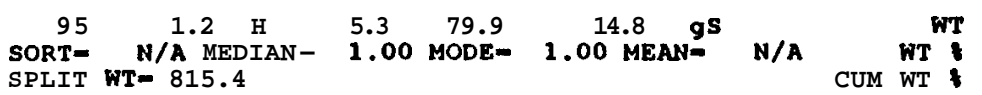

WT:

$\begin{array}{llllclll}100 & 3.3 & \text { H } & 7.3 & 72.1 & 20.6 & \text { gS } & \\ \text { SORT } & \text { N/A MEDIAN- } & 1.00 & \text { MODE- } & 1.00 \mathrm{MEAN}- & \text { N/A } & \text { WTT }\end{array}$

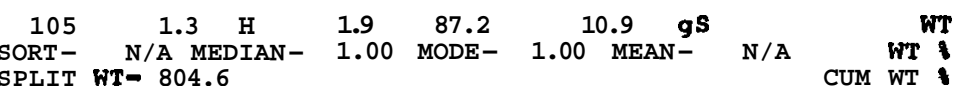

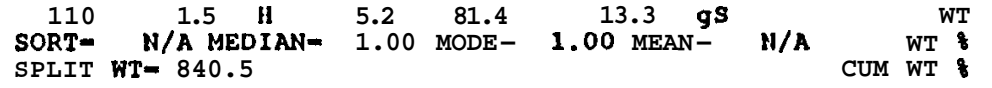

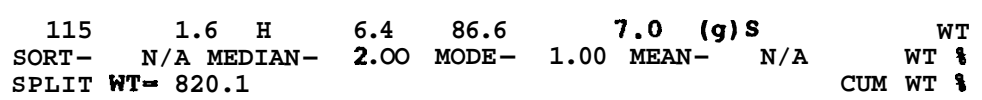
N/A $\begin{array}{llll}80.9 & 136.6 & 114.5 & 80.2\end{array}$ $\begin{array}{rrrr}34.8 & 16.9 & 14.2 & 9.9\end{array}$ $\begin{array}{lllll}\text { N/A } & 212.8 & 124.1 & 192.1 & 150\end{array}$

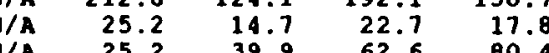
7.3
83.2 90.5 $\begin{array}{lr}\text { N/A } & 9.5 \\ \text { N/A } & 100.0\end{array}$

$\begin{array}{lllll}\text { N/A } & 93.2 & 92.9 & 273.3 & 175.2\end{array}$ $\begin{array}{rrr}92.9 & 273.3 & 175.2 \\ 11.8 & 34.8 & 22.3\end{array}$

91.

5.8 N/A 90.0 $\begin{array}{rrr}5.7 & \text { N/A } & 11.2 \\ 88.9 & \text { N/A } & 100.0\end{array}$

$\begin{array}{rrrrr}\text { N/A } & 80.7 & 118.9 & 254.9 & 182.6 \\ \text { N/A } & 10.0 & 14.8 & 31.7 & 22.7\end{array}$ $.3 \quad 11.3$ $\begin{array}{rrr}33.6 & \text { W/A } & 50.5 \\ 4.0 & \text { N/A } & 6.0 \\ 94.0 & \text { N/A } & 100.0\end{array}$

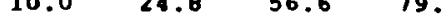

$\begin{array}{lrrrr}\text { N/A } & 120.3 & 102.9 & 253.5 & 175.0 \\ \text { N/A } & 14.8 & 12.6 & 31.2 & 21.5 \\ \text { N/A } & 14.8 & 27.4 & 58.6 & 80.1\end{array}$

175.0
21.5
80.1

79.7
9.9
09.2

25.8

N/A $\quad 35.9$ N/A 14.8

156.7

$\begin{array}{lr}\text { N/A } & 156 \\ \text { N/A } & 20 \\ N / A & 20.6\end{array}$

$120.9 \quad 172.3 \quad 144.2$ 90.1

$\begin{array}{llrrr}N / A & 87.8 & 153.2 & 287.0 & 136.9\end{array}$ $\begin{array}{rrrrr}\text { N/A } & 10.9 & 19.0 & 35.6 & 17.0 \\ \text { N/A } & 10.9 & 29.9 & 65.6 & 02.5\end{array}$

$\begin{array}{llllll}\text { N/A } & 112.0 & 125.3 & 223.6 & 197.1 & 98.0\end{array}$ $\begin{array}{rrrrrrrrr}N / A & 13.3 & 14.9 & 26.6 & 23.5 & 11.7 & 4.8 & N / A & 5.2 \\ N / A & 13.3 & 28.3 & 54.9 & 78.4 & 90.0 & 94.8 & \text { N/A } & 100.0\end{array}$

N/A
N/A
N/A

$\begin{array}{lr}2 & 107 \\ 0 & 13 \\ 0 & 20.2\end{array}$




$$
\text { ROCSAN REPORT }
$$

*** REPORT ON WELL 0299-w09-001 ****

$12 / 11 / 89$

\begin{tabular}{ccccccc} 
DEPTH & ICACO3 & DM & IMUD & $\% S A N D$ & GRAVEL & CLASS \\
\hdashline 120 & 1.2 & H & 5.1 & 84.2 & 10.7 & gS
\end{tabular}

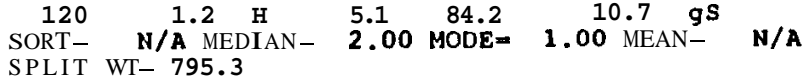

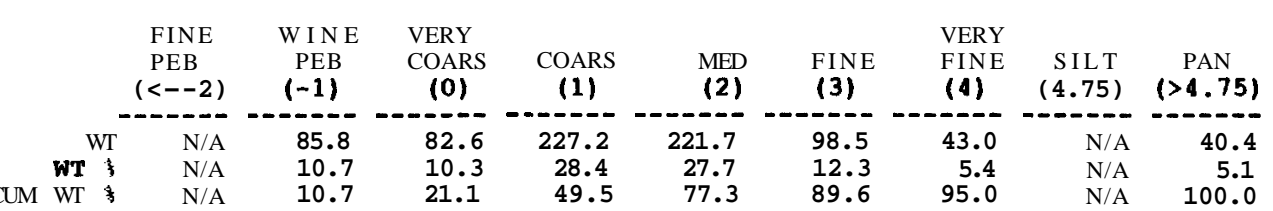

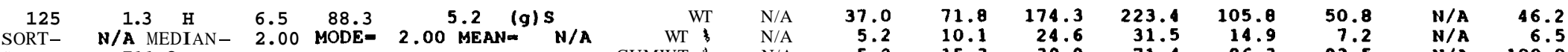

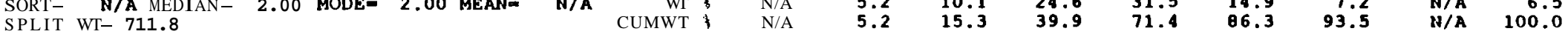

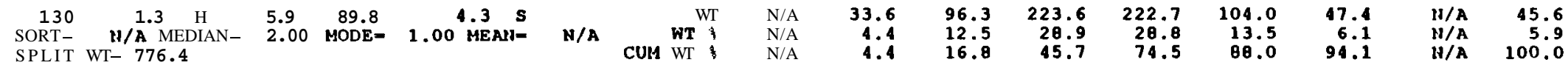

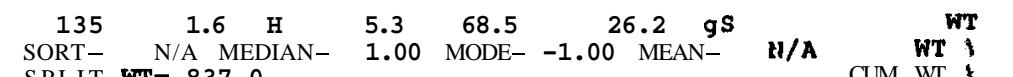

SPLIT WT- 837

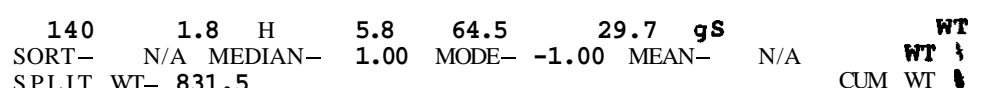

WT ${ }^{\text {WT }}$

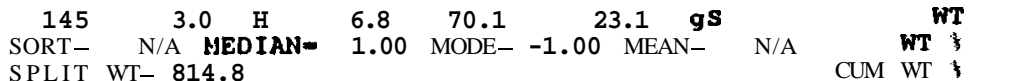

$$
5.8(9) s
$$

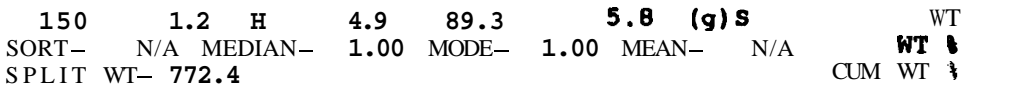

$\begin{array}{llllllr}155 & 3.6 \text { H } & 9.7 & 73.1 & 17.1 & (\mathrm{~m}) \mathrm{gs} & \text { WT } \\ \text { SORT- } & \text { N/A MEDIAN- } & 1.00 & \text { MODE- } & 1.00 \text { MEAN- } & \text { N/A } & \text { WT } \\ \text { SPLIT } & \text { wT- } 774.1 & & & & \text { CUM WT }\end{array}$

N/A $\quad 218.5$

114.6

N/A $\quad 26.2$

90.7

$\begin{array}{rrr}34.9 & \text { N/A } & 44.1 \\ 4.2 & \text { N/A } & 5.3\end{array}$

$246.7 \quad 134.0 \quad 147.1 \quad 142$.

$79.6 \quad 90.5$

$\begin{array}{lr}\text { N/A } & 5.3 \\ \text { N/A } & 100.0\end{array}$

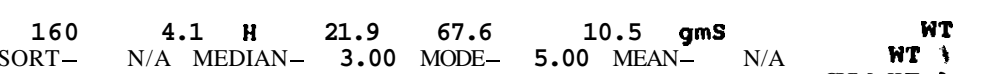

SPLIT WT- 729.7

$\begin{array}{lllllcll}165 & 17.9 & \text { H } & 9.3 & 79.9 & 10.8 & \text { (m) gs } & \text { TT }\end{array}$

SPLIT WT- 732.3

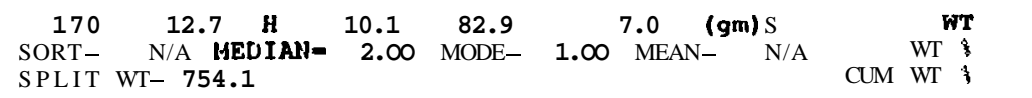


WESTINGHOUSE HANFORD OPERATIONS SIEVE ANALYSIS ROCSAN REPORT

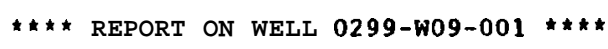

$12 / 11 / 89$

\begin{tabular}{|c|c|c|c|c|c|c|c|c|c|c|c|c|c|c|c|c|c|}
\hline PTH & $8 \mathrm{CACO} 3 \mathrm{DM}$ & IMUD & \&SAND & GRAVEL & CLASS & & & & $\begin{array}{l}\text { INE } \\
\text { IEB } \\
<--2)\end{array}$ & $\begin{array}{l}\text { VFINE } \\
\text { PEB } \\
(-1)\end{array}$ & $\begin{array}{l}\text { VERY } \\
\text { COARS } \\
\text { (0) }\end{array}$ & $\begin{array}{l}\text { COARS } \\
\text { (1) }\end{array}$ & $\begin{array}{r}\text { MED } \\
(2)\end{array}$ & $\begin{array}{l}\text { FINE } \\
\text { (3) }\end{array}$ & $\begin{array}{l}\text { VERY } \\
\text { FINE } \\
\text { (4) }\end{array}$ & $\begin{array}{l}\text { SILT } \\
(4.75)\end{array}$ & $\begin{array}{c}\text { PAN } \\
(>4.75)\end{array}$ \\
\hline $\begin{array}{r}175 \\
\text { SORT- } \\
\text { SPLIT }\end{array}$ & $\begin{array}{l}\text { N/A } \quad \text { H } \\
\text { N/A MEDIAN- } \\
\text { WT }=746.9\end{array}$ & $\begin{array}{l}8.6 \\
2.00\end{array}$ & $\begin{array}{r}86.8 \\
\text { MODE- }\end{array}$ & $2.00 \stackrel{4.6 \mathrm{~S}}{\text { MEAN- }}$ & $s$ & $\mathrm{~N} / \mathrm{A}$ & CUM & $\begin{array}{l}\text { WT } \\
\text { WT }\end{array}$ & $\begin{array}{l}\text { N/A } \\
\text { N/A } \\
\text { N/A }\end{array}$ & $\begin{array}{r}34.3 \\
4.6 \\
4.6\end{array}$ & $\begin{array}{r}38.1 \\
5.1 \\
9.7\end{array}$ & $\begin{array}{r}166.6 \\
22.3 \\
32.1\end{array}$ & $\begin{array}{r}239.0 \\
32.1 \\
64.1\end{array}$ & $\begin{array}{c}136.1 \\
18.3 \\
82.4\end{array}$ & $\begin{array}{r}67.7 \\
9.1 \\
91.4\end{array}$ & $\begin{array}{l}\text { N/A } \\
\text { N/A } \\
\text { N/A }\end{array}$ & $\begin{array}{r}63.8 \\
8.6 \\
100.0\end{array}$ \\
\hline $\begin{array}{r}180 \\
\text { SORT- } \\
\text { SPIIT }\end{array}$ & $\begin{array}{l}5.9 \stackrel{\text { H }}{5} \\
\text { N/A MEDIAN- } \\
\text { WT- } 610.4\end{array}$ & $\begin{array}{l}7.8 \\
2.00\end{array}$ & $\begin{array}{l}90.6 \\
\text { MODE- }\end{array}$ & $2.00 \mathrm{MEAN}-$ & & N/A & CUM & $\begin{array}{l}\text { WT } \\
\text { WT }\end{array}$ & $\begin{array}{l}N / A \\
N / A \\
N / A\end{array}$ & $\begin{array}{r}10.0 \\
1.7 \\
1.7\end{array}$ & $\begin{array}{r}12.6 \\
2.1 \\
3.7\end{array}$ & $\begin{array}{r}41.6 \\
6.9 \\
10.6\end{array}$ & $\begin{array}{r}328.8 \\
54.2 \\
64.7\end{array}$ & $\begin{array}{r}129.0 \\
21.3 \\
86.0\end{array}$ & $\begin{array}{r}38.1 \\
6.3 \\
92.3\end{array}$ & $\begin{array}{l}\text { N/A } \\
\text { N/A } \\
\text { N/A }\end{array}$ & $\begin{array}{r}47.1 \\
7.8 \\
100.0\end{array}$ \\
\hline $\begin{array}{l}185 \\
\text { ORT- } \\
\text { PLIT }\end{array}$ & $\begin{array}{c}1.7 \stackrel{\text { H }}{1.7} \\
\text { N/A MEDIAN- } \\
\text { WT- } 723.9\end{array}$ & $\begin{array}{l}6.4 \\
2.00\end{array}$ & $\begin{array}{c}93.4 \\
\text { MODE- }\end{array}$ & $2.00^{0.2} \mathrm{MEAN}-$ & & $\mathrm{N} / \mathrm{A}$ & CUM & $\begin{array}{l}\text { WT } \\
\text { WT }\end{array}$ & $\begin{array}{l}\text { N/A } \\
\text { N/A } \\
\text { N/A }\end{array}$ & $\begin{array}{l}1.1 \\
0.2 \\
0.2\end{array}$ & $\begin{array}{l}1.4 \\
0.2 \\
0.3\end{array}$ & $\begin{array}{r}27.0 \\
3.7 \\
4.1\end{array}$ & $\begin{array}{r}430.1 \\
59.6 \\
63.6\end{array}$ & $\begin{array}{r}170.6 \\
23.6 \\
87.3\end{array}$ & $\begin{array}{r}45.8 \\
6.3 \\
93.6\end{array}$ & $\begin{array}{l}\text { N/A } \\
\text { N/A } \\
\text { N/A }\end{array}$ & $\begin{array}{r}46.3 \\
6.4 \\
100.0\end{array}$ \\
\hline $\begin{array}{l}190 \\
\text { ORT- } \\
\text { SIIT }\end{array}$ & $\begin{array}{c}1.7 \quad \text { H } \\
\text { N/A MEDIAN- } \\
\text { WT- } 719.0\end{array}$ & $\begin{array}{l}7.0 \\
2.00\end{array}$ & $\begin{array}{r}92.9 \\
\text { MODE- }\end{array}$ & $2.00^{0.1} \mathrm{MEAN}-$ & & N/A & CUM & $\begin{array}{l}\text { WT } \\
\text { WT }\end{array}$ & $\begin{array}{l}\text { N/A } \\
\text { N/A } \\
\text { N/A }\end{array}$ & $\begin{array}{l}0.7 \\
0.1 \\
0.1\end{array}$ & $\begin{array}{l}0.8 \\
0.1 \\
0.2\end{array}$ & $\begin{array}{r}42.9 \\
6.0 \\
6.2\end{array}$ & $\begin{array}{r}400.0 \\
55.9 \\
62.1\end{array}$ & $\begin{array}{r}175.0 \\
24.5 \\
86.5\end{array}$ & $\begin{array}{r}45.9 \\
6.4 \\
93.0\end{array}$ & $\begin{array}{l}\text { N/A } \\
\text { N/A } \\
\text { N/A }\end{array}$ & $\begin{array}{r}50.4 \\
7.0 \\
100.0\end{array}$ \\
\hline $\begin{array}{l}195 \\
\text { ORT- } \\
\text { ORIT }\end{array}$ & $\begin{array}{l}1.4 \underset{H}{\mathrm{H}} \\
\mathrm{N} / \mathrm{A} \text { MEDIAN- } \\
\mathrm{WT}=863.4\end{array}$ & $\begin{array}{l}5.7 \\
2.00\end{array}$ & $\begin{array}{r}74.3 \\
\text { MODE- }\end{array}$ & 2.00 MEAN & $g$ & N/A & CUM & $\begin{array}{l}\text { WT } \\
\text { WT }\end{array}$ & $\begin{array}{l}\text { N/A } \\
\text { N/A } \\
\text { N/A }\end{array}$ & $\begin{array}{r}171.5 \\
20.0 \\
20.0\end{array}$ & $\begin{array}{r}43.8 \\
5.1 \\
25.1\end{array}$ & $\begin{array}{r}76.8 \\
9.0 \\
34.1\end{array}$ & $\begin{array}{r}324.4 \\
37.8 \\
71.9\end{array}$ & $\begin{array}{r}130.1 \\
15.2 \\
87.1\end{array}$ & $\begin{array}{r}61.6 \\
7.2 \\
94.3\end{array}$ & $\begin{array}{l}\text { N/A } \\
\text { N/A } \\
\text { N/A }\end{array}$ & $\begin{array}{r}49.0 \\
5.7 \\
100.0\end{array}$ \\
\hline $\begin{array}{l}200 \\
\text { ORT- } \\
\text { SPIT }\end{array}$ & $\begin{array}{l}0.5 \stackrel{\text { H }}{ } \\
\text { N/A MEDIAN- } \\
\text { WT= } 857.9\end{array}$ & $\begin{array}{l}7.6 \\
2.00\end{array}$ & $\begin{array}{r}65.1 \\
\text { MODE- }\end{array}$ & -1.00 MEAN & $(m) g$ & $\stackrel{S}{S / A}$ & CUM & WT: & $\begin{array}{l}\text { N/A } \\
\text { N/A } \\
\text { N/A }\end{array}$ & $\begin{array}{r}232.7 \\
27.3 \\
27.3\end{array}$ & $\begin{array}{c}83.6 \\
9.8 \\
37.1\end{array}$ & $\begin{array}{r}103.7 \\
12.2 \\
49.2\end{array}$ & $\begin{array}{r}217.3 \\
25.5 \\
74.7\end{array}$ & $\begin{array}{c}102.3 \\
12.0 \\
86.7\end{array}$ & $\begin{array}{r}48.3 \\
5.7 \\
92.4\end{array}$ & $\begin{array}{l}\text { N/A } \\
\text { N/A } \\
\text { N/A }\end{array}$ & $\begin{array}{r}65.1 \\
7.6 \\
100.0\end{array}$ \\
\hline $\begin{array}{l}205 \\
\text { ORT- } \\
\text { PIIT }\end{array}$ & $\begin{array}{c}0.6 \underset{H}{H} \\
N / A \text { MEDIAN- } \\
W T=845.5\end{array}$ & $\begin{array}{l}9.8 \\
2.00\end{array}$ & $\begin{array}{r}74.9 \\
\text { MODE- }\end{array}$ & 2.00 MEAN- & $(m) g$ & $\begin{array}{l}\text { S } \\
\text { N/A }\end{array}$ & CUM & ${ }_{\text {WT }}^{\text {WT }}$ & $\begin{array}{l}\text { N/A } \\
\text { N/A } \\
\text { N/A }\end{array}$ & $\begin{array}{r}129.5 \\
15.4 \\
15.4\end{array}$ & $\begin{array}{r}104.5 \\
12.4 \\
27.7\end{array}$ & $\begin{array}{r}119.0 \\
14.1 \\
41.8\end{array}$ & $\begin{array}{r}236.6 \\
28.0 \\
69.9\end{array}$ & $\begin{array}{r}112.0 \\
13.3 \\
83.2\end{array}$ & $\begin{array}{r}59.7 \\
7.1 \\
90.2\end{array}$ & $\begin{array}{l}\text { N/A } \\
\text { N/A } \\
\text { N/A }\end{array}$ & $\begin{array}{r}82.4 \\
9.8 \\
100.0\end{array}$ \\
\hline $\begin{array}{l}210 \\
\text { ORT- } \\
\text { PIIT }\end{array}$ & $\begin{array}{c}0.4 \quad \mathrm{H} \\
\text { N/A MEDIAN- } \\
\text { WT- } 919.2\end{array}$ & $\begin{array}{c}11.4 \\
1.00\end{array}$ & $\begin{array}{r}60.5 \\
\text { MODE- }\end{array}$ & $-1.00^{28.1}$ MEAN & $(m) g$ & $\begin{array}{l}S \\
\text { N/A }\end{array}$ & CUM & $\underset{\text { WT }}{\text { WT }}$ & $\begin{array}{l}\text { N/A } \\
\text { N/A } \\
\text { N/A }\end{array}$ & $\begin{array}{r}258.0 \\
28.1 \\
28.1\end{array}$ & $\begin{array}{l}99.2 \\
10.8 \\
38.9\end{array}$ & $\begin{array}{l}161.1 \\
17.6 \\
56.5\end{array}$ & $\begin{array}{r}157.8 \\
17.2 \\
73.7\end{array}$ & $\begin{array}{l}93.1 \\
10.2 \\
83.9\end{array}$ & $\begin{array}{r}43.7 \\
4.8 \\
88.6\end{array}$ & $\begin{array}{l}\text { N/A } \\
\text { N/A } \\
\text { N/A }\end{array}$ & $\begin{array}{r}104.4 \\
11.4 \\
100.0\end{array}$ \\
\hline $\begin{array}{l}215 \\
\text { SORT- } \\
\text { SPLIT }\end{array}$ & $\begin{array}{c}0.2 \quad \mathrm{H} \\
\text { N/A MEDIAN- } \\
\text { WT- } 814.8\end{array}$ & $\begin{array}{l}11.9 \\
1.00\end{array}$ & $\begin{array}{r}65.8 \\
\text { MODE- }\end{array}$ & $\begin{array}{c}22.3 \\
-1.00 \text { MEAN }\end{array}$ & $(m) g$ & $\stackrel{g}{N / A}$ & CUM & $\begin{array}{l}\text { WT } \\
\text { WT } \\
\text { WT }\end{array}$ & $\begin{array}{l}N / A \\
N / A \\
H / A\end{array}$ & $\begin{array}{r}100.0 \\
22.3 \\
22.3\end{array}$ & $\begin{array}{l}88.4 \\
10.9 \\
33.2\end{array}$ & $\begin{array}{r}137.1 \\
16.9 \\
50.2\end{array}$ & $\begin{array}{r}142.2 \\
17.6 \\
67.7\end{array}$ & $\begin{array}{r}122.9 \\
15.2 \\
82.9\end{array}$ & $\begin{array}{r}12.0 \\
5.2 \\
88.1\end{array}$ & $\begin{array}{l}\text { N/A } \\
\text { N/A } \\
\text { Is/A }\end{array}$ & $\begin{array}{r}96.6 \\
11.9 \\
100.0\end{array}$ \\
\hline $\begin{array}{l}220 \\
\text { ORT- } \\
\text { PIIT }\end{array}$ & $\begin{array}{c}0.1 \stackrel{\text { H }}{\text { N/A MEDIAN- }} \\
\text { WT- } 590.0\end{array}$ & $\begin{array}{c}12.6 \\
2.00\end{array}$ & $\begin{array}{c}58.1 \\
\text { MODE- }\end{array}$ & $\begin{array}{c}29.3 \\
-1.00 \text { MEAN }\end{array}$ & $(m) g$ & $\mathbb{N}_{N / A}$ & CUM & $\begin{array}{l}\text { WT } \\
\text { WT } \\
\text { WT }\end{array}$ & $\begin{array}{l}\text { N/A } \\
N / A \\
\text { N/A }\end{array}$ & $\begin{array}{r}170.9 \\
29.3 \\
29.3\end{array}$ & $\begin{array}{r}54.5 \\
9.3 \\
38.6\end{array}$ & $\begin{array}{l}64.6 \\
11.1 \\
49.7\end{array}$ & $\begin{array}{l}94.1 \\
16.1 \\
65.8\end{array}$ & $\begin{array}{l}60.8 \\
11.8 \\
77.6\end{array}$ & $\begin{array}{r}57.1 \\
9.8 \\
87.4\end{array}$ & $\begin{array}{l}\text { N/A } \\
\text { H/A } \\
\text { N/A }\end{array}$ & $\begin{array}{r}73.7 \\
12.6 \\
100.0\end{array}$ \\
\hline $\begin{array}{l}225 \\
\text { PRT- } \\
\text { PLIT }\end{array}$ & $\begin{array}{c}0.0 \stackrel{\text { H }}{ } \\
\text { N/A MEDIAN- } \\
\text { WT }=871.6\end{array}$ & $\begin{array}{l}9.8 \\
1.00\end{array}$ & $\begin{array}{r}59.9 \\
\text { MODE- }\end{array}$ & $-1.00^{30.3}$ MEAN- & $\begin{array}{l}\text { maG } \\
v_{-}\end{array}$ & N/A & $M$ & $\begin{array}{l}\text { WT } \\
\text { WT } \\
\text { WT }\end{array}$ & $\begin{array}{l}\text { N/A } \\
\text { N/A } \\
\text { N/A }\end{array}$ & $\begin{array}{r}263.2 \\
30.3 \\
30.3\end{array}$ & $\begin{array}{l}94.7 \\
10.9 \\
41.2\end{array}$ & $\begin{array}{r}100.4 \\
11.6 \\
52.8\end{array}$ & $\begin{array}{r}153.5 \\
17.7 \\
70.4\end{array}$ & $\begin{array}{r}124.2 \\
14.3 \\
84.7\end{array}$ & $\begin{array}{r}47.2 \\
5.4 \\
90.2\end{array}$ & $\begin{array}{l}\text { N/A } \\
\text { N/A } \\
\text { N/A }\end{array}$ & $\begin{array}{r}85.5 \\
9.8 \\
100.0\end{array}$ \\
\hline
\end{tabular}



ROCSAN REPORT

$12 / 11 / 89$

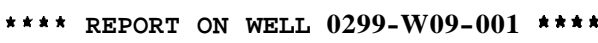

\begin{tabular}{|c|c|c|c|c|c|c|c|c|c|c|c|c|c|c|c|c|c|}
\hline DEPTH & 8ACO3 & DM & IMUD & ISAND & ○GRAVEL C & CLASS & & & $\begin{array}{l}\text { FINE } \\
\text { PEB } \\
(<--2)\end{array}$ & $\begin{array}{l}\text { VF INE } \\
\text { PEB } \\
(-1)\end{array}$ & $\begin{array}{l}\text { VERY } \\
\text { COARS } \\
(0)\end{array}$ & $\begin{array}{l}\text { COARS } \\
\text { (1) }\end{array}$ & $\begin{array}{l}\text { MED } \\
(2)\end{array}$ & $\begin{array}{l}\text { FINE } \\
\text { (3) }\end{array}$ & $\begin{array}{l}\text { VERY } \\
\text { FINE } \\
\text { (4) }\end{array}$ & $\begin{array}{l}\text { SILT } \\
(4.75)\end{array}$ & $\begin{array}{c}\text { PAN } \\
(>4.75)\end{array}$ \\
\hline $\begin{array}{r}230 \\
\text { SORT- } \\
\text { SPIIT }\end{array}$ & $\begin{array}{c}0.1 \\
\text { N/A MED } \\
\text { WT- } 753.0\end{array}$ & $\begin{array}{l}\text { H } \\
\text { DIAN- }\end{array}$ & $\begin{array}{c}8.1 \\
-1.00\end{array}$ & $\begin{array}{r}\mathbf{3 1 . 0} \\
\text { MODE- }\end{array}$ & $\begin{array}{c}60.9 \\
-1.00 \\
\text { MEAN- }\end{array}$ & $\mathrm{I}_{-}^{\mathrm{msG}} \mathrm{N} / \mathrm{A}$ & CUM & $\begin{array}{l}\text { WT } \\
\text { WT } \\
\text { WT }\end{array}$ & $\begin{array}{l}\text { N/A } \\
\text { N/A } \\
\text { N/A }\end{array}$ & $\begin{array}{r}456.3 \\
60.9 \\
60.9\end{array}$ & $\begin{array}{r}74.5 \\
9.9 \\
70.8\end{array}$ & $\begin{array}{r}44.1 \\
5.9 \\
76.7\end{array}$ & $\begin{array}{r}38.9 \\
5.2 \\
81.9\end{array}$ & $\begin{array}{r}41.3 \\
5.5 \\
87.4\end{array}$ & $\begin{array}{r}33.7 \\
4.5 \\
91.9\end{array}$ & $\begin{array}{l}\text { N/A } \\
\text { N/A } \\
\text { M/A }\end{array}$ & $\begin{array}{r}60.6 \\
8.1 \\
100.0\end{array}$ \\
\hline $\begin{array}{r}235 \\
\text { SORT- } \\
\text { SPLIT }\end{array}$ & $\begin{array}{c}\text { O.0 } \\
\text { N/A MED } \\
\text { WT }=927.3\end{array}$ & $\begin{array}{l}\text { H } \\
\text { DIAN- }\end{array}$ & $\begin{array}{c}6.7 \\
-1.00\end{array}$ & $\begin{array}{r}40.1 \\
\text { MODE- }\end{array}$ & $\begin{array}{r}53.2 \\
-1.00 \\
\text { MEAN- }\end{array}$ & $I_{-}^{m s G} \quad$ N/A & cuM & $\begin{array}{l}\text { WT } \\
\text { WT } \\
\text { WT I I }\end{array}$ & $\begin{array}{l}\text { N/A } \\
\text { N/A } \\
\text { N/A }\end{array}$ & $\begin{array}{r}493.1 \\
53.2 \\
53.2\end{array}$ & $\begin{array}{r}135.0 \\
14.6 \\
67.7\end{array}$ & $\begin{array}{r}77.1 \\
8.3 \\
76.0\end{array}$ & $\begin{array}{r}63.6 \\
6.9 \\
02.9\end{array}$ & $\begin{array}{r}57.5 \\
6.2 \\
89.1\end{array}$ & $\begin{array}{r}38.9 \\
4.2 \\
93.3\end{array}$ & $\begin{array}{l}\text { N/A } \\
N / A \\
\text { N/A }\end{array}$ & $\begin{array}{r}62.5 \\
6.7 \\
100.0\end{array}$ \\
\hline $\begin{array}{r}240 \\
\text { SORT- } \\
\text { SPIIT }\end{array}$ & $\begin{array}{c}\mathbf{0 . 1} \\
\text { N/A MED } \\
\text { WT- } 811.8\end{array}$ & $\begin{array}{l}\text { DIAN- } \\
8\end{array}$ & $\begin{array}{c}10.2 \\
1.00\end{array}$ & $\begin{array}{r}64.2 \\
\text { MODE- }\end{array}$ & $\begin{array}{c}25.6 \\
-1.00 \text { MEAN }\end{array}$ & $(m) g S$ & CUM & $\begin{array}{l}\text { WT } \\
\text { WT } \\
\text { WT }\end{array}$ & $\begin{array}{l}\text { N/A } \\
\text { N/A } \\
\text { N/A }\end{array}$ & $\begin{array}{r}207.4 \\
25.6 \\
25.6\end{array}$ & $\begin{array}{l}95.1 \\
11.7 \\
37.3\end{array}$ & $\begin{array}{r}111.1 \\
13.7 \\
51.0\end{array}$ & $\begin{array}{r}137.8 \\
17.0 \\
68.0\end{array}$ & $\begin{array}{r}105.0 \\
12.9 \\
80.9\end{array}$ & $\begin{array}{r}71.8 \\
8.9 \\
89.0\end{array}$ & $\begin{array}{l}\text { N/A } \\
\text { N/A } \\
\text { N/A }\end{array}$ & $\begin{array}{r}83.1 \\
10.2 \\
100.0\end{array}$ \\
\hline $\begin{array}{r}245 \\
\text { SORT- } \\
\text { SPLIT }\end{array}$ & $\begin{array}{c}0.1 \\
\text { N/A MEL } \\
\text { WT- } 766.8\end{array}$ & $\begin{array}{l}\text { H } \\
\text { DIAN- } \\
8\end{array}$ & $\begin{array}{l}8.0 \\
1.00\end{array}$ & $\begin{array}{r}64.5 \\
\text { MODE- }\end{array}$ & $\begin{array}{c}27.6 \\
-1.00 \text { MEAN- }\end{array}$ & $(m) g S$ & $\mathrm{CuM}$ & $\begin{array}{r}\text { WT } \\
\text { WT } \\
1 \mathrm{WT}\end{array}$ & $\begin{array}{l}\text { N/A } \\
\text { N/A } \\
\text { N/A }\end{array}$ & $\begin{array}{r}211.0 \\
27.6 \\
27.6\end{array}$ & $\begin{array}{r}107.3 \\
14.0 \\
41.6\end{array}$ & $\begin{array}{l}01.2 \\
10.6 \\
52.2\end{array}$ & $\begin{array}{r}144.8 \\
18.9 \\
71.2\end{array}$ & $\begin{array}{l}97.7 \\
12.8 \\
84.0\end{array}$ & $\begin{array}{r}61.9 \\
8.1 \\
92.1\end{array}$ & $\begin{array}{l}\text { N/A } \\
N / A \\
\text { N/A }\end{array}$ & $\begin{array}{r}60.8 \\
8.0 \\
100.0\end{array}$ \\
\hline $\begin{array}{r}250 \\
\text { SORT- } \\
\text { SPLIT }\end{array}$ & $\begin{array}{c}0.1 \\
\text { N/A MED } \\
\text { WT- } 783.6\end{array}$ & $\begin{array}{l}\text { H } \\
\text { DIAN- } \\
6\end{array}$ & $\begin{array}{l}9.3 \\
1.00\end{array}$ & $\begin{array}{r}\text { 66.0 } \\
\text { MODE- }\end{array}$ & $\begin{array}{c}24.7 \\
-1.00 \text { MEAN }\end{array}$ & $(m) g S$ & CUM & $\begin{array}{l}\text { WT } \\
\text { WT } \\
\text { WT }\end{array}$ & $\begin{array}{l}\text { N/A } \\
\text { H/A } \\
\text { N/A }\end{array}$ & $\begin{array}{r}193.7 \\
24.7 \\
24.7\end{array}$ & $\begin{array}{r}138.0 \\
17.6 \\
42.3\end{array}$ & $\begin{array}{r}115.9 \\
14.8 \\
57.1\end{array}$ & $\begin{array}{r}104.5 \\
13.3 \\
70.4\end{array}$ & $\begin{array}{l}93.2 \\
11.9 \\
02.3\end{array}$ & $\begin{array}{r}66.3 \\
8.5 \\
90.7\end{array}$ & $\begin{array}{l}\text { N/A } \\
\text { N/A } \\
\text { N/A }\end{array}$ & $\begin{array}{r}72.7 \\
9.3 \\
100.0\end{array}$ \\
\hline $\begin{array}{r}255 \\
\text { SORT- } \\
\text { SPIIT }\end{array}$ & $\begin{array}{c}0.1 \\
\text { N/A MED } \\
\text { WT= } 691 .\end{array}$ & $\begin{array}{l}\text { D } \\
1\end{array}$ & $\begin{array}{r}12.2 \\
2.00\end{array}$ & $\begin{array}{r}73.9 \\
\text { MODE- }\end{array}$ & $\begin{array}{l}13.9 \\
1.00 \text { MEAN- }\end{array}$ & $(m) g s$ & CUM & $\begin{array}{l}\text { WT } \\
\text { WT I } \\
\text { WT } \\
\text { I }\end{array}$ & $\begin{array}{l}\mathbf{N} / \mathbf{A} \\
\mathbf{N} / \mathbf{A} \\
\mathbf{N} / \mathbf{A}\end{array}$ & $\begin{array}{l}95.5 \\
13.9 \\
13.9\end{array}$ & $\begin{array}{r}111.3 \\
16.2 \\
30.1\end{array}$ & $\begin{array}{r}124.9 \\
18.2 \\
48.3\end{array}$ & $\begin{array}{r}108.7 \\
15.8 \\
64.1\end{array}$ & $\begin{array}{r}104.6 \\
15.2 \\
79.3\end{array}$ & $\begin{array}{r}58.1 \\
8.5 \\
87.8\end{array}$ & $\begin{array}{l}N / A \\
N / A \\
N / A\end{array}$ & $\begin{array}{r}03.9 \\
12.2 \\
100.0\end{array}$ \\
\hline $\begin{array}{r}260 \\
\text { SORT- } \\
\text { SPIIT }\end{array}$ & $\begin{array}{c}\mathbf{0 . 3} \\
\text { N/A MED } \\
\text { WT- } 773.5\end{array}$ & $\begin{array}{l}\text { H } \\
\text { DIAN- }\end{array}$ & $\begin{array}{l}9.8 \\
1.00\end{array}$ & $\begin{array}{r}\mathbf{6 7 . 6} \\
\text { MODE- }\end{array}$ & $\begin{array}{c}22.6 \\
-1.00 \\
\text { MEAN- }\end{array}$ & $\underset{N}{(m) g S}$ & CUM & $\begin{array}{l}\text { WT } \\
\text { WT } \\
\text { WT }\end{array}$ & $\begin{array}{l}N / A \\
N / A \\
N / A\end{array}$ & $\begin{array}{r}173.5 \\
22.6 \\
22.6\end{array}$ & $\begin{array}{r}124.7 \\
16.2 \\
38.8\end{array}$ & $\begin{array}{r}120.8 \\
15.7 \\
54.6\end{array}$ & $\begin{array}{r}105.0 \\
13.7 \\
68.3\end{array}$ & $\begin{array}{r}103.2 \\
13.4 \\
81.7\end{array}$ & $\begin{array}{r}65.5 \\
8.5 \\
90.2\end{array}$ & $\begin{array}{l}N / A \\
N / A \\
N / A\end{array}$ & $\begin{array}{r}75.1 \\
9.8 \\
100.0\end{array}$ \\
\hline $\begin{array}{r}265 \\
\text { SORT- } \\
\text { SPLIT }\end{array}$ & $\begin{array}{r}1.2 \\
\text { N/A MED } \\
\text { WT- } 766.2\end{array}$ & ${ }_{\text {DIAN- }}^{\mathrm{H}}$ & $\begin{array}{c}10.2 \\
2.00\end{array}$ & $\begin{array}{r}64.9 \\
\text { MODE- }\end{array}$ & -1.00 MEAN & (m) gs & CUM & $\begin{array}{l}\text { WT } \\
\text { WT I } \\
\text { WT }\end{array}$ & $\begin{array}{l}\mathbf{N} / \boldsymbol{A} \\
\mathbf{N} / \mathbf{A} \\
\mathbf{N} / \mathbf{A}\end{array}$ & $\begin{array}{r}189.5 \\
24.9 \\
24.9\end{array}$ & $\begin{array}{l}87.3 \\
11.5 \\
36.4\end{array}$ & $\begin{array}{l}84.2 \\
11.1 \\
47.5\end{array}$ & $\begin{array}{r}130.8 \\
17.2 \\
64.7\end{array}$ & $\begin{array}{r}112.3 \\
14.8 \\
79.5\end{array}$ & $\begin{array}{l}78.5 \\
10.3 \\
89.8\end{array}$ & $\begin{array}{l}N / A \\
N / A \\
N / A\end{array}$ & $\begin{array}{r}77.6 \\
10.2 \\
100.0\end{array}$ \\
\hline $\begin{array}{r}270 \\
\text { SORT- } \\
\text { SPLIT }\end{array}$ & $\begin{array}{c}1.4 \\
\text { N } / A \text { MED } \\
\mathrm{WT}=699.5\end{array}$ & $\begin{array}{l}\text { DIAN- } \\
5\end{array}$ & $\begin{array}{c}10.8 \\
2.00\end{array}$ & $\begin{array}{r}65.3 \\
\text { MODE- }\end{array}$ & $\begin{array}{c}23.9 \\
-1.00 \text { MEAN }\end{array}$ & (m) gS & CUM & $\begin{array}{l}\text { WT } \\
\text { WT } \\
\text { WT }\end{array}$ & $\begin{array}{l}N / A \\
N / A \\
N / A\end{array}$ & $\begin{array}{r}166.7 \\
23.9 \\
23.9\end{array}$ & $\begin{array}{l}90.8 \\
13.0 \\
36.9\end{array}$ & $\begin{array}{l}74.5 \\
10.7 \\
47.6\end{array}$ & $\begin{array}{r}119.1 \\
17.1 \\
64.7\end{array}$ & $\begin{array}{r}101.8 \\
14.6 \\
79.3\end{array}$ & $\begin{array}{l}69.5 \\
10.0 \\
89.2\end{array}$ & $\begin{array}{l}N / A \\
N / A \\
N / A\end{array}$ & $\begin{array}{r}75.2 \\
10.8 \\
100.0\end{array}$ \\
\hline $\begin{array}{r}275 \\
\text { SORT- } \\
\text { SPIIT }\end{array}$ & $\begin{array}{c}0.9 \\
\text { N/A MED } \\
\text { WT- } 629.8\end{array}$ & $\begin{array}{l}\mathrm{H} \\
\mathrm{DIAN}-\end{array}$ & $\begin{array}{c}10.8 \\
2.00\end{array}$ & $\begin{array}{r}75.2 \\
\text { MODE- }\end{array}$ & 2.00 MEAN- & (m) gS & CUM & $\begin{array}{l}\text { WT } \\
\text { WT } \\
\text { WT }\end{array}$ & $\begin{array}{l}N / A \\
N / A \\
N / A\end{array}$ & $\begin{array}{l}87.1 \\
14.0 \\
14.0\end{array}$ & $\begin{array}{l}74.9 \\
12.0 \\
26.0\end{array}$ & $\begin{array}{l}85.7 \\
13.8 \\
39.7\end{array}$ & $\begin{array}{r}134.2 \\
21.5 \\
61.3\end{array}$ & $\begin{array}{r}103.8 \\
16.7 \\
77.9\end{array}$ & $\begin{array}{l}70.3 \\
11.3 \\
89.2\end{array}$ & $\begin{array}{l}N / A \\
N / A \\
N / A\end{array}$ & $\begin{array}{r}67.4 \\
10.8 \\
100.0\end{array}$ \\
\hline $\begin{array}{r}280 \\
\text { SORT- } \\
\text { SPIIT }\end{array}$ & $\begin{array}{c}0.5 \\
\text { N/A MED } \\
\text { WT- } 727.5\end{array}$ & $\begin{array}{l}\text { DI } \\
5\end{array}$ & $\begin{array}{l}8.1 \\
0.00\end{array}$ & $\begin{array}{r}\mathbf{4 2 . 0} \\
\text { MODE- }\end{array}$ & $1.00^{50.0}{ }^{n}{ }^{n}$ & $\begin{array}{l}\text { msG } \\
-\end{array}$ & & $\begin{array}{l}\text { WT } \\
\text { WT I } \\
\text { WT \& }\end{array}$ & $\begin{array}{l}N / A \\
N / A \\
N / A\end{array}$ & $\begin{array}{r}360.9 \\
50.0 \\
50.0\end{array}$ & $\begin{array}{r}66.3 \\
9.2 \\
59.1\end{array}$ & $\begin{array}{r}53.5 \\
7.4 \\
66.6\end{array}$ & $\begin{array}{l}75.6 \\
10.5 \\
77.0\end{array}$ & $\begin{array}{r}65.2 \\
9.0 \\
86.1\end{array}$ & $\begin{array}{r}42.6 \\
5.9 \\
92.0\end{array}$ & $\begin{array}{l}N / A \\
N / A \\
N / A\end{array}$ & $\begin{array}{r}58.3 \\
8.1 \\
100.0\end{array}$ \\
\hline
\end{tabular}


WESTINGHOUSE HANFORD OPERATIONS SIEVE ANALYSIS ROCSAN REPORT

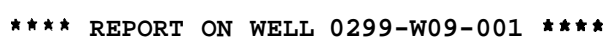

$12 / 11 / 89$

\begin{tabular}{|c|c|c|c|c|c|c|c|c|c|c|c|c|c|c|c|c|c|c|}
\hline PTH & $1 \mathrm{CACO} 3$ & DM & MUD & YSAND & GRAVEL & CLASS & & & & $\begin{array}{l}\text { FINE } \\
\text { PEB } \\
(<--2)\end{array}$ & $\begin{array}{l}\text { VF INE } \\
\text { PEB } \\
(-1)\end{array}$ & $\begin{array}{l}\text { VERY } \\
\text { COARS } \\
(0)\end{array}$ & $\begin{array}{l}\text { COARS } \\
\text { (1) }\end{array}$ & $\begin{array}{l}\text { MED } \\
\text { (2) }\end{array}$ & $\begin{array}{l}\text { FINE } \\
\text { (3) }\end{array}$ & $\begin{array}{l}\text { VERY } \\
\text { FINE } \\
\text { (4) }\end{array}$ & $\begin{array}{c}\text { SILT } \\
(4.75)\end{array}$ & $\begin{array}{c}\text { PAN } \\
(>4.75)\end{array}$ \\
\hline $\begin{array}{r}285 \\
\text { SORT- } \\
\text { SPLIT }\end{array}$ & $\begin{array}{r}0.8 \\
\text { N/A ME } \\
\text { WT- } 658 .\end{array}$ & $\begin{array}{l}\text { H } \\
\text { DIAN- } \\
0\end{array}$ & $\begin{array}{l}7.9 \\
1.00\end{array}$ & $\begin{array}{r}51.7 \\
\text { MODE- }\end{array}$ & $-1.00^{40.4} \mathrm{MEAN}$ & $\mathbf{v}_{-}^{\mathbf{m s G}}$ & N/A & CUM & $\begin{array}{l}\text { WT } \\
\text { WT } \\
\text { WT }\end{array}$ & $\begin{array}{l}\text { N/A } \\
\text { N/A } \\
\text { N/A }\end{array}$ & $\begin{array}{r}263.9 \\
40.4 \\
40.4\end{array}$ & $\begin{array}{r}50.2 \\
7.7 \\
48.1\end{array}$ & $\begin{array}{r}59.5 \\
9.1 \\
57.2\end{array}$ & $\begin{array}{r}109.4 \\
16.7 \\
73.9\end{array}$ & $\begin{array}{l}75.5 \\
11.6 \\
85.5\end{array}$ & $\begin{array}{r}43.5 \\
6.7 \\
92.1\end{array}$ & $\begin{array}{l}\text { N/A } \\
\text { N/A } \\
\text { N/A }\end{array}$ & $\begin{array}{r}51.4 \\
7.9 \\
100.0\end{array}$ \\
\hline $\begin{array}{r}295 \\
\text { SORT- } \\
\text { SPIIT }\end{array}$ & $\begin{array}{r}1.6 \\
\text { N/A ME } \\
\text { WT- } 710 .\end{array}$ & $\begin{array}{l}\text { H } \\
\text { DIAN- } \\
8\end{array}$ & $\begin{array}{l}7.8 \\
0.00\end{array}$ & $\begin{array}{r}48.2 \\
\text { MODE- }\end{array}$ & $-1.00^{44.0}$ MEAN & $\mathrm{msG}$ & N/A & UM & $\begin{array}{l}\text { WT } \\
\text { WT }\end{array}$ & $\begin{array}{l}\text { N/A } \\
\text { N/A } \\
\text { N/A }\end{array}$ & $\begin{array}{c}310.8 \\
44.0 \\
44.0\end{array}$ & $\begin{array}{l}74.0 \\
10.6 \\
54.6\end{array}$ & $\begin{array}{r}62.0 \\
8.0 \\
63.3\end{array}$ & $\begin{array}{l}8 \theta .2 \\
12.5 \\
75.8\end{array}$ & $\begin{array}{l}73.7 \\
10.4 \\
86.2\end{array}$ & $\begin{array}{r}42.1 \\
6.0 \\
92.2\end{array}$ & $\begin{array}{l}\text { N/A } \\
\text { N/A } \\
\text { N/A }\end{array}$ & $\begin{array}{r}55.2 \\
7.8 \\
100.0\end{array}$ \\
\hline
\end{tabular}


$12 / 11 / 89$

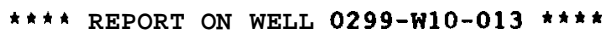

\begin{tabular}{|c|c|c|c|c|c|c|c|c|c|c|c|c|c|c|c|c|c|c|}
\hline EPTH & $8 \mathrm{CAC} 03$ & DM & IMUD & ISAND & ZGRAVEL & CLAS & & & & $\begin{array}{l}\text { FINE } \\
\text { PEB } \\
<<--2)\end{array}$ & $\begin{array}{l}\text { VEINE } \\
\text { PEB } \\
(-1)\end{array}$ & $\begin{array}{l}\text { VERY } \\
\text { COARS } \\
(0)\end{array}$ & $\begin{array}{l}\text { COARS } \\
\text { (1) }\end{array}$ & $\begin{array}{l}\text { MED } \\
(2)\end{array}$ & $\begin{array}{l}\text { FINE } \\
\text { (3) }\end{array}$ & $\begin{array}{l}\text { VERY } \\
\text { FINE } \\
\text { (4) }\end{array}$ & $\begin{array}{c}\text { SILT } \\
(4.75)\end{array}$ & $\begin{array}{c}\text { PAN } \\
(>4.75)\end{array}$ \\
\hline $\begin{array}{l}5 \\
\text { ORT- } \\
\text { PLIT }\end{array}$ & $\begin{array}{c}0.9 \\
\text { N/A MED } \\
\text { WT- } 523.4\end{array}$ & DIAN- & $\begin{array}{l}0.9 \\
-\quad 4.00\end{array}$ & $\begin{array}{c}91.1 \\
\text { MODE- }\end{array}$ & $\begin{array}{c}0.0 \\
4.00 \mathrm{MEAN}\end{array}$ & s & N/A & CUM & $\begin{array}{l}\text { WT } \\
\text { WT } \\
\text { WT }\end{array}$ & $\begin{array}{l}\text { N/A } \\
\text { N/A } \\
\text { N/A }\end{array}$ & $\begin{array}{l}0.2 \\
0.0 \\
0.0\end{array}$ & $\begin{array}{l}4.3 \\
0.0 \\
0.9\end{array}$ & $\begin{array}{c}41.0 \\
8.0 \\
0.9\end{array}$ & $\begin{array}{l}06.5 \\
16.5 \\
25.4\end{array}$ & $\begin{array}{c}114.7 \\
21.9 \\
47.3\end{array}$ & $\begin{array}{r}229.3 \\
43.0 \\
91.1\end{array}$ & $\begin{array}{l}\text { N/A } \\
\text { N/A } \\
\text { N/A }\end{array}$ & $\begin{array}{r}46.5 \\
0.9 \\
100.0\end{array}$ \\
\hline $\begin{array}{r}10 \\
\text { ORT- } \\
\text { PLIT }\end{array}$ & $\begin{array}{c}1.6 \\
\text { N/A MED } \\
\text { WT- } 486.0\end{array}$ & $\begin{array}{l}\text { N/ } \\
\text { DIAN- } \\
0\end{array}$ & $\begin{array}{c}10.3 \\
3.00\end{array}$ & $\begin{array}{r}00.9 \\
\text { MODE- }\end{array}$ & $4.00^{0.0}$ & $(m):$ & $\mathrm{s}_{\mathrm{N} / \mathrm{A}}$ & CUM & $\begin{array}{l}\text { WT } \\
\text { WT } \\
\text { WT }\end{array}$ & $\begin{array}{l}\text { N/A } \\
\text { N/A } \\
\text { N/A }\end{array}$ & $\begin{array}{l}3.8 \\
0.8 \\
0.8\end{array}$ & $\begin{array}{l}2.5 \\
0.5 \\
1.3\end{array}$ & $\begin{array}{r}17.9 \\
3.7 \\
5.0\end{array}$ & $\begin{array}{l}61.3 \\
12.6 \\
17.6\end{array}$ & $\begin{array}{r}170.7 \\
35.1 \\
52.7\end{array}$ & $\begin{array}{c}179.7 \\
37.0 \\
09.7\end{array}$ & $\begin{array}{l}\text { N/A } \\
\text { N/A } \\
\text { N/A }\end{array}$ & $\begin{array}{r}50.1 \\
10.3 \\
100.0\end{array}$ \\
\hline $\begin{array}{r}15 \\
\text { ORT- } \\
\text { PLIT }\end{array}$ & $\begin{array}{c}1.6 \\
\text { N/A MED } \\
\text { WT- } 557.2\end{array}$ & ${ }_{2}^{\text {DIAN- }}$ & $\begin{array}{c}10.9 \\
-\quad 3.00\end{array}$ & $\begin{array}{l}01.5 \\
\text { MODE- }\end{array}$ & $3.00{ }^{7.6}$ & (gm) & ) & CUM & $\begin{array}{l}\text { WT } \\
\text { WT } \\
\text { WT }\end{array}$ & $\begin{array}{l}\text { N/A } \\
\text { N/A } \\
\text { N/A }\end{array}$ & $\begin{array}{r}42.3 \\
7.6 \\
7.6\end{array}$ & $\begin{array}{l}7.5 \\
1.4 \\
9.0\end{array}$ & $\begin{array}{r}24.1 \\
4.3 \\
13.3\end{array}$ & $\begin{array}{l}63.0 \\
11.3 \\
24.6\end{array}$ & $\begin{array}{r}103.3 \\
32.9 \\
57.5\end{array}$ & $\begin{array}{r}176.2 \\
31.6 \\
09.1\end{array}$ & $\begin{array}{l}\text { N/A } \\
\text { N/A } \\
\text { N/A }\end{array}$ & $\begin{array}{r}60.5 \\
10.9 \\
100.0\end{array}$ \\
\hline $\begin{array}{r}20 \\
\text { SORT- } \\
\text { SPLIT }\end{array}$ & $\begin{array}{c}0.4 \\
\text { N/A MED } \\
\text { WT- } 670.6\end{array}$ & $\begin{array}{l}\text { C } \\
\text { DIAN- } \\
6\end{array}$ & $\begin{array}{l}2.8 \\
-\quad 0.00\end{array}$ & $\begin{array}{r}67.7 \\
\text { MODE- }\end{array}$ & $-1.00^{29.5}$ MEAN & gs & N/A & CUM & $\begin{array}{l}\text { WT } \\
\text { WT } \\
\text { WT }\end{array}$ & $\begin{array}{l}\text { N/A } \\
\text { N/A } \\
\text { N/A }\end{array}$ & $\begin{array}{r}200.4 \\
29.5 \\
29.5\end{array}$ & $\begin{array}{r}152.7 \\
22.5 \\
52.1\end{array}$ & $\begin{array}{r}100.3 \\
14.0 \\
66.0\end{array}$ & $\begin{array}{r}105.6 \\
15.6 \\
02.4\end{array}$ & $\begin{array}{l}74.3 \\
11.0 \\
93.4\end{array}$ & $\begin{array}{r}26.3 \\
3.9 \\
97.2\end{array}$ & $\begin{array}{l}\text { W/A } \\
\text { W/A } \\
\text { W/A }\end{array}$ & $\begin{array}{r}18.7 \\
2.8 \\
100.0\end{array}$ \\
\hline $\begin{array}{r}25 \\
\text { ORT- } \\
\text { PLIT }\end{array}$ & $\begin{array}{c}0.9 \\
\text { N/A MED } \\
\text { WT= } 744.7\end{array}$ & $\begin{array}{l}\text { C } \\
\text { DIAN- }\end{array}$ & $\begin{array}{l}3.1 \\
-\quad 1.00\end{array}$ & $\begin{array}{r}74.7 \\
\text { MODE- }\end{array}$ & $1.00^{22.2}$ MEAN & $\mathrm{gs}$ & N/A & CUM & $\begin{array}{l}\text { WT } \\
\text { WT } \\
\text { WT }\end{array}$ & $\begin{array}{l}\text { N/A } \\
\text { N/A } \\
\text { N/A }\end{array}$ & $\begin{array}{r}165.3 \\
22.2 \\
22.2\end{array}$ & $\begin{array}{c}129.0 \\
17.3 \\
39.6\end{array}$ & $\begin{array}{r}215.3 \\
20.9 \\
60.5\end{array}$ & $\begin{array}{r}135.0 \\
10.3 \\
06.0\end{array}$ & $\begin{array}{r}54.9 \\
7.4 \\
94.1\end{array}$ & $\begin{array}{c}20.9 \\
2.0 \\
97.0\end{array}$ & $\begin{array}{l}\text { N/A } \\
\text { W/A } \\
\text { N/A }\end{array}$ & $\begin{array}{r}22.7 \\
3.1 \\
100.0\end{array}$ \\
\hline $\begin{array}{r}30 \\
\text { ORT- } \\
\text { PLIT }\end{array}$ & $\begin{array}{c}0.0 \\
\text { N/A MED } \\
\text { WT- } 080.6\end{array}$ & $\begin{array}{l}\text { C } \\
\text { DIAN- } \\
6\end{array}$ & $\begin{array}{c}13.4 \\
-\quad 0.00\end{array}$ & $\begin{array}{r}46.3 \\
\text { MODE- }\end{array}$ & $-1.00^{40.3}$ MEAN & mse & N/A & CUM & $\begin{array}{l}\text { WT } \\
\text { WT } \\
\text { WT }\end{array}$ & $\begin{array}{l}\text { N/A } \\
\text { N/A } \\
\text { N/A }\end{array}$ & $\begin{array}{r}354.6 \\
40.3 \\
40.3\end{array}$ & $\begin{array}{c}102.2 \\
11.6 \\
51.9\end{array}$ & $\begin{array}{r}70.0 \\
9.0 \\
60.9\end{array}$ & $\begin{array}{r}04.2 \\
9.6 \\
70.5\end{array}$ & $\begin{array}{r}104.0 \\
11.0 \\
02.3\end{array}$ & $\begin{array}{r}38.0 \\
4.3 \\
06.6\end{array}$ & $\begin{array}{l}\text { N/A } \\
\text { N/A } \\
\text { N/A }\end{array}$ & $\begin{array}{r}117.9 \\
13.4 \\
100.0\end{array}$ \\
\hline $\begin{array}{r}35 \\
\text { SORT- } \\
\text { SPLIT }\end{array}$ & $\begin{array}{c}1.1 \\
\text { W/A MED } \\
\text { WT- } 572.7\end{array}$ & $\begin{array}{l}\text { C } \\
\text { DIAN- }\end{array}$ & $\begin{array}{l}6.9 \\
2.00\end{array}$ & $\begin{array}{l}91.3 \\
\text { MODE- }\end{array}$ & $2.00_{\text {MEAN }}^{1.0}$ & 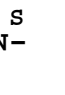 & N/A & CUM & $\begin{array}{l}\text { WT } \\
\text { WT } \\
\text { WT }\end{array}$ & $\begin{array}{l}\text { N/A } \\
\text { N/A } \\
\text { N/A }\end{array}$ & $\begin{array}{r}10.3 \\
1.8 \\
1.8\end{array}$ & $\begin{array}{r}35.4 \\
6.2 \\
0.0\end{array}$ & $\begin{array}{r}124.7 \\
21.0 \\
29.8\end{array}$ & $\begin{array}{r}179.7 \\
31.4 \\
61.2\end{array}$ & $\begin{array}{r}117.4 \\
20.5 \\
81.7\end{array}$ & $\begin{array}{l}65.2 \\
11.4 \\
93.1\end{array}$ & $\begin{array}{l}N / A \\
N / A \\
N / A\end{array}$ & $\begin{array}{r}39.4 \\
6.9 \\
100.0\end{array}$ \\
\hline $\begin{array}{r}40 \\
\text { SORT- } \\
\text { SPIIT }\end{array}$ & $\begin{array}{c}1.5 \\
\text { N/A MED } \\
\text { WT- } 691.9\end{array}$ & $\begin{array}{l}\text { C } \\
\text { DIAN- } \\
9\end{array}$ & $\begin{array}{l}4.5 \\
-\quad 1.00\end{array}$ & $\begin{array}{c}95.0 \\
\text { MODE- }\end{array}$ & $1.00^{0.4}$ MEAN & s- & N/A & CUM & $\begin{array}{c}\text { WT } \\
\text { WT } \\
\text { WT }\end{array}$ & $\begin{array}{l}N / A \\
N / A \\
N / A\end{array}$ & $\begin{array}{l}3.1 \\
0.5 \\
0.5\end{array}$ & $\begin{array}{l}73.3 \\
10.6 \\
11.0\end{array}$ & $\begin{array}{r}337.0 \\
40.7 \\
59.7\end{array}$ & $\begin{array}{r}120.6 \\
17.4 \\
77.2\end{array}$ & $\begin{array}{r}60.1 \\
0.7 \\
05.9\end{array}$ & $\begin{array}{r}66.5 \\
9.6 \\
95.5\end{array}$ & $\begin{array}{l}N / A \\
N / A \\
N / A\end{array}$ & $\begin{array}{r}31.4 \\
4.5 \\
100.0\end{array}$ \\
\hline $\begin{array}{r}45 \\
\text { SORT- } \\
\text { SPLIT }\end{array}$ & $\begin{array}{c}1.7 \\
\text { N/A MED } \\
\text { WT- } 504.0\end{array}$ & $\begin{array}{l}\text { C } \\
\text { DIAN- } \\
0\end{array}$ & $\begin{array}{l}5.3 \\
2.00\end{array}$ & $\begin{array}{r}94.7 \\
\text { MODE- }\end{array}$ & $2 . \infty 0_{\mathrm{MEAN}}^{\mathrm{N} / \mathrm{A}}$ & G- & N/A & CUM & $\begin{array}{l}\text { WT } \\
\text { WT } \\
\text { WT }\end{array}$ & $\begin{array}{l}\text { N/A } \\
\text { N/A } \\
\text { N/A }\end{array}$ & $\begin{array}{l}\text { N/A } \\
\text { N/A } \\
\text { N/A }\end{array}$ & $\begin{array}{l}2.4 \\
0.4 \\
0.4\end{array}$ & $\begin{array}{l}06.6 \\
14.0 \\
15.2\end{array}$ & $\begin{array}{r}270.7 \\
46.3 \\
61.5\end{array}$ & $\begin{array}{r}129.5 \\
22.2 \\
03.7\end{array}$ & $\begin{array}{l}64.1 \\
11.0 \\
94.7\end{array}$ & $\begin{array}{l}N / A \\
N / A \\
N / A\end{array}$ & $\begin{array}{r}31.2 \\
5.3 \\
100.0\end{array}$ \\
\hline $\begin{array}{r}50 \\
\text { ORT- } \\
\text { SPIIT }\end{array}$ & $\begin{array}{c}1.2 \\
\mathrm{~N} / \mathrm{A} \text { MED } \\
\text { WT- } 604.9\end{array}$ & $\begin{array}{l}\text { C } \\
9 \text { DIAN- } \\
9\end{array}$ & $\begin{array}{l}9.0 \\
3.00\end{array}$ & $\begin{array}{r}90.4 \\
\text { MODE- }\end{array}$ & $3.00 \quad 0.7$ & $s$ & $\mathbf{N} / \mathbf{A}$ & CUM & $\begin{array}{r}\text { WT } \\
\text { WT } \\
\text { W' }\end{array}$ & $\begin{array}{l}\text { N/A } \\
\text { N/A } \\
\text { N/A }\end{array}$ & $\begin{array}{l}4.0 \\
0.7 \\
0.7\end{array}$ & $\begin{array}{r}11.1 \\
1.0 \\
2.5\end{array}$ & $\begin{array}{r}20.0 \\
4.6 \\
7.1\end{array}$ & $\begin{array}{l}70.4 \\
11.6 \\
10.0\end{array}$ & $\begin{array}{r}313.0 \\
51.7 \\
70.5\end{array}$ & $\begin{array}{r}124.7 \\
20.6 \\
91.1\end{array}$ & $\begin{array}{l}N / A \\
N / A \\
N / A\end{array}$ & $\begin{array}{r}54.2 \\
9.0 \\
100.0\end{array}$ \\
\hline $\begin{array}{r}55 \\
\text { ORT- } \\
\text { PLIT }\end{array}$ & $\begin{array}{c}1.3 \\
\text { N/A MED } \\
\text { WT- } 510.2\end{array}$ & $\begin{array}{l}\text { C } \\
\text { DIAN- }\end{array}$ & $\begin{array}{l}4.7 \\
2.00\end{array}$ & $\begin{array}{r}94.4 \\
\text { MODE- }\end{array}$ & $2.00 \begin{array}{l}0.9 \\
\text { MEAN }\end{array}$ & $s$ & N/A & 1 & $\begin{array}{l}\text { WT } \\
\text { WT }\end{array}$ & $\begin{array}{l}N / A \\
N / A \\
N / A\end{array}$ & $\begin{array}{l}4.4 \\
0.9 \\
0.9\end{array}$ & $\begin{array}{r}27.9 \\
5.5 \\
6.3\end{array}$ & $\begin{array}{l}95.4 \\
10.7 \\
25.0\end{array}$ & $\begin{array}{c}160.7 \\
31.5 \\
56.4\end{array}$ & $\begin{array}{r}149.5 \\
29.3 \\
05.7\end{array}$ & $\begin{array}{r}40.0 \\
9.6 \\
95.3\end{array}$ & $\begin{array}{l}N / A \\
N / A \\
N / A\end{array}$ & $\begin{array}{r}24.2 \\
4.7 \\
100.0\end{array}$ \\
\hline
\end{tabular}


WESTINGHOUSE HANPORD OPERATIONS SIEVE ANALYSIS ROCSAN REPORT

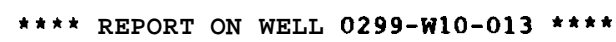

$12 / 11 / 89$

\begin{tabular}{ccccccl} 
DEPTH & 8CAC03 & DM & 8MUD & ISAND & \%GRAVEL & CLASS \\
\hdashline 60 & 2.0 & C & 4.9 & 94.0 & 0.3 & S
\end{tabular} $\begin{array}{lllllll}\text { SORT- N/A MEDIAN- } & 2.00 & \text { MODE- } & 1.00 & 0.3 \mathrm{~S} & \mathrm{~S} & \\ & & & \end{array}$ SPLIT WT- 526.3

$\begin{array}{lllllll}65 & 1.9 & \mathrm{C} & 5.4 & 94.2 & 0.4 \mathrm{~S} & \\ \text { SORT- } & \text { N/A } & \text { MEDIAN- } & 2.00 & \text { MODE- } & 2.00 & \text { MEAN- }\end{array}$

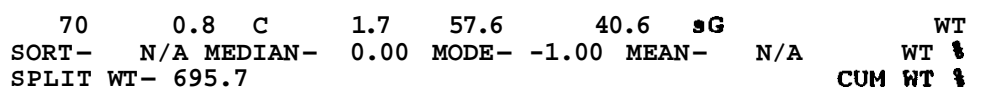

$\begin{array}{lll}\text { FINE } & \text { VFINE } & \text { VERY } \\ \text { PEB } & \text { PEB } & \text { COARS }\end{array}$

COARS MED FINE VERY

FINE SILT PAN (

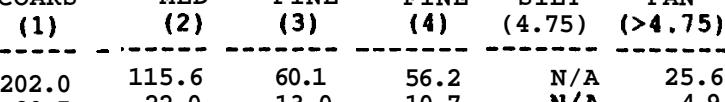

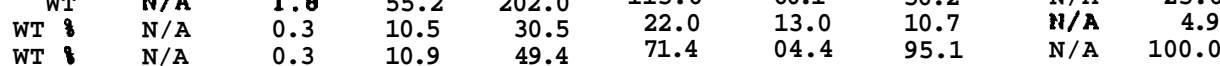
$\begin{array}{lllllll}75 & 0.9 & \mathrm{C} & 2.5 & 79.3 & 10.1 & \mathrm{gS}\end{array}$

N/A $\quad 40.6$

$\begin{array}{lll}0.3 & 10.9 & 0.9 \\ 0.2 & 9.1 & 122.7\end{array}$
NORT- N/A MEDIAN- 1.00 MODE- 1.00 MEAN- N/A WT $1.5 \mathrm{C}$ $\begin{array}{llll}4.0 & 31.6 \quad 63.5 \quad \mathrm{msG}\end{array}$ N/A SPLIT WT- 759.4

WT:

$\begin{array}{lllllll}85 & 1.1 & \mathrm{H} & 18.8 & 43.6 & 37.7 & \mathrm{msG}\end{array}$

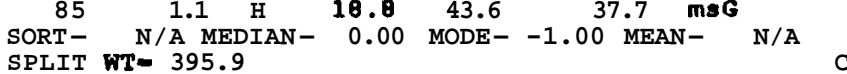

$90-1.0-$

WT
WT
WT
SORT- N/A MEDIAN- 1.00 MODE- -1.00 MEAN- N/A
SPLIT WT- 379.9 $950.9 \quad H$

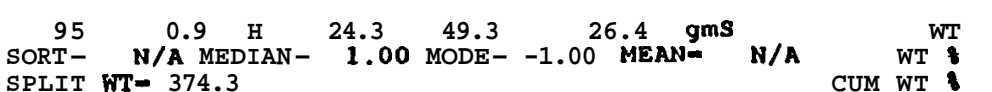

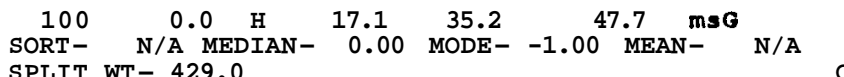

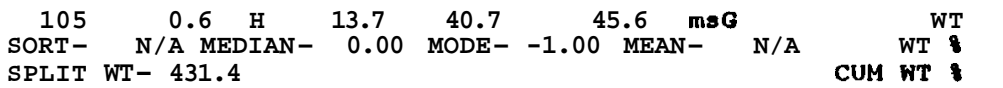

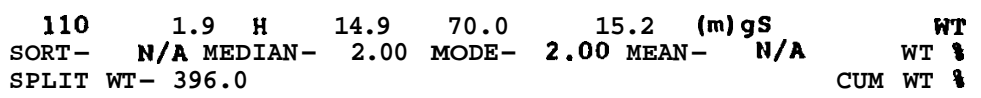

N/A $\quad 124.2$ N/A 10

$\mathrm{N} / \mathrm{A} \quad 401.2$

$\begin{array}{ll}\text { N/A } & 63.5 \\ \text { N/A } & 63.5\end{array}$

N/A 140.3

$\begin{array}{lr}\text { N/A } & 140.3 \\ \text { N/A } & 37.7 \\ \text { N/A } & 37.7\end{array}$

$\begin{array}{rr}\text { N/A } & 112.7 \\ \text { N/A } & 29.7\end{array}$ $\begin{array}{ll}\text { N/A } & 29.7 \\ \text { N/A } & 29.7\end{array}$

\section{N/A 99}

99.0
26.4 $\mathrm{N} / \mathrm{A}$

$\begin{array}{lr}\text { N/A } & 204.9 \\ \text { N/A } & 17.7\end{array}$

$\mathrm{N} / \mathrm{A}$

$\begin{array}{lr}\text { N/A } & 196.7 \\ \text { N/A } & 45.6\end{array}$

196.7
45.6
45.6

$\begin{array}{ll}\text { N/A } & 60.0 \\ \text { N/A } & 15.2\end{array}$

$\begin{array}{ll}\text { N/A } & 60.0 \\ \text { N/A } & 15.2\end{array}$

22.7

$226.1 \quad 105.2$

$\begin{array}{ll}1.6 & 22.2 \\ 2.0 & 24.2\end{array}$

19.0 N/A 29.8 $\begin{array}{rrrrr}40.8 & 19.0 & 10.6 & N / A & 5.4 \\ 65.0 & 04.0 & 94.6 & N / A & 100.0\end{array}$

$\begin{array}{lllll}75.3 & 23.0 & 12.6 & \text { N/A } & 12.1\end{array}$ $\begin{array}{rrrrr}10.9 & 3.3 & 1.0 & \text { N/A } & 1.8 \\ 93.1 & 96.4 & 98.3 & \text { N/A } & 100.0\end{array}$

$\begin{array}{lllll}03.3 & 26.4 & 14.3 & \text { N/A } & 17.3\end{array}$ $\begin{array}{rrrrr}12.2 & 3.9 & 2.1 & \text { N/A } & 2.5 \\ 91.6 & 95.4 & 97.5 & \text { N/A } & 100.0\end{array}$

$38.9 \quad 24.7 \quad 17.2$ $\begin{array}{rrrrr}5.1 & 3.3 & 2.3 & \text { N/A } & 4.9 \\ 89.6 & 92.9 & 95.2 & \text { I/A } & 100.0\end{array}$ $\begin{array}{lllll}32.8 & 22.0 & 16.1 & \text { N/A } & 73.9\end{array}$ $\begin{array}{rrrrr}8.3 & 5.6 & 4.1 & \text { N/A } & 18.8 \\ 71.6 & 77.1 & 81.2 & \text { N/A } & 100.0\end{array}$ $\begin{array}{lllll}35.1 & 26.2 & 21.9 & \text { N/A } & 89.0\end{array}$ $\begin{array}{rrrrr}9.2 & 6.9 & 5.8 & \text { N/A } & 23.9 \\ 63.9 & 70.8 & 76.6 & \text { N/A } & 100.0\end{array}$ $37.4 \quad 27.7 \quad 21.9 \quad N / A \quad 90.9$ $\begin{array}{rrrrr}10.0 & 7.4 & 5.8 & \text { N/A } & 24.3 \\ 62.5 & 69.9 & 75.7 & \text { N/A } & 100.0\end{array}$ $25.7-20.0-17.2$ $\begin{array}{rrrrr}6.0 & 4.7 & 4.0 & \text { N/A } & 17.1 \\ 74.3 & 78.9 & 82.9 & \text { N/A } & 100.0\end{array}$ $\begin{array}{lllll}25.8 & 17.6 & 14.0 & \text { N/A } & 59.2\end{array}$ $\begin{array}{rrrrr}6.0 & 4.1 & 3.3 & \text { N/A } & 13.7 \\ 79.0 & 03.0 & 06.3 & \text { N/A } & 100.0\end{array}$ $98.3 \quad 30.0 \quad 16.3 \quad$ N/A 58.9 $\begin{array}{rrrrr}98.3 & 30.0 & 16.3 & \text { N/A } & 58.9 \\ 24.8 & 7.6 & 4.1 & \text { N/A } & 14.9 \\ 73.4 & 01.0 & 85.1 & \text { N/A } & 100.0\end{array}$ 
ROCSAII REPORT

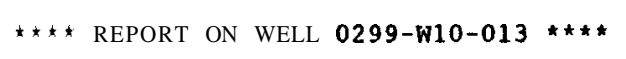

$12 / 11 / 89$

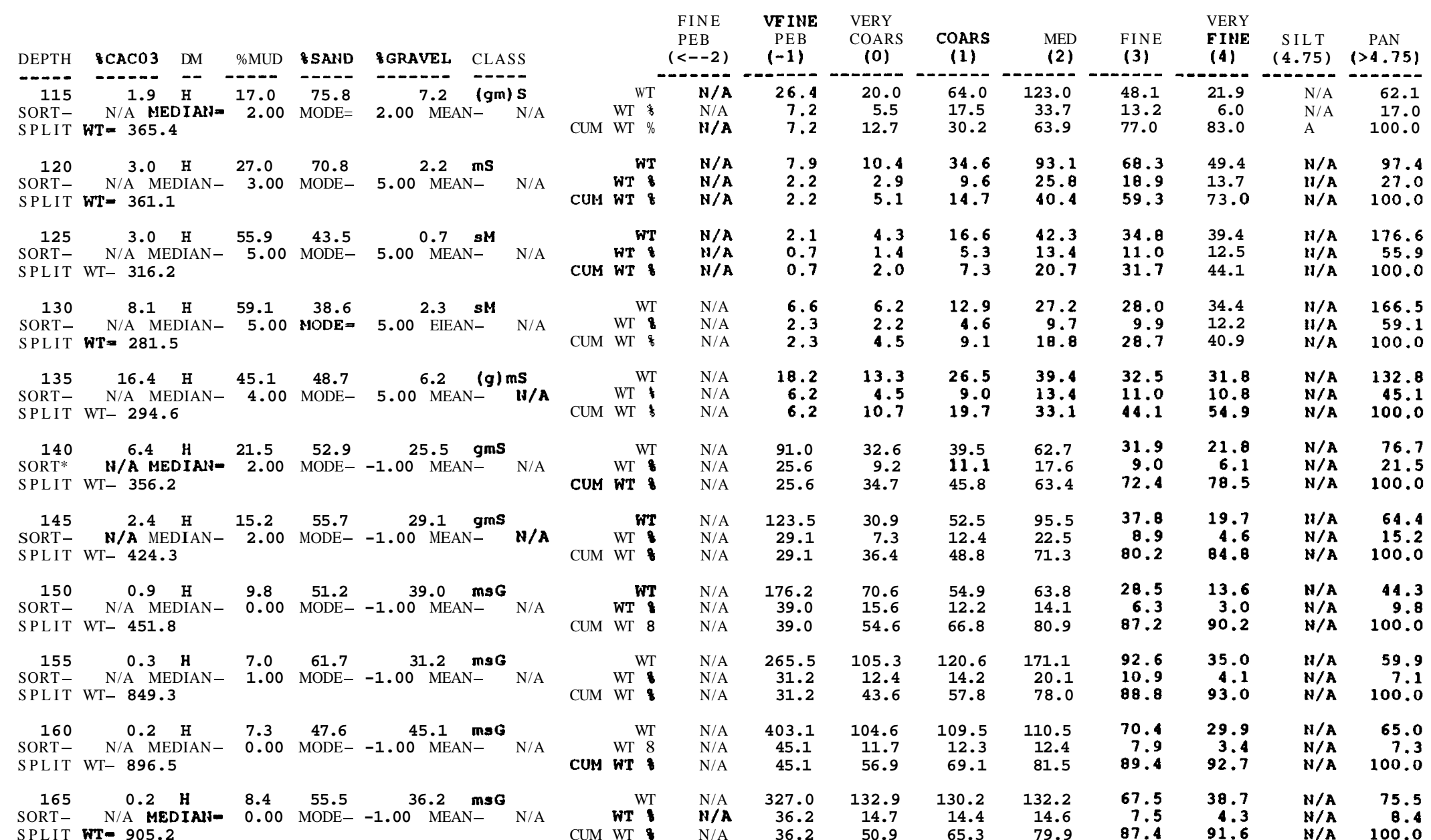


ROCSAN REPORT

$12 / 11 / 89$

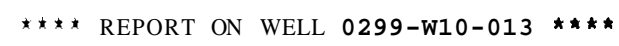

\section{$12 / 11 / 89$}

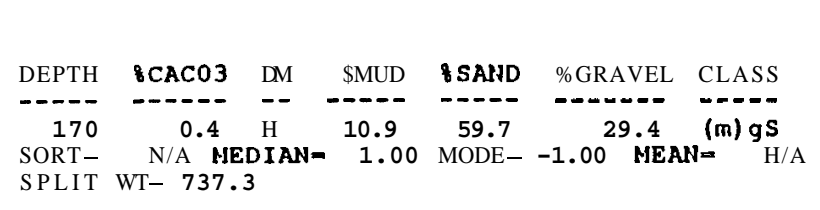

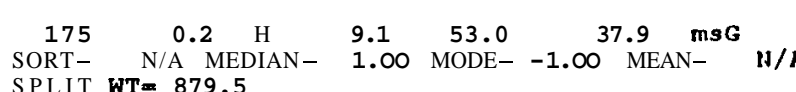

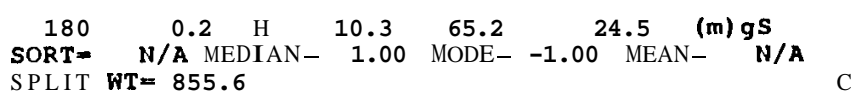

$\begin{array}{lcl}\text { FINE } & \text { VFINE } & \text { VERY } \\ \text { PEB } & \text { PEB } & \text { COARS }\end{array}$

CTITS

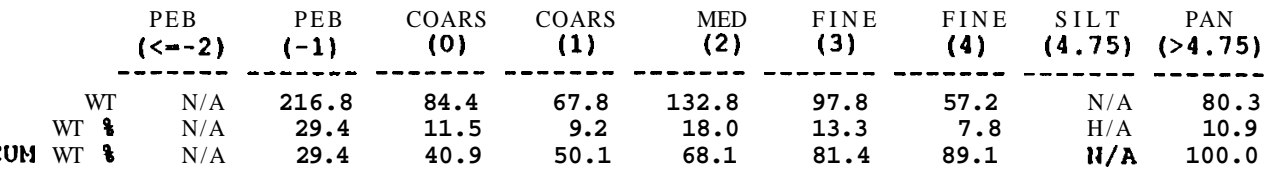
CUN WT

N/A 29.4

11.5
40.9

18.0
68.1

81.4

89.1

$\begin{array}{rr}\mathrm{H} / \mathrm{A} & 10.9 \\ \mathrm{IN} / \mathrm{A} & 100.0\end{array}$

WT
WT
WT

$\begin{array}{lr}\text { N/A } & 333.9 \\ \text { N/A } & 37.9\end{array}$

$92.5 \quad 122.3$

$128.6 \quad 77.2$

45.9 N/A 80.3

WT N/A 209.4

85.7

$\begin{array}{rrr}5.2 & \text { IJ/A } & 9.1 \\ 90.9 & \text { N/A } & 100.0\end{array}$

N/A 209.4

$92.0 \quad 159.9$

$159.9 \quad 122.7 \quad 124.3$

$58.2 \quad \mathrm{~N} / \mathrm{A} \quad 87.6$
$89.7 \quad \mathrm{~N} / \mathrm{A} \quad 100.0$

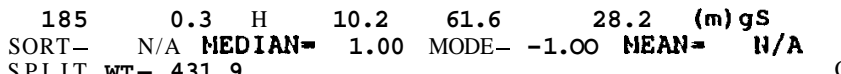

WT

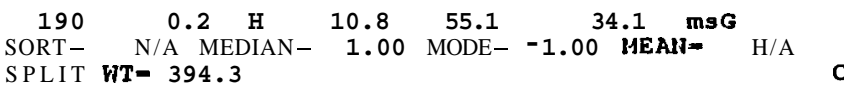

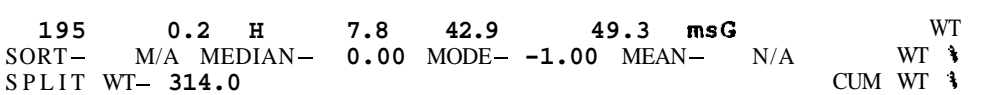

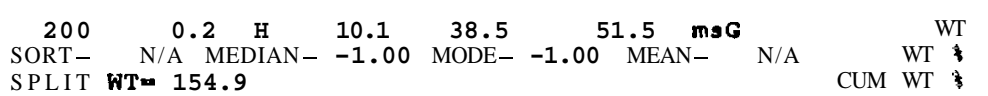

WT

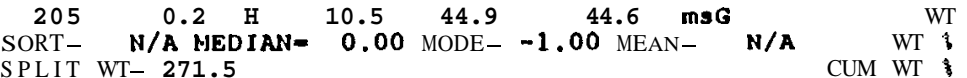

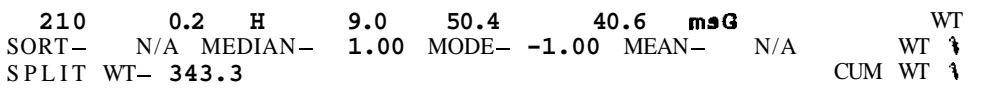

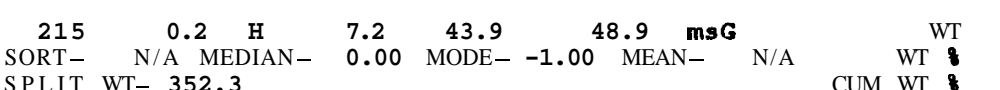

N/A 121

4.060 .4

14.6

6.8

N/A 10.3

$\begin{array}{lllllll}3.5 & 76.2 & 58.3 & 43.1 & 35.0 & \text { N/A } & 44.0\end{array}$

$\begin{array}{rrrrrrr}40.6 & 58.2 & 13.5 & 10.0 & 8.1 & \text { H/A } & 10.2\end{array}$

$\begin{array}{lllllllll}\text { AA } & 134.2 & 41.3 & 40.1 & 57.7 & 47.8 & 29.7 & \text { N/A } & 42.4\end{array}$

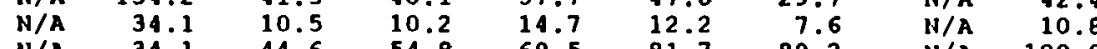

$\begin{array}{lllllllll}\text { N/A } & 34.1 & 44.6 & 54.8 & 69.5 & 81.7 & 09.2 & \text { N/A } & 100.0\end{array}$

$\begin{array}{lllllllll}\text { H/A } & 153.3 & 32.2 & 35.3 & 26.0 & 22.1 & 17.9 & \text { N/A } & 24.2\end{array}$

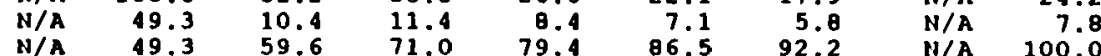

$\begin{array}{rrrrrrrrr}\text { N/A } & 79.8 & 19.5 & 12.5 & 10.1 & 9.5 & 0.1 & \text { N/A } & 15.6 \\ \text { N/A } & 51.5 & 12.6 & 8.1 & 6.5 & 6.1 & 5.2 & \text { N/A } & 10.1 \\ \text { N/A } & 51.5 & 64.0 & 72.1 & 78.6 & 84.7 & 89.9 & \text { N/A } & 100.0\end{array}$

$\begin{array}{lllllllll}\text { N/A } & 121.3 & 25.2 & 18.3 & 32.4 & 28.9 & 17.5 & \text { B } / \mathrm{A} & 28.6\end{array}$

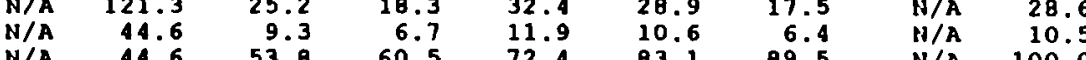

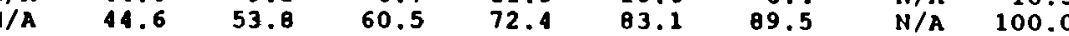

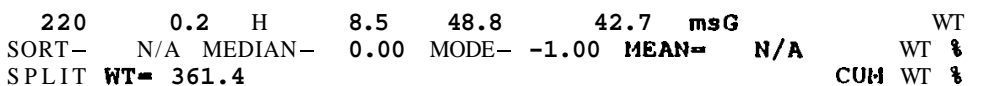

\section{N/A 139}

N/A 40.6

89.

M/A 30.8

N/A $\quad 170.5$

170.5
48.9
48.9

70 .

134

96.

91.0

N/A $\quad 9.0$ N/A $\quad 48$

$\begin{array}{rr}8.5 & 6.0 \\ 57.3 & 63.9\end{array}$

$\begin{array}{rr}\text { N/A } & 154.7 \\ \text { N/A } & 42.7 \\ \text { N/A } & 42.7\end{array}$

42.7
42.7

34.6
9.6

63.

2.5
12.2
76.1

$\begin{array}{rrrr}30.8 & 19.4 & \text { N/A } & 25.1 \\ 11.1 & 5.6 & \text { N/A } & 7.2\end{array}$

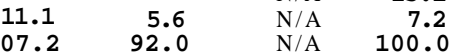

$\begin{array}{llll}42.9 & 22.5 & \text { N/A } & 30.7\end{array}$

$\begin{array}{rrrr}42.9 & 22.5 & \text { N/A } & 30.7 \\ 11.9 & 6.2 & \text { N/A } & 0.5 \\ 85.3 & 91.5 & \text { N/A } & 100.0\end{array}$ 

ROCSAN REPORT

$12 / 11 / 89$

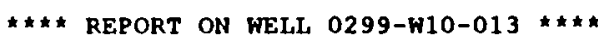

\begin{tabular}{|c|c|c|c|c|c|c|c|c|c|c|c|c|c|c|c|c|c|c|}
\hline DEPTH & $\triangle \mathrm{CACO} 3$ & PM $\%$ & ～U \% & $\sim \mathbf{U U} \mathbf{B}$ & GRAVEL C & CLAS & & & & $\begin{array}{l}\text { FINE } \\
\text { PEB } \\
(<--2)\end{array}$ & $\begin{array}{l}\text { VF INE } \\
\text { P E \& } \\
(-1)\end{array}$ & $\begin{array}{l}\text { VERY } \\
\text { COARS } \\
\text { (0) }\end{array}$ & $\begin{array}{c}\text { COARS } \\
\text { (1) }\end{array}$ & $\begin{array}{l}\text { MED } \\
(2)\end{array}$ & $\begin{array}{l}\text { FINE } \\
(3)\end{array}$ & $\begin{array}{l}\text { VERY } \\
\text { FINE } \\
\text { Id) }\end{array}$ & $\begin{array}{c}\text { SILT } \\
(4.75)\end{array}$ & $\begin{array}{c}\text { PAN } \\
(>4.75)\end{array}$ \\
\hline 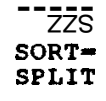 & $\begin{array}{c}0.2 \\
N / A \text { MED } \\
W T=187.3\end{array}$ & $\begin{array}{l}\text { H } \\
\text { DIAN- } \\
3\end{array}$ & $\begin{array}{c}20.7 \\
2.00\end{array}$ & $\begin{array}{r}50.2 \\
M O D E=\end{array}$ & $-1.00^{29.2}$ MEAN & gms & $N / A$ & cum & $\begin{array}{l}\text { WT } \\
\text { WT } \\
\text { WT }\end{array}$ & $\begin{array}{l}N / \Lambda \\
N / A \\
N / M\end{array}$ & $\begin{array}{l}54.6 \\
29.2 \\
29.2\end{array}$ & $\begin{array}{l}22.5 \\
12.0 \\
41.2\end{array}$ & $\begin{array}{r}15.8 \\
8.4 \\
49.6\end{array}$ & $\begin{array}{r}19.6 \\
10.5 \\
60.1\end{array}$ & $\begin{array}{l}22.8 \\
12.2 \\
7 Z . Z\end{array}$ & $\begin{array}{r}13.3 \\
7.1 \\
79.3\end{array}$ & $\begin{array}{l}N / \Lambda \\
N / A \\
N / A\end{array}$ & $\begin{array}{r}38.7 \\
20.7 \\
100.0\end{array}$ \\
\hline $\begin{array}{r}\text { ZヨO } \\
\text { SORT- } \\
\text { SPLIT }\end{array}$ & $\begin{array}{c}0.2 \\
\text { N/A MED } \\
\text { WT- } 247.8\end{array}$ & $\underset{8}{\text { DIAN= }}$ & $\begin{array}{l}8.9 \\
1.00\end{array}$ & $\begin{array}{r}\text { MODE }= \\
\text { MODE }\end{array}$ & $-1.00^{27.5}$ & $(m) g$ & $S / A$ & cum n & $\begin{aligned} & \text { WT } \\
& \text { WT } \\
& \text { WT }\end{aligned}$ & $\begin{array}{l}N / \Lambda \\
N / A \\
N / \Lambda\end{array}$ & $\begin{array}{l}67.4 \\
27.5 \\
Z 7 . S\end{array}$ & $\begin{array}{l}36.7 \\
15.0 \\
42.5\end{array}$ & $\begin{array}{l}30.1 \\
12.3 \\
54.8\end{array}$ & $\begin{array}{l}38.9 \\
15.9 \\
70.7\end{array}$ & $\begin{array}{l}30.9 \\
12.6 \\
83.3\end{array}$ & $\begin{array}{r}19.2 \\
7.8 \\
91.1\end{array}$ & $\begin{array}{l}N / A \\
N / A \\
N / M\end{array}$ & $\begin{array}{r}21.8 \\
8.9 \\
100.0\end{array}$ \\
\hline $\begin{array}{l}\text { ZヨS } \\
\text { SORT- } \\
\text { SPLIT }\end{array}$ & $\begin{array}{c}O . Z \\
N / A M E D \\
W T=148.6\end{array}$ & $\begin{array}{l}\mathrm{H} \\
\text { DIAN= } \\
6\end{array}$ & $\begin{array}{r}22.6 \\
2.00\end{array}$ & MODE- & $-1.000_{\mathrm{MEAN}}^{29,2}$ & $g m s$ & N/A & CUM & $\begin{array}{r}\text { WT } \\
\mathbf{W T} \% \\
\mathbf{W T}\end{array}$ & $\begin{array}{l}N / M \\
N / A \\
N / \Lambda\end{array}$ & $\begin{array}{l}43.4 \\
29.2 \\
29.2\end{array}$ & $\begin{array}{l}15.0 \\
10.1 \\
39.3\end{array}$ & $\begin{array}{r}12.6 \\
8.5 \\
47.8\end{array}$ & $\begin{array}{l}15.3 \\
10.3 \\
58.1\end{array}$ & $\begin{array}{l}16.8 \\
11.3 \\
69.4\end{array}$ & $\begin{array}{r}11.9 \\
8.0 \\
77.4\end{array}$ & $\begin{array}{l}N / A \\
N / A \\
N / A\end{array}$ & $\begin{array}{r}33.6 \\
2 Z .6 \\
100.0\end{array}$ \\
\hline $\begin{array}{l}\text { ZdO } \\
\text { SORT= } \\
\text { SPLIT }\end{array}$ & $\begin{array}{c}0.2 \\
N / A \text { MED } \\
W T=622.5\end{array}$ & $\begin{array}{l}\text { H } \\
\text { DIAN- } \\
5\end{array}$ & $\begin{array}{l}7 . \exists \\
1.00\end{array}$ & $\begin{array}{r}53.2 \\
M O D E=\end{array}$ & $\begin{array}{c}39.5 \\
-1.00^{\prime \prime} \text { MEAN" }\end{array}$ & $m g G$ & $N / A$ & cum & $\begin{array}{l}\text { WT } \\
\text { WT } \\
\text { WT }\end{array}$ & $\begin{array}{l}N / \Lambda \\
N / A \\
N / M\end{array}$ & $\begin{array}{r}245.6 \\
39.5 \\
39.5\end{array}$ & $\begin{array}{r}41.9 \\
6.7 \\
46.3\end{array}$ & $\begin{array}{r}43.6 \\
7.0 \\
53.3\end{array}$ & $\begin{array}{r}134.6 \\
21.7 \\
74.9\end{array}$ & $\begin{array}{r}7 Z .7 \\
11.7 \\
86.6\end{array}$ & $\begin{array}{r}37.6 \\
6.1 \\
92.7\end{array}$ & $\begin{array}{l}N / \Lambda \\
N / A \\
N / A\end{array}$ & $\begin{array}{r}45.4 \\
7.9 \\
100.0\end{array}$ \\
\hline $\begin{array}{r}245 \\
\text { SORT- } \\
\text { SPLIT }\end{array}$ & $\begin{array}{c}0.2 \\
\text { N/A MED } \\
\text { WT- } 241.4\end{array}$ & $\begin{array}{l}\text { H } \\
\text { DIAN- } \\
4\end{array}$ & $\begin{array}{l}Z . Z \\
2.00\end{array}$ & $\begin{array}{c}96.4 \\
\text { MODE- }\end{array}$ & $2.00^{1}$ MEAN & $s=$ & N/A & cum & $\begin{array}{c}\text { WT } \\
\text { WT } \\
\text { WT }\end{array}$ & $\begin{array}{l}N / \Lambda \\
N / A \\
N / M\end{array}$ & $\begin{array}{l}3.3 \\
1.4 \\
1.4\end{array}$ & $\begin{array}{l}1.5 \\
0.6 \\
2.0\end{array}$ & $\begin{array}{l}3.7 \\
1.6 \\
3.6\end{array}$ & $\begin{array}{r}122.4 \\
51.6 \\
55.2\end{array}$ & $\begin{array}{l}91.6 \\
38.6 \\
93.8\end{array}$ & $\begin{array}{r}9.3 \\
3.9 \\
97.8\end{array}$ & $\begin{array}{l}\text { N/A } \\
\text { N/A } \\
\text { N/A }\end{array}$ & $\begin{array}{r}5.3 \\
2.2 \\
100.0\end{array}$ \\
\hline $\begin{array}{r}\text { ZSO } \\
\text { SORT= } \\
\text { SPLIT }\end{array}$ & $\begin{array}{c}O . Z \\
\text { N/A MED } \\
\text { WT- } 275.8\end{array}$ & $\begin{array}{l}\text { DIAN= } \\
\text { DIA } \\
{ }^{H}\end{array}$ & $\begin{array}{l}2.1 \\
2.00\end{array}$ & $\begin{array}{r}97.9 \\
M O D E=\end{array}$ & $2.00^{0.0} \mathrm{MEAN}$ & $s-$ & N/A & CUM & $\begin{array}{l}\text { WT } \\
\text { WT } \\
\text { WT }\end{array}$ & $\begin{array}{l}N / \Lambda \\
N / \Lambda \\
N / A\end{array}$ & $\begin{array}{l}0,0 \\
0,0 \\
0,0\end{array}$ & $\begin{array}{l}1.0 \\
0.4 \\
0.4\end{array}$ & $\begin{array}{l}2.0 \\
0.7 \\
1.1\end{array}$ & $\begin{array}{r}171.6 \\
62.1 \\
63.2\end{array}$ & $\begin{array}{l}98.8 \\
32.1 \\
95.3\end{array}$ & $\begin{array}{r}7.3 \\
2.6 \\
97.9\end{array}$ & $\begin{array}{l}N / \Lambda \\
N / \Lambda \\
N / A\end{array}$ & $\begin{array}{r}5.7 \\
2.1 \\
100.0\end{array}$ \\
\hline
\end{tabular}


WESTINGHOUSE HANFORD OPERRTIONS SIEVE ANALYSIS ROCSAN REPORT

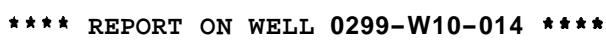

$12 / 11 / 89$

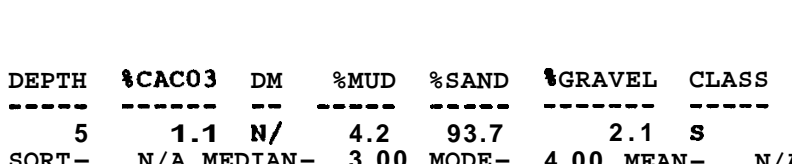

SORT- N/A MED

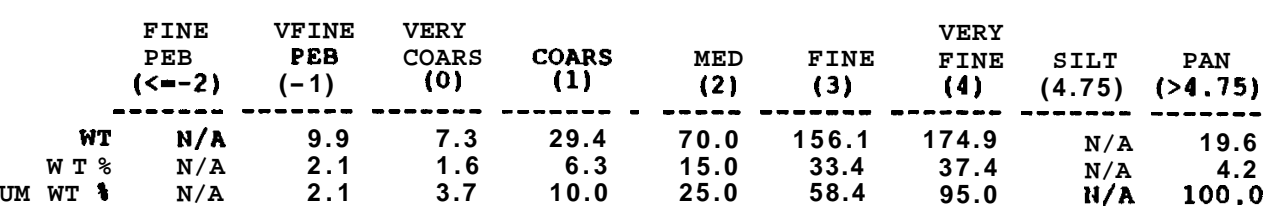

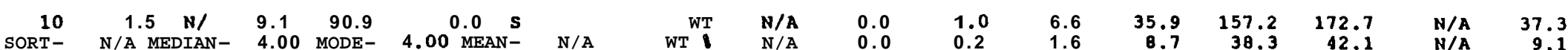

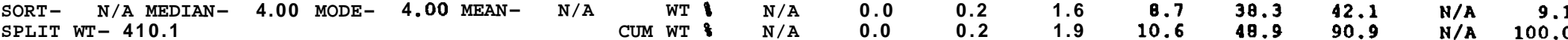

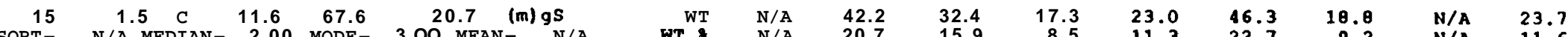

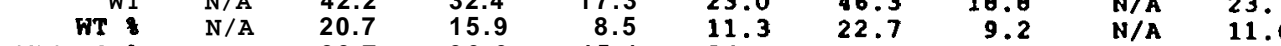

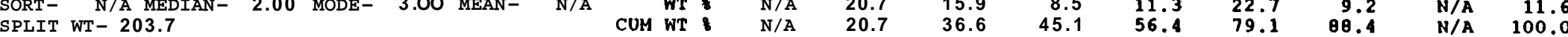

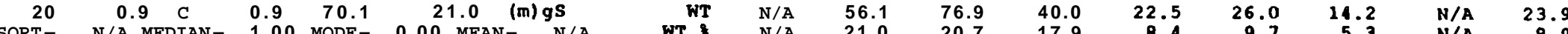

$\stackrel{0}{0}$

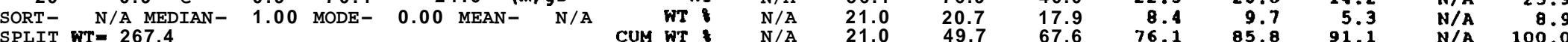

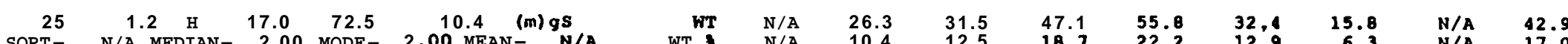

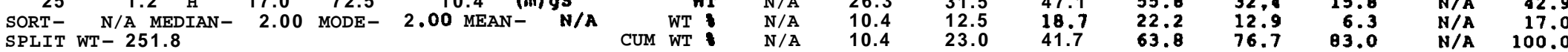

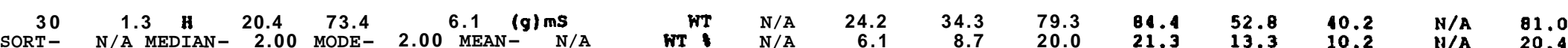

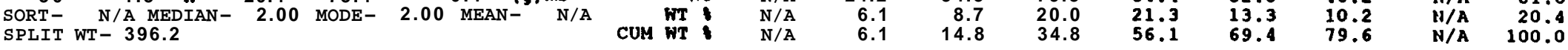

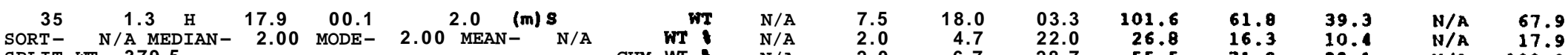

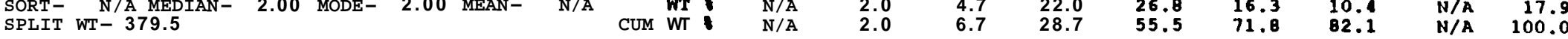

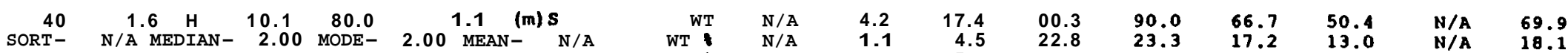

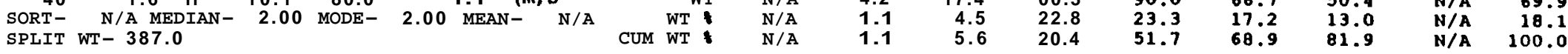

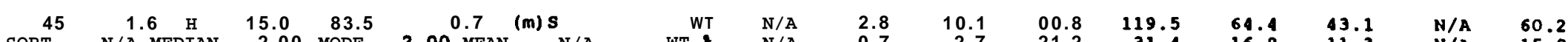

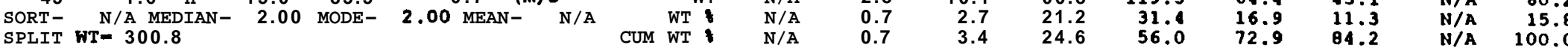

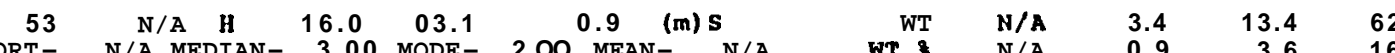

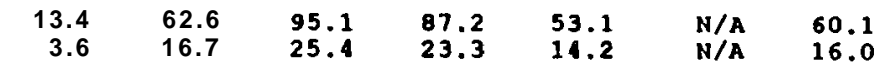

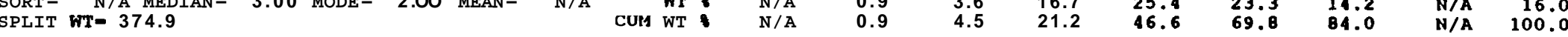

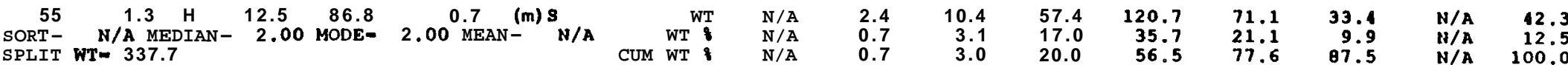



ROCSAN REPORT

$12 / 11 / 89$

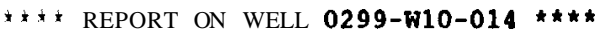

DEPTH

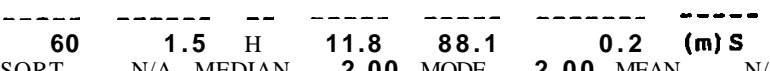
$\begin{array}{lllll}\text { SORT- N/A MEDIAN- } & \mathbf{2} .00 & \text { MODE- } & \mathbf{2} .00 & \text { MEAN- N/A }\end{array}$

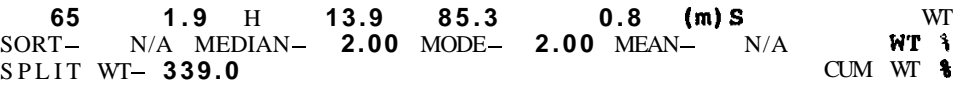

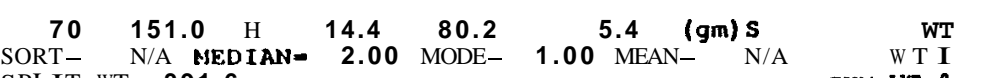
SPLIT WT- 391.6

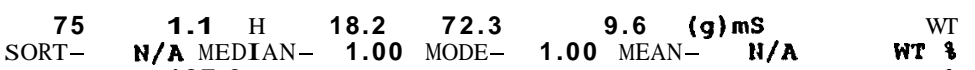

SPLIT WT- 187.0 $\begin{array}{rrrrrrr}80 & 1.3 & \text { H } & 18.9 & 63.5 & 17.6^{\prime} & \mathrm{gms}\end{array}$ SORT - N/A MEDIAN $\mathbf{8 5} \mathbf{1}$ : H
SORT- N/A MEDIA
SPLIT WT- $\mathbf{4 4 4 . 6}$

\section{FINE VTINE VERY}

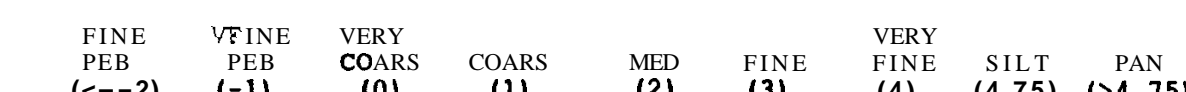

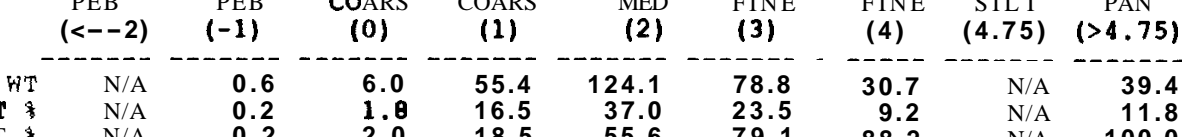

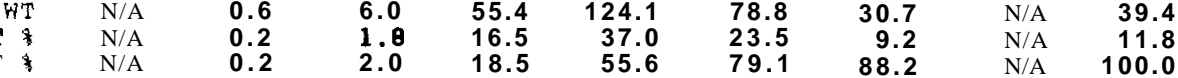

$\stackrel{9}{3}$




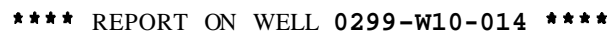

$12 / 11 / 89$

\begin{tabular}{ccccccc} 
DEPTH & ICACO3 & DM & 8MUD & $\%$ SAND & OGRAVEL & CLASS \\
\hdashline 115 & -2.3 & H & -15.8 & 74.8 & 9.4 & (gm) $\mathrm{s}$
\end{tabular} $\begin{array}{ccccccc}115 & 2.3 & \mathrm{H} & 15.8 & \mathbf{7 4 . 8} & 9.4 & (\mathrm{gm}) \mathrm{s} \\ \text { SORT- } & \text { N/A } & \text { MEDIAN- } & 2.00 & \text { MODE* } & 2.00 & \text { MEAN- }\end{array}$ SPLIT WT $=208.0$

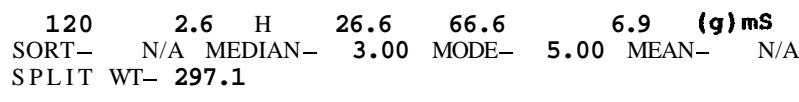

$\begin{array}{ccc}\text { FINE } & \text { VFINE } & \text { VERY } \\ \text { PEB } & \text { PEB } & \text { COARS }\end{array}$

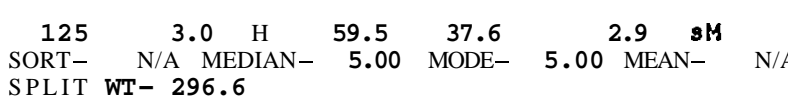

$\begin{array}{lll} & & \\ \text { COARS } & \text { MED } & \text { FINE } \\ \text { FINE }\end{array}$

\begin{tabular}{|c|c|c|c|c|c|c|c|c|c|}
\hline & $\begin{array}{l}\text { PEB } \\
(<--2)\end{array}$ & $\begin{array}{l}\text { PEB } \\
(-1)\end{array}$ & $\begin{array}{c}\text { COARS } \\
\text { (0) }\end{array}$ & $\begin{array}{l}\text { COARS } \\
\text { (1) }\end{array}$ & $\begin{array}{l}\text { MED } \\
\text { (2) }\end{array}$ & $\begin{array}{l}\text { FINE } \\
\text { (3) }\end{array}$ & $\begin{array}{l}\text { FINE } \\
\text { (4) }\end{array}$ & $\begin{array}{r}\text { SILT } \\
(\mathbf{4 . 7 5 )}\end{array}$ & $\begin{array}{c}\text { PAN } \\
(>4.75)\end{array}$ \\
\hline $\begin{array}{r}\text { WT } \\
\text { WT }\end{array}$ & $\begin{array}{l}\text { N/A } \\
\text { N/A }\end{array}$ & $\begin{array}{r}19.6 \\
9.4\end{array}$ & 14.1 & & $\begin{array}{l}68.0 \\
32.7\end{array}$ & $\begin{array}{l}21.2 \\
10.2\end{array}$ & $\begin{array}{l}9.6 \\
4.6\end{array}$ & $\begin{array}{l}\text { N/A } \\
\text { N/A }\end{array}$ & $\begin{array}{l}32.9 \\
15.8\end{array}$ \\
\hline WT & N/A & 9.4 & 16.2 & 36.7 & 69.4 & 79.6 & 84.2 & N/A & 100.0 \\
\hline
\end{tabular}

$\begin{array}{llllll}\text { SORT- N/A MEDIAN- } & 5.00 & \text { MODE- } & 5.00 & \text { MEAN- } & \text { N/A } \\ \text { SPLIT } & \text { WT- } 157.6 & & & \text { CUH WT }\end{array}$

N/A 20

$6.9 \quad 11.8$

.8

.0

79.6

4.6
84.2

$\begin{array}{lr}\text { N/A } & 15.8 \\ \text { N/A } & 100.0\end{array}$

WT WT

N/A

6.9

4.0
10.8

$\begin{array}{ll}34.4 & 75.0 \\ 11.6 & 25.2\end{array}$

46.530.

$\begin{array}{llll}46.5 & 30.1 & \text { N/A } & 79.0 \\ 15.7 & 10.1 & \text { N/A } & 26.6\end{array}$

$\begin{array}{lrrrrrrrrr}\text { N/A } & 8.5 & 5.5 & 13.2 & 31.1 & 26.6 & 35.2 & \text { N/A } & 176.4 \\ \text { N/A } & 2.9 & 1.9 & 4.5 & 10.5 & 9.0 & 11.9 & \text { N/A } & 59.5\end{array}$

N/A

2.9

$4.7 \quad 4.5 \quad 10.5$

WT

N/A $\quad 0.3$

$\begin{array}{lllllll}135 & 10.7 & \mathrm{H} & 36.8 & 58.0 & 5.3 & (\mathrm{~g}) \mathrm{ms}\end{array}$

SORT- N/A MEDIA

WT

SPLIT WT- 347.6 MEDIAN- 2.00 MODE- 2.00 MEAN- N/A WT

$\begin{array}{llllllllll}145 & 5.3 & \text { H } & 16.5 & 49.8 & 33.7 & \text { msG } & \text { WT } & \text { N/A } & 107\end{array}$

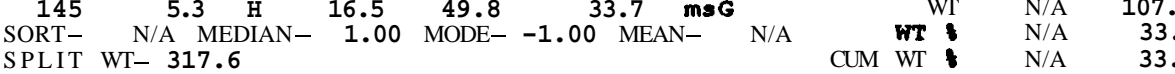

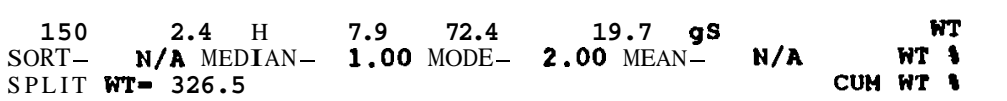

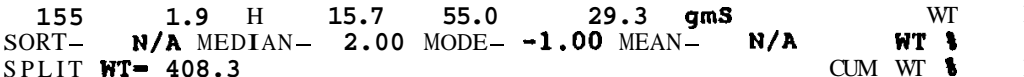

$\begin{array}{llllllll}160 & 0.9 & \text { H } & 15.9 & 42.1 & 42.0 & \text { msG } & \text { WT }\end{array}$ SORT- N/A MEDIAH- 0.00 MODE- -1.00 MEAN- N/A
SPLIT WT $=457.0$

$\begin{array}{lcccccc}165 & \mathbf{0 . 8} & \mathbf{2 6} & \mathbf{2 6} .9 & \mathbf{5 4 . 1} & \mathbf{1 9 . 0} \mathbf{g m g} & \\ \text { SORT- } & \text { N/A } & \text { MEDIAN- } & \mathbf{2 . 0 0} & \text { MODE- } & \mathbf{5 . 0 0} \text { MEAN- } & \text { N/A } \\ \text { SPLIT } & \text { WT- } 403.0 & & & & \text { CUM WT }\end{array}$

$\begin{array}{llll}\text { WT N/A } & 76.7 & 45.3\end{array}$

N/A $\quad 19.0 \quad 11.2$

$\begin{array}{llll}\text { H/A } & 64.5 & 39.1 & 70.6\end{array}$

$\begin{array}{llll} & & & \\ \text { N/A } & 19.0 & 39.1 & 70.6\end{array}$

$\begin{array}{ll}\text { M/A } \quad 19.8 & 31.7\end{array}$

N/A $\quad 119.6 \quad 39.2 \quad 43$.

$\begin{array}{rr}19.6 & 39.2 \\ 29.3 & 9.6\end{array}$

9.6
38.9

10.6
49.5

$43.6 \quad 55.5$

$\begin{array}{rr}9.5 & 12.1 \\ 60.7 & 72.8\end{array}$

$28.6 \quad 40.5$

N/A 100.0

II/A 19.0

$\begin{array}{ll}44.0 & 58.9 \\ 10.9 & 14.6\end{array}$

$\begin{array}{ll}10.9 & 14.6 \\ 41.2 & 55.8\end{array}$

$\begin{array}{llll}17.3 & 23.4 & \text { N/A } & 100.0\end{array}$

$\begin{array}{rrrr}11.0 & 14.9 & \text { N/A } & 63.5 \\ 21.7 & 36.6 & \text { N/A } & 100.0\end{array}$

$\begin{array}{llll}31.6 & 32.0 & \mathrm{~N} / \mathrm{A} \quad & 78.1\end{array}$

$\begin{array}{rrrr} & & & \\ 14.9 & 15.1 & \text { N/A } & 78.1 \\ 48.2 & 63.2 & \text { N/A } & 36.8\end{array}$

(3).

$38.2 \quad 23.0 \quad$ N/A 68.5

$\begin{array}{rrrr}11.0 & 6.6 & \text { N/A } & 19.7 \\ 73.7 & 80.3 & \text { N/A } & 100.0\end{array}$

$26.8 \quad 16.5$ N/A 52.3

$\begin{array}{rrrr}78.4 & 5.2 & \text { N/A } & 16.5 \\ & 83.5 & \text { N/A } & 100.0\end{array}$

$22.3 \quad 7.7$ N/A 25.8

$\begin{array}{rrrr}6.8 & 2.4 & \text { N/A } & 7.9\end{array}$

$38.7 \quad 20.7 \quad$ N/A 64.3

$\begin{array}{rrrr}9.5 & 5.1 & \text { N/A } & 15.8 \\ 79.2 & 84.3 & \text { N/A } & 100.0\end{array}$

$31.120 .4 \quad$ H/A 72.7

$\begin{array}{rrrr}6.8 & 4.5 & \text { N/A } & 72.7 \\ 79.6 & 84.1 & \text { N/A } & 15.9 \\ & & \end{array}$

$39.4 \quad 30.5$ N/A 108.3

$\begin{array}{rrrr}9.8 & 7.6 & \text { N/A } & 26.9 \\ 65.6 & 73.1 & \text { N/A } & 100.0\end{array}$ 

ROCSAI REPORT

$12 / 11 / 89$

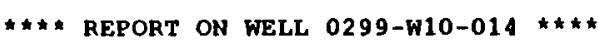

\begin{tabular}{|c|c|c|c|c|c|c|c|c|c|c|c|c|c|c|c|c|c|}
\hline EPTH & $\triangle \mathrm{CACO} 3$ & DM & SMUD & ISAND & GGRAVEL CLAS & & & & $\begin{array}{l}\text { FINE } \\
\text { PEB } \\
(<=-2)\end{array}$ & $\begin{array}{l}\text { VFINE } \\
\text { PEB } \\
(-1)\end{array}$ & $\begin{array}{l}\text { W I V } \\
\text { COARS } \\
\text { (0) }\end{array}$ & $\begin{array}{l}\text { COARS } \\
\text { (1) }\end{array}$ & $\begin{array}{l}\text { MED } \\
(2)\end{array}$ & $\begin{array}{l}\text { FINE } \\
\text { (3) }\end{array}$ & $\begin{array}{l}\text { VERY } \\
\text { FINE } \\
\text { (4) }\end{array}$ & $\begin{array}{l}\text { SILT } \\
(4.75)(\end{array}$ & $\begin{array}{l}\mathbf{p p}_{\mathbf{N}} \mathbf{N} \\
\sim, 7 \exists)\end{array}$ \\
\hline $\begin{array}{l}\text { 17o } \\
\text { SORT= } \\
\text { SPLIT }\end{array}$ & $\begin{array}{r}\text { N/A MED } \\
\mathrm{HE}-287.5\end{array}$ & $\begin{array}{l}\text { DIAN- } \\
5\end{array}$ & $\begin{array}{l}37.1 \\
3.00\end{array}$ & $\begin{array}{r}S 7 . \exists \\
\text { MODE- }\end{array}$ & $5.00 \mathrm{MEAN}=$ & $\mathrm{nS}$ & CUM & $\begin{array}{l}\text { WT } \\
\text { Wr } \\
\text { WT }\end{array}$ & $\begin{array}{l}N / \Lambda \\
N / A \\
N / \Lambda\end{array}$ & $\begin{array}{r}16.1 \\
5.6 \\
5.6\end{array}$ & $\begin{array}{r}26.5 \\
9.2 \\
14.8\end{array}$ & $\begin{array}{l}40.7 \\
19.2 \\
29.0\end{array}$ & $\begin{array}{l}42.7 \\
14.9 \\
43.8\end{array}$ & $\begin{array}{l}30.2 \\
10.5 \\
54.3\end{array}$ & $\begin{array}{r}20.7 \\
8.6 \\
62.9\end{array}$ & $\begin{array}{l}N / A \\
N / A \\
\text { U/A }\end{array}$ & $\begin{array}{r}106.7 \\
37.1 \\
100.0\end{array}$ \\
\hline $\begin{array}{r}175 \\
\text { SORT= } \\
\text { SPLIT }\end{array}$ & $\begin{array}{c}0.6 \\
\text { N/A MED } \\
\text { WT- } 412.7\end{array}$ & ${ }_{7}^{H}$ & $\begin{array}{c}17.3 \\
0.00\end{array}$ & $\begin{array}{r}38.8 \\
\text { MODE }=-\end{array}$ & $-1.00^{43.9}$ MEAN $^{m g G}$ & $\mathbf{N} / \mathbf{A}$ & CUH & $\begin{array}{r}\text { WN } \\
\text { WT } \\
\text { HT }\end{array}$ & $\begin{array}{l}N / \lambda \\
N / A \\
N / A\end{array}$ & $\begin{array}{r}101.1 \\
43.9 \\
43.9\end{array}$ & $\begin{array}{l}42.8 \\
10.4 \\
54.3\end{array}$ & $\begin{array}{r}37.6 \\
9.1 \\
63.4\end{array}$ & $\begin{array}{r}\exists 7 . \exists \\
9.0 \\
72.4\end{array}$ & $\begin{array}{r}24.0 \\
5.8 \\
78.2\end{array}$ & $\begin{array}{r}18.5 \\
4.5 \\
82.7\end{array}$ & $\begin{array}{l}N / A \\
N / A \\
N / A\end{array}$ & $\begin{array}{r}71.4 \\
17.3 \\
100.0\end{array}$ \\
\hline $\begin{array}{r}180 \\
\text { SORT- } \\
\text { SPLIT }\end{array}$ & $\begin{array}{c}0.5 \\
N / A \\
W T=434.2\end{array}$ & $\begin{array}{l}\text { DIASI- } \\
2\end{array}$ & $\begin{array}{l}16.7 \\
1.00\end{array}$ & $\begin{array}{r}46.4 \\
M O D E=\end{array}$ & -1.00 MEAN $^{36.8}$ & $\mathbf{N} / \mathbf{A}$ & CUM & $\begin{array}{r}\text { WT } \\
\text { WT } \\
\text { WT }\end{array}$ & $\begin{array}{l}N / A \\
N / A \\
N / \Lambda\end{array}$ & $\begin{array}{r}160.0 \\
36.8 \\
\mathbf{3 6 . 8}\end{array}$ & $\begin{array}{l}56.0 \\
12.9 \\
49.7\end{array}$ & $\begin{array}{l}54.2 \\
12.5 \\
62.2\end{array}$ & $\begin{array}{l}45.9 \\
10.6 \\
72.8\end{array}$ & $\begin{array}{r}26.6 \\
6.1 \\
78.9\end{array}$ & $\begin{array}{r}19.0 \\
4.4 \\
83.3\end{array}$ & $\begin{array}{l}N / A \\
N / A \\
N / A\end{array}$ & $\begin{array}{r}72.6 \\
16.7 \\
100.0\end{array}$ \\
\hline $\begin{array}{r}185 \\
\text { SORTE } \\
\text { SPLIT }\end{array}$ & $\begin{array}{c}0.4 \\
N / A \text { MED } \\
W T=384.0\end{array}$ & $\begin{array}{l}\text { DIAN- } \\
0\end{array}$ & $\begin{array}{l}23.6 \\
1.00\end{array}$ & $\begin{array}{r}46,3 \\
M O D E=\end{array}$ & -1.00 MEAN & $N / A$ & CUM & WT & $\begin{array}{l}N / A \\
N / A \\
N / A\end{array}$ & $\begin{array}{r}115.5 \\
30.1 \\
30.1\end{array}$ & $\begin{array}{l}42.9 \\
11.2 \\
41.3\end{array}$ & $\begin{array}{l}43.8 \\
11.4 \\
52.7\end{array}$ & $\begin{array}{l}39.9 \\
10.4 \\
63.1\end{array}$ & $\begin{array}{r}28.5 \\
7.4 \\
70.5\end{array}$ & $\begin{array}{r}22.6 \\
5.9 \\
76.4\end{array}$ & $\begin{array}{l}M / A \\
N / A \\
N / A\end{array}$ & $\begin{array}{r}90.8 \\
23.7 \\
100.0\end{array}$ \\
\hline $\begin{array}{r}190 \\
\text { SORT= } \\
\text { SPLIT }\end{array}$ & $\begin{array}{c}0.1 \\
N / A \text { MED } \\
W T=464.9\end{array}$ & $\begin{array}{l}\stackrel{H}{\text { DIAN- }} \\
9\end{array}$ & $\begin{array}{c}18.9 \\
1.00\end{array}$ & MODE- & $-1,00^{36} \dot{M E A N}^{7}=$ & $N / A$ & CUM & WT & $\begin{array}{l}N / A \\
N / A \\
N / A\end{array}$ & $\begin{array}{r}170.4 \\
36.7 \\
36.7\end{array}$ & $\begin{array}{r}44.5 \\
9.6 \\
46.2\end{array}$ & $\begin{array}{l}55.0 \\
12.0 \\
58.2\end{array}$ & $\begin{array}{l}49.6 \\
Y 0.7 \\
68.9\end{array}$ & $\begin{array}{r}32.2 \\
6.9 \\
75.8\end{array}$ & $\begin{array}{r}24.5 \\
5.3 \\
81.1\end{array}$ & $\begin{array}{l}\text { N/A } \\
N / \Lambda \\
\text { N/A }\end{array}$ & $\begin{array}{r}87.9 \\
18.9 \\
100.0\end{array}$ \\
\hline $\begin{array}{r}195 \\
\text { SORT- } \\
\text { SPLIT }\end{array}$ & $\begin{array}{c}0.1 \\
N / A M E D \\
W T=45.8\end{array}$ & $\begin{array}{l}\text { D } \\
\text { DIAN- }\end{array}$ & $\begin{array}{c}20.9 \\
1.00\end{array}$ & $\begin{array}{r}a \exists . O \\
\text { MODE- }\end{array}$ & -1.00 MEAN $=$ & $N / A$ & CUM & $\begin{array}{l}\mathrm{WT} \\
\mathrm{WT}\end{array}$ & $\begin{array}{l}N / A \\
N / A \\
N / A\end{array}$ & $\begin{array}{r}160.9 \\
36.1 \\
36.1\end{array}$ & $\begin{array}{r}43.2 \\
9.7 \\
45.8\end{array}$ & $\begin{array}{r}43.4 \\
9.7 \\
55.5\end{array}$ & $\begin{array}{r}42.3 \\
9.5 \\
65.0\end{array}$ & $\begin{array}{r}9 \\
7.8 \\
72.8\end{array}$ & $\begin{array}{r}28.1 \\
6.3 \\
79.1\end{array}$ & $\begin{array}{l}\text { N/A } \\
N / A \\
N / A\end{array}$ & $\begin{array}{r}93.1 \\
20.9 \\
100.0\end{array}$ \\
\hline $\begin{array}{r}200 \\
\text { SORT- } \\
\text { SPLIT }\end{array}$ & $\begin{array}{c}0.1 \\
N / A \text { MED } \\
W T=348,3\end{array}$ & ${ }_{3}^{H}$ & $\begin{array}{r}27.7 \\
2.00\end{array}$ & MODE- & 5.00 MEANE & $N / A$ & CUM & WT & $\begin{array}{l}N / A \\
N / A \\
N / A\end{array}$ & $\begin{array}{l}84.8 \\
24.4 \\
24.4\end{array}$ & $\begin{array}{l}39.9 \\
11.5 \\
35.8\end{array}$ & $\begin{array}{r}34.3 \\
9.9 \\
45.7\end{array}$ & $\begin{array}{r}33.9 \\
9.7 \\
55.4\end{array}$ & $\begin{array}{r}32.2 \\
9.3 \\
64.7\end{array}$ & $\begin{array}{r}26.5 \\
7.6 \\
72.3\end{array}$ & $\begin{array}{l}N / A \\
N / A \\
N / A\end{array}$ & $\begin{array}{r}96.6 \\
27.7 \\
100.0\end{array}$ \\
\hline $\begin{array}{r}205 \\
\text { SORT- } \\
\text { SPLIT }\end{array}$ & $\begin{array}{c}0.1 \\
\text { N/A MED } \\
\text { WT- } 48.2\end{array}$ & $\begin{array}{l}\mathbf{2}^{H} \\
\text { DIAN- }\end{array}$ & $\begin{array}{c}18.0 \\
1.00\end{array}$ & MODE- & $-1.00^{36.3} \mathrm{MEAN}=$ & $N / A$ & CUM & $\underset{\mathrm{WT}}{\mathrm{Wr}}$ & $\begin{array}{l}H / \lambda \\
N / \lambda \\
N / \lambda\end{array}$ & $\begin{array}{r}162.7 \\
36.3 \\
36.3\end{array}$ & $\begin{array}{l}45.9 \\
10.2 \\
46.5\end{array}$ & $\begin{array}{r}39.0 \\
8.7 \\
55.2\end{array}$ & $\begin{array}{l}50.9 \\
11.4 \\
66.6\end{array}$ & $\begin{array}{r}41.4 \\
9.2 \\
75.8\end{array}$ & $\begin{array}{r}23.9 \\
5.3 \\
81.1\end{array}$ & $\begin{array}{l}n / A \\
\text { N/A } \\
\text { N/A }\end{array}$ & $\begin{array}{r}84.5 \\
18.9 \\
100.0\end{array}$ \\
\hline $\begin{array}{r}210 \\
\text { SORT } \\
\text { SPLIT }\end{array}$ & $\begin{array}{c}0.1 \\
N / A B D D \\
W Z-458.3\end{array}$ & ${ }_{3}^{H}$ & $\begin{array}{r}15.0 \\
0.00\end{array}$ & $\begin{array}{r}\exists a, 7 \\
\text { MODE- }\end{array}$ & $-1.00^{19.5}$ MEAN $^{\text {msG }}$ & $N / A$ & CUM & WT & $\begin{array}{l}N / A \\
N / A \\
N / A\end{array}$ & $\begin{array}{r}226.6 \\
49.5 \\
49.5\end{array}$ & $\begin{array}{r}32.3 \\
7.1 \\
56.5\end{array}$ & $\begin{array}{r}29.0 \\
6.5 \\
63.0\end{array}$ & $\begin{array}{r}11.9 \\
9.1 \\
72.1\end{array}$ & $\begin{array}{r}35.1 \\
7.7 \\
79.8\end{array}$ & $\begin{array}{r}20.1 \\
4.4 \\
64.2\end{array}$ & $\begin{array}{l}N / A \\
N / \Lambda \\
N / A\end{array}$ & $\begin{array}{r}72.4 \\
15.8 \\
100.0\end{array}$ \\
\hline $\begin{array}{r}215 \\
\text { SORT } \\
\text { SPLIT }\end{array}$ & $\begin{array}{c}0.1 \\
N / A \\
W T-439.5\end{array}$ & $\begin{array}{l}\text { DIAN- } \\
5\end{array}$ & $\begin{array}{l}12.2 \\
-1.00\end{array}$ & MODE- & $-1,00$ MEAN $^{\mathrm{mgG}}$ & N/A & CUM & $\underset{\text { WT }}{\text { WT }}$ & $\begin{array}{l}N / \lambda \\
N / A \\
N / A\end{array}$ & $\begin{array}{r}245.1 \\
55.8 \\
55.8\end{array}$ & $\begin{array}{r}33.5 \\
7.6 \\
63.4\end{array}$ & $\begin{array}{r}19.6 \\
4.5 \\
67.9\end{array}$ & $\begin{array}{r}33.8 \\
7.7 \\
75.5\end{array}$ & $\begin{array}{r}\exists 7.0 \\
8.4 \\
84.0\end{array}$ & $\begin{array}{r}16.7 \\
3.8 \\
87.8\end{array}$ & $\begin{array}{l}N / A \\
N / A \\
N / A\end{array}$ & $\begin{array}{r}53.8 \\
12.2 \\
100.0\end{array}$ \\
\hline $\begin{array}{r}220 \\
\text { SORT- } \\
\text { SPLIT }\end{array}$ & $\begin{array}{c}0.1 \\
\text { N/A MED } \\
\text { WE- } 436.3\end{array}$ & ${ }_{3}^{H}$ & $\begin{array}{c}11.6 \\
1.00\end{array}$ & $\begin{array}{r}67.2 \\
\text { MODE- }\end{array}$ & $-1.000_{\text {MEAN }}^{21}=$ & $s / A$ & & $\begin{array}{c}W T \\
W T\end{array}$ & $\begin{array}{l}N / \Lambda \\
N / A \\
N / A\end{array}$ & $\begin{array}{l}92.6 \\
21.2 \\
21.2\end{array}$ & $\begin{array}{l}81.8 \\
18.7 \\
90.0\end{array}$ & $\begin{array}{l}86.9 \\
19.9 \\
59.9\end{array}$ & $\begin{array}{l}69.8 \\
16.0 \\
75.8\end{array}$ & $\begin{array}{r}40.1 \\
9.2 \\
85.0\end{array}$ & $\begin{array}{r}14.0 \\
3.4 \\
88.4\end{array}$ & $\begin{array}{l}\text { N/A } \\
\text { N/A } \\
\text { N/A }\end{array}$ & $\begin{array}{r}50.5 \\
11.6 \\
100.0\end{array}$ \\
\hline
\end{tabular}


WESTINGHOUSE HANFORD OPERATIONS SIEVE ANALYSIS

$* * *$ REPORT ON WHL 0299-W10-014 ****

$12 / 11 / 89$

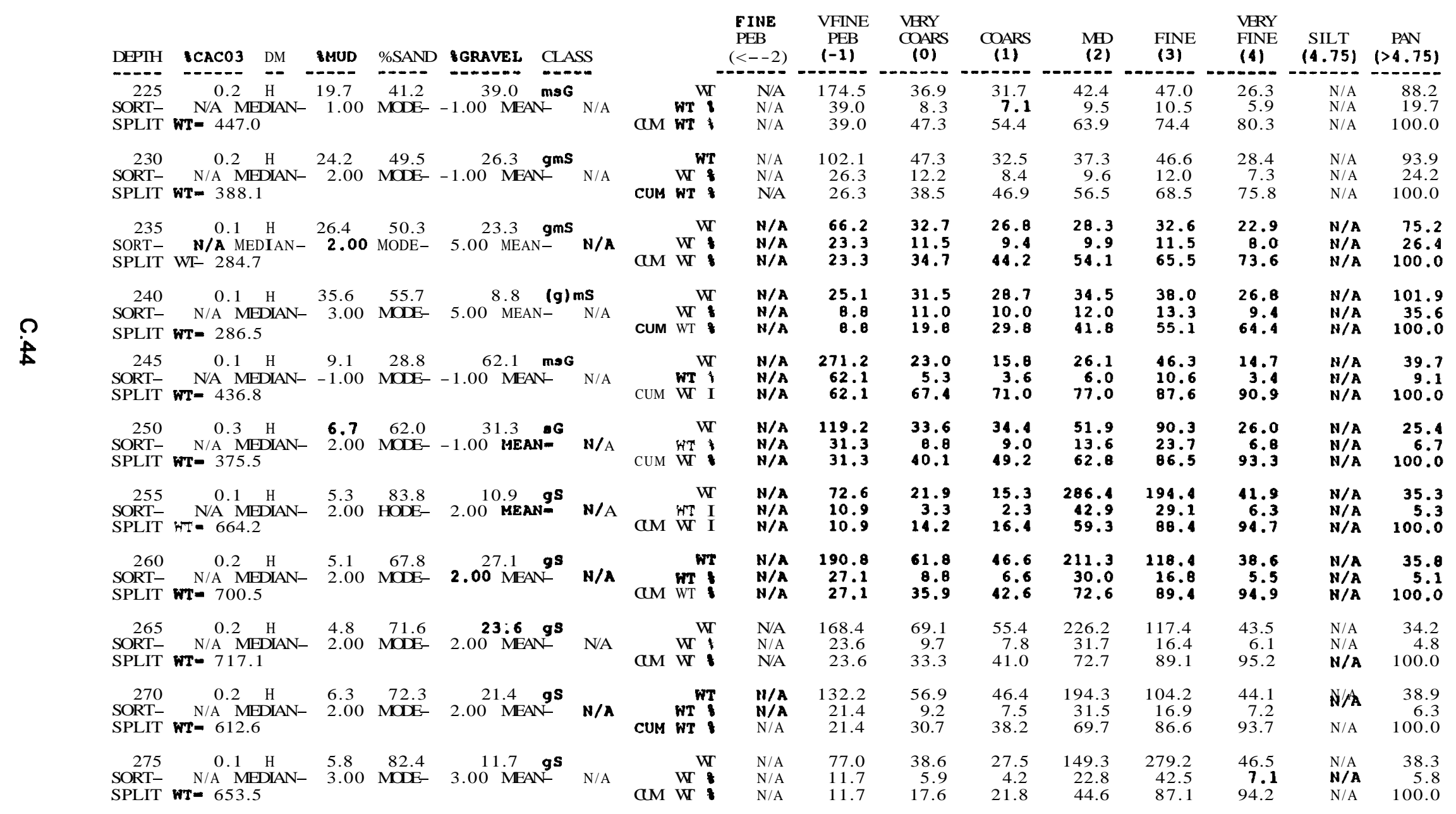



ROCSAN REPORT

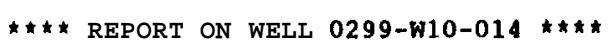

$12 / 11 / 89$

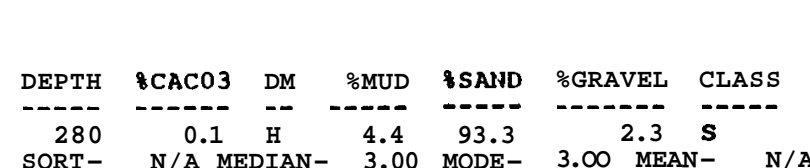

SORT- N/A MEDIAN- 3.00 MODE- 3.00 MEAN- N/A
SPLIT WT- 607.3

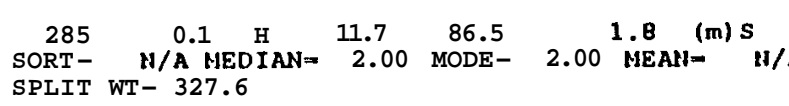

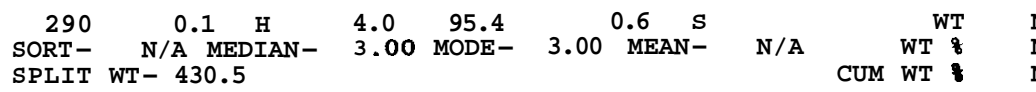

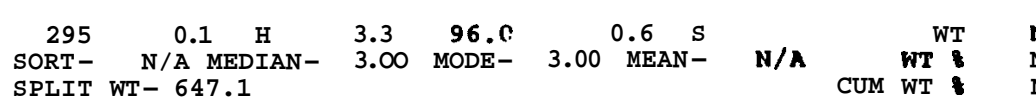

$\stackrel{?}{\dot{1}}$

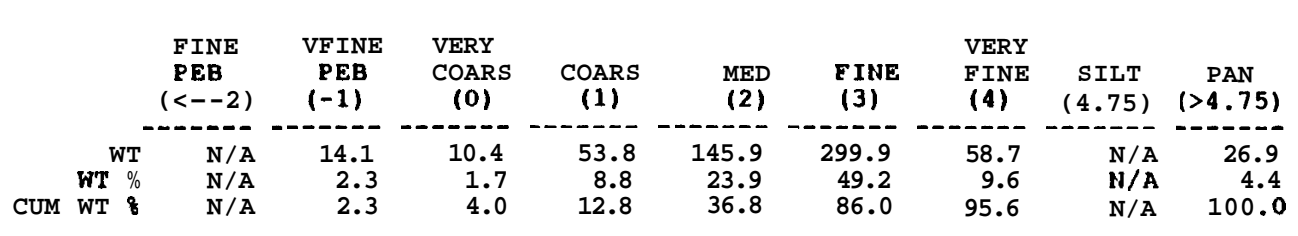

$\begin{array}{lllllllll}\text { N/A } & 5.9 & 7.2 & 5.7 & 153.9 & 91.3 & 25.2 & \text { N/A } & 38.5\end{array}$

$\begin{array}{lllllllll}\text { N/A } & 1.8 & 4.0 & 5.7 & 52.7 & 80.6 & 88.3 & \text { II/A } & 100.0\end{array}$

$\begin{array}{lllrrrrrr}\text { N/A } & 2.6 & 1.8 & 16.5 & 108.5 & 252.5 & 36.5 & \text { N/A } & 17.5\end{array}$

$\begin{array}{lllllllll}\text { N/A } & 0.6 & 0.4 & 3.8 & 24.9 & 57.9 & 8.4 & \text { N/A } & 4.0 \\ \text { N/A } & 0.6 & 1.0 & 4.0 & 29.7 & 87.6 & 96.0 & \text { N/A } & 100.0\end{array}$

$\begin{array}{llllllllll}300 & 0.1 & \mathrm{H} & 3.8 & 82.6 & 13.6 & \mathrm{gS} & \mathrm{WT} & \mathrm{N} / \mathrm{A} & 95.8\end{array}$

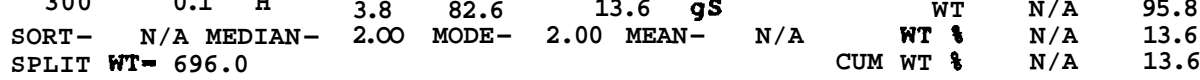

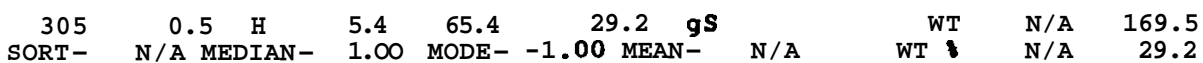

$\begin{array}{llllllll}\text { SORT- N/A MEDIAN- } 1.00 & \text { MODE- }-1.00 \text { MEAN- N/A } & \text { WT } & \text { N/A } & 29.2 \\ \text { SPLIT WT- } 574.3 & & & \text { CUM WT } & \text { N/A } & 29.2\end{array}$

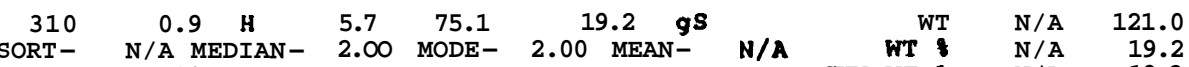

$\begin{array}{llllllll}\text { SORT- NT } & \text { N/A MEDIAN- } 2.00 & \text { MODE- } & 2.00 & \text { MEAN- N/A } & \text { N/A } & 19.2 \\ \text { SPLIT WT- } 629.1 & & & \text { CUM WT } & \text { N/A } & 19.2\end{array}$

$\begin{array}{lllllllllrrr}315 & 1.1 & \text { H } & 6.0 & 64.1 & 29.9 & \text { gS } & & & \text { WT } & \text { N/A } & 209.3 \\ \text { SORT- } & \text { N/A MEDIAN- } & 1.00 & \text { MODE- } & -1.00 & \text { MEAN- } & \text { N/A } & & \text { WT } & \text { N/A } & 29.9 \\ \text { SPLIT } & \text { WT- } 704.5 & & & & & & \text { CUM WT } & \text { N/A } & 29.9\end{array}$

$\begin{array}{lllllllllll}320 & 0.3 & \text { H } & 3.7 & 63.0 & 33.3 & \text { G } & \text { WT } & \text { N/A } & 195.6\end{array}$

SORT- N/A MEDIAN- 2.00 MODE- -1.00 MEAN- N/A $\quad$ WT N N / A 33.3

$\begin{array}{lllllllllll}325 & 0.2 & \mathrm{H} & 2.3 & 97.3 & 0.4 \mathrm{~S} & \mathrm{WT} & \text { N/A } & 2.3 \\ \text { SORT- } & \text { N/A MEDIAN- } & 2.00 & \text { MODE- } & 2.00 & \text { MEAN- } & \text { N/A } & \text { WT } & \text { N/A } & 0.4\end{array}$

$\begin{array}{lllllllll}\text { SORT- N/A MEDIAN- } 2.00 & \text { MODE- } & 2.00 & \text { MEAN- N/A } & \text { WT } & \text { N/A } & 0.4 \\ \text { SPLIT WT- } 619.1 & & & \text { CUM WT } & \text { N/A } & 0.4\end{array}$

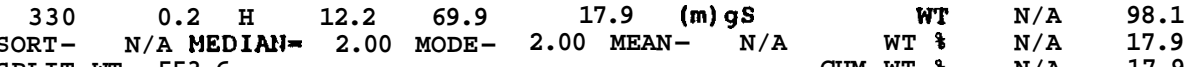

SPLIT WT- 553.6 $\begin{array}{llllllll}.2 & 3.0 & 26.7 & 183.0 & 358 . \theta & 50.1 & \text { N/A } & 21.5\end{array}$

$\begin{array}{llrrrrr}0.5 & 4.1 & 28.3 & 55.4 & 7.7 & \text { N/A } & 3.3\end{array}$

160.9

$\begin{array}{rrrrrrr}32.0 & 54.4 & 289.9 & 168.1 & 36.4 & \text { N/A } & 26.8 \\ 4.6 & 7.7 & 41.2 & 23.9 & 5.2 & \text { N/A } & 3.8\end{array}$

$\begin{array}{rrrrrrr}4.6 & 7.7 & 41.2 & 23.9 & 5.2 & \text { N/A } & 3.8 \\ 18.2 & 25.9 & 67.1 & 91.0 & 96.2 & \text { N/A } & 100.0\end{array}$

$\begin{array}{lllllll}73.6 & 68.0 & 120.9 & 84.1 & 33.4 & \text { N/A } & 31.4\end{array}$

$\begin{array}{rrrrrrr}12.7 & 11.7 & 20.8 & 14.5 & 5.8 & \text { N/A } & 5.4 \\ 41.9 & 53.6 & 74.4 & 88.9 & 94.6 & \text { N/A } & 100.0\end{array}$

$\begin{array}{lllllll}67.1 & 51.0 & 213.1 & 106.5 & 36.5 & \mathrm{~N} / \mathrm{A} & 36.3\end{array}$

$\begin{array}{rrrrrrr}10.6 & 8.1 & 33.8 & 16.9 & 56.5 & \text { N/A } & 36.3 \\ 29.0 & 37.9 & 71.6 & 88.5 & 94.3 & \text { N/A } & 100.0\end{array}$

$\begin{array}{lllllll}89.5 & 68.5 & 167.9 & 01.9 & 40.4 & \text { N/A } & 42.0\end{array}$

$\begin{array}{rrrrrrr}12.8 & 9.8 & 24.0 & 11.7 & 5.8 & \text { N/A } & 42.0 \\ 42.7 & 52.5 & 76.5 & 88.2 & 94.0 & \text { N/A } & 100.0\end{array}$

$\begin{array}{lllllll}53.4 & 32.7 & 169.3 & 90.4 & 23.6 & \text { N/A } & 21.8\end{array}$

$\begin{array}{rrrrrrr}9.1 & 5.6 & 28.9 & 15.4 & 4.0 & \text { N/A } & 3.7 \\ 42.4 & 48.0 & 76.9 & 92.3 & 96.3 & \text { N/A } & 100.0\end{array}$

$\begin{array}{rrrrrrr}12.5 & 80.6 & 283.1 & 192.3 & 37.0 & \text { N/A } & 14.2\end{array}$

$\begin{array}{rrrrrrr}2.4 & 13.0 & 45.5 & 30.9 & 6.0 & \text { N/A } & 2.3\end{array}$

$\begin{array}{llllllll}65.8 & 62.7 & 112.6 & 86.3 & 56.8 & \mathrm{~N} / \mathrm{A} & 67.2\end{array}$

$\begin{array}{rrrrrrr}12.0 & 11.4 & 112.6 & 86.3 & 56.8 & \text { N/A } & 67.2 \\ 29.8 & 41.2 & 61.7 & 77.4 & 10.3 & \text { N/A } & 12.2 \\ & & & & & & \end{array}$ 
WESTINGHOUSE HANFORD OPERATIONS SIEVE ANALYSIS ROCSAN REPORT

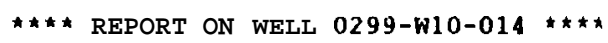

$12 / 11 / 89$

DEPTH $8 \mathrm{CACO}$ DM $\mathrm{MMUD}$ \&SAND \%GRAVEL CLASS

\begin{tabular}{ccccccc} 
DEPTH & 8CACO3 & DM & 8MUD & 8SAND & \%GRAVEL & CLASS \\
\hdashline 335 & 0.4 & H & 8.7 & 66.5 & 24.9 & (m)
\end{tabular} $\begin{array}{llllll}335 & 0.4 & \text { H } & 8.7 & 66.5 & 24.9 \\ \text { SORT- }\end{array}$ SPLIT WT- 680.4

FINE VFINE VERY

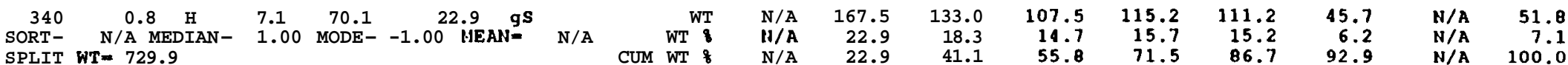

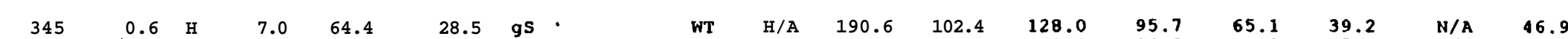

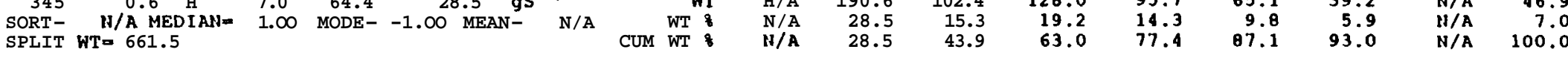

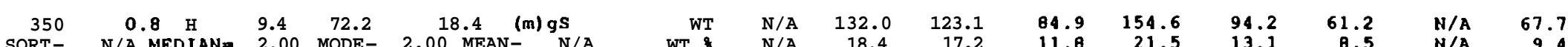

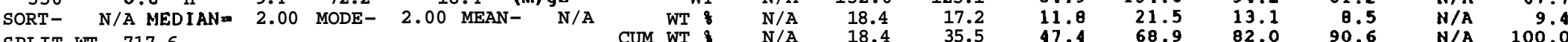
SPLIT WT- 717.6

WT $\quad$ N/A $\quad 65.7 \quad 68.9$

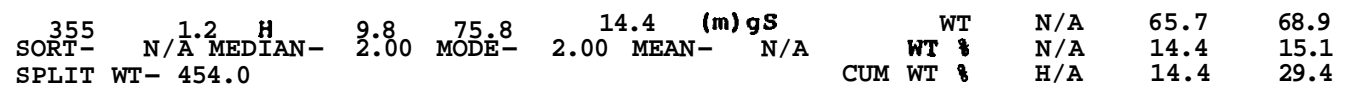

$67.4 \quad 111.2 \quad 63.7 \quad 35.7 \quad$ N/A $\quad 44.8$

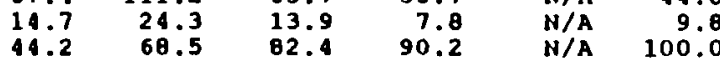

N/A $171.4 \quad 97.9$
$\begin{array}{llllllllllrrr}360 & 1.3 & \text { H } & 5.9 & 60.8 & 25.4 & \text { gS } & & & \text { WT } & \text { N/A } & 171.4 & 97.9 \\ \text { SORT- } & \text { N/A MEDIAN- } & 1.00 & \text { MODE- } & -1.00 & \text { MEAN- } & \text { N/A } & & \text { WT } & \text { N/A } & 25.4 & 14.5 \\ \text { SPLIT } & \text { WT- } 673.6 & & & & \text { CUM WT } & \text { N/A } & 25.4 & 39.8\end{array}$

$74.5 \quad 155.7$

WT

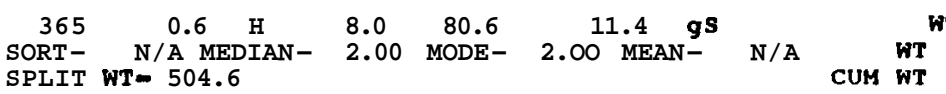

$\begin{array}{llr}\text { N/A } & 57.7 & 95.4 \\ \text { N/A } & 11.4 & 18.8\end{array}$

$\begin{array}{rr}71.5 & 155.7 \\ 11.0 & 23.0 \\ 50.9 & 73.9\end{array}$

14.

$\begin{array}{lll}N / A & 11.4 & 18 \\ N / A & 11.4 & 30.2\end{array}$

$90.1 \quad 119.969 .9$

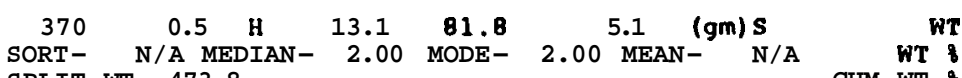

$\begin{array}{lr}\text { N/A } & 24.1 \\ \text { N/A } & 5.1\end{array}$
SPLIT WT- 473.8

$73.9 \quad 133.7$

85.3

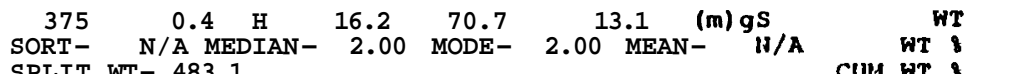

SPIIT

$\mathrm{N} / \mathrm{A} \quad 63.8 \quad 42$.

$33.8 \quad$ N/A 40.6 $\begin{array}{rrr}6.7 & N / A & 0.0\end{array}$

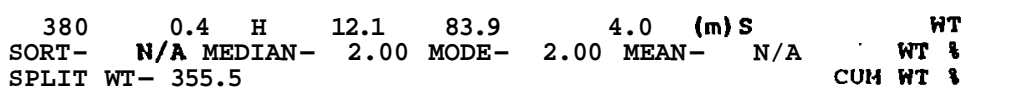

48.8 N/A 78.6 $\begin{array}{llllllll}385 & 0.2 & \mathrm{H} & 6.6 & 82.4 & 11.0 & \mathrm{gS} & \mathrm{WT}\end{array}$ $\begin{array}{llllll}\text { SORT- N/A MEDIAN- } & 2.00 & \text { MODE- } & 2.00 & \text { MEAN- } & \text { M/A } \\ \text { SPLIT WT- } 473.0 & \text { CUM WT }\end{array}$

$\begin{array}{ll}\text { N/A } & 52.2 \\ \text { N/A } & 11.0 \\ \text { N/A } & 11.0\end{array}$



ROCSAN REPORT

$12 / 11 / 89$

$\star * \star *$ REPORT ON WELL 0299-W10-014 $\star \star \star \star *$

DEPTH ВCACO3 DM \$MUD \%SAND \%GRAVEL CLASS

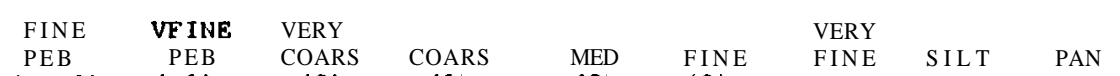

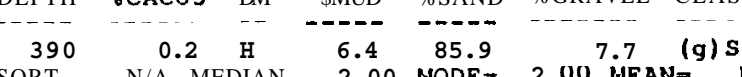

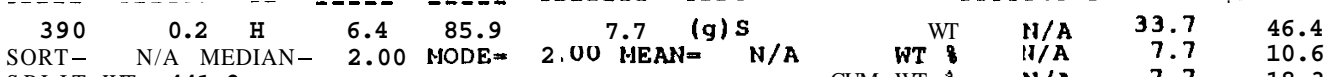
SORT - N/A MEDIAN- 2.00 MODE $* 2.00$ HEAN= N/A
SPLIT WT- 441.9

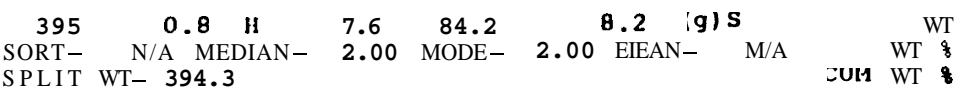

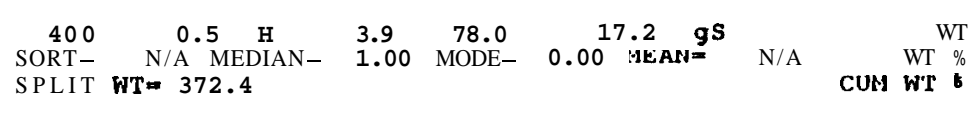
18.3 COARS
$(1)$ MED $\quad$ FINE $(4.75) \quad(>4.75)$

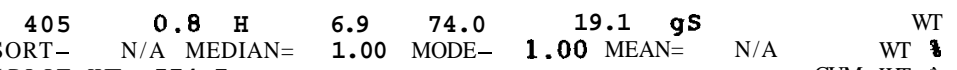
SPLIT WT-574.7

WT

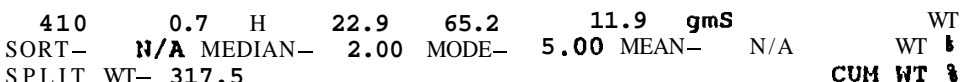

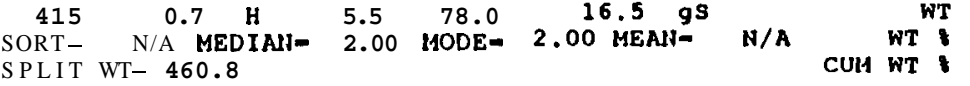

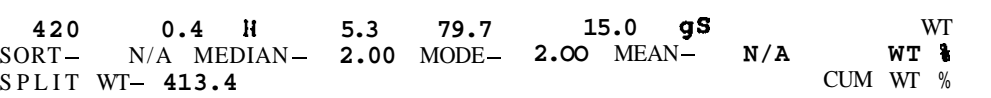

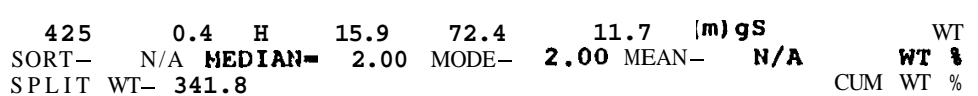

T

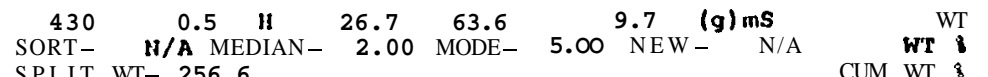

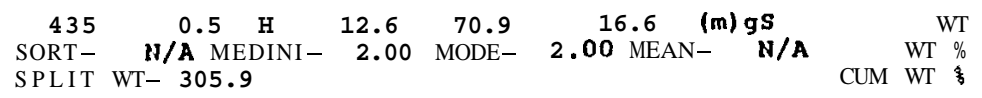

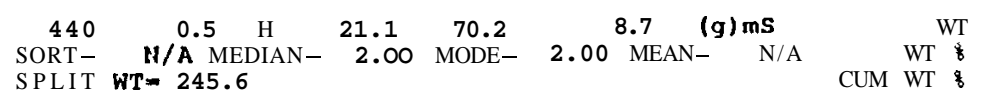
$\begin{array}{lr}\text { N/A } & 32.7 \\ \text { N/A } & 0 \\ \text { N/A } & 0.2\end{array}$ N/A 63.7 57.6
14.4 15.8 172.9 N/A $\quad 17.2$ 22.6

s.t.

80.5
21.
39.

77.

$\begin{array}{lr}\text { N/A } & 110.1 \\ \text { H/A } & 19.1 \\ \text { N/A } & 19.1\end{array}$

90.

90.7
15.7
34.8

N/A 37.9

42.2
13.3

$\begin{array}{lll}\text { H/A } & 11.9 & 25.2\end{array}$

N/A $\quad 77.0$

39 .

39.9
25.6

N/A 62.1

N/A 15.

56.

N/A $\quad 40.0$

20.5

N/A 11.7

29.0
0.7
20.4

$\begin{array}{lr}\text { N/A } & 24.9 \\ \text { N/A } & 9.7\end{array}$

N/A 50.7

$\begin{array}{ll}\text { N/A } & 16 . \\ \text { N/A } & 16.6\end{array}$

M/A 21.3

$\begin{array}{lrr} & & \\ \text { II/A } & 0.7 & 28.1\end{array}$

11.5

21.2
0.3
10.0

40.8
6.0
32.5

0
.54


WESTINGHOUSE HANFORD OPERATIONS SIEVE ANALYSIS ROCSAN REPORT

$12 / 11 / 89$

$\star * *$ REPORT O11 WHL 0299-w10-014 ****

\begin{tabular}{|c|c|c|c|c|c|c|c|c|c|c|c|c|c|c|c|c|c|}
\hline DEPIH & $8 \mathrm{CACO} 3 \mathrm{DM}$ & 8MUD & ZSAND & ZGRAVEL & CLAS & & & & $\begin{array}{l}\text { F IHE } \\
\text { PEB } \\
(<--2)\end{array}$ & $\begin{array}{l}\text { VF INE } \\
\text { PEB } \\
(-1)\end{array}$ & $\begin{array}{l}\text { VERY } \\
\text { COARS } \\
(0)\end{array}$ & $\underset{\text { (1) }}{\text { COARS }}$ & $\begin{array}{l}\mathrm{MD} \\
\text { (2) }\end{array}$ & $\begin{array}{l}\text { FINE } \\
\text { (3) }\end{array}$ & $\begin{array}{l}\text { VERY } \\
\text { FINE } \\
\text { (4) }\end{array}$ & $\begin{array}{c}\text { SILT } \\
(4.75)\end{array}$ & $\underset{(>4.75)}{\text { PAN }}$ \\
\hline 445 & $0.5 \mathrm{H}$ & 24.8 & 55.6 & 19.6 & gms & & & HT & $\mathrm{N} / \mathrm{A}$ & 51.2 & 36.6 & 22.1 & 35.3 & 31.3 & 19.8 & N/A & 64.9 \\
\hline SORT- & N/A MEDIAN= & 2.00 & MODE- & 5.00 MEAN & & N/A & & $\mathrm{W}$ & $\mathrm{N} / \mathrm{A}$ & 15 & 14.0 & 8 & 13.5 & 1 & 7.6 & A & 24.9 \\
\hline SPLIT & WI- 261.3 & & & & & & CUM & WT & $\mathrm{N} / \mathrm{A}$ & 19.6 & 33.6 & 42.1 & 55.6 & 67.6 & 75.1 & N/A & 100.0 \\
\hline $\begin{array}{l}450 \\
\text { SORT- } \\
\text { SPLIT }\end{array}$ & $\begin{array}{l}0.4 \quad \mathrm{H} \\
\text { N/A MEDIAN- } \\
\text { WT- } 258.8\end{array}$ & $\begin{array}{c}36.0 \\
-\quad 3.00\end{array}$ & $\begin{array}{r}49.6 \\
\text { HODE }\end{array}$ & 5.00 HEAN & $g m S$ & $N / A$ & CUIS & $\begin{array}{l}\text { WT } \\
\text { WT } \\
\text { WT }\end{array}$ & $\begin{array}{l}N / A \\
H / A \\
N / A\end{array}$ & $\begin{array}{l}37.2 \\
14.4 \\
14.4\end{array}$ & $\begin{array}{l}30.2 \\
11.7 \\
26.0\end{array}$ & $\begin{array}{r}22.1 \\
8.5 \\
34.6\end{array}$ & $\begin{array}{l}38.2 \\
14.8 \\
49.3\end{array}$ & $\begin{array}{r}25.1 \\
9.7 \\
59.0\end{array}$ & $\begin{array}{r}12.8 \\
4.9 \\
64.0\end{array}$ & $\begin{array}{l}\text { N/A } \\
\text { N/A } \\
\text { N/A }\end{array}$ & $\begin{array}{r}93.3 \\
36.0 \\
100.0\end{array}$ \\
\hline $\begin{array}{l}455 \\
\text { SORT- } \\
\text { SPLIT }\end{array}$ & $\begin{array}{l}0.8 \mathrm{H} \\
\text { II/A MEDIAH- } \\
\text { WI- } 233.5\end{array}$ & $\begin{array}{c}66.9 \\
5.00\end{array}$ & $\begin{array}{r}31.9 \\
\text { MODE- }\end{array}$ & 5.00 MEAN & SM & NI/A & CM & $\begin{array}{l}\mathrm{WT} \\
\mathbf{W T} \\
\mathrm{WT}\end{array}$ & $\begin{array}{l}\text { N/A } \\
\text { N/A } \\
\text { N/A }\end{array}$ & $\begin{array}{l}2.6 \\
1.1 \\
1.1\end{array}$ & $\begin{array}{l}5.8 \\
2.5 \\
3.6\end{array}$ & $\begin{array}{l}7.0 \\
3.3 \\
6.9\end{array}$ & $\begin{array}{l}23.7 \\
10.2 \\
17.1\end{array}$ & $\begin{array}{r}22.7 \\
9.7 \\
26.8\end{array}$ & $\begin{array}{r}14.6 \\
6.3 \\
33.1\end{array}$ & $\begin{array}{l}\text { N/A } \\
\text { II/A } \\
\text { N/A }\end{array}$ & $\begin{array}{r}156.3 \\
66.9 \\
100.0\end{array}$ \\
\hline
\end{tabular}



ROCSAN REPORT

$12 / 11 / 89$

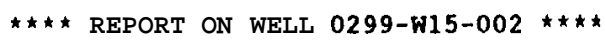

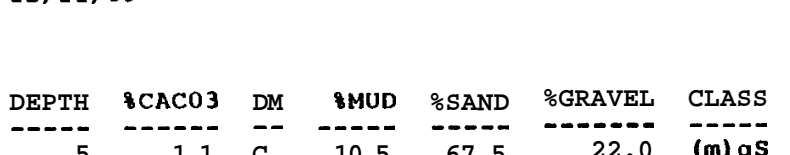

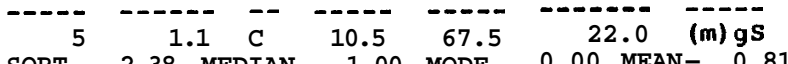
SORT- 2.38 MEDIAN- 1.00 MODE- 0.00 MEAN- 0.81
SPLIT WT- 139.9

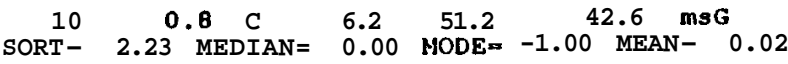

SPLIT WT- 141.3

$\begin{array}{rllllcl}15 & 1.6 & \mathrm{C} & 0.5 & 67.9 & 23.6 & \text { (m) gs } \\ \text { SORT- } & 2.44 & \text { MEDIAN- } & 1.00 & \text { MODE- } & 0.00 & \text { MEAN- }\end{array}$ SPLIT WT- 121.1

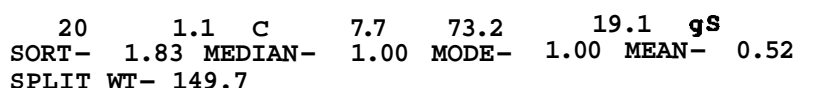

SPLIT WT- 149.7

$\begin{array}{lllllll}25 & 1.1 & \mathrm{C} & 3.7 & 68.5 & 27.8 & \mathrm{gS}\end{array}$

SORT- N/A MEDIAN- 0.00 MODE- 1.00 MEAN- N/A

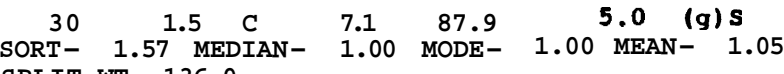

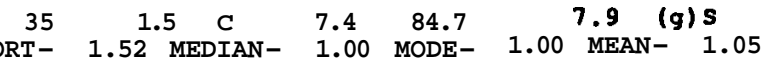

SPLIT WT- 116.1

$\begin{array}{lccccc}40 & 2.2 & \mathrm{C} & 12.8 & 07.0 & 0.2 \mathrm{(m)s} \\ \text { SORT- } & 1.33 .2 \mathrm{MEDIAN}- & 3.00 & \text { MODE- } & 2.00 & \text { MEAN- } 2.29 \\ \text { SPLIT } & \text { WT- } & 108.8\end{array}$

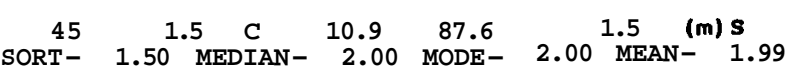

SPLIT WT $=170.0$

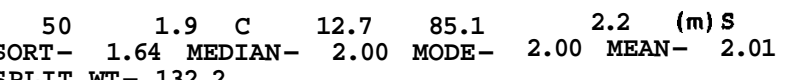

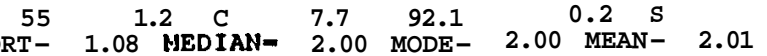

SPLIT WT $=132.5$

\begin{tabular}{|c|c|c|c|c|c|c|c|c|c|}
\hline & $\begin{array}{l}\text { FINE } \\
\text { PEB } \\
(<--2)\end{array}$ & $\begin{array}{c}\text { W I N E } \\
\text { PEB } \\
(-1)\end{array}$ & $\begin{array}{c}\text { VERY } \\
\text { COARS } \\
\text { (0) }\end{array}$ & $\begin{array}{l}\text { COARS } \\
\text { (1) }\end{array}$ & $\begin{array}{l}\text { MED } \\
\text { (2) }\end{array}$ & $\begin{array}{l}\text { FINE } \\
\text { (3) }\end{array}$ & $\begin{array}{l}\text { VERY } \\
\text { FINE } \\
\text { (4) }\end{array}$ & $\begin{array}{c}\text { SILT } \\
(4.75)\end{array}$ & $\begin{array}{c}\text { PAN } \\
(>4.75)\end{array}$ \\
\hline $\begin{array}{r}\text { WT } \\
\text { WT } \% \\
\text { J W T O }\end{array}$ & $\begin{array}{l}6.5 \\
4.7 \\
4.7\end{array}$ & $\begin{array}{l}24.0 \\
17.3 \\
22.0\end{array}$ & $\begin{array}{l}31.0 \\
22.3 \\
44.3\end{array}$ & $\begin{array}{l}22.2 \\
16.0 \\
60.3\end{array}$ & $\begin{array}{l}14.0 \\
10.1 \\
70.3\end{array}$ & $\begin{array}{c}13.6 \\
9.0 \\
80.1\end{array}$ & $\begin{array}{c}13.0 \\
9.4 \\
89.5\end{array}$ & $\begin{array}{r}7.6 \\
5.5 \\
95.0\end{array}$ & $\begin{array}{r}7.0 \\
5.0 \\
100.0\end{array}$ \\
\hline
\end{tabular}

$\begin{array}{rrrrr}\text { WT } & 17.5 & 42.4 & 30.2 & 15.1 \\ \text { WT } & 12.4 & 30.1 & 21.5 & 10.7 \\ \text { CUM WT } & 12.4 & 42.6 & 64.0 & 74.8\end{array}$

$\begin{array}{rrrrr}8.5 & 7.7 & 10.6 & 4.5 & 4.2 \\ 6.0 & 5.5 & 7.5 & 3.2 & 3.0 \\ 80.8 & 86.3 & 93.8 & 97.0 & 100.0\end{array}$

$\begin{array}{llll}\text { WT } & 10.4 & 17.9 & 25.7\end{array}$

$\begin{array}{rrrr}\text { CUM WT } & 0.7 & 14.9 & 21.4 \\ \text { WT } & 0.7 & 23.6 & 45.0\end{array}$

$20.9 \quad 11.0 \quad 9.7$

9.7
8.1
79.6

14.3
11.9
91.5

$4.1 \quad 6.1$

$\begin{array}{llll}\text { WT } & 8.0 & 20.5 & 31.3\end{array}$

$28.4 \quad 18.4 \quad 10.8$

$\begin{array}{lll}28.4 & 12.3 & 7.2\end{array}$

s:

$\begin{array}{rrr}29.9 & 17.5 & 6.4 \\ 23.8 & 13.9 & 5.9 \\ 73.9 & 07.9 & 92.9\end{array}$

6.4
5.1
92.9

96.

$\begin{array}{lll}48.1 & 25.3 & 11 . \\ 35.7 & 18.8 & 8.8\end{array}$

$\begin{array}{rrr}35.7 & 18.8 & 8.8 \\ 58.6 & 77.4 & 86.1\end{array}$

92.

$\begin{array}{lll}34.0 & 30.6 & 11.5 \\ 29.5 & 26.6 & 10.0 \\ 51.3 & 77.9 & 87.8\end{array}$

92.

11.3

40.625

$37.6 \quad 23.6$

23.6

15. CUH WT: 0.0

$\begin{array}{r:r}\text { WT } & 0.4 \\ \text { พT } & 0.2\end{array}$ CUM WT

2.2
1.3
1.5

$7.8 \quad 37.0$

$49.0 \quad 35.1$

$27.8 \quad 19.9$

$35.2 \quad 27.2$

27.2
20.6
74.6

$\begin{array}{rrr}25.8 & 9.6 & 9.6 \\ 14.6 & 5.4 & 5.4 \\ 89.1 & 94.6 & 100.0\end{array}$ $\begin{array}{rrr}\text { WT } & 0.6 \\ \text { WT } & 0.5 \\ \text { CUM WT } & 0.5\end{array}$

2.3
1.7

6.0
4.5

$4.5 \quad 20.6$

27.3

54.0

$\begin{array}{rrr}16.8 & 8.8 & 8.0 \\ 12.7 & 6.7 & 6.1\end{array}$

$\begin{array}{ll}\text { WT } & 0.0 \\ \text { WT } & 0.0\end{array}$

0.2
0.2
0.2

$\begin{array}{ll}2.6 & 17.1 \\ 2.0 & 12.9 \\ 2.1 & 15.1\end{array}$

$\begin{array}{lllll}56.3 & 32.2 & 13.5 & 4.8 & 5.4\end{array}$

$\begin{array}{rrrrr}12.6 & 24.4 & 10.2 & 3.8 & 5.4 \\ 57.7 & 82.1 & 92.3 & 95.9 & 100.0\end{array}$ 
WESTINGHOUSE HANFORD OPERATIONS SIEVE ANALYSIS ROCSAN REPORT

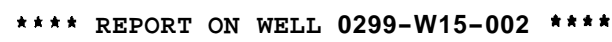

$12 / 11 / 89$

\begin{tabular}{rrrrrrl} 
DEPTH & ICACO3 & DM & IMUD & \%SAND & GRAVEL & CLASS \\
\hdashline 60 & 1.4 & $\mathrm{C}$ & 9.7 & 90.2 & 0.1 & $\mathrm{~S}$
\end{tabular}

ORT- 1.14 MEDIAN- 3.00 MODE- 2.00 MEAN- 2.30 SPLIT WT $=159.7$

$\begin{array}{lllllll}65 & 2.1 & C & 9.1 & 67.6 & 23.2 & \text { (m) gs }\end{array}$ $\begin{array}{llll}\text { SORT- } 2.50 \text { MEDIAN- } & 1.00 \text { MODE- } 1.00 \text { MEAN- } 0.81 \\ \text { SPLIT WT- } 115.7 & \end{array}$

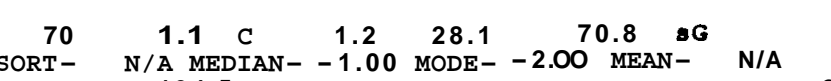

SPLIT WT- 134.5

$\begin{array}{lllllll}75 & 1.7 & \mathrm{C} & 8.9 & 86.1 & 5.0 & \text { (g) } \mathrm{s}\end{array}$

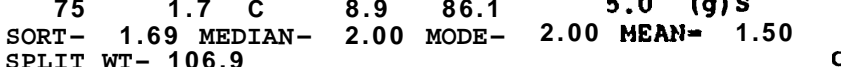

$\begin{array}{rrrrrrr}80 & 1.9 & \mathrm{C} & \mathbf{1 1 . 1} & 83.4 & 5.4 & (\mathrm{gm}) \mathrm{s} \\ \text { SORT- } & 1.71 & \text { MEDIAN- } & 2.00 & \text { MODE- } & 2.00 & \text { MEAN- } 1.65\end{array}$ SPLIT WT- 120.2

$\begin{array}{rlllllll}85 & 1.8 & \mathrm{C} & 9.3 & 86.4 & 4.4 \mathrm{~s} & \\ \text { SORT- } & 1.57 & \text { MEDIAN- } & 2.00 & \text { MODE- } & 2.00 & \text { MEAN- } & 1.64\end{array}$ SPLIT WT- 134.4

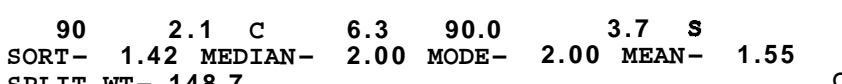

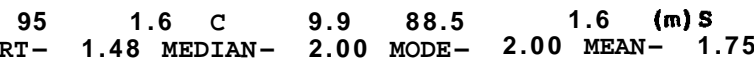

SORT- 1.48 MEDI

$\begin{array}{ccccccc}100 & 1.8 \mathrm{C} & 9.3 & 87.2 & 3.5 \mathrm{~S} & \\ \text { SORT- } & 1.49 \mathrm{MEDIAN}- & 2.00 & \text { MODE- } & 2.00 & \text { MEAN- } & 1.68\end{array}$

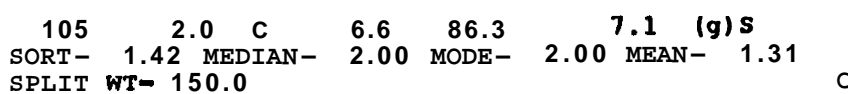

$\begin{array}{lllllll}110 & 1.6 \mathrm{C} & 8.8 & 86.6 & 4.6 & \mathrm{~s} & \\ \text { SORT- } & 1.37 & \mathrm{MEDIAN}- & 2.00 & \text { MODE- } & 2.00{ }_{\text {MEAN- }} & 1.56 \\ \text { SPLIT } & \text { WTT }= & 167.2\end{array}$
FINE VFINE VERY

$\begin{array}{lclllllll}\text { FINE } & \text { VFINE } & \text { VERY } & & & & \text { VERY } & \\ \text { PEB } & \text { PEB } & \text { COARS } & \text { COARS } & \text { MED } & \text { FINE } & \text { FINE } & \text { SILT } & \text { PAN } \\ (<--2) & (-1) & (0) & (1) & (2) & (3) & (4) & (4.75) & (>4.75)\end{array}$

T

\begin{tabular}{cccc}
$\begin{array}{c}\text { PEB } \\
(<--2)\end{array}$ & $\begin{array}{c}\text { PEB } \\
(-1)\end{array}$ & $\begin{array}{c}\text { CORS } \\
(0)\end{array}$ & $\begin{array}{c}\text { COARS } \\
(1)\end{array}$ \\
\hline 0.0 & 0.2 & 1.1 & 9.0 \\
0.0 & 0.1 & 0.7 & 5.7 \\
0.0 & 0.1 & 0.8 & 6.5
\end{tabular}

wT $15.0 \quad 11.7$

11.7
10.2

6.5

$\frac{(2)}{62.5}-\frac{(3)}{50.1}-$

$\begin{array}{rrr} & (4.75) & (>4.75) \\ 21.1 & 7.0 & 8.4 \\ 13.2 & 4.4 & 5.3\end{array}$

WT 13.1

10.2
23.2

13.9
12.1

2.1

20.1

17.5
52.8

39.2
45.7

$\begin{array}{rr}4.4 & 5.3 \\ 94.7 & 100.0\end{array}$

$\begin{array}{rrrr}\text { WT } & 66.5 & 29.1 & 17.6 \\ \text { WT } & 49.2 & 21.5 & 13.0\end{array}$

7.6
3.0
3.8

9.6
7.1

$15.1 \quad 13.1$

15.1
13.1
01.1

$\begin{array}{rrr}11.2 & 5.1 & 5.4\end{array}$

$\begin{array}{rrr}9.8 & 4.4 & 4.7 \\ 90.9 & 95.3 & 100.0\end{array}$ WT I 49

70.8

WT 0.8

4.5
4.2
5.0

$\begin{array}{ll}\text { WT } & 1.2 \\ \text { CUM } & 1.0\end{array}$

4.2
5.0

12.0
11.3
16.3

90.9

5.6
4.2
95.

$\begin{array}{llll}3.0 & 2.1 & 0.9 & 0.7\end{array}$

$\begin{array}{rrrr}2.2 & 1.6 & 0.7 & 0.5 \\ 97.3 & 98.0 & 99.5 & 100.0\end{array}$

COM WT 1.0

$\begin{array}{rr}5.2 & 11.2 \\ 4.4 & 9.5\end{array}$

23.6
22.2
38.5

30.0$$
14.3
$$

$\begin{array}{lll}11.5 & 3.5 & 6.0\end{array}$

\section{9}

66.8

80.2

$\begin{array}{rrr}10.8 & 3.3 & 5.7 \\ 91.1 & 94.4 & 100.0\end{array}$

3.8

.811

11.0
8.3
12.7

22.7
37.6

34.1
28.8
66.4

15.8
13.3

10.8

6.5100 .0

$\begin{array}{ll}29.3 & 42.5 \\ 22.1 & 32.0\end{array}$

42.5
32.0
66.8

19.2

9.1
88.8

$\begin{array}{rr}6.5 & 6.7 \\ 5.5 & 5.7 \\ 94.3 & 100.0\end{array}$

WT 1

WT $\mathrm{T}$

0.5
0.3

3.

5.011 .5

\section{s..}

$35.2 \quad 51.6$

81.2

$12.6 \quad 5.5 \quad 6.8$

$\begin{array}{rrr}9.5 & 4.1 & 5.1 \\ 90.7 & 94.9 & 100.0\end{array}$

$\begin{array}{llll}19.7 & 14.0 & 4.2 & 5.1\end{array}$

$\begin{array}{rrrr}13.4 & 10.0 & 2.9 & 3.5 \\ 83.7 & 93.7 & 96.6 & 100.0\end{array}$

$0.0 \quad 2.6 \quad 11.1$

$\begin{array}{rrrr}28.4 & 14.4 & 8.0 & 8.2 \\ 17.4 & 8.8 & 4.9 & 5.0\end{array}$

$\begin{array}{llll}11.3 & 90.1 & 95.0 & 100.0\end{array}$

CUM WT

WT 1.1

1.6

4.

$1.5 \quad 30.7$

63.9

25.6

13.4

$\begin{array}{ll}6.0 & 8.2 \\ 3.9 & 5.4\end{array}$

WT I 0

$\begin{array}{r:r}\text { WT } & 2.0 \\ \text { WT } & 1.3 \\ \text { WT } & 1.3\end{array}$

$\begin{array}{lll}8.6 & 14.8 & 34.5\end{array}$

33.9
65.1

81.9

90.7

$94.6 \quad 100.0$

WT 0.0

8.6
5.0
7.1

$\begin{array}{rr}4.8 & 34.5 \\ 9.9 & 23.1\end{array}$

52.1
34.9
74.9

$\begin{array}{llll}18.2 & 9.4 & 4.7 & 5.2\end{array}$

$\begin{array}{rrrr}12.2 & 6.3 & 3.1 & 5.2 \\ 87.1 & 93.4 & 96.5 & 100.0\end{array}$

$\begin{array}{rrrr}7.8 & 11.9 & 32.0 & 65.0 \\ 1.7 & 7.1 & 19.1 & 38.7\end{array}$

$\begin{array}{rrrrr}65.0 & 25.3 & 11.2 & 7.5 & 7.2 \\ 8.7 & 15.1 & 6.7 & 4.5 & 4.3\end{array}$

$\begin{array}{rrrrr}6.0 & 25.3 & 11.2 & 7.5 & 7.2 \\ 8.7 & 15.1 & 6.7 & 4.5 & 4.3 \\ 9.5 & 04.6 & 91.3 & 95.7 & 100.0\end{array}$


WESTINGHOUSE HANFORD OPERATIONS SIEVE ANALYSIS ROCSAN REPORT

*** REPORT ON WELL 0299-W15-002 ****

$12 / 11 / 89$

\begin{tabular}{|c|c|c|c|c|c|c|c|c|c|c|c|c|c|c|c|c|}
\hline DEPTH & ICACO3 DM & 8MUD & $\%$ SAND & IGRAVEL C & CLASS & & & $\begin{array}{l}\text { FINE } \\
\text { PEB } \\
(<--2)\end{array}$ & $\begin{array}{l}\text { VFINE } \\
\text { PEB } \\
(-1)\end{array}$ & $\begin{array}{l}\text { VERY } \\
\text { COARS } \\
(0)\end{array}$ & $\begin{array}{l}\text { COARS } \\
\text { (1) }\end{array}$ & $\begin{array}{l}\text { MED } \\
(2) \\
-\end{array}$ & $\begin{array}{l}\text { FINE } \\
\text { (3) }\end{array}$ & $\begin{array}{l}\text { VERY } \\
\text { FINE } \\
\text { (4) }\end{array}$ & \begin{tabular}{c} 
SILT \\
$(\mathbf{4 . 7 5})$ \\
\hdashline
\end{tabular} & $\begin{array}{l}\text { PAN } \\
(>4.75)\end{array}$ \\
\hline $\begin{array}{l}\mathbf{1 1 5} \\
\text { SORT- } \\
\text { SPLIT }\end{array}$ & $\begin{array}{c}\mathbf{7 . 5} \mathrm{C} \\
\mathbf{1 . 7 6} \text { MEDIAN- } \\
\text { WT }=\mathbf{1 7 1 . 0}\end{array}$ & $\begin{array}{c}17.8 \\
3.00\end{array}$ & $\begin{array}{r}\mathbf{7 9 . 8} \\
\text { MODE- }\end{array}$ & $2.00^{2.4}$ MEAN & $\left\{\begin{array}{l}(m) s \\
=2.31\end{array}\right.$ & CUM & $\begin{array}{l}\text { WT } \\
\text { WT } \\
\text { WT }\end{array}$ & $\begin{array}{l}0.5 \\
0.3 \\
0.3\end{array}$ & $\begin{array}{l}3.6 \\
2.1 \\
2.4\end{array}$ & $\begin{array}{l}9.2 \\
5.4 \\
7.8\end{array}$ & $\begin{array}{l}22.3 \\
13.1 \\
20.8\end{array}$ & $\begin{array}{l}45.5 \\
26.6 \\
47.5\end{array}$ & $\begin{array}{l}28.9 \\
16.9 \\
64.4\end{array}$ & $\begin{array}{l}30.5 \\
17.9 \\
82.2\end{array}$ & $\begin{array}{r}15.4 \\
9.0 \\
91.2\end{array}$ & $\begin{array}{r}15.0 \\
8.8 \\
100.0\end{array}$ \\
\hline $\begin{array}{l}120 \\
\text { SORT- } \\
\text { SPLIT }\end{array}$ & 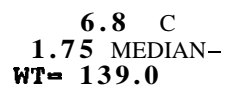 & $\begin{array}{c}22.4 \\
3.00\end{array}$ & $\begin{array}{r}\mathbf{7 6 . 6} \\
\text { MODE- }\end{array}$ & 2.00 MEAN & $\begin{array}{l}\mathrm{mS} \\
\mathrm{V}-\quad 2.64\end{array}$ & CUM & $\begin{array}{l}\text { WT } \\
\text { WT } \\
\text { WT }\end{array}$ & $\begin{array}{l}0.0 \\
\text { 0.0 } \\
0.0\end{array}$ & $\begin{array}{l}1.3 \\
0.9 \\
0.9\end{array}$ & $\begin{array}{l}4.8 \\
3.5 \\
4.4\end{array}$ & $\begin{array}{l}16.5 \\
12.0 \\
16.4\end{array}$ & $\begin{array}{l}38.6 \\
28.0 \\
44.4\end{array}$ & $\begin{array}{l}16.3 \\
11.8 \\
56.2\end{array}$ & $\begin{array}{l}29.5 \\
21.4 \\
77.6\end{array}$ & $\begin{array}{l}13.9 \\
10.1 \\
87.7\end{array}$ & $\begin{array}{r}17.0 \\
12.3 \\
100.0\end{array}$ \\
\hline $\begin{array}{l}130 \\
\text { SORT- } \\
\text { SPLIT }\end{array}$ & $\begin{array}{c}\mathbf{1 8 . 7} \mathrm{C} \\
\text { N/A MEDIAN- } \\
\text { wT }=\mathbf{1 6 4 . 1}\end{array}$ & $\begin{array}{c}16.7 \\
2.00\end{array}$ & $\begin{array}{r}\mathbf{5 2 . 4} \\
\text { MODE- }\end{array}$ & $\begin{array}{c}31.0 \\
-\mathbf{2 . 0 0} \text { MEAN }\end{array}$ & $\begin{array}{l}\text { msG } \\
\mathrm{N}-\quad \mathrm{N} / \mathbf{A}\end{array}$ & CUM & $\begin{array}{l}\text { WT } \\
\text { WT } \\
\text { WT }\end{array}$ & $\begin{array}{l}26.5 \\
16.3 \\
16.3\end{array}$ & $\begin{array}{l}24.0 \\
14.7 \\
31.0\end{array}$ & $\begin{array}{r}14.7 \\
9.0 \\
40.0\end{array}$ & $\begin{array}{r}12.0 \\
7.4 \\
47.3\end{array}$ & $\begin{array}{l}20.0 \\
12.3 \\
59.6\end{array}$ & $\begin{array}{l}17.7 \\
10.9 \\
70.4\end{array}$ & $\begin{array}{l}21.0 \\
12.9 \\
83.3\end{array}$ & $\begin{array}{r}16.2 \\
9.9 \\
93.3\end{array}$ & $\begin{array}{r}11.0 \\
6.7 \\
100.0\end{array}$ \\
\hline $\begin{array}{l}\mathbf{1 3 5} \\
\text { SORT- } \\
\text { SPLIT }\end{array}$ & $\begin{array}{c}\mathbf{7 . 5} \mathrm{C} \\
\text { N/A MEDIAN- } \\
\text { wT- } \mathbf{1 5 2 . 3}\end{array}$ & $\begin{array}{l}6.5 \\
0.00\end{array}$ & $\begin{array}{r}\mathbf{5 4 . 6} \\
\text { MODE- }\end{array}$ & -2.00 MEAH & $\underset{d=}{\mathbf{m s G}} \mathrm{N} / \mathrm{A}$ & CUMY & $\begin{array}{l}\text { WT } \\
\text { WT } \\
\text { WTO }\end{array}$ & $\begin{array}{l}30.0 \\
19.6 \\
19.6\end{array}$ & $\begin{array}{l}29.5 \\
19.3 \\
38.9\end{array}$ & $\begin{array}{l}25.3 \\
16.6 \\
55.5\end{array}$ & $\begin{array}{l}16.2 \\
10.6 \\
66.1\end{array}$ & $\begin{array}{r}15.0 \\
9.8 \\
75.9\end{array}$ & $\begin{array}{r}10.0 \\
6.5 \\
82.5\end{array}$ & $\begin{array}{l}16.9 \\
11.1 \\
93.5\end{array}$ & $\begin{array}{r}4.6 \\
3.0 \\
96.5\end{array}$ & $\begin{array}{r}5.3 \\
3.5 \\
100.0\end{array}$ \\
\hline $\begin{array}{l}\mathbf{1 4 0} \\
\text { SORT- } \\
\text { S PLIT }\end{array}$ & $\begin{array}{l}\mathbf{2 . 9} \text { C } \\
\mathbf{1 . 9 7} \text { MEDIAN- } \\
\text { WT- } \mathbf{1 5 3 . 5}\end{array}$ & $\begin{array}{l}5.1 \\
0.00\end{array}$ & $\begin{array}{r}\mathbf{6 0 . 5} \\
\text { MODE- }\end{array}$ & 0.00 MEAN & $\begin{array}{l}=96 \\
V-0.06\end{array}$ & CUM & $\begin{array}{l}\text { WT } \\
\text { WT } \\
\text { WT T }\end{array}$ & $\begin{array}{l}23.9 \\
15.5 \\
15.5\end{array}$ & $\begin{array}{l}29.1 \\
18.9 \\
34.4\end{array}$ & $\begin{array}{l}29.6 \\
19.2 \\
53.6\end{array}$ & $\begin{array}{l}25.1 \\
16.3 \\
69.9\end{array}$ & $\begin{array}{l}22.0 \\
14.3 \\
84.2\end{array}$ & $\begin{array}{r}9.6 \\
6.2 \\
90.5\end{array}$ & $\begin{array}{r}6.9 \\
4.5 \\
94.9\end{array}$ & $\begin{array}{r}4.2 \\
2.7 \\
97.7\end{array}$ & $\begin{array}{r}3.6 \\
2.3 \\
100.0\end{array}$ \\
\hline $\begin{array}{l}\mathbf{1 4 5} \\
\text { SORT- } \\
\text { SPLIT }\end{array}$ & $\begin{array}{l}\underset{2.0 \quad C}{2.0} \\
\mathbf{2 . 4 9} \text { MEDIAN- } \\
\text { WT- } \mathbf{1 5 6 . 8}\end{array}$ & $\begin{array}{c}10.8 \\
1.00\end{array}$ & $\begin{array}{r}\mathbf{6 0 . 8} \\
\text { MODE- }\end{array}$ & $\begin{array}{l}28.4 \\
2.00 \text { MEAN }\end{array}$ & $(\mathrm{m}) \mathrm{gs}$ & CUM & $\begin{array}{l}\text { WT } \\
\text { wT : } \\
\text { WTO }\end{array}$ & $\begin{array}{l}21.2 \\
13.6 \\
13.6\end{array}$ & $\begin{array}{l}23.2 \\
14.8 \\
28.4\end{array}$ & $\begin{array}{l}22.2 \\
14.2 \\
42.6\end{array}$ & $\begin{array}{l}22.5 \\
14.4 \\
57.0\end{array}$ & $\begin{array}{l}25.7 \\
16.4 \\
73.4\end{array}$ & $\begin{array}{r}15.2 \\
9.7 \\
83.2\end{array}$ & $\begin{array}{r}9.4 \\
6.0 \\
89.2\end{array}$ & $\begin{array}{r}6.8 \\
4.4 \\
93.5\end{array}$ & $\begin{array}{r}10.1 \\
6.5 \\
100.0\end{array}$ \\
\hline $\begin{array}{l}\mathbf{1 5 0} \\
\text { SORT- } \\
\text { SPLIT }\end{array}$ & $\begin{array}{l}\mathbf{1 . 6} \text { C } \\
\mathbf{2 . 5 6} \text { MEDIAN- } \\
\text { WT- } \mathbf{1 5 3 . 0}\end{array}$ & $\begin{array}{l}8.6 \\
1.00\end{array}$ & $\begin{array}{r}\mathbf{6 0 . 5} \\
\text { MODE- }\end{array}$ & $\begin{array}{c}30.9 \\
-1.00 \text { MEAN }\end{array}$ & $\begin{array}{l}\mathrm{msc} \\
\mathrm{N}-0.59\end{array}$ & CUM & $\begin{array}{l}\text { WT } \\
\text { WT } \\
\text { WT }\end{array}$ & $\begin{array}{l}23.0 \\
15.1 \\
15.1\end{array}$ & $\begin{array}{l}24.2 \\
15.9 \\
30.9\end{array}$ & $\begin{array}{l}18.9 \\
12.4 \\
13.3\end{array}$ & $\begin{array}{l}20.0 \\
13.1 \\
56.4\end{array}$ & $\begin{array}{l}23.8 \\
15.6 \\
72.0\end{array}$ & $\begin{array}{l}15.6 \\
10.2 \\
82.3\end{array}$ & $\begin{array}{r}14.0 \\
9.2 \\
91.4\end{array}$ & $\begin{array}{r}5.4 \\
3.5 \\
95.0\end{array}$ & $\begin{array}{r}7.7 \\
5.1 \\
100.0\end{array}$ \\
\hline $\begin{array}{l}\mathbf{1 5 5} \\
\text { SORT- } \\
\text { SPLIT }\end{array}$ & $\begin{array}{l}\mathbf{0 . 9} \text { C } \\
\mathbf{2 . 4 7} \text { MEDIAN- } \\
\text { wT- } 139.7\end{array}$ & $\begin{array}{l}9.3 \\
1.00\end{array}$ & $\begin{array}{r}\mathbf{5 8 . 3} \\
\text { MODE- }\end{array}$ & $\begin{array}{c}32.4 \\
-1.00 \mathrm{MEAN}\end{array}$ & $\mathrm{msG}_{-} \quad 0.43$ & CUM & $\begin{array}{l}\text { WT } \\
\text { WT } \\
\text { WT }\end{array}$ & $\begin{array}{l}21.8 \\
15.6 \\
15.6\end{array}$ & $\begin{array}{l}23.4 \\
16.8 \\
32.4\end{array}$ & $\begin{array}{l}17.9 \\
12.8 \\
45.2\end{array}$ & $\begin{array}{l}21.6 \\
15.5 \\
60.6\end{array}$ & $\begin{array}{l}20.2 \\
14.5 \\
75.1\end{array}$ & $\begin{array}{r}12.9 \\
9.2 \\
84.3\end{array}$ & $\begin{array}{r}8.9 \\
6.4 \\
90.7\end{array}$ & $\begin{array}{r}6.0 \\
4.3 \\
95.0\end{array}$ & $\begin{array}{r}7.0 \\
5.0 \\
100.0\end{array}$ \\
\hline $\begin{array}{l}\mathbf{1 6 0} \\
\text { SORT- } \\
\text { SPLIT }\end{array}$ & $\begin{array}{l}\mathbf{0 . 8} \mathrm{C} \\
\mathbf{1 . 9 0} \text { MEDIAN- } \\
\text { WT- } \mathbf{1 3 7 . 8}\end{array}$ & $\begin{array}{l}4.8 \\
1.00\end{array}$ & $\begin{array}{r}71.8 \\
\text { MODE- }\end{array}$ & $\begin{array}{c}23.4 \\
1.00 \text { MEAN }\end{array}$ & $\mathrm{gs}_{-} \mathbf{0 . 3 7}$ & cum: & $\begin{array}{l}\text { WT } \\
\text { WT }\end{array}$ & $\begin{array}{l}14.4 \\
10.5 \\
10.5\end{array}$ & $\begin{array}{l}17.9 \\
13.0 \\
23.4\end{array}$ & $\begin{array}{l}23.2 \\
16.8 \\
40.3\end{array}$ & $\begin{array}{l}30.8 \\
22.4 \\
62.6\end{array}$ & $\begin{array}{l}26.8 \\
19.5 \\
82.1\end{array}$ & $\begin{array}{r}11.0 \\
6.0 \\
90.1\end{array}$ & $\begin{array}{r}7.1 \\
5.2 \\
95.2\end{array}$ & $\begin{array}{r}3.5 \\
2.5 \\
97.8\end{array}$ & $\begin{array}{r}3.1 \\
2.3 \\
100.0\end{array}$ \\
\hline $\begin{array}{l}\mathbf{1 6 5} \\
\text { SORT- } \\
\text { SPLIT }\end{array}$ & $\begin{array}{c}\mathbf{0 . 5} \mathbf{C} \\
\text { N/A MEDIAN- } \\
\text { WT- } \mathbf{1 6 3 . 2}\end{array}$ & $\begin{array}{l}2.4 \\
0.00\end{array}$ & $\begin{array}{r}\mathbf{5 5 . 5} \\
\text { MODE- }\end{array}$ & $\begin{array}{c}\mathbf{4 2 . 0} \\
-\mathbf{1 . 0 0} \text { MEAN }\end{array}$ & $\begin{array}{l}\mathbf{B G} \\
\mathrm{N}-\end{array}$ & CUM & $\begin{array}{l}\text { WT } \\
\text { WT } \\
\text { W T O }\end{array}$ & $\begin{array}{l}31.7 \\
19.4 \\
19.4\end{array}$ & $\begin{array}{l}36.9 \\
22.6 \\
42.0\end{array}$ & $\begin{array}{l}26.1 \\
16.0 \\
58.0\end{array}$ & $\begin{array}{l}27.6 \\
16.9 \\
74.9\end{array}$ & $\begin{array}{l}22.3 \\
13.7 \\
88.6\end{array}$ & $\begin{array}{r}10.0 \\
6.1 \\
94.7\end{array}$ & $\begin{array}{r}4.7 \\
2.9 \\
97.6\end{array}$ & $\begin{array}{r}2.2 \\
1.4 \\
98.9\end{array}$ & $\begin{array}{r}1.8 \\
1.1 \\
100.0\end{array}$ \\
\hline $\begin{array}{l}\mathbf{1 7 0} \\
\text { SORT- } \\
\text { SPLIT }\end{array}$ & $\begin{array}{c}\mathbf{0 . 7} \mathrm{C} \\
\mathbf{1 . 3 0} \text { MEDIAN- } \\
\text { wT }=\mathbf{1 5 5 . 0}\end{array}$ & $\begin{array}{l}0.8 \\
0.00\end{array}$ & $\begin{array}{r}\mathbf{6 5 . 2} \\
\text { MODE- }\end{array}$ & 0.00 34.0 & $\begin{array}{l}s G \\
y=-0.46\end{array}$ & CUM & $\begin{array}{l}\text { WT } \\
\text { WT } \\
\text { WT }\end{array}$ & $\begin{array}{r}14.9 \\
9.8 \\
9.8\end{array}$ & $\begin{array}{l}36.9 \\
24.2 \\
34.0\end{array}$ & $\begin{array}{l}50.5 \\
33.1 \\
67.1\end{array}$ & $\begin{array}{l}29.5 \\
19.4 \\
86.5\end{array}$ & $\begin{array}{r}12.3 \\
8.1 \\
94.6\end{array}$ & $\begin{array}{r}5.5 \\
3.6 \\
98.2\end{array}$ & $\begin{array}{r}1.6 \\
1.1 \\
99.2\end{array}$ & $\begin{array}{r}0.7 \\
0.5 \\
99.7\end{array}$ & $\begin{array}{r}0.5 \\
0.3 \\
100.0\end{array}$ \\
\hline
\end{tabular}


WESTINGHOUSE HANFORD OPERATIONS SIEVE ANALYSIS ROCSAN REPORT

$\star \star \star \star$ REPORT ON WELL 0299-พ15-002 ****

$12 / 11 / 89$

\begin{tabular}{ccccccc} 
DEPTH & CACO3 & DM & $\%$ MUD & ISAND & GRAVEL & CLASS \\
\hdashline 175 & 0.8 & C & 10.6 & 51.0 & 38.4 & maG
\end{tabular} SORT- N/A MEDIAN- 1.00 MODE- $\mathbf{- 2 . 0 0}$ MEAN $=$ N/A

$\begin{array}{lllllll}180 & 0.8 & \mathrm{C} & 0.0 & 22.7 & 77.3 & \text { sG }\end{array}$

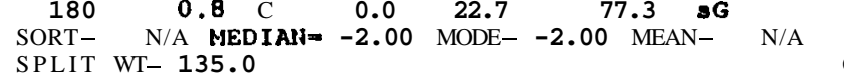

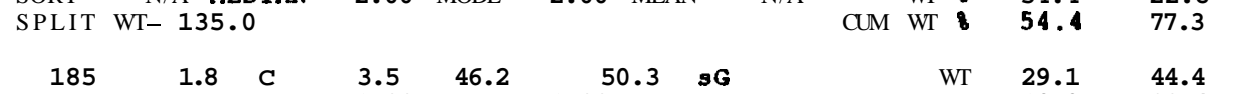

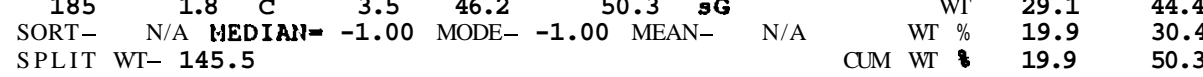

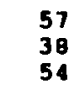

$215 \quad 0.6 \quad \mathrm{C}$ SORT - N/A MEDI
SPLIT WT $=153.6$

WT $38.0 \quad 55.5$

\section{$220 \quad 0.5 \quad C$}

SORT 1.32 MED
SPLIT WT $=153.1$

W'T 24

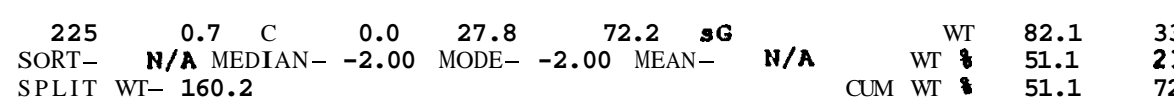




$$
\text { ROCSAN REPORT }
$$

$12 / 11 / 89$

\begin{tabular}{|c|c|c|c|c|c|c|c|c|c|c|c|c|c|c|c|c|c|c|}
\hline DEPTH & $8 \mathrm{CACO} 3$ & DM & FMUD & ISAND & GGRAVEL & CLAS & & & & $\begin{array}{l}\text { FINE } \\
\text { PEB } \\
(<m-2)\end{array}$ & $\begin{array}{c}\text { VF INE } \\
\text { P\&g } \\
(-1)\end{array}$ & $\begin{array}{l}\text { VERY } \\
\text { COARS } \\
(0)\end{array}$ & $\begin{array}{l}\text { COARS } \\
\text { (1) }\end{array}$ & $\begin{array}{l}\text { MED } \\
(2)\end{array}$ & $\begin{array}{l}\text { FINE } \\
(3)\end{array}$ & $\begin{array}{l}\text { VERY } \\
\text { FINE } \\
(d)\end{array}$ & $\begin{array}{l}\text { SILT } \\
(4.75)\end{array}$ & $\begin{array}{c}\text { PAN } \\
(>4.75)\end{array}$ \\
\hline $\begin{array}{r}230 \\
\text { SORT- } \\
\text { SPLIT }\end{array}$ & $\begin{array}{c}0.4 \\
1.67 \mathrm{MED} \\
W 7-132 .\end{array}$ & $\begin{array}{l}\varepsilon \\
0 I A N= \\
0\end{array}$ & $\frac{1.7}{0.00}$ & MODE= & -1.00 MEAN & $s G 0$ & 0.00 & CUM & $\begin{array}{l}\text { WT } \\
\text { WT } \\
\text { WT }\end{array}$ & $\begin{array}{r}10.1 \\
7.6 \\
7.6\end{array}$ & $\begin{array}{l}29.9 \\
22.6 \\
\exists O . Z\end{array}$ & $\begin{array}{l}28.5 \\
21.5 \\
51.7\end{array}$ & $\begin{array}{l}24.8 \\
18.7 \\
70.4\end{array}$ & $\begin{array}{l}24.9 \\
18.8 \\
89.2\end{array}$ & $\begin{array}{r}10.7 \\
7.6 \\
96.0\end{array}$ & $\begin{array}{r}2.0 \\
1.5 \\
98.3\end{array}$ & $\begin{array}{r}1.2 \\
0.9 \\
99.3\end{array}$ & $\begin{array}{r}1.0 \\
0.8 \\
100.0\end{array}$ \\
\hline $\begin{array}{r}Z \exists S \\
\text { sORT= } \\
\text { SPLIT }\end{array}$ & $\begin{array}{c}1.54 \\
W T=150 .\end{array}$ & $\begin{array}{l}\varepsilon \\
7 \\
7 \text { IAN }=\end{array}$ & $\begin{array}{l}0.2 \\
0.00\end{array}$ & $\begin{array}{r}66,9 \\
\text { MODE }=\end{array}$ & $0.00^{32,9}$ MEAN= & $\begin{array}{l}s=-0 \\
=-6\end{array}$ & 0.23 & CUM & $\begin{array}{l}\text { WT } \\
\text { WT } \\
\text { WT }\end{array}$ & $\begin{array}{r}12.6 \\
0.3 \\
8.3\end{array}$ & $\begin{array}{l}\exists 7 . Z \\
24.6 \\
32.9\end{array}$ & $\begin{array}{l}42.9 \\
28.3 \\
61.2\end{array}$ & $\begin{array}{l}Z Z . S \\
14.9 \\
76.1\end{array}$ & $\begin{array}{l}31.1 \\
Z 0.5 \\
96.6\end{array}$ & $\begin{array}{r}4.1 \\
2.7 \\
99.3\end{array}$ & $\begin{array}{r}0.7 \\
0 . S \\
99.8\end{array}$ & $\begin{array}{r}0 . \exists \\
0.2 \\
100.0\end{array}$ & $\begin{array}{r}0.0 \\
0.0 \\
100.0\end{array}$ \\
\hline $\begin{array}{r}240 \\
\text { SORT } \\
\text { SPLIT }\end{array}$ & $\begin{array}{c}0.4 \\
\text { N/A MED } \\
\text { W7- } 119 .\end{array}$ & $\underbrace{\varepsilon}_{7}$ & $\begin{array}{r}0.2 \\
-2.00\end{array}$ & $\begin{array}{l}13,0 \\
\text { MODE- }\end{array}$ & $\begin{array}{c}86.8 \\
-2.00 \text { NEAN }\end{array}$ & & N/A & сим & $\begin{array}{l}\text { ผT } \\
\text { ผT } \\
\text { WT }\end{array}$ & $\begin{array}{l}97.2 \\
80.9 \\
80.9\end{array}$ & $\begin{array}{r}7.1 \\
5.9 \\
86.0\end{array}$ & $\begin{array}{r}1.8 \\
1.5 \\
\theta 8.3\end{array}$ & $\begin{array}{r}1.8 \\
1.5 \\
09.8\end{array}$ & $\begin{array}{r}9.4 \\
7.0 \\
97.6\end{array}$ & $\begin{array}{r}Z .0 \\
1.7 \\
99.3\end{array}$ & $\begin{array}{r}0.6 \\
0.5 \\
99.0\end{array}$ & $\begin{array}{r}0 . \exists \\
0 . \exists \\
100.0\end{array}$ & $\begin{array}{r}0.0 \\
0.0 \\
100.0\end{array}$ \\
\hline $\begin{array}{r}245 \\
\text { SORT } \\
\text { SPLIT }\end{array}$ & $\begin{array}{c}0.7 \\
N / \lambda \text { ME } \\
\mathrm{WT}=179.2\end{array}$ & $\begin{array}{l}\varepsilon \\
2 \\
2 I A H]=\end{array}$ & $\begin{array}{l}6.8 \\
1.00\end{array}$ & $\begin{array}{l}S Z . I \\
\text { MODE= }\end{array}$ & $-2.00^{41.0}$ MEAN & $\mathrm{mgG}$ & $N / \lambda$ & CUM & $\begin{aligned} & \text { WT } \\
\text { WT } & 8 \\
\text { WT } & 8\end{aligned}$ & $\begin{array}{l}\mathbf{5 4 . 5} \\
\exists 0.9 \\
\exists 0 . \exists\end{array}$ & $\begin{array}{l}19.2 \\
10.7 \\
41.0\end{array}$ & $\begin{array}{r}15.3 \\
8.5 \\
49.5\end{array}$ & $\begin{array}{r}14.9 \\
8.3 \\
57.8\end{array}$ & $\begin{array}{l}35.7 \\
19.9 \\
77.7\end{array}$ & $\begin{array}{l}18.7 \\
10.4 \\
68.1\end{array}$ & $\begin{array}{r}9.1 \\
5.1 \\
93.2\end{array}$ & $\begin{array}{r}6.1 \\
3.4 \\
96.5\end{array}$ & $\begin{array}{r}6.2 \\
\exists . S \\
100.0\end{array}$ \\
\hline $\begin{array}{r}250 \\
\text { SORT }- \\
\text { SPLIT }\end{array}$ & $\begin{array}{c}0.6 \\
N / A \mathrm{MED} \\
\mathrm{WT}=159 .\end{array}$ & $\begin{array}{l}\text { C } \\
\text { DIAN- } \\
0\end{array}$ & $\begin{array}{c}0.0 \\
-1.00\end{array}$ & $\begin{array}{r}26.6 \\
\text { MODE- }\end{array}$ & $\begin{array}{c}73.4 \\
-2.00 \text { MEAN }\end{array}$ & 96 & N/A & CUM & $\underset{W T}{W T}$ & $\begin{array}{l}69.7 \\
43.8 \\
43.8\end{array}$ & $\begin{array}{l}47.0 \\
29.6 \\
73.4\end{array}$ & $\begin{array}{l}22.5 \\
14.2 \\
87.6\end{array}$ & $\begin{array}{r}9.1 \\
5.7 \\
93.3\end{array}$ & $\begin{array}{r}8.8 \\
5.5 \\
98.8\end{array}$ & $\begin{array}{r}1.6 \\
1.0 \\
99.0\end{array}$ & $\begin{array}{r}0 . \exists \\
0.2 \\
100.0\end{array}$ & $\begin{array}{r}0.0 \\
0.0 \\
100.0\end{array}$ & $\begin{array}{r}0.0 \\
0.0 \\
100.0\end{array}$ \\
\hline $\begin{array}{r}255 \\
\text { SORT- } \\
\text { SPLIT }\end{array}$ & $\begin{array}{c}0.7 \\
2.18 \mathrm{MED} \\
\text { WT- } 164 .\end{array}$ & $\sum_{7}^{\varepsilon}$ & $\begin{array}{l}9.8 \\
2.00\end{array}$ & $\begin{array}{r}72.9 \\
\text { MODE- }\end{array}$ & $2,00^{17.3}$ MEAN & $(m) g$ & $\begin{array}{l}g s \\
1.20\end{array}$ & сUM & $\underset{W T}{W T}$ & $\begin{array}{l}6.4 \\
3.9 \\
3.9\end{array}$ & $\begin{array}{l}22.0 \\
13.4 \\
17.3\end{array}$ & $\begin{array}{l}18.0 \\
11.0 \\
28.3\end{array}$ & $\begin{array}{r}13.6 \\
8.3 \\
36.6\end{array}$ & $\begin{array}{l}51.1 \\
31.2 \\
67.8\end{array}$ & $\begin{array}{l}22.9 \\
14.0 \\
81.8\end{array}$ & $\begin{array}{r}13.8 \\
8.4 \\
90.2\end{array}$ & $\begin{array}{r}7.9 \\
4.8 \\
95.1\end{array}$ & $\begin{array}{r}9.1 \\
5.0 \\
100.0\end{array}$ \\
\hline $\begin{array}{r}Z 60 \\
\text { SORT= } \\
\text { SPLIT }\end{array}$ & $\begin{array}{c}0.7 \\
N / A \\
W T\end{array}$ & $\begin{array}{l}\varepsilon \\
\text { DIAN= } \\
{ }_{0}\end{array}$ & $\begin{array}{r}1.2 \\
-2.00\end{array}$ & MODE- & -2.00 MEAN & g- & N/A & CUM & $\begin{array}{l}\text { WT } \\
\text { WT } \\
\text { WT }\end{array}$ & $\begin{array}{l}97.0 \\
59.7 \\
59.7\end{array}$ & $\begin{array}{l}37.2 \\
22.9 \\
82.6\end{array}$ & $\begin{array}{r}8.6 \\
5.9 \\
87.9\end{array}$ & $\begin{array}{r}\exists . Z \\
2.0 \\
89.8\end{array}$ & $\begin{array}{r}9.6 \\
5.9 \\
95.8\end{array}$ & $\begin{array}{r}3.7 \\
Z . \exists \\
98.0\end{array}$ & $\begin{array}{r}1.2 \\
0.7 \\
98.0\end{array}$ & $\begin{array}{r}1.0 \\
0.6 \\
99.4\end{array}$ & $\begin{array}{r}1.0 \\
0.6 \\
100.0\end{array}$ \\
\hline
\end{tabular}


ROCSAN REPORT

$12 / 11 / 89$

\begin{tabular}{|c|c|c|c|c|c|c|c|c|c|c|c|c|c|c|c|}
\hline ЕPTH $8 \mathrm{CAC03}$ DM & MUD & CSAND & GRAVEL CLAS & & & & $\begin{array}{l}\text { FINE } \\
\text { PEB } \\
(<--2)\end{array}$ & $\begin{array}{l}\text { VFINE } \\
\text { PEB } \\
(-1)\end{array}$ & $\begin{array}{l}\text { VERY } \\
\text { COARS } \\
(0)\end{array}$ & $\begin{array}{c}\text { COARS } \\
(1)\end{array}$ & $\begin{array}{l}\text { MED } \\
(2)\end{array}$ & $\begin{array}{l}\text { INE } \\
31 \\
-\end{array}$ & $\begin{array}{l}\text { VERY } \\
\text { FINE } \\
\text { (4) } \\
-.-\end{array}$ & $\begin{array}{l}\text { SILT } \\
(4.75)\end{array}$ & $\begin{array}{c}\text { PAN } \\
(>4.75)\end{array}$ \\
\hline 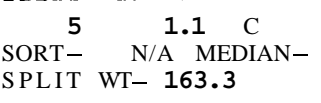 & $\begin{array}{r}14.0 \\
1.00\end{array}$ & $\begin{array}{r}57.8 \\
\text { MODE- }\end{array}$ & -2.00 MEAN $-^{28.2}$ (m) & $\underset{N / A}{g s}$ & cum & $\begin{array}{l}\text { WT } \\
\text { WT } \\
\text { WT }\end{array}$ & $\begin{array}{l}29.5 \\
18.0 \\
18.0\end{array}$ & & 15 & & & & & $\begin{array}{r}2.5 \\
1.5 \\
87.5\end{array}$ & $\begin{array}{r}20.5 \\
12.5 \\
100.0\end{array}$ \\
\hline 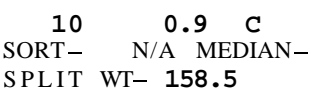 & $\begin{array}{c}10.8 \\
1.00\end{array}$ & $\begin{array}{r}50.8 \\
\text { MODE- }\end{array}$ & $-2.00{ }^{38.5}$ MEAN - & N/A & CUM & $\begin{array}{l}\text { WT T } \\
\text { WT } \\
\text { WT }\end{array}$ & $\begin{array}{l}9.7 \\
25.0 \\
25.0\end{array}$ & & & & & & & $\begin{array}{r}1.0 \\
0.6 \\
89.9\end{array}$ & $\begin{array}{r}16.1 \\
10.2 \\
100.0\end{array}$ \\
\hline $\begin{array}{cc}15 & 0.5 \quad \mathrm{C} \\
\text { RT- } & \text { N/A MEDIAN- } \\
\text { PLIT } & \text { WT- } 163.3\end{array}$ & $\begin{array}{c}3.3 \\
-1.00\end{array}$ & $\begin{array}{r}33.2 \\
\text { MODE- }\end{array}$ & -2.00 MEAN & N/A & & $\begin{array}{l}\text { WT } \\
\text { WT } \\
\text { WT }\end{array}$ & & & & & 9.2 & $\begin{array}{l}5.0 \\
3.1\end{array}$ & & $\begin{array}{l}1.1 \\
0.7 \\
37.4\end{array}$ & $\begin{array}{r}4.3 \\
2.6 \\
100.0\end{array}$ \\
\hline $\begin{array}{lc}20 & 0.6 \mathrm{C} \\
\text { SORT- } & \text { N/A MEDIAN- } \\
\text { SPLIT } & \text { WN- } 163.7\end{array}$ & $\begin{array}{l}5.7 \\
0.00\end{array}$ & $\begin{array}{r}44.8 \\
\text { MODE- }\end{array}$ & -2.00 MEAN- & N/A & CUM & $\begin{array}{l}\text { WT } \\
\text { WT } \\
\text { WT }\end{array}$ & & & & & & & & $\begin{array}{r}3.3 \\
2 . .0 \\
96.3\end{array}$ & $\begin{array}{r}6.0 \\
3.7 \\
100.0\end{array}$ \\
\hline 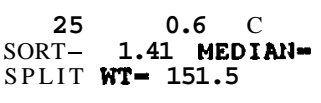 & $\begin{array}{l}5.8 \\
1.00\end{array}$ & $\begin{array}{r}84.3 \\
\text { MODE- }\end{array}$ & $1.00^{9.9}(\mathbf{g}) \mathbf{s}$ & $\begin{array}{l}s \\
0.72\end{array}$ & & & 5.2 & & & & & & 6.1 & $\begin{array}{r}2.7 \\
1.8 \\
95.9\end{array}$ & $\begin{array}{r}6.2 \\
4.1 \\
100.4\end{array}$ \\
\hline 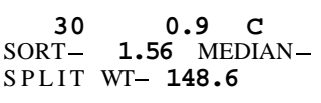 & $\begin{array}{l}5.4 \\
1.00\end{array}$ & $\begin{array}{r}80.7 \\
\text { MODE- }\end{array}$ & 1.00 MEAN -0 & 0.62 & cUM & $\underset{W T}{\mathbf{W T}^{V}}$ & & 14. & & & & & $\begin{aligned} 6.1 \\
94.1 \\
94.7\end{aligned}$ & $\begin{array}{r}2.5 \\
1.7 \\
96.4\end{array}$ & \\
\hline 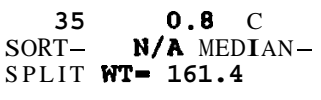 & $\begin{array}{l}5.6 \\
1.00\end{array}$ & $\begin{array}{r}66.0 \\
\text { MODE- }\end{array}$ & $2.00 \mathrm{MegN}$ & N/A & $\mathrm{CuM}$ & & & $\begin{array}{l}15.8 \\
98.9\end{array}$ & $\begin{array}{r}15 \\
9 \\
38\end{array}$ & $\begin{array}{l}33 \\
20 \\
58\end{array}$ & & & $\begin{aligned} 7.3 \\
4.5 \\
94.4\end{aligned}$ & $\begin{array}{r}1.3 \\
0.8 \\
95.2\end{array}$ & $\begin{array}{r}7 \\
100\end{array}$ \\
\hline $\begin{array}{cc}40 & 1.1 \mathrm{C} \\
\text { SORT= } & \text { N/A MEDIAN- } \\
\text { SPLIT } & \text { WI- } 171.0\end{array}$ & $\begin{array}{l}4.6 \\
1.00\end{array}$ & $\begin{array}{r}55.4 \\
\text { MODE- }\end{array}$ & -2.00 MEAN- & N/A & CUM & $\begin{array}{l}\text { WT } \\
\text { WT }\end{array}$ & & & & & & & 5 & $\begin{array}{r}2.8 \\
1.6 \\
97.1\end{array}$ & 100 \\
\hline $\begin{array}{lc}\quad 41 & 0.0 \mathrm{C} \\
\text { SORT- } & \text { N/A MEDIAN- } \\
\text { SPLIT } & \text { WI- } 154.4\end{array}$ & $\begin{array}{c}6.6 \\
-2.00\end{array}$ & $\begin{array}{r}17.6 \\
\text { MODE- }\end{array}$ & $-2.00{ }^{75.8} \mathrm{MEAN}-$ & $\mathrm{N} / \mathrm{A}$ & & 1 & & $\begin{array}{l}18 \\
12 \\
75\end{array}$ & $\begin{array}{r}10 \\
6 \\
82\end{array}$ & $\begin{array}{r}6 \\
4 \\
86\end{array}$ & & & & $\begin{array}{r}1.9 \\
1.2 \\
94.7\end{array}$ & 100 \\
\hline $\begin{array}{lc}45 & 0.9 \quad \mathrm{C} \\
\text { SORT- } & \text { N/A MEDIAN- } \\
\text { SPLIT } & \text { WT- } 168.2\end{array}$ & $\begin{array}{r}6.1 \\
-1.00\end{array}$ & $\begin{array}{r}31.5 \\
\text { MODE- }\end{array}$ & $-2.00^{62.4}$ MEAN $-^{\mathrm{msG}}$ & $N / A$ & CUM & & $\begin{array}{l}80.8 \\
47.9 \\
47.9\end{array}$ & & & & 8 & 5 & 93 & $\begin{array}{r}0.8 \\
0.5 \\
94.4\end{array}$ & $\begin{array}{r}9 \\
5 \\
100\end{array}$ \\
\hline 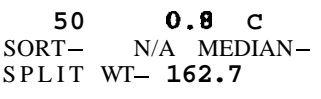 & $\begin{array}{c}6.2 \\
-1.00\end{array}$ & $\begin{array}{r}33.4 \\
\text { MODE- }\end{array}$ & $-2.00^{60.4}$ MEAN- & $3^{N}$ & & & $\begin{array}{l}70 . \\
43 . \\
43 .\end{array}$ & $\begin{array}{l}27 \\
17 \\
60\end{array}$ & $\begin{array}{l}19 \\
12 \\
72\end{array}$ & & $\begin{array}{r}8.7 \\
5.4 \\
86.6\end{array}$ & $\begin{array}{r}6.3 \\
3.9 \\
90.5\end{array}$ & $\begin{array}{r}5.4 \\
3.3 \\
93.8\end{array}$ & $\begin{array}{r}2.5 \\
1.6 \\
95.4\end{array}$ & 100.0 \\
\hline
\end{tabular}




$$
\text { ROCSAN REPORT }
$$

\section{$12 / 11 / 89$}

$* *$ * REPORT ON WELL 0699-045-070 ***

DEPTH \&CACO3 DM \%MUD \&SAND \%RAVEL CLASS

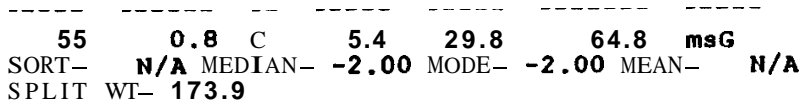

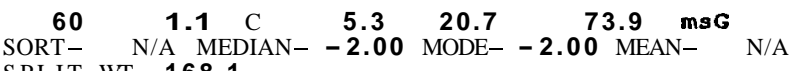
SPLIT WT- 168.1

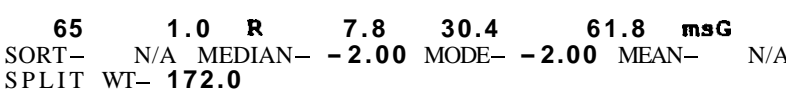

$\begin{array}{lllllll}70 & 0.8 & R & 2.0 & 23.3 & 74.7 & \text { sG }\end{array}$

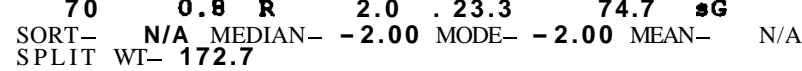

$\begin{array}{lllllcr}75 & 0.9 & R & 7.1 & 44.2 & 48.7 \quad \mathrm{mgG}\end{array}$ SORT - N/A MED

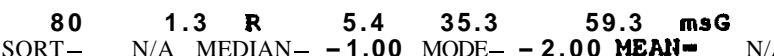
SPLIT WT- $\mathbf{1 6 8 . 5}$

$\begin{array}{lllllll}85 & 1.5 & R & 7.9 & 52.7 & 39.4 & \mathbf{m a G}\end{array}$ $\begin{array}{lllll}\text { SORT- N/A MEDIAN- } & \mathbf{0} .00 & \text { MODE- } & \mathbf{- 2 . 0 0} \mathrm{MEAN}^{-} \text {N/A } \\ \text { SPLIT WT- } \mathbf{1 6 6 . 9} & & & \end{array}$

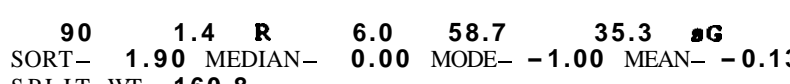
SPLIT WT- 160.8

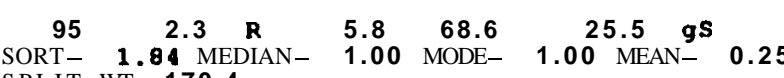

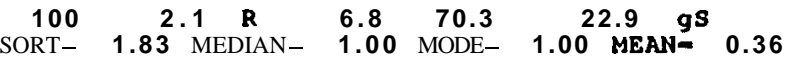
SPLIT WT- 162.1

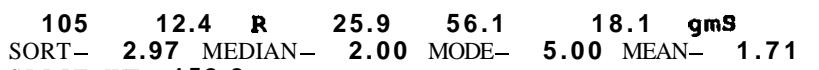
SPLIT WT- 158.8

\begin{tabular}{|c|c|c|c|c|c|c|c|c|}
\hline $\begin{array}{l}\text { FINE } \\
\text { PEB } \\
(<=-2)\end{array}$ & $\begin{array}{c}\text { VF INE } \\
\text { PEB } \\
(-1)\end{array}$ & $\begin{array}{c}\text { VERY } \\
\text { COARS } \\
(0)\end{array}$ & $\begin{array}{c}\text { COARS } \\
\text { (1) }\end{array}$ & $\begin{array}{r}\text { MED } \\
(\mathbf{2}) \\
\end{array}$ & $\begin{array}{l}\text { FINE } \\
\text { (3) }\end{array}$ & $\begin{array}{l}\text { VERY } \\
\text { FINE } \\
\text { (4) }\end{array}$ & $\begin{array}{c}\text { SILT } \\
(4.75) \\
\end{array}$ & $\begin{array}{c}\text { PAN } \\
(>4.75)\end{array}$ \\
\hline $\begin{array}{l}91.4 \\
52.4 \\
52.4\end{array}$ & $\begin{array}{l}21.7 \\
12.4 \\
64.8\end{array}$ & $\begin{array}{l}19.1 \\
10.9 \\
75.7\end{array}$ & $\begin{array}{r}14.8 \\
8.5 \\
84.2\end{array}$ & $\begin{array}{r}9.0 \\
5.2 \\
89.4\end{array}$ & $\begin{array}{r}5.2 \\
3.0 \\
92.3\end{array}$ & $\begin{array}{r}3.9 \\
2.2 \\
94.6\end{array}$ & $\begin{array}{r}4.6 \\
2.6 \\
97.2\end{array}$ & $\begin{array}{l}4.9 \\
2.8 \\
00.0\end{array}$ \\
\hline
\end{tabular}

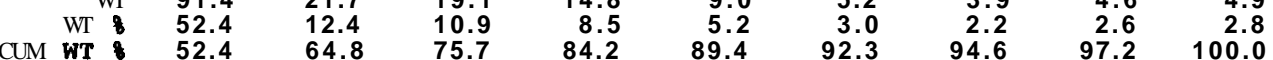

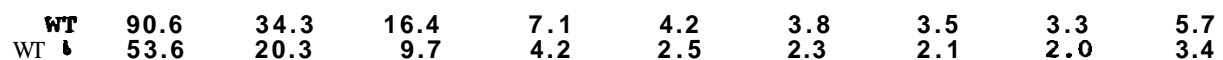

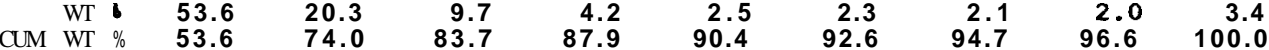

$\begin{array}{rlll}\text { WT } & 86.5 & 20.3 & 17 \\ \text { WT } & 50.0 & 11.7 & 1 \\ \text { WT } \% & 50.0 & 61.8 & 71.7\end{array}$

13.5
7.8
79.5

9.2
5.3
84.9

7.0
4.1
88.9

5.7
3.3
92.2

$4.2 \quad 9.3$

$\begin{array}{llll}\text { WT } & 97.6 & 30.4 & 17.4\end{array}$

9.0

6.2

4.2

$94.6 \quad 100.0$

CUM WT 56

90.1

$\begin{array}{lllll}\text { WT } & 58.5 & 25.2 & 25.2 & 22.7\end{array}$ CUM WT 34.0

14.7

13.2

93.7

96.2

3.1
1.0
9.0

$\begin{array}{rr}1.4 & 2.1 \\ 0.8 & 1.2\end{array}$$$
\begin{array}{r}
8.0 \\
84.5
\end{array}
$$

4.9

$\begin{array}{rrr}5.9 & 5.1 & 7.1 \\ 3.1 & 3.0 & 4.1 \\ 92.9 & 95.9 & 100.0\end{array}$

$\begin{array}{rrrr}\text { WT } & 73.8 & 26.1 & 20.5 \\ \text { WT } & 43.8 & 15.5 & 12 .\end{array}$

14.3$$
\begin{aligned}
& 11.8 \\
& 7.0 \\
& 87.0
\end{aligned}
$$

$\begin{array}{llll}7.8 & 5.1 & 3.7 & 5.4\end{array}$ CUM WT 43.8

$\begin{array}{rl}\text { WT } & 38.8 \\ \text { WT } & 23 . \\ \mathrm{WT} & 23.1\end{array}$

80.

4.6
91.6

$\begin{array}{rrr}5.1 & 3.7 & 5.4 \\ 3.0 & 2.2 & 3.2 \\ 94.6 & 96.8 & 100.0\end{array}$

$\begin{array}{ll}24.9 & 25.0 \\ 14.0 & 14.9\end{array}$

17.6
10.5

12.3
76.9

$$
6.0 \quad 7.2
$$

WT 24.6

$\begin{array}{rrr}\text { WT } & 15.3 & 20.0 \\ \text { CUM WT } & 15.3 & 35.3\end{array}$

31.9

19.8

31.7
19.7

17.6

10.9

85.7

8.1

8.1
5.0
90.7

5.2
92.1

3.6
95.7

7.2
4.3
100.0

$\begin{array}{rrr}\text { WT } 14.1 & 29.6 \\ \text { WT } & 8.2\end{array}$

34.2

42.2
24.7

$\begin{array}{lll}\text { WT } & 8.2 & 17.3 \\ \text { CUM W'r } & 8.2 & 25.5\end{array}$

$\begin{array}{lll}\text { WT } & 8.7 & 28.7 \\ \text { WT } & 5.3 & 17.6\end{array}$

$\begin{array}{rlll}\text { WT } & 5.3 & 17.6 \\ \text { CUM พT } & 5.3 & 22.9\end{array}$ $\begin{array}{ccc} & \text { WT } & 11 . \\ \text { WT } & 7.3 \\ \text { CUM WT } & 7.3\end{array}$

$\begin{array}{ll}7.3 & 17.2 \\ 7.3 & 10.8 \\ & \end{array}$

45.5

\section{6.}

22.3
45.2

24.7
70.2

22.1
12.9
83.1

11.8
6.9
90.0

$\begin{array}{lll}5.2 & 3.5 & 6.2\end{array}$ $\begin{array}{rrr}3.2 & 2.2 & 3.9 \\ 94.0 & 96.1 & 100.0\end{array}$

$\begin{array}{rrr}7.1 & 5.8 & 4.2 \\ 4.2 & 3.4 & 2.5 \\ 94.2 & 97.6 & 100.0\end{array}$

$39.2 \quad 21.2$

11.2

$\begin{array}{lll}6.8 & 3.9 & 7.2\end{array}$

$\begin{array}{lllr}89.0 & 93.2 & 95.6 & 100.0\end{array}$

$\begin{array}{rrrrrrrr}19.2 & 19.3 & 22.2 & 16.8 & 13.7 & 17.3 & 15.5 & 25.7\end{array}$

$\begin{array}{lll}12.8 & 12.1 & 13.9 \\ 8.1 & 30.2 & 44\end{array}$

16.8
10.6

8.6
63.3

10.9
74.2

$\begin{array}{rr}9.7 & 16.1 \\ 83.9 & 100.0\end{array}$ 


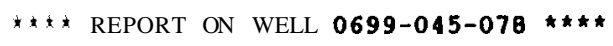

\section{$12 / 11 / 89$}

\begin{tabular}{|c|c|c|c|c|c|c|c|c|c|c|c|c|c|c|c|c|}
\hline EPTH & $8 \mathrm{CACO} 3$ DM & YMUD & SAND & ZGRAVEL & $\begin{array}{l}\text { CLASS } \\
-\cdots--\end{array}$ & & & $\begin{array}{l}\text { FINE } \\
\text { PEB } \\
(<--2)\end{array}$ & $\begin{array}{l}\text { VEINE } \\
\text { PEB } \\
(-1)\end{array}$ & $\begin{array}{l}\text { VERY } \\
\text { COARS } \\
\text { (0) }\end{array}$ & $\begin{array}{c}\text { COARS } \\
(1)\end{array}$ & $\begin{array}{l}\text { MED } \\
(2) \\
-\end{array}$ & $\begin{array}{l}\text { FINE } \\
(3)\end{array}$ & $\begin{array}{l}\text { VERY } \\
\text { FINE } \\
\text { (4) }\end{array}$ & $\begin{array}{c}\text { SILT } \\
(\mathbf{4 . 7 5 )}\end{array}$ & $\begin{array}{c}\text { PAN } \\
(>4.75)\end{array}$ \\
\hline $\begin{array}{l}110 \\
\text { SORT- } \\
\text { SPLIT }\end{array}$ & $\begin{array}{l}2.6 \quad R \\
1.64 \text { MEDIAN= } \\
\text { WT- } 168.2\end{array}$ & $\begin{array}{l}0.9 \\
2.00\end{array}$ & $\begin{array}{r}80.3 \\
\text { MODE- }\end{array}$ & $\begin{array}{c}10.9 \\
1.00 \text { MEAN }\end{array}$ & gs 1.14 & Cum & $\begin{array}{l}\text { WT } \\
\text { WT } \\
\text { WT }\end{array}$ & $\begin{array}{l}9.1 \\
5.4 \\
5.4\end{array}$ & $\begin{array}{r}9.1 \\
5.4 \\
10.9\end{array}$ & $\begin{array}{r}15.3 \\
9.1 \\
20.0\end{array}$ & $\begin{array}{l}49.0 \\
29.2 \\
49.2\end{array}$ & $\begin{array}{l}45.8 \\
27.3 \\
76.6\end{array}$ & $\begin{array}{r}14.9 \\
8.9 \\
05.5\end{array}$ & $\begin{array}{r}9.5 \\
5.7 \\
91.1\end{array}$ & $\begin{array}{r}5.3 \\
3.2 \\
94.3\end{array}$ & $\begin{array}{r}9.6 \\
5.7 \\
100.0\end{array}$ \\
\hline $\begin{array}{r}115 \\
\text { SORT- } \\
\text { SPLIT }\end{array}$ & $\begin{array}{l}2.0 \mathbf{R} \\
1.00 \text { MEDIAN- } \\
\text { WT- } 139.8\end{array}$ & $\begin{array}{l}8.4 \\
2.00\end{array}$ & $\begin{array}{r}79.3 \\
\text { MODE- }\end{array}$ & $2.00^{12.3}$ MEAN & $\mathrm{gS}$ & CuM & $\begin{array}{l}\text { WT } \\
\text { WT } \\
\text { WT }\end{array}$ & $\begin{array}{l}4.5 \\
3.2 \\
3.2\end{array}$ & $\begin{array}{r}12.7 \\
9.1 \\
12.3\end{array}$ & $\begin{array}{r}12.9 \\
9.2 \\
21.5\end{array}$ & $\begin{array}{l}31.9 \\
22.7 \\
44.2\end{array}$ & $\begin{array}{l}32.7 \\
23.3 \\
67.5\end{array}$ & $\begin{array}{l}21.0 \\
15.0 \\
82.5\end{array}$ & $\begin{array}{r}12.8 \\
9.1 \\
91.6\end{array}$ & $\begin{array}{r}4.8 \\
3.4 \\
95.0\end{array}$ & $\begin{array}{r}7.0 \\
5.0 \\
100.0\end{array}$ \\
\hline $\begin{array}{r}120 \\
\text { SORT- } \\
\text { SPLIT }\end{array}$ & $\begin{array}{l}2.6 \quad \mathbf{R} \\
1.95 \text { MEDIAN- } \\
\text { WT- } 134.5\end{array}$ & $\begin{array}{l}8.1 \\
2.00\end{array}$ & $\begin{array}{r}76.9 \\
\text { MODE- }\end{array}$ & $1.00^{15.0}$ MEAN & $\underset{v-}{g s} 1.07$ & curs & $\begin{array}{l}\text { WT } \\
\text { WT } \\
\text { WT }\end{array}$ & $\begin{array}{l}6.0 \\
4.5 \\
4.5\end{array}$ & $\begin{array}{l}14.1 \\
10.5 \\
15.0\end{array}$ & $\begin{array}{r}12.0 \\
9.6 \\
24.6\end{array}$ & $\begin{array}{l}31.0 \\
23.1 \\
47.7\end{array}$ & $\begin{array}{l}30.5 \\
22.8 \\
70.4\end{array}$ & & $\begin{array}{r}10.6 \\
7.9 \\
91.9\end{array}$ & $\begin{array}{r}4.2 \\
3.1 \\
95.1\end{array}$ & $\begin{array}{r}6.6 \\
4.9 \\
100.0\end{array}$ \\
\hline $\begin{array}{r}125 \\
\text { SORT- } \\
\text { SPLIT }\end{array}$ & $\begin{array}{l}1.6 \quad \mathbf{R} \\
1.49 \stackrel{\text { MEDIAN- }}{1.6}= \\
\text { WT- } 128.0\end{array}$ & $\begin{array}{l}5.0 \\
2.00\end{array}$ & $\begin{array}{r}85.9 \\
\text { MODE- }\end{array}$ & $1.00^{8.3}$ MEAN & $\begin{array}{l}(g) S \\
v-\quad 1.12\end{array}$ & CUM & $\begin{array}{l}\text { WT } \\
\mathrm{WT} \\
\mathrm{WT}\end{array}$ & $\begin{array}{l}1.0 \\
0.8 \\
0.8\end{array}$ & $\begin{array}{l}9.6 \\
7.5 \\
8.3\end{array}$ & $\begin{array}{l}14.0 \\
11.6 \\
19.8\end{array}$ & $\begin{array}{l}37.5 \\
29.3 \\
49.1\end{array}$ & $\begin{array}{l}34.0 \\
26.6 \\
75.7\end{array}$ & $\begin{array}{l}16.2 \\
12.7 \\
88.4\end{array}$ & $\begin{array}{r}7.5 \\
5.9 \\
94.2\end{array}$ & $\begin{array}{r}2.9 \\
2.3 \\
96.5\end{array}$ & $\begin{array}{r}4.5 \\
3.5 \\
100.0\end{array}$ \\
\hline $\begin{array}{r}130 \\
\text { SORT- } \\
\text { SPLIT }\end{array}$ & $\begin{array}{c}1.3 \mathbf{R} \\
1.34 \text { MEDIAN- } \\
\text { WT- } 139.4\end{array}$ & $\begin{array}{l}5.9 \\
2.00 .\end{array}$ & $\begin{array}{r}89.5 \\
\text { MODE- }\end{array}$ & $1.00 \stackrel{4.6}{\text { MEAN }}$ & $\begin{array}{ll}\mathrm{S} & \\
\mathrm{v}- & \mathbf{1 . 2 5}\end{array}$ & CUM & $\begin{array}{l}\text { WT } \\
\text { WT } \\
\text { WT }\end{array}$ & $\begin{array}{l}0.7 \\
0.5 \\
0.5\end{array}$ & $\begin{array}{l}5.8 \\
4.1 \\
4.6\end{array}$ & $\begin{array}{l}16.0 \\
11.4 \\
16.1\end{array}$ & & $\begin{array}{l}39.7 \\
20.3 \\
75.5\end{array}$ & & $\begin{array}{r}8.3 \\
5.9 \\
94.2\end{array}$ & $\begin{array}{r}3.2 \\
2.3 \\
96.4\end{array}$ & $\begin{array}{r}5.0 \\
3.6 \\
100.0\end{array}$ \\
\hline $\begin{array}{r}135 \\
\text { SORT- } \\
\text { SPLIT }\end{array}$ & $\begin{array}{l}0.7 \quad R \\
1.22 \quad \text { MEDIAN- } \\
\mathbf{w T}=149.0\end{array}$ & $\begin{array}{l}3.0 \\
1.00\end{array}$ & $\begin{array}{r}87.0 \\
\text { MODE- }\end{array}$ & $1.00^{10.1}$ MEAN & $\mathrm{gs}$ & CUM & $\begin{array}{l}\text { WT } \\
\text { WT } \\
\mathrm{WT}\end{array}$ & $\begin{array}{l}3.0 \\
2.0 \\
2.0\end{array}$ & $\begin{array}{r}12.1 \\
8.1 \\
10 .\end{array}$ & $\begin{array}{l}21 \\
14 \\
24\end{array}$ & $\begin{array}{l}62 \\
41 \\
65\end{array}$ & $\begin{array}{l}32 . \\
21 . \\
07 .\end{array}$ & $\begin{array}{l}10.7 \\
7.1 \\
94.1\end{array}$ & $\begin{array}{r}1.3 \\
2.9 \\
97.0\end{array}$ & $\begin{array}{r}1.7 \\
1.1 \\
9 \theta .1\end{array}$ & $\begin{array}{r}2.0 \\
1.9 \\
100.0\end{array}$ \\
\hline $\begin{array}{r}140 \\
\text { SORT- } \\
\text { SPLIT }\end{array}$ & $\begin{array}{c}0.9 \mathbf{R} \\
1.83 \text { MEDIAN- } \\
\text { WT- } 151.2\end{array}$ & $\begin{array}{l}0.7 \\
1.00\end{array}$ & $\begin{array}{r}79.2 \\
\text { MODE- }\end{array}$ & $1.00^{12.1}$ MEAN & $\mathrm{vs}_{-}^{\mathrm{gs}} 0.00$ & CUM & $\begin{array}{l}\text { WT: } \\
\text { WW TZ }\end{array}$ & $\begin{array}{l}3.2 \\
2.1 \\
2.1\end{array}$ & $\begin{array}{l}15.2 \\
10.0 \\
12.1\end{array}$ & $\begin{array}{l}34.0 \\
22.4 \\
34.6\end{array}$ & $\begin{array}{l}35.0 \\
23.6 \\
58.2\end{array}$ & $\begin{array}{l}26.7 \\
17.6 \\
75.0\end{array}$ & $\begin{array}{r}15.0 \\
9.9 \\
85.7\end{array}$ & $\begin{array}{r}8.5 \\
5.6 \\
91.3\end{array}$ & $\begin{array}{r}4.6 \\
3.0 \\
94.3\end{array}$ & $\begin{array}{r}9.6 \\
5.7 \\
100.0\end{array}$ \\
\hline $\begin{array}{r}145 \\
\text { SORT- } \\
\text { SPLIT }\end{array}$ & $\begin{array}{c}0.6 \mathbf{R} \\
2.16 \text { MEDIAN- } \\
\text { WT- } 158.2\end{array}$ & $\begin{array}{l}8.3 \\
1.00\end{array}$ & $\begin{array}{r}63.1 \\
\text { MODE- }\end{array}$ & $\begin{array}{c}20.6 \\
0.00^{2} \text { MEAN }\end{array}$ & $\begin{array}{l}\text { (m) } g s \\
g-\quad 0.27\end{array}$ & Cum & $\begin{array}{l}\text { WT } \\
\mathrm{WT} \\
\mathrm{WT}\end{array}$ & $\begin{array}{l}19.9 \\
12.5 \\
12.5\end{array}$ & $\begin{array}{l}25.5 \\
16.1 \\
20.6\end{array}$ & $\begin{array}{l}32.6 \\
20.5 \\
49.2\end{array}$ & & $\begin{array}{l}18.4 \\
11.6 \\
80.1\end{array}$ & $\begin{aligned} 11.3 \\
7.1 \\
07.2\end{aligned}$ & $\begin{array}{r}7.2 \\
4.5 \\
91.7\end{array}$ & $\begin{array}{r}4.0 \\
2.5 \\
94.3\end{array}$ & $\begin{array}{r}9.1 \\
5.7 \\
100.0\end{array}$ \\
\hline $\begin{array}{r}150 \\
\text { SORT- } \\
\text { SPLIT }\end{array}$ & $\begin{array}{l}0.5 \mathbf{R} \\
2.24 \text { MEDIAN- } \\
\text { WT- } 167.8\end{array}$ & $\begin{array}{l}0.9 \\
1.00\end{array}$ & $\begin{array}{r}61.1 \\
\text { MODE- }\end{array}$ & $1.00^{30.1}$ MEAN & $\mathrm{Nm}_{\mathrm{mg}}^{\mathrm{mg}} \mathbf{0 . 3 3}$ & CUM & $\begin{array}{l}\text { WT } \\
\text { Wr } \\
\text { WT }\end{array}$ & $\begin{array}{l}21.0 \\
12.5 \\
12.5\end{array}$ & $\begin{array}{l}29.6 \\
17.6 \\
30.1\end{array}$ & $\begin{array}{l}30.0 \\
17.8 \\
47.9\end{array}$ & $\begin{array}{l}31 \\
10 \\
66\end{array}$ & $\begin{array}{l}20.7 \\
12.3 \\
79.0\end{array}$ & $\begin{array}{r}12.5 \\
7.4 \\
86.4\end{array}$ & $\begin{array}{r}0.0 \\
4.0 \\
91.2\end{array}$ & $\begin{array}{r}4.2 \\
2.5 \\
93.7\end{array}$ & $\begin{array}{r}10.7 \\
6.4 \\
100.0\end{array}$ \\
\hline $\begin{array}{r}155 \\
\text { SORT- } \\
\text { SPLIT }\end{array}$ & $\begin{array}{l}1.4 \underset{R}{R} \\
2.22 \text { NED IAH }= \\
\text { WT- } 157.6\end{array}$ & $\begin{array}{l}0.4 \\
1.00\end{array}$ & $\begin{array}{r}61.0 \\
\text { MODE- }\end{array}$ & $\begin{array}{c}30.6 \\
1.00 \mathrm{MEAN}\end{array}$ & $\begin{array}{l}\mathrm{mgG} \\
\mathrm{va} \quad 0.32\end{array}$ & CUM : & $\begin{aligned} & \text { WT } \\
& \text { WT } \\
& \text { HT }\end{aligned}$ & $\begin{array}{l}20.0 \\
12.7 \\
12.7\end{array}$ & $\begin{array}{l}28.1 \\
17.9 \\
30.6\end{array}$ & $\begin{array}{l}25.9 \\
16.5 \\
47.1\end{array}$ & $\begin{array}{l}29.7 \\
10.9 \\
66.0\end{array}$ & $\begin{array}{l}20.0 \\
13.2 \\
79.2\end{array}$ & $\begin{array}{r}12.0 \\
7.6 \\
86.8\end{array}$ & $\begin{array}{r}7.5 \\
4.8 \\
91.6\end{array}$ & $\begin{array}{r}4.1 \\
2.6 \\
94.2\end{array}$ & $\begin{array}{r}9.1 \\
5.8 \\
100.0\end{array}$ \\
\hline $\begin{aligned} 160 \\
\text { SORT- } \\
\text { SPLIT }\end{aligned}$ & $\begin{array}{c}0.6 \quad \mathbf{R} \\
2.16 \text { MEDIAN- } \\
\text { wT }=142.1\end{array}$ & $\begin{array}{c}11.2 \\
1.00\end{array}$ & $\begin{array}{r}72.4 \\
\text { MODE- }\end{array}$ & $\begin{array}{c}16.3 \\
1.00^{\text {MEAN }}\end{array}$ & $\begin{array}{l}\text { (m) } g s \\
V_{-}\end{array}$ & CUM & $\begin{array}{l}\text { WT } \\
\text { WT } \\
\text { WT }\end{array}$ & $\begin{array}{l}6.9 \\
4.8 \\
4.0\end{array}$ & $\begin{array}{l}16.4 \\
11.5 \\
16.3\end{array}$ & $\begin{array}{l}21.5 \\
15.1 \\
31.4\end{array}$ & $\begin{array}{l}29.4 \\
20.6 \\
52.0\end{array}$ & $\begin{array}{l}18.4 \\
70.5\end{array}$ & $\begin{array}{l}16.3 \\
11.4 \\
81.9\end{array}$ & $\begin{array}{r}9.8 \\
6.9 \\
88.8\end{array}$ & $\begin{array}{r}5.0 \\
3.5 \\
92.3\end{array}$ & $\begin{array}{r}11.0 \\
7.7 \\
100.0\end{array}$ \\
\hline
\end{tabular}



ROCSAII REPORT

$12 / 11 / 89$

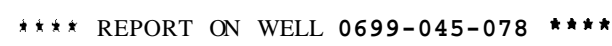

\begin{tabular}{|c|c|c|c|c|c|c|c|c|c|c|c|c|c|c|c|c|}
\hline DEPTH & $8 \mathrm{CAC} 03 \mathrm{DM}$ & $\%$ MUD & BSAIJD & \%GRAVEL CLAS & & & & $\begin{array}{l}\text { FINE } \\
\text { PEB } \\
(<--2)\end{array}$ & $\begin{array}{l}\text { VFINE } \\
\text { PEB } \\
(-1)\end{array}$ & $\begin{array}{l}\text { VERY } \\
\text { COARS } \\
\text { (0) }\end{array}$ & COARS & $\begin{array}{l}\text { MED } \\
(2)\end{array}$ & $\begin{array}{l}\text { FINE } \\
\text { (3) }\end{array}$ & $\begin{array}{l}\text { VERY } \\
\text { FINE } \\
\text { (4) }\end{array}$ & $\begin{array}{r}\text { SILT } \\
(4.75) \\
\end{array}$ & $\begin{array}{l}\text { PAN } \\
(>4.75)\end{array}$ \\
\hline $\begin{array}{r}170 \\
\text { SORT- } \\
\text { SPLIT }\end{array}$ & $\begin{array}{c}0.8 \mathrm{R} \\
2.19 \text { MEDIAN= } \\
\text { WT- } 158.0\end{array}$ & $\begin{array}{l}9.5 \\
2.00\end{array}$ & $\begin{array}{l}74.8 \\
\text { MODE- }\end{array}$ & $1.00^{15.7}$ MEAH $={ }^{(m)} \mathrm{g}$ & $\begin{array}{l}\text { gS } \\
1.14\end{array}$ & CUM & $\begin{aligned} \text { WT } \\
\mathrm{WT} \\
\mathrm{WT} \%\end{aligned}$ & $\begin{array}{c}10.8 \\
6.8 \\
6.8\end{array}$ & $\begin{array}{r}14.1 \\
8.9 \\
15.7\end{array}$ & $\begin{array}{l}18.5 \\
11.7 \\
27.4\end{array}$ & $\begin{array}{l}35.6 \\
22.5 \\
49.8\end{array}$ & $\begin{array}{l}27.3 \\
17.2 \\
67.1\end{array}$ & $\begin{array}{l}20.1 \\
12.7 \\
79.7\end{array}$ & $\begin{array}{l}17.0 \\
10.7 \\
90.5\end{array}$ & $\begin{array}{r}6.1 \\
3.9 \\
94.3\end{array}$ & $\begin{array}{r}9.0 \\
5.7 \\
100.0\end{array}$ \\
\hline $\begin{array}{l}175 \\
\text { SORT- } \\
\text { SPLIT }\end{array}$ & $\begin{array}{l}0.8 \text { R } \\
1.64 \text { MEDIAN- } \\
\text { WT- } 138.5\end{array}$ & $\begin{array}{l}8.8 \\
1.00\end{array}$ & $\begin{array}{r}86.3 \\
\text { NODE- }\end{array}$ & $1.00^{4.9} \stackrel{S}{\text { MEAH- }} 1$ & 1.25 & CUM & $\begin{array}{l}\text { WT } \\
\text { WT } \\
\text { WT \% }\end{array}$ & $\begin{array}{l}0.1 \\
0.1 \\
0.1\end{array}$ & $\begin{array}{l}6.6 \\
4.8 \\
4.9\end{array}$ & $\begin{array}{l}20.9 \\
15.2 \\
20.0\end{array}$ & $\begin{array}{l}41.4 \\
30.0 \\
50.0\end{array}$ & $\begin{array}{l}29.7 \\
21.5 \\
71.6\end{array}$ & $\begin{array}{l}17.0 \\
12.3 \\
83.9\end{array}$ & $\begin{array}{r}10.0 \\
7.3 \\
91.2\end{array}$ & $\begin{array}{r}4.1 \\
3.0 \\
94.1\end{array}$ & $\begin{array}{r}8.1 \\
5.9 \\
100.0\end{array}$ \\
\hline $\begin{array}{r}180 \\
\text { SORT- } \\
\text { SPLIT }\end{array}$ & $\begin{array}{c}0.7 \text { R } \\
\begin{array}{c}\mathbf{R} \\
\text { WT }\end{array}=154.3 \\
\text { MEDIAI= }\end{array}$ & $\begin{aligned} & 3.0 \\
&-1.00\end{aligned}$ & $\begin{array}{r}44.6 \\
\text { MODE- }\end{array}$ & $-1.00^{52.4} \mathbf{M E A H}=-0$ & -0.59 & CUH & $\begin{array}{l}\text { WT } \\
\text { WT } \% \\
\text { WT }\end{array}$ & $\begin{array}{l}17.4 \\
11.3 \\
11.3\end{array}$ & $\begin{array}{l}63.6 \\
41.1 \\
52.4\end{array}$ & $\begin{array}{l}29.5 \\
19.1 \\
71.5\end{array}$ & $\begin{array}{l}17.5 \\
11.3 \\
82.8\end{array}$ & $\begin{array}{r}11.6 \\
7.5 \\
90.3\end{array}$ & $\begin{array}{r}6.8 \\
4.4 \\
94.7\end{array}$ & $\begin{array}{r}3.5 \\
2.3 \\
97.0\end{array}$ & $\begin{array}{r}1.6 \\
1.0 \\
98.0\end{array}$ & $\begin{array}{r}3.1 \\
2.0 \\
100.0\end{array}$ \\
\hline $\begin{array}{r}190 \\
\text { SORT- } \\
\text { SPLIT }\end{array}$ & $\begin{array}{l}0.3 \mathbf{R} \\
2.02 \text { MEDIAN- } \\
\text { WT- } 155.3\end{array}$ & $\begin{array}{l}7.7 \\
0.00\end{array}$ & $\begin{array}{r}58.3 \\
\text { MODE- }\end{array}$ & $\begin{array}{c}34.0 \quad \mathbf{m s G} \\
-1.00 \text { MEAN- } 0\end{array}$ & 0.17 & CUM & $\begin{aligned} & \text { WT } \\
\text { WT } & 8 \\
\text { W' } & 8\end{aligned}$ & $\begin{array}{l}5.2 \\
3.3 \\
3.3\end{array}$ & $\begin{array}{l}47.6 \\
30.6 \\
34.0\end{array}$ & $\begin{array}{l}39.3 \\
25.3 \\
59.2\end{array}$ & $\begin{array}{l}15.8 \\
10.2 \\
69.4\end{array}$ & $\begin{array}{l}17.9 \\
11.5 \\
80.9\end{array}$ & $\begin{array}{r}10.5 \\
6.8 \\
87.6\end{array}$ & $\begin{array}{r}7.2 \\
4.6 \\
92.3\end{array}$ & $\begin{array}{r}3.9 \\
2.5 \\
94.8\end{array}$ & $\begin{array}{r}8.1 \\
5.2 \\
100.0\end{array}$ \\
\hline $\begin{aligned} & 195 \\
& \text { SORT- } \\
& \text { SPLIT }\end{aligned}$ & $\begin{array}{l}0.3 \underset{\mathbf{R}}{\mathbf{R}} \\
1.91 \text { MEDIAN- } \\
\text { WT- } 162.0\end{array}$ & $\begin{array}{l}6.8 \\
-0.00\end{array}$ & $\begin{array}{r}57.7 \\
\text { MODE- }\end{array}$ & $-1.000_{\text {HEAN }}^{35.4}$ mgG & 0.08 & CUM & $\begin{array}{l}\text { WT } \\
\text { WT } \\
\text { WT }\end{array}$ & $\begin{array}{l}4.1 \\
2.5 \\
2.5\end{array}$ & $\begin{array}{l}53.5 \\
32.9 \\
35.4\end{array}$ & $\begin{array}{l}39.0 \\
24.0 \\
59.4\end{array}$ & $\begin{array}{l}18.9 \\
11.6 \\
71.1\end{array}$ & $\begin{array}{l}18.6 \\
11.5 \\
82.5\end{array}$ & $\begin{array}{r}10.3 \\
6.3 \\
88.9\end{array}$ & $\begin{array}{r}7.0 \\
4.3 \\
93.2\end{array}$ & $\begin{array}{r}3.7 \\
2.3 \\
95.5\end{array}$ & $\begin{array}{r}7.4 \\
4.6 \\
100.0\end{array}$ \\
\hline $\begin{aligned} 200 \\
\text { SORT- } \\
\text { SPLIT }\end{aligned}$ & $\begin{array}{c}0.2 \stackrel{R}{R} \\
\text { W/A MEDIAN- } \\
\text { WT- } 161.7\end{array}$ & $\begin{array}{c}1.6 \\
-1.00\end{array}$ & $\begin{array}{l}11.5 \\
\text { MODE- }\end{array}$ & $-1.00^{86.9} \mathrm{~g} G \mathrm{G}$ & $11 / \mathrm{A}$ & CUM & $\begin{array}{l}\text { WT } \\
\text { WT } \\
\text { WT }\end{array}$ & $\begin{array}{l}54.2 \\
33.6 \\
33.6\end{array}$ & $\begin{array}{l}86.1 \\
53.4 \\
86.9\end{array}$ & $\begin{array}{r}8.1 \\
5.0 \\
92.0\end{array}$ & $\begin{array}{r}3.6 \\
2.2 \\
94.2\end{array}$ & $\begin{array}{r}3.1 \\
1.9 \\
96.1\end{array}$ & $\begin{array}{r}2.3 \\
1.4 \\
97.5\end{array}$ & $\begin{array}{c}1.4 \\
0.9 \\
98.4\end{array}$ & $\begin{array}{r}0.8 \\
0.5 \\
98.9\end{array}$ & $\begin{array}{r}1.8 \\
1.1 \\
100.0\end{array}$ \\
\hline $\begin{array}{l}205 \\
\text { SORT- } \\
\text { SPLIT }\end{array}$ & $\begin{array}{c}0.2 \text { R } \\
2.45 \text { MEDIAN- } \\
\text { WT }=147.1\end{array}$ & $\begin{array}{l}7.6 \\
0.00\end{array}$ & $\begin{array}{r}42.9 \\
\text { MODE- }\end{array}$ & $-1.00^{49.5}$ MEAN" $\mathrm{Mg}$ & 0.07 & CU14 & $\begin{array}{l}\text { WT } \\
\text { WT } \\
\text { WT }\end{array}$ & $\begin{array}{l}16.1 \\
11.0 \\
11.0\end{array}$ & $\begin{array}{l}56.6 \\
38.5 \\
49.5\end{array}$ & $\begin{array}{r}13.6 \\
9.3 \\
58.8\end{array}$ & $\begin{array}{r}13.5 \\
9.2 \\
67.9\end{array}$ & $\begin{array}{r}12.0 \\
8.2 \\
76.1\end{array}$ & $\begin{array}{r}11.2 \\
7.6 \\
83.7\end{array}$ & $\begin{array}{r}12.7 \\
8.7 \\
92.4\end{array}$ & $\begin{array}{r}4.7 \\
3.2 \\
95.6\end{array}$ & $\begin{array}{r}6.5 \\
4.4 \\
100.0\end{array}$ \\
\hline $\begin{array}{r}210 \\
\text { SORT- } \\
\text { SPLIT }\end{array}$ & 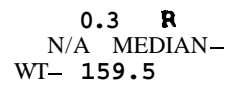 & $\begin{array}{c}2.6 \\
-1.00\end{array}$ & $\begin{array}{c}28.7 \\
\text { MODE- }\end{array}$ & $\begin{array}{c}68.7 \quad \mathbf{~ s G} \\
-1.00^{\mathrm{MEAN}-}\end{array}$ & N/A & CUM & $\begin{array}{l}\text { WT } \\
\text { WT } \\
\text { WT }\end{array}$ & $\begin{array}{l}42.7 \\
26.4 \\
26.4\end{array}$ & $\begin{array}{l}68.4 \\
42.3 \\
68.8\end{array}$ & $\begin{array}{l}19.9 \\
12.3 \\
81.1\end{array}$ & $\begin{array}{r}12.6 \\
7.8 \\
88.9\end{array}$ & $\begin{array}{r}7.7 \\
4.8 \\
93.6\end{array}$ & $\begin{array}{r}3.7 \\
2.3 \\
95.9\end{array}$ & $\begin{array}{r}2.4 \\
1.5 \\
97.4\end{array}$ & $\begin{array}{r}1.3 \\
0.8 \\
98.2\end{array}$ & $\begin{array}{r}2.9 \\
1.8 \\
100.0\end{array}$ \\
\hline $\begin{aligned} & 215 \\
& \text { SORT- } \\
& \text { SPLIT }\end{aligned}$ & $\begin{array}{l}0.2 \quad \mathbf{R} \\
\text { N/A MEDIAN- } \\
\text { WT- } 153.5\end{array}$ & $\begin{array}{l}4.1 \\
-1.00\end{array}$ & $\begin{array}{r}24.0 \\
\text { MODE- }\end{array}$ & $\begin{array}{l}72.0 \text { mSG } \\
-2.00 \text { MEAN- }\end{array}$ & $\mathrm{N} / \mathrm{A}$ & CUM & $\begin{array}{l}\text { WT } \\
\text { WT } \\
\text { WT }\end{array}$ & $\begin{array}{l}65.9 \\
43.2 \\
43.2\end{array}$ & $\begin{array}{l}44.0 \\
28.8 \\
72.0\end{array}$ & $\begin{array}{r}14.1 \\
9.2 \\
81.2\end{array}$ & $\begin{array}{r}8.8 \\
5.8 \\
87.0\end{array}$ & $\begin{array}{r}6.5 \\
4.3 \\
91.2\end{array}$ & $\begin{array}{r}4.4 \\
2.9 \\
94.1\end{array}$ & $\begin{array}{r}2.8 \\
1.8 \\
95.9\end{array}$ & $\begin{array}{r}1.9 \\
1.2 \\
97.2\end{array}$ & $\begin{array}{r}4.3 \\
2.8 \\
100.0\end{array}$ \\
\hline $\begin{array}{r}220 \\
\text { SORT- } \\
\text { SPLIT }\end{array}$ & $\begin{array}{l}\mathbf{0 . 2} \underset{\mathbf{R}}{\mathbf{R}} \\
\text { H/A MEDIAN- } \\
\text { WT- } \quad \mathbf{7 8 . 9}\end{array}$ & $\begin{array}{c}1.4 \\
-2.00\end{array}$ & $\begin{array}{r}12.7 \\
\text { MODE- }\end{array}$ & $\begin{array}{r}85.9 \text { G } \\
-2.00 \text { IEEAN- }\end{array}$ & N/A & CUM & 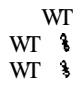 & $\begin{array}{l}51.6 \\
65.1 \\
65.1\end{array}$ & $\begin{array}{l}16.5 \\
20.8 \\
85.9\end{array}$ & $\begin{array}{r}4.7 \\
5.9 \\
91.8\end{array}$ & $\begin{array}{r}1.9 \\
2.4 \\
94.2\end{array}$ & $\begin{array}{r}1.6 \\
2.0 \\
96.2\end{array}$ & $\begin{array}{r}1.2 \\
1.5 \\
97.7\end{array}$ & $\begin{array}{r}0.7 \\
0.9 \\
98.6\end{array}$ & $\begin{array}{r}0.4 \\
0.5 \\
99.1\end{array}$ & $\begin{array}{r}0.7 \\
0.9 \\
100.0\end{array}$ \\
\hline $\begin{array}{r}235 \\
\text { SORT- } \\
\text { SPLIT }\end{array}$ & $\begin{array}{l}0.3 \mathbf{R} \\
\text { N/A MEDIAII- } \\
\text { WT- } 154.6\end{array}$ & $\begin{array}{c}2.3 \\
-1.00\end{array}$ & $\begin{array}{r}16.5 \\
M O D E=\end{array}$ & $\begin{array}{r}81.2 \mathrm{G} \\
-1.00 \mathrm{MEAN}-\end{array}$ & N/A & CUM & $\begin{array}{ll} & \text { WT } \\
\text { WT } & \$ \\
\text { WT } & 8\end{array}$ & $\begin{array}{l}50.7 \\
32.9 \\
32.9\end{array}$ & $\begin{array}{l}74.6 \\
48.4 \\
81.2\end{array}$ & $\begin{array}{r}7.6 \\
4.9 \\
86.1\end{array}$ & $\begin{array}{r}4.8 \\
3.1 \\
09.3\end{array}$ & $\begin{array}{r}4.9 \\
3.2 \\
92.4\end{array}$ & $\begin{array}{r}4.1 \\
2.7 \\
95.1\end{array}$ & $\begin{array}{r}4.0 \\
2.6 \\
97.7\end{array}$ & $\begin{array}{r}1.6 \\
1.0 \\
98.7\end{array}$ & $\begin{array}{r}2.0 \\
1.3 \\
100.0\end{array}$ \\
\hline
\end{tabular}


$12 / 11 / 89$

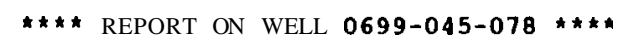

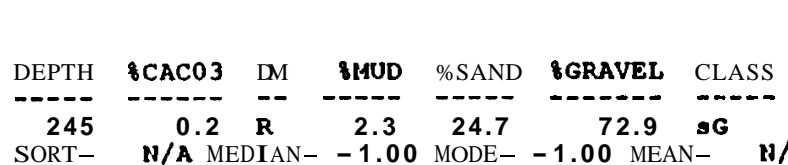
SORT - N/A MEDI
SPLIT

$\begin{array}{lllllll}250 & 0.2 & R & 3.5 & 31.0 & 64.0 & \text { sG }\end{array}$

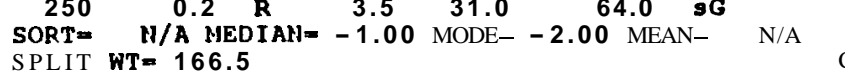

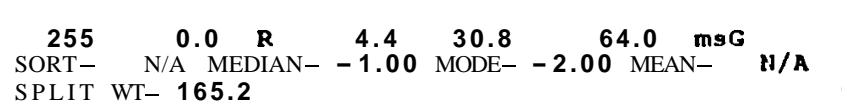

$\begin{array}{lllllll}260 & 0.1 & \mathrm{R} & 2.6 & 25.4 & 72.1 & \mathrm{sG}\end{array}$

$\bigcap_{\infty}$

WT- 158.2

HT
WT $\quad 74.3$

FINE VEIHE VERY

$\begin{array}{rr}\text { WT } & 43.7 \\ \mathrm{WT} & 32.1 \\ \mathrm{WT} & 32.1\end{array}$

PEB COARS

\begin{tabular}{rr}
$(-1)$ & $(0)$ \\
\hline 55.7 & 13.5
\end{tabular}

3.5

COARS MED FINE FINE

$\begin{array}{lll}32.1 & 72.9 & 82.0\end{array}$

8.0

7.0
5.9

7.0

\begin{tabular}{rrrr} 
FINE & FINE & SILT & \multicolumn{1}{c}{ PAN } \\
$(3)$ & $(4)$ & $(4.75)$ & $(>4.75)$ \\
\hline 3.3 & 1.9 & 1.0 & $-r .2$ \\
2.4 & 1.4 & 0.7 & 1.6 \\
96.3 & 97.7 & 98.4 & 100.0
\end{tabular}

$\begin{array}{ll}8.0 & 50.6 \\ 4.6 & 30.2\end{array}$

50.6

$.2 \quad 11.9$

3.0

$\begin{array}{ll}2.4 & 97.4\end{array}$

0.7
98.4

$\begin{array}{rr}0.7 & 1.6 \\ 8.4 & 100.0\end{array}$

MT

4.6

211

$\begin{array}{ll}18.7 & 12 \\ 11.2 & 7.2 \\ 75.9 & 83\end{array}$

11.9
7.1
90.3

94.

6.8
4.1
4.3

$\begin{array}{lll}3.7 & 1.9 & 3.9\end{array}$

$43.1 \quad 17.7$

$10.8 \quad 12.0$

3

96.5

$\begin{array}{rr}1.9 & 3.9 \\ 1.1 & 2.3 \\ 97.7 & 100.0\end{array}$

$\begin{array}{llll}\text { WT } & 63.8 & 43.1 & 17.7 \\ \text { WT } & 38.7 & 26.1 & 10.7\end{array}$

$\begin{array}{rr}6.6 & 7.3 \\ 82.1 & 89.3\end{array}$

93.2

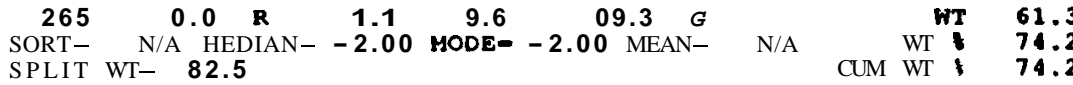

$50.4 \quad 19.6$

19.6

7.766 .5

6.54 .0

$\begin{array}{llll}3.9 & 2.3 & 5.0\end{array}$

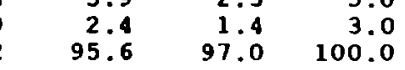

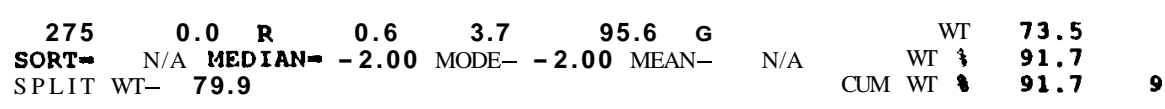

$12.5 \quad 2.9$

4.3

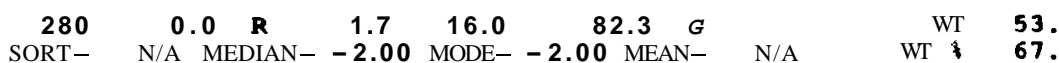

SORT- N/A MEDIAN- $\mathbf{- 2 . 0 0}$ MODE- $\mathbf{- 2 . 0 0}$ MEAN- N/A CUM WT 67.6

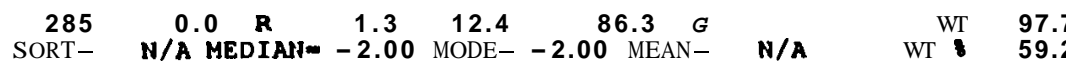

SORT $N / A$ MEDIAN- -2.00 MODE- -2.00 MEAN- N/A
SPLIT WT- 164.5

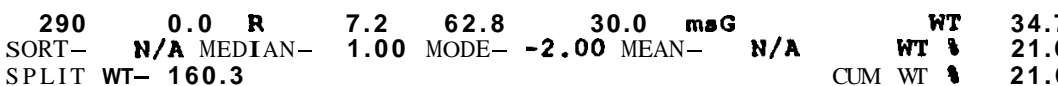

$\begin{array}{lllllllll}295 & 0.4 & \text { R } & \mathbf{1 . 1} & 19.7 & 79.1 & \text { SG } & \text { WT } & 96.4\end{array}$

$\begin{array}{llllll}\text { SORT- N/A MEDIAN- } \mathbf{- 2 . 0 0} \text { MODE- } \mathbf{- 2 . 0 0} \text { MEAN- } & \text { N/A } & \text { WT } & \mathbf{5 8 . 4} \\ \text { SPLIT } & \text { WT- } \mathbf{1 6 4 . 7} & & \text { CUM WT } & \mathbf{5 0}\end{array}$

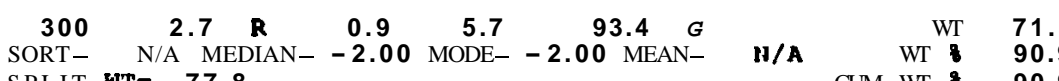

SPLIT WT $=77.8$ 
ROCSAN REPORT

$\star \star \star *$ REPORT ON WELL 0699-045-078 ****

$12 / 11 / 89$

\begin{tabular}{lllllll} 
DEPTH & 8CACO3 & DM & IMUD & \&SAND & $\%$ GRAVEL & CLASS \\
\hdashline-05 & -0.4 & -- & -7.7 & -14.7 & -983.7 & G
\end{tabular}

$\begin{array}{rrrrrrr}305 & 0.4 & \text { R } & 1.7 & 14.7 & 83.7 \text { G } & \\ \text { SORT- } & \text { N/A MEDIAN- } & \mathbf{- 2 . 0 0} & \text { MODE- } & \mathbf{- 2 . 0 0} \text { MEAN- } & \text { N/A }\end{array}$

SORT - N/A MED

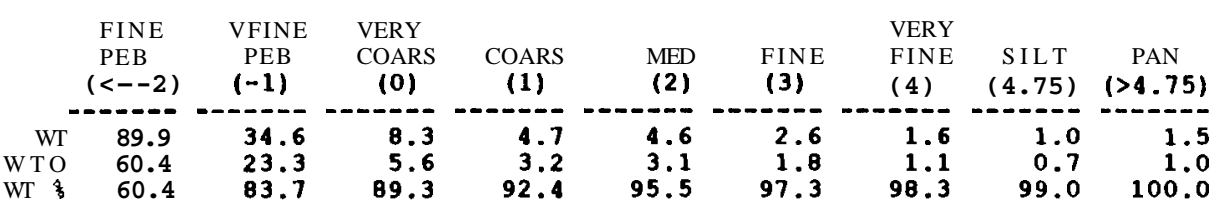

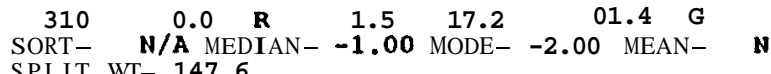

WT 65.1

10.8

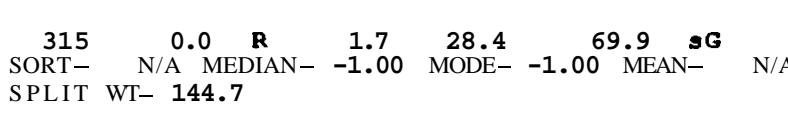

$\begin{array}{rr}\text { WT } & 35.3 \\ \text { WT } & 24.3\end{array}$

$\begin{array}{ll}55.8 & 10 \\ 37.6 & 7 \\ 81.4 & 88.6\end{array}$

6.

96

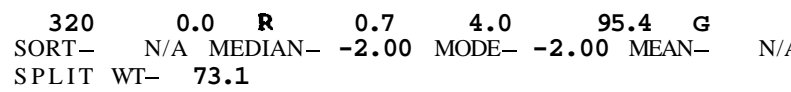

WT 64.1

$\begin{array}{ll}66.4 & 19.2 \\ 45.6 & 13.2\end{array}$

19.2
13.2
3.1

89.

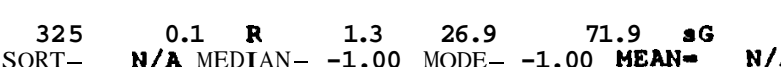

SORT- N/A MED

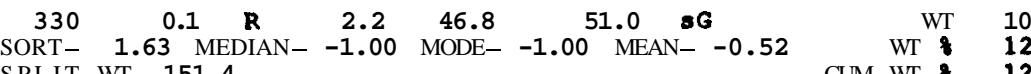

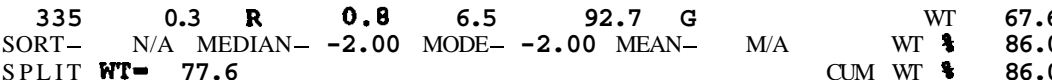

$\begin{array}{llllllll}340 & 1.5 & \text { R } & 2.2 & 18.9 & 78.9 & \mathbf{m g G}\end{array}$

SORT- N/A MEDIAN

SPLIT WT- 142.9

WT 62

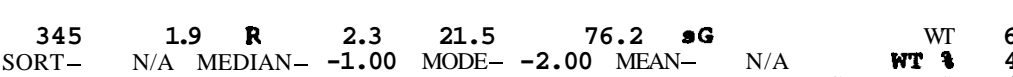

SORLIT WT- 133.6
SPLIN- -1.00 MODE- -2.00 MEAN- N/A CUMWT
WT

$\begin{array}{lllllllll}350 & 0.6 & R & 4.0 & 42.6 & 52.6 & \mathrm{msG} & \mathrm{WT} & 49\end{array}$

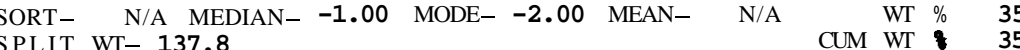

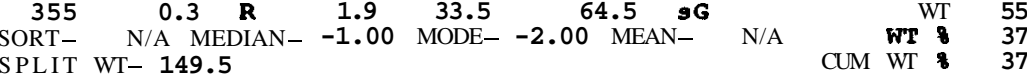

$5.8 \quad 1.0$

$\begin{array}{lll}1.0 & 0.6 \\ 1.4 & 0.8 & 0 \\ 96.7 & 97.5\end{array}$

.3

$\begin{array}{rrrr}2.4 & 1.3 & 0.8 & 1.4 \\ 1.6 & 0.9 & 0.5 & 0.9 \\ 7.7 & 98.5 & 99.1 & 100.0\end{array}$

$\begin{array}{rr}7.9 & 1.4 \\ 95.4 & 96.7\end{array}$

$0.6 \quad 0.7$

2.

$\begin{array}{lll}1.6 & 0.9 & 1.6\end{array}$

$60.7 \quad 15.9$

15.9
10.6
02.4

97.5

0.7
1.0
98.5

0.4

98.3

$\begin{array}{rr}0.9 & 1.6 \\ 98.6 & 100.1\end{array}$

8.

$\begin{array}{ll}59.5 & 26.7 \\ 38.9 & 17.5\end{array}$

08.1

11.0
7.3
95.4

99.0

$\begin{array}{lll}0.2 & 0.2 & 0.3 \\ 0.3 & 0.3 & 0.4\end{array}$

$\begin{array}{rr}0.3 & 0.4 \\ 99.6 & 100.0\end{array}$

$\begin{array}{rrrr}2.4 & 0.9 & 0.5 & 1.2 \\ 97.8 & 90.8 & 99.2 & 100.0\end{array}$

$\begin{array}{llllll}6.7 & 10.9 & 6.9 & 2.3 & 1.1 & 2.2\end{array}$

68.5

91.8

96.3

97.9

$\begin{array}{rr}0.7 & 1.4 \\ 98.6 & 100.0\end{array}$

$\begin{array}{llllll}1.2 & 1.1 & 0.6 & 0.4 & 0.3 & 0.3\end{array}$

$\begin{array}{rr}6.7 & 2.3 \\ 95.0 & 95\end{array}$

1.5
96.6

98.0

0.6
0.8
98.7

0.5
99.2

$\begin{array}{rr}0.3 & 0.3 \\ 0.4 & 0.4 \\ 99.6 & 100.0\end{array}$

$\begin{array}{lr}24.2 & 11.8 \\ 16.9 & 8.2\end{array}$

5.1
3.6
90.7

5.5
3.0
94.5

3.0
2.1
96.6

$\begin{array}{lll}1.8 & 1.0 & 2.1 \\ 1.3 & 0.7 & 1.5\end{array}$

$37.6 \quad 10.2$

3.

$$
\begin{array}{r}
7.5 \\
52.8
\end{array}
$$

4.3

$\begin{array}{rrr}1.3 & 0.7 & 1.5 \\ 97.8 & 90.5 & 100.0\end{array}$

76.2

$23.2 \quad 11.3$

$\begin{array}{ll}7.0 & 23.5 \\ 5.6 & 17.0\end{array}$

96.

2.3
1.7
97.7

$\begin{array}{rr}1.1 & 2.0 \\ 0.8 & 1.5\end{array}$

$\begin{array}{rrrr}11.4 & 4.9 & 2.2 & 4.4 \\ 8.3 & 3.6 & 1.6 & 3.2 \\ 91.7 & 95.2 & 96.8 & 100.0\end{array}$

$\begin{array}{ll}41.0 & 18.0 \\ 27.5 & 12.1\end{array}$

13.1
8.8
85.4

11.1
7.4
92.8

5.5
3.7
96.5

2.3
1.5
98.1

$\begin{array}{rr}1.0 & 1.9 \\ 0.7 & 1.3 \\ 98.7 & 100.0\end{array}$ 

\begin{tabular}{ccccccc} 
& & & & & & \\
DEPTH & ICACO3 & DM & \&MUD & ISAND & IGRAVEL & CLASS \\
\hdashline 360 & 0.1 & R & -5.0 & 37.4 & 57.5 & mgG
\end{tabular} SPLIT WT- 122.5

$\begin{array}{lllllll}365 & 0.1 & R & 6.6 & 52.4 & 40.9 & \mathrm{msG}\end{array}$ $\begin{array}{lllllll}365 & 0.1 & R & 6.6 & 52.4 & 40.9 & \mathrm{msG} \\ \text { SORT- } & \text { N/A MEDIAN- } & 1.00 & \text { MODE- } & 2.00 & \text { MEAN- } & \text { N/A } \\ \text { SPLIT } & \text { WT- } 148.7\end{array}$

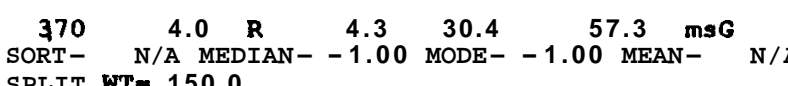
SPLIT WT $=150.0$

$\begin{array}{llllll}375 & 1.2 & R & 5.0 & 46.9 & 47.4\end{array}$ SORT- 1.99 MEDIAN- 0.00 MODE $=-1.00$ MEN $=-0.26$

\begin{tabular}{|c|c|c|c|c|c|c|c|c|c|c|}
\hline & & $\begin{array}{l}\text { FINE } \\
\text { PEB } \\
(<=-2)\end{array}$ & $\begin{array}{l}\text { VFINE } \\
\text { PEB } \\
(-1)\end{array}$ & $\begin{array}{l}\text { VERY } \\
\text { COARS } \\
\text { (0) }\end{array}$ & $\begin{array}{l}\text { COARS } \\
\text { (1) }\end{array}$ & $\begin{array}{l}\text { MED } \\
\text { (2) }\end{array}$ & $\begin{array}{l}\text { FINE } \\
\text { (3) }\end{array}$ & $\begin{array}{l}\text { VERY } \\
\text { FINE } \\
\text { (4) }\end{array}$ & $\begin{array}{c}\text { SILT } \\
(4.75)\end{array}$ & $\begin{array}{c}\text { PAN } \\
(>4.75)\end{array}$ \\
\hline CUM & $\begin{array}{l}\text { WT } \\
\text { WT } \\
\text { WT }\end{array}$ & $\begin{array}{l}47.3 \\
30.4 \\
30.4\end{array}$ & $\begin{array}{l}23.5 \\
19.1 \\
57.5\end{array}$ & $\begin{array}{l}13.6 \\
11.1 \\
60.6\end{array}$ & $\begin{array}{r}5.9 \\
4.8 \\
73.4\end{array}$ & $\begin{array}{l}14.0 \\
11.4 \\
04.7\end{array}$ & $\begin{array}{r}8.1 \\
6.6 \\
91.3\end{array}$ & $\begin{array}{r}4.5 \\
3.7 \\
95.0\end{array}$ & $\begin{array}{r}2.3 \\
1.9 \\
96.0\end{array}$ & $\begin{array}{r}3.9 \\
3.2 \\
100.0\end{array}$ \\
\hline CUM & $\begin{array}{l}\text { WT } \\
\text { WT } \\
\text { WT }\end{array}$ & $\begin{array}{l}32.2 \\
21.6 \\
21.6\end{array}$ & $\begin{array}{l}20.8 \\
19.3 \\
40.9\end{array}$ & $\begin{array}{r}12.8 \\
0.6 \\
49.5\end{array}$ & $\begin{array}{r}10.9 \\
7.3 \\
56.9\end{array}$ & $\begin{array}{l}32.9 \\
22.1 \\
70.9\end{array}$ & $\begin{array}{r}14.3 \\
9.6 \\
08.5\end{array}$ & $\begin{array}{r}7.2 \\
4.0 \\
93.4\end{array}$ & $\begin{array}{r}3.5 \\
2.4 \\
95.7\end{array}$ & $\begin{array}{r}6.4 \\
4.3 \\
100.0\end{array}$ \\
\hline CUM & $\begin{array}{l}\text { WT } \\
\text { WT } \\
\text { WT }\end{array}$ & $\begin{array}{l}35.9 \\
23.9 \\
23.9\end{array}$ & $\begin{array}{l}50.1 \\
33.4 \\
57.3\end{array}$ & $\begin{array}{r}13.6 \\
9.1 \\
66.3\end{array}$ & $\begin{array}{r}14.0 \\
9.9 \\
76.2\end{array}$ & $\begin{array}{l}17.1 \\
11.4 \\
07.5\end{array}$ & $\begin{array}{r}8.0 \\
5.3 \\
92.9\end{array}$ & $\begin{array}{r}4.2 \\
2.8 \\
95.7\end{array}$ & $\begin{array}{r}2.2 \\
1.5 \\
97.1\end{array}$ & $\begin{array}{r}4.3 \\
2.9 \\
100.0\end{array}$ \\
\hline CUM & $\begin{array}{l}\text { WT } \\
\text { WT } \\
\text { WT }\end{array}$ & $\begin{array}{l}24.2 \\
15.8 \\
15.8\end{array}$ & $\begin{array}{l}48.2 \\
31.5 \\
47.4\end{array}$ & $\begin{array}{l}17.7 \\
11.6 \\
58.9\end{array}$ & $\begin{array}{r}14.2 \\
9.3 \\
68.2\end{array}$ & $\begin{array}{l}24.4 \\
16.0 \\
84.2\end{array}$ & $\begin{array}{r}10.1 \\
6.6 \\
90.8\end{array}$ & $\begin{array}{r}5.3 \\
3.5 \\
94.3\end{array}$ & $\begin{array}{r}3.7 \\
2.4 \\
96.7\end{array}$ & $\begin{array}{r}5.1 \\
3.3 \\
100.0\end{array}$ \\
\hline CUM & $\begin{array}{r}\text { WT } \\
\text { WT } \\
\text { WT }\end{array}$ & $\begin{array}{l}58.2 \\
74.6 \\
74.6\end{array}$ & $\begin{array}{l}10.4 \\
13.3 \\
80.0\end{array}$ & $\begin{array}{r}2.1 \\
2.7 \\
90.6\end{array}$ & $\begin{array}{r}1.8 \\
2.3 \\
93.0\end{array}$ & $\begin{array}{r}2.2 \\
2.8 \\
95.8\end{array}$ & $\begin{array}{r}1.1 \\
1.4 \\
97.2\end{array}$ & $\begin{array}{r}0.7 \\
0.9 \\
98.1\end{array}$ & $\begin{array}{r}0.8 \\
1.0 \\
99.1\end{array}$ & $\begin{array}{r}0.7 \\
0.9 \\
100.0\end{array}$ \\
\hline CUM & $\begin{array}{l}\text { WT } \\
\text { WT } \\
\text { WT }\end{array}$ & $\begin{array}{l}74.2 \\
54.0 \\
54.0\end{array}$ & $\begin{array}{l}27.7 \\
20.2 \\
74.2\end{array}$ & $\begin{array}{r}10.2 \\
7.4 \\
01.6\end{array}$ & $\begin{array}{r}7.6 \\
5.5 \\
87.2\end{array}$ & $\begin{array}{r}9.9 \\
7.2 \\
94.4\end{array}$ & $\begin{array}{r}3.6 \\
2.6 \\
97.0\end{array}$ & $\begin{array}{r}1.7 \\
1.2 \\
98.3\end{array}$ & $\begin{array}{r}0.9 \\
0.7 \\
98.9\end{array}$ & $\begin{array}{r}1.5 \\
1.1 \\
100.0\end{array}$ \\
\hline CUM & $\begin{array}{l}\text { WT } \\
\text { WT } \\
\text { WT }\end{array}$ & $\begin{array}{l}52.6 \\
73.0 \\
73.0\end{array}$ & $\begin{array}{r}7.8 \\
10.8 \\
03.0\end{array}$ & $\begin{array}{r}3.7 \\
5.1 \\
00.9\end{array}$ & $\begin{array}{r}2.7 \\
3.7 \\
92.6\end{array}$ & $\begin{array}{r}2.7 \\
3.7 \\
96.4\end{array}$ & $\begin{array}{r}1.2 \\
1.7 \\
90.0\end{array}$ & $\begin{array}{r}0.6 \\
0.8 \\
90.9\end{array}$ & $\begin{array}{r}0.3 \\
0.4 \\
99.3\end{array}$ & $\begin{array}{r}0.5 \\
0.7 \\
100.0\end{array}$ \\
\hline CUM & $\begin{array}{l}\text { WT } \\
\text { WT } \\
\text { WT }\end{array}$ & $\begin{array}{l}50.3 \\
35.0 \\
35.0\end{array}$ & $\begin{array}{r}9.4 \\
6.6 \\
41.6\end{array}$ & $\begin{array}{r}4.5 \\
3.1 \\
44.7\end{array}$ & $\begin{array}{l}21.3 \\
14.0 \\
59.5\end{array}$ & $\begin{array}{l}15.6 \\
10.9 \\
70.4\end{array}$ & $\begin{array}{r}11.5 \\
8.0 \\
70.4\end{array}$ & $\begin{array}{r}10.6 \\
7.4 \\
05.0\end{array}$ & $\begin{array}{r}6.4 \\
4.5 \\
90.3\end{array}$ & $\begin{array}{r}14.0 \\
9.0 \\
100.0\end{array}$ \\
\hline CUts & $\begin{array}{l}\text { WT } \\
\text { WT } \\
\text { WT }\end{array}$ & $\begin{array}{l}96.0 \\
66.7 \\
66.7\end{array}$ & $\begin{array}{l}23.2 \\
16.1 \\
82.8\end{array}$ & $\begin{array}{r}9.9 \\
6.9 \\
89.7\end{array}$ & $\begin{array}{r}5.1 \\
3.5 \\
93.3\end{array}$ & $\begin{array}{r}4.1 \\
2.9 \\
96.1\end{array}$ & $\begin{array}{r}2.2 \\
1.5 \\
97.6\end{array}$ & $\begin{array}{r}1.3 \\
0.9 \\
98.5\end{array}$ & $\begin{array}{r}0.7 \\
0.5 \\
99.0\end{array}$ & $\begin{array}{r}1.4 \\
1.0 \\
100.0\end{array}$ \\
\hline CUIS & $\begin{array}{l}\text { WT } \\
\text { WT }\end{array}$ & $\begin{array}{l}55.4 \\
75.1 \\
75.1\end{array}$ & $\begin{array}{r}4.5 \\
6.1 \\
81.2\end{array}$ & $\begin{array}{r}3.8 \\
5.2 \\
86.3\end{array}$ & $\begin{array}{r}3.3 \\
4.5 \\
90.8\end{array}$ & $\begin{array}{r}2.8 \\
3.8 \\
94.6\end{array}$ & $\begin{array}{r}1.5 \\
2.0 \\
96.6\end{array}$ & $\begin{array}{r}0.9 \\
1.2 \\
97.8\end{array}$ & $\begin{array}{r}0.5 \\
0.7 \\
98.5\end{array}$ & $\begin{array}{r}1.1 \\
1.5 \\
100.0\end{array}$ \\
\hline CUM & $\begin{array}{r}\text { WT } \\
\text { WT } \\
\text { WT }\end{array}$ & $\begin{array}{l}93.0 \\
68.2 \\
68.2\end{array}$ & $\begin{array}{l}18.1 \\
13.3 \\
81.5\end{array}$ & $\begin{array}{r}8.5 \\
6.2 \\
87.8\end{array}$ & $\begin{array}{r}5.6 \\
4.1 \\
91.9\end{array}$ & $\begin{array}{r}4.9 \\
3.6 \\
95.5\end{array}$ & $\begin{array}{r}2.5 \\
1.8 \\
97.3\end{array}$ & $\begin{array}{r}1.4 \\
1.0 \\
98.3\end{array}$ & $\begin{array}{r}0.8 \\
0.6 \\
98.9\end{array}$ & $\begin{array}{r}1.5 \\
1.1 \\
100.0\end{array}$ \\
\hline
\end{tabular}




$$
\text { ROCSAN REPORT }
$$

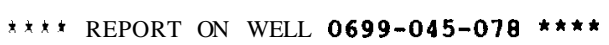

$12 / 11 / 89$

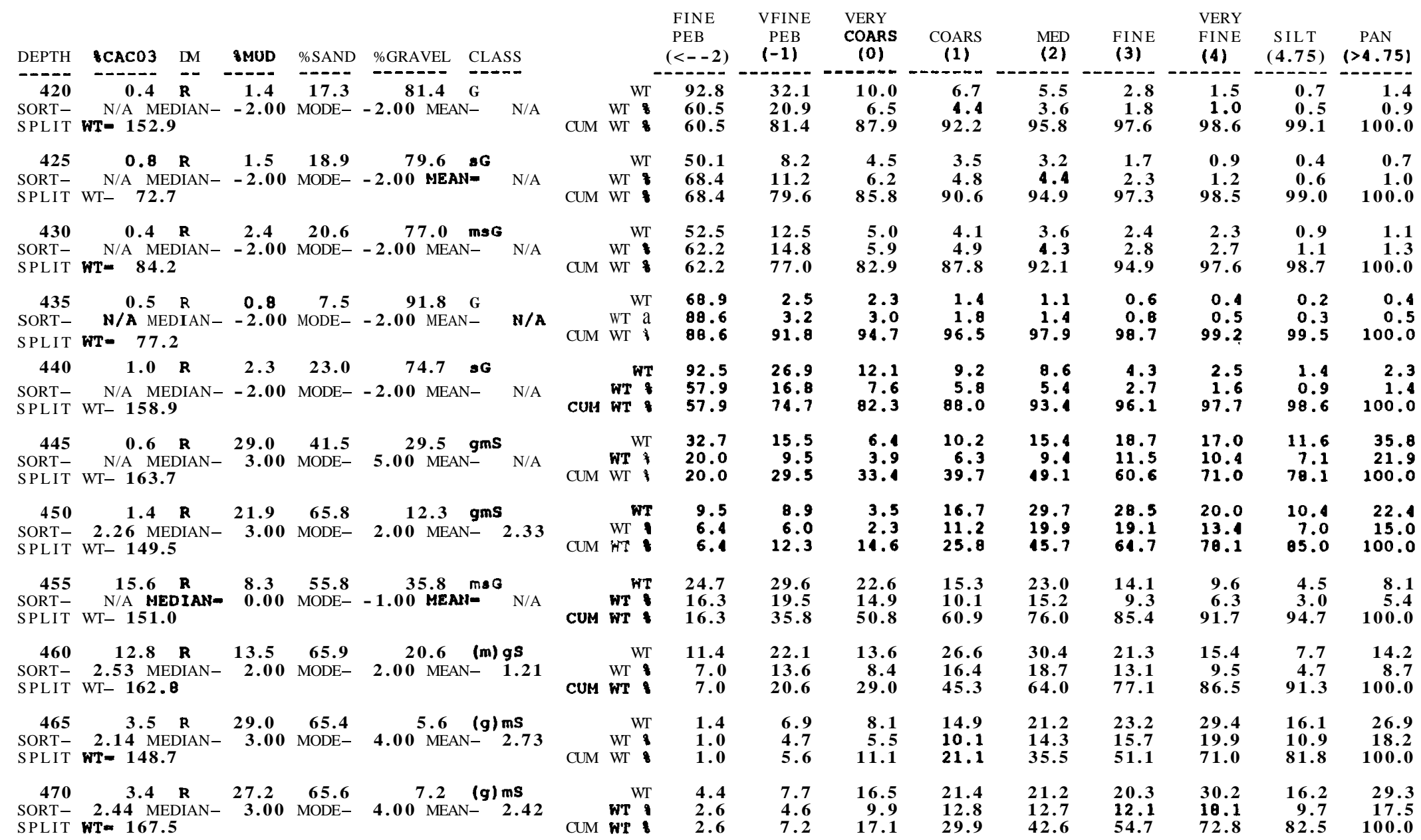


WESTINGHOUSE HANFORD OPERATIONS SIEVE ANALYSIS ROCSAN REPORT

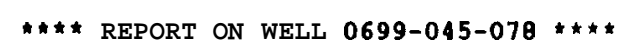

$12 / 11 / 89$

DEPTH 8 CACO3 DM IMUD 8 SAND $\%$ GRAVEL CLASS

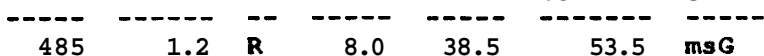
$\begin{array}{cccccc}485 & 1.2 & R & 8.0 & 38.5 & 53.5 \mathrm{msG}^{2} \\ \text { SORT- N/A MEDIAN- } & -1.00 & \text { MODE- } & -1.00 \mathrm{MEAN}- & \mathrm{N} / \mathrm{A}\end{array}$

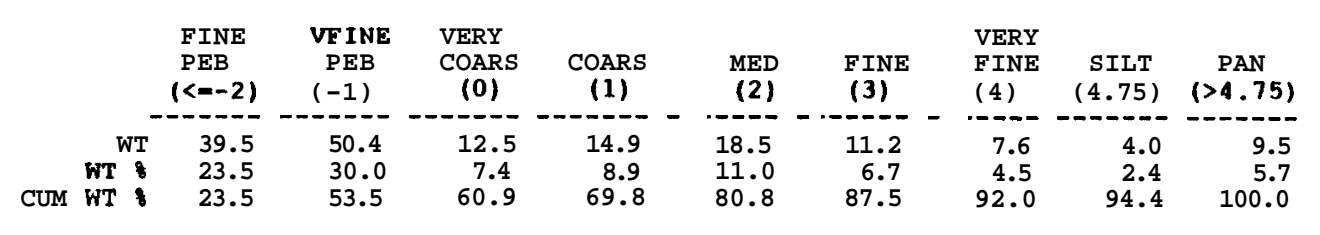

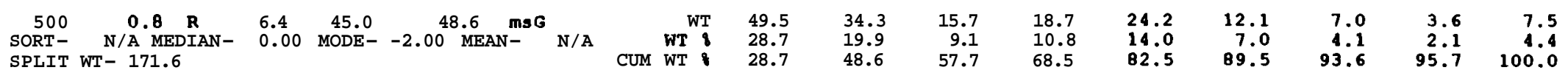

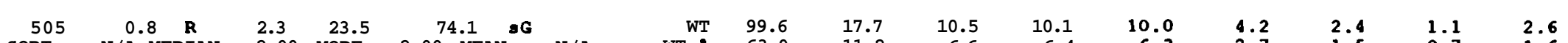

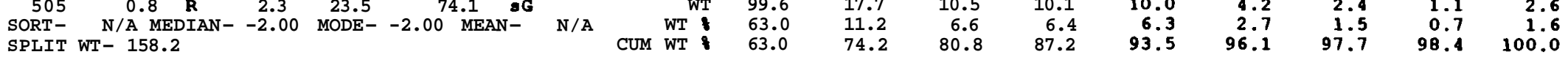

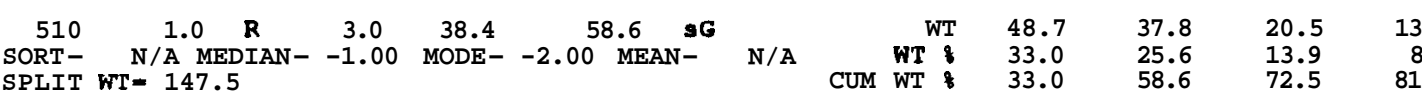

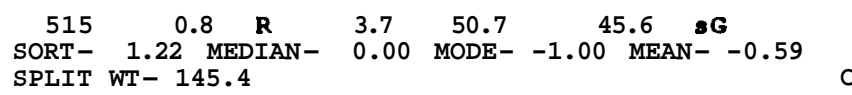
$\begin{array}{rrr}\text { WT } & 2.7 \\ \text { WT } & 1.9 \\ \text { CUM } & 1.9\end{array}$ $\begin{array}{rr}\text { WT } & 15.0 \\ \text { CUM WT } & 10.6 \\ 10.6\end{array}$ $\begin{array}{llllll}520 & 0.9 & R & 3.3 & 28.8 & 68.0 \quad \mathrm{msG}\end{array}$ SORT- 0.95 MEDIA

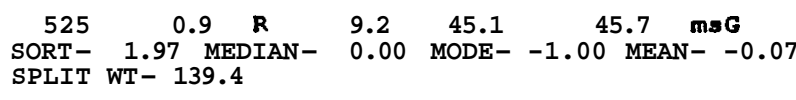

$\begin{array}{c:cc}\text { WT } & \text { WT } \\ \text { WT } & 2.0\end{array}$

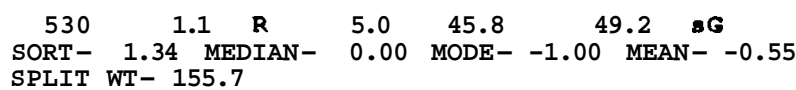

WT:

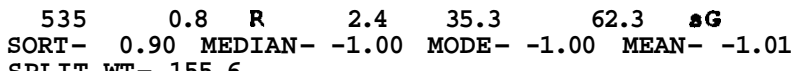

WT 7.9

SPLIT WT- 155.6 $\begin{array}{lll}\text { WT: } & 5.1 \\ \text { CUM WT } & 5.1\end{array}$

$\begin{array}{lllllll}540 & 0.9 & R & 3.3 & 40.0 & 56.7 & \text { SG }\end{array}$ SORT- 1.19 MEDIAN-
SPLIT $W T=144.0$

CUM WT:

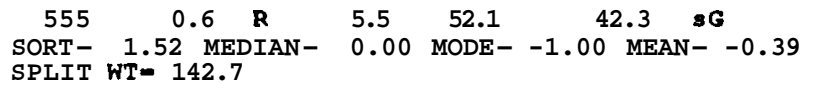
CUM WT WT

$\begin{array}{lll}63.7 & 45.9 & 13.1\end{array}$

43.8
45.6

45.9
31.6
77.2

80.9
57.3
68.0

22.8
16.2
84.1

9.0
86.2

93.5

12.6
8.5 8.5
90.0 6.7
4.5
94.5

7.5
5.2
91.3

94

4.7
93.1

3.
95.

$60.9 \quad 28.1 \quad 14.9$

43.7

28.1

14.9
10.7

76.5

8:

$\begin{array}{lr}12.9 & 11.5 \\ 27.5 & 7.4\end{array}$

33.9
21.8
84.1

84.0

10.7
6.9
90.9

$\begin{array}{rrr}77.7 & 31.7 & 11.9 \\ 54.1 & 22.1 & 8.3\end{array}$

$\begin{array}{rrr}54.1 & 22.1 & 8.3 \\ 56.7 & 78.8 & 87.1\end{array}$

87.1

$\begin{array}{lll}50.6 & 42.5 & 14.6 \\ 35.5 & 29.8 & 10.2 \\ 42.3 & 72.1 & 82.3\end{array}$ $\begin{array}{rrrrr}.8 & 4.5 & 3.4 & 4.1 & 8.8 \\ & 07.3 & 90.8 & 93.7 & 6.3\end{array}$

$\begin{array}{lllll}7.6 & 5.6 & 1.0 & 2.4 & 5.4\end{array}$ $\begin{array}{lllll}80.9 & 92.5 & 95.0 & 96.6 & 100.0\end{array}$

$\begin{array}{lllll}5.2 & 3.0 & 2.1 & 1.3 & 2.5\end{array}$ $\begin{array}{rrrrr}3.3 & 1.9 & 1.4 & 0.8 & 1.6 \\ 94.3 & 96.2 & 97.6 & 98.4 & 100.0\end{array}$

$\begin{array}{lllll}6.7 & 4.3 & 2.8 & 1.5 & 3.3\end{array}$ $\begin{array}{rrrrr}6.7 & 3.0 & 2.0 & 1.0 & 2.3 \\ 91.7 & 94.7 & 96.7 & 97.7 & 100.0\end{array}$

$\begin{array}{rrrrr}7.6 & 5.7 & 4.0 & 2.3 & 5.6 \\ 5.3 & 4.0 & 2.8 & 1.6 & 3.9\end{array}$


WESTINGHOUSE HANFORD OPERATIONS SIEVE ANALYSIS

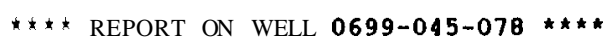

\section{$12 / 11 / 89$}

\begin{tabular}{ccccccc} 
DEPTH & 8CAC03 & DM & \%MUD & ISAND & $\%$ GRAVEL & CLASS \\
\hdashline 560 & 0.6 & $\mathbf{R}$ & -5.2 & 35.5 & 59.4 & msG
\end{tabular}

SORT - N/A MEDIAN $=-1.00$ MODE- -1.00 MEAN $=$ N/A

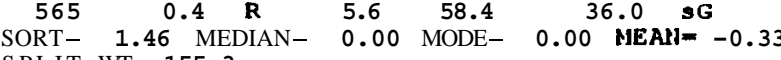

$\begin{array}{ll}\text { SORT- } 1.46 & \text { MED } \\ \text { SPLIT } & \text { WT- } 155.3\end{array}$

\begin{tabular}{|c|c|c|c|c|c|c|c|c|c|}
\hline & $\begin{array}{l}\text { FINE } \\
\text { PEB } \\
(<-2)\end{array}$ & $\begin{array}{l}\text { VFINE } \\
\text { PEB } \\
(-1)\end{array}$ & $\begin{array}{c}\text { VERY } \\
\text { COARS } \\
\text { (0) }\end{array}$ & $\begin{array}{l}\text { COARS } \\
\text { (1) }\end{array}$ & $\begin{array}{l}\text { MED } \\
\text { (2) }\end{array}$ & $\begin{array}{l}\text { FINE } \\
\text { (3) }\end{array}$ & $\begin{array}{l}\text { VERY } \\
\text { FINE } \\
\text { (4) }\end{array}$ & $\begin{array}{c}\text { SILT } \\
(\mathbf{4 . 7 5 )}\end{array}$ & $\begin{array}{c}\text { PAN } \\
(>4.75)\end{array}$ \\
\hline $\begin{array}{l}\text { WT } \\
\text { WT } \\
\text { WT }\end{array}$ & $\begin{array}{l}29.7 \\
20.5 \\
20.5\end{array}$ & $\begin{array}{l}56.3 \\
38.9 \\
59.4\end{array}$ & $\begin{array}{l}24.4 \\
16.8 \\
76.2\end{array}$ & $\begin{array}{r}10.3 \\
7.1 \\
83.3\end{array}$ & $\begin{array}{r}7.1 \\
4.9\end{array}$ & $\begin{array}{r}5.5 \\
3.8\end{array}$ & $\begin{array}{r}4.1 \\
2.8\end{array}$ & $\begin{array}{r}2.5 \\
1.7 \\
96.6\end{array}$ & $\begin{array}{r}5.0 \\
3.5\end{array}$ \\
\hline $\begin{array}{l}\text { WT } \\
\text { WT } \\
\text { WT }\end{array}$ & $\begin{array}{l}8.7 \\
5.6 \\
5.6\end{array}$ & $\begin{array}{l}47.1 \\
30.4 \\
36.0\end{array}$ & $\begin{array}{l}52.8 \\
34.0 \\
70.0\end{array}$ & $\begin{array}{l}19.4 \\
12.5 \\
82.5\end{array}$ & $\begin{array}{r}8.5 \\
5.5 \\
68.0\end{array}$ & $\begin{array}{r}5.8 \\
3.7 \\
91.8\end{array}$ & $\begin{array}{r}4.1 \\
2.6 \\
94.4\end{array}$ & $\begin{array}{r}2.5 \\
1.6 \\
96.0\end{array}$ & $\begin{array}{r}6.2 \\
4.0 \\
100.0\end{array}$ \\
\hline
\end{tabular}


APPENDIX D

MOISTURE CHARACTERISTIC CURVES 


\section{APPENDIX D}

\section{MOISTURE CHARACTERISTIC CURVES}

This appendix presents moisture-characteristiccurves for 15 samples collected from the 200 Areas reported by Last et al. (1989). The characteristic curves are based on water-retentiondata presented in Table 10. Four of these samples are from the Hanford formation in the 200-East Area. The remaining 11 samples are from 200-West Area wells: seven of these are from the Hanford formation, two are from the middle Ringold, one is from the upper Ringold unit, and one is from the Plio-Pleistocene unit. Only two of the wells sampled (299-W7-2 and 299-W10-13) are within $1000 \mathrm{ft}$ of W-5. Samples from the other wells are all from the Hanford formation; these may not be representative of conditions at W-5, but they are included here to indicate the variable nature inherent to the Hanford formation.

D.1 
Well \#: 299-W7-2

Depth: $65 \mathrm{ft}$

Stratigraphic Unit: Plio-Pleistocene Unit

Lithofacies: Calcic Soil

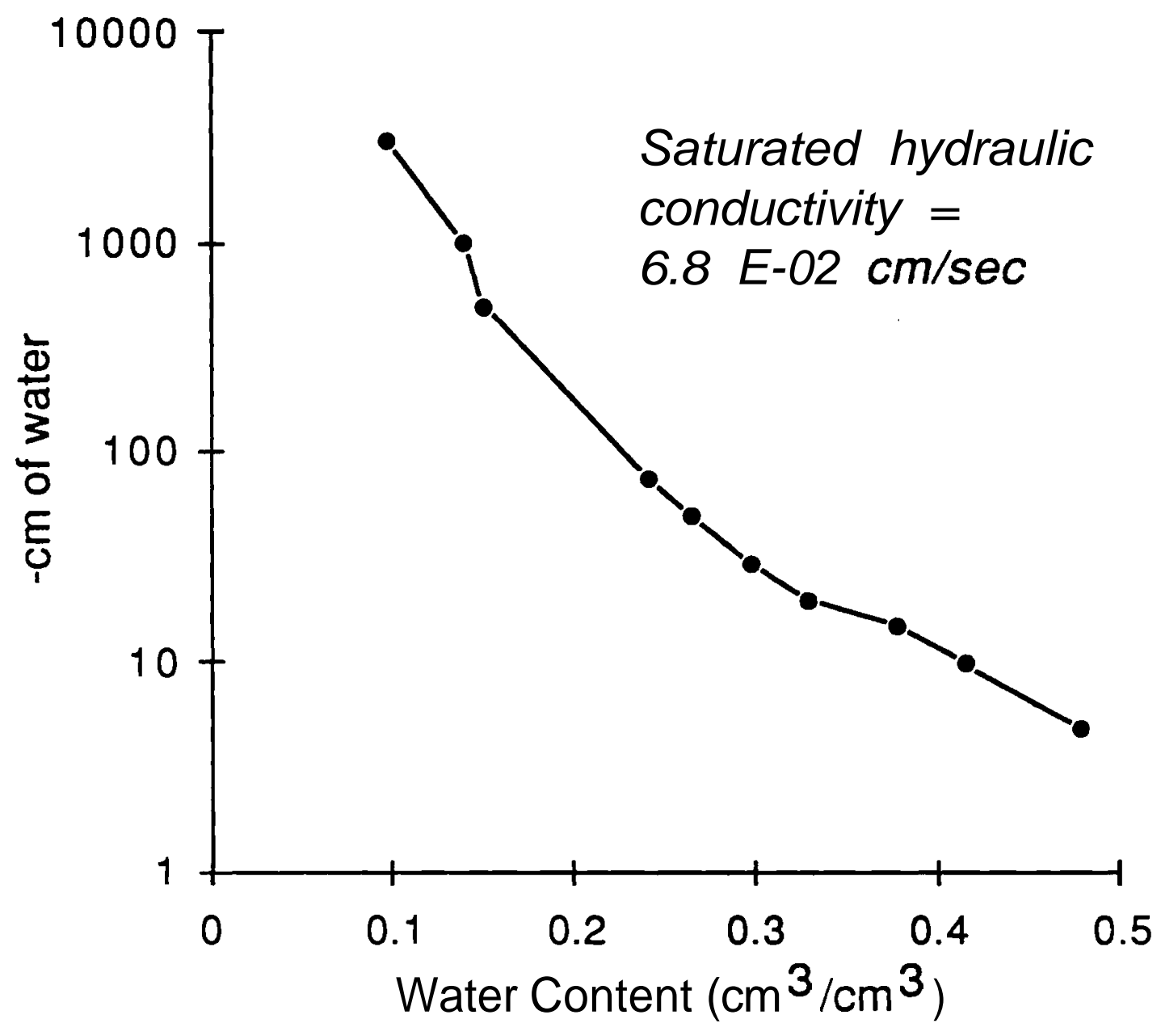


Well \#: 299-W7-2

Depth: $94-95 \mathrm{ft}$

Stratigraphic Unit: Upper Ringold

Lithofacies: ms G (Muddy Sandy Gravel)

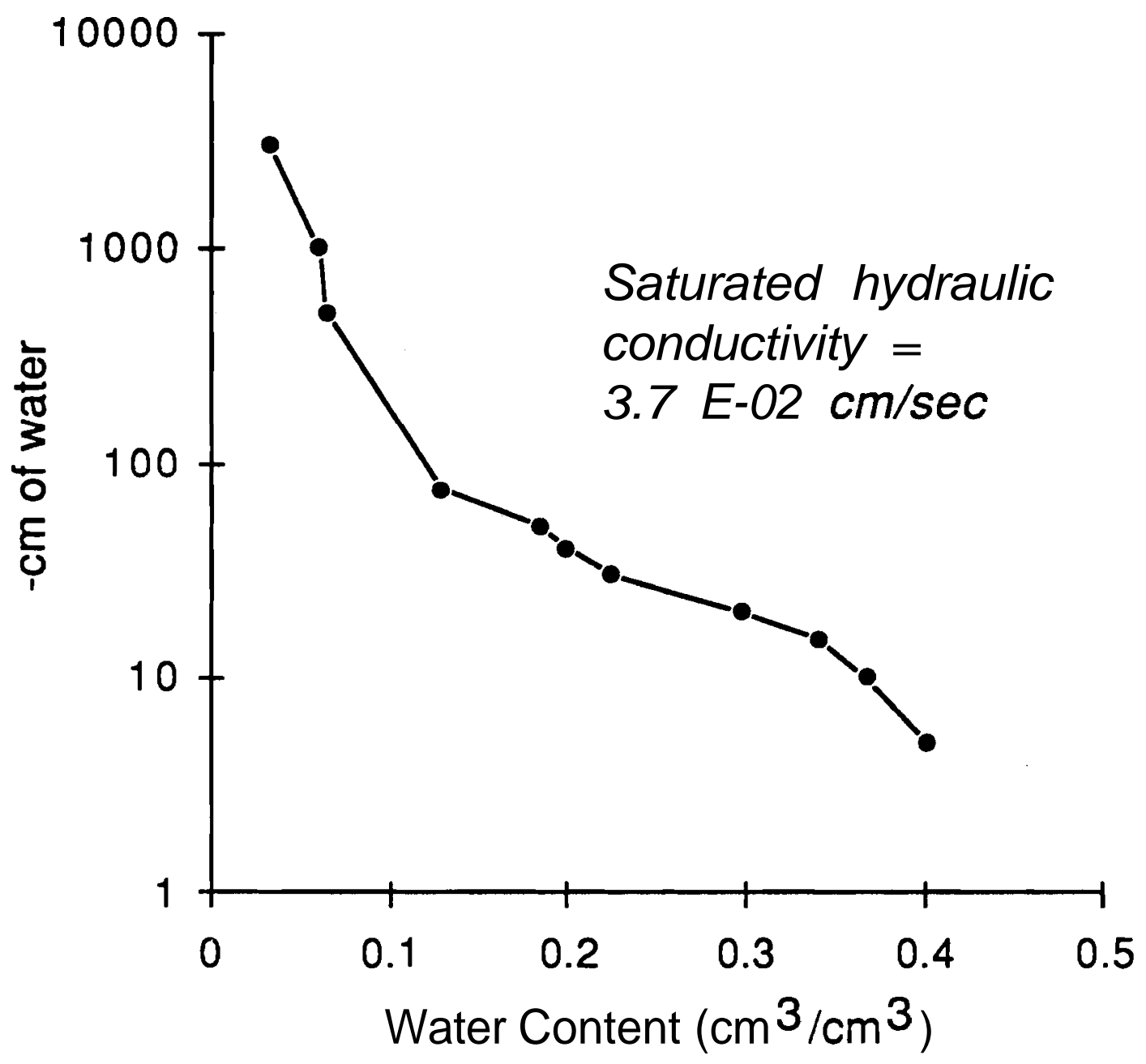


Well \#: 299-W7-2

Depth: $154-155 \mathrm{ft}$

Stratigraphic Unit: Middle Ringold

Lithofacies: ms G (Muddy Sandy Gravel)

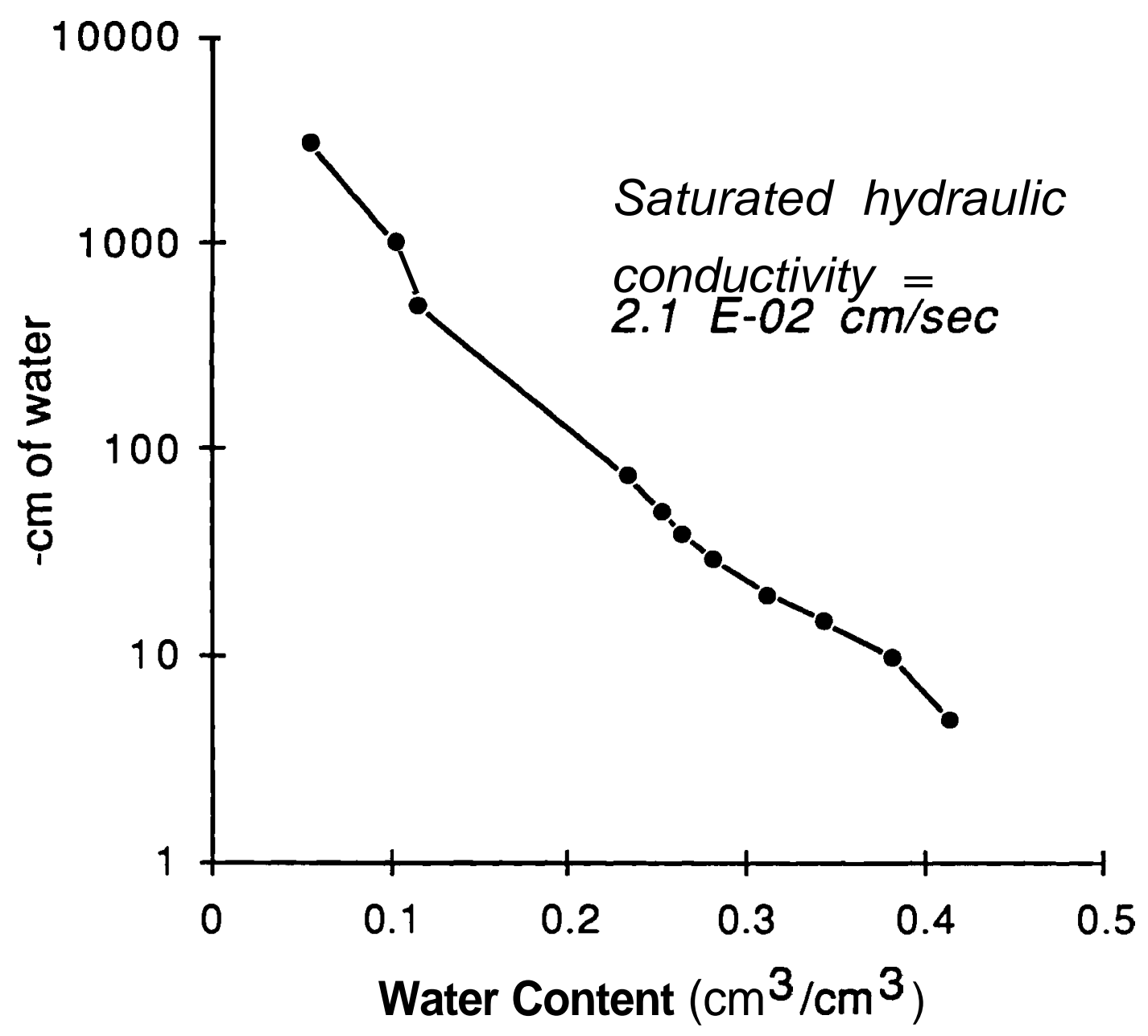


Well \#: 299-W7-2

Depth: $219-220 \mathrm{ft}$

Stratigraphic Unit: Middle Ringold

Lithofacies: ms G (Muddy Sandy Gravel)

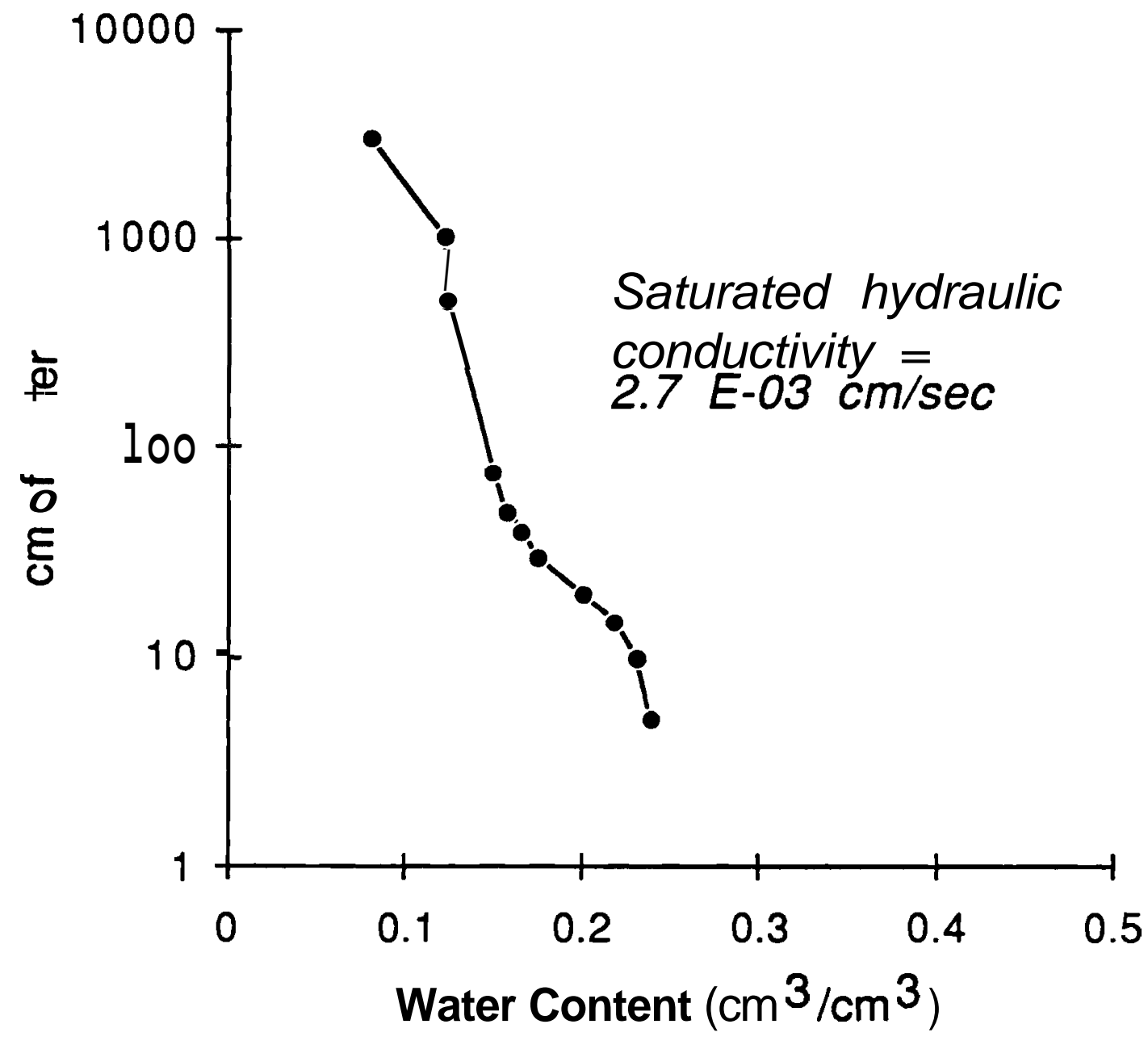


Well \#: 299-W7-5

Depth: $10 \mathrm{ft}$

Stratigraphic Unit: Hanford Formation

Lithofacies: G (Gravel)

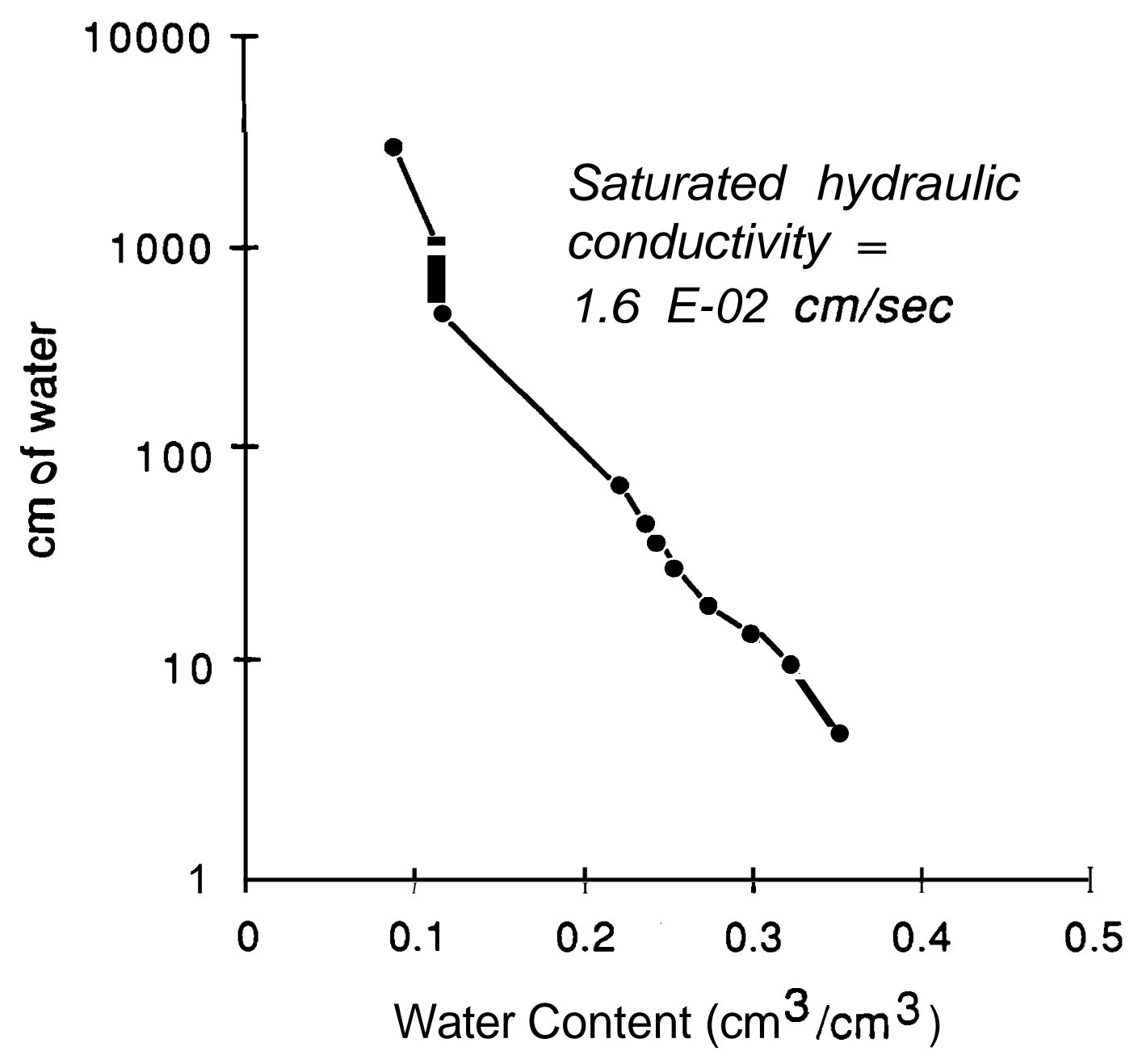


Well \#: 299-W10-13

Depth: $45 \mathrm{ft}$

Stratigraphic Unit: Hanford Formation

Lithofacies: G (Gravel)

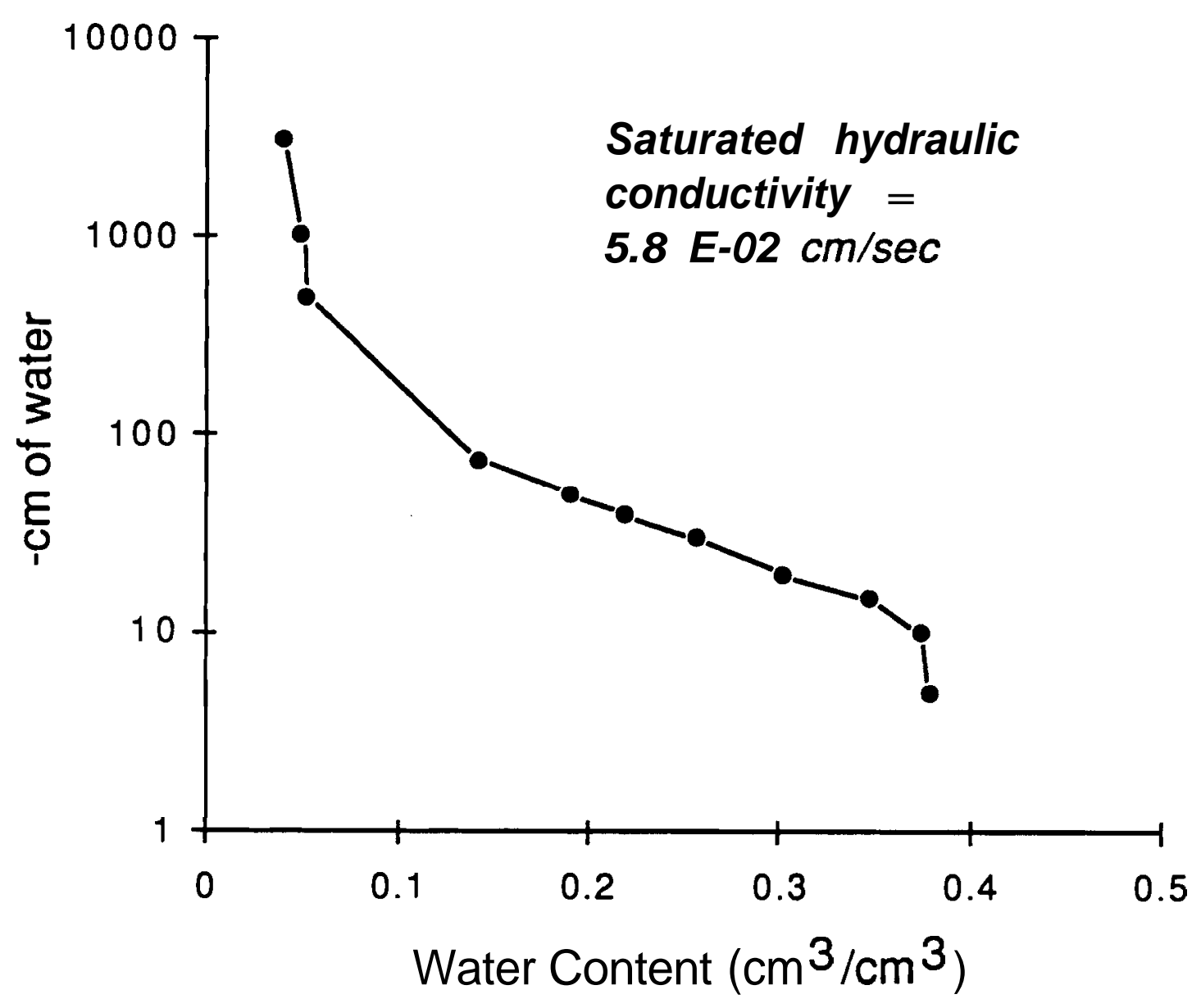


Well \#: 299-W10-13

Depth: $80 \mathrm{ft}$

Stratigraphic Unit: Hanford Formation

Lithofacies: ms G (Muddy Sandy Gravel)

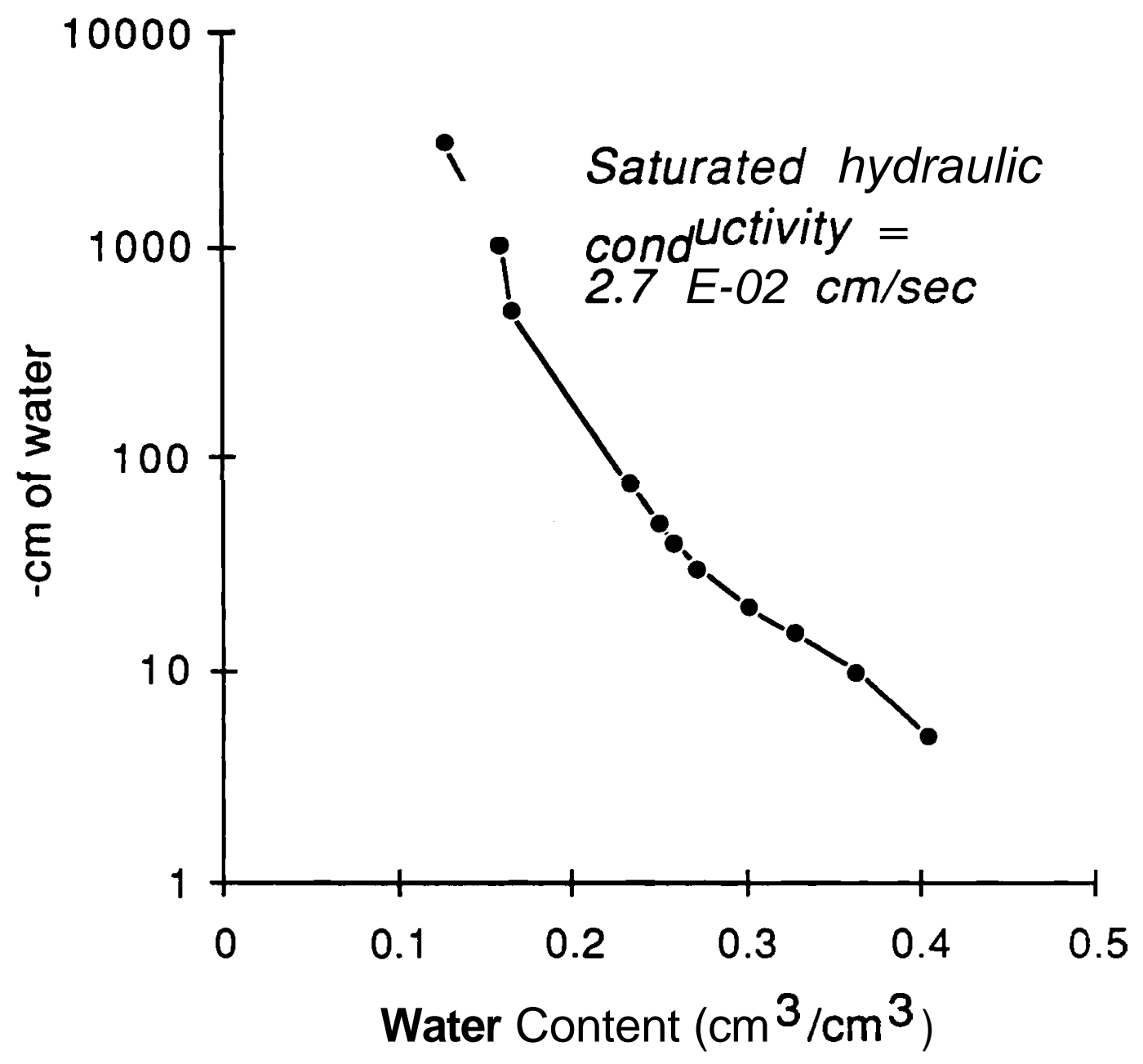

D.8 
Well \#: 299-W15-16

Depth: $40 \mathrm{ft}$

Stratigraphic Unit: Hanford Formation

Lithofacies: S (Sand)

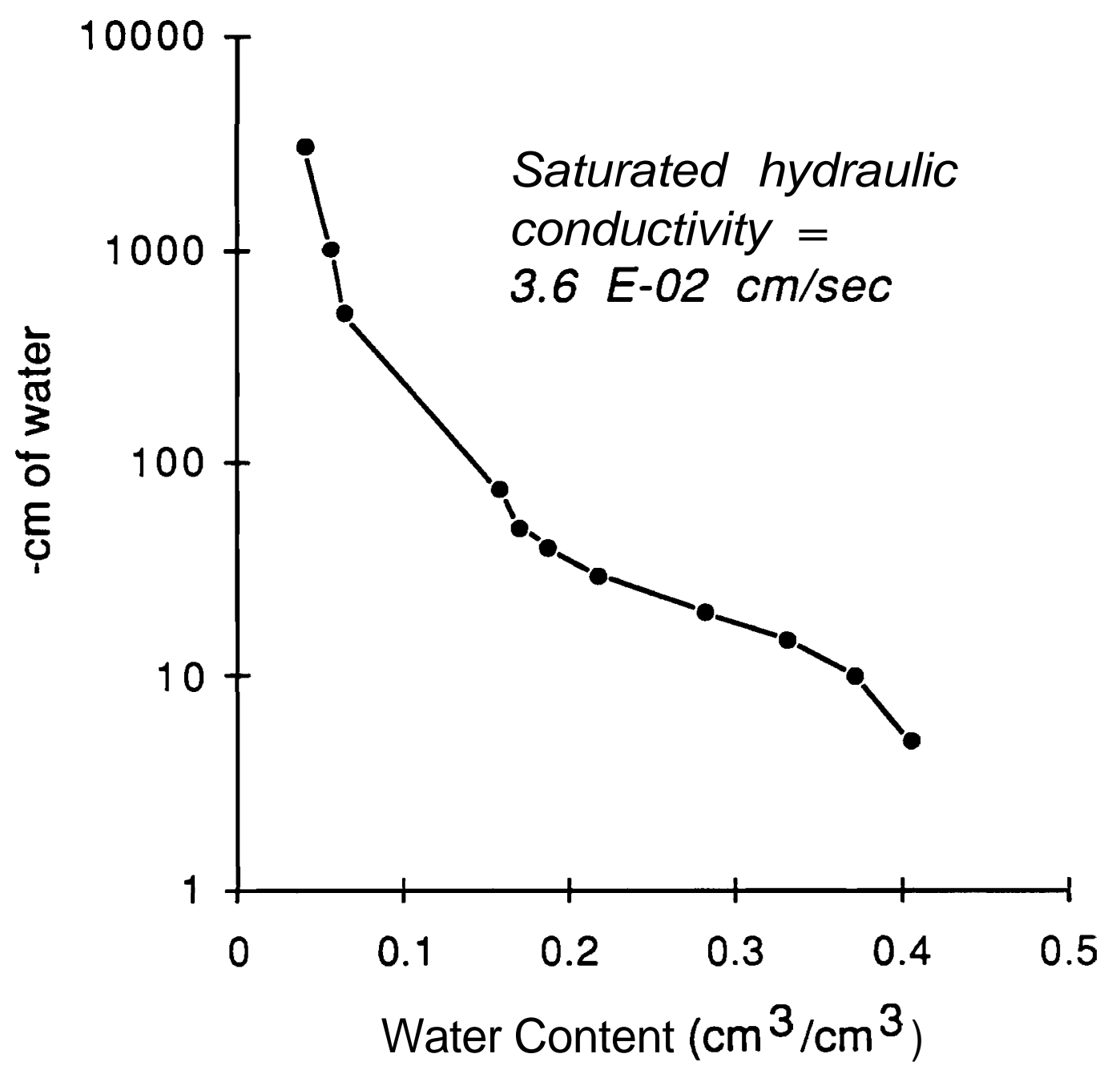


Well \#: 299-W15-16

Depth: $110 \mathrm{ft}$

Stratigraphic Unit: Hanford Formation

Lithofacies: ms G (Muddy Sandy Gravel)

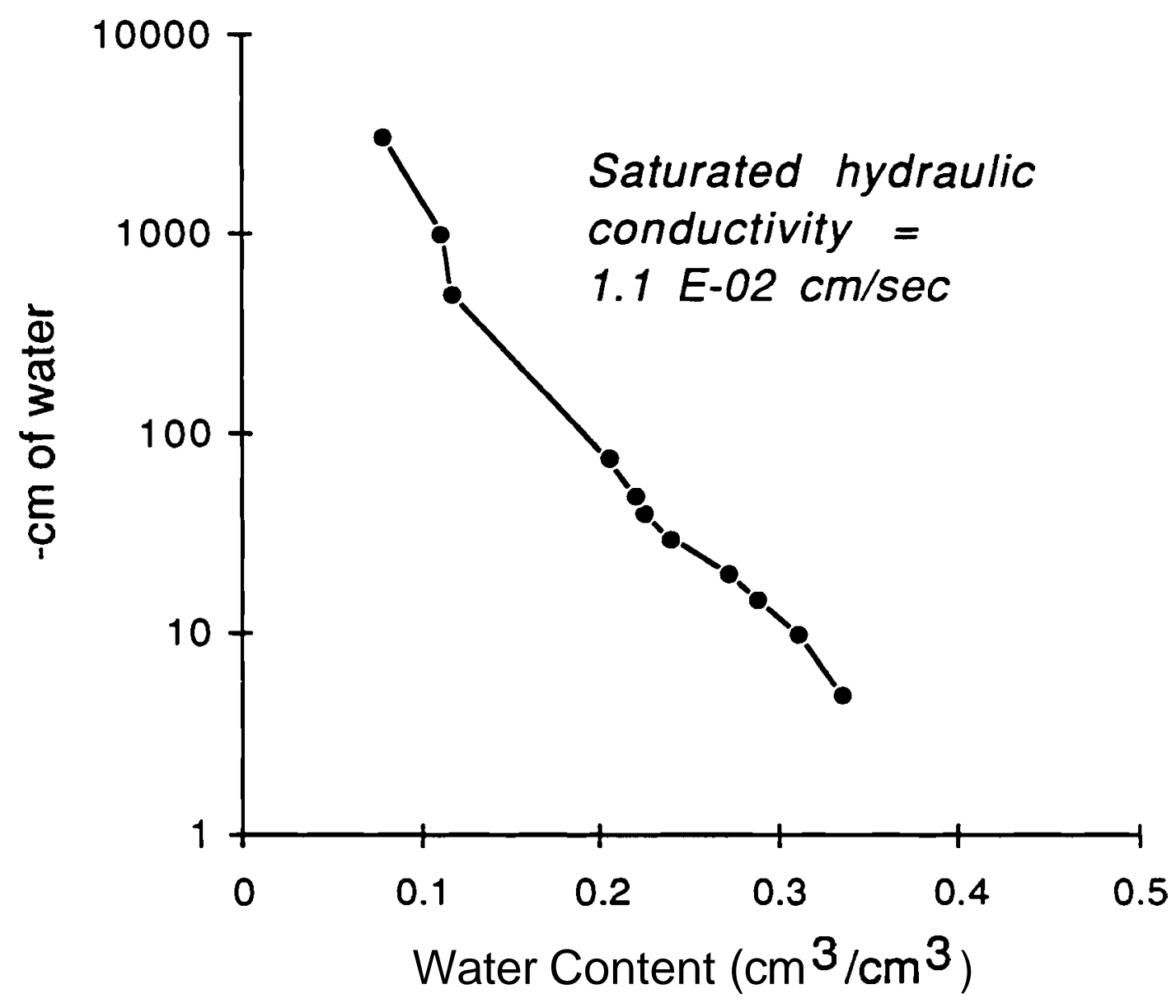


Well \#: 299-W18-21

Depth: $25 \mathrm{ft}$

Stratigraphic Unit: Hanford Formation

Lithofacies: (m)g S (Slightly Muddy Gravelly Sand)

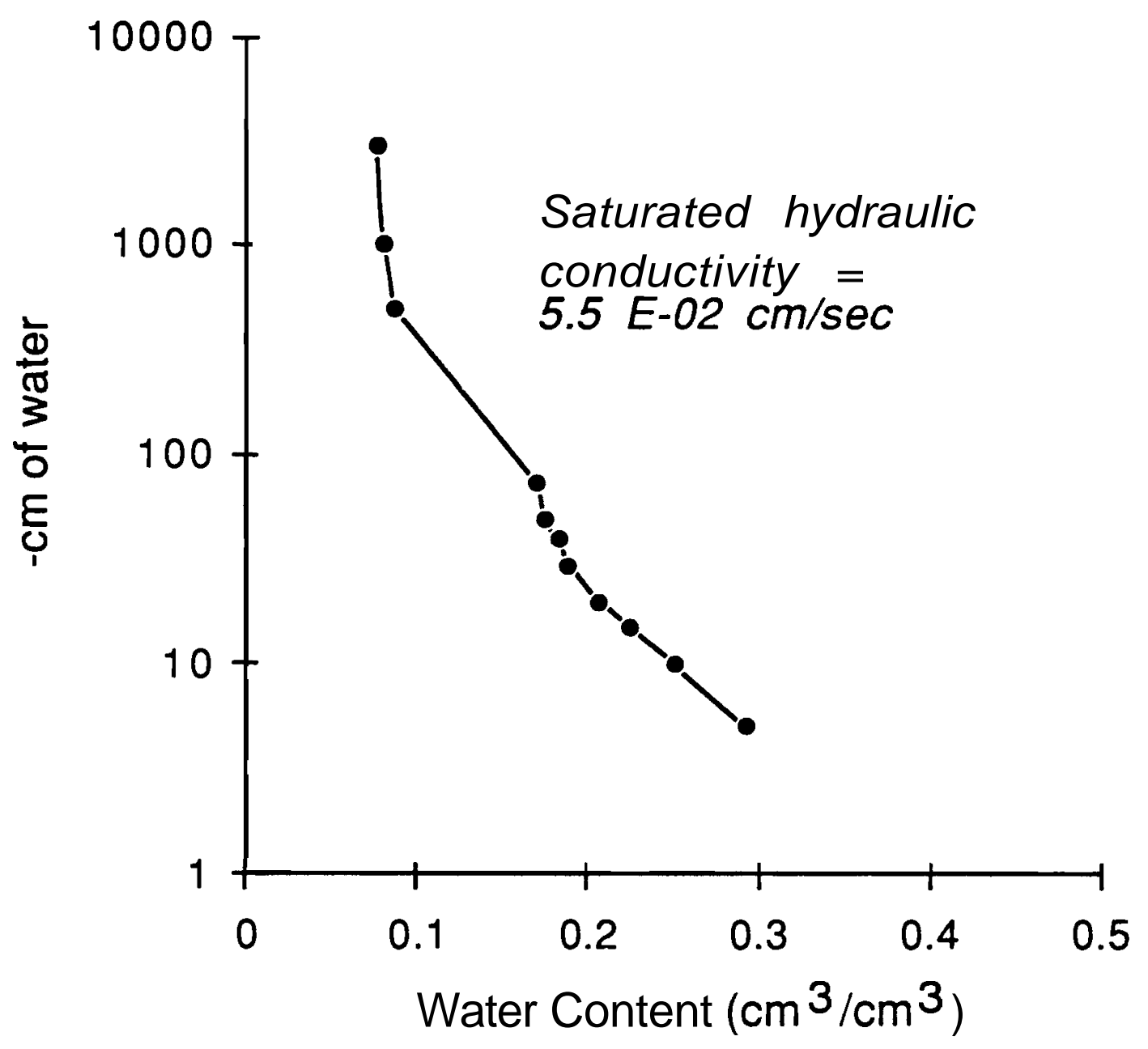


Well \#: 299-W18-21

Depth: $40 \mathrm{ft}$

Stratigraphic Unit: Hanford Formation

Lithofacies: (g) S (Slightly Gravelly Sand)

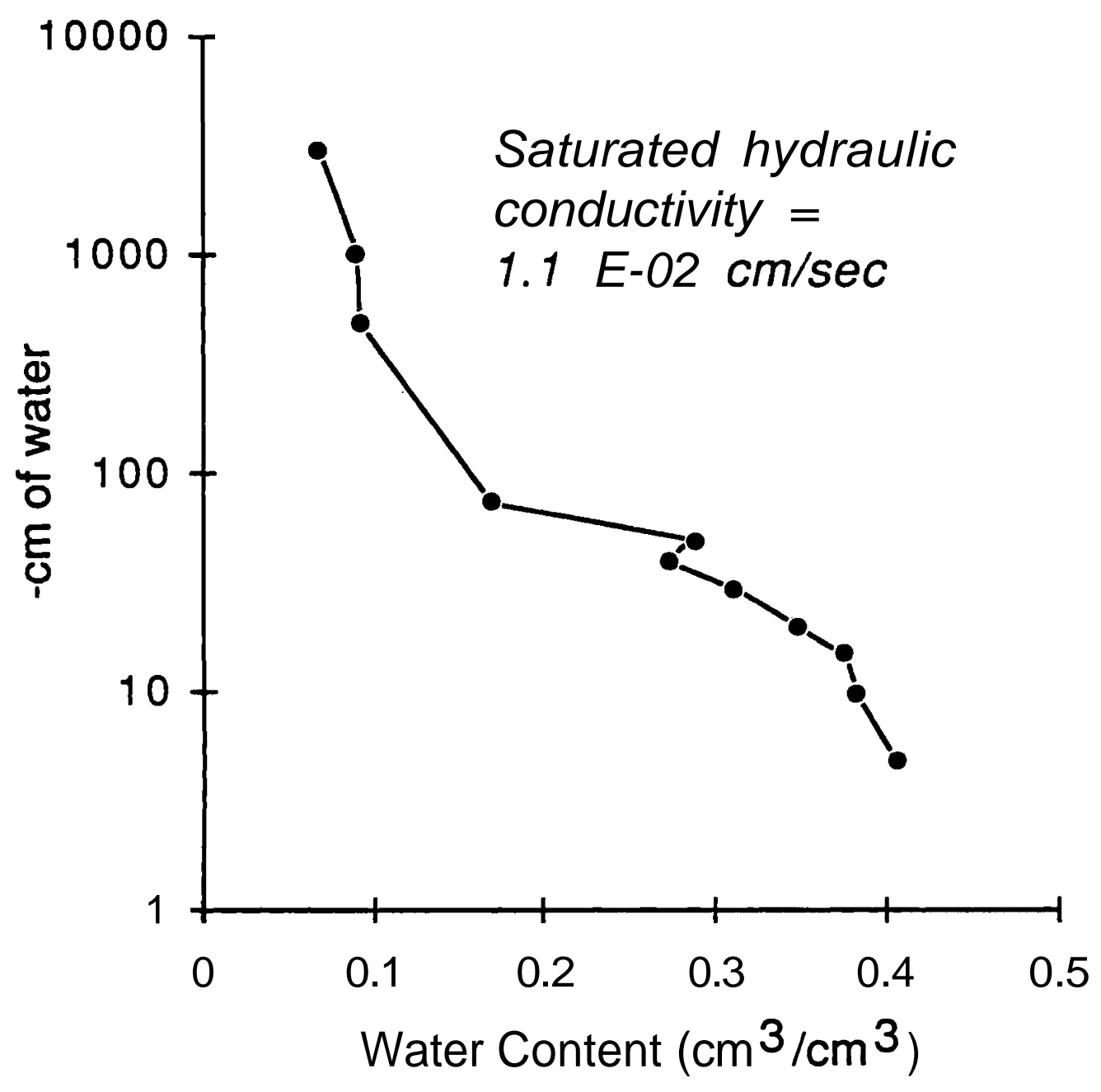


Well \#: 299-E28-26

Depth: $129-130 \mathrm{ft}$

Stratigraphic Unit: Hanford Formation

Lithofacies: (g) S (Slightly Gravelly Sand)

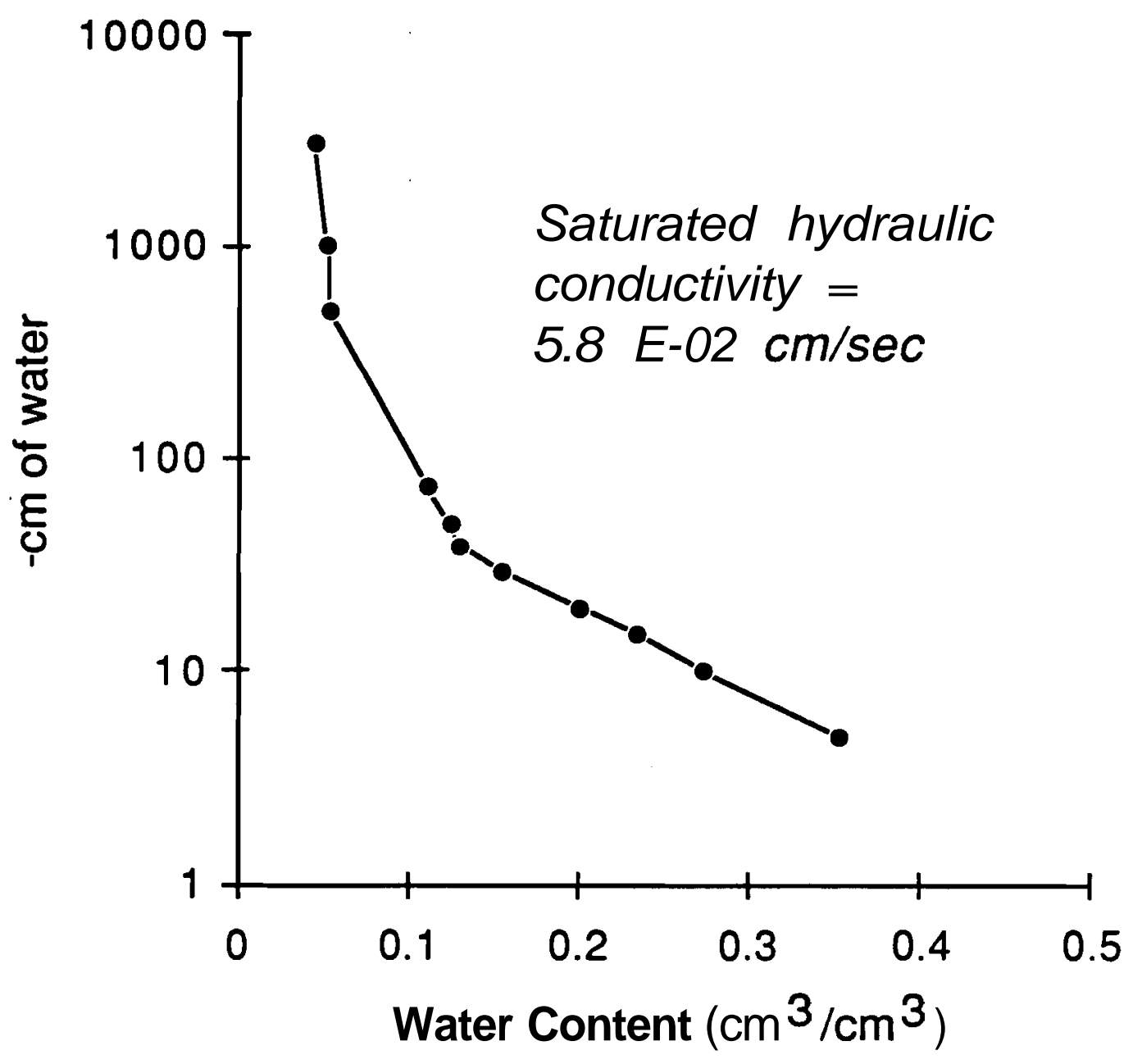


Well \#: 299-E28-26

Depth: $230 \mathrm{ft}$

Stratigraphic Unit: Hanford Formation

Lithofacies: s G (Sandy Gravel)

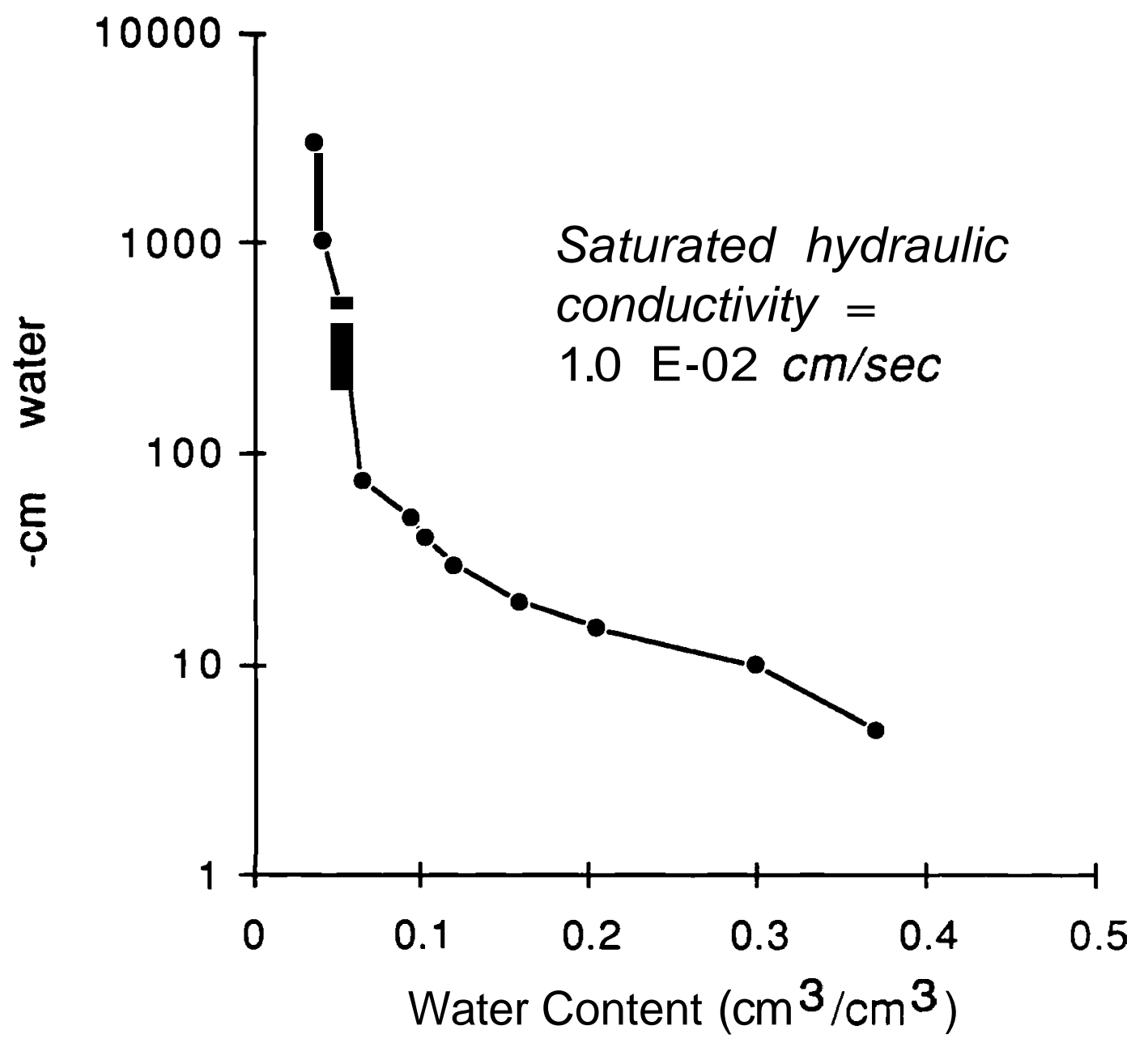


Well \#: 299-E33-30

Depth: $139-140 \mathrm{ft}$

Stratigraphic Unit: Hanford Formation

Lithofacies: S (Sand)

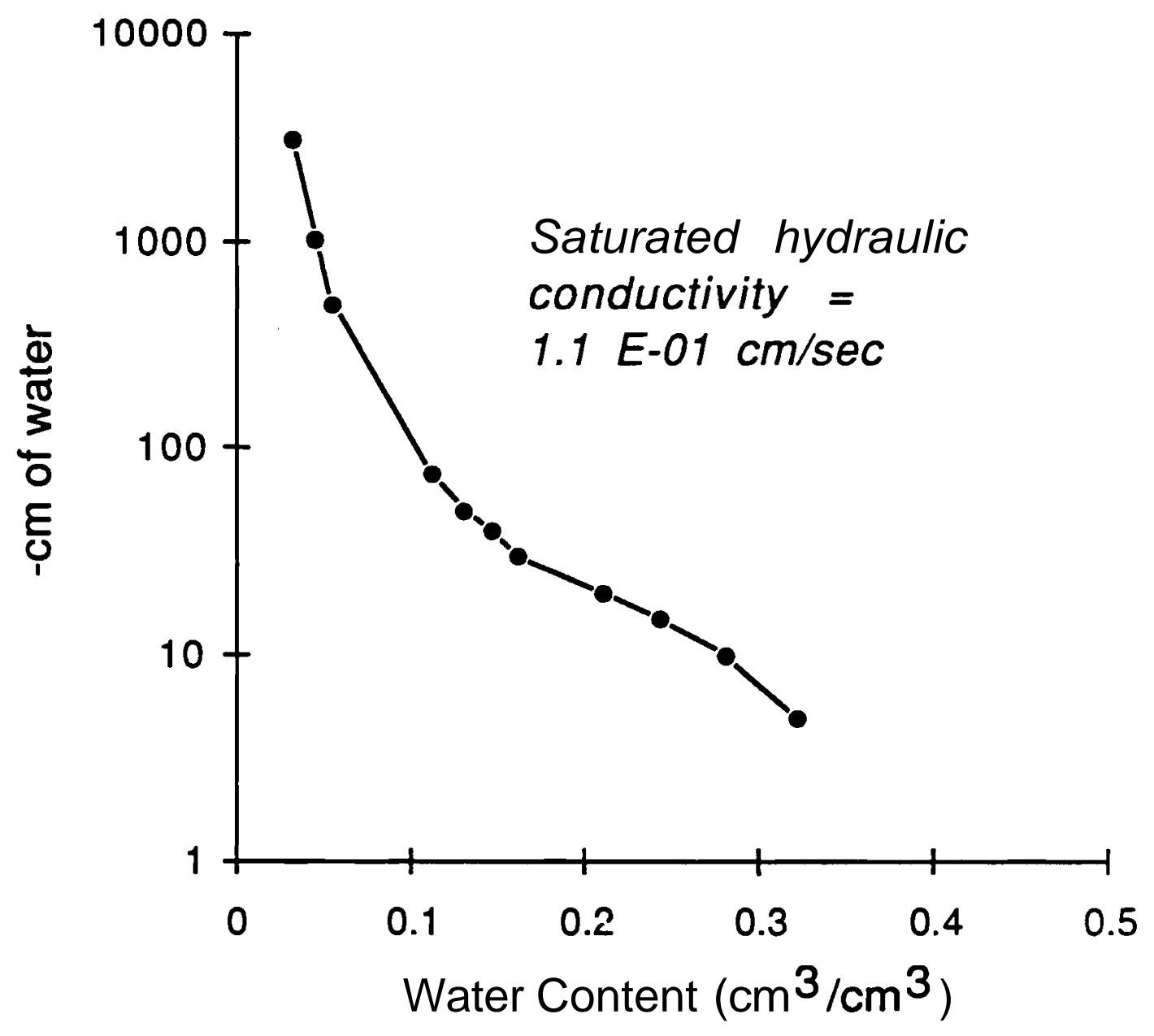


Well \#: 299-E33-30

Depth: $239-240 \mathrm{ft}$

Stratigraphic Unit: Hanford Formation

Lithofacies: ms G (Muddy Sandy Gravel)

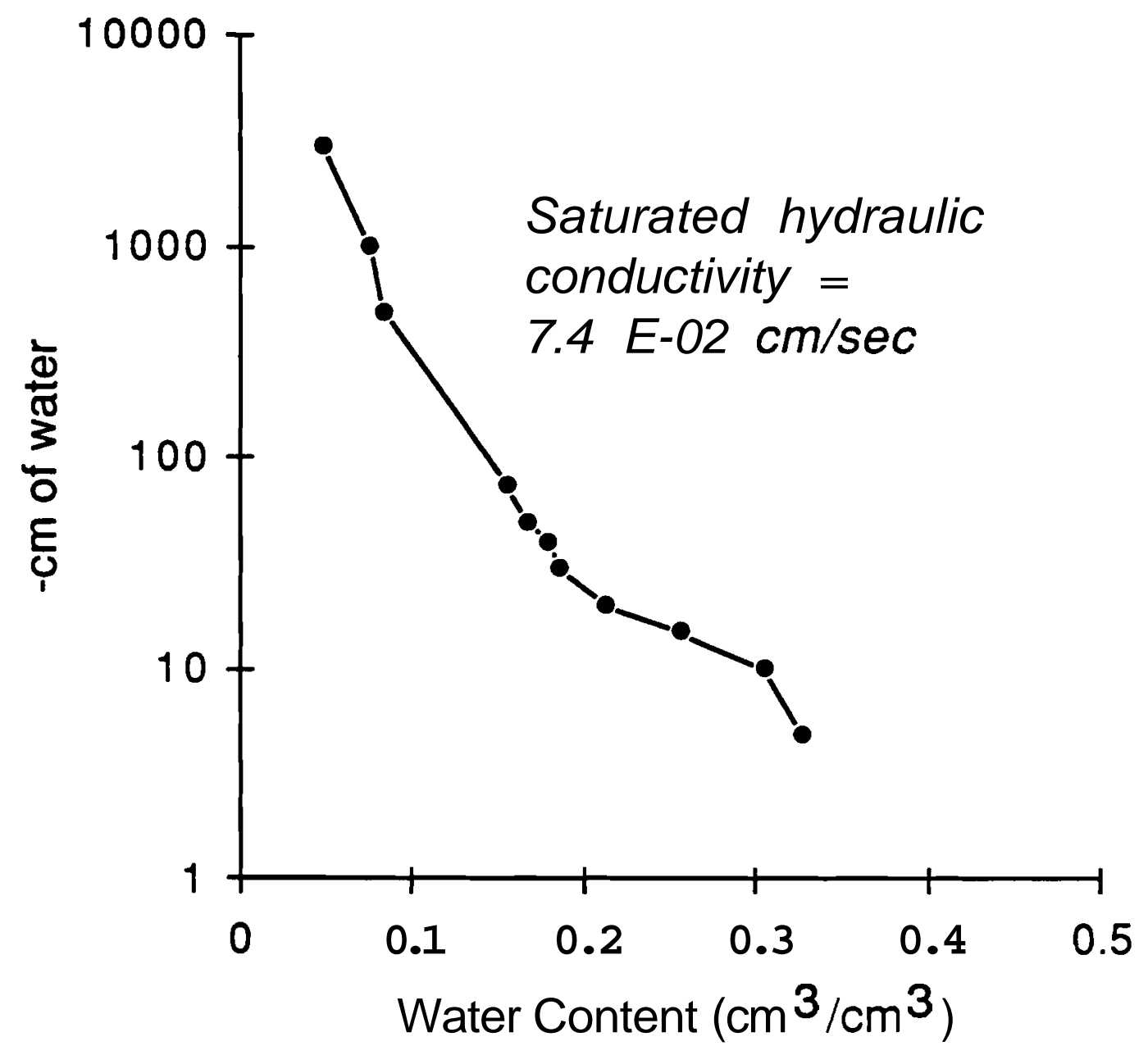




\section{DISTRIBUTION}

No. of

Copies

OFFSITE

2 DOE Office of Scientific and Technical Information

R. R. Seitz

Idaho National Engineering Laboratory P.O. Box 1625

Idaho Falls, Idaho 83415

\section{QNSITE}

DOE Richland Operations Office

D. M. Wanek

8 Westinahouse Hanford Company

J. Cammann

K. R. Fecht

R. Khaleel

R. B. Mercer

J. F. Relyea

R. J. Roberts

J. Scott

M. I. Wood

\section{Pacific Northwest Laboratory}

R. L Aaberg

J. E. Amonette
No. of

Copies

M. P. Bergeron

B. N. Bjornstad (5)

J. V. Borghese

M. A Chamness

P. J. Doctor

L. A. Doremus

J. W. Falco

M. J. Fayer

R. E. Gephart

L. K. Grove

J. M. Hales

P. C. Hays

E. J. Jensen

W. E. Kennedy

G. V. Last

P. E. Long

S. P. Luttrell

V. L. McGhan

W. T. Pennell

L. E. Rogers

R. Schalla

J. A. Schramke

P. A. Scott

R. J. Serne

R. L. Skaggs

R. M. Smith

F. A. Spane

Publishing Coordination

Technical Report Files (5) 